\title{
SELF-HEALING BACTERIAL CEMENTITIOUS CONCRETE COMPOSITES: DEVELOPMENT AND PERFORMANCE EVALUATION
}

By

\section{SINI BHASKAR}

M.A.Sc., Ryerson University, Toronto, Canada, 2009

M. Tech., Cochin University of Science and Technology, India, 2005

\author{
A Dissertation \\ presented to Ryerson University \\ in partial fulfillment of the \\ requirements for the Degree of \\ Doctor of Philosophy \\ in the Program of \\ Civil Engineering
}

Toronto, Ontario, Canada, 2016

(C) Sini Bhaskar 2016 


\begin{abstract}
AUTHOR'S DECLARATION
AUTHOR'S DECLARATION FOR ELECTRONIC SUBMISSION OF A DISSERTATION

I hereby declare that I am the sole author of this dissertation. This is a true copy of the dissertation, including any required final revisions, as accepted by my examiners.

I authorize Ryerson University to lend this dissertation to other institutions or individuals for the purpose of scholarly research

I further authorize Ryerson University to reproduce this dissertation by photocopying or by other means, in total or in part, at the request of other institutions or individuals for the purpose of scholarly research.
\end{abstract}

I understand that my dissertation may be made electronically available to the public. 


\title{
SELF-HEALING BACTERIAL CEMENTITIOUS CONCRETE COMPOSITES: DEVELOPMENT AND PERFORMANCE EVALUATION
}

Sini Bhaskar, Doctor of philosophy, 2016

Department of Civil Engineering, Ryerson University

\begin{abstract}
The principal objective of the research is to contribute towards attaining the goal of developing self-healing cementitious concrete composites by incorporating bacteria as healing agent. Since the root cause of the majority of structural failure is attributed to concrete cracking, there is a compelling economic incentive to develop a concrete that can treat and repair the damage all by itself. Even though some research has been carried out in this area, a major breakthrough in identifying the types of bacteria, modes to protect this bacteria from high $\mathrm{pH}$ concrete environment and nutrients for effective healing are yet to materialise. For the present study, three different bacteria namely, Sporosarcina ureae, Sporosarcina pasteurii and Bacillus subtilis subsp. spizizenii and two protective vehicles such as zeolite and pumice were selected to determine the best combination among them for self-healing. Normal and fibre reinforced mortar and engineered cementitious composite (ECC) specimens were employed for the study. In order to develop selfhealing bacterial concrete based materials, it is crucial to understand whether the introduction of mineral producing bacteria and nutrients adversely affect the properties. Thus, various concentrations of bacteria and nutrients were tested to determine the best possible combinations without sacrificing concrete properies. Evaluation of healing effect was determined by comparing compressive strength, sorptivity and rapid chloride permeability (RCPT), four point bending and ultrasonic pulse velocity (UPV) properties of sound and damaged specimens at different ages. Healing associated with crack closure was visualised and analysed using scanning electron
\end{abstract}


microscopy (SEM), Energy Dispersive Spectrum Energy (EDS) and X-ray diffraction (XRD) studies. Finally, an attempt was made to employ statistical models for parameter optimization of self-healing characteristics in terms of compressive strength, sorptivity, RCPT and UPV by design and analysis of experiments. Evaluation of results to determine self-healing efficiency indicated that a significant amount of self-healing was achieved by all three selected bacteria, out of which Sporosarcina pasteurii and Bacillus subtilis subsp. spizizenii found to be promising choices. Both zeolite and pumice turned out to be effective protective vehicles. Statistical modelling of the experiment proved to be the ideal choice for modelling self-healing characteristics. 


\section{Acknowledgments}

Completing the $\mathrm{PhD}$ and writing this thesis was an amazing journey that would not have been possible without the support and encouragement of many outstanding people. In pursuit of this academic endeavor, I have been fortunate because inspiration, guidance, direction, cooperation, love and care - all came in my way in abundance and it seems like an impossible task for me to express the gratitude in words.

At the outset, I wish to express my deep sense of gratitude towards my supervisor Dr. Mohamed Lachemi for providing inspiration, motivation and encouragement for the present work. I am highly indebted for his support and thought provoking suggestions during needful circumstances.

I would like to express my appreciation to my supervisor Dr. Khandaker M. Anwar Hossain for his advice during my doctoral research endeavor for the past four years. As my supervisor, he has constantly forced me to remain focused on achieving my goal. His observations and comments helped me to establish the overall direction of the research and to move forward with investigation in depth. His relentless support immensely helped me in the development of this thesis and enhanced my scientific writing skills. My sincerest thanks to him for making me a better person, guiding me and improving trivial mistakes.

I also express my gratitude to my third supervisor Dr. Gideon Wolfaardt for accepting me as his student and providing me an opportunity to work in the microbiology laboratory. Without his support and help this research would not be possible.

Special thanks must also be extended to Dr. William Yeung and Evan Ronan who helped and taught me important microbiological laboratory techniques. Without their guidance, this endeavor would not have been fruitful. I would like to express my thanks to Dr. Otini Kroukamp who was always available with invaluable suggestions during my microbiological experiments. I cannot help but express my gratitude to my colleagues in the microbiology lab Wendy, Lindsey, Alex, Roshnak and Romeo, who gave me encouragement and kind assistance in performing the microbiological techniques which were unfamiliar to me.

Financial support for this research from Natural Sciences and Engineering Research Council (NSERC) of Canada is gratefully acknowledged. Special thanks to Dr. Hamou, Dr. Medhat 
Shehata, Dr. Martina Hausner and Dr. Aburrahmaan Lofty for being examiners of the thesis. I wish to thank all my colleagues and friends in the Civil Engineering Department at Ryerson University especially Mohamed Sherir, Dr. Hocine Siad and Ahmed Alyousef for their valuable support and help during my experimental study. I take this opportunity to express my immense thankfulness to the laboratory technicians Min Yao and Mohamad Aldardari for their all-around support. A special thanks must be extended to my lab helpers Muhammed Anwar, Rahim, Clement, Rougaya, Ahmed and Tharunbir singh who were available at any time during my experimental work.

I am deeply indebted to my family, especially my four year old daughter Amelia and my husband Cirosh who was extremely supportive during the entire study period. Their love and support provided me the energy to persevere. My heartfelt gratitude especially to my parents and sisters who always supported me during this period.

Above all, I wholeheartedly thank Almighty God for giving me the vision, power, spirit and endurance to complete this interesting research. 


\section{DEDICATION}

To

My family 


\section{TABLE OF CONTENTS}

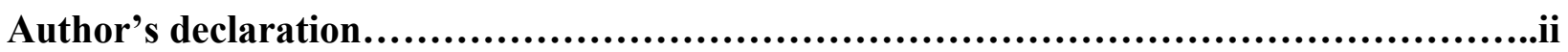

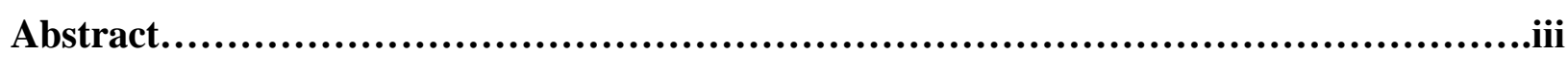

Acknowledgments................................................................................

Dedication...................................................................................

Table of Contents.........................................................................ii

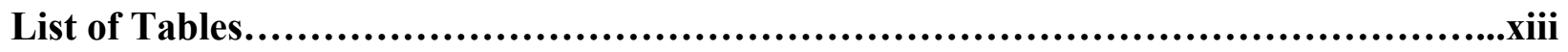

List of Figures.............................................................................

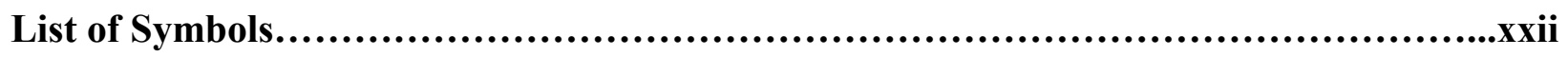

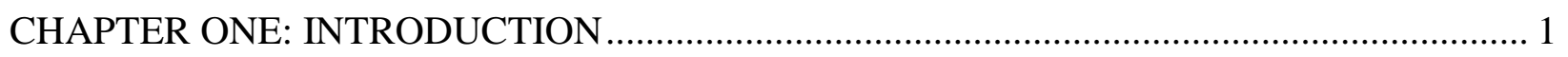

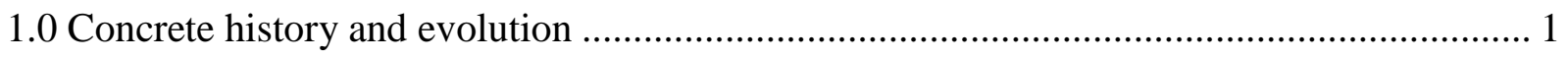

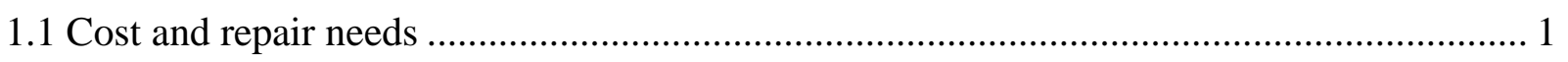

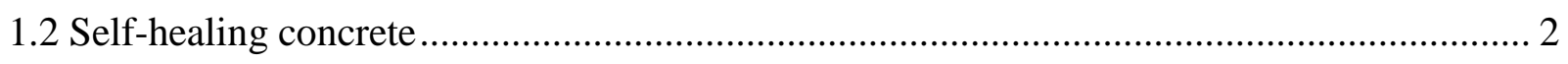

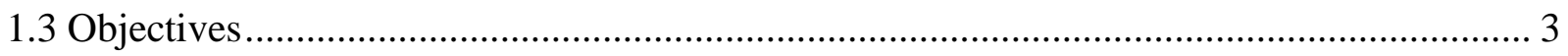

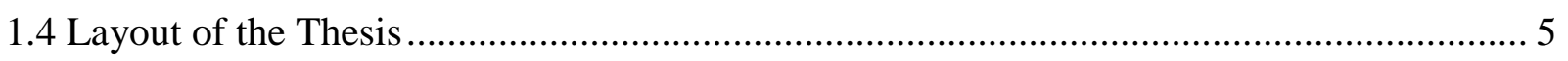

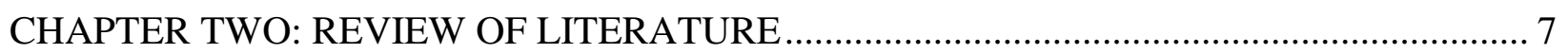

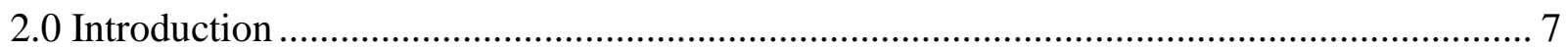

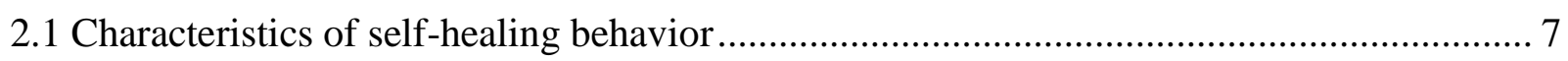

2.1.1 Basic underlying principles and mechanism of self-healing materials ......................... 8

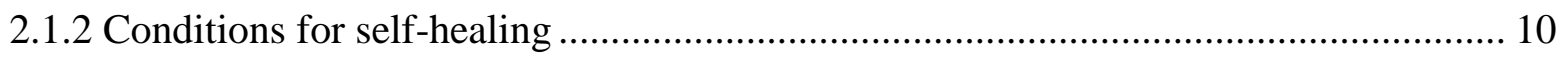

2.1.3 Classification of materials based on their self-healing behaviour............................... 10

2.2 Characteristics of engineered cementitious composite ………......................................... 11

2.3 Background on design and analysis of experiments ................................................... 13

2.3.1 Previous application of design and analysis of experiments on cementitious materials

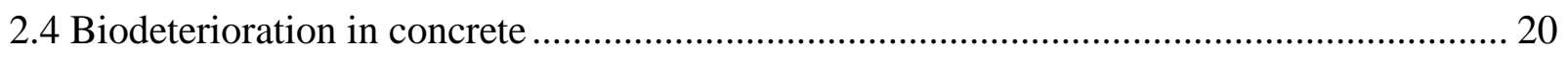

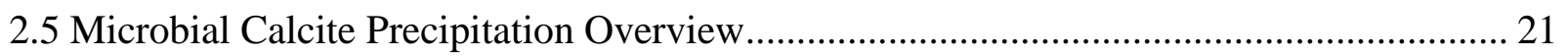


2.5.1 Biological processes of Microbial Induced Calcite Precipitation (MICP) ................. 21

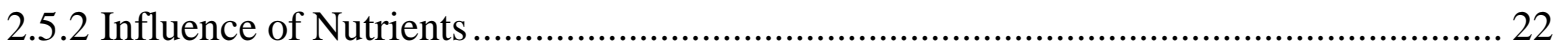

2.5.3 Influence of the type of microorganisms ............................................................. 22

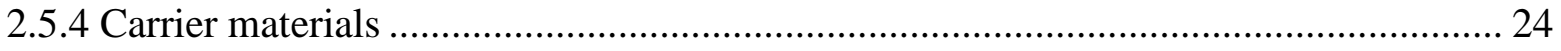

2.6 Microbial concrete in cementitious materials ........................................................... 25

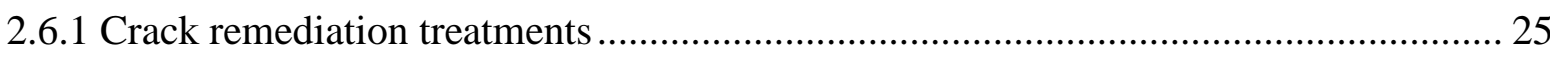

2.6.2 Improvement of concrete compressive strength .............................................. 26

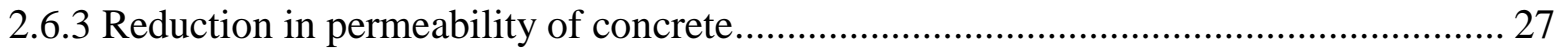

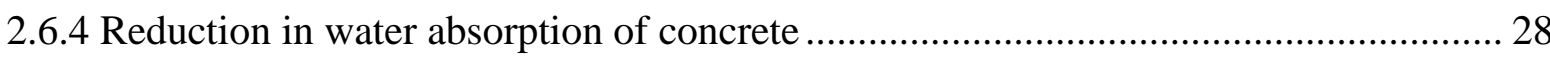

2.6.5 Improvement of mechanical performance of concrete ......................................... 29

2.6.6 Corrosion reduction in reinforced concrete ........................................................... 29

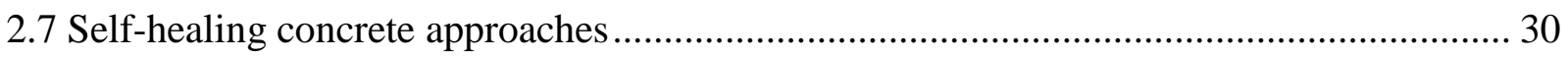

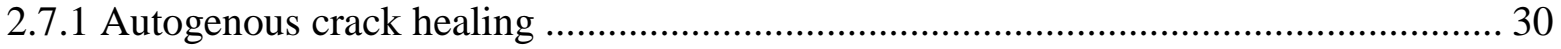

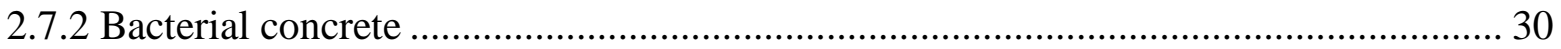

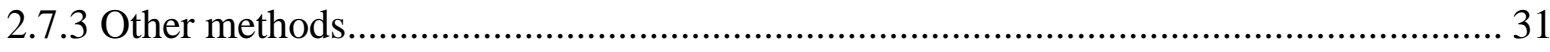

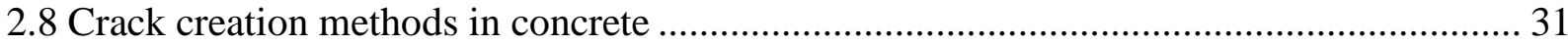

2.9 Testing and visualization aspects of self-healing ..................................................... 32

2.10 Numerical modeling of Self-healing mechanism.................................................. 33

2.11 Future considerations for studying self-healing concrete ........................................... 34

CHAPTER THREE: MATERIALS AND METHODS ….................................................... 36

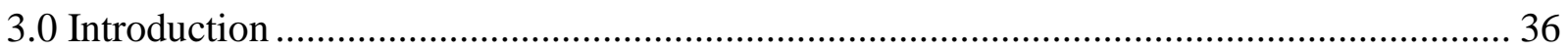

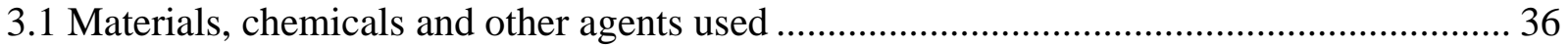

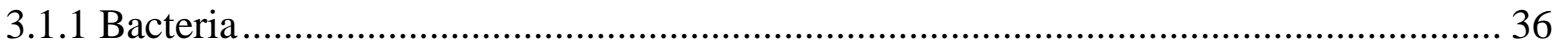

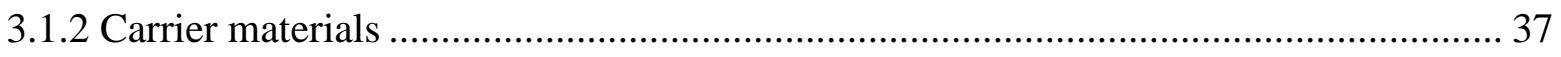

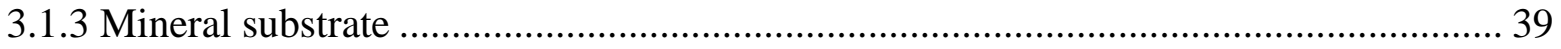

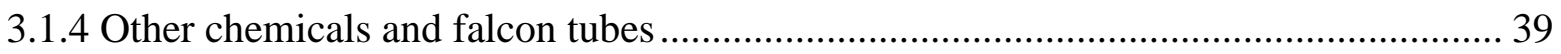

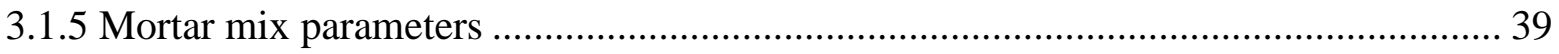

3.2 Research Phase 1: Culturing and survival testing of bacteria ........................................ 42

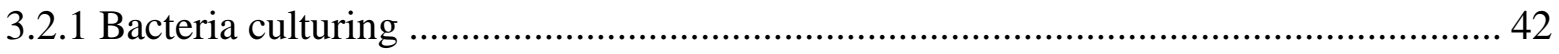

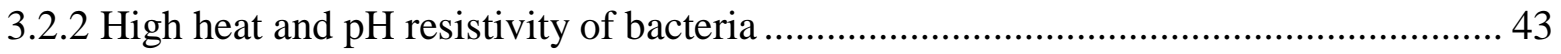

3.2.3 Mineral producing capability of bacteria.............................................................. 43 
3.3 Research Phase 2: Ureolytic activity testing of bacteria ............................................ 44

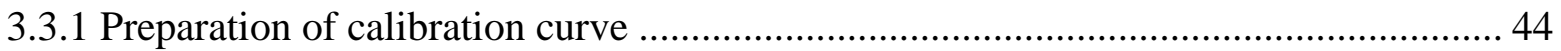

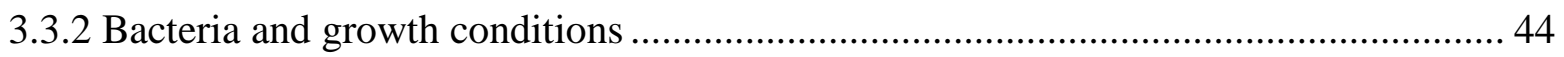

3.3.3 Activity of immobilized bacteria under neutral and high $\mathrm{pH}$ conditions ................... 44

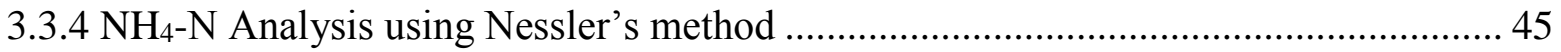

3.4 Research Phase 3: Investigation of influence of healing agent additions on self-healing based on compressive strength of mortar cubes ............................................................ 46

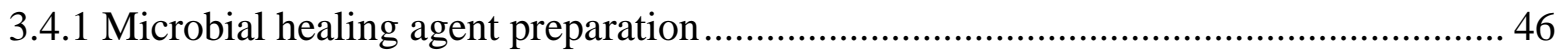

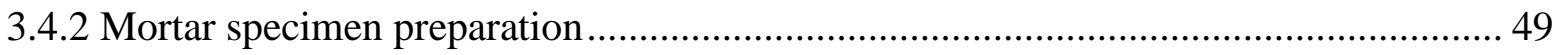

3.4.3 Effect of self-healing on compressive strength ................................................. 51

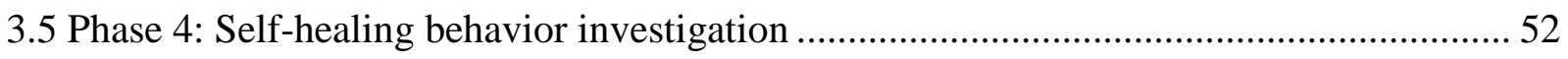

3.5.1 Four-point bending tests and UPV measurements ............................................ 52

3.5.2 Effect of self-healing on permeation properties .................................................. 55

3.5.3. Scanning Electron Microscopy (SEM), Energy Dispersive Spectrum (EDS) and X-

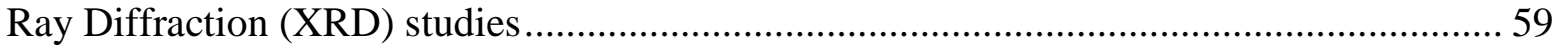

3.6 Research Phase 5: Self-healing investigation on ECC materials ................................... 61

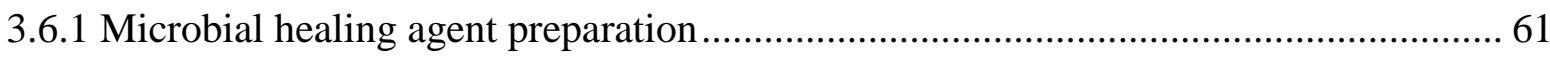

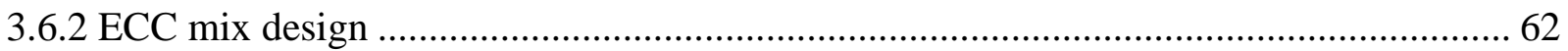

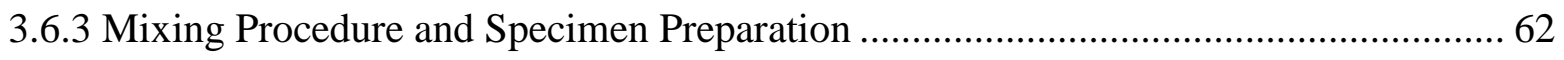

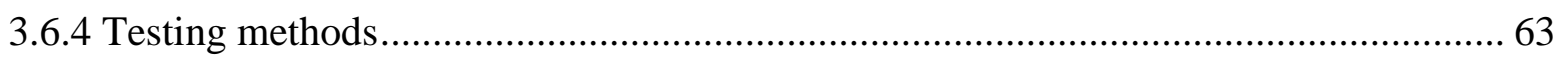

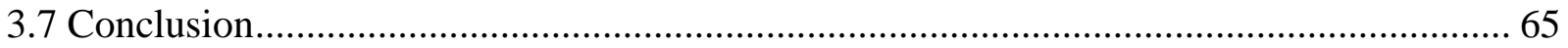

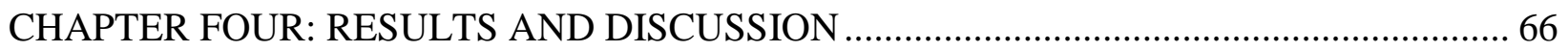

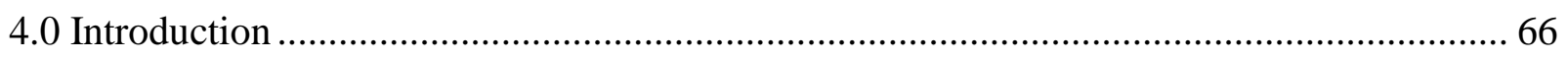

4.1 Research Phase 1: Culturing and survival testing of bacteria ......................................6 66

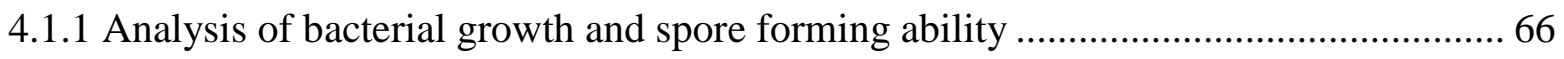

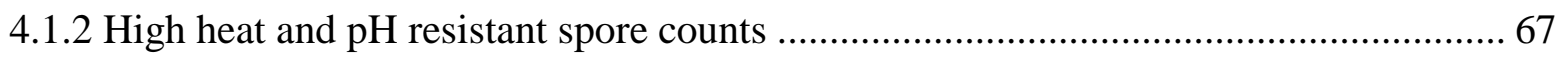

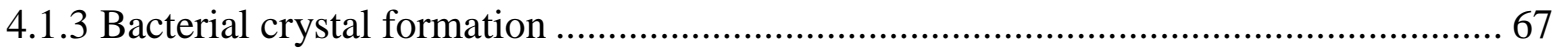

4.2 Research Phase 2: Ureolytic activity testing of bacteria ............................................. 68

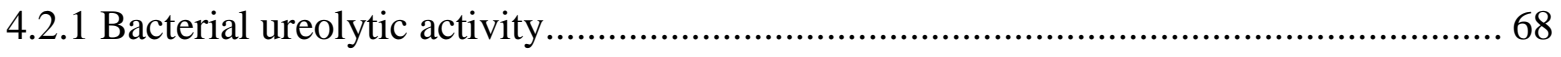

4.3 Research Phase 3: Effect of healing agents on self-healing based on compressive strength

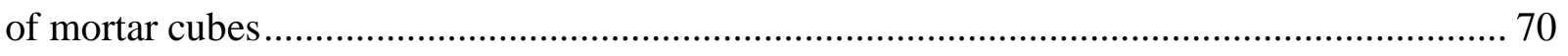

4.3.1 Effect of the addition of nutrients on compressive strength of mortar cubes ............. 70 
4.3.2 Effect of different concentration of bacteria on compressive strength of mortar cubes 71

4.3.3 Effect of bacteria on compressive strength of fibre reinforced mortar..................... 76

4.4 Research Phase 4: Self-healing behavior investigation ............................................... 78

4.4.1 Effect of bacteria induced self-healing on sorptivity and water absorption properties 79

4.4.2 Effect of bacteria induced self-healing on rapid chloride permeability ..................... 97

4.4.3 Self-healing quantification based on bending strength evolution of fiber reinforced mortar.

4.4.4. Healing quantification based on Ultrasonic Pulse Velocity (UPV) ........................ 107

4.4.5 Photographic images to visualise self-healing.................................................. 109

4.4.6 SEM and EDS studies for self-healing characterization ..................................... 110

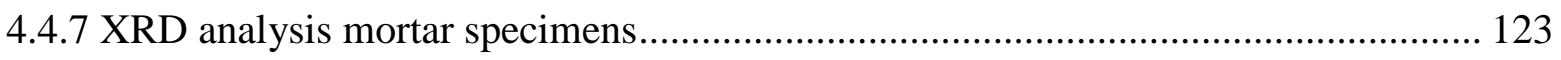

4.5 Research Phase 5: Self-healing efficiency of bacteria based ECC ................................ 129

4.5.1 Self-healing in terms of compressive strength recovery of ECC mix cubes ............. 129

4.5.2 Self-healing in terms of flexural strength recovery of ECC mix............................ 130

4.5.3 Self-healing measurements of ECC using Ultrasonic Pulse Velocity (UPV) ........... 137

4.5.4 Visualisation of crack healing of ECC materials................................................ 138

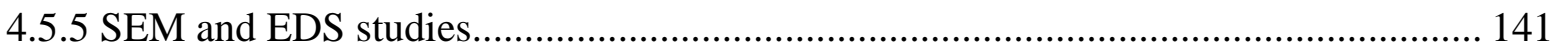

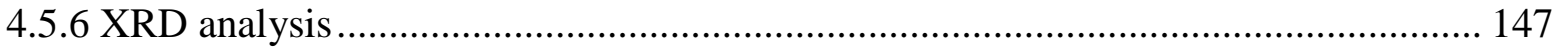

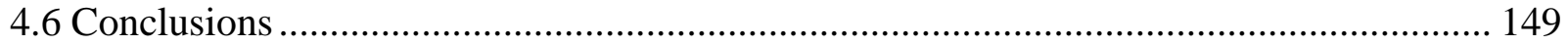

CHAPTER FIVE: MATHEMATICAL MODELING OF SELF-HEALING PROPERTIES ... 150

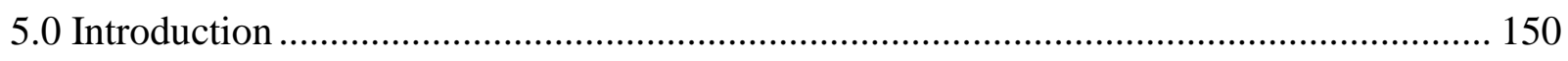

5.1 Modeling of compressive strength of normal mortar with different bacterial concentration

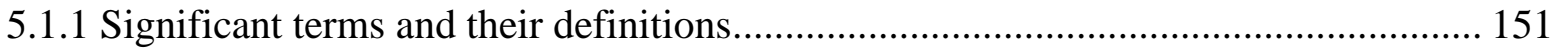

5.1.2 Compressive strength based on full factorial design ........................................ 153

5.1.3 Regression model for compressive strength ...................................................... 158

5.1.4 Validation of the proposed statistical model .................................................. 158

5.2 Modeling self-healing behavior of bacteria incorporated fiber reinforced mortar........... 161

5.2.1 Full Factorial Experimental Design................................................................. 162

5.2.2 Statistical Analysis results when the response is RCP ........................................ 163

5.2.3 Statistical Analysis results when the response is mean primary sorptivity ............... 169 
5.2.4 Statistical Analysis results when the response is mean UPV ............................... 174

5.2.5 Statistical Analysis results when the response is mean compressive strength .......... 178

5.2.6 Validation of the proposed model ...................................................................... 184

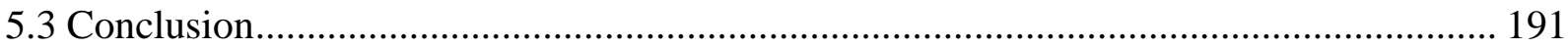

CHAPTER SIX: CONCLUSIONS AND RECOMMENDATIONS ................................... 193

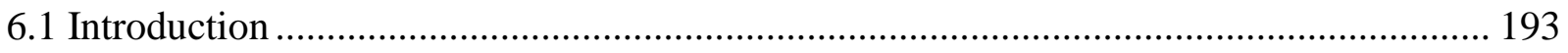

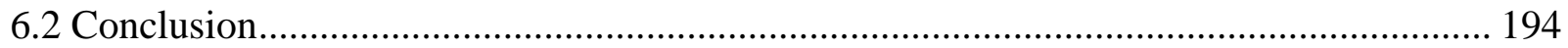

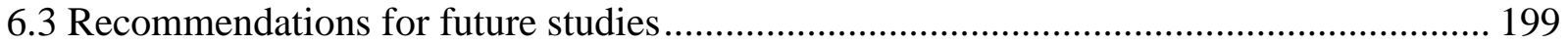

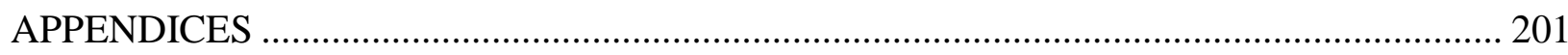

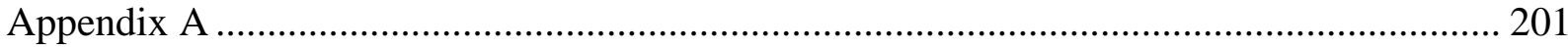

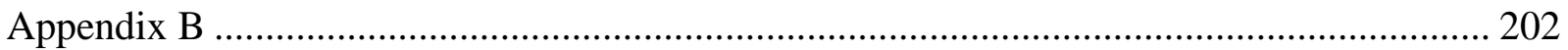

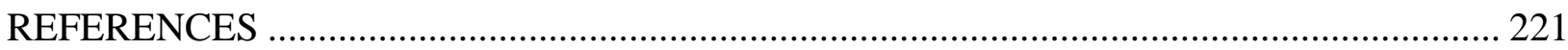




\section{LIST OF TABLES}

Table 3.1: Physical and chemical properties of zeolite ......................................

Table 3.2: Physical and chemical properties of cement and Fly ash .........................40

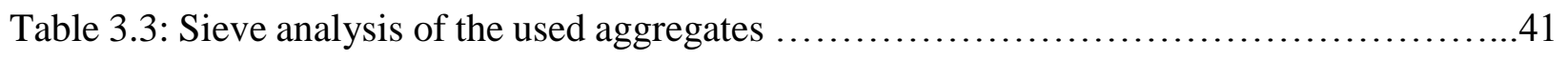

Table 3.4: Characteristics of HRWRA ........................................... 41

Table 3.5: Mix proportions of mortar cubes in terms of cement weight $\ldots \ldots \ldots \ldots \ldots \ldots \ldots \ldots \ldots . .52$

Table 3.6: Mix proportions of FR mortar in terms of cement weight ........................53

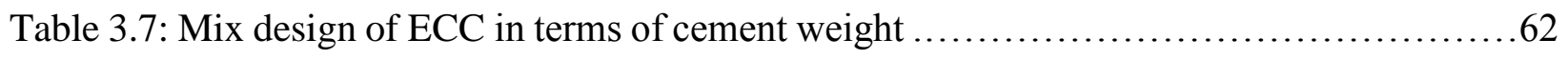

Table 4.1: Compressive strength of mortar cubes with different concentration of calcium lactate

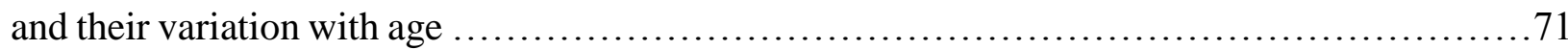

Table 4.2: Compressive strength of mortar cubes with different concentration of S. pasteurii and

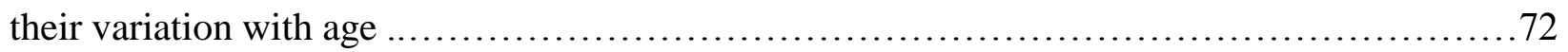

Table 4.3: Compressive strength of mortar cubes with different concentration of B. subtilis and

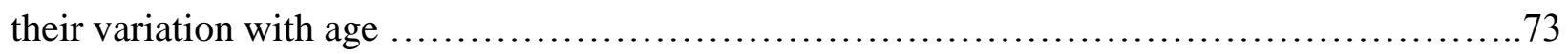

Table 4.4: Compressive strength of mortar cubes with different concentration of $S$. ureae and their

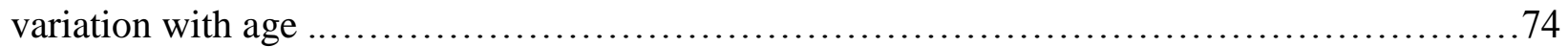

Table 4.5: Primary sorptivity of cracked FR mortar and their variation with age ..............91

Table 4.6: Secondary sorptivity of cracked FR mortar and their variation with age ............93

Table 4.7: Primary sorptivity of normal mortar with holes and their variation with age .........95

Table 4.8: Secondary sorptivity of normal mortar with holes and their variation with age ........97

Table 4.9: RCPT values of normal mortar with holes and their variation with age ..............100

Table 4.10: RCPT values of cracked FR mortar and their variation with age ...................101

Table 4.11: Flexural strength recovery of FR mortar due to self-healing .....................104

Table 4.12: Deflection recovery of FR mortar due to self-healing ........................ 105

Table 4.13: Flexural toughness recovery of FR mortar due to self-healing ..................106

Table 4.14: UPV performed before loading, after loading, and after healing at various ages .....108

Table 4.15: Flexural strength at preload level and recovery after healing . ....................134

Table 4.16: Mid-span deflection at preload level and recovery after healing ..................134

Table 4.17: UPV performed before loading, after loading and after healing ...................137

Table 5.1: Factors and their levels ................................................. 151 
Table 5.2: ANOVA table for the mean compressive strength based on the full factorial design ..154 Table 5.3: Regression equation for compressive strength .............................. 158 Table 5.4: Comparison of Predicted strength (P) of B. subtilis and Experimental strength (E) of

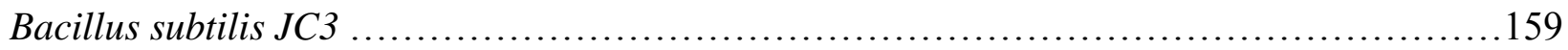
Table 5.5: Comparison of Predicted strength (P) of S. pasteurii and Experimental strength (E) of Bacillus subtilis JC3

Table 5.6: Comparison of Predicted strength (P) of S. ureae and Experimental strength (E) of

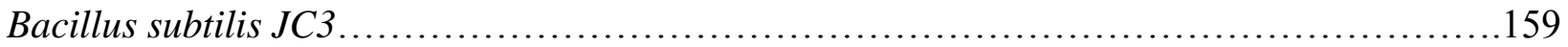

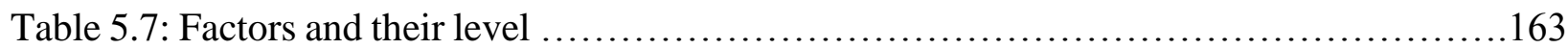

Table 5.8: ANOVA table for the mean RCP based on the full factorial design .................164

Table 5.9: Statistical results of analysis of variance (ANOVA) for regression analysis ..........168

Table 5.10: ANOVA table for the mean sorptivity based on the full factorial design ...........170

Table 5.11: ANOVA table for the mean UPV based on the full factorial design ................175

Table 5.12: ANOVA table for the mean compressive strength ............................ 180

Table 5.13: Comparison of predicted (Y') and experimental (Y) RCP of FR mortar and normal mortar for healing period of 7 days 185

Table 5.14: Comparison of predicted (Y') and experimental (Y) RCP of FR mortar and normal

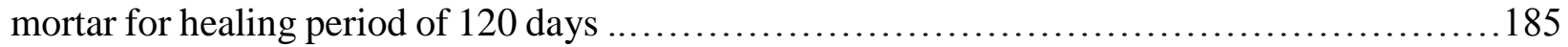
Table 5.15: Comparison of predicted (Y') and experimental (Y) RCP of FR mortar and normal

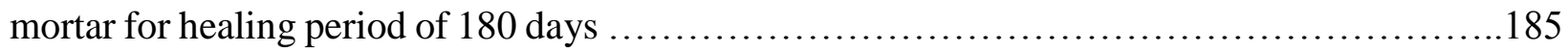
Table 5.16: Comparison of predicted (Y') and experimental (Y) RCP of FR mortar and normal mortar for healing period of 240 days 186 Table 5.17: Comparison of predicted ( $\left.\mathrm{Y}^{\prime}\right)$ and experimental (Y) primary sorptivity of FR mortar and normal mortar for healing period of 7 days .186 Table 5.18: Comparison of predicted ( $\mathrm{Y}^{\prime}$ ) and experimental (Y) primary sorptivity of FR mortar and normal mortar for healing period of 120 days Table 5.19: Comparison of predicted ( $\mathrm{Y}^{\prime}$ ) and experimental (Y) primary sorptivity of FR mortar and normal mortar for healing period of 180 days Table 5.20: Comparison of predicted ( $\mathrm{Y}^{\prime}$ ) and experimental (Y) primary sorptivity of FR mortar and normal mortar for healing period of 240 days .187 
Table 5.21: Comparison of predicted (Y') and experimental (Y) UPV of FR mortar for healing

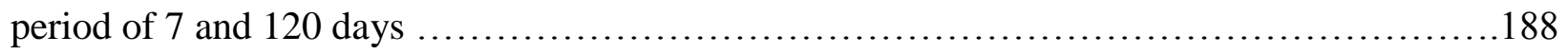

Table 5.22: Comparison of predicted (Y') and experimental (Y) UPV of FR mortar for healing

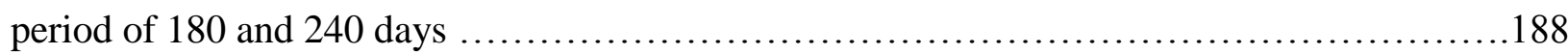

Table 5.23: Comparison of predicted ( $\mathrm{Y}^{\prime}$ ) and experimental (Y) compressive strength of normal

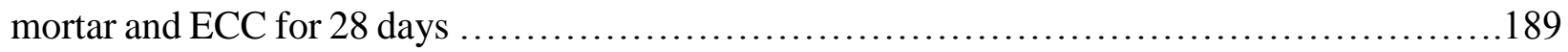
Table 5.24: Comparison of predicted (Y') and experimental (Y) compressive strength of normal

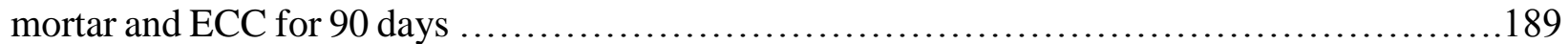
Table 5.25: Comparison of predicted ( $\mathrm{Y}^{\prime}$ ) and experimental (Y) compressive strength of normal

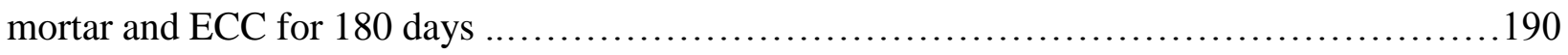




\section{LIST OF FIGURES}

Figure 2.1: Common basic principles of self-healing materials ........................... 9

Figure 2.2: Types of failures in cementitious materials ................................. 12

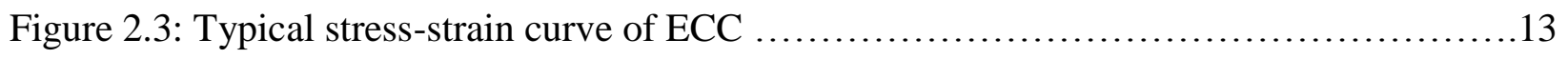

Figure 3.1: The streaked agar plates of Sporosarcina pasteurii (left), Sporosarcina ureae (centre) and Bacillus subtilis subsp. spizizenii (right) culture ..................................43

Figure 3.2: Zeolite (left) and pumice (right) ...................................... 45

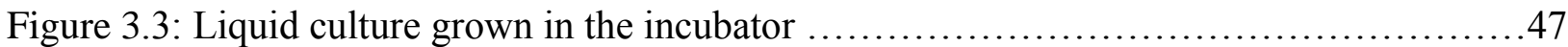

Figure 3.4: Grown culture transferred into $50 \mathrm{ml}$ falcon tubes ...........................47

Figure 3.5: Centrifuge machine (left), falcon tubes kept in the rotor inside the centrifuge machine for centrifuging (right), rotor with cover ready for centrifuging (bottom) ..................48

Figure 3.6: Falcon tubes with harvested cells (left), falcon tube with bacterial cells (right) ......48

Figure 3.7: Bacterial cells immobilised in zeolite/pumice ...............................49

Figure 3.8: Dissolved nutrient solution used as part of mixing water $\ldots \ldots \ldots \ldots \ldots \ldots \ldots \ldots \ldots \ldots$

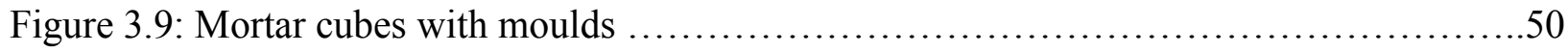

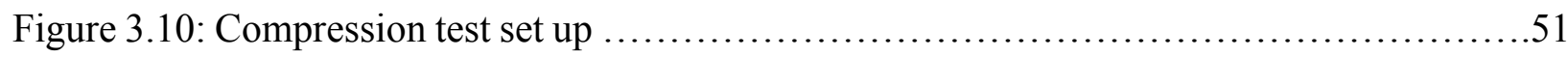

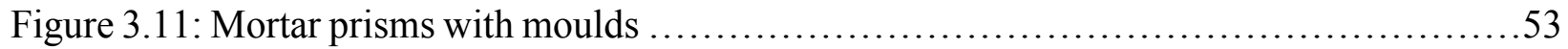

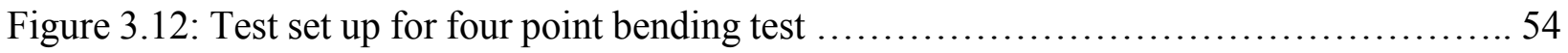

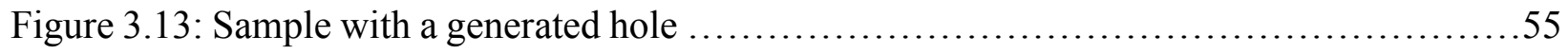

Figure 3.14: Tensile splitting test set up to induce cracks (left) and the induced crack (right) ....56

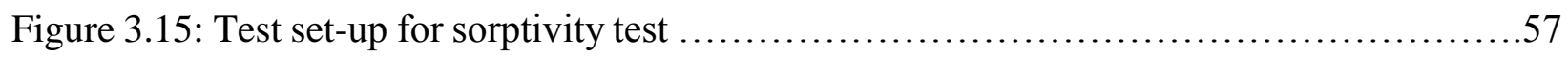

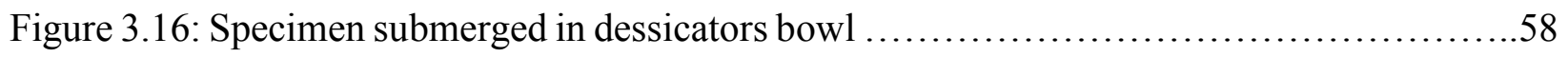

Figure 3.17: Denton vacuum Desk IV coating system (left), specimen ready for coating (right) ..60 Figure 3.18: X Pert PRO apparatus (left), samples mounted on the X Pert PRO apparatus .......61

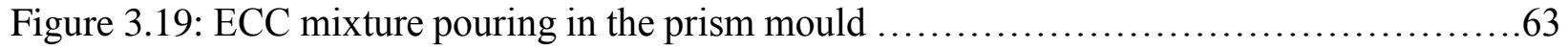

Figure 3.20: Typical failed sample of bacteria based ECC with macro crack and multiple micro

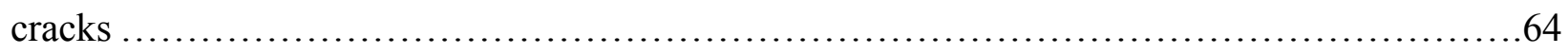

Figure 3.21: Typical preloaded bacteria based ECC specimen with multiple cracks ............65 
Figure 4.1: Light microscopy of (63x magnification) of (A) Sporosarcina ureae, (B) Sporosarcina pasteurii and (C) Bacillus subtilis subsp. Spizizenii cultures showing vegetative cells with intracellular spores

Figure 4.2: Light microscopy of (10x magnification) of crystal formation on plates for (A) Sporosarcina pasteurii and (B) Sporosarcina ureae .68

Figure 4.3: Ureolytic activity of zeolite and pumice immobilized S. pasteurii in neutral and high$\mathrm{pH}$ cement slurry

Figure 4.4: Ureolytic activity of zeolite and pumice immobilized S. ureae in neutral and high-pH cement slurry

Figure 4.5: Compressive strength of mortar cubes with different calcium lactate concentrations .71 Figure 4.6: 28-day compressive strength of mortar cubes with different bacterial concentration .75 Figure 4.7: Cube compressive strength of all 9 mortar mixes at different ages with $10^{6}$ cells $/ \mathrm{ml} .76$ Figur4.8: Cube compressive strength of all 9 FR mortar mixes at different ages ................78

Figure 4.9: Water absorption of cracked FR mortar in 7 days with pumice ...................80

Figure 4.10: Water absorption of cracked FR mortar in 7 days with zeolite ....................80

Figure 4.11: Water absorption of cracked FR mortar after 120 days of healing with pumice .....81

Figure 4.12: Water absorption of cracked FR mortar after 120 days of healing with zeolite .....81

Figure 4.13: Water absorption of cracked FR mortar after 180 days of healing with pumice .....82

Figure 4.13: Water absorption of cracked FR mortar after 180 days of healing with zeolite ......82

Figure 4.15: Water absorption of cracked FR mortar after 240 days of healing with pumice ........83

Figure 4.16: Water absorption of cracked FR mortar after 240 days of healing with zeolite .......83

Figure 4.17: Water absorption of 7 days old normal mortar with holes with pumice ............85

Figure 4.18: Water absorption of 7 days old normal mortar with holes with zeolite ..............85

Figure 4.19: Water absorption of 120 days healed normal mortar with holes with pumice ........86

Figure 4.20: Water absorption of 120 days healed normal mortar with holes with zeolite ........86

Figure 4.21: Water absorption of 180 days healed normal mortar with holes with pumice .......87

Figure 4.22: Water absorption of 180 days healed normal mortar with holes with zeolite ........87

Figure 4.23: Water absorption of 240 days healed normal mortar with holes with pumice ........88

Figure 4.24: Water absorption of 240 days healed normal mortar with holes with zeolite ........88

Figure 4.25: Primary sorptivity of FR mortar with pumice .............................90

Figure 4.26: Primary sorptivity of FR mortar with zeolite .............................90 
Figure 4.27: Secondary sorptivity of FR mortar with pumice $\ldots \ldots \ldots \ldots \ldots \ldots \ldots \ldots \ldots \ldots \ldots . \ldots . \ldots \ldots 2$

Figure 4.28: Secondary sorptivity of FR mortar with zeolite $\ldots \ldots \ldots \ldots \ldots \ldots \ldots \ldots \ldots \ldots \ldots \ldots 2$

Figure 4.29: Primary sorptivity of normal mortar with holes and pumice ....................94

Figure 4.30: Primary sorptivity of Normal mortar with holes and zeolite ...................95

Figure 4.31: Secondary sorptivity of Normal mortar with holes and pumice ..................96

Figure 4.32: Secondary sorptivity of normal mortar with holes and zeolite ..................96

Figure 4.33: Rapid chloride permeability of normal mortar with pumice .....................98

Figure 4.34: Rapid chloride permeability of normal mortar with zeolite ....................99

Figure 4.35: Rapid chloride permeability of FR mortar with zeolite .......................99

Figure 4.36: Rapid chloride permeability of FR mortar with pumice ...................... 100

Figure 4.37: Observation of crack healing in bacteria incorporated specimen .................103

Figure 4.38: Flexural stress- deflection curve for the fibre reinforced mortar specimens including virgin specimen loaded to failure and reloaded curve after 4 months of healing ...............107

Figure 4.39: Changes in UPV values with time ...................................... 109

Figure 4.40: Photographic images of crack healing of specimen treated bacteria and control (a) control, (b) no bacteria + nutrients, (c) and (d) Bacillus subtilis treated (e) and (f) Sporosarcina ureae treated (g) and (h) Sporosarcina pasteurii treated ................................. 110

Figure 4.41: SEM observation of cracked samples with Sporosarcina pasteurii + pumice .......113

Figure 4.42: EDS analysis of Sporosarcina pasteurii + pumice ............................... 113

Figure 4.43: SEM observation of cracked samples with Sporosarcina pasteurii + zeolite .....114

Figure 4.44: EDS analysis of Sporosarcina pasteurii + zeolite ............................114

Figure 4.45: SEM observation of $\mathrm{CaCO}_{3}$ crystals formed by Sporosarcina pasteurii ...........115

Figure 4.46: SEM observation of cracked samples with $B$. subtilis subsp. spizizenii + zeolite ....115

Figure 4.47: EDS analysis of B. subtilis subsp. spizizenii + zeolite ........................116

Figure 4.48: SEM observation of cracked samples with B. subtilis subsp. spizizenii + pumice ...116

Figure 4.49: SEM observation of $\mathrm{CaCO}_{3}$ crystals formed by Bacillus subtilis subsp. spizizenii

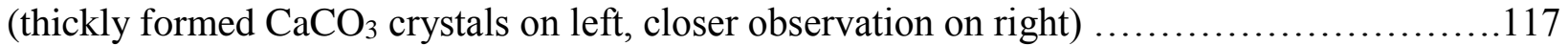

Figure 4.50: EDS analysis of B. subtilis subsp. spizizenii + pumice .......................117

Figure 4.51: SEM observation of cracked samples with Sporosarcina ureae + zeolite .........118

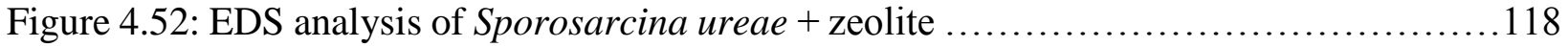

Figure 4.53: SEM observation of control sample with no healing .........................119 
Figure 4.54: SEM observation of sample with nutrients + pumice with lesser healing ....

Figure 4.55: Thin section observation of crack healing at the interior region of crack (Sporosarcina pasteurii based mortar) .... 120

Figure 4.56: Thin section observation of crack healing at the interior region of crack (Bacillus subtilis subsp. Spizizenii based mortar) .....

Figure 4.57: Thin section observation of crack healing at the interior region of crack (Sporosarcina ureae based mortar) 121

Figure 4.58: Microstructure of mortar matrix with bacteria and nutrients .................... 122

Figure 4.59: Microstructure of mortar matrix without bacteria but with nutrients ................123

Figure 4.60: XRD analysis of Control sample (without bacteria and nutrients) ..................124

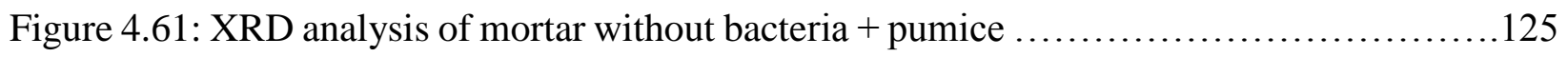

Figure 4.62: XRD analysis of mortar without bacteria + zeolite ........................... 125

Figure 4.63: XRD analysis of mortar with Sporosarcina pasteurii + pumice .....................126

Figure 4.64: XRD analysis of mortar with Sporosarcina pasteurii + zeolite .....................126

Figure 4.65: XRD analysis of mortar Bacillus subtilis + pumice .............................127

Figure 4.66: XRD analysis of mortar Bacillus subtilis + zeolite ...............................127

Figure 4.67: XRD analysis of mortar Sporosarcin ureae + pumice ........................... 128

Figure 4.68: XRD analysis of mortar Sporosarcina ureae + zeolite ........................... 128

Figure 4.69: Compressive strength of bacteria based ECC mix at 7 and 28 days ...............130

Figure 4.70: Flexural stress - deflection curve for Control ECC …........................132

Figure 4.71: Flexural stress - deflection curve for unprotected Sporosarcina pasteurii based ECC

Figure 4.72: Flexural stress - deflection curve for zeolite + Sporosarcina pasteurii based ECC 133 Figure 4.73: Flexural stress - deflection curve for zeolite + Bacillus subtilis based ECC ........133 Figure 4.74: Relative flexural stiffness of healed specimens as a percentage of the reference virgin

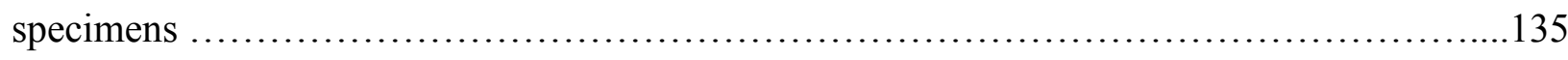

Figure 4.75: Healed specimen of zeolite immobilised Bacillus subtilis before (top) and after reloading (bottom) showing the deviation of crack formation ..............................136

Figure 4.76: Control specimen with no visible crack healing .............................. 138

Figure 4.77: ECC specimen with unprotected Sporosarcina pasteurii .........................139 
Figure 4.78: ECC specimens with Sporosarcina pasteurii immobilised into zeolite healed cracks (top) and all micro cracks appeared to be filled with white precipitates (bottom) ..............140

Figure 4.79: Healed specimens with Bacillus subtilis immobilised into zeolite .... 141

Figure 4.80: SEM observation of cracked ECC specimen with S. pasteurii + zeolite after 1 month of healing .143

Figure 4.81: EDS analysis of cracked ECC specimen with $S$. pasteurii+zeolite after 1 month of healing 143

Figure 4.82: SEM observation of cracked ECC specimen with B. subtilis + zeolite after 1 month of healing

Figure 4.83: EDS analysis of cracked ECC specimen with $B$. subtilis+zeolite after 1 month of healing .144

Figure 4.84: SEM observation of cracked ECC specimen (control) after 1 month of healing ....145

Figure 4.85: EDS analysis of cracked ECC specimen (control) after I month of healing .145

Figure 4.86: SEM observation of cracked ECC specimen with unprotected S. pasteurii after 1 month of healing .146

Figure 4.87: EDS analysis of cracked ECC specimen with unprotected S. pasteurii after 1 month of healing .... 146

Figure 4.88: XRD analysis of ECC mix with unprotected S.pasteurii 147

Figure 4.89: XRD analysis of ECC mix with zeolite immobilised B. subtilis 148

Figure 4.90: XRD analysis of ECC mix with zeolite immobilised S. pasteurii 148

Figure 4.91: XRD analysis of control ECC mix 149

Figure 5.1: Two-way interaction plots for the compressive strength 153

Figure 5.2: The normal probability plot for the full factorial model found by ANOVA for the compressive strength 155

Figure 5.3: The residuals versus fitted values of the full factorial model found by ANOVA for the compressive strength .155

Figure 5.4: Main effects plot based on the full factorial design for the compressive strength ....156 Figure 5.5: Contour plot of the compressive strength

Figure 5.6: Comparison of Predicted strength of B. subtilis and Experimental strength of B. subtilis JC3 .160

Figure 5.7: Two-way interaction plots for the mean RCP of fibre reinforced mortar .... 165 
Figure 5.8: The residuals versus fitted values of the full factorial model found by ANOVA for the $\mathrm{RCP}$ 165

Figure 5.9: The residual normal probability plot for the full factorial model found by ANOVA for the RCP 166

Figure 5.10: Main effect plot for the mean RCP of fibre reinforced mortar .167

Figure 5.11: Two-way interaction plots for the mean sorptivity of fibre reinforced mortar ......171

Figure 5.12: The residuals versus fitted values of the full factorial model found by ANOVA for the primary sorptivity 171 Figure 5.13: The residual normal probability plot for the full factorial model found by ANOVA for the primary sorptivity .172 Figure 5.14: Main effect plot for the sorptivity of fibre reinforced mortar 173 Figure 5.15: Two-way interaction plots for the UPV of fibre reinforced mortar 175 Figure 5.16: The residuals versus fitted values of the full factorial model found by ANOVA for the UPV 176 Figure 5.17: The residual normal probability plot for the full factorial model found by ANOVA

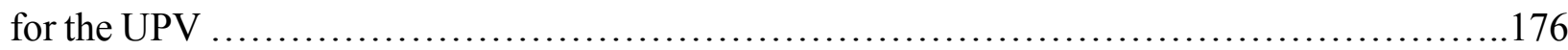

Figure 5.18: Main effect plot for the UPV of fibre reinforced mortar $\ldots \ldots \ldots \ldots \ldots \ldots \ldots \ldots \ldots . \ldots 178$

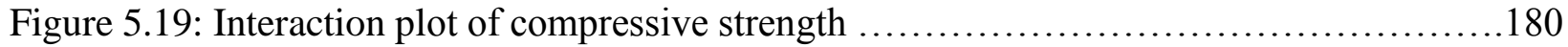

Figure 5.20: The residuals versus fitted values for the compressive strength .................181

Figure 5.21: The residual normal probability plot for the compressive strength ...............181

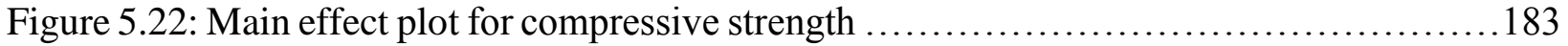




\section{LIST OF SYMBOLS AND ABBREVIATIONS}

$\varepsilon$

$\eta$

a

A

$\mathrm{A}_{\mathrm{i}}$

AASHTO

$\mathrm{AB}_{\mathrm{ij}}$

$\mathrm{AE}$

ANOVA

ASTM

B

$\mathrm{B}_{\mathrm{j}}$

C

$\mathrm{Ca}$

$\mathrm{Ca}^{2+}$

$\mathrm{CaC}_{6} \mathrm{H}_{10} \mathrm{O}_{6}$

$\mathrm{Ca}\left(\mathrm{CH}_{3} \mathrm{COO}\right)_{2} \cdot \mathrm{H}_{2} \mathrm{O}$

$\mathrm{CaCl}_{2} \cdot 2 \mathrm{H}_{2} \mathrm{O}$

$\mathrm{Ca}\left(\mathrm{NO}_{3}\right)_{2} \cdot 4 \mathrm{H}_{2} \mathrm{O}$
Error

Response surface

Cross-sectional area of the standpipe

Cross-sectional area subject to flow

Total of all measurements of parameter A at level i

American association of state highway and transportation officials

Total of all measurements at the $i^{\text {th }}$ level of parameter $A$ and at the $j^{\text {th }}$ level of parameter B

Acoustic emission

Analysis of variance

American society for testing and materials

Bacillus

Total of all measurements of parameter B at level $\mathrm{j}$

Carbon

Calcium

Calcium ion

Calcium lactate

Calcium acetate

Calcium chloride

Calcium nitrate 


\begin{tabular}{|c|c|}
\hline $\mathrm{CaCO}_{3}$ & Calcium carbonate \\
\hline $\mathrm{CO}_{2}$ & Carbon dioxide \\
\hline $\mathrm{CO}_{3}{ }^{2-}$ & Carbonate ion \\
\hline $\mathrm{CO}\left(\mathrm{NH}_{2}\right)_{2}$ & Urea \\
\hline $\mathrm{CF}$ & Correction factor \\
\hline$d_{f}$ & Degrees of freedom \\
\hline $\mathrm{DE}$ & Diatomaceous earth \\
\hline DOE & Design and analysis of experiments \\
\hline DSMZ & German collection of microorganisms and cell cultures \\
\hline ECC & Engineered cementitious composite \\
\hline EDS & Energy dispersive spectrum \\
\hline ESEM & Environmental scanning electron microscopy \\
\hline FA & Fly ash \\
\hline $\mathrm{Fe}$ & Iron \\
\hline FEA & Finite element analysis \\
\hline FR & Fibre reinforced \\
\hline $\mathrm{h}_{0}$ & Initial hydraulic head \\
\hline$h_{f}$ & Final hydraulic head \\
\hline $\mathrm{H}_{2} \mathrm{O}$ & Water \\
\hline HAC & High-alumina cement \\
\hline HRWRA & High-range water-reducing admixtures \\
\hline
\end{tabular}




\begin{tabular}{|c|c|}
\hline $\mathrm{k}$ & Permeability coefficient \\
\hline $\mathrm{K}$ & Potassium \\
\hline $\mathrm{L}$ & Specimen thickness in the direction of flow \\
\hline LVDT & Linear variable differential transformer \\
\hline LWSCC & Lightweight self-consolidating concrete \\
\hline $\mathrm{Mg}$ & Magnesium \\
\hline MICP & Microbial induced calcite precipitation \\
\hline MTS & Material testing system \\
\hline MS & Mean squares \\
\hline $\mathrm{n}$ & Total number of measurements \\
\hline $\mathrm{N}$ & Nitrogen \\
\hline $\mathrm{NaCl}$ & Sodium chloride \\
\hline $\mathrm{NaHCO}_{3}$ & Sodium bicarbonate \\
\hline $\mathrm{NaOH}$ & Sodium hydroxide \\
\hline $\mathrm{NH}_{4}^{+}$ & Ammonium ion \\
\hline $\mathrm{NH}_{4} \mathrm{Cl}$ & Ammonium chloride \\
\hline $\mathrm{O}$ & Oxygen \\
\hline OPC & Ordinary portland cement \\
\hline $\mathrm{P}$ & Phosphorus \\
\hline PU & Polyurethanes \\
\hline PVA & Polyvinyl alcohol \\
\hline
\end{tabular}




$\begin{array}{ll}\text { RCP } & \text { Rapid chloride permeability } \\ \text { R-Sq } & \text { R-squared } \\ \text { SCC } & \text { Sporosarcina } \\ \text { SEM } & \text { Self-consolidating concrete } \\ \text { SMA } & \text { Scanning electron microscopy } \\ \text { SS } & \text { Shape memory alloy } \\ \text { SS } & \text { Sum of squares of parameter A } \\ t_{f} & \text { Sum of squares of parameter B } \\ \text { UPV } & \text { Test duration } \\ \text { XRD } & \text { Ultrasonic pulse velocity } \\ y_{i} & \text { X-Ray Diffraction }\end{array}$




\section{CHAPTER ONE: INTRODUCTION}

\subsection{Concrete history and evolution}

Concrete is a strong and relatively cheap construction material and is therefore; presently the most used construction material worldwide (Emmons and Sordyl, 2006). It is composed of aggregates, binder, water and admixtures in different proportions depending on the required strength and functionality. Concrete was first used by the Egyptians who used mud mixed with straw to bind dried bricks (Mindess, 2003; Neville, 1996). Later, the Romans made many developments in concrete technology including the use of hydraulic cements made from slaked lime and made famous landmark such as Roman aqueduct and Colosseum that are still standing today. Modern concrete, a $\$ 30$ billion per year industry in sales alone in the U.S. (National Ready Mixed Concrete Association), is based on hydraulic Portland cement involving sintering of limestone and clay at high temperature (Mindess, 2003; Neville, 1996); however, this modern process is still an analogous to that of lime-based mortars that was used by the Romans.

\subsection{Cost and repair needs}

Concrete structures often suffer from cracking that leads to deterioration and shorten their service life. Cracks can occur at any stage of the service life due to volume instabilities within concrete or external factors such as extreme loading, harsh environmental exposure, poor construction procedures or design error. Micro-cracks permit the penetration of water and other impurities such as chloride and sulphate ions in to the concrete matrix leading to premature matrix degradation, corrosion of embedded reinforcement etc. which in turn hinders structural integrity. Generally, huge expenses are incurred in maintenance and repair of concrete structures. It is estimated that damage due to corrosion of concrete in the US is $\$ 276$ billion (Koch et al., 2001) with annual cost in repairs to be $\$ 18-21$ billion per year (Emmons and Sordyl, 2006). Moreover, indirect costs due to traffic jams and associated loss of productivity due to reparation are even 10 times higher than the direct costs of maintenance and repair (Freyermuth, 2001). Therefore, it is imperative that crack propagation in concrete must be minimized to extend the longevity thus reducing maintenance. There is a compelling economic incentive to develop a concrete that can treat and repair the damage all by itself. 


\subsection{Self-healing concrete}

Animals and plants have a natural capability to heal small bodily damages by themselves in a relatively short amount of time without any external influence. Similarly, natural self-healing ability of concrete known as the autogenous healing has been observed for many years (Neville, 2002; Li, and Yang, 2007). It has been noticed that the micro cracks in old structures were selfhealed by the recrystallization of calcite (Edvardsen, 1999). This reveals that under the right environment concrete is able to seal the cracks by itself with the augmentation of some chemical and/or biological additives and with the presence of moisture. In general, under the right environment, carbon dioxide in the air is dissolved in water, and this carbon dioxide reacts with the calcium ions in the concrete to produce calcium carbonate crystals. The calcium carbonate crystallization made in this way is attached and grown on the crack surface. This leads to the reduction in crack width and eventual repair of the whole crack (Ramm and Biscoping, 1998; Edvardsen, 1999). However, depending on this natural process alone, only a limited crack with width up to $100 \mu \mathrm{m}$ can be repaired (Neville, 2002; Li and Yang, 2007). However, to repair larger cracks with better healing consistence, addition of both chemical and biological amendments might be needed.

Previous studies by various researchers have concluded that self-healing behaviour can be achieved by the introduction of bacteria into the concrete matrix (Jonkers and Schlangen, 2009; Wang et al., 2012). In brief, it was hypothesized that once moisture enters through freshly formed cracks, dormant but viable bacterial spores immobilized in the concrete matrix becomes metabolically active. Then, these cracks will be healed through microbial calcite precipitation, impeding further ingression of water and other chemicals. Most of the applications of bacterial concrete done so far were for crack remediation treatments, which cannot be considered purely 'self-healing' because it was applied after the cracking occurred. (Ramakrishnan et al, 2001; Day et al, 2003; De Muynck et al, 2008; Patil et al, 2008; Raijiwala et al, 2009; Tittelboom et al, 2009). In these studies, an efficient plugging of cracks and recovery of mechanical strength was observed which resulted from the presence of adequate amount of organic substances in the matrix due to microbial biomass. Only limited studies have been performed on pure self-healing bacterial concrete (Jonkers and Schlangen, 2009; Jonkers et al., 2010; Wang et al., 2012; Jing and Wu, 2014) in which very limited attempts have been made to develop a bacteria based self-healing 
Engineered Cementitious Composites (ECC) (Navneet and Rafat, 2013; Sierra-Beltran et al., 2014). The material ingredients of ECC include cement, sand, water, small volume of fibers (around 2\%) and some chemical additives. The basic properties of engineered cementitious composites (ECC) and fiber reinforced (FR) mortar is that after cracking ECC strain hardens while FR mortar does not exhibit such behavior. Strain-hardening materials are characterized by their ability to sustain increasing levels of loading after first cracking while undergoing large deformation. Hence the material has the ability to undergo multiple cracking before failure occurs.

It was reported that the bacteria incorporated self-healing concrete leads to a superior self-healing capacity compared to conventional or engineered non-sustainable self-healing cementitious systems and with this method it is possible to seal the crack width up to $300 \mu \mathrm{m}$ (Jonkers et al., 2010). In order to increase the bacterial activity and viability in high $\mathrm{pH}$ concrete environment, bacteria has to be immobilised in some carrier materials rather than directly incorporating in the concrete mix (Jonkers and Thijssen, 2010). For the incorporated bacteria to precipitate calcium carbonate, it is necessary to provide suitable calcium compound while casting. Also, for the bacteria to become metabolically active, certain mineral substrate is to be added as a growth medium along with bacteria and the calcium source (Jonkers et al., 2010). Eventhough various researchers conducted experiments using different types of bacteria, a major breakthrough to pin point the perfect combinations of bacteria and conditions such as types of bacteria, types of mineral substrate, types of carrier materials and the quantities of each is yet to be identified.

\subsection{Objectives}

The current research is aimed at investigating the potential of different mineral producing bacteria for their long-term viability, their incorporation, their survival in the mortar/ECC matrix and their self-healing ability for crack repair. Three different types of bacteria (along with zeolite/pumice as career materials and calcium lactate, urea and yeast extract as nutrients) are incorporated in cementitious concrete composites (normal mortar, fiber reinforced mortar and ECC) and their performance in inducing self-healing characteristics (strength/durability properties recovery with crack repair) are investigated. Extensive experimental investigations including micro-structural testing (such as scanning electron microscopy 'SEM', Energy Dispersive Spectrum 'EDS', X-ray diffraction 'XRD') and non-destructing testing (such as ultra-sonic pulse velocity 'UPV) as well 
as statistical/mathematical modeling are used. In order to develop a self-healing bacterial concrete based composites, it is crucial to understand how the introduction of the mineral inducing bacteria and nutrients affect the properties, giving emphasis on closure of cracks and regaining of mechanical properties. Thus, the prime goal of this study is to identify the real potential of realizing the idea 'Self-healing Concrete Composites' and this purpose is accomplished by fulfilling some key objectives. The objectives are to:

i) evaluate the growth, crystal forming ability, spore formation, germination, and percentage of survival of three different bacterial species in high temperature and $\mathrm{pH}$ treatments.

ii) investigate the efficiency of zeolite, and pumice as carriers or protective vehicle for bacteria and calcium lactate as nutrient.

iii) check the ureolytic activity of the zeolite and pumice immobilised bacteria in a high $\mathrm{pH}$ concrete environment.

iv) investigate the effect of healing agent additions on mechanical properties such as compressive strength and flexural strength of mortars (normal/fiber based mortar and $\mathrm{ECC})$,

v) evaluate the efficiency of crack healing in terms of recovery of permeation properties such as sorptivity and rapid chloride permeability (RCP), ultrasonic pulse velocity (UPV) and strength properties - both compressive and flexural strength.

vi) visualize the crack healing and characterize mineral constituents including crack healing products using SEM, EDS and XRD studies

vii) develop mathematical/statistical models based on experimental results to quantify selfhealing and to determine mechanical and durability properties of self-healed cement concrete composites using material parameters. Validate the performance of such models based on current experimental results and available data from previous research studies. 


\subsection{Layout of the Thesis}

Following this introduction Chapter, a literature review on the self-healing concrete based materials is presented in Chapter 2. A close attention to the understanding of basic underlying principles of self-healing mechanism is discussed. Various parameters influencing the microbial induced calcite precipitation (MICP) is narrated in this chapter. Along with this a background of the behavior of engineered cementitious composites (ECC) are mentioned as well. A background on design and analysis of experiments (DOE) and previous applications of DOE on cementitious materials are introduced. Previous experimental studies on bacteria incorporated crack remediation process with particular reference to concrete materials are described. The importance of current research in the light of existing technology and development in the area of self-healing concrete materials is highlighted.

Chapter 3 describes the experimental research methodology, testing procedures and materials used by describing various activities of the research: i) selection and cultivation of bacteria and their various initial testing, ii) testing the urease activity of selected carrier materials, iii) investigating the optimum quantity of bacteria and mineral substrates, iv) self-healing investigations with various techniques and v) self-healing investigation on ECC materials. The description of the experimental work in this chapter includes the mortar mix parameters, the physical, chemical, and mechanical properties of the materials used, microbial healing agent preparation, casting of mortar specimens, and description of specimen's preparation and the tests setup.

Chapter 4 outlines a detailed analysis of self-healing conducted for normal mortar, fibre reinforced mortar extensively and for engineered cementitious composites to a certain extent. This chapter initially discusses observed results on growth, spore formation, germination, and percentage of survival in high temperature and $\mathrm{pH}$ treatments and ureolytic activity of different bacteria. Effect of healing agents such as bacteria, carrier materials and nutrients on self-healing based on compressive strength of mortar cubes are discussed in subsequent sections. Both qualitative and quantitative analysis of the self-healing was performed using various testing tools. SEM, EDS and XRD studies were performed for qualitative analysis and compressive strength test, sorptivity, rapid chloride permeability, ultrasonic pulse velocity tests and test to identify flexural properties for detailed quantitative analysis. This chapter presents detailed analysis and discussion of the results obtained in all the tests conducted to assess self-healing. 
Chapter 5 presents the development of mathematical/statistical models to quantify the bacteria mediated self-healing properties of cementitious concrete composites based on experimental results. Statistical analyses were carried out using design of experiments to model the influence of key parameter such as age of healing, types of bacteria and types of carrier materials on selfhealing efficiency of mortar and ECC in terms of mechanical and durability properties recovery after induced cracking damage. Such properties/responses include compressive strength, sorptivity, rapid chloride permeability (RCP) and ultrasonic pulse velocity (UPV).

Finally, a brief summary of the research project is given in Chapter 6. The outcomes and conclusions of this research are described and recommendations are made for future research. 


\section{CHAPTER TWO: REVIEW OF LITERATURE}

\subsection{Introduction}

This chapter provides an introduction to the self-healing mechanism by describing the characteristics of self-healing behavior and the basic underlying principles of self-healing mechanism. In addition to this, a background of the behavior of engineered cementitious composites (ECC) are mentioned as well. A detailed description of several parameters influencing the microbial induced calcite precipitation (MICP) and previous experimental studies on bacteria incorporated crack remediation process with particular reference to concrete materials are described. Apart from these, an introduction to the background on design and analysis of experiments (DOE) and some previous applications of DOE on cementitious materials are discussed in detail. This chapter also contains, some highlights on the importance of current research in the light of existing technology and development in the area of self-healing concrete materials so far.

\subsection{Characteristics of self-healing behavior}

Self-healing materials are specific types of materials designed to regain its strength from low-level damage done to the material over the course of its service life. The self-healing technique is especially useful when applied to composite materials, since composites have low damage detectability and is susceptible to sudden and brittle failure (Van der Zwaag, 2010). Modern day man-made materials have shown excellent mechanical properties. However, it lacks the selfrepairing property. Therefore, when damage occurs, there is the possibility of losing its mechanical strength and gradually it loses its functional strength with the time unless appropriate human intervention has taken place (Ghosh, 2009).

The damage formation is considered as the internal separation of a material into two material segments separated by a crack (Van der Zwaag, 2010). Until now, all engineering materials are developed using the concept of 'damage prevention' paradigm rather than the concept of 'damage management' (Van der Zwaag, 2010). This means that the strength and stiffness properties of these materials are optimised in order to prevent or delay the occurrence of damage (Van der Zwaag, 2010). However, it will be rather impossible to prevent the damage formation completely in the course of use. On the other hand, in living organisms, the process of damage management is 
executed through an intact autonomic process which requires no human intervention. That is, by means of closed circulatory systems, damage is perceived biochemically and apposite agents are supplied to the damaged site. Researchers are well inspired by this pungent biological function which leads them to create man-made self-healing materials by imparting virtually similar properties.

\subsubsection{Basic underlying principles and mechanism of self-healing materials}

For self-healing to occur, the responsive materials must not only sense damage, but also actuate repair. As a prerequisite, it is essential to have the presence of a fluid component within the system in order to initiate a self-healing action in a material (Van der Zwaag, 2010). This fluidity makes the transportation of agents to the damaged site and applies the agents all over the affected region. However, the advanced materials and composites needed for engineering applications are hard solids. Therefore, the primary challenge is to introduce fluidity or fluidic components into these materials without sacrificing the mechanical properties of the system, in order to bring the selfhealing characteristics (Van der Zwaag, 2010). There are many options to introduce the fluid component into the structural system. One viable option is to introduce an 'artificial circulatory system' (Van der Zwaag, 2010; Mookhoek et al., 2009). Researchers have recently developed some micron-scale conduits carrying fluid healing agents which extend throughout the material. Up on cracking, this fluid can flow to the damaged region and repair the crack. Another option is to use the same external agent which produces the damage to introduce the fluidity (Van der Zwaag, 2010; Mookhoek et al., 2009; Ghosh, 2009).

The self-healing process of different man-made materials like metals, ceramics, polymers, composite materials, etc. is based on the same common general principle and underlying concepts even if they have different intrinsic properties. Figure 2.1 below shows the common basic principles of self-healing properties of different classes of materials. The step by step procedure is as follows (Hager et al., 2010):

a) As the foremost stage, as shown in Figure 2.1a-b, a crack is formed on the material due to the mechanical load. 
b) As per the general principle, a "mobile phase" is generated as shown in Figure 2.1c which is triggered either by the occurrence of damage or by external stimuli.

c) Consequently, mass transport takes place towards the damaged site and due to the following local mending reaction the damage can be removed as shown in Figure 2.1d. This is achieved by adhering of the crack planes by physical interactions or chemical bonds.

d) Once the healing is attained with fully restored mechanical properties, the previously mobile material is again immobilised which is shown in Figure 2.1e.

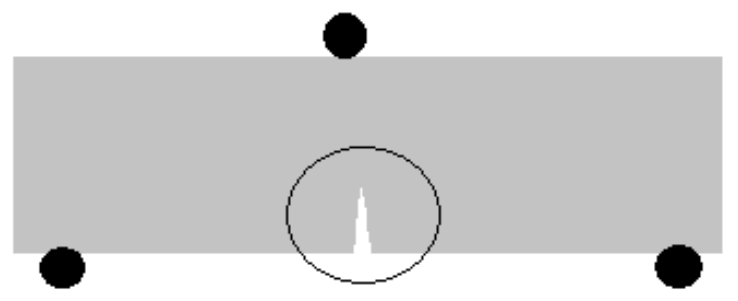

a)

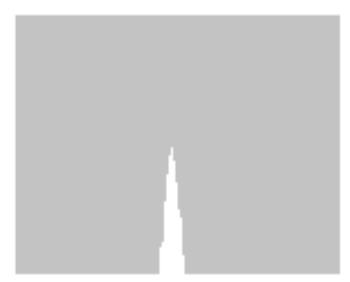

b)

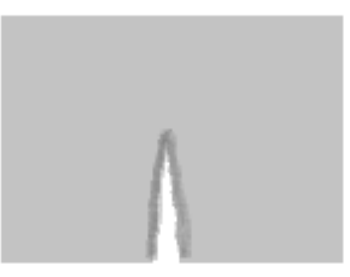

c)

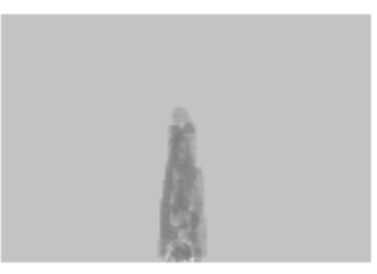

d)

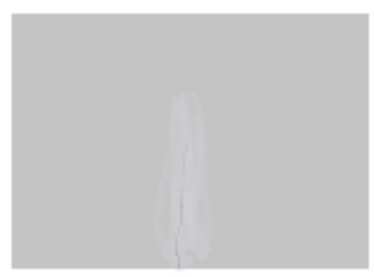

e)

Figure 2.1: Common basic principles of self-healing materials: (a) the mechanical load induces a crack; (b) view of the crack; (c) a "mobile phase" is induced;(d) closure of the crack by the "mobile phase"; (e) immobilisation after healing (Hager et al., 2010)

Although this general principle is identical for all materials, due to their intrinsic properties, certain characteristics such as the effective temperature and the healed damage size varies depending on the material class. Also, the size of damage able to be healed depends on the size and number of species being transported (Hager et al., 2010). 


\subsubsection{Conditions for self-healing}

It should be pointed out from the literature study that in order to ensure self-healing, five general criteria should be satisfied. The five necessary conditions to happen for the healing of cracks are given below:

i. Presence of water: All of the studies conducted so far stated that the presence of water is essential to facilitate healing of the cracks (Qian et al., 2009).

ii. Presence of chemical species: Adequate concentrations of certain critical chemical species essentially carbonate ions or bicarbonate ions and free dissolved calcium ions, play an important role to exhibit healing mechanisms (van der Zwaag, 2007).

iii. Crack width: Controlled crack width is another vital condition. It is actually associated with the efficiency of self-healing in cementitious materials. The crack width to engage distinct self-healing behavior falls below $150 \mu \mathrm{m}$ and preferably lower than $50 \mu \mathrm{m}$ (Yingzi et al., 2009). Smaller crack width requires less self-healing products to fill the crack and it will be easier to grow from both surfaces of the crack to get connected.

iv. Water pressure: Self-healing will not happen, if the water flows quickly through the crack. Therefore the water pressure should not be too high and for a certain crack width, this condition is influenced by the ratio between water height and the thickness of the structure (Schlangen, 2007).

v. Stable crack: In order to guarantee that a crack is not to get damaged again, the crack should be under stable condition and the crack width has to be constant rather varying with time (Hua, 2010).

\subsubsection{Classification of materials based on their self-healing behaviour}

Depending on the underlying chemistry employed in the operation of the self-healing process, selfhealing materials can be categorised into two distinct classes, namely, non-autonomic and autonomic (Ghosh, 2009; Hager et al., 2010). Non-autonomic self-healing materials require an external stimulus, like heat or light in order to drive the healing process. However, autonomic selfhealing materials do not require any external stimulus where the damage itself is the stimulus for the healing (Ghosh, 2009; Hager et al., 2010). 
Another classification is extrinsic and intrinsic self-healing materials. In extrinsic self-healing materials, the healing process is based on external healing components like micro or nano capsules (Yuan et al., 2008; Van der Zwaag, 2010; Ghosh, 2009). In order to make the materials selfhealing, these capsules are deliberately embedded into the matrix of materials and the content of these capsules acts as the mobile phase when damage occurs. Conversely, no separate healing agents are required in intrinsic self-healing materials. Usually, the preferable process is intrinsic self-healing; however, it is not always feasible (Hager et al., 2010).

\subsection{Characteristics of engineered cementitious composite}

Concrete with increasingly high compressive strength have been applied to civil engineering applications in the last decades. Even though, the addition of steel fiber and powders improves a number of concrete properties, most of these materials still remain brittle. Moreover, in some cases, the brittleness increases as the compressive strength goes up, which poses potential dangers or fracture failures of the concrete. A specially designed cementitious material termed as Engineered Cementitious Composites (ECC) has been developed by Li and continuously evolved over the last twenty years (Zhou et al., 2009). ECC is characterized by a high ductility in the range of $3-7 \%$, a tight crack width of around $60 \mu \mathrm{m}$ and relatively low fiber content of $2 \%$ or less by volume (Zhou et al., 2009). In terms of main material constituents, ECC has characteristics similar to regular Fiber Reinforced Concrete (FRC), including water, cement, sand, fiber and some additives. So far, various fiber types and different cementitious matrixes have been used in ECC, but the detail composition of ECC must obey certain principles imposed by micromechanics considerations. The most fundamental mechanical property of ECC is of the ability to carry higher levels of loading after first cracking while undergoing large deformation. The fibers used in ECC are designed to work with the matrix for the purpose of constraining localized brittle fracture and guaranteeing more uniform distribution of micro cracks. Due to the slip-hardening behavior of fibers, ECC can take increasing load that generates new cracks at other sites. It can be observed from Figure 2.2 that first cracking in ECC is followed by increasing stress accompanied by a rise in strain. This strain-hardening behavior of ECC is similar to ductile metals. 


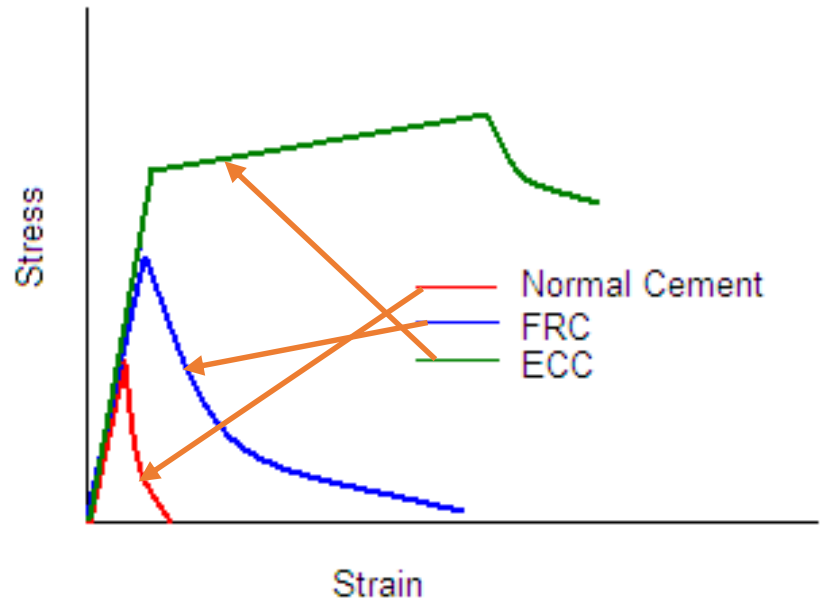

Figure 2.2: Types of failures in cementitious materials $(\mathrm{Li}, 1998)$

The crack width is another important factor, reflecting the durability of a concrete structure. ECC exhibits a tight crack width self-controlled in terms of a flat steady state micro cracks propagation, see Figure 2.2. After the tensile deformation up to around $1 \%$ strain, the early micro cracks stop widening and remain more or less constant with crack width of around $60 \mu \mathrm{m}$. ECC material can be designed to form numerous closely spaced micro cracks. The crack width is much smaller than the typical ones normally observed in the reinforced concrete. Moreover, the self-control of crack width can be seen as intrinsic properties of ECC material, rather than depending on steel reinforcement ratio and structural dimensions (Yang et al., 2009). Figure 2.3 also shows the tensile strain capacity of 5\% that is about $300-500$ times great than normal concrete (Sahmaran and Li, 2009). 


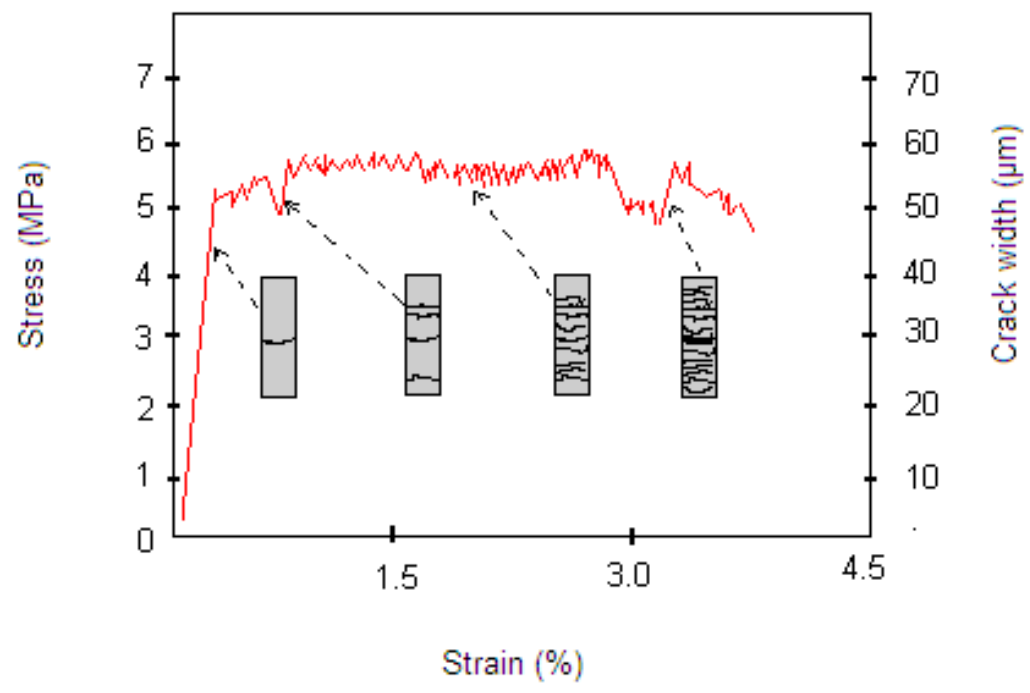

Figure 2.3: Typical stress-strain curve of ECC (Sahmaran and Li, 2009)

From the aspect of ECC-concrete repair system, the advantage of using ECC as repair material is that the trapping mechanism of ECC can serve as a means for enhancing the durability of repair system. It was reported that micro cracks originated from the tips of defects on the ECC-concrete interface and subsequently were trapped in the ECC material. Due to the rapidly rising toughness of the ECC, additional load can drive further crack extension into the interface after kinked crack arrest, followed by subsequent kink and arrest (Kamada and Li, 2000)

\subsection{Background on design and analysis of experiments}

Analysis of variance (ANOVA) and general regression models are the analysis techniques used in this study with the full factorial statistical experimental designs.

The use of orthogonal arrays can be traced back to Euler's Greco-Latin Squares. Sir Ronald Fisher, who introduced ANOVA, was the primary promoter of the use of statistically designed experiments between the First and Second World Wars, 1918-1939. Since that time statistically designed experiments have played an increasingly important role in medical and R\&D activities 
and have been a primary source in the use of statistics in industry (ITT Total Quality Management Group, 1992).

A balanced matrix experiment consists of a set of experimental conditions where the settings of multiple product or process parameters are changed. The objective of these experiments is to be capable of studying the effect that these changes to settings have on the system under study. After conducting the matrix experiment, the data collected from these experiments can then be analyzed to separate and quantify the size and direction of the effects that each product or process parameter had on the system.

The objectives of the analysis of experiments may include (Montgomery, 1997):

i) Determining which variables are most influential on the effect of self-healing response y, which may be (as used in this research) are compressive strength, sorptivity, Rapid Chloride permeability (RCPT) and Ultrasonic Pulse velocity (UPV) of fiber reinforced mortar,

ii) Determining where to set the influential $x$ 's so that $y$ is almost near the desired nominal value. The x's are the controllable factors, namely age of healing, types of bacteria and types of carrier as used in this study.

iii) Determining where to set the influential $x$ 's so that the variability in y is small.

iv) Determining where to set the influential x's so that the effects of the uncontrollable variables $\mathrm{z}_{1}, \mathrm{z}_{2}, \ldots ., \mathrm{z}_{\mathrm{q}}$ are minimized. There are several uncontrollable variables which cannot be introduced in the design, for example environmental conditions, human factors, and etc.

One strategy of experimentation that is extensively used in concrete testing is the one factor at a time approach. This method consists of selecting a starting point or baseline set of levels for each factor, then successively varying each factor over its range with the other factors held constant at the baseline level (Montgomery, 1997). After all tests are performed, a series of graphs are usually constructed showing how the response variable is affected by varying each factor with all other factors held constant. The major disadvantage of this strategy is that it fails to consider any possible interaction between the factors. 
The correct approach to dealing with several factors is to conduct a factorial experiment. A factorial design is more efficient than one factor at a time experiments. A factorial design is necessary when interactions are present to avoid misleading conclusions. Also factorial designs allow the effects of a factor to be estimated at several levels of the other factors, yielding conclusions that are valid over a range of experimental conditions. By a full factorial design, in each complete trial or replication of the experiment all possible combinations of the levels of the factors are investigated. Consideration of all main factor and factor interaction effects generally produces good results. However, number of required experiments increases rapidly with an increase in number of analyzed parameters making usage of the full factorial designs infeasible. If it is reasonably assumed that certain high order interactions are negligible, then information on the main effects and low order interactions may be obtained by running only a fraction of the complete factorial experiment. This approach is known as fractional factorial design. It saves considerable time and money but requires rigorous mathematical treatment, both in the design of the experiment and in the analysis of the results. Each experimenter may design a different set of fractional factorial experiments (Van Nostrand, 1990).

The analysis of variance (ANOVA) is the statistical treatment most commonly applied to the results of the experiment to determine the percent contribution of each factor and factor interactions. Study of the ANOVA table for a given analysis helps to determine which of the factors need control and which do not (Van Nostrand, 1990). ANOVA employs sums of squares which are mathematical abstracts that are used to separate the overall variance in the response into variances due to the processing parameters and measurement errors. The correction factor for mean (CF), total, main effects, the interaction and error sum of squares are calculated as in the following equations:

$$
\begin{aligned}
& C F=\frac{\left(\sum_{i=1}^{n} y_{i}\right)^{2}}{n} \\
& S S_{T}=\sum_{i=1}^{n} y_{i}^{2}-C F \\
& S S_{A}=\frac{\sum_{i=1}^{n a} A_{i}^{2}}{b r}-C F \\
& S S_{B}=\frac{\sum_{j=1}^{b} B_{j}^{2}}{b r}-C F
\end{aligned}
$$


$S S_{A B}=\frac{\sum_{j=1}^{b} \sum_{i=1}^{a} A B_{i j}^{2}}{r}-S S_{A}-S S_{B}-C F$

$S S_{e}-S S_{T}-S S_{A}-S S_{B}-S S_{A B}$

where:

$$
\begin{aligned}
& \mathrm{y}_{\mathrm{i}}=\text { individual observation } \\
& \mathrm{a}=\text { number of levels of parameter } \mathrm{A} \\
& \mathrm{b}=\text { number of levels of parameter } \mathrm{B} \\
& \mathrm{r}=\text { number of measurements for each pair of levels of parameter A and } \mathrm{B} \\
& \mathrm{n}=\text { total number of measurements }=\text { abr } \\
& \mathrm{A}_{\mathrm{i}}=\text { total of all measurements of parameter A at level } i ;(i=1,2, \ldots, a) \\
& \mathrm{B}_{\mathrm{j}}=\text { total of all measurements of parameter } \mathrm{B} \text { at level } j ;(j=1,2, \ldots, b)
\end{aligned}
$$

$\mathrm{AB}_{\mathrm{ij}}=$ total of all measurements at the $\mathrm{i}^{\text {th }}$ level of parameter $\mathrm{A}$ and at the $\mathrm{j}^{\text {th }}$ level of parameter $B ;(i=1,2, \ldots, a ; j=1,2, \ldots, b)$

Mean squares (MS) for each factor is obtained by dividing the sum of squares by their respective degrees of freedom $\left(\mathrm{d}_{\mathrm{f}}\right)$.

$M S_{A}=\frac{S S_{A}}{d f_{A}}$

F ratios are calculated by dividing the mean squares by the mean square of error.

$F_{A}=\frac{M S_{A}}{M S_{e}}$

The relationship between the response variable and the factors is characterized by a mathematical model called a regression model. It provides a technique for building a statistical predictor of a response and places a bound on the error of prediction (Mendenhall and Sincich, 1996). By employing least squares method it tries to find the levels of the design variables that result in the best values of the response. Response surface methodology is a collection of mathematical and statistical techniques that are useful for the modeling and analysis of problems in which a response 
of interest is influenced by several variables and the objective is to optimize the response (Montgomery, 1997). If the general regression model is:

$y=f\left(x, x_{2}\right)+\varepsilon$

where $\varepsilon$ represents the noise or error observed in the response y. If the expected response is denoted by $E(y)=f\left(x, x_{2}\right)=\eta$, then the surface represented by

$\eta=f\left(x, x_{2}\right)$

is called a response surface (Montgomery, 1997).

\subsubsection{Previous application of design and analysis of experiments on cementitious materials}

The application of experimental design and analysis techniques to self-healing is very rare. No one has attempted to model the self-healing of concrete using this design and analysis of experiments. Some of the previous civil engineering application of this method is presented here even though these studies do not come under self-healing modeling.

An attempt has been made by Srinivasan et al. (2003) to make cost effective rapid-set high strength cement. The experiments were designed using orthogonal array technique in L9 array with three factors, namely ordinary portland cement (OPC)/high-alumina cement (HAC)/anhydrous calcium sulphate, fineness of the cement, and type of additives, at three levels each. The responses studied were initial setting time, final setting time, and compressive strength. The response data were analyzed using analysis of variance (ANOVA) technique with a software package, ANOVA by Taguchi Method. In the case of setting time, fineness of the cement and OPC/HAC/anhydrous calcium sulphate ratio plays a significant role. Additive type and the OPC/HAC/anhydrous calcium sulphate are significant factors affecting the compressive strength at different ages. The confirmatory trial results clearly indicate that the setting time and compressive strength at different ages targeted were achieved using design of experiments.

Patel et al. (2004) investigated 21 statistically balanced concrete mixtures to minimize the use of high-range water-reducing admixtures (HRWRA) and to optimize the use of fly ash in Self Consolidating Concrete (SCC). Four independent variables such as total binder content (350 to $450 \mathrm{~kg} / \mathrm{m} 3$ ), percentage of FA as cement replacement (30 to $60 \%$ by mass), percentage of HRWRA 
( 0.1 to $0.6 \%$ by solid mass), and water-binder ratio w/b (0.33 to 0.45$)$ were used for the design of SCC mixtures. The fresh concrete properties were determined from slump flow, V-funnel flow, filling capacity, bleeding, and air content, and segregation tests. The mechanical properties and durability characteristics of SCC such as compressive strength, freezing-and-thawing resistance, rapid chloride permeability, surface scaling resistance, and drying shrinkage were determined to evaluate the performance of SCC. Four statistical models to predict the slump flow, 1- and 28-day compressive strength, and the rapid chloride permeability of SCC were developed and their performances were validated. The models can be used as economical tools for the optimized design of FA SCC mixtures with desired properties in practical applications.

Arabi et al. (2009) carried out statistical experimental design for predicting workability and hardened properties of Self-Compacting Concrete (SCC). Four key parameters such as cement, water to powder ratio, fly ash and super plasticizer that can have significant influence on the mix characteristics of SCC were selected to derive the mathematical models for evaluating relevant properties. The responses included compressive strength at 3, 7 and 28 days and modulus of elasticity. Thirty one mixtures were prepared to derive the numerical models and evaluate the accuracy. The models were valid for a wide range of mixture proportioning. The research presented derived numerical models that can be useful to reduce the test procedures and trials needed for the proportioning of self-compacting concrete. The qualities of these models were evaluated based on several factors such as level prediction, residual error, residual mean square and correlation coefficients.

Nabil et al. (2010) adopted design of experiment method to study different procedures to avoid plastic shrinkage cracking of concrete in hot climates. This method has been employed by the researcher in order to reduce the number of tests, increase the number of studied factors and to study the interaction between the factors. The key factors chosen for the study were plastic sheets, curing agent, cold water and polypropylene fiber and each factor has two levels. Secondary factors selected were environmental factors such as temperature and wind. The output response parameters were plastic shrinkage cracks, evaporation rate, tensile and compressive strengths. 
Cihan et al. (2013) developed response surface for compressive strength of concrete. For adequate optimization the main, quadratic and interaction terms of influence levels of controllable variables on concrete compressive strength should be determined and response surfaces should be established in ready-mixed concrete production where a large number of effect parameters were there. Firstly influence levels of the main and interaction terms of effect variables were determined using $2^{7-3}$ fractional factorial design in order to reduce the number of simultaneously controllable variables. Then, quadratic terms were determined using D-Optimal design, and response surface graphics were plotted.

A step-by-step statistical approach is proposed by Shamsad and Saeid (2014) to obtain optimum proportioning of concrete mixtures using the data obtained through a statistically planned experimental program. The utility of the proposed approach for optimizing the design of concrete mixture was illustrated considering a typical case in which trial mixtures were considered according to a full factorial experiment design involving three factors and their three levels with three replicates. The response considered was compressive strength and the key factors affecting compressive strength of concrete considered were water to cementitious materials ratio, cementitious materials content, and fine/total aggregate ratio. The experimental data were utilized to carry out analysis of variance (ANOVA) and to develop a polynomial regression model for compressive strength in terms of the three design factors considered in this study. The developed statistical model was used to show how optimization of concrete mixtures can be carried out with different possible options.

Lotfy et al. (2015) developed statistical models to study the influence of key mix design parameters on the properties of lightweight self-consolidating concrete (LWSCC) with expanded shale (ESH) aggregates. Responses included slump flow diameter, V-funnel flow time, J-ring flow diameter, Jring height difference, L-box ratio, filling capacity, sieve segregation, unit weight and compressive strength. The developed models were valid for mixes with $0.30-0.40$ water-to-binder ratio, high range water reducing admixture of $0.3-1.2 \%$ (by total content of binder) and total binder content of $410-550 \mathrm{~kg} / \mathrm{m}^{3}$. The models were able to identify the influential mix design parameters and their interactions which can be useful to reduce the test protocol needed for proportioning of LWSCCs. Three industrial class ESH-LWSCC mixtures were developed using statistical models 
and their performance was validated through test results with good agreement. The developed ESH-LWSCC mixtures were able to satisfy the European EFNARC criteria for self-consolidating concrete.

\subsection{Biodeterioration in concrete}

Concrete is potentially vulnerable to a variety of biodeterioration processes promoted by microbial activities (Lajili et al., 2008). Biodegradability of concrete is mostly due to the increased concentration of carbonates and inorganic sulfur compounds, as well as other chemically aggressive reagents of either abiotic or biotic nature (Beata, 2008). The microorganisms most frequently involved in biodeterioration of cementitious materials are both autotrophic and heterotrophic bacteria, cyanobacteria, fungi and algae (Gaylarde et al., 2003). There has been some discussion in the literature regarding the role of sulfur reducing bacterial strains such as Thiobacillus thiooxidans and Thiobacillus ferrooxidans, iron and sulphur oxidising bacteria genera Acidithiobacillus in sewer and water treatment facilities in transportation structures (Covino, 1999; Roberts et al., 2002). Acidophilic organisms are known to cause severe damage in concrete sewer pipes. In sewers, hydrogen sulfide, generated by anaerobic sulfate reducing bacteria, oxidises the sulfur to sulfuric acid. The acid then reacts with calcium hydroxide, weakens the concrete (Islander, 1999). If any bacteria do become viable, the localized reduction in $\mathrm{pH}$ by the bacteria itself will lead to acid attack of the concrete (Islander, 1999).

However, many researchers investigated the application of bacterial calcite in enhancing the durability of cementitious structures (Ramachandran et al., 2001; Bang et al., 2001; Ghosh et al., 2005; Ramakrishnan., 2007; Jonkers et al., 2007; Achal et al., 2011). A novel technique was developed for the remediation of damaged structural formations that employs a selective microbial plugging process, in which microbial metabolic activities promote precipitation of calcium carbonate in the form of calcite (Gollapudi et al., 1995). As a microbial sealant, $\mathrm{CaCO}_{3}$ exhibited its positive potential to selectively consolidate simulated fractures and surface fissures in granites and sand plugging (Zhong and Islam, 1995; Achal et al., 2009). Many studies have been reported to assess the application of bacteria in concrete for the remediation of cracks (Stocks-Fisher et al., 1999; Ramakrishnan et al., 2001; Day J L et al., 2003; Patil et al., 2008; Raijiwala et al., 2009). 
Because of the highly alkaline environment exists in the concrete matrix, it is appeared to be unfavourable for life at first. However, bacterial spores, as mentioned previously, can survive in this high alkaline environment. When the water seeps into the structure through cracks and with the necessary nutrients either in the form of carriers or brought in by the water, the surrounding $\mathrm{pH}$ would lowered to values in the range ( $\mathrm{pH} 10$ to 11.5), and by this process the bacterial spores become activated.

\subsection{Microbial Calcite Precipitation Overview}

Microbial calcite precipitation is a form of autonomic intrinsic self-healing mechanism that got more of the attention recently. A number of bacteria which can be found in soil, sand and natural minerals have the ability to precipitate calcium carbonate both in natural and in laboratory conditions (Krumbein, 1979; Rodriguez et al., 2003). In nature 3 groups of organisms can induce Microbial Calcite Precipitation (MCP): (i) Photosynthetic organisms- such as cyanobacteria and algae that remove $\mathrm{CO}_{2}$, (ii) Sulphate reducing bacteria- that are responsible for dissimilatory reduction of sulphates and (iii) organisms that are involved in the nitrogen cycle either ammonification of amino acids/ nitrate reduction/ hydrolysis of urea (Castanier et al. 1999; Hammes and Verstraete, 2002). Among these, hydrolysis of urea by the enzyme urease is the simplest of all the Microbial Induced Calcite Precipitation (MICP) mechanisms (Whiffin 2004).

\subsubsection{Biological processes of Microbial Induced Calcite Precipitation (MICP)}

Different types of bacteria as well as abiotic factors such as salinity, surrounding $\mathrm{pH}$, temperature and available nutrients composition of the medium play a significant role in the precipitation of calcium carbonate in a wide range of different environments (Knorre and Krumbein, 2000; Rivadeneyra et al., 2004). Four key factors which govern the MICP are: (i) the calcium concentration, (ii) the concentration of dissolved inorganic carbon, (iii) the $\mathrm{pH}$ and (iv) the availability of nucleation sites (Hammes and Verstraete, 2002).

The urease enzymes produced by the bacteria decompose urea into ammonium and carbonate ions. The chemical reaction is given below (Ng et al., 2012):

$$
\begin{aligned}
& \mathrm{CO}\left(\mathrm{NH}_{2}\right)_{2}+2 \mathrm{H}_{2} \mathrm{O} \rightarrow 2 \mathrm{NH}_{4}^{+}+\mathrm{CO}_{3}^{2-} \\
& \mathrm{Ca}^{2+} \mathrm{CO}_{3}{ }^{2-} \rightarrow \mathrm{CaCO}_{3}
\end{aligned}
$$


The ammonium ions $\left(\mathrm{NH}_{4}{ }^{+}\right)$released from urea hydrolysis increases local $\mathrm{pH}$ and commence the precipitation of calcium carbonate. The high $\mathrm{pH}$ at localised area increases the tendency for bacteria itself to serve as nucleation site for calcite crystal. Calcite is precipitated through the combination of carbonate ions $\left(\mathrm{CO}_{3}{ }^{2-}\right)$ from the hydrolysis of urea and the calcium ion $\left(\mathrm{Ca}^{2+}\right)$ from supplied calcium compound (Stocks-Fisher et al., 1999).

\subsubsection{Influence of Nutrients}

Since nutrients are the main energy sources for bacteria, it is very important to provide proper and sufficient nutrient for calcite producing bacteria. Nutrients are supplied to bacteria during culture stage and concrete casting or treatment stage depending on the specific application. The common nutrients for bacteria include $\mathrm{CO}_{2}, \mathrm{~N}, \mathrm{P}, \mathrm{K}, \mathrm{Mg}, \mathrm{Ca}$ and Fe (Mitchell and Santamarina, 2005). Rodriguez-Navarro et al. (2003) observed a reduced efficiency of calcium carbonate deposition with a very rapid bacterial carbonate precipitation and also observed that rhombohedral calcite crystals showed a very good consolidating effect compared to the small calcite crystals. Numerous researches on calcite precipitation have been carried out using different calcium sources such as calcium chloride $\left(\mathrm{CaCl}_{2} .2 \mathrm{H}_{2} \mathrm{O}\right)$, calcium lactate, calcium glutamate, calcium acetate $\left(\mathrm{Ca}\left(\mathrm{CH}_{3} \mathrm{COO}\right)_{2} . \mathrm{H}_{2} \mathrm{O}\right)$ and calcium nitrate $\left(\mathrm{Ca}\left(\mathrm{NO}_{3}\right)_{2} .4 \mathrm{H}_{2} \mathrm{O}\right)$, (Bang et al. 2001; Tittelboom et al., 2009; Ramachandran et al. 2001; Jonkers \& Erik Schlangen, 2008; Wang et al, 2012). It has been reported that calcium lactate is a good choice because it starts to dissolve during the mixing process and does not interfere with the setting time of concrete (Jonkers et al, 2010). However, calcium chloride as the calcium source is not ideal because the chloride ions maybe harmful for the concrete reinforcement (Jonkers and Schlangen, 2009). However, further studies are needed to identify the effects from different types of nutrients and metabolic products used for growing calcifying microorganisms, and their influence on survival, growth, biofilm and crystal formation.

\subsubsection{Influence of the type of microorganisms}

It was reported that the microbial mineral plugging system depends on the precipitation of carbonate ions (Ferris and Stehmeier, 1992; Zhong and Islam, 1995). The hydrolysis of urea is the most suitable pathway for the production of carbonate ions due to its ability to alkalinize the environment. Therefore, the bacteria should be able to act as a catalyst in the urea hydrolysis and they are usually urease positive bacteria. The ability to hydrolyze urea is widely distributed among indigenous bacteria in soils and groundwater systems (Mobley and Hausinger, 1989; Fujita et al., 
2000). Most urease positive bacteria belong genera Bacillus, Sporosarcina, Clostridium and Desulfotomaculum (Kucharski et al, 2008). Freshly made concrete is highly alkaline and is typically characterized by $\mathrm{pH}$ values between 11 and 13. Therefore the added bacteria should be able to withstand the mechanical stresses due to mixing and also to survive the high alkalinity for long term. The addition of bacterial cells directly in to the concrete specimen cannot be possible because of two reasons. One is due to the decrease in bacterial activity in the high alkaline environment $(\mathrm{pH}>12)$ present in concrete. Other one is because of the possibility of bacterial cells destruction during the process of hydration (Jonkers and Thijssen, 2010). It was reported that the bacteria did not survive due to the decreasing of pore diameters during the hydration of the cement materials (Jonkers et al, 2010). It was found that strains of the bacteria genus Bacillus were able to survive in the high-alkaline environment. These alkali-resistant bacteria typically form spores, which are specialized cells able to resist high mechanically and chemically induced stresses (Sagripanti and Bonifacino 1996). Also, these spores have low-metabolic activity and extremely long lifetimes and some species are known to produce spores which are viable for up to 200 years (Schlegel 1993). Also the incorporated bacteria need to be oxygen tolerant because the ingress of oxygen causes the concrete matrix to be oxic. Therefore, most promising bacterial agents appear to be aerobic alkaliphilic spore-forming bacteria of genus Bacillus (Jonkers et al, 2010) and similar genera like Sporosarcina. Some of the Bacillus were reclassified and Sporosarcina is one among them. In the previous literature, urea-utilizing bacteria Sporosarcina pasteurii (Santhosh et al., 2001, Bang et al., 2001 and Ramakrishnan et al,. 2005; Achal et al., 2011) and Bacillus sphaericus (Ramachandran et al., De Muynck et al., 2008; Wang et al., 2011) were the most commonly used bacteria in cementious materials and concrete while Shewanella species (Ghosh et al., 2005), Bacillus lentus (Dick et al., 2006), Bacillus halodurans, Bacillus psuedofirmus, Bacillus cohnii (Jonkers and Erik Schlangen, 2008; Jonkers et al., 2010), Arthrobacter crystallopoietes, Lysinibacillus fusiformis (Park et al., 2010), Bacillus sp. CT-5 (Achal et al., 2011), Bacillus alkalinitrilicus (Wiktor and Jonkers et al., 2011) have also been tested to a certain extent. It was also reported that various sub species of Bacillus subtilis involved in precipitation of calcite (Barabesi et al., 2007; Achal et al., 2011). However, it was noticed that the bacterial microorganism namely Escherichia coli do not have any role in improving the healing property. From this, it can be inferred that the selection of micro-organism plays a chief role in the improvement of compressive strength (Jonkers, 2007). 


\subsubsection{Carrier materials}

Bacterial spores can be further protected in concrete environment by immobilizing them in to carriers. Polyurethanes (PU) and silica gel have been widely used as a vehicle for immobilization of enzymes and whole cells because of its mechanically strong and biochemically inert characteristics (Fukushima et al., 1978; Wang and Ruchenstein, 1993, Wang et al., 2011). In general, silica gel immobilized bacteria showed a higher activity than PU immobilized bacteria; however, higher strength regaining and lower water permeability was observed with the PU immobilisation (Wang et al., 2011). Although metabolic activities of cells remain high in PU matrices, it is however uncertain whether the rate of cell growth remains the same (O'Reilly and Crawford, 1989; Sumino et al., 1992). Jonkers et al. (2010) used clay pellets that were around 2$4 \mathrm{~mm}$ wide as carrier material for the bacteria and the nutrients, but it was observed that the clay pellets occupy too much volume of the matrix and in turn negatively influenced the strength of the concrete. Diatomaceous earth (DE) consists of diatom skeletons which are highly porous, light in weight and chemically stable and inert with size ranging typically from 10 to $200 \mu \mathrm{m}$ was successfully used as a suitable carrier material for aerobic and anaerobic bacteria in bioscrubber (Sorial et al., 1997; Sorial et al., 1998; Wright, 2005), wastewater treatment applications (Durham et al, 1994; Peres, 1999; Kim, 2002; Bertin et al., 2004), and a high-pH concrete environment (Wang et al., 2012). The porous cells of DE pellets can provide home for microbes and oxygen, water and nutrients to help to protect the life of the bacterial colonies incorporated in the pellets. Previous literature revealed the successful use of pumice as carrier vehicle for removal of toxic compounds from industrial waste water (Di Lorenzo et al., 2005; Kitis et al., 2005). Based on the quite high and stable denitrification activity over time, the good mechanical properties, the low cost and the low energy requirements for production, pumice can be considered as a potential material for microbial immobilisation. Zeolite clinoptilolite is also a promising material for immobilization of microorganisms due to its roughness, large surface and high porosity. Zeolite was largely used as a bacterial immobilization material on waste water treatment on the base of its widespread occurrence in the nature, accessibility and feasibility, cost effectiveness, large surface area, rigidity, surface functionality, thermal, mechanical and radiation stability (Jasna et al, 2003).

There are many factors responsible for the adhesion of cells to inert surfaces. Of these factors,

carrier pore size has been described as the most important having a greater effect than macroscopic 
surface roughness and total surface area (Huysman et al., 1983; Seth et al., 1995). Optimal pore diameter is in the range of one to five times the diameter of the microbe, such that materials with pores in the range of $1-10 \mu \mathrm{m}$ are ideal for the immobilization of bacterial cells (Ince et al., 2000).

\subsection{Microbial concrete in cementitious materials}

The quality of concrete structures depends mainly on three parameters: compressive strength, permeability and corrosion resistance. Crack problems in concrete are mostly dealt by manual inspections and repair by impregnation of cracks with epoxy based fillers, latex binding agents like acrylic, polyvinyl acetate, butadiene styrene etc. But there are many disadvantageous aspects of traditional repair systems such as different thermal expansion coefficient compared to concrete, weak bonding, environmental and health hazards along with being costly. So many researchers investigated the application of bacterial calcite in enhancing the durability of cementitious buildings and restoration of structures (Dhami et al., 2012).

\subsubsection{Crack remediation treatments}

Even if a variety of techniques are available for crack repair, the traditional repair systems have a number of disadvantages such as different thermal expansion coefficient compared to concrete and environmental and health hazards. Microbiologically enhanced crack remediation was conducted by using a soil bacterium Bacillus pasteurii immobilized and protected in polyurethane polymer, lime, silica fume and fly ash. As a result, the remediated concrete has shown a significant increase in compressive strength and stiffness (Ramakrishnan et al., 2001; Day et al., 2003; Patil et al., 2008; Raijiwala et al., 2009). However, it was seen that the compressive strength was around 5\% higher than cells without immobilization. This supports that immobilization of cells increases cell retentivity and provides space to bacteria for more bacteriogenic activity.

De Belie \& De Muynck (2008) reported positive potential of microbiologically induced carbonate precipitation for the repair of cracks in concrete by $S$. sphaericus. The research team reported a complete sealing of artificial cracks of $0.3 \mathrm{~mm}$ wide and $10 \mathrm{~mm}$ deep and observed that the concrete permeability was reduced much more than that by a cement grout repair technique. Qian 
et al. (2010) also reported that compressive strength of treated specimens could be restored to $84 \%$ upon treatment of bacterial calcite.

\subsubsection{Improvement of concrete compressive strength}

One of the most important characteristics of the concrete which decides its durability is the compressive strength. Therefore, the influence of the application of bacterial concrete on compressive strength is a critical area to be investigated in order to realize the actual potential of the bacterial concrete. A considerable improvement in the compressive strength was observed by the incorporation of bacteria in concrete and mortar. It is believed that calcium carbonate crystals would have precipitated on the surface of cells and ultimately within the pores which in turn would result in sealing and blockage of oxygen and nutrients flow in to the cells. The cells either die or turn into endospores and act as organic fibers that contribute to improve the compressive strength of concrete or mortar cubes (Ramachandran et al., 2001).

Numerous tests have been carried out in order to study the applicability of biological concrete to have an effect on the compressive strength of mortar and concrete (Bang et al., 2001; Ramachandran et al., 2001; Ghosh et al., 2005; De Muynck et al., 2008; Jonkers et al., 2010; Achal et al., 2011). In these various experimental studies, different micro-organisms have been applied in the concrete mixture. Ramchandran et al. (2001) conducted studies by incorporating various concentrations of the bacterial species Bacillus pasteurii in the cement mortar cubes. They found that there was a considerable increment in compressive strength at 7 and 28 days which resulted from the presence of adequate amount of organic substances in the matrix due to microbial biomass. Ghosh et al. (2005) studied the positive potential of a new type of thermophilic anaerobic microorganism belonging to Shewanella species on compressive strength of mortar specimens. They observed an increase in compressive strength of 25-30\% after 28 days. This is due to growth of filler material within the pores of the cement-sand matrix. Jonkers (2007) selected Bacillus pseudofirmus and Bacillus cohnii to concrete specimens and found a $10 \%$ increase in the compressive strength while Achal et al. (2009) selected the bacterial species Sporosarcina pasteurii with mortar cubes and noticed a $17 \%$ increase in compressive strength. A $22 \%$ increase in the compressive strength was observed by Park et al. (2010) when the mortar cubes were treated by the species Arthrobacter crystallopoietes. In their study, out of four selected microorganisms, namely Sporosarcina soli, Bacillus massiliensis, Arthrobacter crystallopoietes and Lysinibacillus 
fusiformis, the strength increment exhibited by Arthrobacter crystallopoietes was the highest. However, a 36\% increase in compressive strength was noticed by Achal et al. (2011) with the mixing of Bacillus sp. CT-5 to mortar specimen.

Navneet et al (2012) studied the influence of the bacteria Sporosarcina pasteurii on the compressive strength of fly ash concrete and reported a maximum increase of $22 \%$ in compressive strength. In this study, cement was replaced with three percentages (10, 20 and 30) with fly ash by weight and three different bacterial cell concentration, $10^{3}, 10^{5}$ and $10^{7}$ cells $/ \mathrm{ml}$ were used. This improvement in compressive strength was observed due to deposition on the bacteria cell surfaces within the pores.

\subsubsection{Reduction in permeability of concrete}

Another important feature which affects the durability of concrete is permeability. Concrete with

high permeability leads to the percolation of water and pollutants into the concrete, affects the structural integrity and ultimately affects the durability of the concrete. Therefore, it is reported that concrete with low permeability has longer service life (Nolan et al., 1995). Since bacterial precipitation mainly takes place on the surface layer, this calcite precipitation can be regarded as a covering system (Ramakrishnan et al., 1998). Permeability can be examined by carbonation tests because it is known that decrease in gas permeability due to surface treatment results leads to an increase in resistance towards carbonation and chloride ingress. Ramakrishnan et al. (1998) reported an increase in resistance of concrete towards alkali, freeze thaw attack, drying shrinkage and reduction in permeability upon application of bacterial cells. The influence of the precipitation of calcium carbonate on permeability is studied by De Muynck et al. (2008) using the microorganism S. Sphaericus on mortar cubes and observed a significant reduction in permeability when compared with untreated mortar samples.

Achal et al. (2011) observed a considerable reduction in water permeability in cement mortar cubes by using Sporosarcina pasteurii. It is believed that this lower permeability of bacteria incorporated cubes may be due to the presence of a denser interfacial zone formed between the aggregate and the concrete matrix by calcite precipitation. Due to the better compaction and closing of pores at the top, they observed more water permeability at the sides than that at the top. This gives a more believable insight on the influence of microbial calcite on the permeability of concrete. The same 
group studied the effect of Bacillus pasteurii on water impermeability and observed the similar result (Achal et al., 2010). In addition, six times reduction in absorption of water in mortar cubes were observed when compared with untreated specimen up on incorporation with Bacillus sp. CT5 (Achal et al., 2011). About eight times reduction in chloride permeability were observed by Navneet et al (2012) when studies were conducted on the Influence of the bacteria Sporosarcina pasteurii on fly ash concrete. It is believed that this reduction in permeability might be due to the presence of calcite deposition in concrete. Also, a $68 \%$ reduction in water permeability was reported by Wang et al (2014) when hydrogel encapsulated Bacillus sphaericus spores were applied into the mortar specimen to investigate self-healing.

\subsubsection{Reduction in water absorption of concrete}

Navneet et al. (2012) observed a four times reduction in water absorption of bacteria based fly ash concrete. Water absorption test at 7-days was conducted as per ASTM C 642. It was noticed that with the inclusion of bacteria, water absorption capacity of fly ash concretes decreased with the increase in bacteria concentration. Maximum reduction in water absorption was observed with $10^{5}$ cells $/ \mathrm{ml}$ for all fly ash concretes including concrete with $10 \%$ fly ash concrete gave $3.25 \%$ water absorption (minimum). In this study, the presence of bacteria resulted in a significant decrease in the water uptake compared to control specimens. The deposition of a layer of calcium carbonate on the surface and inside pores of the concrete specimens resulted in a decrease of water absorption.

Farshad et al. (2014) reported that the value of sorptivity coefficient for 28 days old biologically treated mortar specimens not amended and amended with silica fume was 42-48 \% and 57-64 \% lower, respectively, than the corresponding value determined for untreated specimens. Their results showed that in the case of normal specimens, even though the sorptivity was very much reduced, bacterial treatment of cracked specimens did not reduce the value of sorptivity down to the same value as uncracked specimens. However, they have reported that bacterially treated cracked specimens that contained silica fume had sorptivity coefficients that were very similar to bacterial treated uncracked specimens. 


\subsubsection{Improvement of mechanical performance of concrete}

In spite of a number of prior works, reported results on mechanical performance of concrete treated by bacterial self-healing were very rare. Jing $\mathrm{Xu}$ and $\mathrm{Wu}$ (2014) focused on the mechanical behavior of concrete incorporating a non-ureolytic bacteria-based healing agent at multi scale levels. In the research, four-point bending and ultrasonic pulse velocity (UPV) as macro scale mechanical measurements were performed to evaluate the mechanical properties of concrete during the processes of damaging and healing. In addition, nano indentation as a nano scale mechanical test was carried out to investigate the nano-mechanical properties of mineral precipitation and its bonding to concrete. Their results showed that the type of calcium source has a profound impact on healing effectiveness. Ultrasonic pulse velocity and four-point bending tests demonstrated that the highest healing ratio and recovery ratio of flexural strength and modulus were obtained by the two-component self-healing with calcium glutamate.

Flexural behavior of bio-based the ECC materials were evaluated by Sierra-Beltran et al. (2014). After cracking and healing the mixtures with bio-based healing agent show a slightly better recovery of both flexural strength and deflection capacity from control mixtures without bio-based healing agent.

\subsubsection{Corrosion reduction in reinforced concrete}

Corrosion of steel in concrete is considered to be one of the main reasons for the structural failure. Permeability and corrosion are considered to be interrelated because permeability leads to the ingress of chloride ions and impurities into the concrete leading to corrosion of steel which in turn affects the durability of the concrete. It was observed that the bacterial calcium carbonate precipitation aided to reduce the permeability by plugging the path of percolation of impurities into the concrete matrix (Jonkers et al., 2007). Mukherjee et al. (2010) observed a considerable reduction in corrosion of steel reinforcement by reducing the water and chloride ion percolation because of the precipitation of calcite using the microorganism Sporosarcina pasteurii and Bacillus sp. CT-5. 


\subsection{Self-healing concrete approaches}

\subsubsection{Autogenous crack healing}

Autogenous healing is the natural process of crack repair that can occur in concrete in the presence of moisture, and the absence of tensile stress. Due to the autogenous healing, there will be a gradual reduction in the water flows through the cracks and, in extreme cases; there will be a complete closure of cracks (Edvardsen, 1999). The major reasons for autogenous healing were swelling and hydration of cement paste, precipitation of calcium carbonate crystals, blocking of flow path by water impurities and blocking of flow path by concrete particles broken from the cracks surfaces due to cracking (Clear et al., 1985; Guppy, 1988). However, the most significant factor which influences autogenous healing is the precipitation of calcium carbonate (Edvardsen, 1999). As suggested by many previous studies (Clear 1985; Edvardsen 1999; Reinhardt and Joos 2003), the crack width of the concrete material was found to be critical for self-healing to take place. The requirement of crack width to promote self-healing falls roughly below $200 \mu \mathrm{m}$, preferably lower than $50 \mu \mathrm{m}$ (Li and Yang 2007).

\subsubsection{Bacterial concrete}

A more advanced technology was introduced in the recent literature in which a biological concrete was being developed that uses specially selected bacteria of the genus Bacillus, along with a combination of nutrients to create a healing agent within the concrete (Jonkers and Schlangen, 2008; De Muynck et al., 2008; Jonkers, 2011, Wang et al., 2011). With this bacteria mediated selfhealing concrete it is possible to seal the crack width of more than $100 \mu \mathrm{m}$ (Jonkers, 2011). Therefore, this area of research appears to be promising alternative to non-sustainable cement based healing systems. In this approach, the bacteria convert $\mathrm{CO}_{2}$ into carbonate ions under alkaline condition and subsequently these react with $\mathrm{Ca}$ ions from the concrete matrix leading to the formation of calcium carbonate crystals. In addition, locally produced $\mathrm{CO}_{2}$ directly reacts with calcium hydroxide in the matrix which leads to the calcite precipitation (Jonkers and Schlangen, 2008; Wang et al., 2011). This production of large sized calcium carbonate crystals in the bacteria incorporated self-healing concrete leads to a superior self-healing capacity compared to

conventional or engineered non-sustainable self-healing cementitious systems (Jonkers and Schlangen, 2009). 


\subsubsection{Other methods}

In order to create a self-healing concrete, several chemical approaches have been proposed by various researchers (Thao et al., 2007; Nishiwaki et al., 2000 ; Tittelboom et al., 2011; Pelletier et al, 2010; Zhengxian Yang et al, 2011). In those approaches, a healing agent was encapsulated inside a microcapsule and upon cracking the microcapsule ruptured, released the healing agent and ultimately healed the crack. Numerous healing agents, such as epoxy resins (Nishiwaki et al., 2006; Thao et al., 2007), cyanacrylates (Li et al., 2009; Dry et al., 2001; Joseph et al., 2007), and alkalisilica solutions (Mihashi et al., 2000), were tested and found that the material could be able to regain its mechanical strength almost similar to the strength regained by manual crack healing. These healing agents all have some common characteristics like low viscosity to guarantee a wider repair area, excellent bond between crack surfaces and enough capillary forces to move the agent into the crack. Also, there must be enough capillary forces in order to move the agent into the crack. Several methods were proposed to encapsulate the healing agent into the matrix such as the use of micro-capsules (Tittelboom et al., 2011; Pelletier et al, 2010), a continuous glass supply tube (Mihashi et al., 2000) and capillary tubes. The healing of cracks in multiple locations was possible with the use of microcapsules. However, after consuming the whole capsule, a permanent cavity was formed at that location in the concrete (Tittelboom et al., 2011). Capillary tubes used in the medical profession for blood testing can also be used as encapsulating tubes in concrete (Joseph et al., 2007). Another approach is the use of continuous glass supply pipes to heal larger cracks in which it is possible to change the healing agent. Additional healing agent can also be provided in this approach (Dry et al., 2001). However, the concrete becomes more brittle with the addition of some chemicals which are originally intended to seal the cracks. Hence, there is a chance of reduced durability of concrete with the use of chemical approach (Dry et al., 2001; Mihashi et al., 2000).

\subsection{Crack creation methods in concrete}

Several methods have been employed to create cracks in concrete or mortar specimen out of which most widely used method was crack width controlled three-point bending test (Titleboom et al., 2010; Pelletier et al., 2010; Yang et al., 2011; Wang et al., 2011). In this method, a linear variable differential transformer (LVDT) attached at the bottom of the specimen measures the crack width. 
Wiktor and Jonkers (2011) employed a computer controlled application of tensile force to create multiple cracks. In order to make this possible, a zinc plated steel bar was placed at the centre horizontal axis of the mould with the bar extending on both sides. Some researchers conducted splitting tensile tests to create cracks on concrete cylinders wrapped in fiber reinforced polymer (Titleboom et al, 2010; Wang et al., 2011). In another approach, the mortar samples were casted with a cut to simulate cracks with average width of $3.175 \mathrm{~mm}$ and depths of 12.7, 19.05 and 25.4 mm (Ramachandran et al., 2002). Titleboom et al., (2010) and De Belie and De Muynck (2009) introduced a thin copper plate of $0.3 \mathrm{~mm}$ thickness in the fresh concrete paste up to a depth of 10 $\mathrm{mm}$ or $20 \mathrm{~mm}$ which resulted in the formation of a narrow groove on the upper surface upon demoulding after 24 hours.

\subsection{Testing and visualization aspects of self-healing}

Numerous techniques have been employed in order to examine the self-healing quality of concrete materials. Two most common techniques which come under this category are water permeability test (Tittelboom et al., 2009; De Muynck et al., 2008) and acoustic emission technology (De Muynck et al., 2008; De Belie and De Muynck, 2009). The cracked and uncracked specimens can be subjected to a water permeability test in order to investigate the efficiency of the healing mechanism. The permeability test can be used to examine self-healing of a single crack as well as multiple cracks through water permeability. In this method, the drop in water level due to the water flow through the specimen can be measured at regular time intervals on a vaccum saturated specimen and the permeability coefficient $(\mathrm{k})$ can be determined using the formula given in eq. 2.13:

$$
k=\frac{a \cdot L}{A \cdot t_{f}}\left(\frac{h_{0}}{h_{f}}\right)
$$

where, $\mathrm{a}$ is the cross-sectional area of the standpipe, $\mathrm{L}$ is the specimen thickness in the direction of flow, $\mathrm{A}$ is the cross-sectional area subject to flow, $\mathrm{t}_{\mathrm{f}}$ is the test duration, $\mathrm{h}_{\mathrm{o}}$ is the initial hydraulic head and $h_{f}$ is the final hydraulic head.

Acoustic emission (AE) is a micro seismic (elastic) wave generated from dislocations, micro cracking and other irreversible changes in a stressed material. The transmitted waves are detected by transducers on the surface of a specimen. An acoustic emission technology is based on the 
ultrasonic pulse velocity (UPV) measurement. However with UPV measurement, it is difficult to determine the extent of the accurate crack healing (Van der Zwaag, 2010). Also, other techniques such as resonant frequency or dynamic modulus measurements and pulse echo technique have been used to measure the self-healing process (Van der Zwaag, 2010). These methods will be beneficial to measure both the rate and extent of self-healing within the cracked concrete specimen. Recently, one-sided stress wave transmission measurements were used to describe the self-healing process (Ghosh, 2009; Van der Zwaag, 2010). Although this measurement method has the advantage of being relatively fast, the main drawback is that it fails to explicitly differentiate the crack widths of over 100 $\mathrm{m}$ (Van der Zwaag, 2010). However, with the above mentioned methods, it is difficult to clearly distinguish between the exact natures of self-healing, because it is difficult to predict whether recovery of mechanical and/or transport properties have actually occurred. Therefore, a group of the self-healing examination methods, such as dynamic modulus measurements, uniaxial tension test, water permeability test, surface chemical analysis (XEDS) and environmental scanning electron microscopy (ESEM), should be used in combination to have an extensive perception of self-healing behavior in concrete materials (Van der Zwaag, 2010). The dynamic modulus measurements impart rapid measures to evaluate the presence of self-healing while the self-healing of mechanical properties can be determined using the uniaxial tension test (Van der Zwaag, 2010). In order to observe the recovery of transport properties through penetration, the water permeability test can be employed (Tittelboom et al., 2009, De Muynck et al., 2008). The chemical composition and morphology of self-healing product can be examined through X-ray diffraction and environmental scanning electron microscopy (ESEM) (De Muynck et al., 2008; Jonkers and Schlangen, 2009; Titleboom et al, 2010).

\subsection{Numerical modeling of Self-healing mechanism}

Though the research in the field of self-healing materials focused primarily on laboratory and experimental work, there are a few publications devoted to the mathematical modeling of the selfhealing processes. Zemskov et al (2011) considered two mathematical models for bacterial selfhealing of a crack. The first model concerned an analytic formulation to compute the probability that a crack hits an encapsulated particle. Hence, it was supposed to predict the probability that the self-healing process starts. The second model of the self-healing process was based on a moving boundary problem. A Galerkin finite-element method was used to solve the diffusion equations. 
The functions built in this paper allow to estimate combinations of crack lengths, capsule size, and mean intercapsule distance in order to analyse the efficiency of a self-healing material. The study is performed in the framework of the investigation of the potential of bacteria to act as a catalyst of the self-healing process in concrete.

A self-healing, metal matrix composite reinforced by shape memory alloy wires (SMA) was simulated using finite element analysis by Gao et al. (2004). A one-dimensional constitutive model for SMA behavior is implemented as a user-defined truss element in ABAQUS by the team. In this method, a mode I crack was allowed to propagate through the brittle specimen upon loading. During the loading process the wires underwent a martensitic phase transformation, bridging of the crack took place. A simple heating was required to heal the crack which reverse transforms the wires and brought the crack faces back into contact. When using prestrained SMA wires for reinforcement, the reverse transformation of the wires during heating caused a closure force across the crack. The results gave some idea on design of self-healing composites using shape memory alloys.

Crack expanding calculation in composite materials with microcapsules was made by the means of FEA software ANSYS by Li et al (2009). In this method, when cracks travelled through microcapsules, stress was concentrated at the crack end and microcapsule ruptured, and then the encapsulated liquid came out to fill the crack by the capillary and polymerization with catalyst in the composite. As a result, the healing of crack happened.

\subsection{Future considerations for studying self-healing concrete}

Most of the applications of bacterial concrete done so far were for crack remediation treatments, which cannot be considered purely 'self-healing' because it was applied after the cracking occurred (Ramakrishnan et al, 2001; Day et al, 2003; De Muynck et al, 2008; Patil et al, 2008; Raijiwala et al, 2009; Tittelboom et al, 2009). In these studies, an efficient plugging of cracks and recovery of mechanical strength was observed which resulted from the presence of adequate amount of organic substances in the matrix due to microbial biomass. Only limited studies have been performed on pure self-healing bacterial concrete (Jonkers and Schlangen, 2009; Wang et al, 2009; Jonkers, 2011; Navneet et al, 2012; Jing Xu and Wu, 2014). Also, very limited studies have been conducted 
on bacteria based self-healing on ECC materials (Sierra-Beltran et al, 2014). The results of the experiment with microbial self- healing concrete showed that immobilized bacteria mediate the precipitation of minerals and, moreover, the bacteria and certain classes of needed food sources do not negatively affect concrete strength characteristics (Jonkers and Schlangen, 2009; Jonkers et al, 2010; Wang et al, 2011).

It can therefore be concluded that bacterially controlled crack-healing in concrete by mineral precipitation is potentially feasible. However, this concept needs further developments on some areas. It should still be clarified whether bacterial mineral precipitation effectively seals larger cracks, that is, significantly reduces the permeability of cracked concrete in order to protect the embedded reinforcement from corrosion and thus increases the durability of the material. Furthermore, bacterial species must be selected which, when part of the concrete matrix, remain viable for at least the expected lifetime of the construction. Even though no major breakthrough has been achieved so far in the field of self-healing concrete, it is a very promising area of research and the potential gains are enormous. 


\section{CHAPTER THREE: MATERIALS AND METHODS}

\subsection{Introduction}

In order to realize the aim of attaining a true self-healing bacterial concrete material which can cure the cracks by itself, right selection of ingredients, processes and their sequences are of utmost importance. This chapter intends to identify, select, prepare and quantify the materials necessary for the research as well as to design the required methodology that would produce favorable results. An in-depth description of the materials involved and the experimental methods employed are discussed in this chapter. Subsequently, various testing methodologies employed to examine the effectiveness of self-healing are also discussed in detail. The research is mainly divided into five phases: 1) study culturing, spore formation capability, germination characteristics, and percentage of survival in high temperature and $\mathrm{pH}$ treatments of different bacteria, 2) evaluating the ureolytic activity of selected immobilised bacteria in high pH cement slurry, 3) testing the influence of healing agent additions on the compressive strength of mortar cubes, 4) investigating self-healing behavior of cracked specimens which include: preparing and testing of cracked cement mortar cylinders to study the self-healing effect of bacteria on permeation properties as well as preparing and testing of cement mortar beam specimens (in which realistic cracks are made) to determine and quantify the efficiency of crack healing coupled with structural performance (in terms of loaddeflection response, strength etc.) with time, and 5) self-healing investigation on ECC materials. This chapter will cover experimental methods, bacteria and their characteristics as well as materials and their properties

\subsection{Materials, chemicals and other agents used}

\subsubsection{Bacteria}

As mentioned in the literature review, the most promising bacterial agents which can withstand the various extreme environmental conditions such as temperature, salinity, $\mathrm{pH}$, and oxygen concentration appear to be aerobic alkaliphilic spore-forming bacteria of genus Bacillus (Jonkers et al., 2010). Also mentioned in the previous chapter that in order to produce the carbonate ions which are essential for the microbial mineral precipitation (Ferris and Stehmeier, 1992; Zhong and Islam, 1995), the selected bacteria should be a urease positive bacteria (Santhosh et al., 2001, Bang et al., 2001; Ramakrishnan et al., 2005; Achal et al., 2011). A urease positive bacterial species such as Sporosarcina pasteurii is selected as our control for our study because it has been widely 
used as a standard spore forming calcite bacteria in crack treatments in concrete. Sporosarcina ureae is another bacteria selected for the study as it has a promising urease activity (McCoy et al., 1992; Gruninger and Goldman, 1988) and to our knowledge even with this promising urease activity, it has not been used in concrete self-healing experiments. Sporosarcina ureae is chosen because this microbe is known to be very closely related to Sporosarcina pasteurii and possess high levels of nickel containing urease (McCoy et al., 1992). It was specified in the literature review that various sub species of Bacillus subtilis involved in precipitation of calcite (Barabesi et al., 2007, Achal et al., 2011). Therefore, Bacillus subtilis subsp. Spizizenii is also selected for the present study. In the genome, it has ure $\mathrm{A}$, ure $\mathrm{B}$ and $u r e \mathrm{C}$ genes and for all three subunits alpha, beta and gamma. Therefore, genetic potential wise, this species has the gene to do the work (Earl et al., 2012). All the above mentioned bacteria are spore forming bacterial species commonly isolated from soil.

Three different bacterial species, Sporosarcina ureae (DSM 2281), Sporosarcina pasteurii (DSM 33) and Bacillus subtilis subsp. Spizizenii (DSM 15029) were purchased from German Collection of Microorganisms and Cell Cultures (DSMZ), Braunschweig, Germany.

\subsubsection{Carrier materials}

The bacterial cells could not be added to concrete directly (Jonkers and Thijssen, 2010) in order to increase the bacterial activity and viability. Therefore, in this study, potential use of different materials such as zeolite and pumice as protective vehicle for bacteria are to be compared. It was mentioned in the literature review that the successful use of pumice as carrier vehicle for removal of toxic compounds from industrial waste water (Di Lorenzo et al., 2005; Kitis et al., 2005). Pumice is an inert aluminosilicate mineral of volcanic origin characterized by high porosity and, by low density (Boertje, 1995; Challinor, 1996). However, no one used this material as bacterial carrier in concrete. Based on the quite high and stable denitrification activity over time, the good mechanical properties, the low cost and the low energy requirements for production, pumice is also selected as a potential material for microbial immobilisation for this study. Another selected carrier material is zeolite clinoptilolite, which is also a promising material for immobilization of microorganisms due to its roughness, large surface and high porosity. These are crystalline, microporous, hydrated alumino-silicate minerals with pore size ranging from $0.3-0.1 \mathrm{~nm}$ (Bogdevov et 
al., 2009). Even though no one used this material as bacterial carrier in concrete, zeolite is largely used as a bacterial immobilization material on wastewater treatment on the base of its widespread occurrence in the nature (Jasna et al., 2003).

Zeolite (zeosand) was supplied by Zeo Inc, McKinney, TX, USA and Pumice was supplied by Garibaldi Pumice Ltd, Burnaby, BC, Canada. Table 3.1 presents the physical and chemical properties of Zeolite and Pumice.

Table 3.1: Physical and chemical properties of zeolite

\begin{tabular}{|l|c|c|}
\hline \multicolumn{3}{|c|}{ ZHYSICAL PROPERTIES } \\
\hline Particle size & $\begin{array}{c}0.42 \mathrm{~mm} \text { to } 1.4 \mathrm{~mm} \text {. Less than } 2 \% \\
\text { smaller than 0.42mm and less than } 2 \% \\
\text { larger than 1.4 mm }\end{array}$ & 0.1 to $0.3 \mathrm{~mm}$ \\
\hline Effective size & $0.62 \mathrm{~mm}$ & \\
\hline Uniformity coefficient & 1.6 & \\
\hline Average size & $0.8 \mathrm{~mm}$ & $608 \mathrm{~kg} / \mathrm{m}^{3}$ \\
\hline Bulk density & $880 \mathrm{~kg} / \mathrm{m}^{3}$ & Light grey white \\
\hline Color & Light grey green & 1.66 \\
\hline BET surface area & $29.3 \mathrm{~m}^{2} / \mathrm{g}$ & \\
\hline Specific gravity & & $60 \%$ \\
\hline & CHEMICAL PROPERTIES \\
\hline Silicon dioxide & $64-70 \%$ & $16 \%$ \\
\hline Alumina & $10-12 \%$ & $2 \%$ \\
\hline Potassium oxide & $3-5 \%$ & $5 \%$ \\
\hline Calcium oxide & $1-3 \%$ & $6 \%$ \\
\hline Sodium oxide & $2-0.5 \%$ & \\
\hline Moisture & $<10$ & \\
\hline Zeolite type & Clinoptilolite & \\
\hline
\end{tabular}




\subsubsection{Mineral substrate}

For the incorporated bacteria to precipitate limestone, a suitable mineral substrate is to be provided along with bacteria during casting. Calcium lactate was selected for the present study as a calcium source because of its successful use in the concrete (Jonkers et al, 2010). Bacteria with ureolytic activity use urea as a source of nitrogen, where urease hydrolyses urea releasing two ammonium molecules and carbonate ions (Whiffin, 2004). Therefore, urea is selected as the nitrogen source for the bacteria. In addition, yeast extract is selected as a medium supplement for the study as a growth medium for bacteria because of its successful use in concrete (Wang et al., 2005).

Calcium lactate was purchased from Sigma Aldrich Canada Ltd. (Oakville, Ontario) and urea and yeast extract were purchased from Bio basic Canada Inc. (Markham, Ontario).

\subsubsection{Other chemicals and falcon tubes}

All the other chemicals (unless otherwise specified) were analytical grade and obtained from Fisher Scientific Ltd. (Nepean, Ontario), Sigma Aldrich Canada Ltd. (Oakville, Ontario) and Bio basic Canada Inc. (Markham, Ontario). Falcon tubes were purchased from Fisher Scientific Ltd. (Nepean, Ontario).

\subsubsection{Mortar mix parameters}

\subsubsection{Cement}

Cement used was Type GU/10 Normal Portland Cement manufactured and supplied by St. Marys cement. A summary of physical and chemical properties of cement is presented in Table 3.2.

\subsubsection{Fly sh}

Class-CI fly ash conforming to ASTM C-618 (2012) requirements obtained from Lafarge plant was used for engineered cementitious composite (ECC) mixes. The chemical and physical properties are given in Table 3.2.

\subsubsection{Fine aggregate}

Concrete sand was used as fine aggregate for normal mortar mixes and mortar sand is used for ECC mixes. Sieve analysis of the used aggregates is shown in Table 3.3. Fineness modulus for concrete sand is 2.45 and mortar sand is 1.56 . Absorption and bulk relative density for concrete 1sand is 1.08 and $2.66 \mathrm{~kg} / \mathrm{m}^{3}$, respectively. 
Table 3.2: Physical and chemical properties of cement and Fly ash

\begin{tabular}{|c|c|c|}
\hline & Cement & Fly ash CI \\
\hline \multicolumn{3}{|c|}{ PHYSICAL PROPERTIES } \\
\hline Specific Gravity & 3.15 & 2.43 \\
\hline \multicolumn{3}{|l|}{ Fineness } \\
\hline Residue $45 \mu \mathrm{m}(\%)$ & 3 & 17.5 \\
\hline Specific surface, Blaine, $\mathrm{m}^{2} / \mathrm{kg}$ & 410 & \\
\hline \multicolumn{3}{|l|}{ Compressive strength (MPa) } \\
\hline 7- day & 32.5 & \\
\hline 28-day & 40.5 & \\
\hline \multicolumn{3}{|l|}{ Setting time (min) } \\
\hline Initial setting time & 91 & \\
\hline Final setting time & 198 & \\
\hline Air content of mortar (volume \%) & 8.6 & \\
\hline Autoclave expansion (\%) & 0.333 & \\
\hline \multicolumn{3}{|c|}{ CHEMICAL PROPERTIES (\%) } \\
\hline Silicon dioxide & 19.86 & 41.57 \\
\hline Aluminium oxide & 4.45 & 26.12 \\
\hline Ferric oxide & 2.64 & 3.9 \\
\hline Calcium oxide & 62.13 & 14.3 \\
\hline Magnesium oxide & 3.27 & 3.4 \\
\hline Sulphur trioxide & 3.88 & 1.55 \\
\hline Total alkali as $\mathrm{Na}_{2} \mathrm{O}$ & 0.6 & 0.71 \\
\hline Loss on ignition & 2 & 1.49 \\
\hline
\end{tabular}


Table 3.3: Sieve analysis of the used aggregates

\begin{tabular}{|c|c|c|c|}
\hline & & Concrete sand & Mortar sand \\
\hline Sieve No & Sieve size $(\mathbf{m m})$ & \% retained & \% retained \\
\hline No. 4 & $4.75 \mathrm{~mm}$ & 0 & - \\
\hline No. 8 & $2.36 \mathrm{~mm}$ & 10.3 & 0 \\
\hline No.16 & $1.18 \mathrm{~mm}$ & 23 & 3 \\
\hline No.30 & $600 \mu \mathrm{m}$ & 41.7 & 12.6 \\
\hline No.50 & $300 \mu \mathrm{m}$ & 71.7 & 49.4 \\
\hline No.100 & $150 \mu \mathrm{m}$ & 95.7 & 91.4 \\
\hline No.200 & $75 \mu \mathrm{m}$ & 99.7 & 99.6 \\
\hline PAN & - & 100 & 100 \\
\hline
\end{tabular}

\subsubsection{Admixtures}

ADVA ${ }^{\circledR}$ CAST 575 from Grace Construction Products was used as High Range Water Reducing Admixture (HRWRA) in order to improve the workability of ECC mixture. ADVA $®$ CAST 575 is poly carboxylic-ether with approximately 30\% solid content of and conforming to ASTM C 494 type F. The characteristics of this HRWRA are given in Table 3.4.

Table 3.4: Characteristics of HRWRA

\begin{tabular}{|c|c|}
\hline Description & Property \\
\hline Color & Turkish blue \\
\hline State & Liquid \\
\hline $\mathrm{pH}$ & $2.7-6.5$ \\
\hline Boiling point & $100^{\circ} \mathrm{C}$ \\
\hline Freezing point & $0^{\circ} \mathrm{C}$ \\
\hline Specific gravity & 1.1 \\
\hline
\end{tabular}




\subsubsection{Polyvinyl Alcohol (PVA) Fiber}

PVA fibers with a length of $8 \mathrm{~mm}$ and a diameter of $40 \mu \mathrm{m}$ were used for ECC mixture. The tensile strength of the PVA fiber is $1600 \mathrm{MPa}$ and the density is $1,300 \mathrm{~kg} / \mathrm{m}^{3}$. The fiber surface is coated with $1.2 \%$ oil by weight to reduce the fiber/matrix chemical and friction bond.

\subsection{Research Phase 1: Culturing and survival testing of bacteria}

This section discusses the testing methods to assess the growth, spore formation, germination, and percentage of survival in high temperature and $\mathrm{pH}$ treatments of different bacteria

\subsubsection{Bacteria culturing}

Liquid culture of Sporosarcina ureae (DSM 2281) and Bacillus subtilis subsp. Spizizenii (DSM 15029) were grown on media consisted of $8 \mathrm{~g} / \mathrm{L}$ nutrient broth (Peptone: $5 \mathrm{~g} / \mathrm{L}$; and Meat extract: $3 \mathrm{~g} / \mathrm{L}$ ) at the $\mathrm{pH} 7$ as instructed by DSMZ. For S. ureae, $\mathrm{pH}$ was adjusted to 7 after the addition of $20 \mathrm{~g} / \mathrm{L}$ of urea. Liquid culture of Sporosarcina pasteurii (DSM 33) was grown in media consisted of $30 \mathrm{~g} / \mathrm{L}$ Trypic Soy broth (Peptone from casein: $15 \mathrm{~g}$; Peptone from soyameal: $5 \mathrm{~g}$ and NaCl: 5 g). The $\mathrm{pH}$ was adjusted to 7.3 and after the addition of $20 \mathrm{~g} / \mathrm{L}$ of urea. According to the DSMZ's recommendation, each medium was supplemented with $10 \mathrm{mg} / \mathrm{L}$ of $\mathrm{MnSO}_{4} \mathrm{X} \mathrm{H}_{2} \mathrm{O}$ to enhance the sporulation. All liquid media were sterilized by autoclaving for $20 \mathrm{~min}$ at $120^{\circ} \mathrm{C}$. All cultures were incubated aerobically at $30^{\circ} \mathrm{C}$ for $24 \mathrm{~h}$ with shaking at $250 \mathrm{rpm}$. Growth and sporulation yield of bacteria was checked regularly and quantified by light microscopic analysis. The culture was streaked on nutrient agar plates and kept at room temperature. Figure 3.1 shows the streaked agar plates of Sporosarcina pasteurii, Bacillus subtilis subsp. Spizizenii and Sporosarcina ureae culture. The pure culture was maintained in liquid, on nutrient agar plate, and cryopreserved in $20 \%$ glycerol at $-80^{\circ} \mathrm{C}$. 

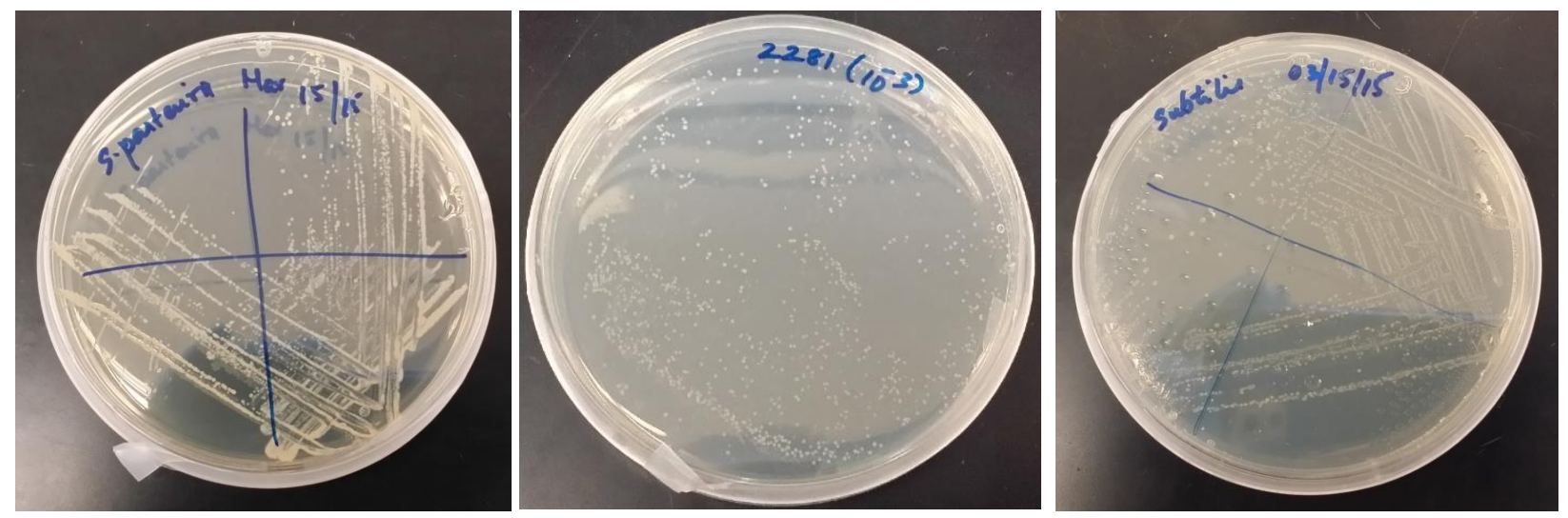

Figure 3.1: The streaked agar plates of Sporosarcina pasteurii (left), Sporosarcina ureae (centre) and Bacillus subtilis subsp. spizizenii (right) culture

\subsubsection{High heat and pH resistivity of bacteria}

In order to investigate the high heat and $\mathrm{pH}$ resistivity of the bacterial spores, the bacterial solutions were passed through high heat $\left(65^{\circ} \mathrm{C}\right)$ and high $\mathrm{pH}(\mathrm{pH} \mathrm{10)}$ for almost $1 \mathrm{hr}$. All cultures were incubated at $30^{\circ} \mathrm{C}$ on a shaker at $250 \mathrm{rpm}$ for $48 \mathrm{~h}$. Natural samples were suspended in a sterile physiological solution ( $9 \mathrm{~g}$ of $\mathrm{NaCl}$ per litre) diluted appropriately and plated on an agar containing the required growth medium specified by the suppliers. Viable counts were obtained by plating diluted cells on the surface of nutrient agar plates for Bacillus subtilis subsp. Spizizenii (DSM 15029), nutrient agar and urea plates for Sporosarcina ureae (DSM 2281), and Trypic Soy agar and urea plates for Sporosarcina pasteurii (DSM 33). Heat resistant spore counts were obtained by plating after heating at $65^{\circ} \mathrm{C}$ for $15 \mathrm{~min}$ and $45 \mathrm{~min}$.

\subsubsection{Mineral producing capability of bacteria}

In order to investigate the calcite crystal formation potential of all these selected bacteria, natural samples were suspended in a sterile physiological solution ( $9 \mathrm{~g}$ of $\mathrm{NaCl}$ per litre) diluted appropriately and plated on an agar containing $3 \mathrm{~g} / \mathrm{l}$ of Nutrient Broth; $20 \mathrm{~g} / \mathrm{l}$ of urea; $2.12 \mathrm{~g} / \mathrm{l}$ of $\mathrm{NaHCO}_{3} ; 10 \mathrm{~g} / \mathrm{l}$ of $\mathrm{NH}_{4} \mathrm{Cl}$ and $30 \mathrm{mM} \mathrm{CaCl} 22 \mathrm{H}_{2} \mathrm{O}$. Crystal formation was observed after 7 days and 14days. A sample of soil liquid was also plated to try to get environmental crystal formers. 


\subsection{Research Phase 2: Ureolytic activity testing of bacteria}

This section investigates the ureolytic activity of zeolite or pumice immobilised bacteria in high $\mathrm{pH}$ cement slurry.

\subsubsection{Preparation of calibration curve}

A series of standards containing the following volumes of ammonia nitrogen solution diluted to $5 \mathrm{~mL}$ with water was prepared: $0.0,100.0,200.0,300.0,400.0$ and $500.0 \mu \mathrm{L}$. Added $100 \mu \mathrm{L}$ of Nessler's reagent and mixed. After $30 \mathrm{~min}$, using a photometer absorbance measurements were taken at $425 \mathrm{~nm}$ in which distilled water were treated as the blank. Calibration curves were prepared based on these series values and presented in APPENDIX A.

\subsubsection{Bacteria and growth conditions}

The medium used to grow Sporosarcina ureae (DSM 2281) and Sporosarcina pasteurii (DSM 33) consisted of yeast extract and urea. The yeast extract medium was first autoclaved for $20 \mathrm{~min}$ at $120^{\circ} \mathrm{C}$ and then the sterilized urea solution was added, which was obtained by means of filtration through a sterile $0.2-\mu \mathrm{m}$ Millipore filter. The final concentrations of yeast extract and urea in the growth medium were $20 \mathrm{~g} / \mathrm{L}$ each. Cultures were incubated at $30^{\circ} \mathrm{C}$ on shaker at $150 \mathrm{rpm}$ for $24 \mathrm{~h}$. Bacterial cells were harvested by centrifuging the $24 \mathrm{~h}$ old grown cultures (5000 g, $5 \mathrm{~min}$ ) and were re-suspended in a physiological solution $(\mathrm{NaCl}, 9 \mathrm{~g} / \mathrm{L})$. The concentration of bacterial cells in the suspension was $10^{8}$ cells $/ \mathrm{ml}$.

\subsubsection{Activity of immobilized bacteria under neutral and high $\mathrm{pH}$ conditions}

The ureolytic activity of two different bacterial species, Sporosarcina ureae (DSM 2281) and Sporosarcina pasteurii (DSM 33) with and without immobilization into two different carrier materials such as zeolite and pumice was examined in different kinds of $\mathrm{pH}$ environments. Bacterial suspension obtained was mixed with the two different sterile carriers such as zeolite and pumice in a $50 \mathrm{ml}$ falcon tube. In each falcon tube, $30 \mathrm{ml}$ of bacterial suspension was mixed with the $4 \mathrm{~g}$ of carrier material and was put on a shaker for $1 \mathrm{~h}$ (Wang et al, 2005). Samples of zeolite and pumice are shown in Figure 3.2.

Urea medium with neutral $\mathrm{pH}$ was obtained by adjusting the $\mathrm{pH}$ by using a $1 \mathrm{M} \mathrm{NaOH}$ solution. High $\mathrm{pH}$ concrete environment was created by adding cement powder to the urea medium. In order 
to make sure that the cement powder reacted completely with water, the cement suspension was put on the shaker for one day and the $\mathrm{pH}$ was measured as 12.5(Wang et al, 2012). Then the zeolite or pumice immobilised bacteria was transferred to this cement suspension and this mixture is referred to as cement slurry. The cement slurry was put on the shaker for 5 days. Unimmobilised bacterial cells were also added to the cement suspension in order to compare the ureolytic activity of immobilised and unimmobilised bacterial cells. The ureolytic activity of the bacteria was indicated by the amount of urea decomposed by the bacteria, which was determined by the total ammonium nitrogen in the urea media. One mole of urea produces $2 \mathrm{~mol} \mathrm{of} \mathrm{NH}_{4}{ }^{+}$and hence the amount of $\mathrm{NH}_{4}{ }^{+}$can show the amount of urea decomposed. Amount of urea decompose in 1, 3 and 5 days was measured calorimetrically by the method of Nessler (Figurovskaya et al, 2005) which is described in section 3.3.4.
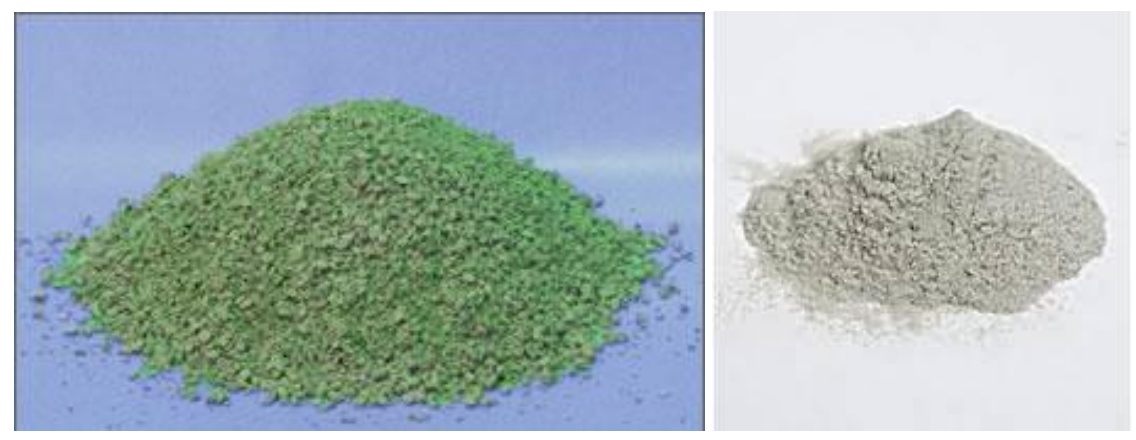

Figure 3.2: Zeolite (left) and pumice (right)

\subsubsection{NH4-N Analysis using Nessler's method}

Ammonuim concentration in the pumice/zeolite immobilised biomass in cement slurry was determined spectrophotometrically by the method of Nessler (Figurovskaya et al, 2005). Samples were immediately centrifuged and the resulting supernatant was transferred into a clean tube and frozen prior to analysis. The samples were thawed before dilution to be in the range of $0-0.5 \mathrm{mM}$. $2 \mathrm{ml}$ of diluted sample was mixed with $100 \mu \mathrm{l}$ of Nessler's reagent and allowed to react for exactly 1 minute before reading the absorbance at $425 \mathrm{~nm}$. Absorbance values were compared to those from ammonium chloride standards measured under the same method (section 3.3.1). 


\subsection{Research Phase 3: Investigation of influence of healing agent additions on self-healing based on compressive strength of mortar cubes}

This section mainly covers the preparation of healing agents and mortar specimen and testing of the compressive strength of mortar cubes.

\subsubsection{Microbial healing agent preparation}

Sporosarcina ureae (DSM 2281) and Sporosarcina pasteurii (DSM 33) were grown by the same method as described in "Bacteria and growth conditions" section and Bacillus subtilis subsp. Spizizenii (DSM 15029) were grown by the same method as described in "Bacteria culturing" section. Figure 3.3 shows the liquid culture grown in the incubator. For removing the bacterial cells from medium residues, $30 \mathrm{ml}$ of the bacterial culture was put in separate $50 \mathrm{ml}$ falcon tubes and the bacterial cells were harvested by centrifuging each falcon tube containing the grown cultures (5000 g, $5 \mathrm{~min}$ ). Figure 3.4 and Figure 3.5 show the grown culture transferred into $50 \mathrm{ml}$ falcon tubes and centrifuge machine, respectively. The harvested cells were re-suspended in a physiological solution $(\mathrm{NaCl}, 9 \mathrm{~g} / \mathrm{L})$. Figure 3.6 shows the falcon tubes with harvested cells. The obtained clean bacterial suspension was subsequently diluted with physiological solution to obtain different final cell densities. Three different bacterial cell concentrations such as $10^{4}, 10^{6}$ and $10^{8} \mathrm{cell} / \mathrm{s} / \mathrm{ml}$ were selected to investigate the optimum bacterial cell concentration which gave the maximum strength. Three different concentration of bacterial suspension $\left(10^{4}, 10^{6}\right.$ and $\left.10^{8} \mathrm{cells} / \mathrm{ml}\right)$ obtained after washing was mixed with sterile zeolite/pumice powders in a 50-ml falcon tube (30 $\mathrm{ml}$ of bacterial solution was mixed with $12 \mathrm{~g}$ zeolite/pumice in each falcon tube and total of 40 falcon tubes were used for 1.2 litre of bacterial solution). Figure 3.7 shows the bacterial cells immobilised in zeolite/pumice. Subsequently, the falcon tube was put on a shaker at $100 \mathrm{rpm}$ for $1 \mathrm{~h}$ (Wang et al., 2005).

Calcium lactate $\left(\mathrm{CaC}_{6} \mathrm{H}_{10} \mathrm{O}_{6}\right)$ was used as calcium carbonate precursor. Besides, urea as urease enzyme source and yeast extract was added as nutritional carbon and nitrogen source for bacteria. Individual ingredients were autoclaved separately and mixed afterwards to avoid precipitation. The final $\mathrm{pH}$ of the media was adjusted to 9 in order to avoid possible chemical precipitation of calcium carbonate. 
These bacteria immobilised in zeolite/pumice together with the nutrient solution constitutes the healing agent.

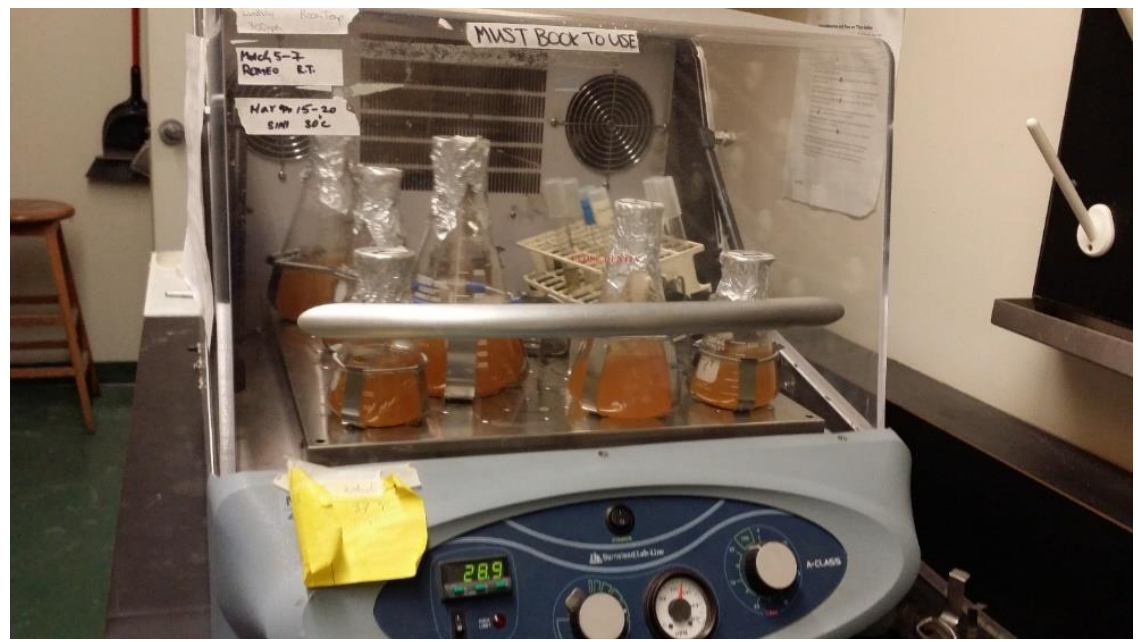

Figure 3.3: Liquid culture grown in the incubator

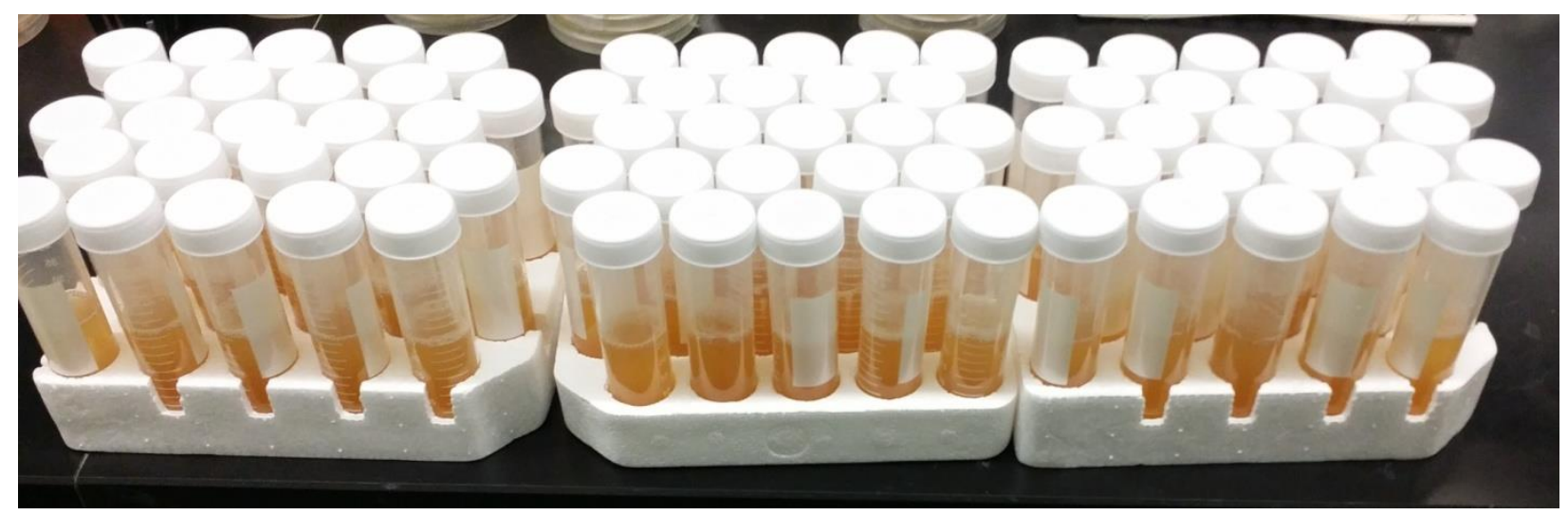

Figure 3.4: Grown culture transferred into $50 \mathrm{ml}$ falcon tubes 

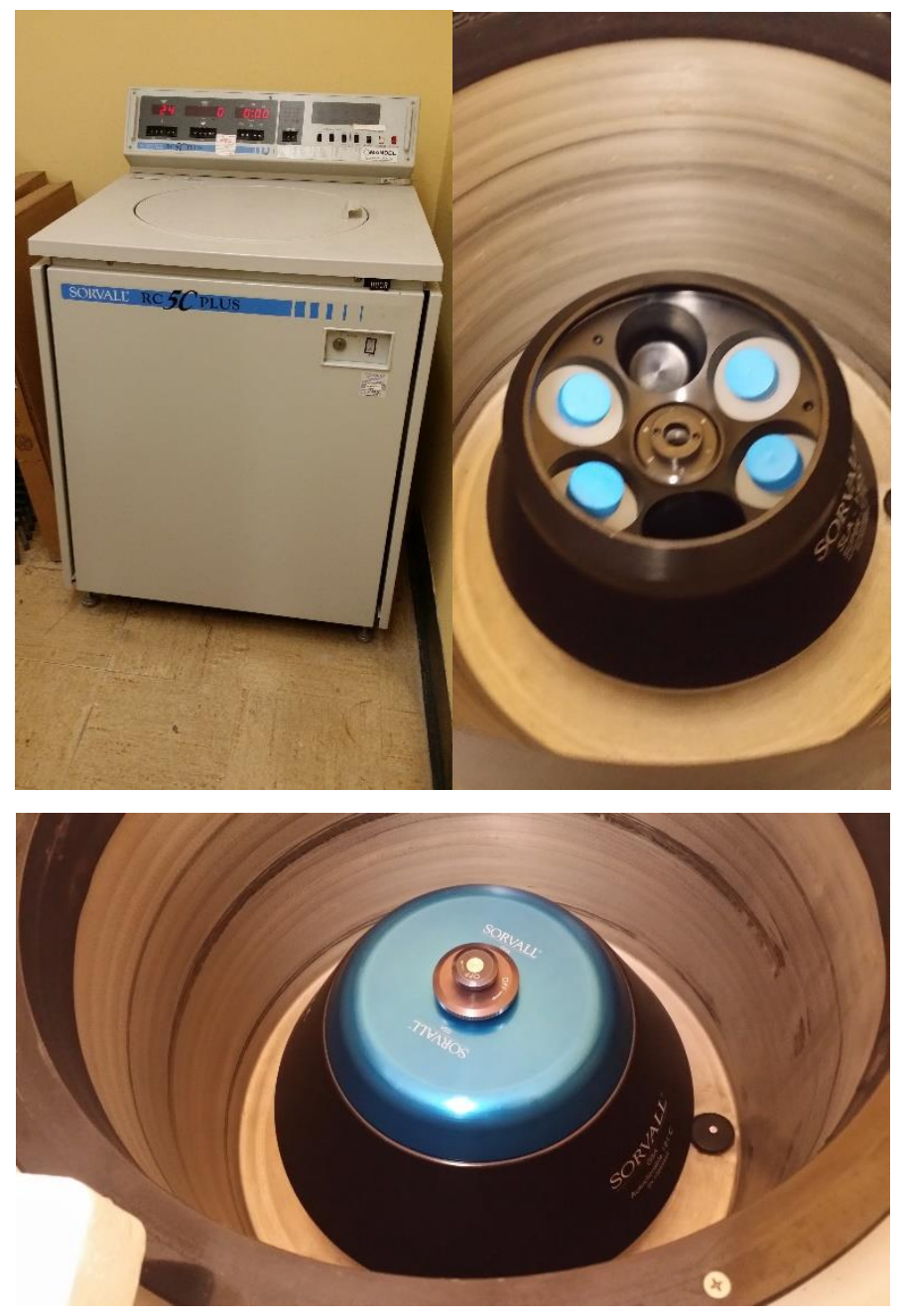

Figure 3.5: Centrifuge machine (left), falcon tubes kept in the rotor inside the centrifuge machine for centrifuging (right), rotor with cover ready for centrifuging (bottom)

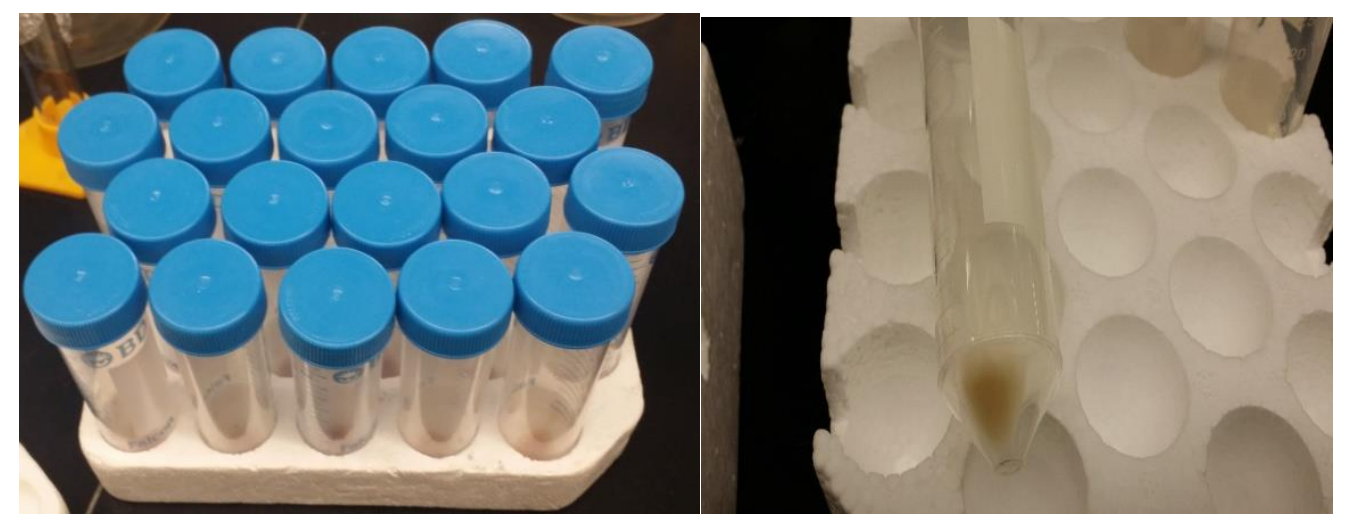

Figure 3.6: Falcon tubes with harvested cells (left), falcon tube with bacterial cells (right) 


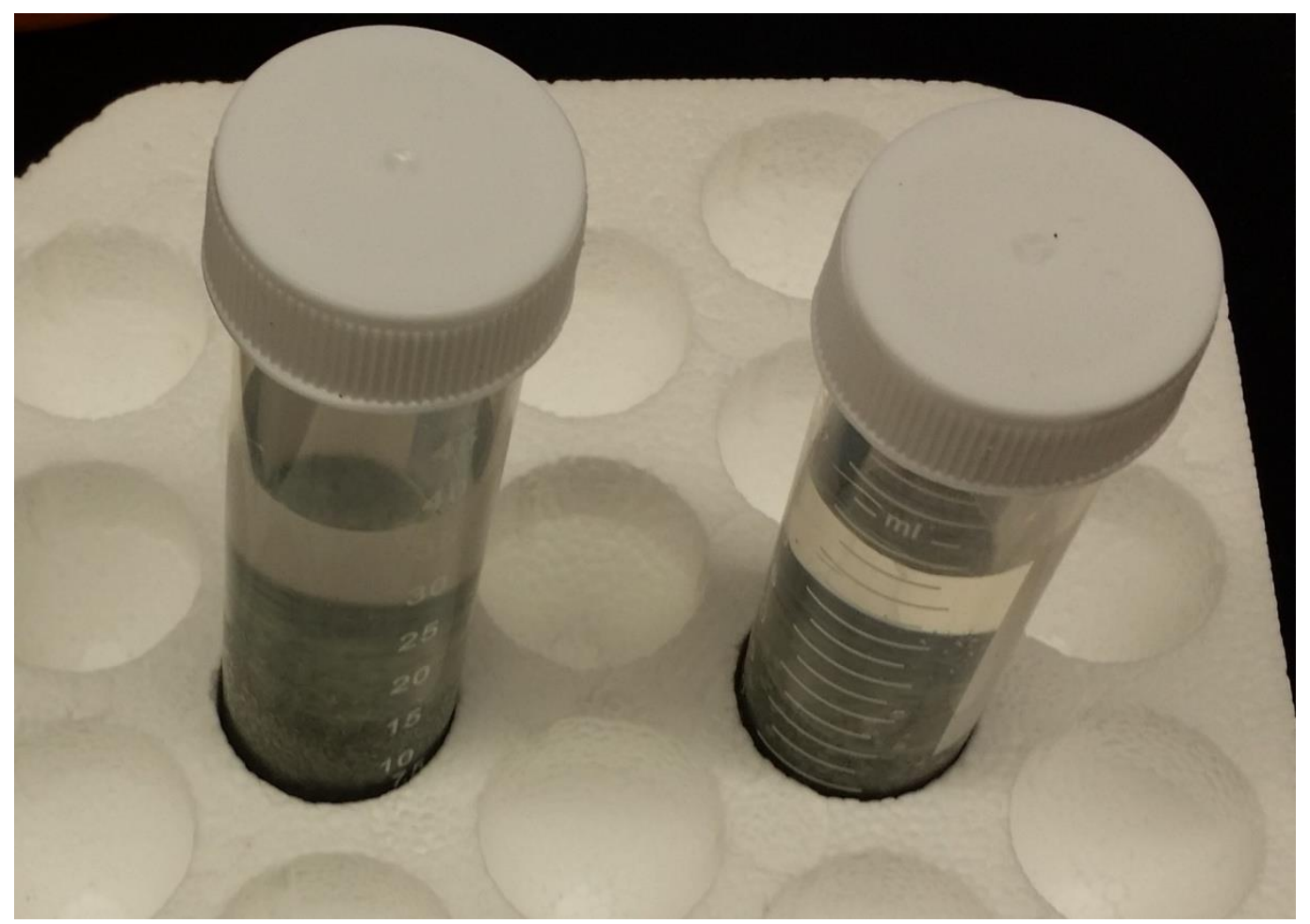

Figure 3.7: Bacterial cells immobilised in zeolite/pumice

\subsubsection{Mortar specimen preparation}

Since bacteria and nutrients should be incorporated in mortar matrix to achieve self-healing, the compatibility between mortar and components of healing agent must be evaluated by determining the compressive strength in advance. A series of tests were performed in order to determine the potential effects of the addition of bacteria (three different types) and organic compounds on strength characteristics of cement mortar. In order to determine the effects of healing agent additions on strength property, mortar cube specimens were prepared with and without (control) incorporating bacteria. During the process of mixing, nutrients (yeast extract, urea, and Calcium lactate with a concentration of $0.2 \%, 2 \%$ and $2 \%$ of cement mass, respectively) were firstly dissolved in part of the mixing water and part of the mixing water was replaced by zeolite/pumiceimmobilised bacterial suspension.

Figure 3.8 shows the dissolved nutrient solution used as part of mixing water. For determining the optimum quantity of the calcium compound to be used in mortar specimen, 3 different percentages such as $1 \%, 2 \%$ and $3 \%$ and $4 \%$ of cement mass of calcium lactate were tested. However, the percentages of other nutrients such as urea and yeast extract were kept constant throughout the test 
in order to reduce the number of combinations. For this preliminary test, total of 36 mortar cubes were prepared. The nutrients solution was mixed with cement and sand. The mixture containing zeolite/pumice-immobilized bacteria were mixed with cement, sand, and the nutrient solution. Figure 3.9 shows the mortar cubes with moulds.

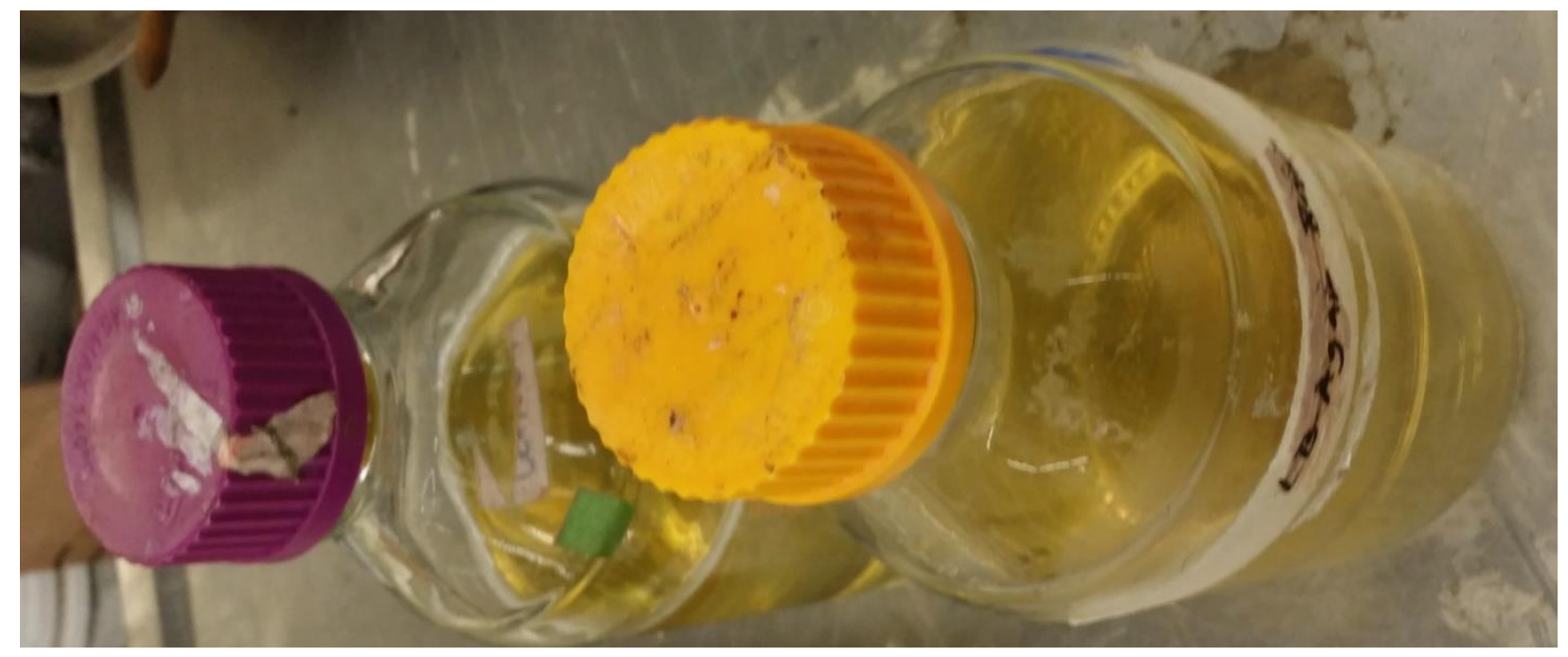

Figure 3.8: Dissolved nutrient solution used as part of mixing water

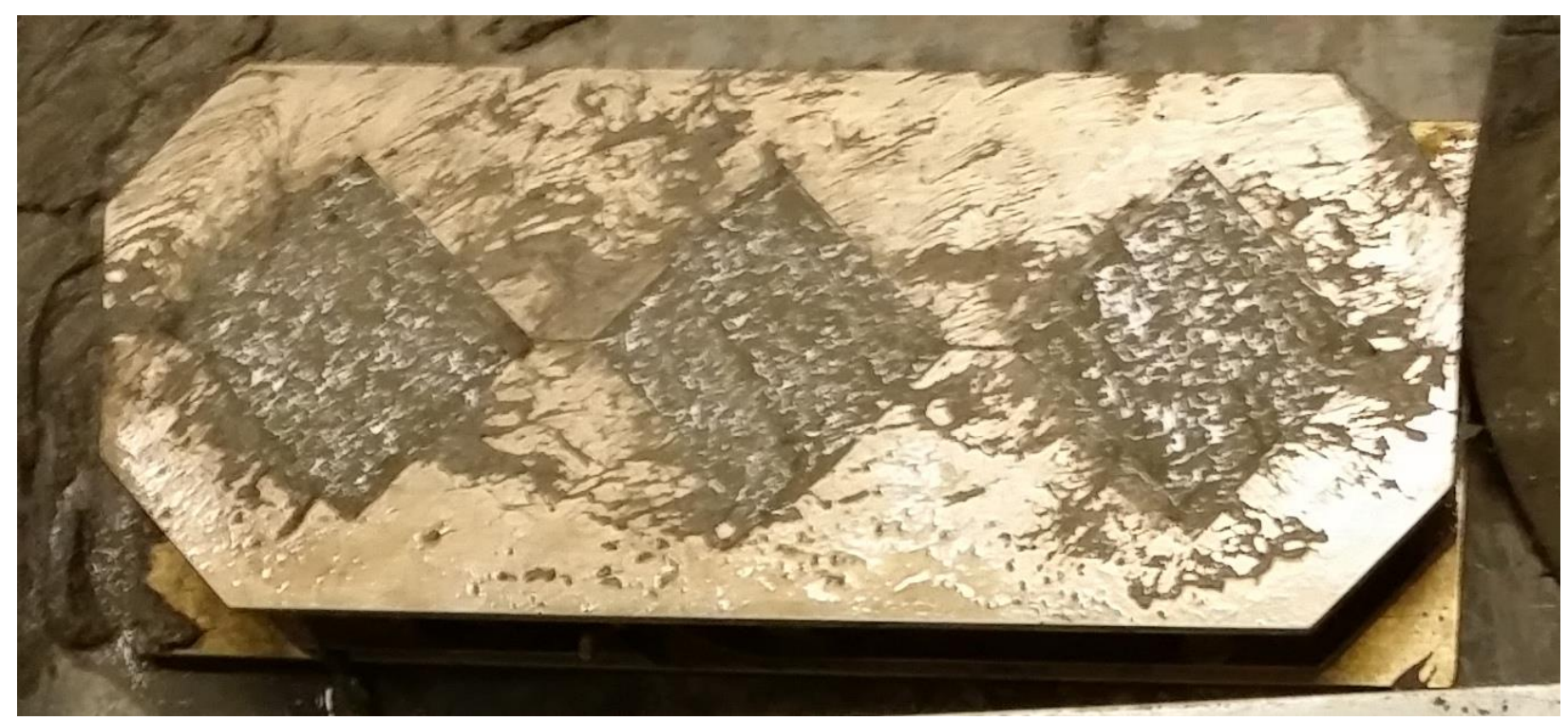

Figure 3.9: Mortar cubes with moulds 


\subsubsection{Effect of self-healing on compressive strength}

For each mixture, three specimens were prepared for the compressive strength test. Ordinary Portland cement with water to cement ratio of 0.5 and cement to sand ratio of 0.333 were used to prepare the cement mortar cubes with dimensions of $50.8 \mathrm{~mm}$ x $50.8 \mathrm{~mm}$ x $50.8 \mathrm{~mm}$. Minimum of three replicate mortar cubes were prepared for each cell concentration, mineral substrate and carrier material. Control specimens were also prepared in a similar way without adding bacterial cells. There were 6 different mixes with 3 different bacterial cell concentrations and total of 324 mortar cubes were prepared. Table 3.5 shows components of all the 9 mixes for nine mortar cubes. All specimens were demolded at the age of $24 \mathrm{~h}$, and then cured at the air conditioned room until testing. Compressive strength of cement mortar cubes at 7, 14, 28, 60, 180 and 270 days were determined in order to investigate the effect of healing agent addition on the strength with age. Figure 3.10 shows the compression test set up.

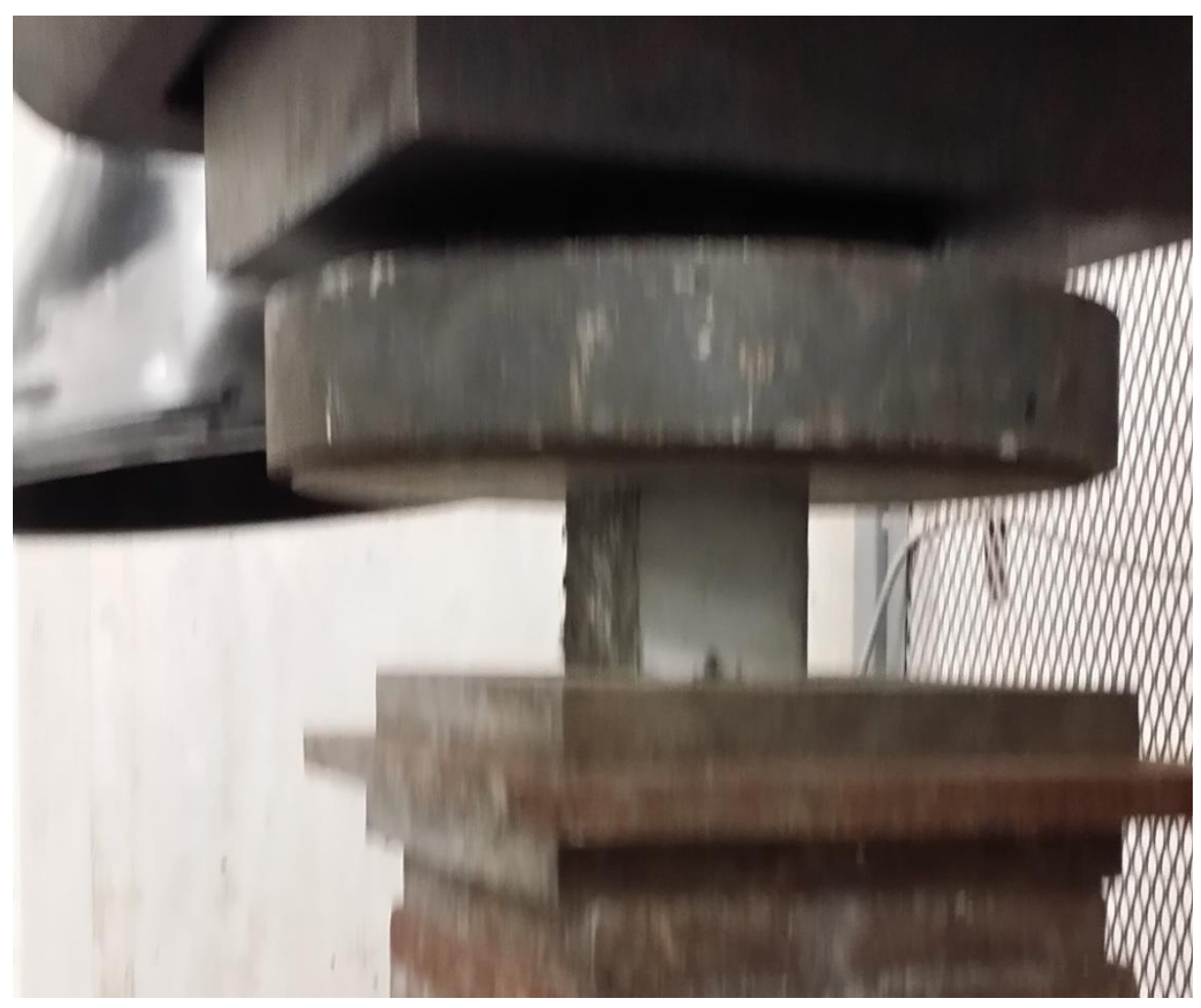

Figure 3.10: Compression test set up 
Table 3.5: Mix proportions of normal mortar cubes in terms of cement weight

\begin{tabular}{|c|c|c|c|c|c|c|c|c|c|}
\hline Mix & Specimen & Cement & Sand & Water & Carrier & BS & NS & w/c & c/s \\
\hline $\mathbf{1}$ & Control & 1 & 3 & 0.5 & - & - & - & 0.5 & 0.333 \\
\hline $\mathbf{2}$ & NS + zeolite & 1 & 2.836 & 0.25 & 0.122 & - & 0.25 & 0.5 & 0.333 \\
\hline $\mathbf{3}$ & NS + pumice & 1 & 2.836 & 0.25 & 0.122 & - & 0.25 & 0.5 & 0.333 \\
\hline $\mathbf{4}$ & S. pasteurii + NS + zeolite & 1 & 2.836 & - & 0.122 & 0.25 & 0.25 & 0.5 & 0.333 \\
\hline $\mathbf{5}$ & S. pasteurii + NS + pumice & 1 & 2.836 & - & 0.122 & 0.25 & 0.25 & 0.5 & 0.333 \\
\hline $\mathbf{6}$ & B. subtilis + NS + zeolite & 1 & 2.836 & - & 0.122 & 0.25 & 0.25 & 0.5 & 0.333 \\
\hline $\mathbf{7}$ & B. subtilis + NS + pumice & 1 & 2.836 & - & 0.122 & 0.25 & 0.25 & 0.5 & 0.333 \\
\hline $\mathbf{8}$ & S. ureae + NS + zeolite & 1 & 2.836 & - & 0.122 & 0.25 & 0.25 & 0.5 & 0.333 \\
\hline $\mathbf{9}$ & S. ureae + NS + pumice & 1 & 2.836 & - & 0.122 & 0.25 & 0.25 & 0.5 & 0.333 \\
\hline
\end{tabular}

NS: Nutrient solution and NS in terms of cement weight included 0.02 calcium lactate, 0.02 urea and 0.002 yeast extract; Carrier: zeolite/pumice; BS: Bacterial solution

\subsection{Phase 4: Self-healing behavior investigation}

The self-healing efficiency of bacteria incorporated mortar specimens was evaluated by measuring the strength regain using four point bending test, Ultrasonic pulse velocity test (UPV), Permeation properties such as sorptivity test and rapid Chloride Permeability test (RCPT).

\subsubsection{Four-point bending tests and UPV measurements}

For each mixture, fiber reinforced mortar prisms with dimensions of $50 \mathrm{~mm} \times 75 \mathrm{~mm}$ x360 mm were prepared for strength regain and UPV tests. Figure 3.11 shows the mortar prisms with moulds. The use of fiber reinforcement was to facilitate large size cracks created followed by mechanical loading while keeping the integrity of the sample. For 9 different mixes, total of 90 prisms were casted. Table 3.6 shows mix proportions of all the 9 mixes. All specimens were demolded at the age of $24 \mathrm{~h}$, and then cured at the air conditioned room until testing. After 28 days of curing, fourpoint bending test was conducted with a loading rate of $0.125 \mathrm{~mm} / \mathrm{min}$ using MTS machine. The full span length was $300 \mathrm{~mm}$ with a middle span of $100 \mathrm{~mm}$. Figure 3.12 shows the test set up for four point bending test. During the test, the load and the midspan deflection were recorded on the computerized data recording system and the load-displacement curve was obtained. All specimens were tested after peak load and the final deflection was controlled as the load stopped at $0.6 \mathrm{~mm}$, which resulted in the formation of cracks at the midspan region of the specimen. Crack width was measured using a Crack Scope and varied in the range of $0.2-0.6 \mathrm{~mm}$ for each specimen. 


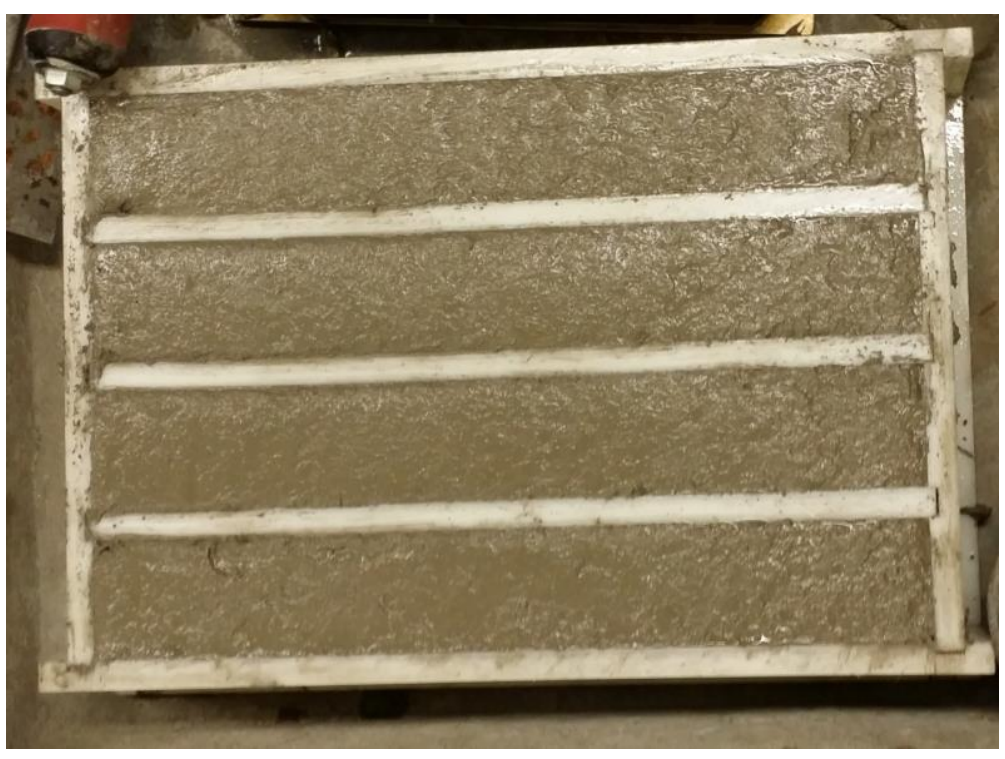

Figure 3.11: Mortar prisms with moulds

Table 3.6: Mix proportions of FR mortar in terms of cement weight

\begin{tabular}{|c|c|c|c|c|c|c|c|c|c|}
\hline Mix & Specimen & Cement & Sand & Water & Carrier & BS & NS & PVA & HRWRA \\
\hline $\mathbf{1}$ & Control & 1 & 1 & 0.44 & - & - & - & 0.03 & 0.02 \\
\hline $\mathbf{2}$ & NS + zeo & 1 & 0.91 & 0.22 & 0.06 & - & 0.22 & 0.03 & 0.02 \\
\hline $\mathbf{3}$ & NS + pum & 1 & 0.91 & 0.22 & 0.06 & - & 0.22 & 0.03 & 0.02 \\
\hline $\mathbf{4}$ & S. pasteurii + NS + zeo & 1 & 0.91 & - & 0.06 & 0.22 & 0.22 & 0.03 & 0.02 \\
\hline $\mathbf{5}$ & S. pasteurii + NS + pum & 1 & 0.91 & - & 0.06 & 0.22 & 0.22 & 0.03 & 0.02 \\
\hline $\mathbf{6}$ & B. subtilis + NS + zeo & 1 & 0.91 & - & 0.06 & 0.22 & 0.22 & 0.03 & 0.02 \\
\hline $\mathbf{7}$ & B. subtilis + NS + pum & 1 & 0.91 & - & 0.06 & 0.22 & 0.22 & 0.03 & 0.02 \\
\hline $\mathbf{8}$ & S. ureae + NS + zeo & 1 & 0.91 & - & 0.06 & 0.22 & 0.22 & 0.03 & 0.02 \\
\hline $\mathbf{9}$ & S. ureae + NS + pum & 1 & 0.91 & - & 0.06 & 0.22 & 0.22 & 0.03 & 0.02 \\
\hline
\end{tabular}

NS: Nutrient solution and NS in terms of cement weight included 0.02 calcium lactate, 0.02 urea and 0.002 yeast extract; Carrier: zeolite/pumice; BS: Bacterial solution; zeo: zeolite; pum: pumice 


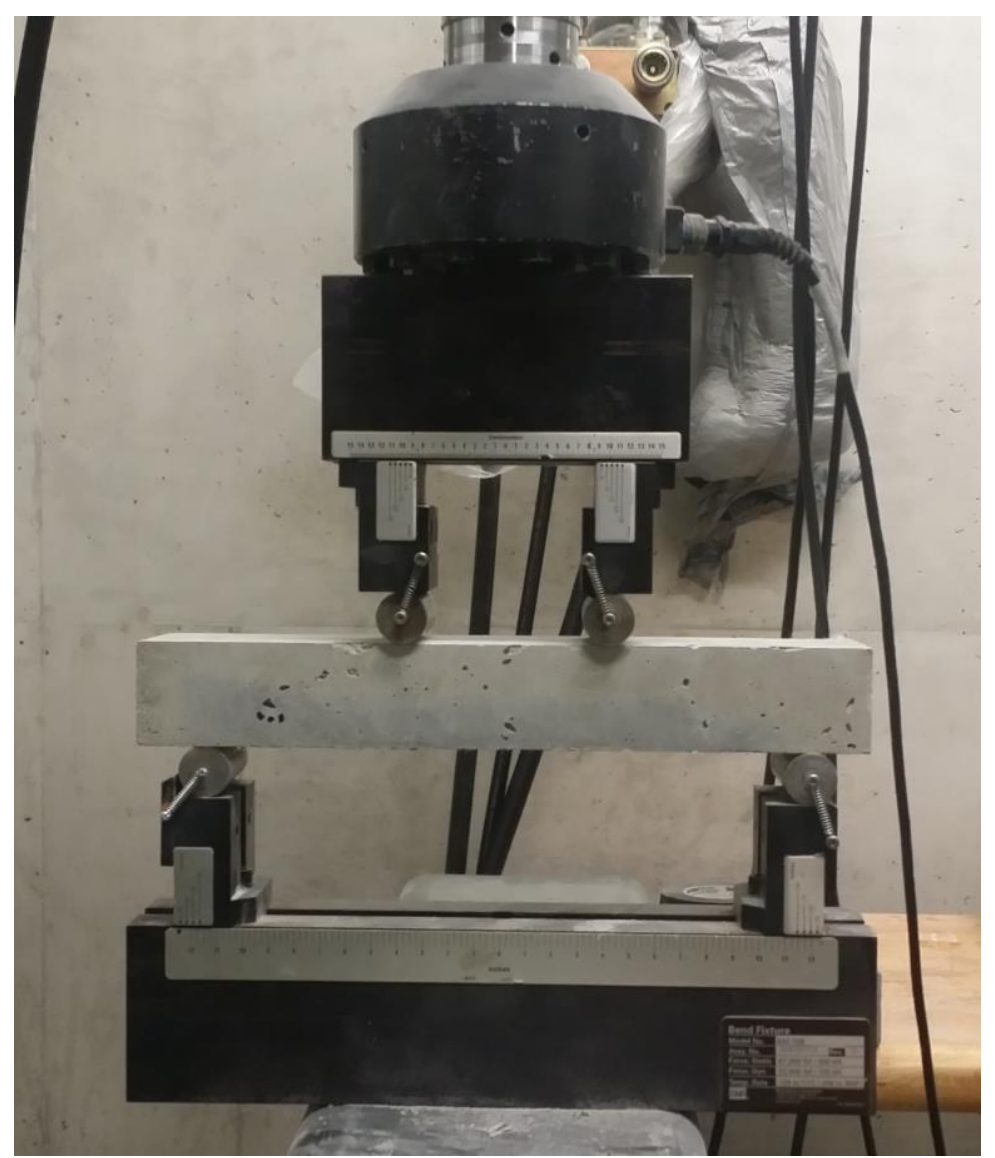

Figure 3.12: Test set up for four point bending test

\subsubsection{Effect of self-healing on strength gain using four-point bending test}

Each specimen with cracks was immersed horizontally in tap water in plastic containers which were kept open to the atmosphere during the whole incubation period. About $1 \mathrm{~cm}$ water column covered the specimens to allow the diffusion of oxygen for the bacteria. After 120 days of incubation, specimens were removed and four-point flexural tests were carried out again and all specimens were loaded until failure.

\subsubsection{Effect of self-healing on UPV measurements}

UPV is considered as a reliable non-destructive technique to assess damage in concrete. UPV results were used as an indicator of damage and healing in mortar prisms. UPV was carried out on prisms before loading, after loading, and each month after healing. 


\subsubsection{Effect of self-healing on permeation properties}

Another way to measure the extent of self-healing is by measuring the permeation properties. Penetration of water or chloride ions into concrete can adversely affect its durability. Firstly the penetration of water can give rise to the freeze thaw effect, and can lead to corrosion of the steel reinforced bars in concrete. Thus the rate at which concrete absorbs water or chloride ions of salt water, becomes an important property to be tested.

Two different types of mortar mixes were prepared for the RCPT and sorptivity tests; normal mortar with holes and fibre reinforced mortar. Normal mortar was prepared with ordinary Portland cement with water to cement ratio of 0.5 and cement to sand ratio of 0.333 . Holes were created by using fishing line of diameter $0.25 \mathrm{~mm}$ which was inserted into the moulds during casting and were removed after $24 \mathrm{hrs}$. Figure 3.13 shows the picture of a sample with hole. Both for rapid chloride penetration test (RCPT) and sorptivity test, 6 cylinder specimens were prepared for each mixture with diameter and thickness of $100 \mathrm{~mm}$ and $50 \mathrm{~mm}$, respectively. Originally, cylindrical specimens of diameter $100 \mathrm{~mm}$ and height $200 \mathrm{~mm}$ were prepared from which three $50 \mathrm{~mm}$ thick discs were extracted by using a diamond blade saw from the central portion of the cylinder specimen. For 18 different mixes, total of 108 cylinders were prepared. After 7 days curing, three specimens were kept as control while three other specimens of fiber reinforced mortar were pre-loaded by tensile splitting test so as to produce cracks. Figure 3.14 shows the tensile splitting test set up to induce cracks. Before the test, crack width of all cracked specimens was measured using crack scope. For all the mixtures, first round of both tests were conducted after 7 days curing and then the specimens were cured in water for 120 days before conducting the second round of tests.

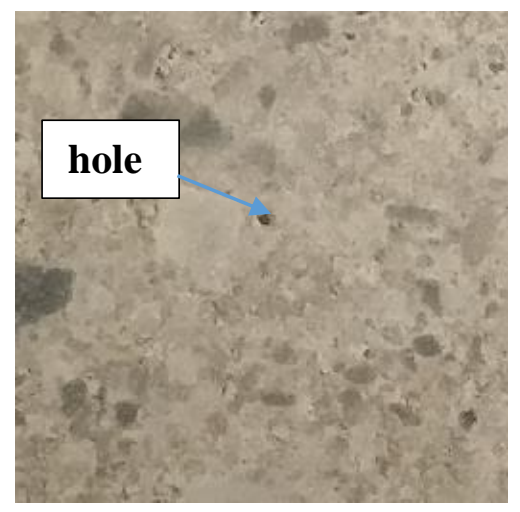

Figure 3.13: Sample with a generated hole 


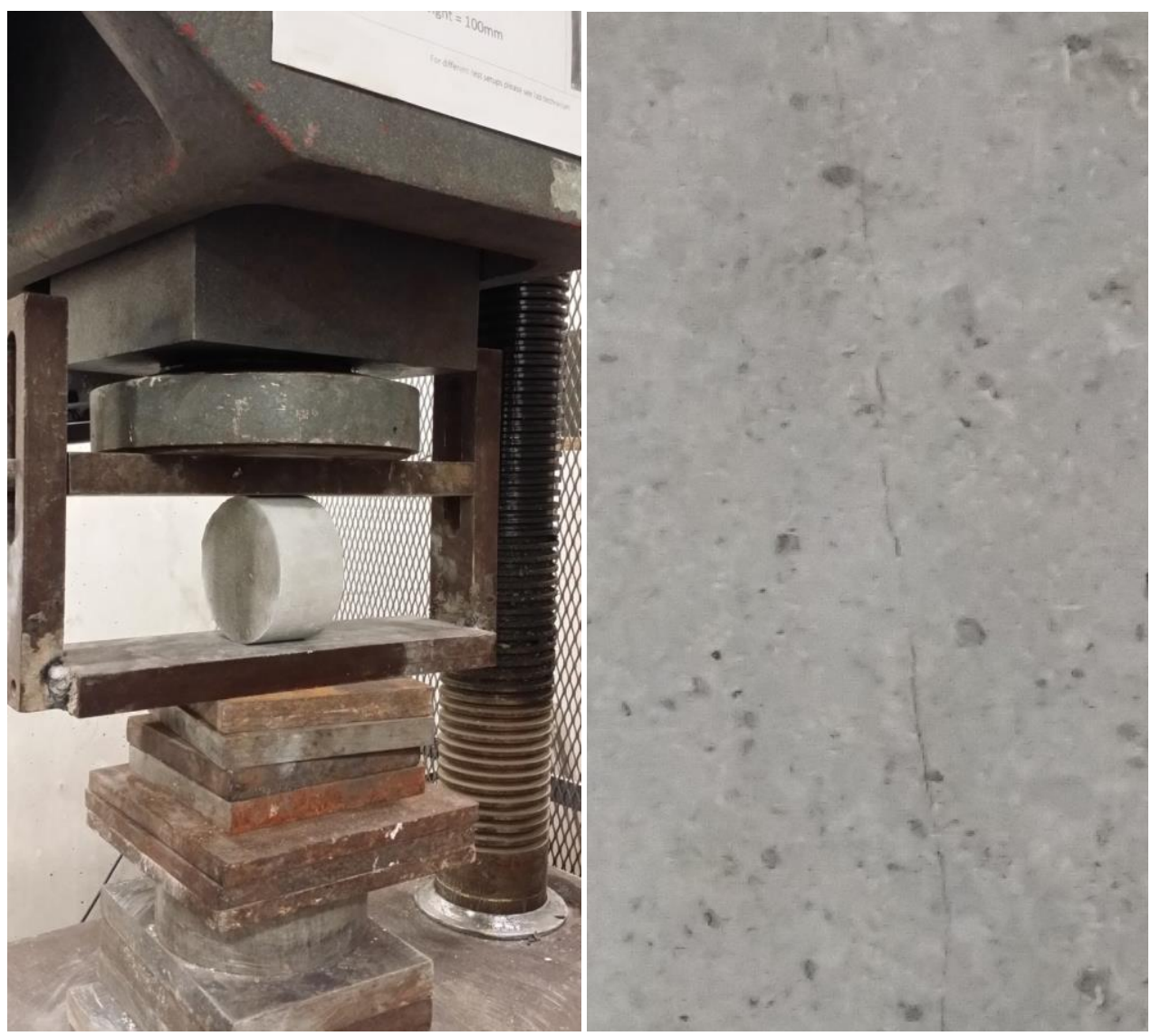

Figure 3.14: Tensile splitting test set up to induce cracks (left) and the induced crack (right)

\subsubsection{Sorptivity test}

The sorptivity test was based on ASTM C1585. The increase in mass of a cylindrical specimen $(100 \times 50 \mathrm{~mm})$ at given intervals of time when permitted to absorb water by capillary suction was registered. The specimens were dried in an oven at $50^{\circ} \mathrm{C}$ for 3 days before each test. Only one surface of the specimen was allowed to be in contact with water, with the depth of water between 1 and $3 \mathrm{~mm}$. The sides of the specimen were sealed with an epoxy coating in order to guarantee one directional flow through the specimen. Figure 3.15 shows the test set-up for sorptivity test. Measurements were taken at regular intervals of 1, 5, 10, 15, 20, $30 \mathrm{~min} ; 1,2,3,4,5$ and $6 \mathrm{hrs} ; 1$, 2, 3, 4, 5, 6 and 7 days. Immediately after the measurement, the test specimens were re-submerged. The test was performed in triplicate. The rate of absorption $\left(\mathrm{mm}^{3} / \mathrm{mm}^{2}\right)$, defined as the change in mass $(\mathrm{g})$ divided by the cross sectional area of the test specimen $\left(\mathrm{mm}^{2}\right)$ and the density of water at 
the recorded temperature $\left(\mathrm{g} / \mathrm{mm}^{3}\right)$, was plotted against the square root of time $\left(\mathrm{sec}^{1 / 2}\right)$. The slope of the resulting curve defines the sorptivity of the specimen during the period of testing.

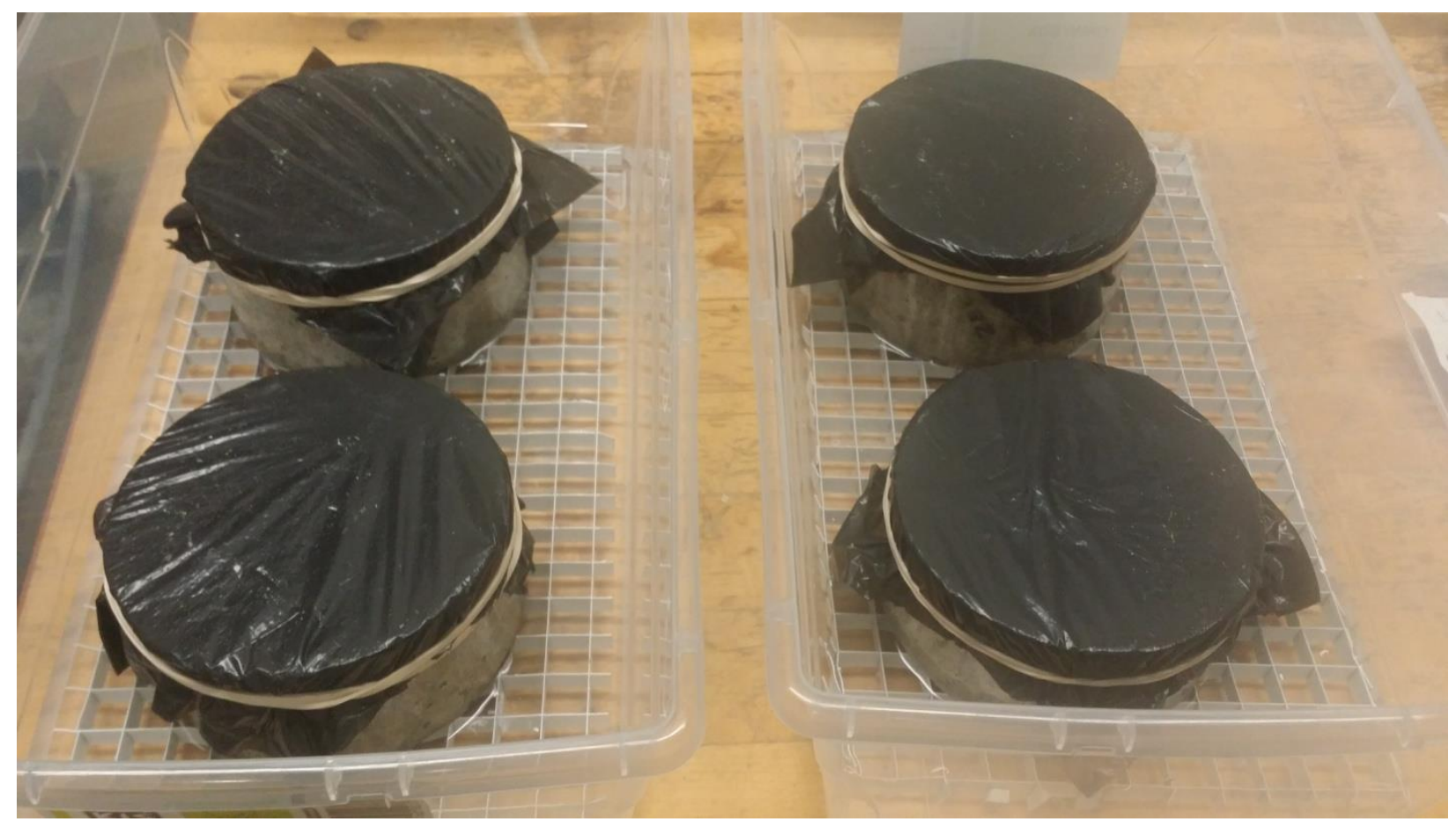

Figure 3.15: Test set-up for sorptivity test

\subsubsection{Rapid chloride permeability test}

Chlorides can penetrate into the concrete by different kinds of processes such as capillary absorption, hydrostatic pressure and diffusion. However, diffusion is the predominant one. When the outside concentration of chloride ion is greater than the inside concentration, diffusion occurs. This leads to the penetration of chloride ions into the concrete matrix. Permeation is another mechanism for chloride ingress which is impelled by pressure gradients. When water containing chlorides come upon a dry surface, it will percolate into the pore structure due to capillary suction. This absorption is driven by moisture gradients. The main factor which controls the rate of chloride ion penetration into the concrete matrix is internal pore structure. This pore structure depends on many factors such as mix design, degree of hydration, curing conditions and use of other supplementary materials. Due to all these reasons, there is a potential risk of chloride induced corrosion. This insists that the concrete should be evaluated for chloride ion permeability. Rapid chloride permeability test is the simple test which is used to test the chloride ion permeability. This 
test is based on electrical conductivity of concrete. The concrete sample is subjected to a potential difference of $60 \mathrm{~V}$. The total charge passing through the sample at the end of $6 \mathrm{hrs}$ is measured and expressed in terms of Coulombs. Lower permeability and a better resistance to chloride ion penetration can be indicated by a reduction in this total charge value.

Rapid chloride permeability test (RCPT) has been developed as a quick test able to measure the rate of transport of chloride ions in concrete. Rapid Chloride Permeability Test (RCPT) was conducted according to two very similar standards AASHTO T 277 and ASTM C 1202. Specimens were placed in the vacuum desiccator's bowl and the vacuum was maintained in the desiccators bowl for $3 \mathrm{~h}$. Then the distilled water was allowed to flow into the desiccator, so that it completely covered the specimens and no air was allowed to enter. Again the vacuum was maintained for another $1 \mathrm{~h}$. Subsequently, the specimens were left to soak in the container water for another $18 \mathrm{~h}$. Figure 3.16 shows the specimen submerged in dessicators bowl.

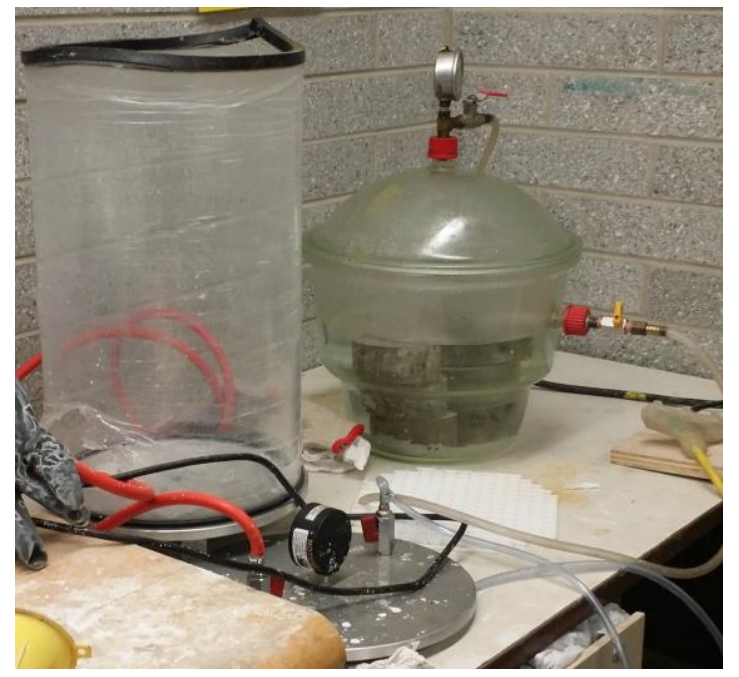

Figure 3.16: Specimen submerged in dessicators bowl

The specimens were removed from the desiccator, dried and placed in gasket. One side of the container was filled with $3 \%$ sodium chloride solution (that side of the cell will be connected to the cathode terminal of the power supply) and other side sodium hydroxide solution $(0.3 \mathrm{~N})$ was poured and connected to anode terminal. The total charge that passed through the samples was determined (expressed in terms of coulombs) at the end of $6 \mathrm{~h}$. Chloride penetrability is directly proportional to the charge passed. The interpretation is that the larger the Coulomb number or the charge transferred during the test, the greater the permeability of the sample. When the test was 
carried out at $60 \mathrm{~V}$, the RCPT machine was unable to provide any result and the results were showing as OVF because the additions of different ions and fibres. Therefore, for the current study, test was conducted at $30 \mathrm{~V}$ even though the standard voltage for the RCPT according to ASTM was $60 \mathrm{~V}$.

\subsubsection{Scanning Electron Microscopy (SEM), Energy Dispersive Spectrum (EDS) and X- Ray Diffraction (XRD) studies}

SEM is essentially a high magnification microscope, which uses a focused scanned electron beam to produce images of the sample, both top-down and cross-sections. To investigate the morphology and chemical constituents of self-healing products and to observe the self-healing process, selected specimens were examined by Scanning Electron Microscope (SEM) and Energy Dispersive Spectroscopy (EDS). SEM is a powerful instrument which permits the characterization of heterogeneous materials and surfaces.

After four months of healing in water, the selected specimens of each mixture were subjected to Scanning Electron Microscopy observation (SEM, JEOL JSM-6380LV, 20V). Back scattered Electron Imaging (BES) was used for electron micrography. Crack healed samples were cut into small cubes and were completely dried at $50^{\circ} \mathrm{C}$ in an oven for three days before the SEM observation. An Energy Dispersive Spectrometer (EDS) connected with SEM was used to detect the components of precipitation. All the selected samples were gold coated with a Denton vacuum Desk IV coating system prior to examination. An energy dispersive spectrometer (EDS) connected with SEM was used simultaneously to detect the components of the precipitation. Figure 3.17 shows the picture of Denton vacuum Desk IV coating system and the specimen ready for coating. 

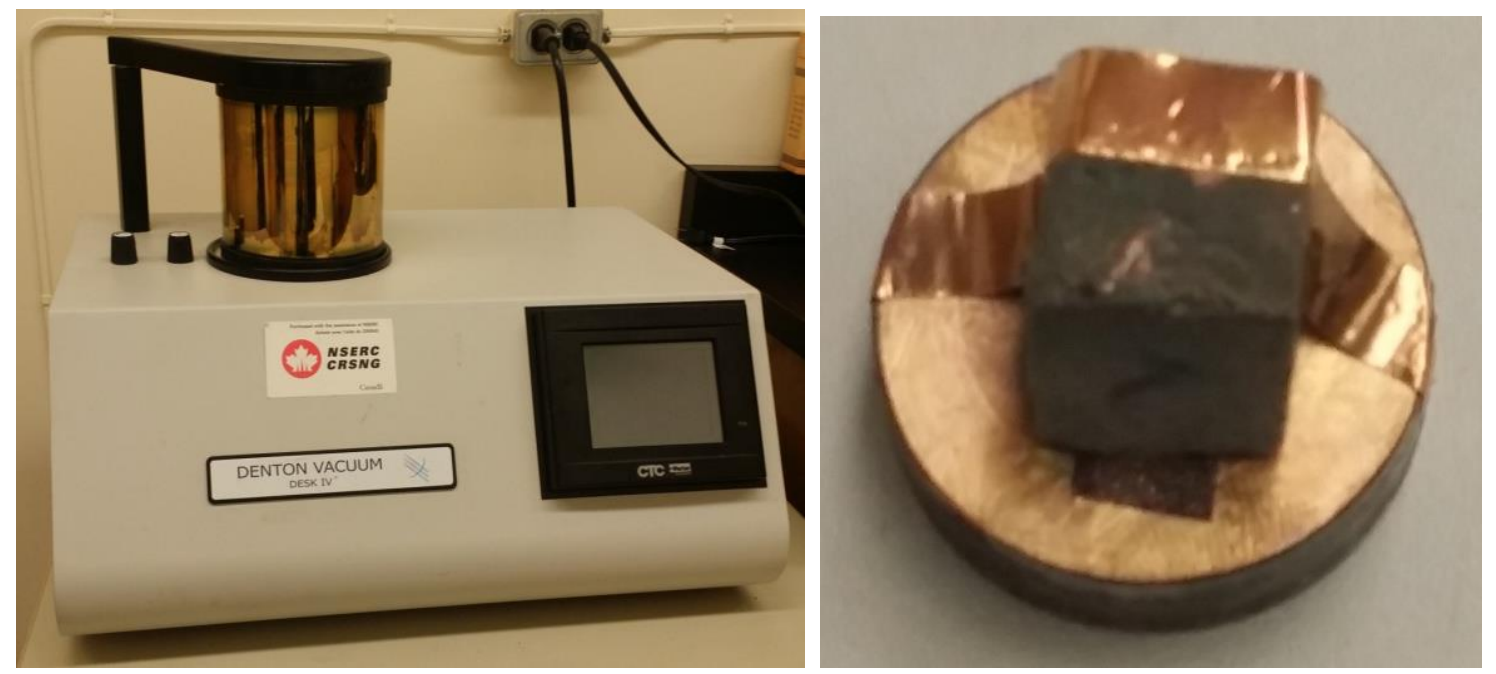

Figure 3.17: Denton vacuum Desk IV coating system (left), specimen ready for coating (right)

$\mathrm{X}$-ray diffraction is a non-destructive technique used to determine the elements present in any particular substance. X-ray powder diffraction technique is the most prominent technique used for unraveling the structure of the materials in bulk and thin film forms. XRD-spectra were obtained using an X'Pert PRO diffractometer with a X-ray tube of PW3373/10Cu LLF DK 400324 shutter (45 kV and $40 \mathrm{~mA}$ ) and scanning from 3 to $60^{\circ} 2 \theta$. Calcium carbonate layer near the crack area of the selected samples of 9 mixes were collected and grinded before mounting on to a glass fibre filter. The components of the sample were identified by comparing them with standards established by the International Centre for Diffraction data. X-ray diffraction is based on the fact that, in a mixture, the measured intensity of a diffraction peak is directly proportional to the content of the substance producing it. The samples for X-ray diffraction analysis were prepared in powdered form. Figure 3.18 shows the X Pert PRO apparatus and the samples monuted on the X Pert PRO apparatus. 

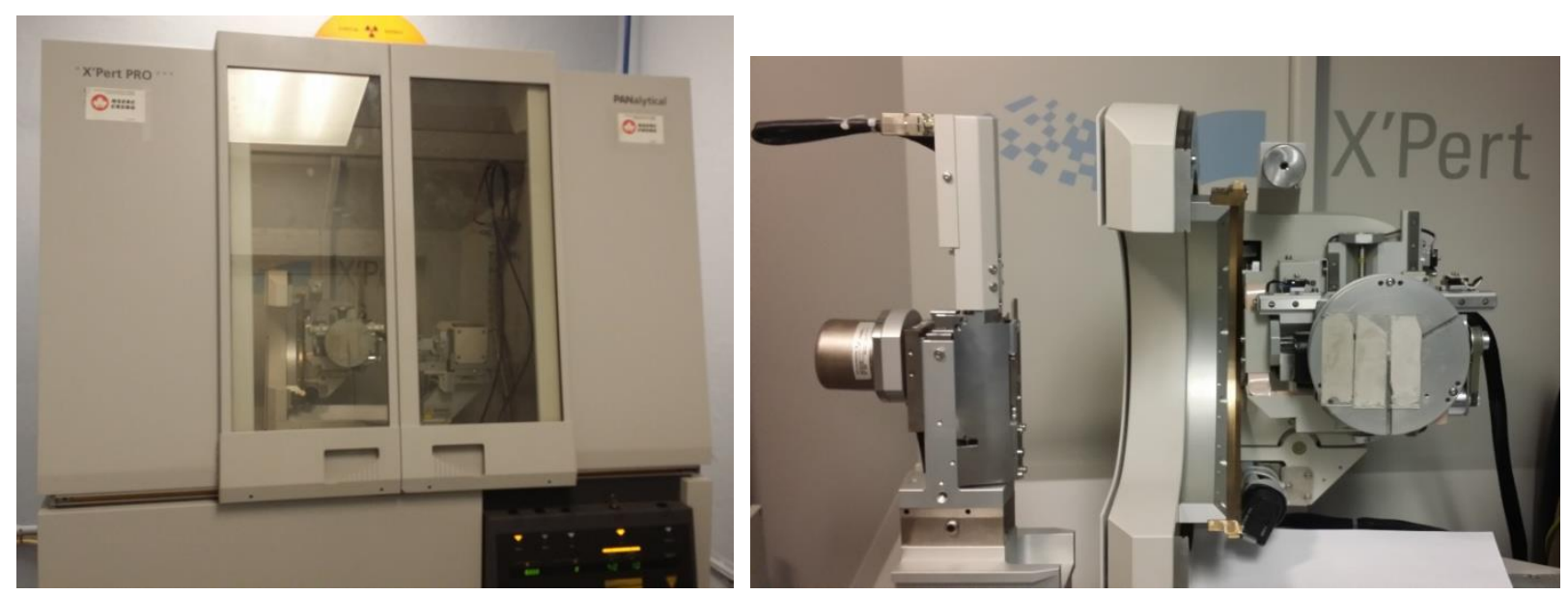

Figure 3.18: $X$ Pert PRO apparatus (left), samples mounted on the X Pert PRO apparatus

\subsection{Research Phase 5: Self-healing investigation on ECC materials}

The experimental program which consisted of the self-healing behaviour investigation of bacteria based ECC mainly focused on investigating the flexural properties of prisms. The self-healing efficiency of bacteria incorporated ECC specimens was evaluated by measuring the strength regain using four point bending test and conducting the Ultrasonic Pulse Velocity test (UPV). Four different mixes were selected for the study. Mix 1 (M1) was a normal ECC mixture which was selected as the control. Unlike the mixes selected for normal mortar and fiber reinforced (PVA fibres) mortar specimens for earlier study, ECC mix with Sporosarcina pasteurii without immobilized in carrier material was also considered in this study (M2). This was to test the efficiency of self-healing effect provided by bacteria without any protective vehicle. ECC Mix with Sporosarcina pasteurii immobilized in zeolite and Bacillus subtilis subsp. spizizenii immobilized in zeolite were selected as M3 and M4, respectively.

\subsubsection{Microbial healing agent preparation}

Two bacterial species (Sporosarcina pasteurii and Bacillus subtilis subsp. spizizenii) and one carrier material (zeolite) which gave the best self-healing effect for the previous study in both normal mortar and fibre reinforced mortar specimens were selected. Sporosarcina pasteurii (DSM 33) were grown by the same method as described in "Bacteria and growth conditions" section and Bacillus subtilis subsp. spizizenii (DSM 15029) were grown by the same method as described in "Bacteria culturing" section. For the M2 mix, the harvested cells re-suspended in a physiological 
solution $(\mathrm{NaCL}, 9 \mathrm{~g} / \mathrm{L})$ were considered as the part of mixing water. Other preparations for the microbial healing agent were done in the same manner as described in section 3.4.1.

\subsubsection{ECC mix design}

Type GU/10 Normal Portland cement manufactured and supplied by St. Mary's cement, Class-CI fly ash (FA) and local crushed sand with maximum size of $1.18 \mathrm{~mm}$ have been used. The mix design is given in Table 3.7. In the mix design, FA and Portland cement were considered as binder materials. Polyvinyl alcohol (PVA) fibres with a length of $8 \mathrm{~mm}$ and diameter of $40 \mu \mathrm{m}$ had been used.

Table 3.7: Mix design of ECC in terms of cement weight

\begin{tabular}{|l|c|c|c|}
\hline Type & Mix 1 & Mix 2 & Mix 3 \\
\hline Water & 0.581 & - & - \\
\hline Cement & 1 & 1 & 1 \\
\hline FA & 1.2 & 1.2 & 1.2 \\
\hline Sand & 0.798 & 0.660 & 0.563 \\
\hline PVA & 0.046 & 0.046 & 0.046 \\
\hline HRWRA & 0.009 & 0.009 & 0.009 \\
\hline w/b & 0.27 & 0.27 & 0.27 \\
\hline Zeolite & - & - & 0.096 \\
\hline NS & - & 0.290 & 0.290 \\
\hline BS & - & 0.290 & 0.290 \\
\hline \multicolumn{4}{|l|}{$\begin{array}{l}\text { HRWRA: High range water reducing admixture, FA: Fly ash CI, NS: Nutrient } \\
\text { solution; NS in terms of cement weight included 0.002 yeast extract, } 0.02 \text { urea } \\
\text { and 0.02 kg/m }{ }^{3} \text { calcium lactate, BS: bacterial solution }\end{array}$} \\
\hline
\end{tabular}

\subsubsection{Mixing Procedure and Specimen Preparation}

The specimens were prepared in a Hobart mixer with 20-liter capacity following different mixing sequences for each mix. For Mix 1 (M1) first all solid materials including sand, cement and fly ash were pre-mixed for $1 \mathrm{~min}$. Then $90 \%$ of water was added into the mixer and the mixer speed was increased for another 2 minutes. The remaining 10\% of water and Super plasticiser (SP) were added until a consistent and uniform ECC mixture was obtained. The final step of mixing procedure was to add the PVA fiber into the mixer in two patches. The mixing was done for another 3 minutes. It should be noted that a slight adjustment in the amount of SP in each mixture was performed to achieve better fiber dispersion and workability. For the bacteria based ECC mixtures (M2, M3 and M4), after the mixing of dry materials, $75 \%$ of the Nutrient solution and $100 \%$ of the bacterial solution was added and the mixer speed was increased for 2 minutes. The remaining 
$25 \%$ of nutrient solution and SP were added until a consistent and uniform ECC mixture was obtained. Then the mixing sequence was continued as that of M1. Figure 3.20 shows ECC mixture pouring in the prism mould. Workability of the mix was measured by conventional slump test. As shown in Figure 3.19, ECC exhibited excellent workability and eliminate the need for vibration. However, a small adjustment by hand was required to obtain a smooth surface.

For each mixture, 9 cube specimens with dimensions of $50.8 \mathrm{~mm}$ x $50.8 \mathrm{~mm}$ x $50.8 \mathrm{~mm}$ and 12 prisms with dimensions of $50 \mathrm{~mm} \times 75 \mathrm{~mm} \times 360 \mathrm{~mm}$ were prepared for the compressive strength and four point bending tests respectively. All specimens were demolded at the age of $24 \mathrm{~h}$, and then cured at the air conditioned room until testing.

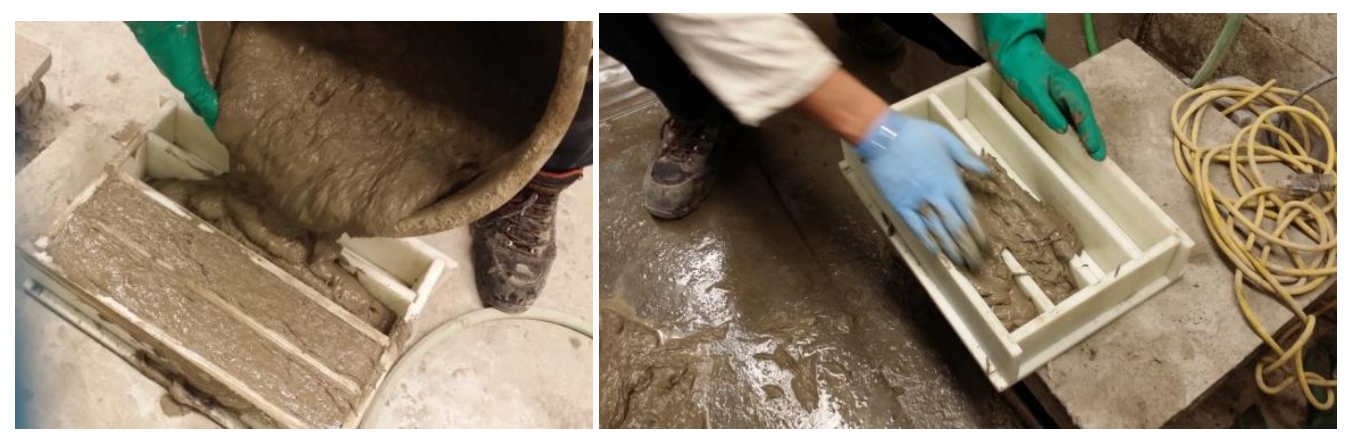

Figure 3.19: ECC mixture pouring in the prism mould

\subsubsection{Testing methods}

\subsubsection{Compression test}

Compressive strength of cubic specimens with dimensions of $50.8 \mathrm{~mm}$ x $50.8 \mathrm{~mm}$ x $50.8 \mathrm{~mm}$ were conducted according to ASTM C109, 1997 by testing at least 3 cubes at different ages.

\subsubsection{Flexural test}

Four point bending test was conducted on mortar prisms with dimensions of $50 \mathrm{~mm} \times 75 \mathrm{~mm} \times$ $360 \mathrm{~mm}$ in order to study the effect of self-healing on flexural properties. Four-point bending test was conducted with a loading rate of $0.125 \mathrm{~mm} / \mathrm{min}$ using MTS machine. The full span length was $300 \mathrm{~mm}$ with a middle span of $100 \mathrm{~mm}$. Test set is same as mentioned in the section 3.5.1. During the test, the load and the mid span deflection were recorded. Initially, all specimens were preloaded up to a deflection of $50 \%$ of the maximum deflection of the failed sample. When this deflection was reached the load was released, after which the specimens were removed and cured in water 
until age 56 days to test the self-healing. This preloading was done after 28 days to deliberately introduce a number of micro-cracks. Figure 3.20 and Figure 3.21 show the failed and preloaded samples with multiple cracks formed in the bacteria based ECC specimen. The crack width was measured by crack scope. Specimens with and without bacteria based agent were kept in separate water containers to avoid cross contamination. Reference specimens from each mixture were cured under the same conditions as the pre-loaded specimens and were tested at 56 days. After 28 days of healing in water, reloading of all specimen under four-point bending test was done to characterise residual mechanical behavior of bacteria based ECC after self-healing.

In order to roughly estimate the preloading deflection, the reference sample was tested until final failure to derive the flexural stress-deflection relation. Deflection of $1 \mathrm{~mm}$ was selected since it is approximately equal to deflection corresponding to $50 \%$ of ultimate strength.

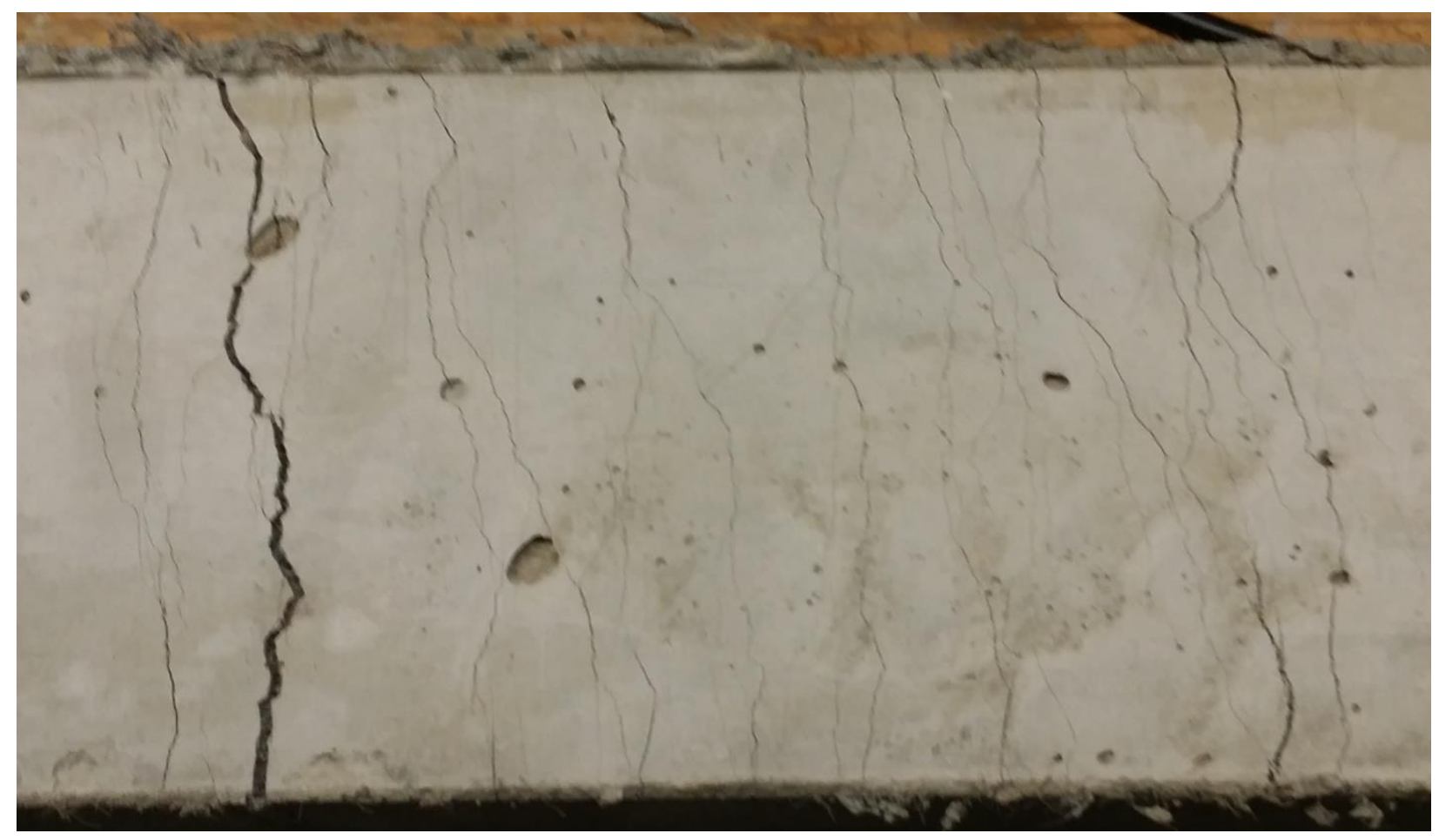

Figure 3.20: Typical failed sample of bacteria based ECC with macro crack and multiple micro cracks 


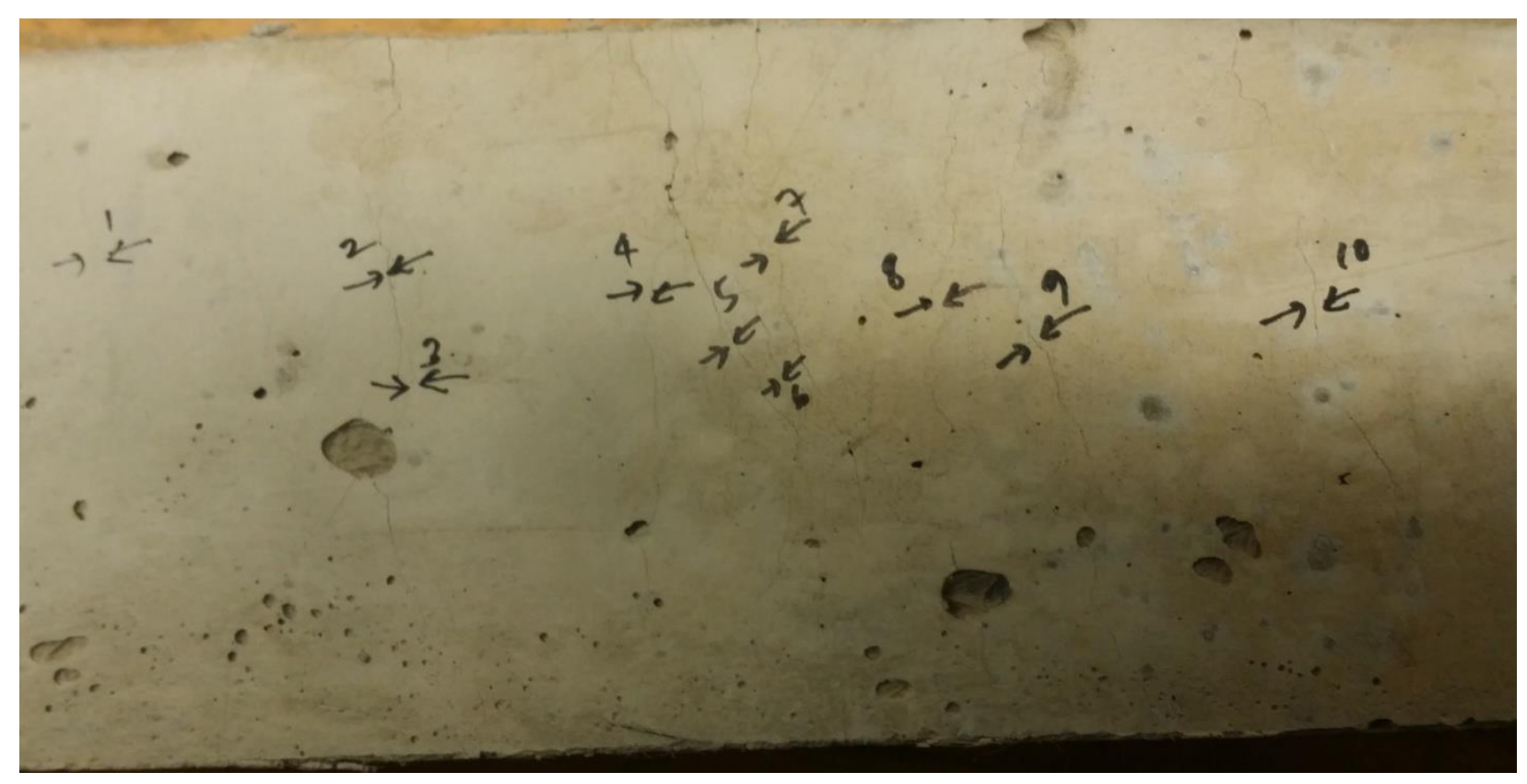

Figure 3.21: Typical preloaded bacteria based ECC specimen with multiple cracks

\subsection{Conclusion}

This chapter was intended to describe materials and experimental methods in quantifying selfhealing activities and processes in cementitious concrete composites. Identifying the optimum concentration of healing agents involved laboratory experimentations at both microbiology and concrete labs at Ryerson University. These included few trial and error tests, some self-designed experiments and implementation of knowledge gleaned from in-depth study of previous literature. In order to assess the effectiveness of self-healing, various testing methods were employed. A detailed analysis and discussion of the results of various experiments and tests will be presented in the following chapter. 


\section{CHAPTER FOUR: RESULTS AND DISCUSSION}

\subsection{Introduction}

This chapter contains a detailed analysis of the results of various experiments conducted on normal mortar, fibre reinforced mortar and engineered cementitious composites to quantify self-healing. The initial challenge of the experiment was to check whether the chosen bacteria was able to grow well in the medium, forms spores, and survive in high temperature and $\mathrm{pH}$ environment. It was also important to see whether they have high urease activity so that they can produce carbonate ions which is needed for calcium carbonate precipitation. The results of those pre-requisites are discussed in detail in this chapter. After finding out the optimum concentration of bacteria, that cell concentration was used for all subsequent self-healing experiments. Both qualitative and quantitative analyses of the self-healing was performed using various testing tools. SEM, EDS and XRD studies were performed for qualitative analysis. Compressive strength, sorptivity, rapid chloride permeability, ultrasonic pulse velocity tests and flexural property tests were conducted for detailed quantitative analysis. This chapter presents detailed analysis and discussion of all the test results to assess self-healing.

\subsection{Research Phase 1: Culturing and survival testing of bacteria}

This sections discusses research on growth, spore formation, germination, and percentage of survival in high temperature and $\mathrm{pH}$ treatments of different bacteria.

\subsubsection{Analysis of bacterial growth and spore forming ability}

Three different bacterial species, Sporosarcina ureae (DSM 2281), Sporosarcina pasteurii (DSM 33) and Bacillus subtilis subsp. Spizizenii (DSM 15029) were cultured in liquid and solid media according to the DSMZ's instruction mentioned in the bacteria culturing section. All three sporeforming alkali-resistant species grew well in the recommended medium.

Addition of manganese to the growth medium simulated the formation of bacterial spores substantially. Light microscopic analysis of growth cultures revealed that spores were produced within vegetative cells (endospores). Figure 4.1 (A-B-C), respectively shows the light microscopy examination (63 x magnification) of Sporosarcina ureae (DSM 2281), Sporosarcina pasteurii (DSM 33) and Bacillus subtilis subsp. Spizizenii (DSM 15029) culture showing vegetative cells with intracellular spores. 

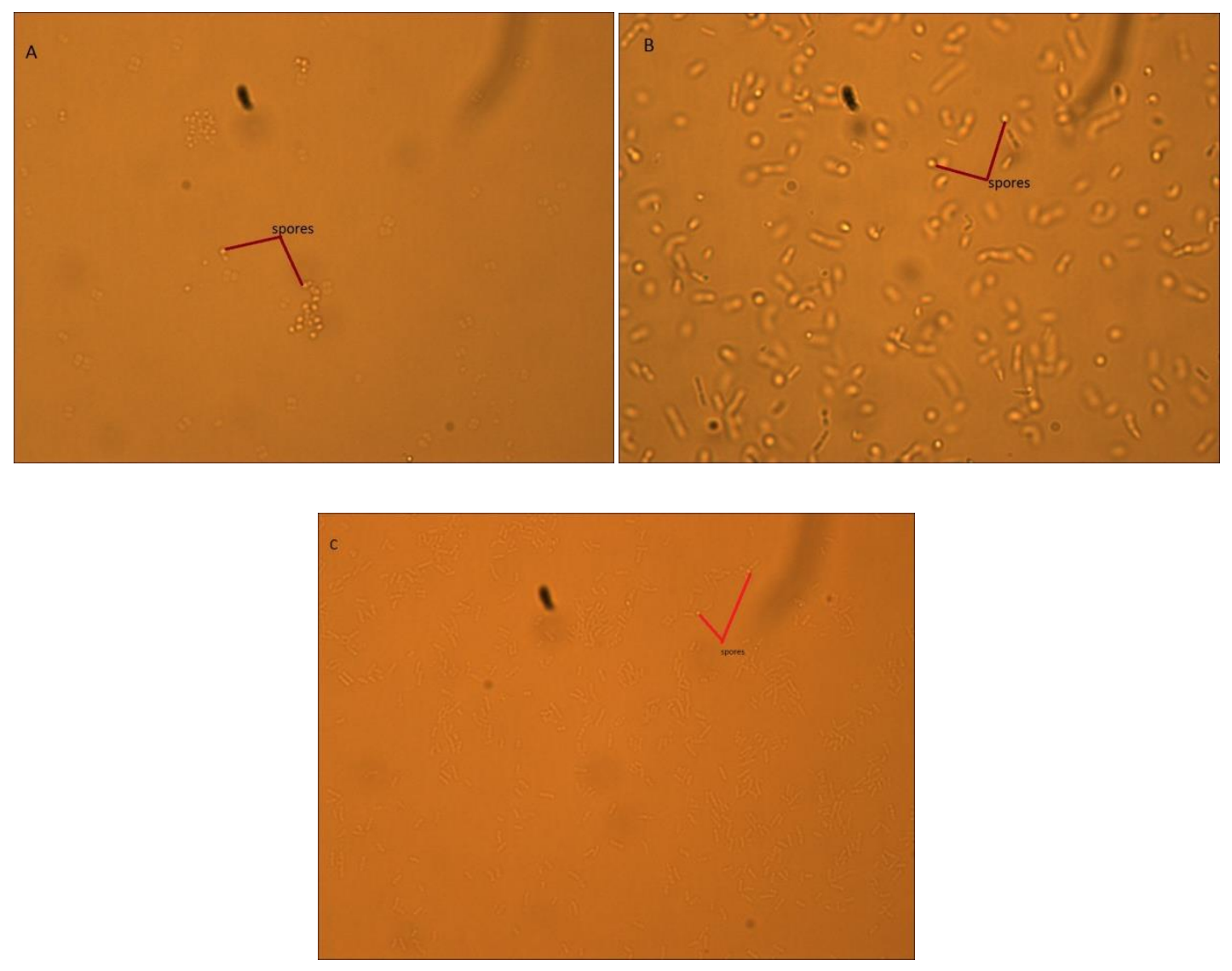

Figure 4.1: Light microscopy of (63x magnification) of (A) Sporosarcina ureae, (B)

Sporosarcina pasteurii and (C) Bacillus subtilis subsp. Spizizenii cultures showing vegetative cells with intracellular spores.

\subsubsection{High heat and $\mathrm{pH}$ resistant spore counts}

Viable counts were obtained by plating the cells after passing the bacterial spores through high heat and high $\mathrm{pH}(\mathrm{pH} \mathrm{10).} \mathrm{A} \mathrm{substantial} \mathrm{amount} \mathrm{of} \mathrm{heat} \mathrm{and} \mathrm{pH}$ resistant bacterial spores were observed on the plates after 2 days.

\subsubsection{Bacterial crystal formation}

Calcium carbonate crystal formation was observed on agar plates by providing appropriate nutrients required for the formation of crystals for the bacterial solution. After 5days, no visible crystal formation was observed however, after 14days, the light microscopic examination confirmed the formation of copious amount of crystals. Figure 4.2 shows the light microscopy examination of the calcite crystals formed on the agar plates. 

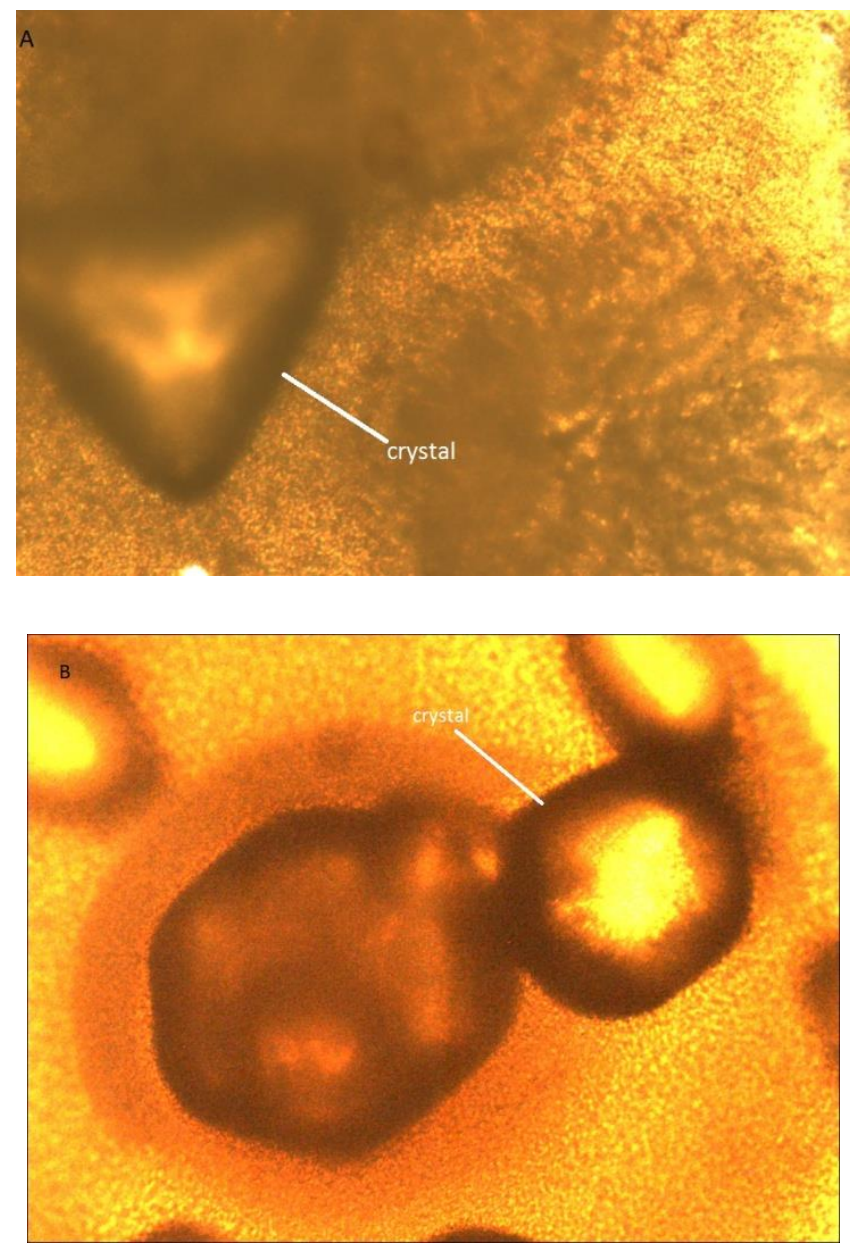

Figure 4.2: Light microscopy of (10x magnification) of crystal formation on plates for (A)

Sporosarcina pasteurii and (B) Sporosarcina ureae

\subsection{Research Phase 2: Ureolytic activity testing of bacteria}

This section details investigation on the ureolytic activity of zeolite/pumice immobilised bacteria in high $\mathrm{pH}$ cement slurry.

\subsubsection{Bacterial ureolytic activity}

In neutral $\mathrm{pH}$ environment, a very high ureolytic activity (more than 95\% urea was decomposed) was observed both for Sporosarcina ureae (DSM 2281) and Sporosarcina pasteurii (DSM 33). Both of them almost show the same activity. However for Sporosarcina pasteurii (DSM 33), it was much higher. There was not much difference in ureolytic activity between unimmobilised and immobilized bacterial cells. However, in high $\mathrm{pH}$ cement slurry, the amount of urea decomposed by the unimmobilised bacterial cells was only less than $5 \%$. About $70 \%$ of the urea was 
decomposed by the zeolite immobilized bacteria whereas around 55\% of the urea was decomposed by pumice immobilized bacteria. Slightly lower values observed on the measured decomposed urea in the $3^{\text {rd }}$ and $5^{\text {th }}$ day might be due to volatization losses. Figure 4.3 and Figure 4.4 show the ureolytic activity of zeolite and pumice immobilized bacteria in neutral and high-pH cement slurry. In Figure 4.3 and Figure 4.4, 'BS' stands for bacterial solution and 'zeo' stands for zeolite

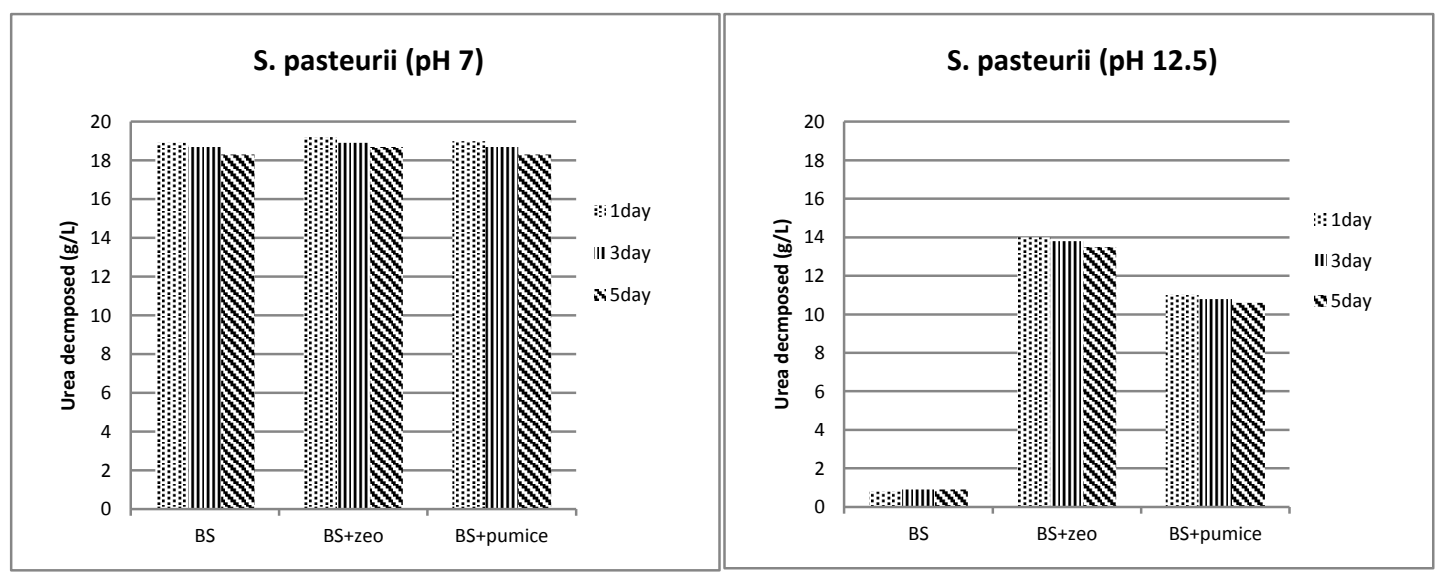

Figure 4.3: Ureolytic activity of zeolite and pumice immobilized S. pasteurii in neutral and high-pH cement slurry

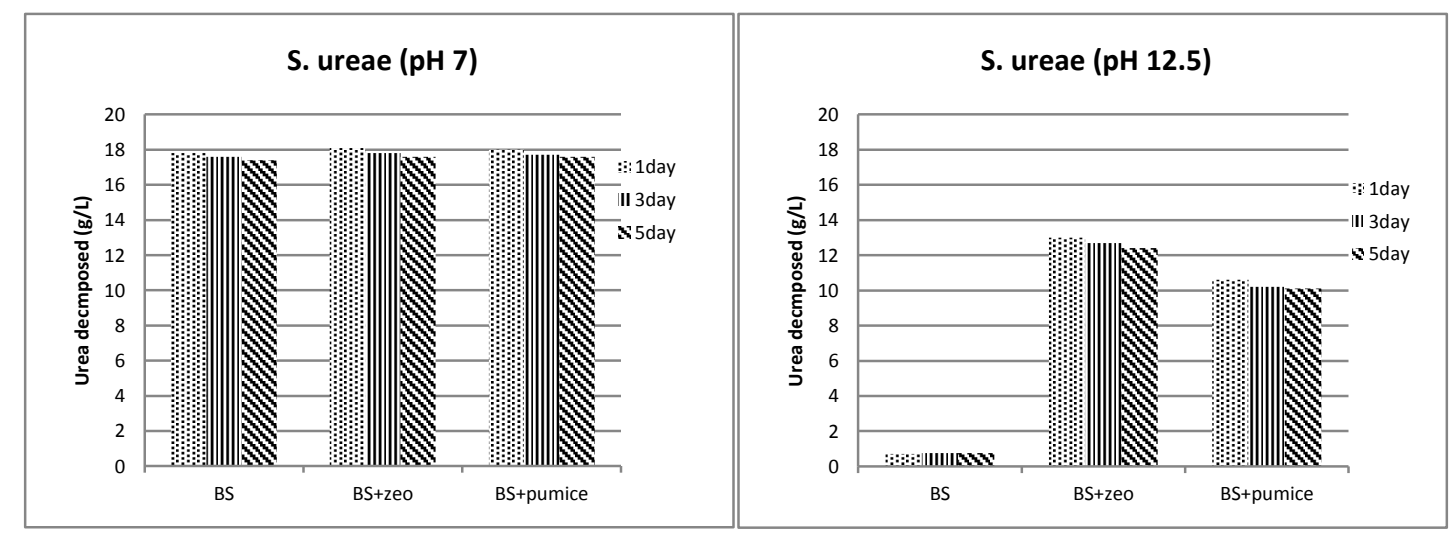

Figure 4.4: Ureolytic activity of zeolite and pumice immobilized S. ureae in neutral and high-pH cement slurry

It was found that both the selected bacterial species had high ureolytic activity in neutral $\mathrm{pH}$. However, this activity was much decreased in the high $\mathrm{pH}$ cement slurry, which demands some kinds of protection for the bacteria in high $\mathrm{pH}$ concrete environment. Zeolite and pumice immobilized bacteria showed a profound protective effect on the bacteria in the high $\mathrm{pH}$ cement slurry, which was made to mimic the real high $\mathrm{pH}$ environment inside the concrete. Similar results 
were reported by Wang et al (2011) in which Bacillus sphaericus immobilised in diatomaceous earth showed good protective effect and high urease activity compared to the activity of the unprotected bacteria. Certain degree of urease activity was observed for zeolite and pumice immobilized bacteria which showed that these materials provided a kind of micro environment for bacteria, in which the local $\mathrm{pH}$ around the bacteria was not as high as that in the cement slurry (Vandamme et al, 1998). However, zeolite showed more protective effect than pumice. This might be due to the variation in particle size distribution of these two material types in which zeolite was more coarser compared to pumice. When the particle size was bigger, bacteria can enter inside the pores of the material to adhere there and this could provide high protective effect from the surrounding harsh environment.

\subsection{Research Phase 3: Effect of healing agents on self-healing based on compressive strength of mortar cubes}

Healing agents mainly composed of the bacteria along with their carrier material and nutrients. A detailed discussion of the effects of different concentration of these materials on self-healing based on compressive strength of mortar cubes are provided in this section.

\subsubsection{Effect of the addition of nutrients on compressive strength of mortar cubes}

Figure 4.5 and Table 4.1 shows the 7, 14 and 28 days compressive strength of mortar cubes without bacteria but with varying concentration of calcium lactate while keeping the concentration of other nutrients constant. It can be seen from the Figure 4.5 that the addition of these nutrients, slightly reduced the compressive strength of mortar cubes compared to those of control mortar cubes. For $1 \%$ calcium lactate, the reduction in compressive strength compared to the normal (control) mortar is $14.4 \%, 15.5 \%$ and $9.4 \%$ in 7,14 and 28 days respectively. At the same time, for $2 \%$ calcium lactate, the reduction is $19 \%, 11 \%$ and $6.5 \%$ and for $3 \%$ calcium lactate, the reduction is $22 \%$, $15.4 \%$ and $11 \%$ in 7,14 , and 28 days respectively. From this data, it is evident that with the increase of age, the reduction of compressive strength in percentage was found to be decreasing. That is, the percentage reduction of compressive strength compared to the normal (control) mortar in 28 days was found to be less compared to that of 7 days for all the mixes. The addition of calcium lactate might have somewhat inhibited the hydration at early ages. Even though the variations were minor, out of the three different concentration of calcium lactate, $2 \%$ calcium lactate gave a strength gain of around $5 \%$ compared to that of the $1 \%$ and $3 \%$ calcium lactate. 
Therefore, $2 \%$ calcium lactate was considered as the optimum concentration for further study. However, it was found that more than $4 \%$ of calcium lactate as nutrient greatly affected the properties of mortar specimens. This may be due to the weak bonding in the mortar matrix as higher calcium lactate concentration probably hindered the bond formation.

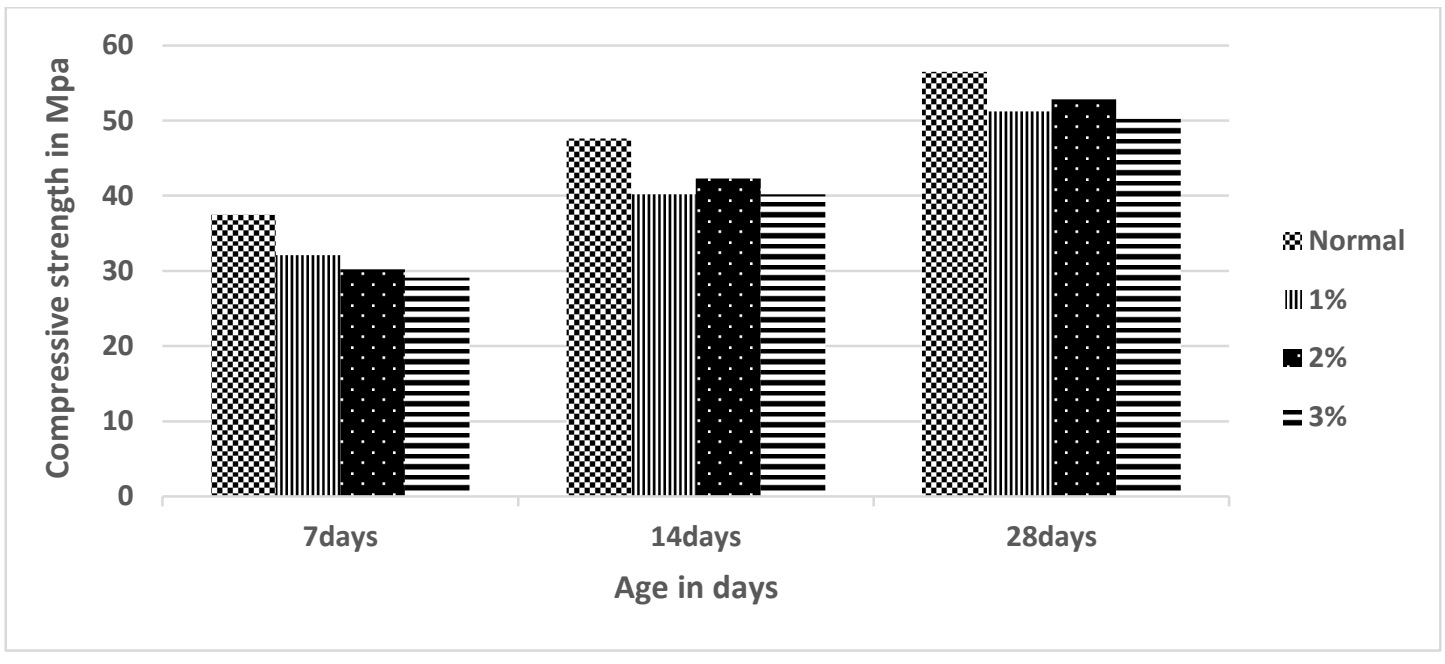

Figure 4.5: Compressive strength of mortar cubes with different calcium lactate concentrations

Table 4.1: Compressive strength of mortar cubes with different concentration of calcium lactate and their variation with age

\begin{tabular}{|c|c|c|c|c|c|c|}
\hline \multirow{2}{*}{$\begin{array}{l}\text { Concentration } \\
\text { of calcium } \\
\text { lactate (\% of } \\
\text { cement mass) }\end{array}$} & \multicolumn{2}{|c|}{ Compressive strength (MPa) at different ages } & \multicolumn{3}{|c|}{$\begin{array}{c}\text { \% reduction of compressive strength at } \\
\text { different ages (\%) }\end{array}$} \\
\cline { 2 - 7 } & $\mathbf{7 ~ d a y s}$ & $\mathbf{1 4}$ days & $\mathbf{2 8 ~ d a y s}$ & $\mathbf{7}$ days & $\mathbf{1 4}$ days & $\mathbf{2 8 ~ d a y s ~}$ \\
\hline Normal (0\%) & 37.5 & 47.6 & 56.5 & - & - & - \\
\hline $1 \%$ & 32.1 & 40.2 & 51.2 & 14.4 & 15.5 & 9.4 \\
\hline $2 \%$ & 30.2 & 42.3 & 52.8 & 19.4 & 11 & 6.5 \\
\hline $3 \%$ & 29.1 & 40.2 & 50.2 & 22.4 & 15.5 & 11 \\
\hline
\end{tabular}

\subsubsection{Effect of different concentration of bacteria on compressive strength of mortar cubes}

Another important objective was to test the effect of addition of bacteria together with nutrients on the compressive strength of mortar cubes. This study was also aimed at investigating the effect 
of the variation in the bacterial concentration on the compressive strength and also to find out the optimum concentration of bacteria for further studies. The effects of all the three bacterial species with three different cell concentrations on the 28-day compressive strength is given in Figure 4.6. Tables 4.2, 4.3 and 4.4 show the compressive strength of mortar cubes at different concentration of chosen bacterial species immobilised in zeolite and pumice and their increment in compressive strength compared to that of the mortar cubes without bacteria at different ages for S. pasteurii, $B$. subtilis and S. ureae respectively.

Table 4.2: Compressive strength of mortar cubes with different concentration of S. pasteurii and their variation with age

\begin{tabular}{|c|c|c|c|c|c|c|c|}
\hline \multirow{2}{*}{ Age } & \multicolumn{4}{|c|}{$\begin{array}{c}\text { Compressive strength at different cell } \\
\text { concentrations (MPa) }\end{array}$} & \multicolumn{3}{|c|}{$\begin{array}{l}\% \text { increase in compressive strength at } \\
\text { different cell concentrations }(\%)\end{array}$} \\
\hline & $\begin{array}{c}0 \\
\text { cells/ml }\end{array}$ & $\begin{array}{c}10^{4} \\
\text { cells/ml }\end{array}$ & $\begin{array}{c}10^{6} \\
\text { cells/ml }\end{array}$ & $\begin{array}{c}10^{8} \\
\text { cells/ml }\end{array}$ & $10{ }^{4}$ cells $/ \mathrm{ml}$ & $10^{6}$ cells $/ \mathrm{ml}$ & $10^{8}$ cells $/ \mathrm{ml}$ \\
\hline \multicolumn{8}{|c|}{ S. pasteurii + zeolite } \\
\hline 7 days & 29.8 & 32.2 & 33.2 & 32.7 & 8.1 & 11.4 & 9.7 \\
\hline 14 days & 40.3 & 44.8 & 46.5 & 44.9 & 11.2 & 15.4 & 11.4 \\
\hline 28 days & 48.9 & 54.8 & 56.9 & 55 & 12.1 & 16.4 & 12.5 \\
\hline \multicolumn{8}{|c|}{ S. pasteurii + pumice } \\
\hline 7 days & 30.1 & 33 & 33.9 & 33.3 & 9.6 & 12.6 & 10.6 \\
\hline 14 days & 41.1 & 45.6 & 47.4 & 45.8 & 10.9 & 15.3 & 11.4 \\
\hline 28 days & 51.7 & 57.9 & 60.6 & 58.8 & 12.0 & 17.2 & 13.7 \\
\hline
\end{tabular}

As shown in Table 4.2, for the specimens with $S$. pasteurii immobilised in zeolite, percentage increase in compressive strength for 7 days was found to be $8.1 \%, 11.4 \%$ and $9.7 \%$ for cell concentration of $10^{4}, 10^{6}$ and $10^{8}$ cells/ml respectively. For 14 days of curing, the increase was found to be bit higher compared to that of 7 days old specimen and were $11.2 \%, 15.4 \%$ and $11.4 \%$ for cell concentrations of $10^{4}, 10^{6}$ and $10^{8}$ cells $/ \mathrm{ml}$ respectively. Highest percentage increase in the compressive strength was observed for 28 days old specimen and was found to be $12.1 \%, 16.4 \%$ and $12.5 \%$ respectively for cell concentration of $10^{4}, 10^{6}$ and $10^{8}$ cells $/ \mathrm{ml}$. Therefore, these results confirmed that there was an increase in the percentage increment in compressive strength with the age. Similar results were observed for the specimen with $S$. pasteurii immobilised in pumice where the highest increment in the compressive strength were also observed for the 28 days old specimen 
and was found to be $12 \%, 17.2 \%$ and $13.7 \%$ respectively for cell concentration of $10^{4}, 10^{6}$ and $10^{8}$ cells $/ \mathrm{ml}$. At the same time, the increment was found to be $9.6 \%, 12.6 \%$ and $10.6 \%$ for 7 days and $10.9 \%, 15.3 \%$ and $11.4 \%$ for 14 days old specimen respectively for cell concentration of $10^{4}$, $10^{6}$ and $10^{8}$ cells $/ \mathrm{ml}$. This upward trend in percentage increment with ages justifies the effects of self-healing by bacteria treated specimen.

Similar to the results observed for the specimen treated with S. pasteurii (Table 4.2), the highest percentage increase in the compressive strength was observed for 28 days old specimen both for B. subtilis immobilised in zeolite and pumice (Table 4.3) and was found as $12.7 \%, 18.2 \%$ and $14.3 \%$ for zeolite immobilised B. subtilis and 13.9\%, 20.1\% and $15.3 \%$ for pumice immobilised B. subtilis respectively for cell concentration of $10^{4}, 10^{6}$ and $10^{8}$ cells $/ \mathrm{ml}$. However, for 7 days old specimen, the percentage increase in compressive strength was found to be $10.1 \%, 14.1 \%$ and $10.7 \%$ for B. subtilis immobilised in zeolite and $10.3 \%, 14.3 \%$ and $11.3 \%$ for B. subtilis immobilised in pumice whereas for 14 days old specimen, the percentage increase in compressive strength was noticed as $10.7 \%, 15.1 \%$ and $11.9 \%$ for B. subtilis immobilised in zeolite and $10.3 \%$, $14.3 \%$ and $11.3 \%$ for B. subtilis immobilised in pumice for cell concentration of $10^{4}, 10^{6}$ and $10^{8}$ cells/ml respectively (Table 4.3 ).

Table 4.3: Compressive strength of mortar cubes with different concentration of $B$. subtilis and their variation with age

\begin{tabular}{|c|c|c|c|c|c|c|c|}
\hline \multirow{2}{*}{ Age } & \multicolumn{4}{|c|}{$\begin{array}{c}\text { Compressive strength at different cell } \\
\text { concentrations (MPa) }\end{array}$} & \multicolumn{3}{|c|}{$\begin{array}{l}\% \text { increase in compressive strength at } \\
\text { different cell concentrations }(\%)\end{array}$} \\
\hline & $\begin{array}{c}0 \\
\text { cells/ml }\end{array}$ & $\begin{array}{c}10^{4} \\
\text { cells/ml }\end{array}$ & $\begin{array}{c}10^{6} \\
\text { cells/ml }\end{array}$ & $\begin{array}{c}10^{8} \\
\text { cells/ml }\end{array}$ & $10{ }^{4}$ cells $/ \mathrm{ml}$ & $10^{6}$ cells $/ \mathrm{ml}$ & $10^{8}$ cells $/ \mathrm{ml}$ \\
\hline \multicolumn{8}{|c|}{ B. subtilis + zeolite } \\
\hline 7 days & 29.8 & 32.8 & 34 & 33 & 10.1 & 14.1 & 10.7 \\
\hline 14 days & 40.3 & 44.6 & 46.4 & 45.1 & 10.7 & 15.1 & 11.9 \\
\hline 28 days & 48.9 & 55.1 & 57.8 & 55.9 & 12.7 & 18.2 & 14.3 \\
\hline \multicolumn{8}{|c|}{ B. subtilis + pumice } \\
\hline 7 days & 30.1 & 33.2 & 34.4 & 33.5 & 10.3 & 14.3 & 11.3 \\
\hline 14 days & 41.1 & 45.7 & 47.6 & 46.3 & 11.2 & 15.8 & 12.7 \\
\hline 28 days & 51.7 & 58.9 & 62.1 & 59.6 & 13.9 & 20.1 & 15.3 \\
\hline
\end{tabular}


Table 4.4: Compressive strength of mortar cubes with different concentration of $S$. ureae and their variation with age

\begin{tabular}{|c|c|c|c|c|c|c|c|}
\hline \multirow{2}{*}{ Age } & \multicolumn{4}{|c|}{$\begin{array}{c}\text { Compressive strength at different cell } \\
\text { concentrations (MPa) }\end{array}$} & \multicolumn{3}{|c|}{$\begin{array}{l}\% \text { increase in compressive strength at } \\
\text { different cell concentrations }(\%)\end{array}$} \\
\hline & $\begin{array}{c}\mathbf{0} \\
\text { cells/ml }\end{array}$ & $\begin{array}{c}10^{4} \\
\text { cells/ml }\end{array}$ & $\begin{array}{c}10^{6} \\
\text { cells } / \mathrm{ml}\end{array}$ & $\begin{array}{c}10^{8} \\
\text { cells } / \mathrm{ml}\end{array}$ & $10^{4}$ cells $/ \mathrm{ml}$ & $10^{6}$ cells $/ \mathrm{ml}$ & $10^{8}$ cells $/ \mathrm{ml}$ \\
\hline \multicolumn{8}{|c|}{ S. ureae + zeolite } \\
\hline 7 days & 29.8 & 31.7 & 32.8 & 32.1 & 6.4 & 10.1 & 7.7 \\
\hline 14 days & 40.3 & 43.4 & 44.7 & 43.8 & 7.7 & 10.9 & 8.7 \\
\hline 28 days & 48.9 & 53.1 & 55.5 & 53.8 & 8.6 & 13.5 & 10.0 \\
\hline \multicolumn{8}{|c|}{ S. ureae + pumice } \\
\hline 7 days & 30.1 & 32.1 & 33.1 & 32.6 & 6.6 & 10.0 & 8.3 \\
\hline 14 days & 41.1 & 44.4 & 45.9 & 45 & 8.0 & 11.7 & 9.5 \\
\hline 28 days & 51.7 & 56.8 & 59.3 & 57.1 & 9.9 & 14.7 & 10.4 \\
\hline
\end{tabular}

In the case of $S$. ureae (Table 4.4), percentage increase in compressive strength for 28 days old specimen (highest increment observed in the cases of S. pasteurii and B. subtilis) were 8.6\%, $13.5 \%$ and $10 \%$ for zeolite immobilised S. ureae and $9.9 \%, 14.7 \%$ and $10.4 \%$ for pumice immobilised $S$. ureae for cell concentration of $10^{4}, 10^{6}$ and $10^{8}$ cells $/ \mathrm{ml}$ respectively. Respective values for zeolite immobilised $S$. ureae were only $6.4 \%, 10.1 \%$ and $7.7 \%$ for 7 days old specimen and $7.7 \%, 10.9 \%$ and $8.7 \%$ for 14 days old specimen. Similarly, for pumice immobilised S. ureae , percentage increment in compressive strength were found as $6.6 \%, 10 \%$ and $8.3 \%$ for 7 days and $8 \%, 11.7 \%$ and $9.5 \%$ in the case of 14 days old specimens for cell concentration of $10^{4}, 10^{6}$ and $10^{8}$ cells/ml respectively.

It can be concluded from the Tables 4.2, 4.3 and 4.4 that pumice immobilised bacteria showed better increment in compressive strength (17.2\% for S. pasteurii, $20.1 \%$ for B. subtilis and $14.7 \%$ for $S$. ureae in 28 days) compared to that of zeolite immobilised bacteria (16.4\% for S. pasteurii, $18.4 \%$ for B. subtilis and $13.5 \%$ for S. ureae in 28 days). Similar trend can be noticed for 7 days and 14 days old specimen for all the three selected bacterial species (Tables 4.2, 4.3 and 4.4). Even though these variations are minor, it can be inferred that the better protective effect for bacteria was provided by the pumice compared to that of zeolite. Hence pumice can be considered as the preferred carrier material for the bacteria. 
From Tables 4.2, 4.3 and 4.4, it was also sighted that the percentage increment in compressive strength was maximum at the cell concentration of $10^{6}$ cells $/ \mathrm{ml}$ for both zeolite $(17.2 \%, 15.3 \%$ and $12.6 \%$ for 28,14 and 7 days respectively) and pumice (17.2\%, $15.3 \%$ and $12.6 \%$ for 28,14 and 7 days respectively) immobilised $S$. pasteurii (Table 4.2). Similar trends were observed in the cases of zeolite/pumice immobilised B. subtilis (Table 4.3) and S. ureae (Table 4.4). Figure 4.6 shows that the compressive strength increased with increase in bacteria cell concentration up to $10^{6}$ cells $/ \mathrm{ml}$, and then there was a reduction in the strength at $10^{8}$ cells $/ \mathrm{ml}$ for all the selected bacterial species. Maximum increase in compressive strengths was achieved at $10^{6}$ cells $/ \mathrm{ml}$ which was selected as the optimum cell concentration for the further study.

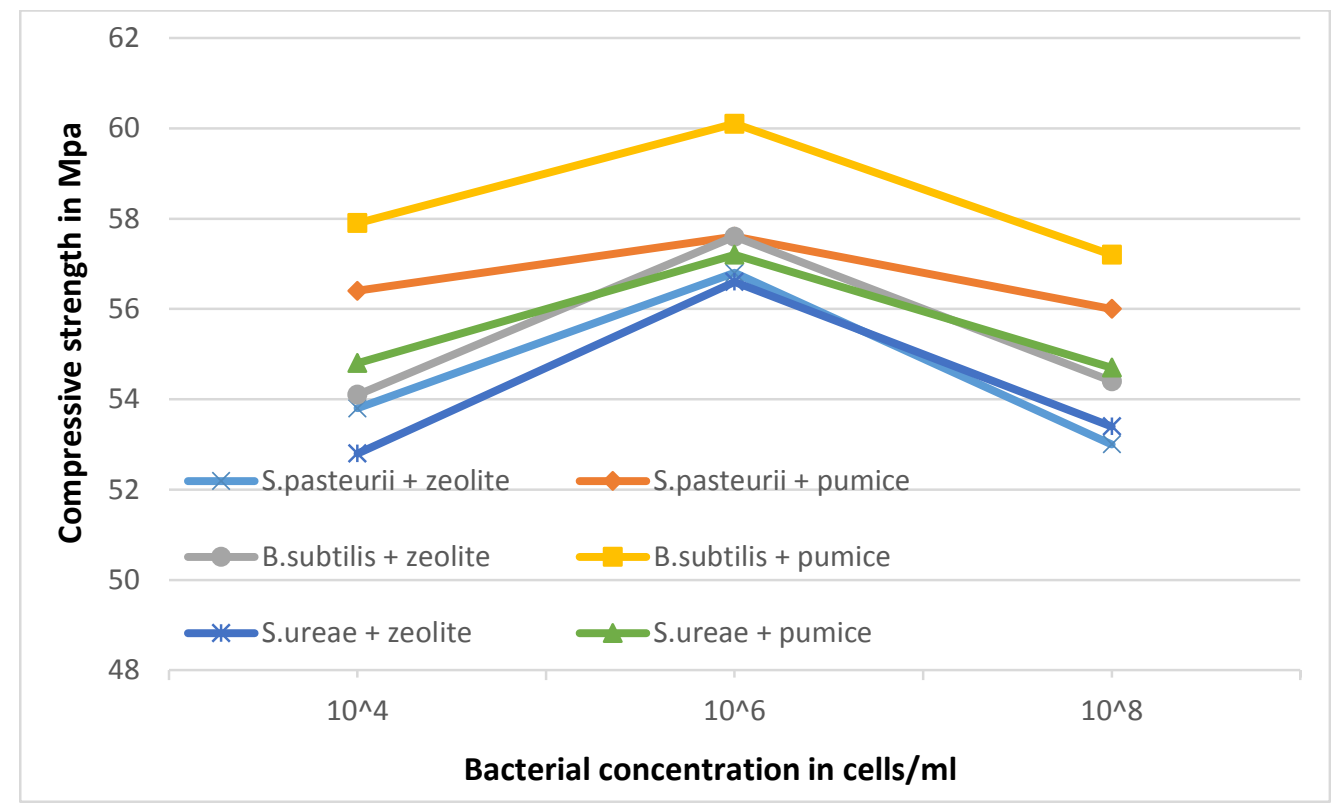

Figure 4.6: 28-day compressive strength of mortar cubes with different bacterial concentration

As far as the bacteria are concerned, B. subtilis provided highest percentage of increment in compressive strength (20\%) followed by S. pasteurii (17.2\%). S. ureae (14.7\%) provided the least increment in compressive strength compared to the other two selected bacterial species (Tables 4.2, 4.3 and 4.4). Figure 4.6 also shows that the maximum compressive strength was given by $B$. subtilis $(62 \mathrm{MPa})$ for a cell concentration of $10^{6} \mathrm{cells} / \mathrm{ml}$. Therefore all the three selected bacterial 
species can be considered as healing agent in the bacterial self-healing process in which $B$. subtilis can be selected as the most preferred among them.

Figure 4.7 compares the compressive strength of mortar cubes from all the 9 mixes with a cell concentration of $10^{6}$ cells $/ \mathrm{ml}$ at different ages. It is found that different bacterial species showed different compressive strength and Sporosarcina pasteurii immobilised into zeolite gave the maximum compressive strength for long term followed by Bacillus subtilis subsp. Spizizenii. The compressive strength had significantly increased for the mortar cubes that contained microbial cells irrespective of the types compared to that of control (normal mortar) specimen. Therefore, it can be concluded that the addition of bacteria and nutrients in the mortar matrix is not affecting the structural integrity in the long term in a detrimental manner.

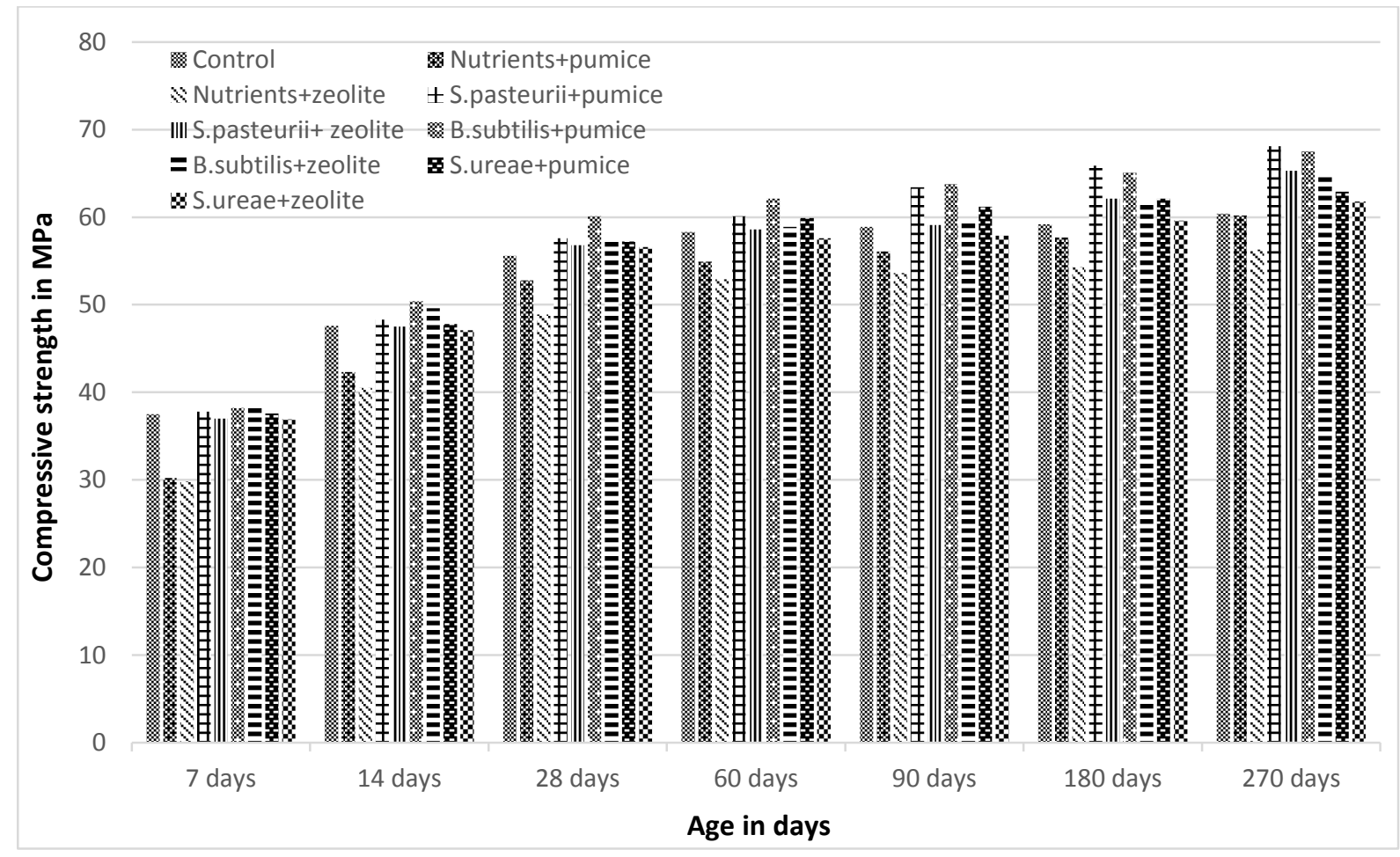

Figure 4.7: Cube compressive strength of all 9 mortar mixes at different ages with $10^{6}$ cells $/ \mathrm{ml}$

\subsubsection{Effect of bacteria on compressive strength of fibre reinforced mortar}

Experimenting with normal mortar with different types and concentrations of bacteria and carrier materials and analyzing the results in-depth, the preferred bacterial concentration for further 
research was identified. Building on this information, further experimentation was done on fibre reinforced mortar to identify the effect of bacterial incorporation on the compressive strength and the extent of healing. In the case of fibre reinforced mortar, the only cell concentration used was $10^{6}$ cells/ml for all three different types of bacteria - because it was identified as the optimal concentration based on the previous test results as described in previous section. The results obtained are depicted on Figure 4.8.

Careful observation of Figure 4.8 indicates that the trend observed for normal mortar is repeated in the cased fiber reinforced mortar as well. Even though the addition of nutrients slightly decreases the compressive strength, addition of bacteria neutralises this reduction and slightly improves the compressive strength overall. It can be concluded at this point that addition of bacteria and nutrients does not affect the structural integrity of the mortar, in fact it improves the strength properties. It also shows that the compressive strength gradually increases with the increase of specimen age. Best results were obtained for the combination of S. pasteuriit zeolite, the compressive strength observed was $100 \mathrm{MPa}$, which was $11 \%$ higher than that of control specimen at the age of 270 days. It is interesting to note that initially, the choice of pumice as the carrier material provided better strength up to the age of 90 days, but afterwards zeolite (as the protective vehicle) performed better. This confirms that long term viability of the bacteria is ensured by zeolite as the protective carrier material.

Another important observation is that, in the case of the choice of bacteria, B. subtilis performs slightly better in terms of compressive strength (around 2\% higher) compared to S. pasteurii up to 90 days of curing and S. pasteurii provided better strength (around 4\% higher) when the curing age was 270 days. The performance of S. ureae was around 10\% lower compared to the other bacteria in the long term (270 days). Initially, at the age of 28 days, the performance of $S$. ureae was relatively close to that of other two bacteria (around 4\%). It is inferred from these observations that $S$. ureae is probably not an ideal choice for long term viability compared to other bacteria chosen. 


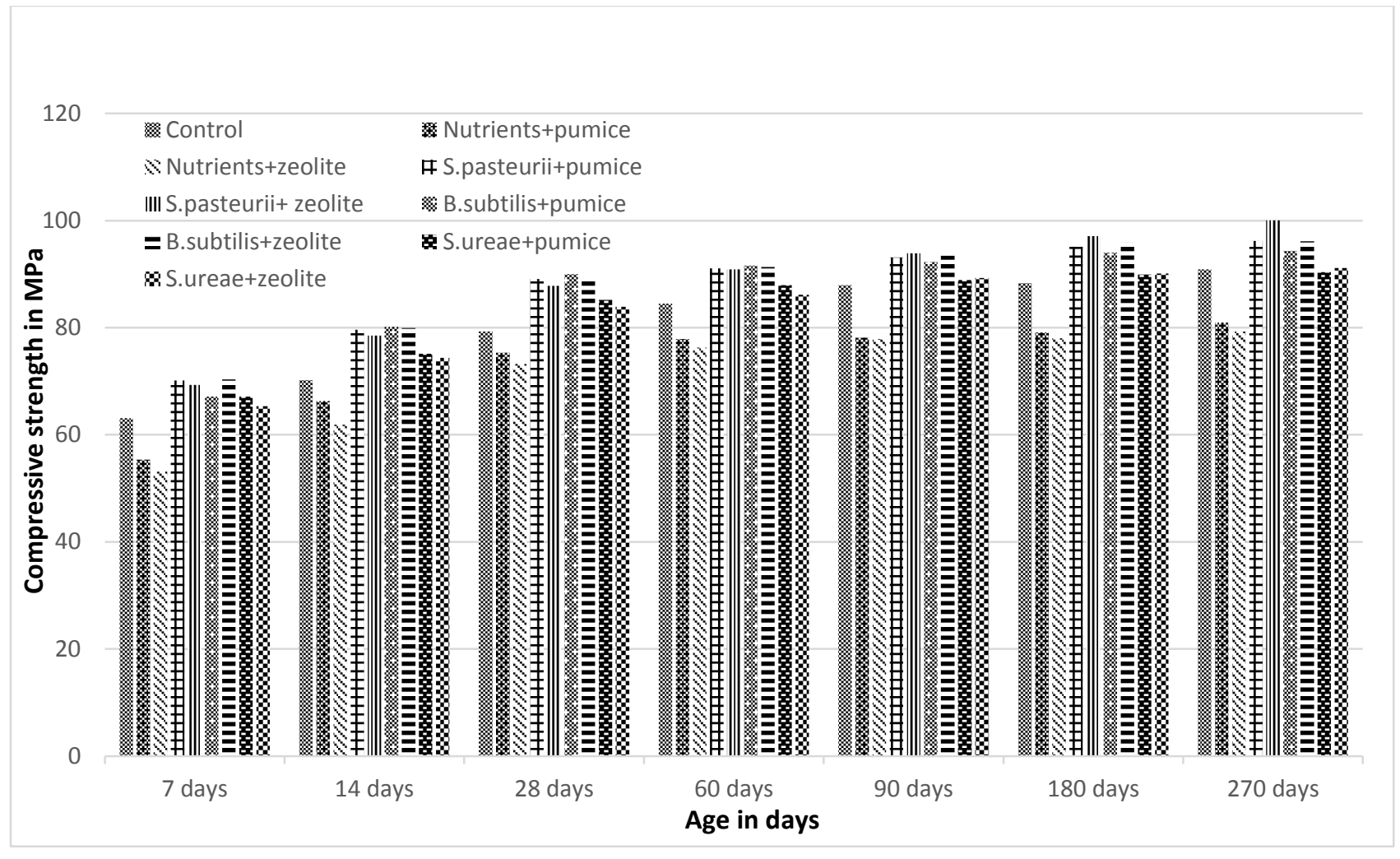

Figure 4.8: Cube compressive strength of all 9 FR mortar mixes at different ages

In this research work, the improvement in compressive strength by the selected bacteria was probably due to deposition of $\mathrm{CaCO}_{3}$ on the micro-organism cell surfaces and within the pores of the mortar. Similar results were published by other researchers (Achal et. al, 2009; Ghosh et. al, 2005; Ramakrishnan et al, 1998). These results have shown that concrete with enhanced strength and low-permeability could be produced with the aid of bacteria. The increase in the matrix strength of mortar made with bacterial cells would eventually increase the overall durability performance.

\subsection{Research Phase 4: Self-healing behavior investigation}

A detailed analysis and discussion of the results of the self-healing investigation conducted on permeation properties of both normal and fibre reinforced mortar and flexural properties of cracked fibre reinforced (FR) mortar are presented in this section. 


\subsubsection{Effect of bacteria induced self-healing on sorptivity and water absorption properties}

The assessment of self-healing on the pre-cracked specimens (both FR and normal mortar) was performed using the sorptivity test. Since it is hard to create cracks in normal mortar, small holes were created to mimic the behavior of cracks during casting. Fibre reinforced mortar was also selected for the study in order to observe the healing effect in sorptivity properties of cracked specimens. Both primary (performed within the first 6 hours of initiation of the testing) and secondary (performed after 24 hours up to 7 days) sorptivity tests were conducted and the results were analysed. In the normal mortar specimens, sorptivity test was conducted on 7 days old cylinders and subsequently immersed in water for self-healing. In the case of fibre reinforced mortar, sorptivity test was conducted immediately after crack creation on the $7^{\text {th }}$ day and then immersed in water for self-healing. The test was performed again at the ages of 120, 180 and 240 days of healing on both these mixes.

The result of water absorption tests on all cracked FR mortar (Figures 4.9 to 4.16 ) and normal mortar with holes (Figures 4.17 and 4.24) in 7 days and after 120, 180 and 240 days of healing are presented and discussed. Figures 4.9 and 4.10 show the plots of water absorption (mm) versus square root of time (sec) of control specimen with that of pumice and zeolite immobilised bacteria respectively for 7 days (just after cracking). It can be seen that the water absorption of all the bacteria treated specimens were very close to that of control specimen. At the same time, the speed of water absorption in the specimens without bacteria was much faster than the ones with bacteria after 120 days of healing (Figures 4.11 and 4.12). Specimen treated with different kinds of bacterial species showed different rates of absorption (with low variation compared to the control specimens). The sequence in 120 days was Bacillus subtilis subsp. Spizizenii immobilised in pumice < Sporosarcina pasteurii immobilised in pumice < Bacillus subtilis subsp. Spizizenii immobilised in zeolite < Sporosarcina pasteurii immobilised in zeolite < Sporosarcina ureae immobilised in pumice $<$ Sporosarcina ureae immobilised in zeolite $<$ Nutrients + pumice $<$ Nutrients + zeolite $<$ Control. The calcium carbonate precipitation in cracks might have profoundly decreased the water absorption of the cracked specimens. Same trend can be observed for 180 days healed specimen (Figures 4.13 and 4.14). However, when it comes to 240 days of healing, it can be observed that the self-healing effect provided by the Sporosarcina pasteurii + pumice was the best, followed by the Bacillus subtilis subsp. Spizizenii + pumice. (Figures 4.15 and 4.16). 
Specimens without bacteria but with nutrients showed slightly lower rate of water absorption compared to the control specimen. The reason for this might be the presence of calcium lactate which can precipitate calcium carbonate upon reaction with carbonate ions. However, the ones with bacteria showed much less rate of absorption compared to the nutrients only specimen which indicated that the specimen with highest precipitation had the lowest rate of water absorption.

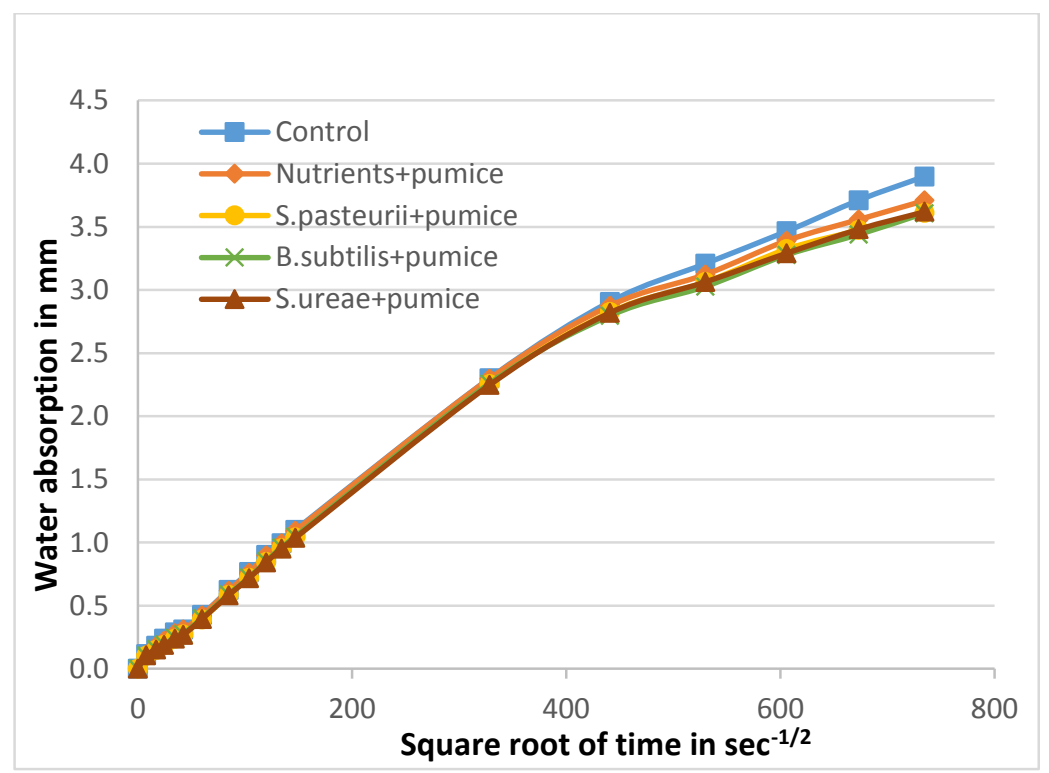

Figure 4.9: Water absorption of cracked FR mortar in 7 days with pumice

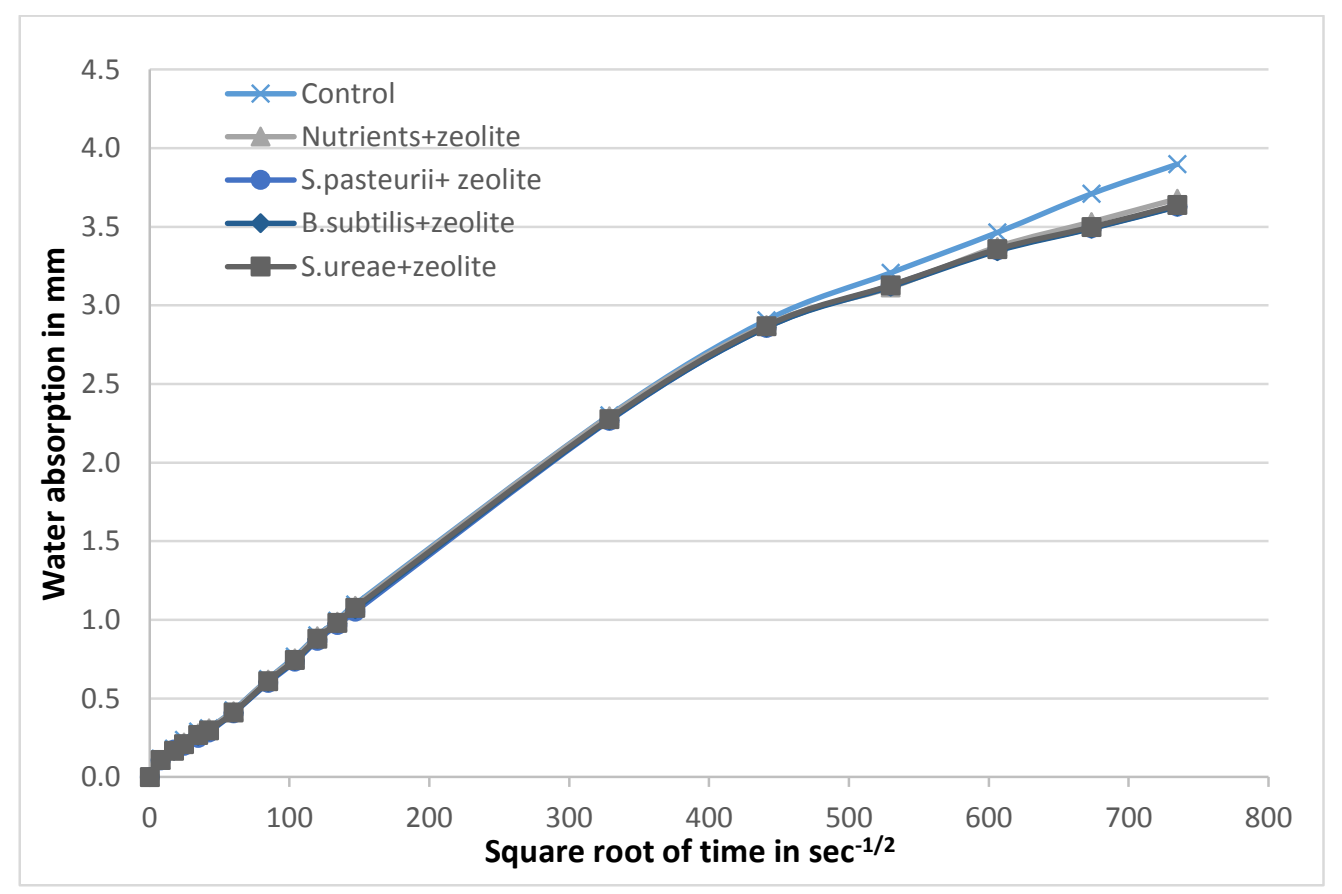

Figure 4.10: Water absorption of cracked FR mortar in 7 days with zeolite 


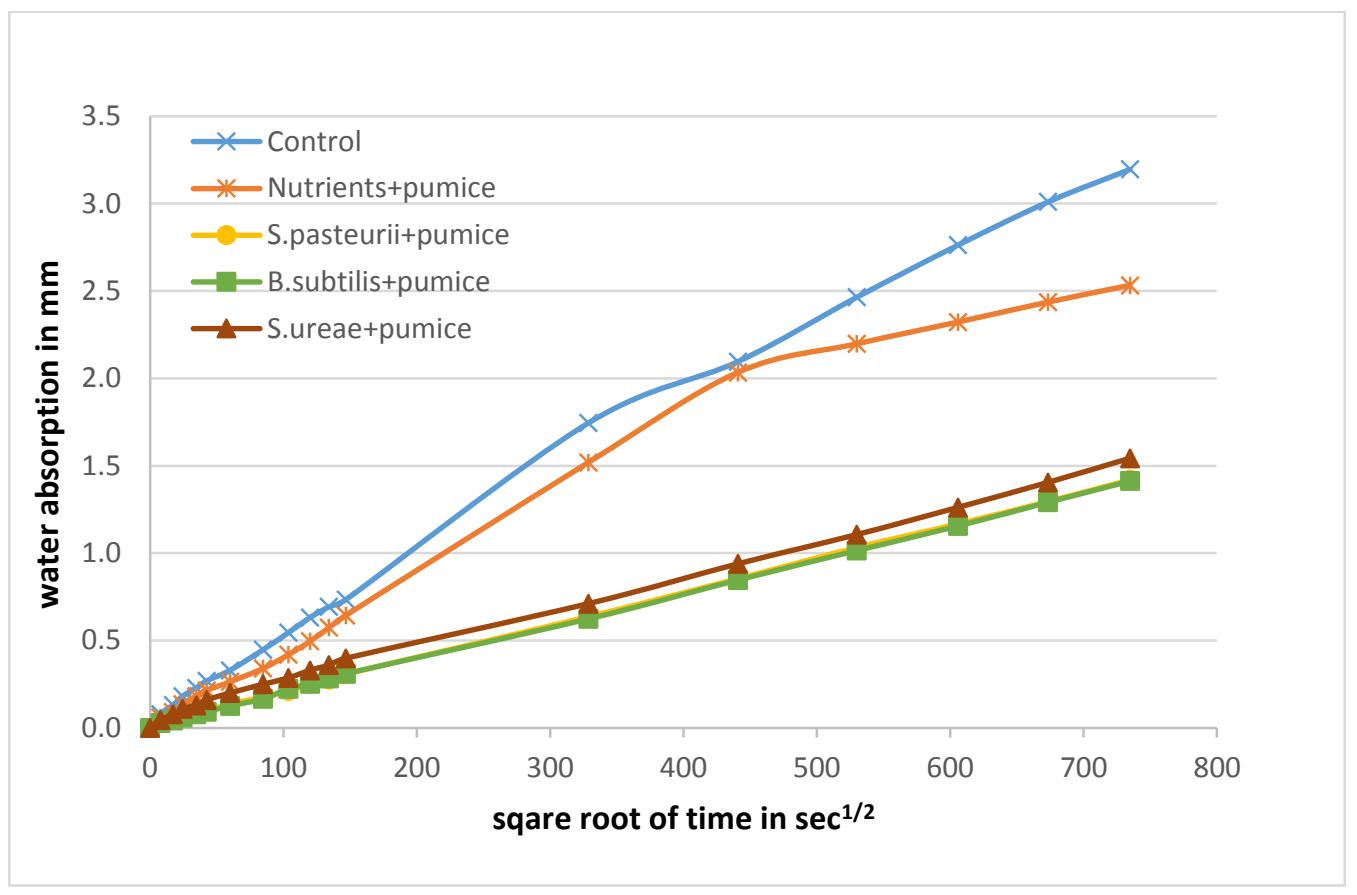

Figure 4.11: Water absorption of cracked FR mortar after 120 days of healing with pumice

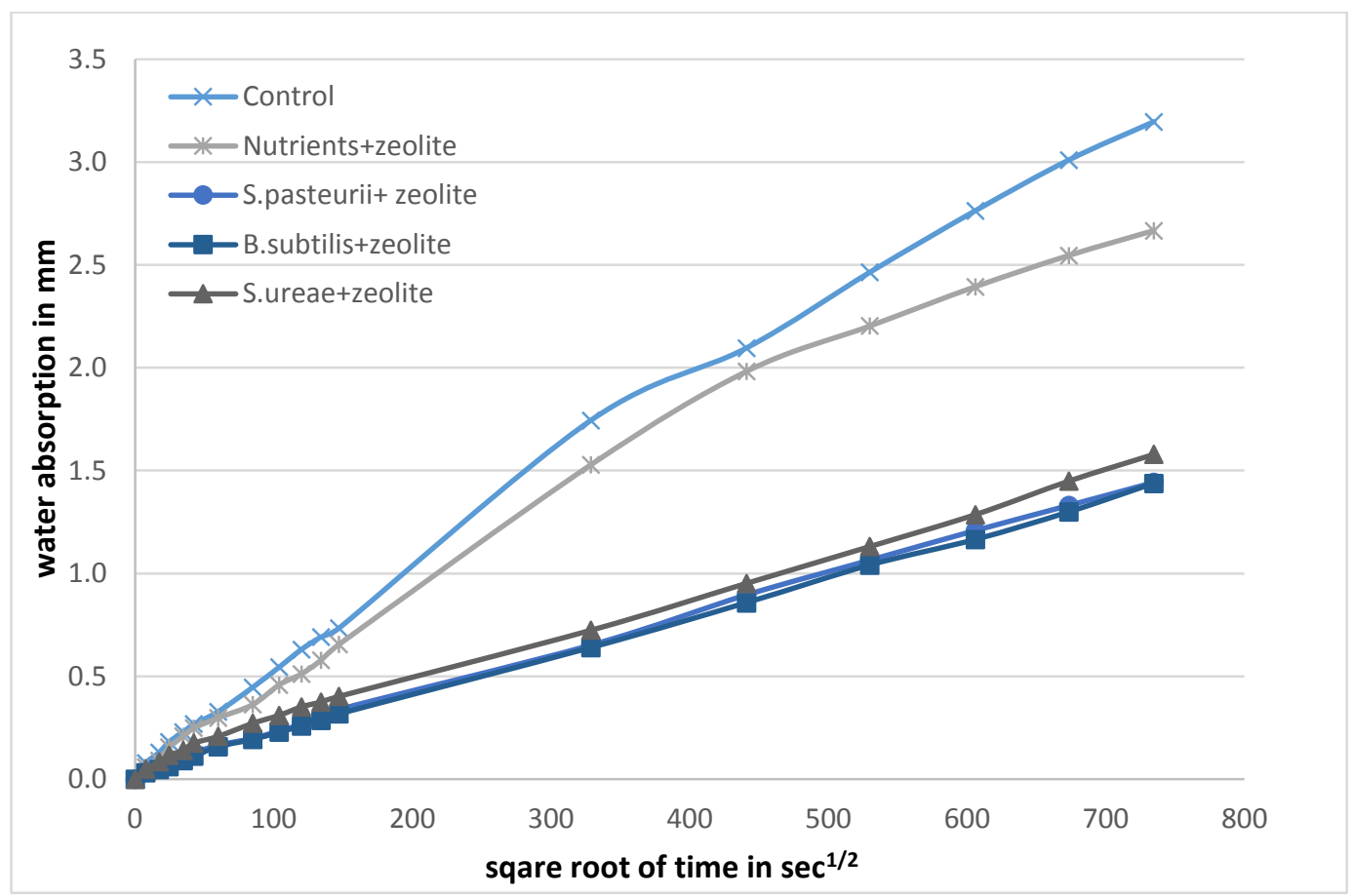

Figure 4.12: Water absorption of cracked FR mortar after 120 days of healing with zeolite 


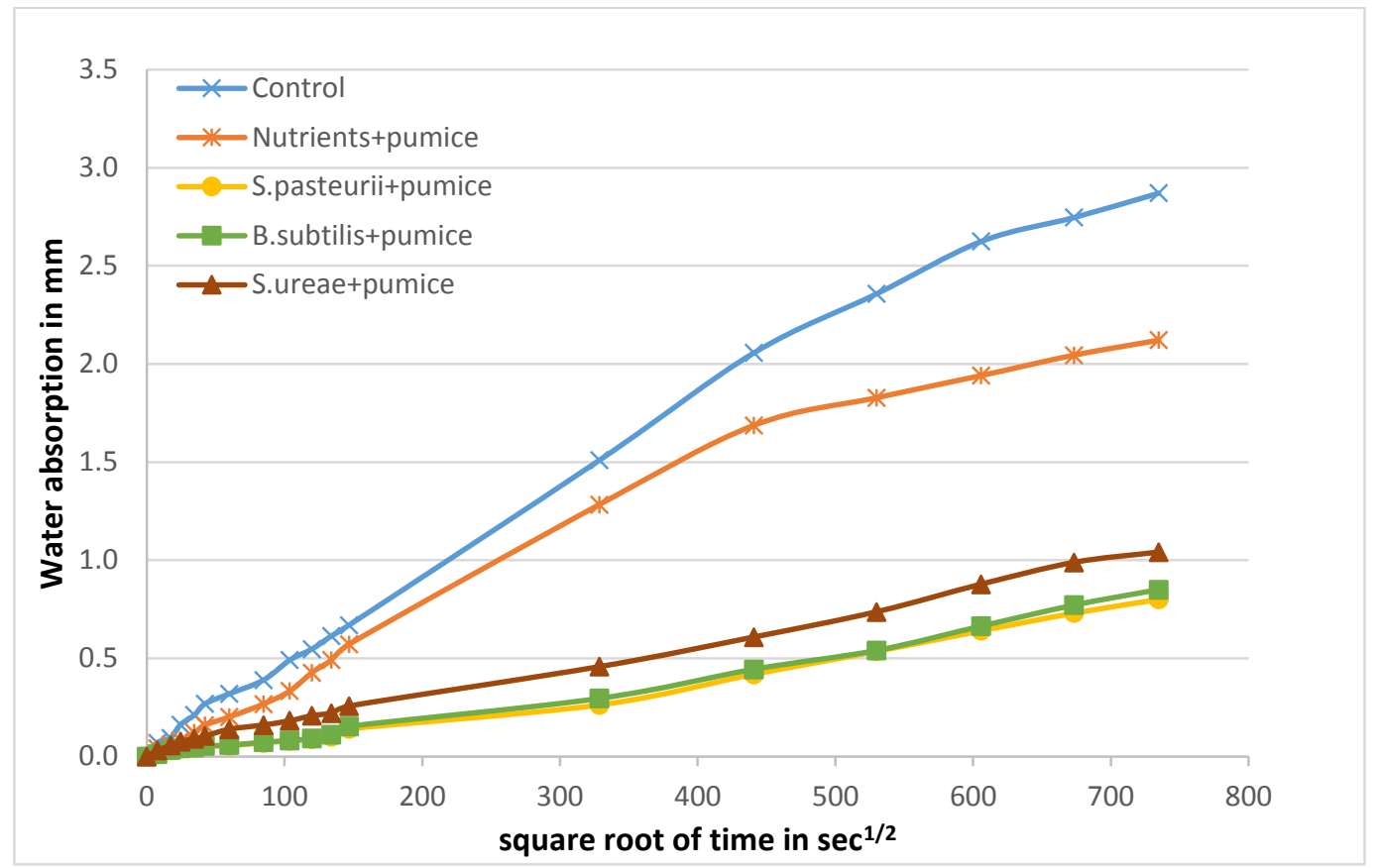

Figure 4.13: Water absorption of cracked FR mortar after 180 days of healing with pumice

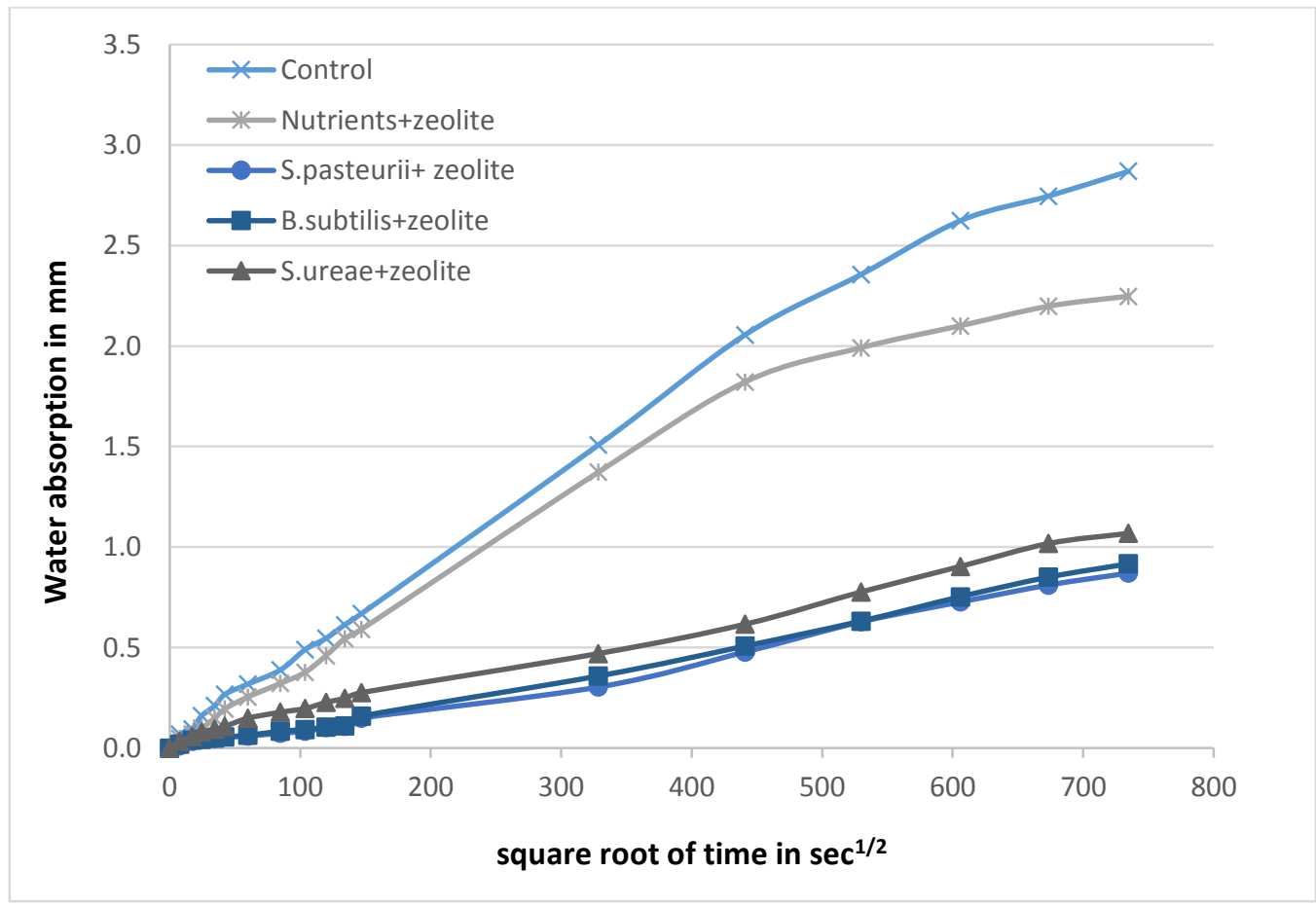

Figure 4.14: Water absorption of cracked FR mortar after 180 days of healing with zeolite 


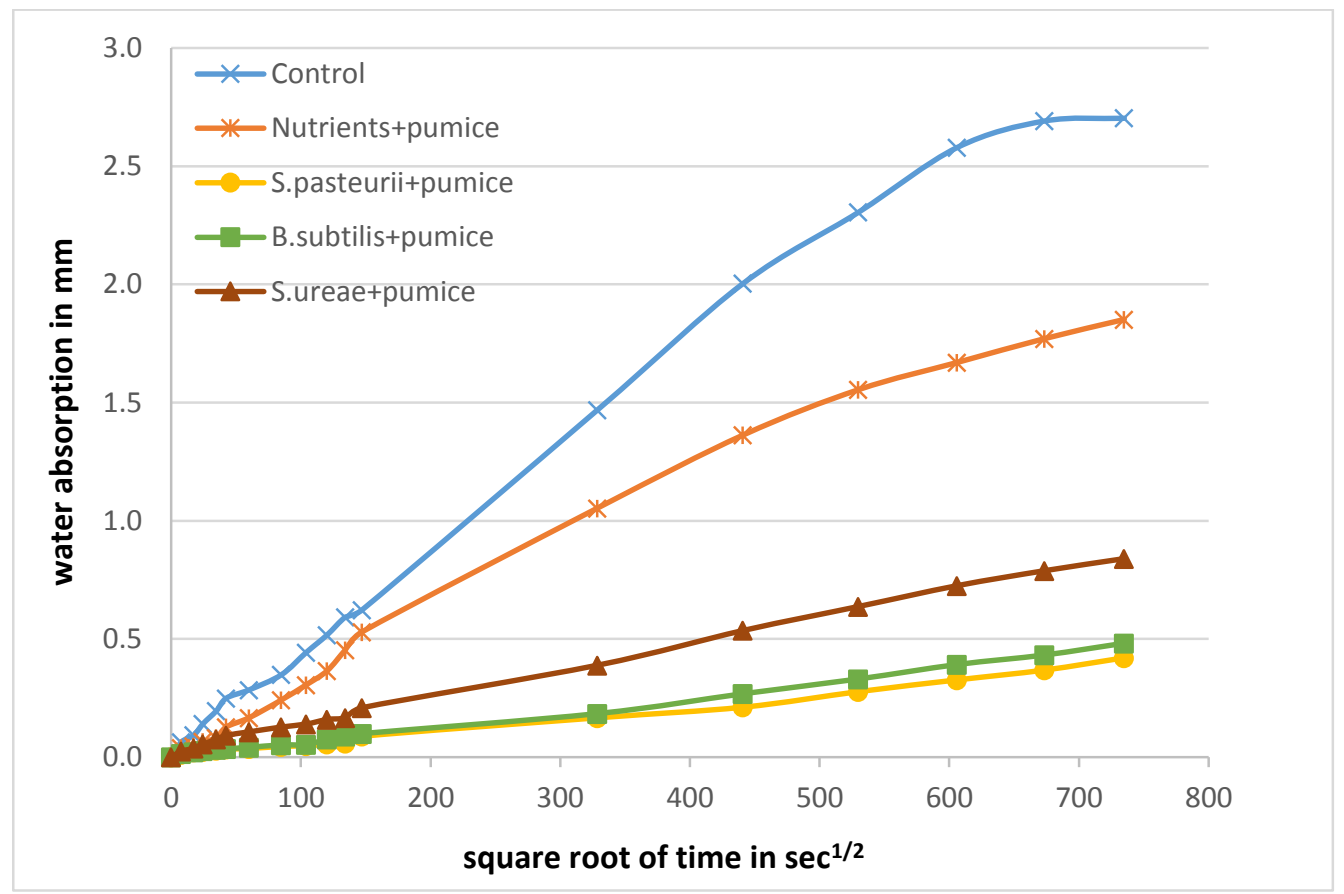

Figure 4.15: Water absorption of cracked FR mortar after 240 days of healing with pumice

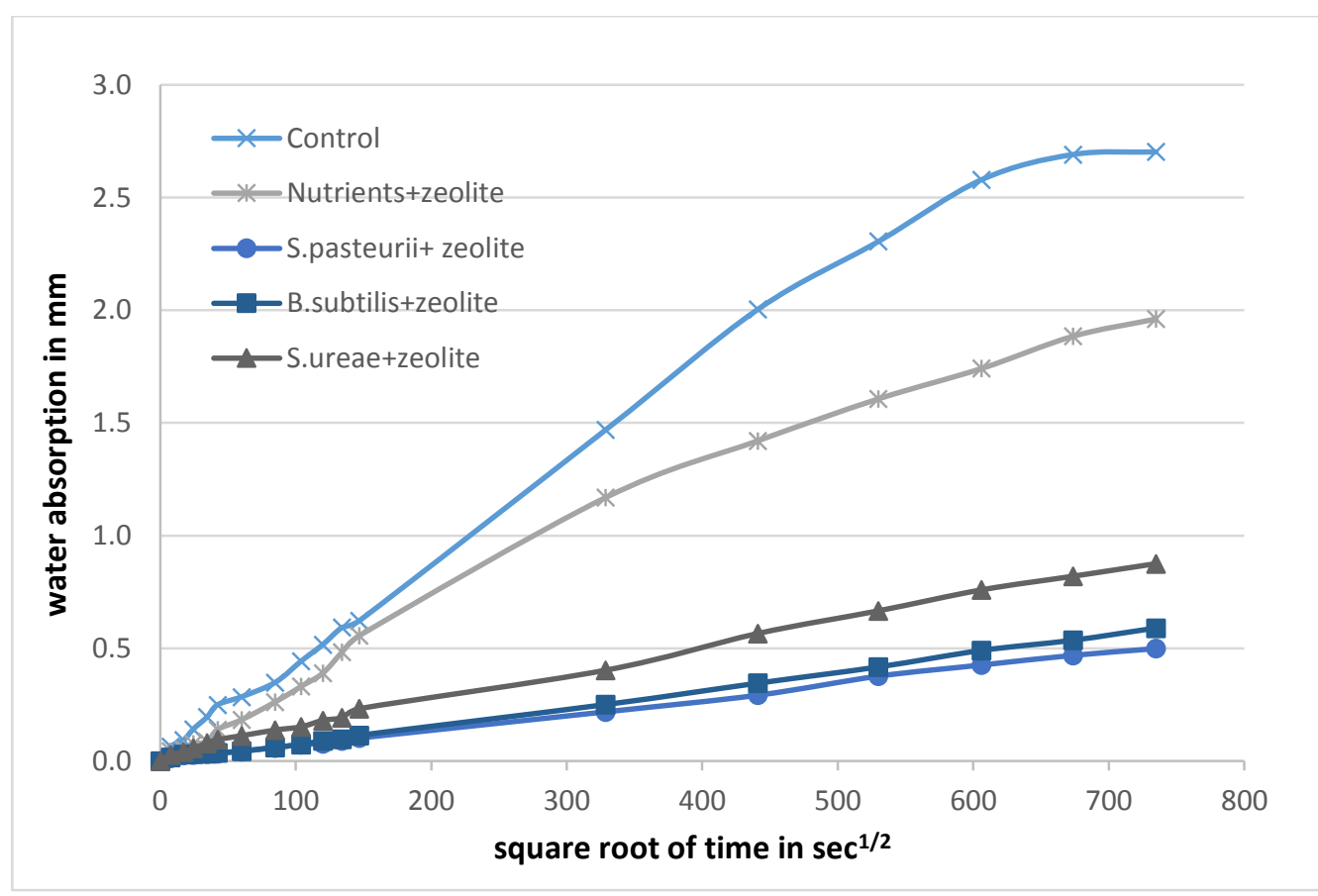

Figure 4.16: Water absorption of cracked FR mortar after 240 days of healing with zeolite 
Similar trend was observed for the water absorption plots of normal mortar with holes (Figures 4.17 and 4.24). It can be observed from Figures 4.9, 4.10, 4.17 and 4.18 (7 days old specimen) that for all the selected mixes, the water absorbed per unit surface area $\left(\mathrm{mm}^{3} / \mathrm{mm}^{2}\right)$ increased with the square root of time. Moreover, the presence of cracks increased the water absorbed per unit surface area. This can be explained by the fact that the micro-cracks act as capillaries which can absorb and reserve water in the crack. Consequently, it increases the absorbed water weight for the specimen. It can be noticed that the water absorption $(\mathrm{mm})$ of all the bacteria treated specimen with pumice and zeolite were significantly reduced for the 120 days old specimen compared to the specimens without bacteria (Figure 4.19 and 4.20) however, the water absorption of all the 9 mixes were relatively close in 7 days old specimen (Figures 4.17 and 4.18). Just like the trend observed in FR mortar specimens (Figures 4.11 to 4.16), despite the B. subtilis + pumice showed the lowest water absorption for 120 days (Figures 4.19 and 4.20) and 180 days (Figures 4.21 and 4.22) healed specimens, it was found that S. pasteurii + pumice gave the lowest absorption of water for the 240 days healed specimen (Figures 4.23 and 4.24). Still, the healing efficiency of $S$. ureae appeared to be less compared to that of S. pasteurii and B. subtilis for 120, 180 and 240 days healed specimens. It was reported that $\mathrm{CaCO}_{3}$ formation and depth of cementation were more intense for Sporosarcina pasteurii compared to Sporosarcina ureae due to the higher urease activity of the enzyme in Sporosarcina pasteurii even though both of them appreared to be very close in characteristics (Sarmast et. al., 2014). 


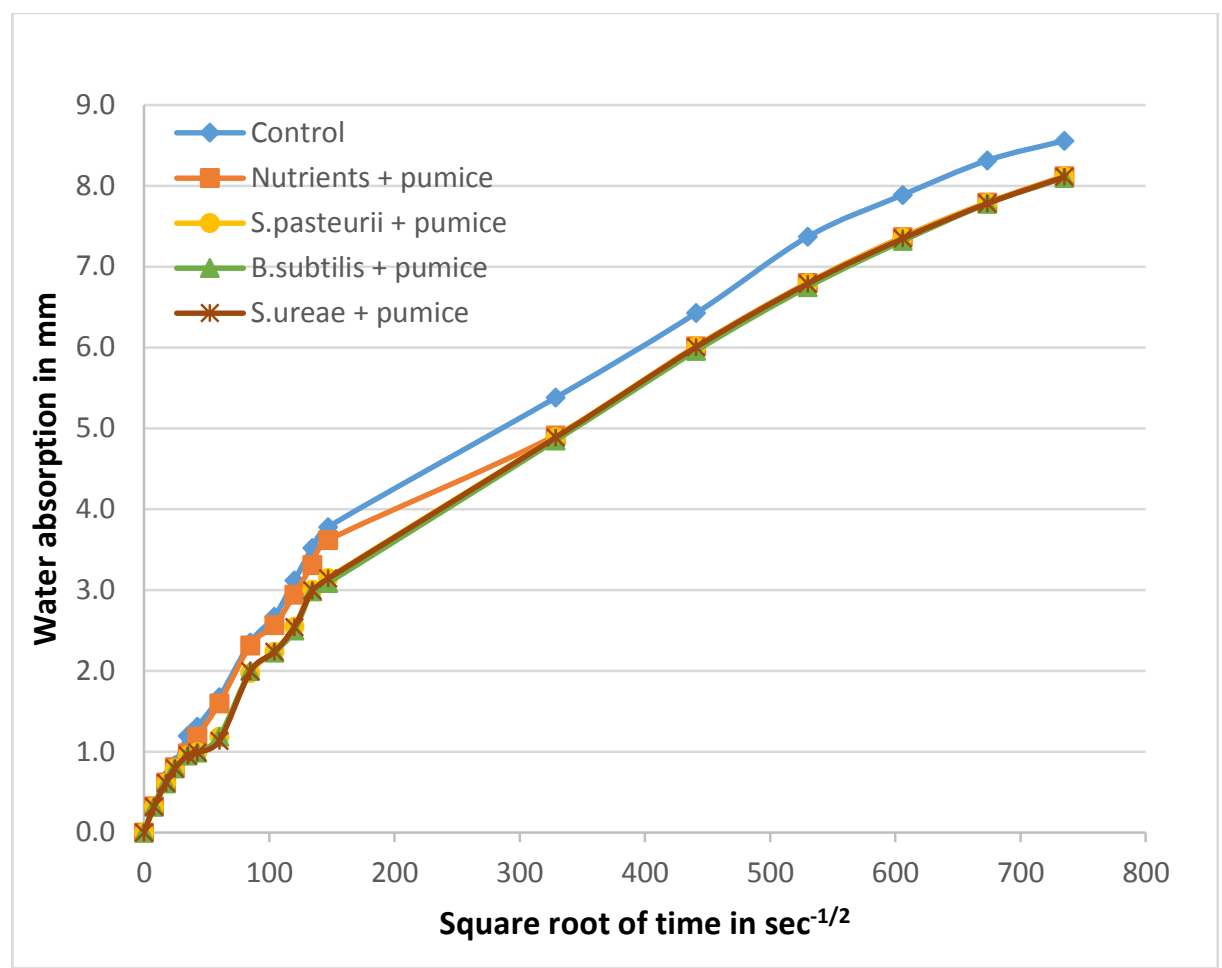

Figure 4.17: Water absorption of 7 days old normal mortar with holes with pumice

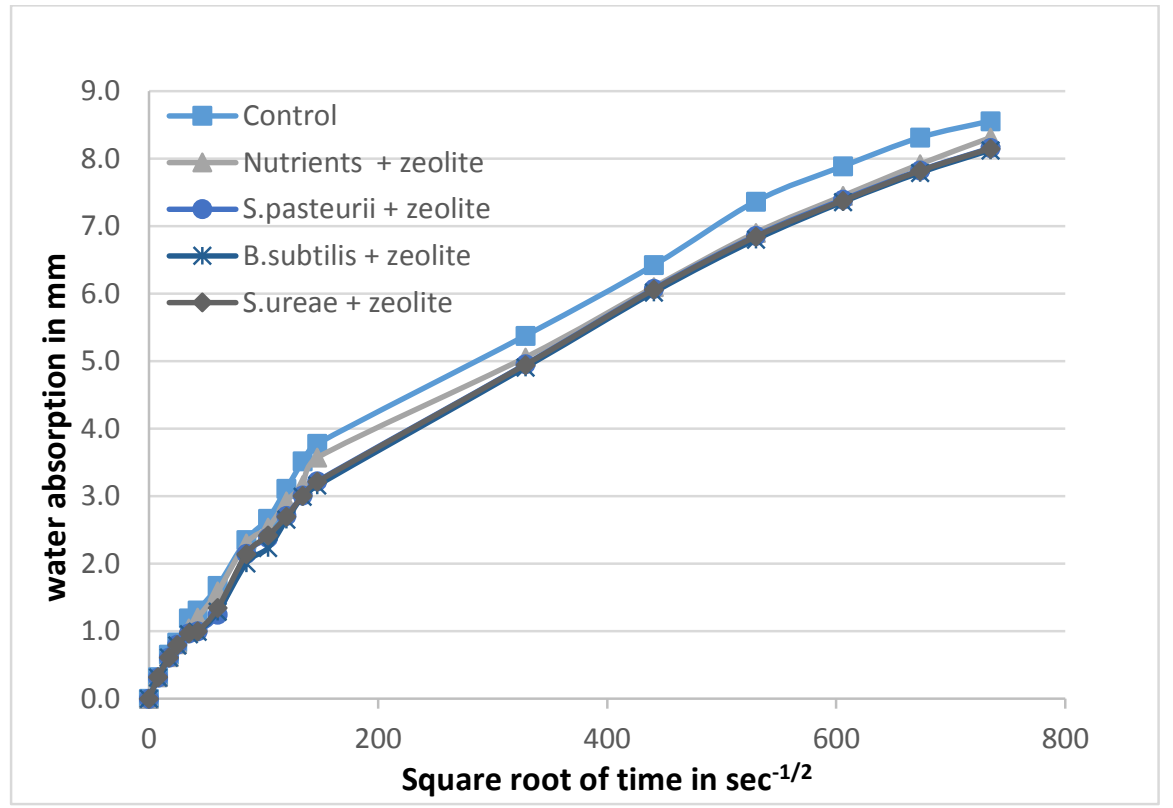

Figure 4.18: Water absorption of 7 days old normal mortar with holes with zeolite 


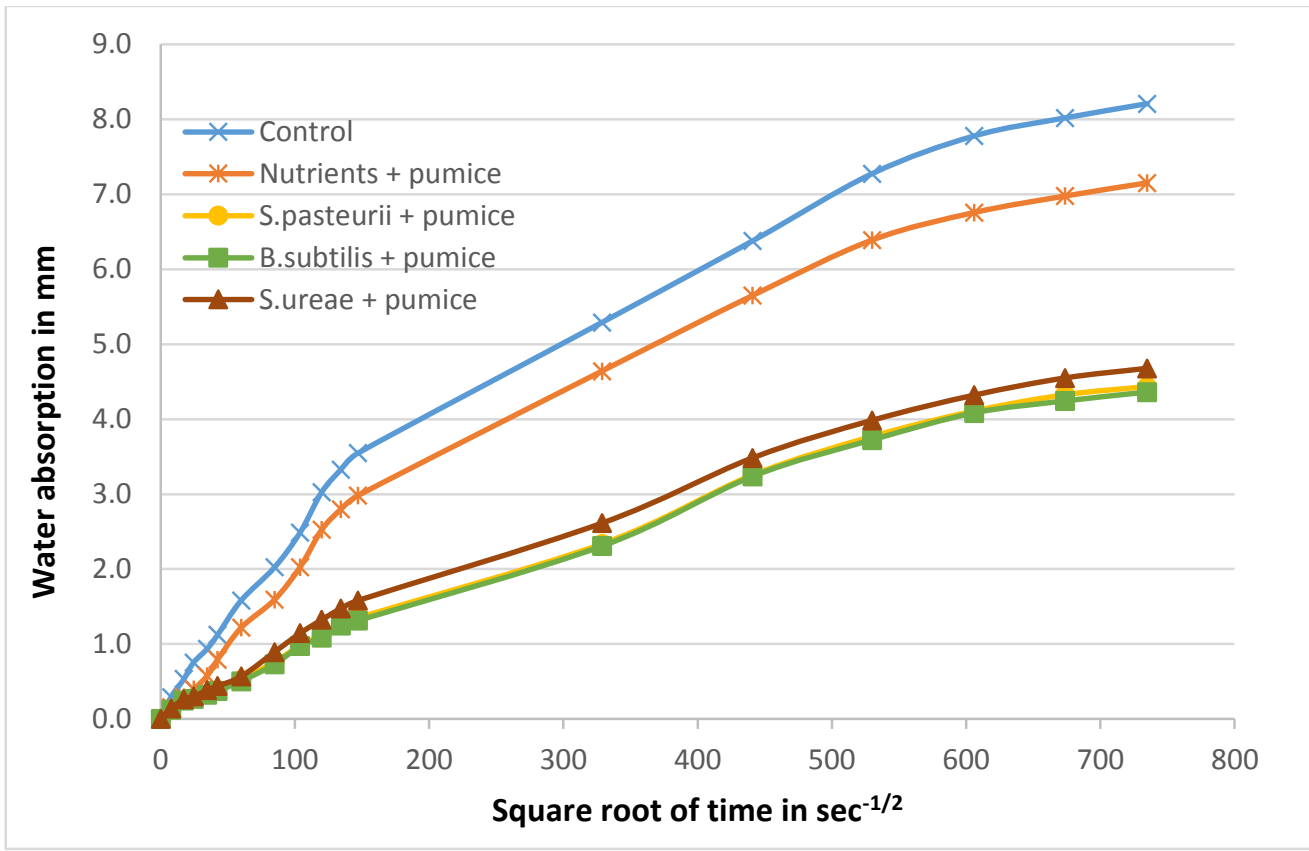

Figure 4.19: Water absorption of 120 days healed normal mortar with holes with pumice

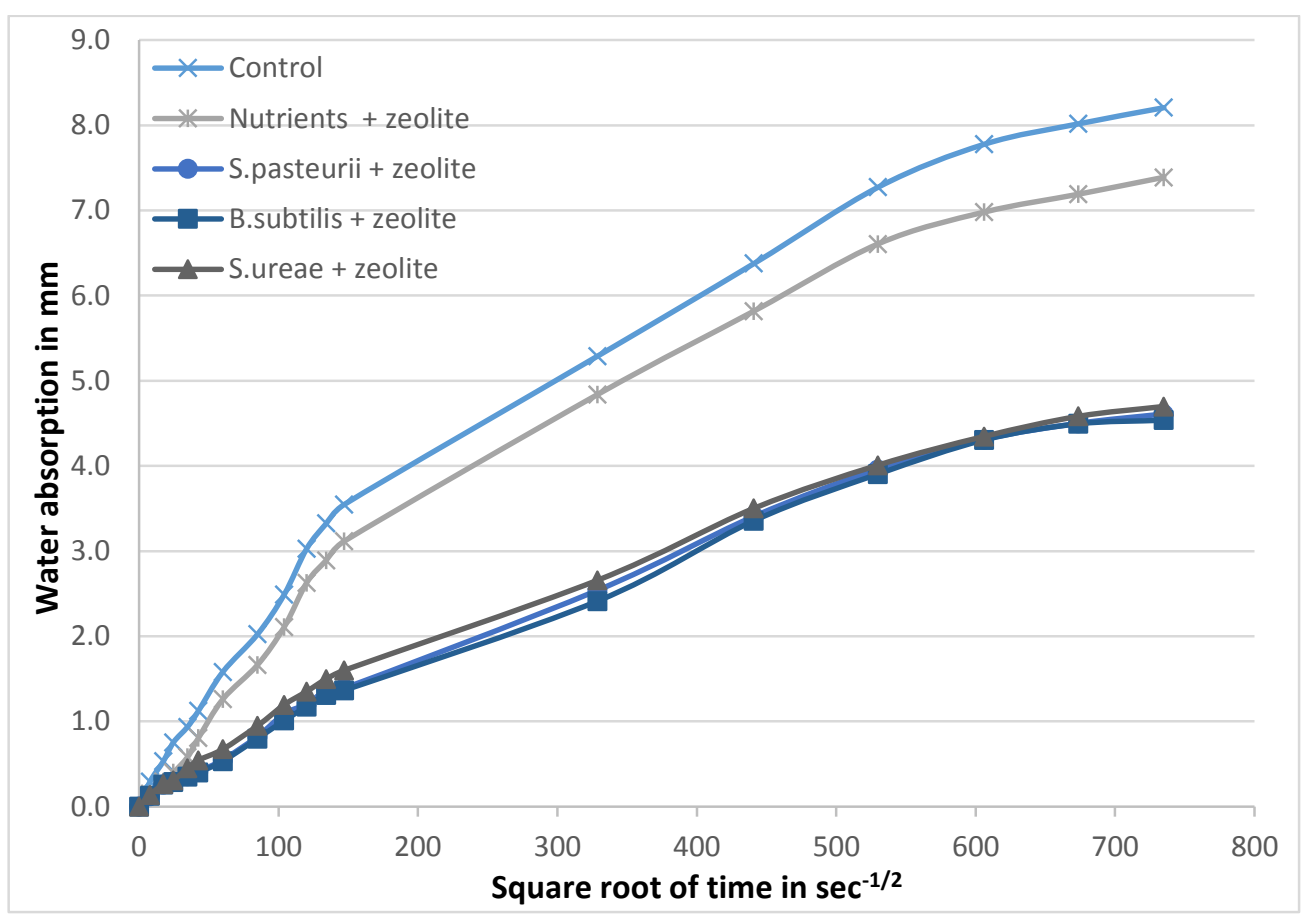

Figure 4.20: Water absorption of 120 days healed normal mortar with holes with zeolite 


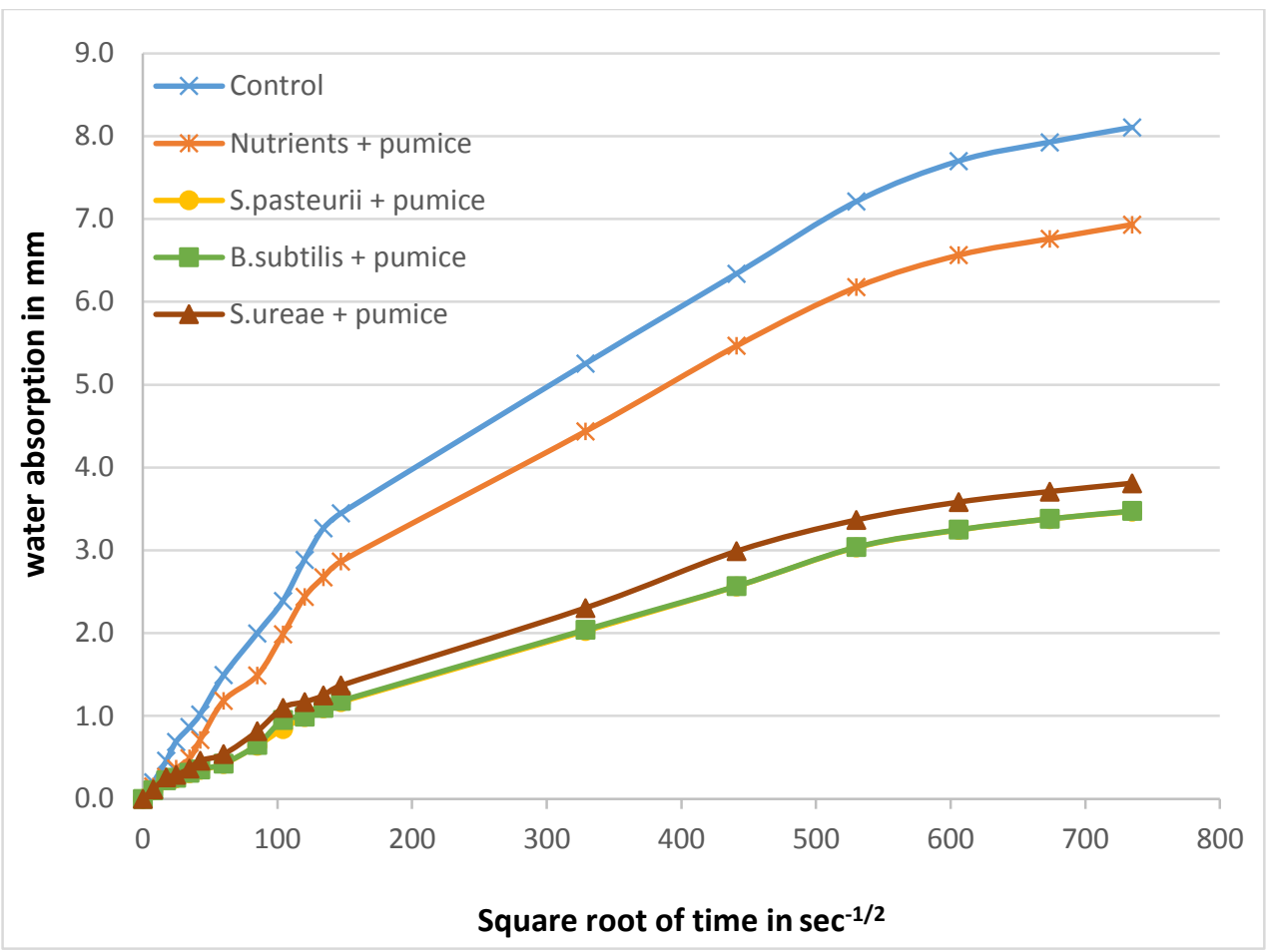

Figure 4.21: Water absorption of 180 days healed normal mortar with holes with pumice

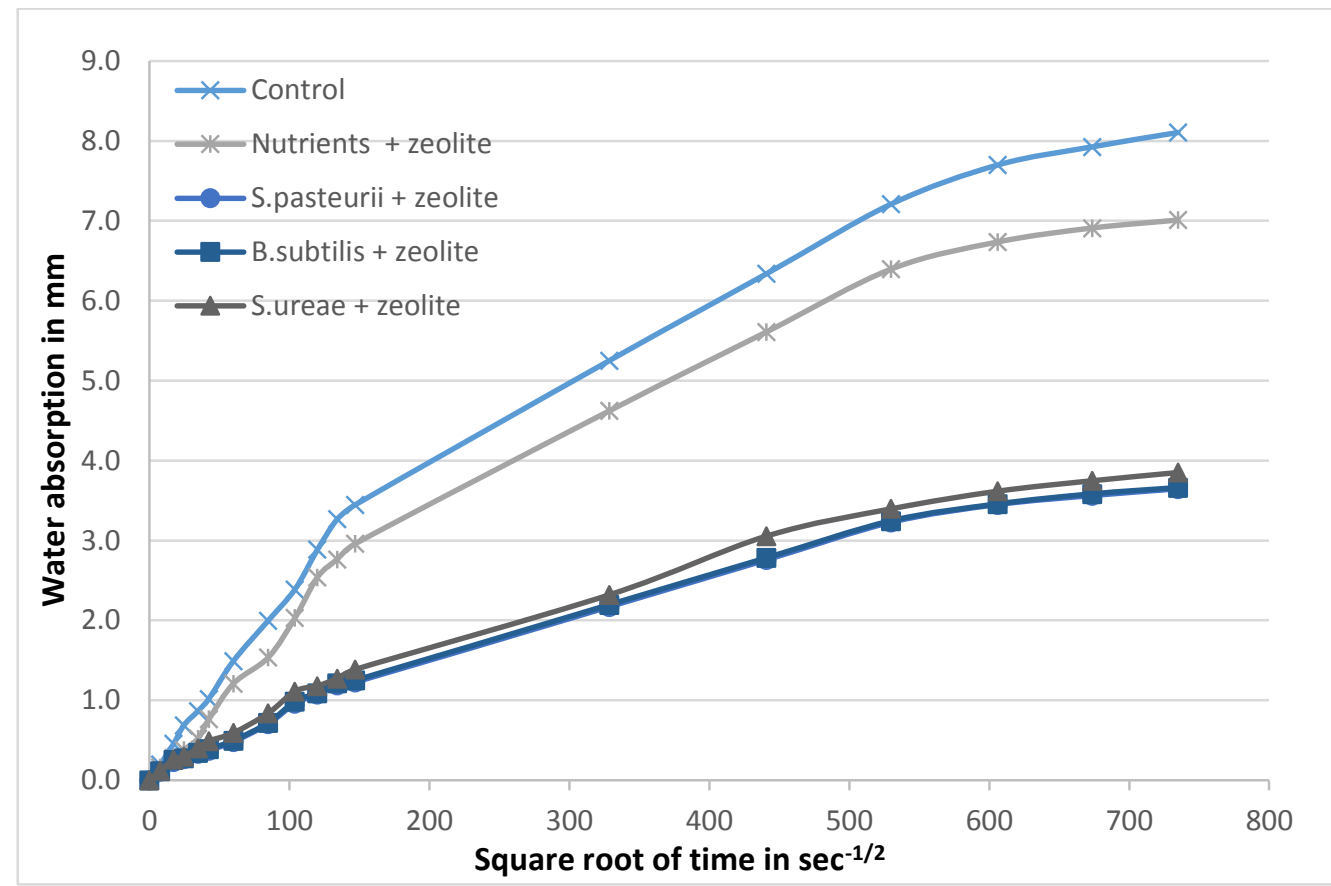

Figure 4.22: Water absorption of 180 days healed normal mortar with holes with zeolite 


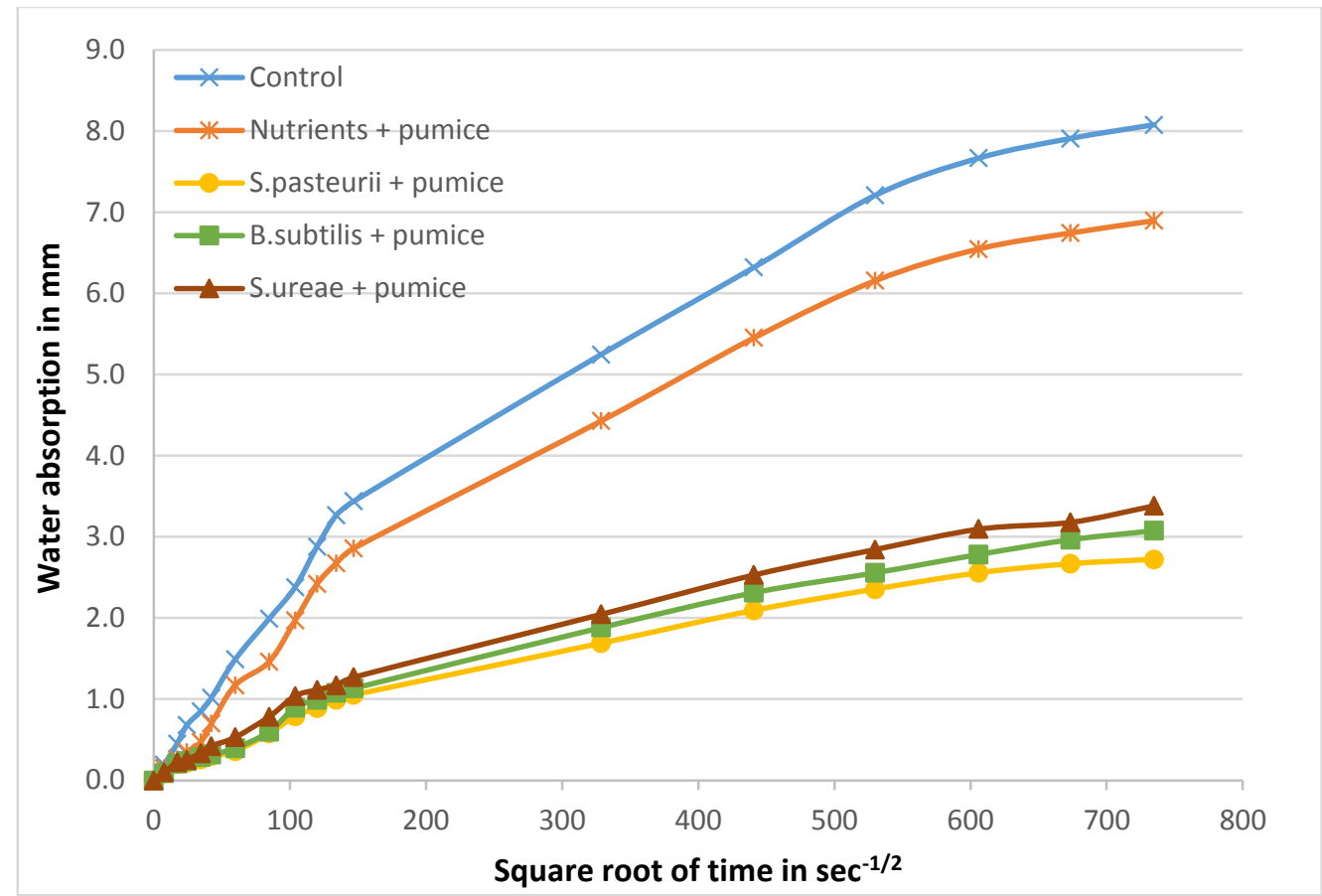

Figure 4.23: Water absorption of 240 days healed normal mortar with holes with pumice

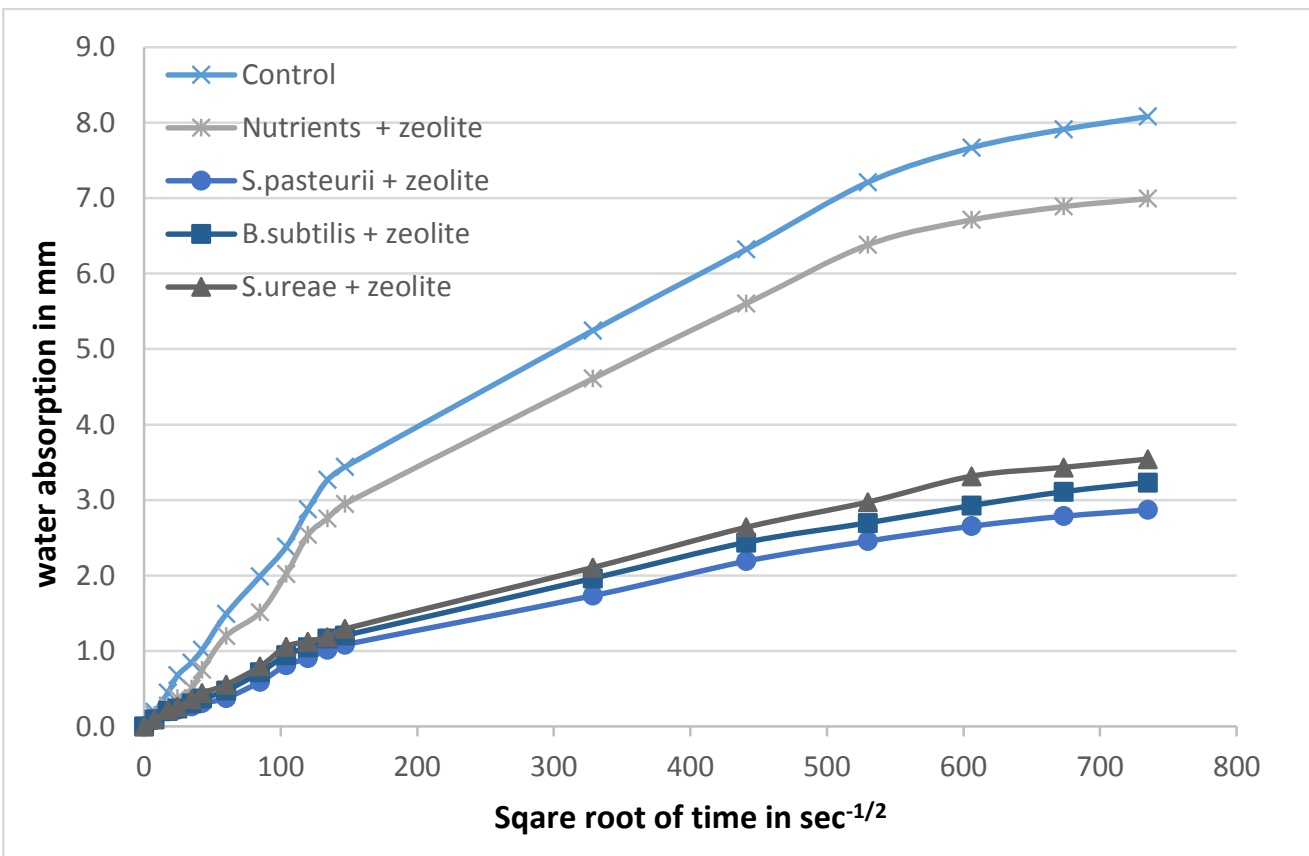

Figure 4.24: Water absorption of 240 days healed normal mortar with holes with zeolite 
Figures 4.25 and 4.26 show the variation of primary sorptivity for bacteria incorporated fibre reinforced mortar with pumice and zeolite respectively along with control specimen for different time periods. For each mix, the primary sorptivity decreased with time. One possible reason is that the continuous hydration of cement particles and precipitation due to bacteria (in the bacteria treated specimen) improve the pore structure and lead to reduced capillary suction effect. Table 4.5 depicts the percentage reduction of primary sorptivity of fiber reinforced mortar at different ages of healing compared to those of 7 days of curing. It can be observed that the percentage reduction of primary sorptivity in 120, 180 and 240 days of healing respectively were $33 \%$, 39\% and $43 \%$ for control specimen while around $40 \%, 45 \%$ and $49 \%$ for nutrients with pumice/zeolite. However, the respective reductions in 120, 180 and 240 days in bacteria treated specimens were $60-70 \%, 70-86 \%$ and $80-92 \%$. This confirmed the self-healing efficiency of bacteria treated specimens. Figure 4.25 and 4.26 indicate that sorptivity decreases rapidly during the first 120 days of healing and it can be inferred from this observation that the pronounced self-healing occurred in the specimens during this stage. After that the slope was relatively less which implies that merely less self-healing activity occurred in the later stage. It is worthwhile to notice that the highest percentage reduction (92\%) of primary sorptivity among the bacteria treated specimens was achieved by $S$. pasteurii + pumice at 240 days period of healing. This amount of healing might be due to the internal moisture provided by the pumice and zeolite.

In comparison with the healing efficiency of carrier materials (zeolite and pumice immobilised bacteria), it can be observed that pumice gave better sorptivity value than zeolite (Table 4.5), however the variations appeared to be very small (around 2\%). The reason for this might be the difference in their particle size distribution. The particle size distribution of pumice (considered for the present study) is finer than that of zeolite. 


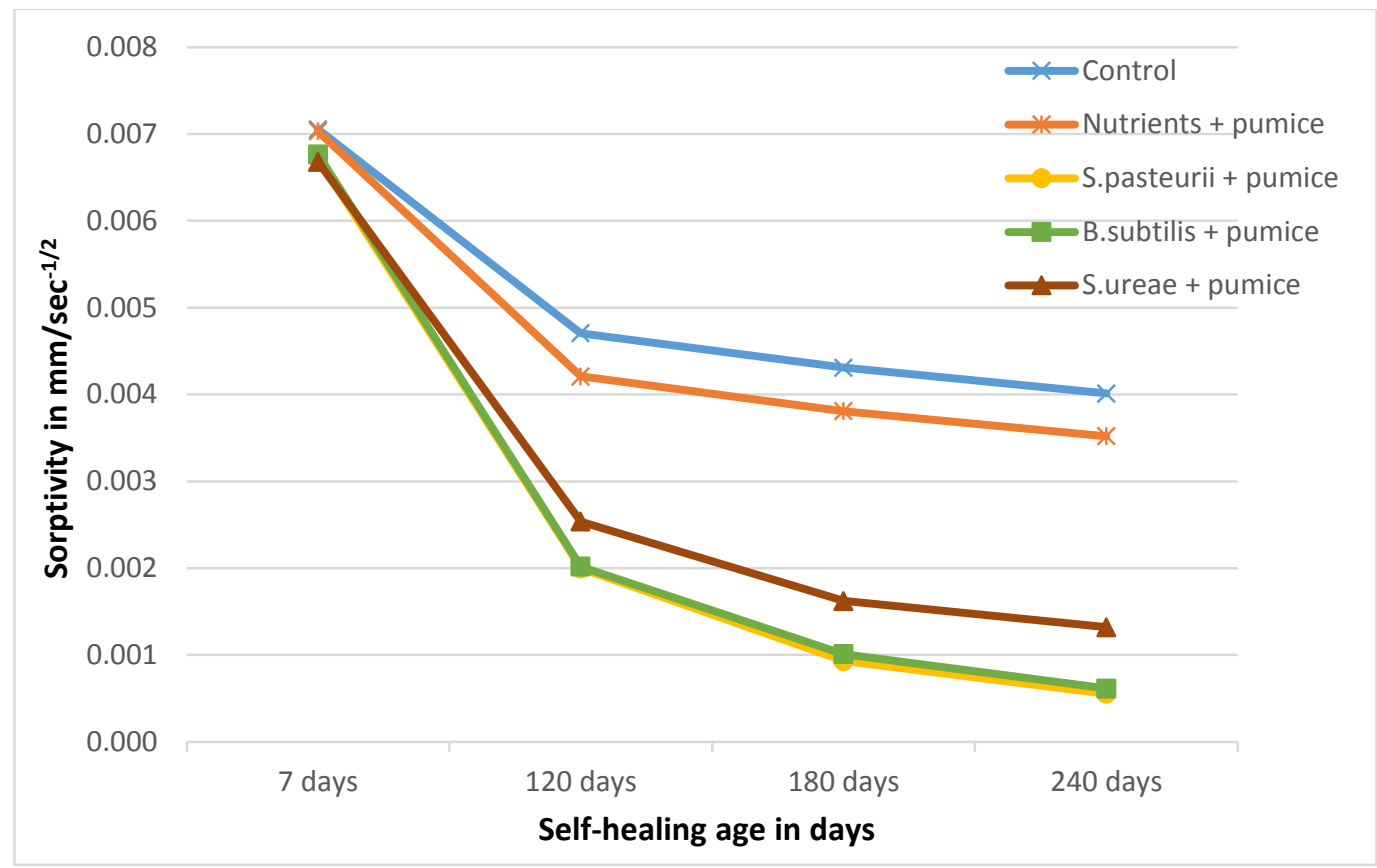

Figure 4.25: Primary sorptivity of FR mortar with pumice

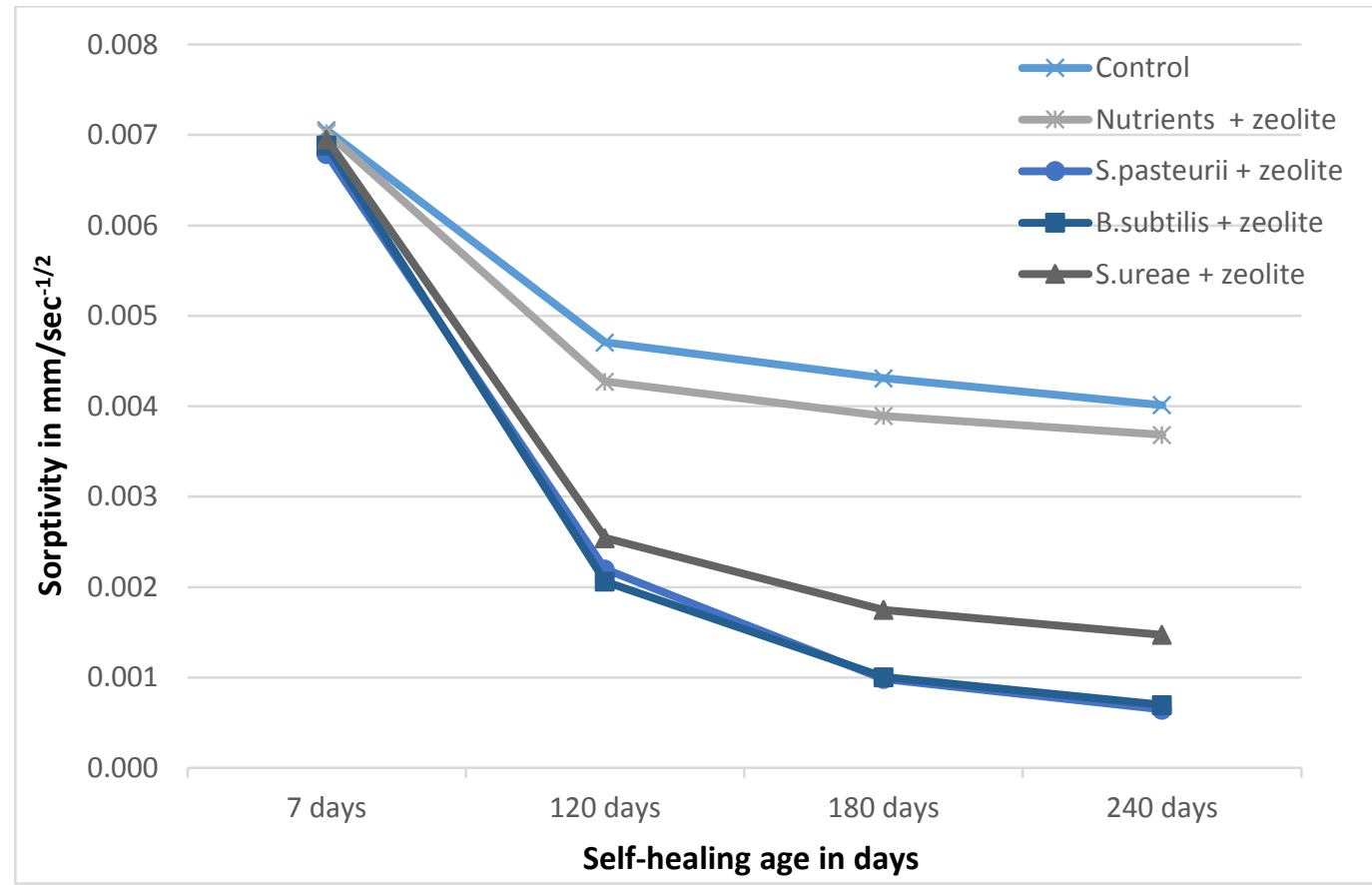

Figure4.26: Primary sorptivity of FR mortar with zeolite 
Table 4.5: Primary sorptivity of cracked FR mortar and their variation with age

\begin{tabular}{|c|c|c|c|c|c|c|c|}
\hline \multirow{2}{*}{ Specimen } & \multicolumn{4}{|c|}{$\begin{array}{l}\text { Primary sorptivity at various ages of } \\
\text { healing }\left(\mathrm{mm} / \mathrm{sec}^{-1 / 2}\right)\end{array}$} & \multicolumn{3}{|c|}{$\begin{array}{l}\% \text { reduction in primary sorptivity } \\
\text { at various ages of healing }(\%)\end{array}$} \\
\hline & 7days & 120 days & 180days & 240 days & 120 days & 180 days & 240 days \\
\hline Control & 0.00705 & 0.0047 & 0.00431 & 0.00401 & 33.33 & 38.87 & 43.12 \\
\hline Nutrients+zeolite & 0.00702 & 0.00427 & 0.00389 & 0.00368 & 39.17 & 44.59 & 47.58 \\
\hline Nutrients+pumice & 0.00703 & 0.00421 & 0.00381 & 0.00352 & 40.11 & 45.80 & 49.93 \\
\hline S. pasteurii+zeolite & 0.00679 & 0.0022 & 0.00098 & 0.00065 & 67.60 & 85.57 & 90.43 \\
\hline S. pasteurii+pumice & 0.00672 & 0.002 & 0.00093 & 0.00055 & 70.24 & 86.16 & 91.82 \\
\hline B. subtilis+zeolite & 0.00689 & 0.00206 & 0.00101 & 0.0007 & 70.10 & 85.34 & 89.84 \\
\hline B. subtilis+pumice & 0.00677 & 0.00202 & 0.00101 & 0.00062 & 70.16 & 85.08 & 90.84 \\
\hline S. ureae+zeolite & 0.00695 & 0.00254 & 0.00175 & 0.00147 & 63.45 & 74.82 & 78.85 \\
\hline S. ureae+pumice & 0.00668 & 0.00254 & 0.00162 & 0.00132 & 61.98 & 75.75 & 80.24 \\
\hline
\end{tabular}

Comparison of secondary sorptivity of pumice and zeolite immobilised bacteria treated specimens with that of control are illustrated in Figures 4.27 and 4.28 respectively. As observed in the case of primary sorptivity, a rapid reduction in the secondary sorptivity was observed from 7 days to 120 days of healing period for the bacteria treated specimen while for the control specimen, the reduction appeared to be less. Table 4.6 supports this argument in which the percentage reduction of secondary sorptivity of cracked fibre reinforced mortar at different ages of healing compared to those of 7 days of curing is illustrated. It was noticed that the maximum percentage reduction of $84 \%$ was achieved by bacteria treated specimen (S. pasteurii + pumice) at 240 days period of healing which is much higher than the reduction achieved by the control (24\%) and the specimens without bacteria but with nutrients $(45 \%)$. 


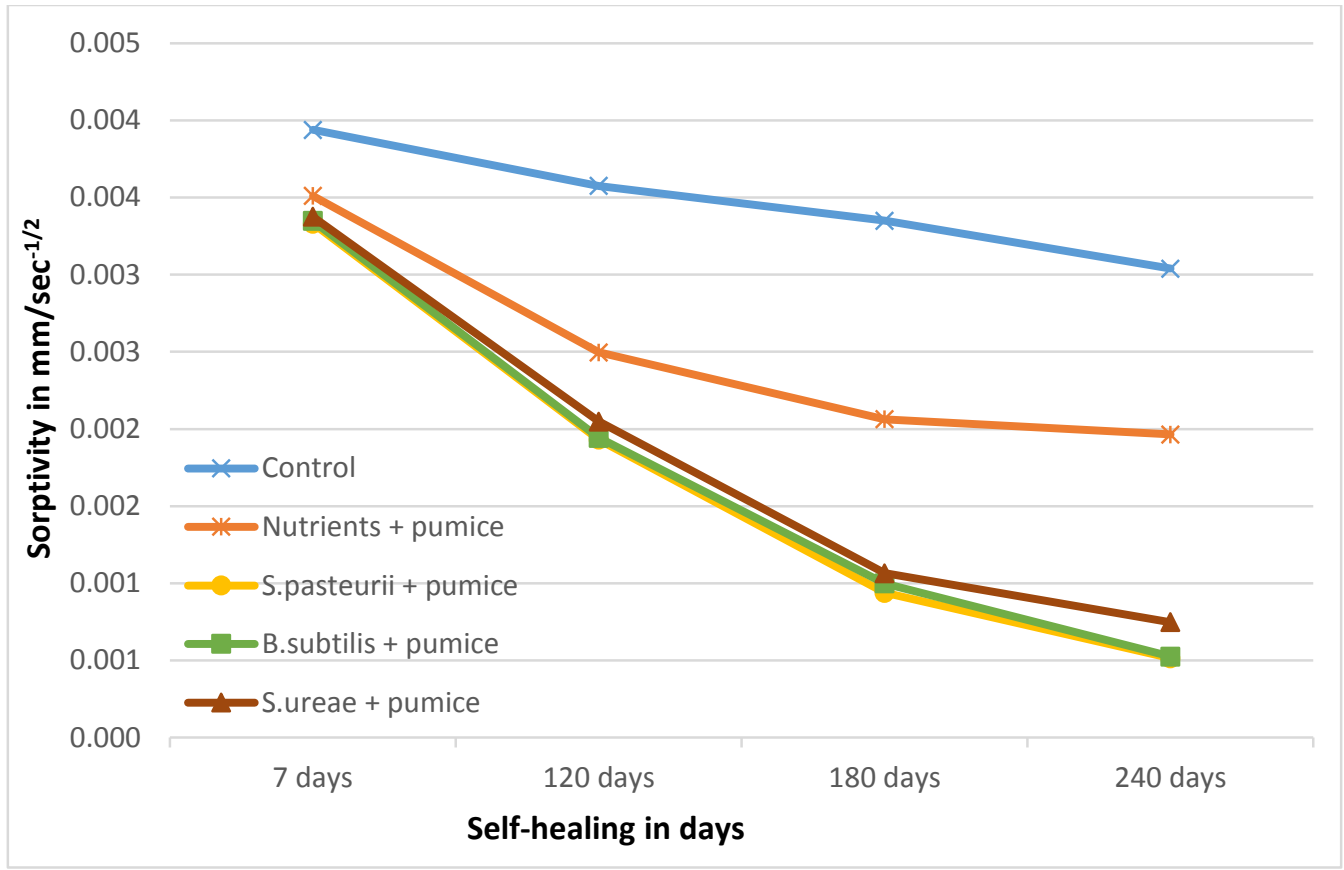

Figure 4.27: Secondary sorptivity of FR mortar with pumice

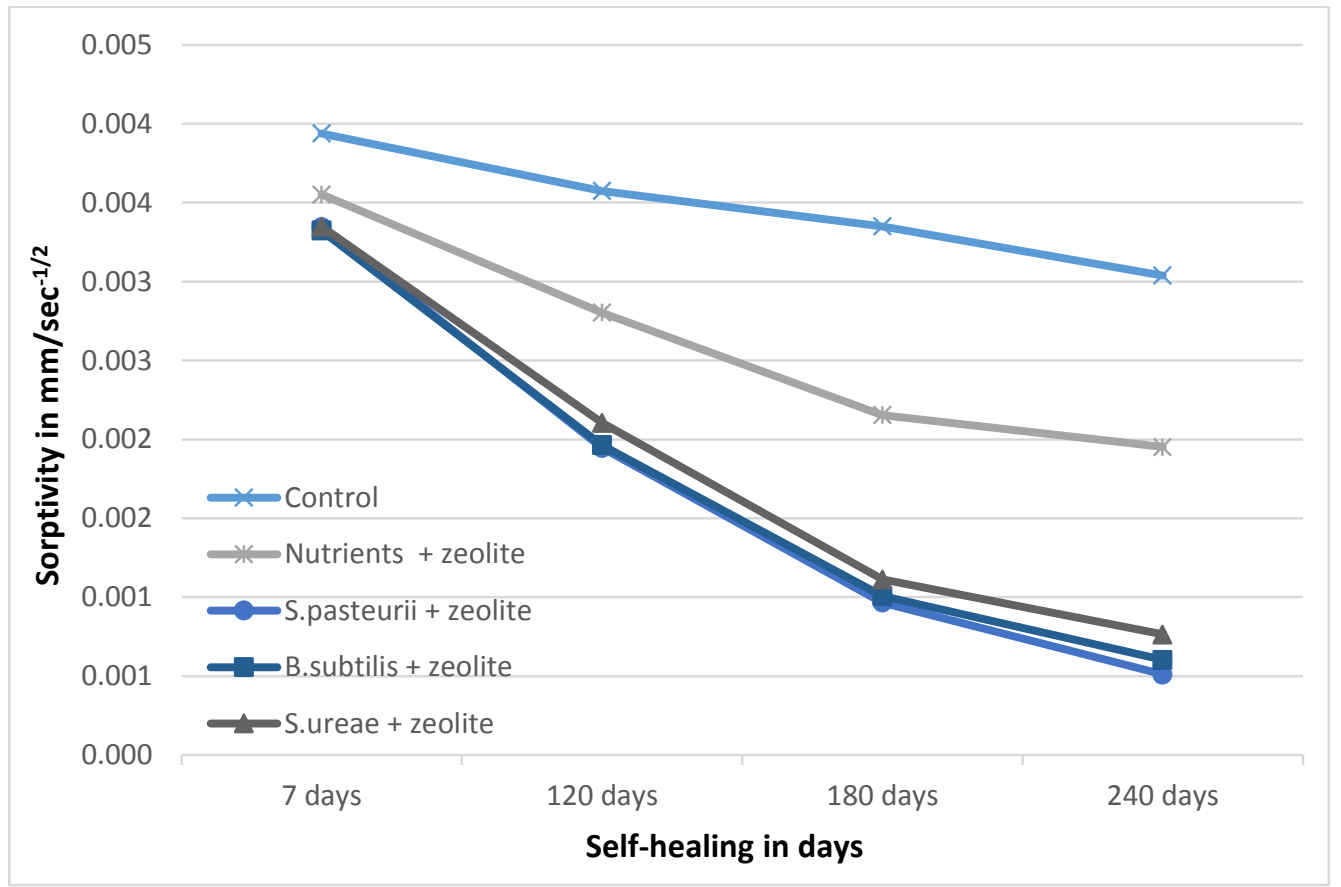

Figure 4.28: Secondary sorptivity of FR mortar with zeolite 
Table 4.6: Secondary sorptivity of cracked FR mortar and their variation with age

\begin{tabular}{|c|c|c|c|c|c|c|c|}
\hline \multirow{2}{*}{ Specimen } & \multicolumn{3}{|c|}{$\begin{array}{c}\text { Secondary sorptivity at various ages of } \\
\text { healing }\left(\mathbf{m m} / \mathbf{s e c}^{-1 / 2}\right)\end{array}$} & \multicolumn{2}{c|}{$\begin{array}{c}\text { reduction in secondary sorptivity } \\
\text { at various ages of healing (\%) }\end{array}$} \\
\cline { 2 - 9 } & $\mathbf{7 d a y s}$ & $\mathbf{1 2 0}$ days & $\mathbf{1 8 0 d a y s}$ & $\mathbf{2 4 0}$ days & $\mathbf{1 2 0}$ days & $\mathbf{1 8 0}$ days & $\mathbf{2 4 0}$ days \\
\hline Control & 0.00394 & 0.00347 & 0.00321 & 0.00301 & 11.93 & 18.53 & 23.60 \\
\hline Nutrients+zeolite & 0.00355 & 0.0028 & 0.00215 & 0.00195 & 21.13 & 39.44 & 45.07 \\
\hline Nutrients+pumice & 0.00351 & 0.0025 & 0.00206 & 0.00196 & 28.77 & 41.31 & 44.16 \\
\hline S. pasteurii+zeolite & 0.00335 & 0.00195 & 0.00097 & 0.00051 & 41.79 & 71.04 & 84.78 \\
\hline S. pasteurii+pumice & 0.00333 & 0.00193 & 0.00094 & 0.00051 & 42.04 & 71.77 & 84.68 \\
\hline B. subtilis+zeolite & 0.00332 & 0.00196 & 0.00101 & 0.0006 & 40.96 & 69.58 & 81.93 \\
\hline B. subtilis+pumice & 0.00335 & 0.00194 & 0.001 & 0.00053 & 42.09 & 70.15 & 84.18 \\
\hline S. ureae+zeolite & 0.00335 & 0.00206 & 0.00109 & 0.00076 & 38.51 & 67.46 & 77.31 \\
\hline S. ureae+pumice & 0.00338 & 0.00205 & 0.00107 & 0.00075 & 39.35 & 68.34 & 77.81 \\
\hline
\end{tabular}

Figures 4.29 and 4.30 show the primary sorptivity of normal mortar with pumice and zeolite respectively. For the specimens with bacteria, initially the downward trend became steeper up to 120 days of healing, then gradually flattened with the age. It can be observed that the control specimen and nutrients + carrier specimens did not exhibit significant reduction in sorptivity compared to the bacteria treated specimens. Table 4.7 shows the percentage reduction of primary sorptivity of normal mortar with holes at various ages compared to those of 7 days of curing. It was observed that both S. pasteurii and B. subtilis with pumice/zeolite showed $57 \%$ of reduction in primary sorptivity whereas $S$. ureae with pumice/zeolite showed $50 \%$ of reduction at 4 months of healing. However, the control, nutrients + zeolite and nutrients + pumice specimens exhibited only $6 \%, 9 \%$ and $14 \%$ respectively for a healing period of 4 months. For 8 months period of healing, bacteria based specimens displayed $59-65 \%$ of reduction in primary sorptivity. These results conclude that significant amount of self-healing had occurred on the bacteria based specimens.

Figures 4.31 and 4.32 present the secondary sorptivity of normal mortar with pumice and zeolite respectively. Similar trend as primary sorptivity was observed in the case of secondary soprtivity. Table 4.8 shows the percentage reduction of secondary sorptivity of normal mortar with holes at various ages. Maximum percentage reduction in secondary sorptivity was achieved by $S$. 
pasteurii+pumice (68\%) while the control specimen achieved only $10 \%$ during 8 months of healing period.

From these obtained results, it can be confirmed that the transport mechanisms in mortar will be affected by microbial induced calcite precipitation. It is evident that the presence of a calcium carbonate layer on the surface by bacterial action has the ability to enhance the resistance of cementitious materials towards degradation.

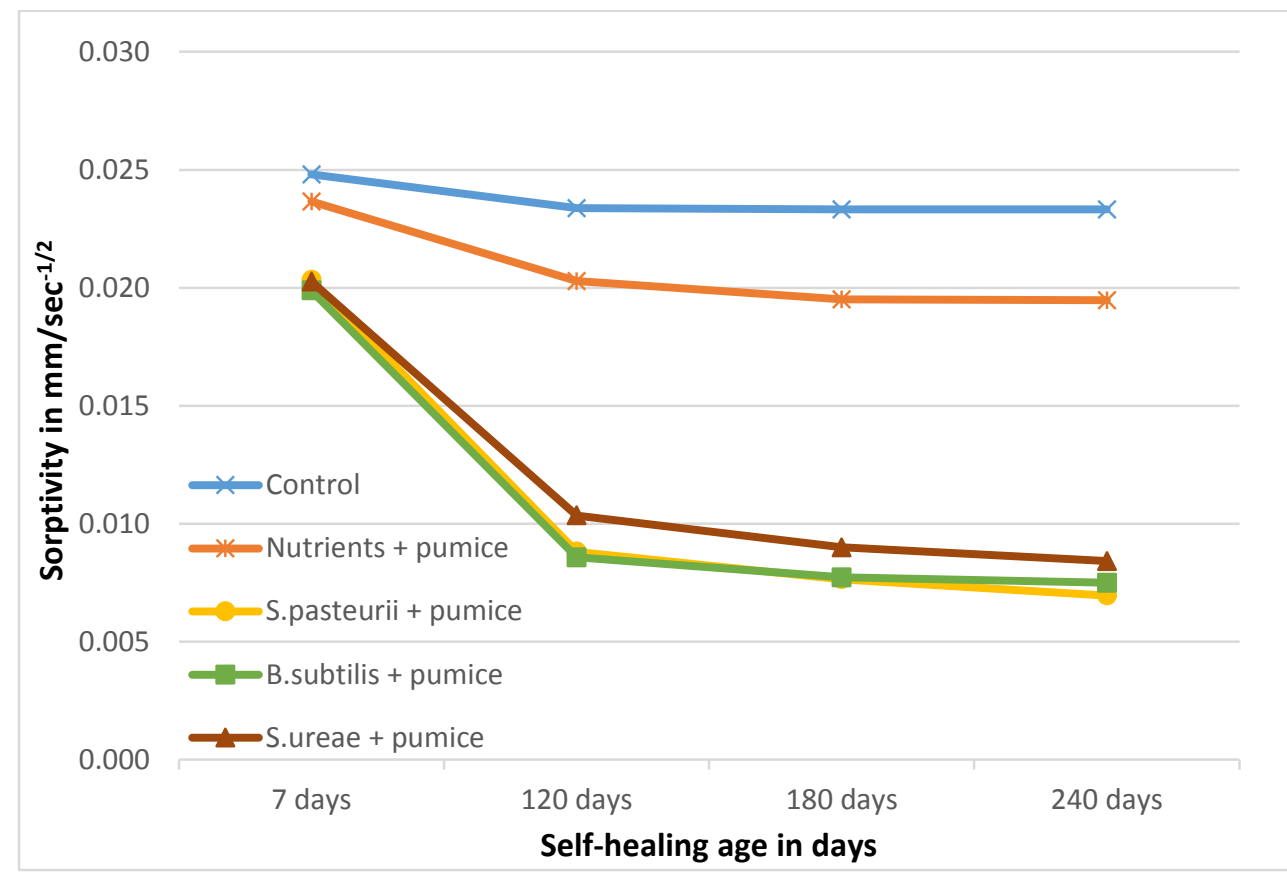

Figure 4.29: Primary sorptivity of normal mortar with holes and pumice 


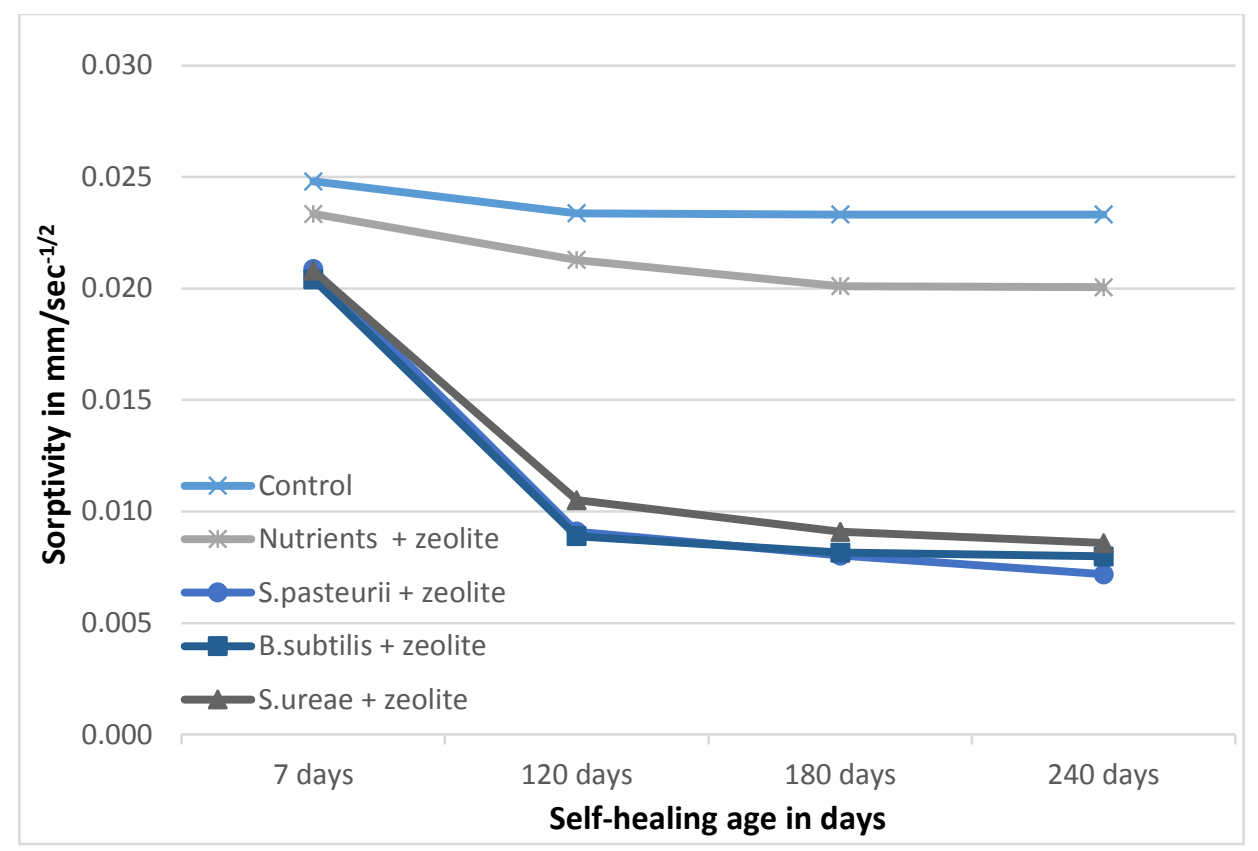

Figure 4.30: Primary sorptivity of Normal mortar with holes and zeolite

Table 4.7: Primary sorptivity of normal mortar with holes and their variation with age

\begin{tabular}{|c|c|c|c|c|c|c|c|}
\hline \multirow{2}{*}{ Specimen } & \multicolumn{4}{|c|}{$\begin{array}{c}\text { Primary sorptivity at various ages of } \\
\text { healing }\left(\mathbf{m m} / \mathbf{s e c}^{-1 / 2}\right)\end{array}$} & \multicolumn{2}{c|}{$\begin{array}{c}\text { reduction in primary sorptivity } \\
\text { at various ages of healing (\%) }\end{array}$} \\
\cline { 2 - 8 } & $\mathbf{7 d a y s}$ & $\mathbf{1 2 0}$ days & $\mathbf{1 8 0 d a y s}$ & $\mathbf{2 4 0}$ days & $\mathbf{1 2 0}$ days & $\mathbf{1 8 0}$ days & $\mathbf{2 4 0}$ days \\
\hline Control & 0.0248 & 0.02337 & 0.02332 & 0.02332 & 5.77 & 5.97 & 5.97 \\
\hline Nutrients+zeolite & 0.02335 & 0.02128 & 0.0201 & 0.02005 & 8.87 & 13.92 & 14.13 \\
\hline Nutrients+pumice & 0.02366 & 0.02028 & 0.0195 & 0.01947 & 14.29 & 17.58 & 17.71 \\
\hline S. pasteurii+zeolite & 0.02087 & 0.0091 & 0.00802 & 0.00718 & 56.40 & 61.57 & 65.60 \\
\hline S. pasteurii+pumice & 0.02032 & 0.00881 & 0.00764 & 0.00695 & 56.64 & 62.40 & 65.80 \\
\hline B. subtilis+zeolite & 0.02041 & 0.0089 & 0.00816 & 0.00799 & 56.39 & 60.02 & 60.85 \\
\hline B. subtilis+pumice & 0.01988 & 0.00857 & 0.00773 & 0.0075 & 56.89 & 61.12 & 62.27 \\
\hline S. ureae+zeolite & 0.0208 & 0.01051 & 0.00909 & 0.00858 & 49.47 & 56.30 & 58.75 \\
\hline S. ureae+pumice & 0.02026 & 0.01034 & 0.00899 & 0.00841 & 48.96 & 55.63 & 58.49 \\
\hline
\end{tabular}




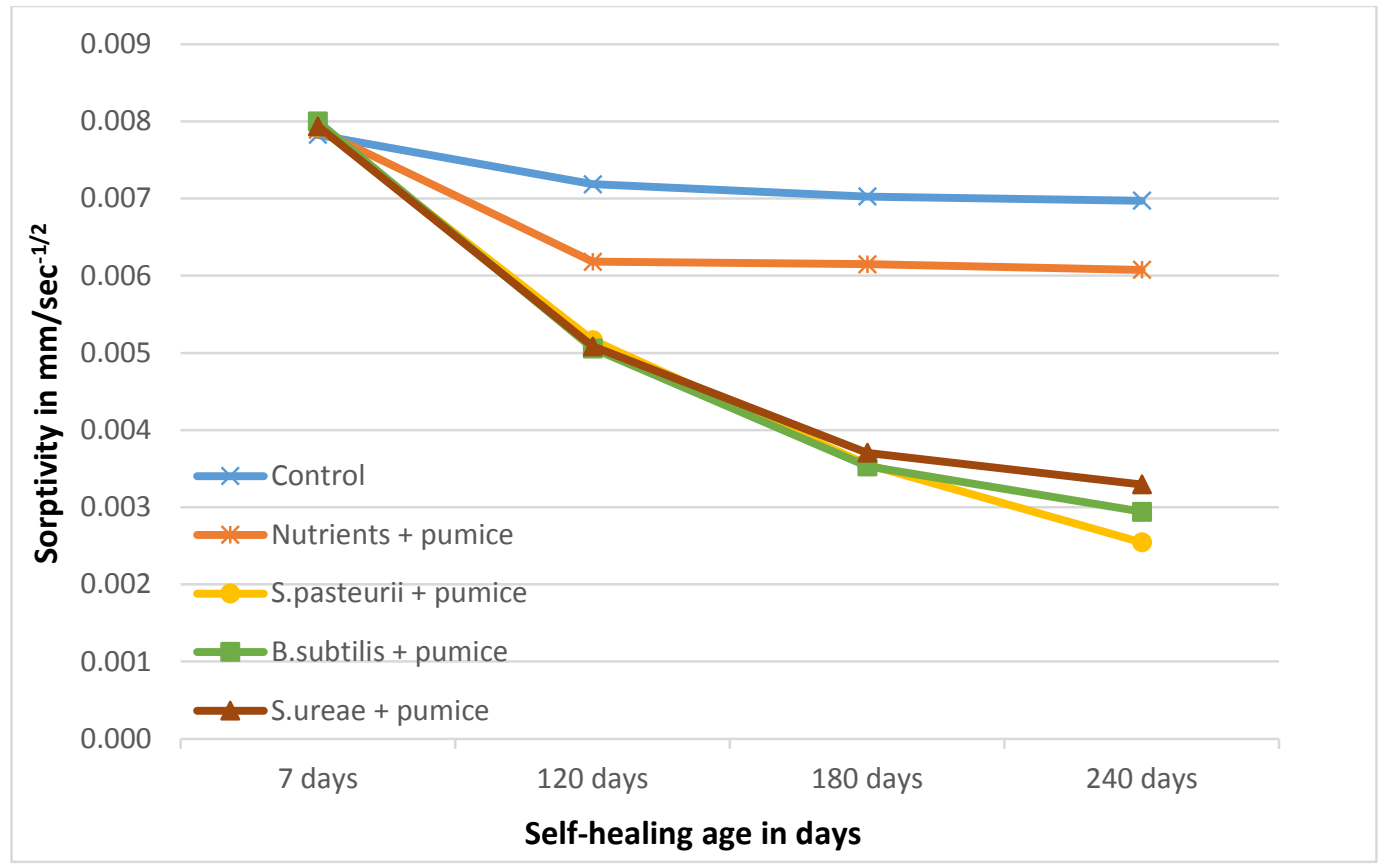

Figure 4.31: Secondary sorptivity of Normal mortar with holes and pumice

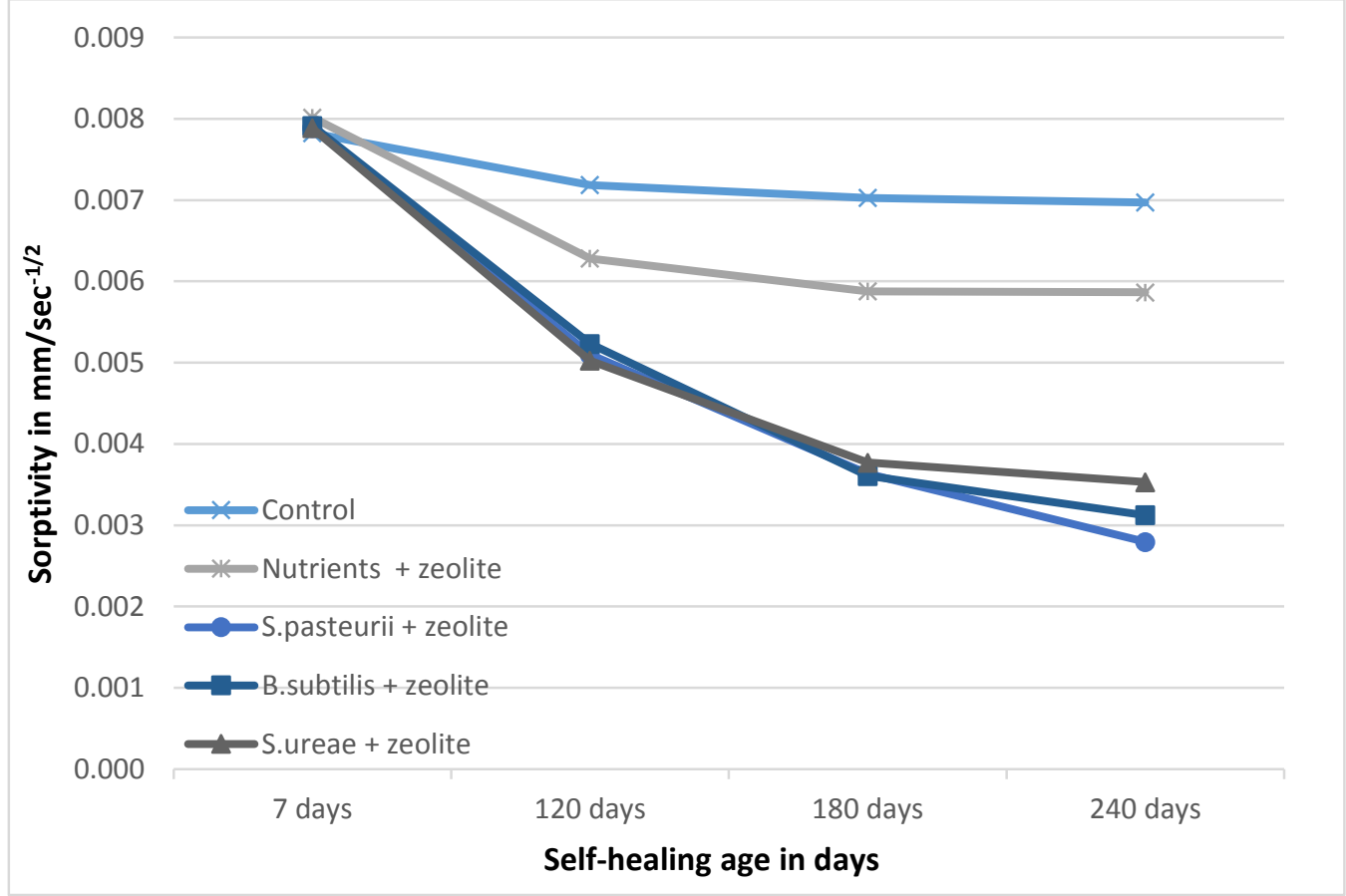

Figure 4.32: Secondary sorptivity of normal mortar with holes and zeolite 
Table 4.8: Secondary sorptivity of normal mortar with holes and their variation with age

\begin{tabular}{|c|c|c|c|c|c|c|c|}
\hline \multirow{2}{*}{ Specimen } & \multicolumn{3}{|c|}{$\begin{array}{c}\text { Secondary sorptivity at various ages of } \\
\text { healing }\left(\mathbf{m m} / \mathbf{s e c}^{-1 / 2}\right)\end{array}$} & \multicolumn{2}{c|}{$\begin{array}{c}\text { reduction in secondary sorptivity } \\
\text { at various ages of healing (\%) }\end{array}$} \\
\cline { 2 - 8 } & $\mathbf{7 d a y s}$ & $\mathbf{1 2 0}$ days & $\mathbf{1 8 0 d a y s}$ & $\mathbf{2 4 0}$ days & $\mathbf{1 2 0}$ days & $\mathbf{1 8 0}$ days & $\mathbf{2 4 0}$ days \\
\hline Control & 0.00782 & 0.00719 & 0.00703 & 0.00697 & 8.06 & 10.10 & 10.87 \\
\hline Nutrients+zeolite & 0.00801 & 0.00628 & 0.00588 & 0.00586 & 21.60 & 26.59 & 26.84 \\
\hline Nutrients+pumice & 0.00789 & 0.00618 & 0.00615 & 0.00608 & 21.67 & 22.05 & 22.94 \\
\hline S. pasteurii+zeolite & 0.00789 & 0.0051 & 0.00363 & 0.0028 & 35.36 & 53.99 & 64.51 \\
\hline S. pasteurii+pumice & 0.00793 & 0.00517 & 0.00355 & 0.00254 & 34.80 & 55.23 & 67.97 \\
\hline B. subtilis+zeolite & 0.00791 & 0.00523 & 0.00361 & 0.00312 & 33.88 & 54.36 & 60.56 \\
\hline B. subtilis+pumice & 0.008 & 0.00506 & 0.00353 & 0.00294 & 36.75 & 55.88 & 63.25 \\
\hline S. ureae+zeolite & 0.00789 & 0.00502 & 0.00377 & 0.00353 & 36.38 & 52.22 & 55.26 \\
\hline S. ureae+pumice & 0.00794 & 0.00509 & 0.00371 & 0.00329 & 35.89 & 53.27 & 58.56 \\
\hline
\end{tabular}

Comparison of the percentage reduction in sorptivity values of fibre reinforced and normal mortar reveals that bacteria based fibre reinforced mortar attained substantial reduction in sorptivity (up to $92 \%$ ) whereas normal mortar achieved up to $67 \%$ reduction. The reason might be due to the presence fibre in the fibre reinforced mortar. It can be inferred that the fibre reinforced mortar may be able to achieve improved self-healing ability in the presence of bacteria.

\subsubsection{Effect of bacteria induced self-healing on rapid chloride permeability}

Figures 4.33 to 4.36 show the evolution of chloride ion permeability at different ages of healing on normal mortar with holes and cracked FR mortar with and without the addition of bacteria. It can be seen that the chloride ion penetration decreased with time for all the selected mortar mixes. With the inclusion of bacteria, chloride ingress capacity of both the normal mortar with holes (Figures 4.33 and 4.34) and cracked FR mortar (Figures 4.35 and 4.36) was significantly decreased. Decrease in chloride ion permeability of the specimens with all kinds of bacteria was much more apparent after 120,180 and 240 days of healing. Therefore, the reduction in the amount of charge passed (which is a measure of chloride ion permeability) ideally reflected the self-healing behavior of mortar specimens. That is, the effect of self-healing agents on the chemistry of pore solution is also an important parameter for RCPT test results. 
It was observed that the reduction in RCP between bacteria incorporated specimens and control specimen was widening quickly in the first 120 days of curing. It can be inferred from this observation that significant self-healing occurred in the specimens during this stage. After that, the rate of change of RCP deteriorated for all the curves which implies that only modest self-healing activity was happening in the later stage. Identical to the sorptivity results, the sequence in the reduction of charge passed was Bacillus subtilis subsp. Spizizenii immobilised in pumice < Sporosarcina pasteurii immobilised in pumice < Bacillus subtilis subsp. Spizizenii immobilised in zeolite < Sporosarcina pasteurii immobilised in zeolite < Sporosarcina ureae immobilised in pumice $<$ Sporosarcina ureae immobilised in zeolite $<$ Nutrients + pumice $<$ Nutrients + zeolite $<$ Control.

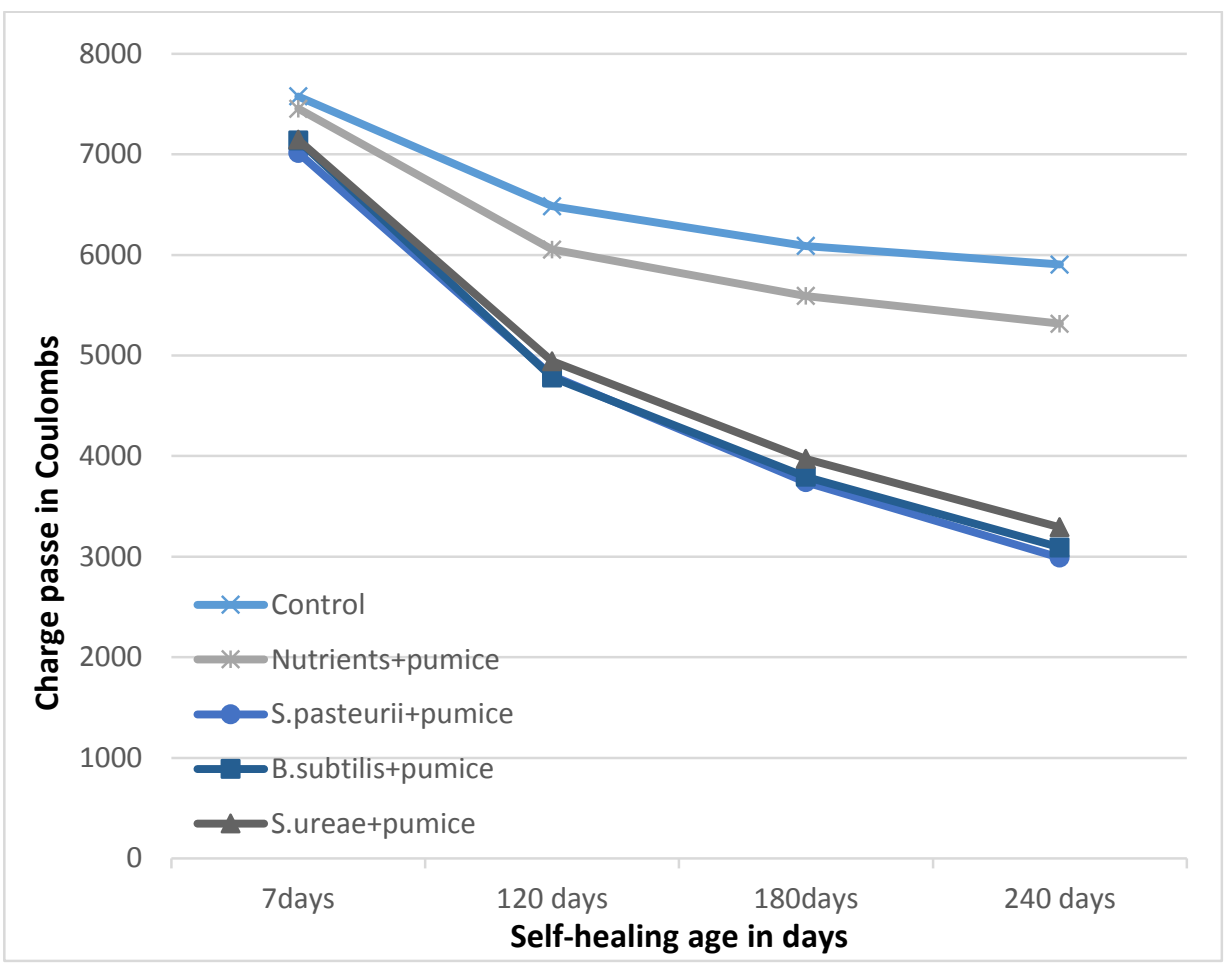

Figure 4.33: Rapid chloride permeability of normal mortar with pumice 


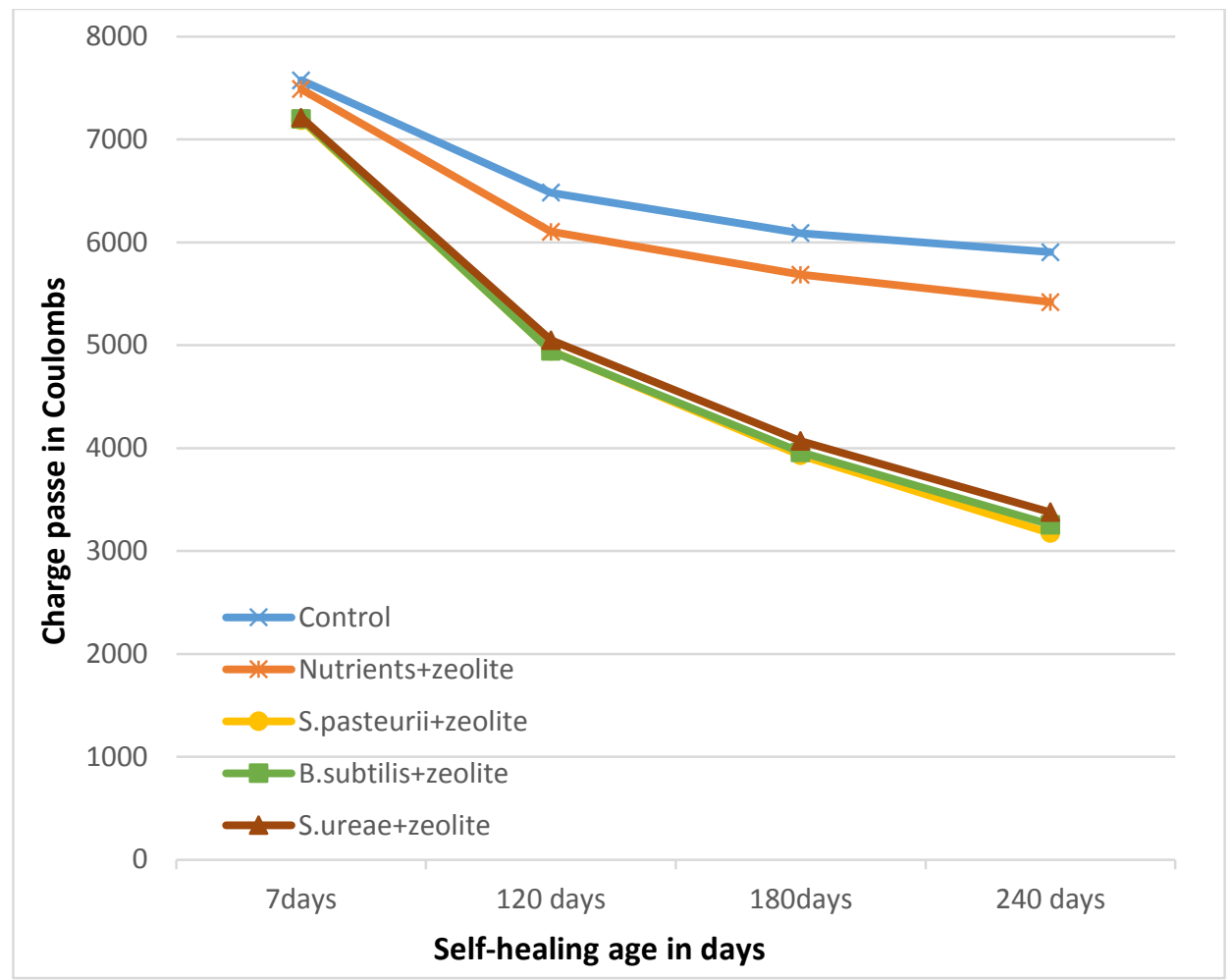

Figure4.34: Rapid chloride permeability of normal mortar with zeolite

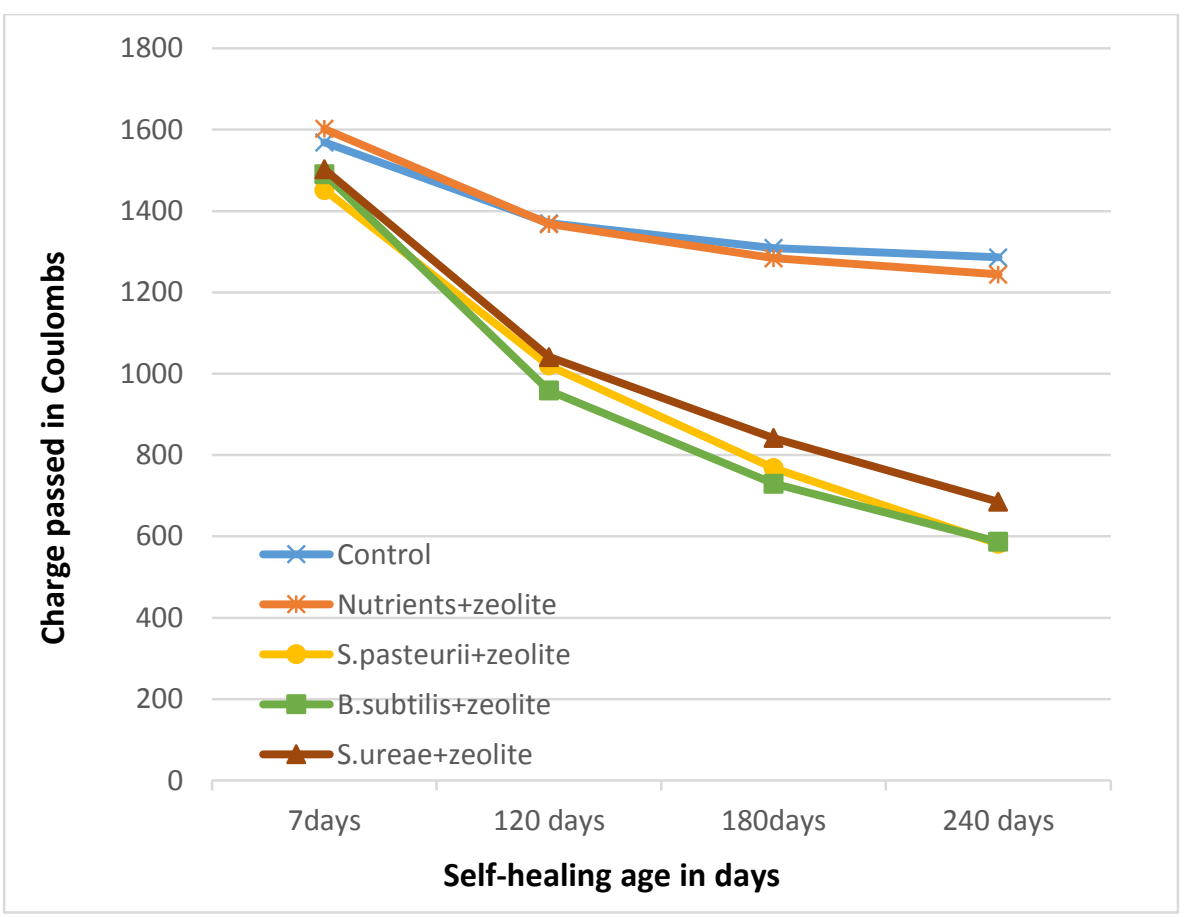

Figure 4.35: Rapid chloride permeability of FR mortar with zeolite 


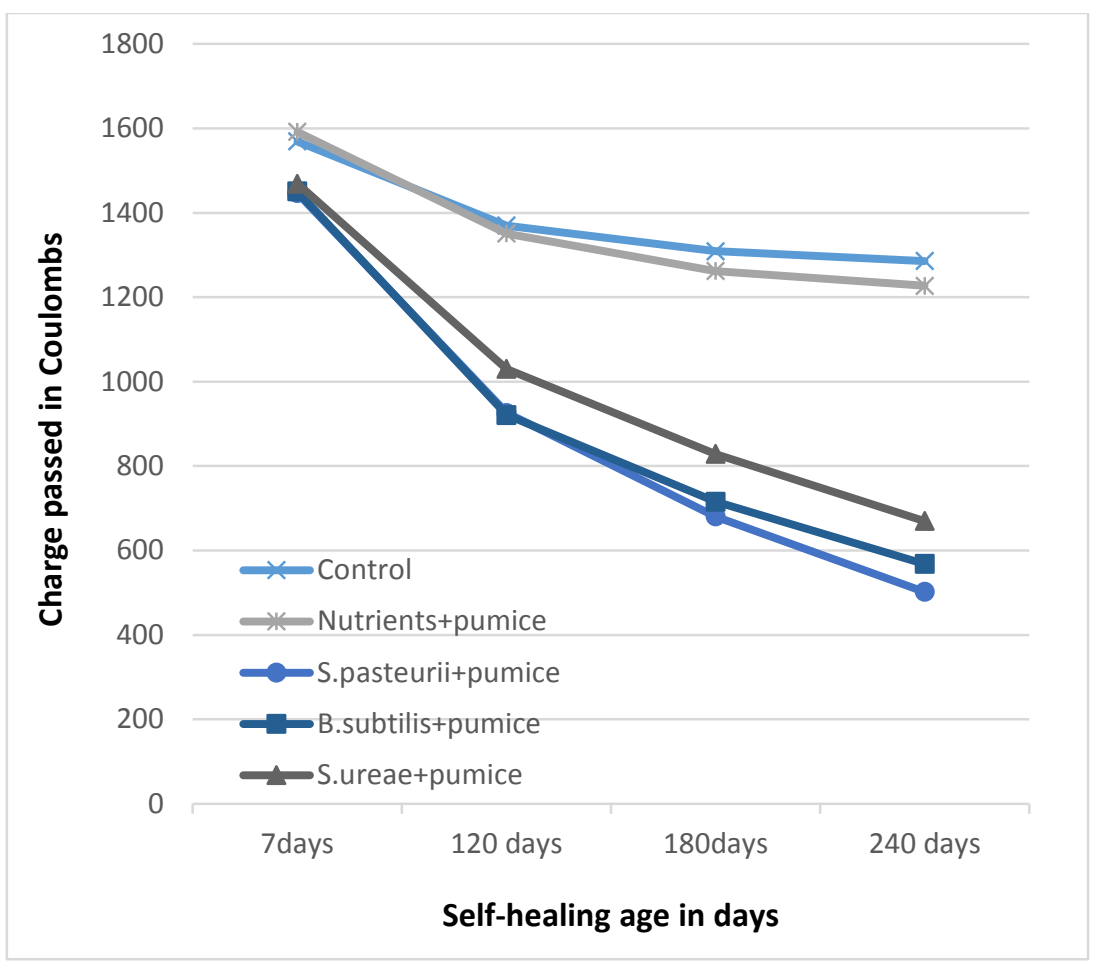

Figure 4.36: Rapid chloride permeability of FR mortar with pumice

Table 4.9: RCPT values of normal mortar with holes and their variation with age

\begin{tabular}{|c|c|c|c|c|c|c|c|}
\hline \multirow{2}{*}{ Specimen } & \multicolumn{3}{|c|}{$\begin{array}{c}\text { Chloride ion permeability at various ages of } \\
\text { healing (Coulomb) }\end{array}$} & \multicolumn{3}{c|}{$\begin{array}{c}\text { \% reduction in chloride ion } \\
\text { permeability at various ages of } \\
\text { healing (\%) }\end{array}$} \\
\cline { 2 - 8 } & $\mathbf{7 d a y s}$ & $\mathbf{1 2 0}$ days & $\mathbf{1 8 0 d a y s}$ & $\mathbf{2 4 0}$ days & 120 days & 180 days & $\mathbf{2 4 0}$ days \\
\hline Control & 7575 & 6484 & 6089 & 5904 & 12.68 & 16.57 & 18.04 \\
\hline Nutrients+zeolite & 7490 & 6104 & 5686 & 5420 & 14.61 & 19.85 & 22.35 \\
\hline Nutrients+pumice & 7454 & 6053 & 5592 & 5317 & 15.14 & 20.73 & 22.93 \\
\hline S. pasteurii+zeolite & 7189 & 4946 & 3930 & 3174 & 31.20 & 45.33 & 55.85 \\
\hline S. pasteurii+pumice & 7015 & 4798 & 3739 & 2990 & 31.60 & 46.70 & 57.38 \\
\hline B. subtilis+zeolite & 7201 & 4944 & 3960 & 3255 & 31.34 & 45.01 & 54.80 \\
\hline B. subtilis+pumice & 7138 & 4782 & 3793 & 3092 & 33.01 & 46.86 & 56.68 \\
\hline S. ureae+zeolite & 7213 & 5049 & 4069 & 3378 & 30.00 & 43.59 & 53.17 \\
\hline S. ureae+pumice & 7145 & 4944 & 3972 & 3292 & 30.80 & 44.41 & 53.93 \\
\hline
\end{tabular}

Table 4.9 shows RCPT values and their percentage reduction at different ages of healing compared to those of 7days of curing for normal mortar with holes. The charge passed for each mixture was 
average values of the test results conducted on six replicates. For the control sample, the decrease in chloride ion permeability was $12.7 \%$ in 120 days, $16.6 \%$ in 180 days and $18.4 \%$ in 240 days whereas, nutrients + zeolite and nutrients + pumice had $14.6 \%$ in 120 days, $19.9 \%$ in 180 days, $22.4 \%$ in 240 days and $15.1 \%$ in 120 days, $20.8 \%$ in 180 days, $22.9 \%$ in 240 days respectively. However, the specimens with bacteria, showed an average decrease in permeability of around $31 \%$ in 120 days, $45 \%$ in 180 days and 55\% in 240 days. Out of the 6 mixes with bacteria, Sporosarcina pasteurii + pumice showed the maximum reduction in chloride ion permeability of around $57 \%$ in 240 days.

Table 4.10: RCPT values of cracked FR mortar and their variation with age

\begin{tabular}{|c|c|c|c|c|c|c|c|}
\hline \multirow{2}{*}{ Specimen } & \multicolumn{3}{|c|}{$\begin{array}{c}\text { Chloride ion permeability at various ages of } \\
\text { healing (Coulomb) }\end{array}$} & \multicolumn{3}{c|}{$\begin{array}{c}\text { \% reduction in chloride ion } \\
\text { permeability at various ages of } \\
\text { healing (\%) }\end{array}$} \\
\cline { 2 - 8 } & $\mathbf{7 d a y s}$ & $\mathbf{1 2 0}$ days & $\mathbf{1 8 0 d a y s}$ & $\mathbf{2 4 0}$ days & $\mathbf{1 2 0}$ days & 180 days & $\mathbf{2 4 0}$ days \\
\hline Control & 1569 & 1370 & 1309 & 1286 & 14.40 & 19.62 & 22.06 \\
\hline Nutrients+zeolite & 1602 & 1368 & 1284 & 1244 & 18.50 & 24.09 & 27.64 \\
\hline Nutrients+pumice & 1592 & 1351 & 1262 & 1227 & 18.80 & 24.98 & 28.67 \\
\hline S. pasteurii+zeolite & 1452 & 1020 & 768 & 582 & 29.75 & 47.11 & 59.92 \\
\hline S. pasteurii+pumice & 1447 & 926 & 681 & 502 & 36.01 & 52.94 & 65.31 \\
\hline B. subtilis+zeolite & 1491 & 959 & 730 & 587 & 35.68 & 51.04 & 60.63 \\
\hline B. subtilis+pumice & 1451 & 921 & 716 & 568 & 36.53 & 50.65 & 60.85 \\
\hline S. ureae+zeolite & 1503 & 1041 & 842 & 685 & 30.74 & 43.98 & 54.42 \\
\hline S. ureae+pumice & 1469 & 1030 & 829 & 670 & 29.88 & 43.57 & 54.39 \\
\hline
\end{tabular}

Table 4.10 shows RCPT values and their reduction at different ages of healing compared to the 7 days of curing for FR mortar. For the control specimen, the decrease in chloride ion permeability was $14.4 \%$ in 120 days, $19.6 \%$ in 180 days and $22.1 \%$ in 240 days. Nutrients + zeolite and nutrients + pumice had $18.5 \%$ in 120 days, $24 \%$ in 6 months, $27.6 \%$ in 240 days and $18.8 \%$ in 120 days, $25 \%$ in 180 days, $28.7 \%$ in 240 days, respectively. It is worthwhile to note that the specimens with bacteria, showed an average decrease in permeability of around 33\% in 120 days, $48 \%$ in 180 days and $59 \%$ in 240 days. Out of the 6 mixes with bacteria, Sporosarcina pasteurii + pumice showed the maximum reduction in chloride ion permeability of around $65 \%$ in 240 days. 
It can be seen that for all the selected mixes, the RCPT values decreased with increase in age regardless of the presence of bacteria. However, the rate of chloride permeability reduction was much higher in the case of mixes with bacteria than mixes without bacteria. Similar to the sorptivity results, it can be seen that the self-healing efficiency of Sporosarcina pasteurii + pumice is the best, followed by Bacillus subtilis subsp. Spizizenii+pumice.

Comparison of the percentage reduction in RCP of fibre reinforced and normal mortar proves that bacteria based fibre reinforced mortar showed a maximum of $65 \%$ reduction of RCP while normal mortar showed 57\% reduction. Presence of fibre in the fibre reinforced mortar helped the specimen to attain more reduction compared to that of normal mortar. However, this variation was observed to be relatively small compared to the results of sorptivity where fibre reinforced mortar exhibited excellent reduction. This may be due to the fact that the test was conducted at $30 \mathrm{~V}$ and presence of calcium and other conductive ions in the mix might have affected the accuracy of the results. It can be inferred that excellent self-healing ability might be achievable by the fibre reinforced mortar in the presence of bacteria.

The lower chloride permeability of the specimens containing bacteria are probably due to the denser microstructure resulted from the microbial induced calcite precipitation. The bacterial precipitation may lead to lower amount of capillary pores and clogging of the pores, which substantially reduces the penetration of chloride ions in concrete. Enhancement in the aggregate cement paste interface by the microbial induced calcite precipitation also probably play a vital role in reducing the chloride ion permeability. Micro-structural investigations presented latter will support this argument.

\subsubsection{Self-healing quantification based on bending strength evolution of fiber reinforced mortar}

After 28 days of curing, cracks were induced on the specimens of fibre reinforced mortar using four-point bending. Uniform control of the crack width was difficult to achieve as all the specimens were loaded after the peak until a deflection of $0.7 \mathrm{~mm}$ was obtained. The crack widths observed ranged from 0.2 to $0.6 \mathrm{~mm}$. Three specimens of each mix were tested again after 120 days of healing and rest of the specimens after 240 days of healing. It was observed that even though the cracks were not completely healed at the tip of the crack, there was visible healing at the root of 
the crack and due to that the existing cracks continued to expand when it was tested after healing. Figure 4.37 shows a sample picture of a semi healed crack. Since the crack widths varied from 0.1 up to $0.6 \mathrm{~mm}$ in single specimen, it was hard to quantify the crack recovery.

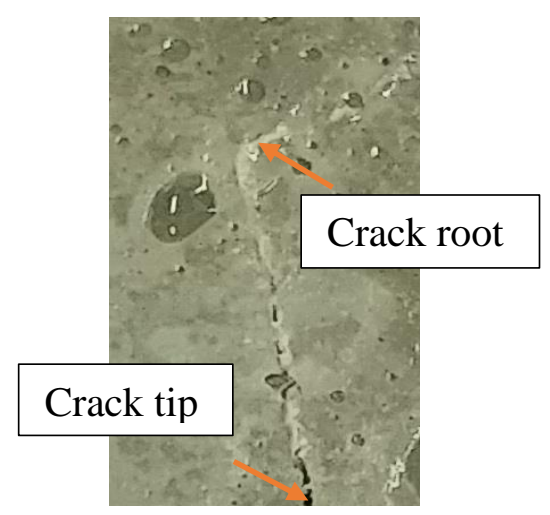

Figure 4.37: Observation of crack healing in bacteria incorporated specimen

Results of the four-point bending tests performed are summarized in Table 4.11. This experiment was used to evaluate whether the mortar specimens were able to recover its strength and deflection after induced damage by cracking. The bending strengths were restored to varying degrees after treatment with different kinds of bacteria in comparison with those of control.

According to Table 4.11, strength recovery is reported as a percentage of the recovered strength of self-healed mortar specimen compared to the strength of sound samples without damage. The control specimens had about 20-23\%of its initial bending strength recovered in 8 months after the damage had occurred. The samples containing the bacteria, however, had $41-48 \%$ recovered strength in 4 months after the damage and about 49-59\% recovered strength in 8 months. This was an indication of the partial healing of the specimens treated with bacteria. It has been noted that the increase in strength recovery was reduced significantly after 4 months of healing for bacteria treated specimens. At the same time, for control specimen, the strength recovery was almost negligible after 4 months. Therefore, it can be inferred that more healing had been taken place in the initial 4 months period. The reason might be that the bacteria residing in the deeper levels of the mortar may not be receiving enough moisture and oxygen due to the formation of a thin layer of calcium carbonate on the inner walls of the crack. Besides, as expected, it is found that the bacterial species Sporosarcina pasteurii (59\%) and Bacillus subtilis (56\%) provided more strength recovery compared to the specimen treated with Sporosarcina ureae (49\%). Similar to the previous 
results, this might be due to the less healing potential of Sporosarcina ureae compared to the other two selected bacterial species. It can be seen that the types of carrier material such as zeolite and pumice do not have much influence on the bacterial healing potential. However, ultimately, this type of healing would be able to promote a longer life of the material since it is prolonging the time to failure.

Table 4.11: Flexural strength recovery of FR mortar due to self-healing

\begin{tabular}{|c|c|c|c|c|c|c|}
\hline \multirow{3}{*}{ Specimen } & \multicolumn{4}{|c|}{ Average Max. Load } & \multirow{2}{*}{\multicolumn{2}{|c|}{ Strength recovered $(\%)$}} \\
\hline & \multicolumn{2}{|c|}{ sound specimen $(\mathbf{k N})$} & \multicolumn{2}{|c|}{$\begin{array}{c}\text { cracked specimen after } \\
\text { healing }(\mathbf{k N})\end{array}$} & & \\
\hline & 4 months & 8 months & 4 months & 8 months & 4 months & 8 months \\
\hline Pumice+nutrients & 8.33 & 7 & 1.72 & 1.69 & 20.6 & 23.9 \\
\hline Zeolite+nutrients & 8.01 & 7.32 & 1.75 & 1.71 & 21.9 & 23.4 \\
\hline S. pasteurii+zeolite & 9.5 & 8.5 & 4.55 & 5 & 47.9 & 58.8 \\
\hline B. subtilis+zeolite & 6.28 & 6.87 & 3.06 & 3.87 & 48.8 & 56.3 \\
\hline S. ureae+zeolite & 6.04 & 6.99 & 2.72 & 3.58 & 45.1 & 51.2 \\
\hline S. pasteurii+pumice & 9 & 9.5 & 3.82 & 5.5 & 46.6 & 57.9 \\
\hline B. subtilis+pumice & 7.66 & 7.22 & 3.37 & 3.98 & 44 & 55.1 \\
\hline S. ureae+pumice & 7.6 & 7.31 & 3.22 & 3.60 & 41.3 & 49.2 \\
\hline
\end{tabular}

Table 4.12 shows the deflection recovered after 4 months and 8 months of healing. Percentage recovery of deflection for 4 months of healing was observed to be a maximum of $68.42 \%$ for $S$. pasteurii+pumice followed by B. subtilis+pumice (62\%). However, for the specimens without bacteria, the recovery was observed to be in the range of $31-37 \%$. This confirmed the effect of self-healing in bacteria based specimen. Highest percentage recovery was observed for $B$. subtilis+pumice $(73 \%)$ for a healing period of 8 months. However, there was a no obvious percentage recovery of deflection observed for S. pasteurii from 4 months to 8 months period of healing. 
Table 4.12: Deflection recovery of FR mortar due to self-healing

\begin{tabular}{|l|c|c|c|c|c|c|}
\hline \multirow{2}{*}{ Specimen } & \multicolumn{2}{|c|}{$\begin{array}{c}\text { Mid-span deflection of the } \\
\text { Sound specimen (mm) }\end{array}$} & $\begin{array}{c}\text { Mid-span deflection of } \\
\text { the cracked specimen } \\
\text { after healing (mm) }\end{array}$ & \multicolumn{2}{c|}{ Deflection recovered (\%) } \\
\cline { 2 - 7 } & 4 months & $\mathbf{8}$ months & $\mathbf{4}$ months & $\mathbf{8}$ months & 4 months & 8 months \\
\hline Pumice+nutrients & 0.84 & 0.91 & 0.26 & 0.3 & 30.95 & 32.97 \\
\hline Zeolite+nutrients & 0.82 & 0.79 & 0.3 & 0.28 & 36.59 & 35.44 \\
\hline S. pasteurii+zeolite & 0.8 & 0.85 & 0.5 & 0.56 & 62.50 & 65.88 \\
\hline B. subtilis+zeolite & 0.81 & 0.79 & 0.49 & 0.53 & 60.49 & 67.09 \\
\hline S. ureae+zeolite & 0.79 & 0.84 & 0.41 & 0.46 & 51.90 & 54.76 \\
\hline S. pasteurii+pumice & 0.76 & 0.8 & 0.52 & 0.53 & 68.42 & 66.25 \\
\hline B. subtilis+pumice & 0.79 & 0.74 & 0.49 & 0.54 & 62.03 & 72.97 \\
\hline S. ureae+pumice & 0.73 & 0.85 & 0.36 & 0.42 & 49.32 & 49.41 \\
\hline
\end{tabular}

Table 4.13 shows the flexural toughness (derived as area under the load-deflection curves) recovered after self-healing of all the bacteria treated specimens compared to that of sound specimens loaded to failure. It can be seen that percentage recovery of flexural toughness for bacteria based specimen were in the range of $41-55 \%$ while that of specimens without bacteria was $26 \%$ for pumice/zeolite + nutrients after 4 months of healing. It can be seen that the percentage recovery after 8 months of healing for bacteria treated specimen were improved and were in the range of 46-68\%. At the same time there was no improvement observed for the specimen without bacteria and were only $27-28 \%$ which was almost same as that of 4 months healed specimens. This difference in the percentage recovery of toughness between the specimen with and without bacteria might be due to the self-healing efficiency of bacteria treated specimen. Partial filling of cracks with calcium carbonate crystals may improve the toughness of the cracked specimen to a certain extend. Highest percentage of recovery was observed for zeolite + S. pasteurii (68\%) followed by pumice + S. pasteurii (66\%). B. subtilis also showed good recovery (around 63\%), however, $S$. ureae treated specimen showed relatively less recovery compared to specimen with other two bacterial species. 
Table 4.13: Flexural toughness recovery of FR mortar due to self-healing

\begin{tabular}{|c|c|c|c|c|c|}
\hline \multirow{3}{*}{ Specimen } & \multicolumn{3}{|c|}{ Flexural toughness (kN-mm) } & \multirow{2}{*}{\multicolumn{2}{|c|}{$\begin{array}{l}\text { Flexural toughness } \\
\text { recovered after } \\
\text { healing }(\%)\end{array}$}} \\
\hline & \multirow{2}{*}{$\begin{array}{c}\text { sound } \\
\text { specimen }\end{array}$} & \multicolumn{2}{|c|}{ after healing } & & \\
\hline & & 4 months & 8 months & 4 months & 8 months \\
\hline pumice+nutrients & 3.79 & 1.001 & 1.016 & 26.41 & 26.81 \\
\hline zeolite+nutrients & 3.85 & 1.01 & 1.11 & 26.23 & 28.83 \\
\hline zeolite $+S$. pasteurii & 3.91 & 2.12 & 2.66 & 54.22 & 68.03 \\
\hline zeolite + B. subtilis & 3.12 & 1.56 & 1.94 & 50.00 & 62.18 \\
\hline zeolite $+S$. ureae & 3.18 & 1.44 & 1.72 & 45.28 & 54.09 \\
\hline pumice $+S$. pasteurii & 3.89 & 2.02 & 2.55 & 51.93 & 65.55 \\
\hline pumice $+B$. subtilis & 3.47 & 1.67 & 2.08 & 48.13 & 59.94 \\
\hline pumice $+S$. ureae & 3.25 & 1.33 & 1.5 & 40.92 & 46.15 \\
\hline
\end{tabular}

Flexural stress versus deflection curves for the fibre reinforced mortar specimens including sound specimens loaded to failure and the reloaded damaged specimens after 4 months of healing are presented in Figure 4.38. Graph includes loading curves for virgin specimens loaded to failure and the reloading curves of damaged specimens without bacteria but with zeolite (1- control), Sporosrcina ureae with zeolite (2), Bacillus subtilis with zeolite (3) and Sporosarcina pasteurii with zeolite (4). It can be seen from the Figure 4.38 that flexural stress of reloaded specimen of no bacteria + zeolite is very less $(2.8 \mathrm{MPa})$ compared to that of sound specimen without bacteria (12MPa). At the same time, reloaded flexural stresses of $S$. pasteurii + zeolite (6.1 MPa), B. subtilis + zeolite (5.5 MPa) and S. ureae +zeolite (4.7 MPa) treated specimen were found to be higher compared to that of sound specimen (around 8 to11MPa). From these observations, the effect of self-healing in bacteria based specimen may be confirmed. It is evident that inclusion of bacteria provided sufficient healing to regain some of the original strength. 


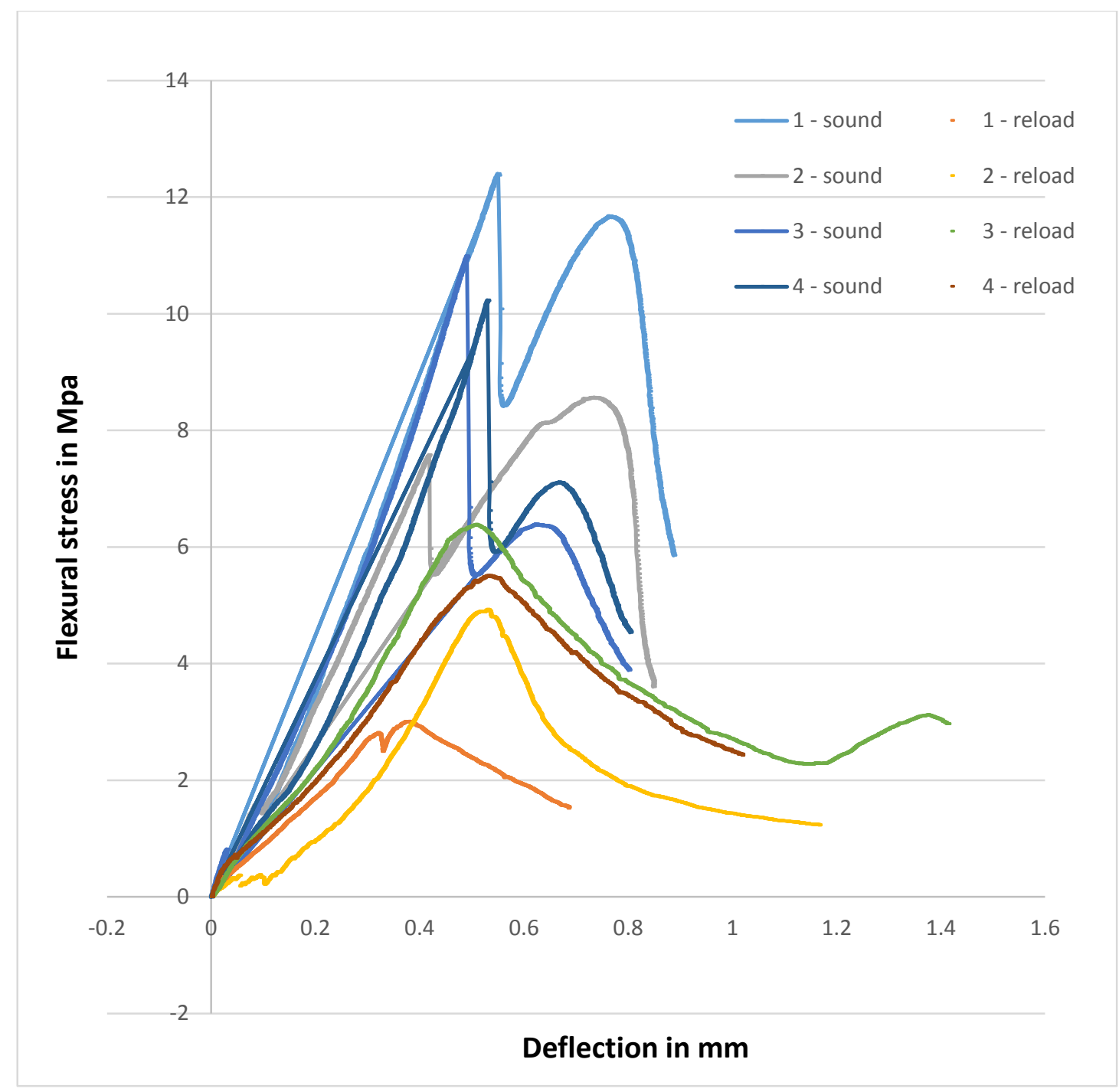

Figure 4.38: Flexural stress- deflection curve for the fibre reinforced mortar specimens including virgin specimen loaded to failure and reloaded curve after 4 months of healing

\subsubsection{Healing quantification based on Ultrasonic Pulse Velocity (UPV)}

Results of UPV performed before loading, after loading to damage, and after healing are shown in Table 4.14. It can be inferred that for the control specimen, the velocity increase in 8 months of healing was found to be $51 \mathrm{~m} / \mathrm{s}$, while that of nutrients + pumice was $84 \mathrm{~m} / \mathrm{s}$ and that of nutrients + zeolite was found to be $75 \mathrm{~m} / \mathrm{s}$. At the same time, the increase in UPV was $401 \mathrm{~m} / \mathrm{s}$ for $S$. pasteurii, $372 \mathrm{~m} / \mathrm{s}$ for $B$. subtilis and $300 \mathrm{~m} / \mathrm{s}$ for $S$. ureae. This increase in UPV for the bacteria treated specimen indicates the effectiveness of self-healing in cracked specimen. The difference in UPV 
for the same bacteria with different carrier materials was found to be negligibly small (around $20 \mathrm{~m} / \mathrm{s}$ ). Figure 4.37 shows the changes in UPV values with 1, 2, 3, 4, 5, 6, 7 and 8 months of healing. Increase in UPV values shows the healing efficiency. It can be seen from the graph that for the control, nutrients + pumice and nutrients + zeolite specimens, increase in UPV values are negligible compared to that treated with selected bacterial species. The highest increase in UPV was observed for the bacteria $S$. pasteurii with pumice followed by $S$. pasteurii + zeolite. It can be observed from the Figure 4.37 that initially (up to 2 months of healing), B. subtilis + pumice showed slightly better increment in UPV than S. pasteurii + zeolite. However, after 2 months of healing, S. pasteurii + zeolite surpassed in UPV. At the same time, UPV values of S. ureae remained less significant compared to the other two bacterial species. These results conclude that S. pasteurii have better healing potential in long term healing. The increase in UPV in the initial months up to the $5^{\text {th }}$ month shows a gradual upward trend, then it starts to slightly flatten.

Table 4.14: UPV performed before loading, after loading, and after healing at various ages

\begin{tabular}{|c|c|c|c|c|c|}
\hline \multirow{2}{*}{ Specimen } & \multirow{2}{*}{$\begin{array}{l}\text { UPV before } \\
\text { cracking } \\
(\mathrm{m} / \mathrm{s})\end{array}$} & \multirow{2}{*}{$\begin{array}{l}\text { UPV immediately } \\
\text { after cracking } \\
(\mathrm{m} / \mathrm{s})\end{array}$} & \multicolumn{3}{|c|}{ UPV after healing $(\mathrm{m} / \mathrm{s})$} \\
\hline & & & 4 months & 6 months & 8 months \\
\hline Control & 3822 & 3502 & 3511 & 3552 & 3553 \\
\hline Nutrients+pumice & 3928 & 3628 & 3649 & 3710 & 3712 \\
\hline Nutrients+zeolite & 3848 & 3548 & 3566 & 3618 & 3623 \\
\hline Zeolite $+S$. pasteurii & 3996 & 3685 & 3793 & 4063 & 4086 \\
\hline Zeolite $+B$. subtilis & 3866 & 3544 & 3640 & 3901 & 3923 \\
\hline Zeolite+S. ureae & 3887 & 3628 & 3718 & 3912 & 3919 \\
\hline Pumice $+S$. pasteurii & 4082 & 3732 & 3831 & 4127 & 4152 \\
\hline Pumice $+B$. subtilis & 3964 & 3634 & 3728 & 3986 & 4006 \\
\hline Pumice $+S$. ureae & 3948 & 3644 & 3733 & 3928 & 3934 \\
\hline
\end{tabular}




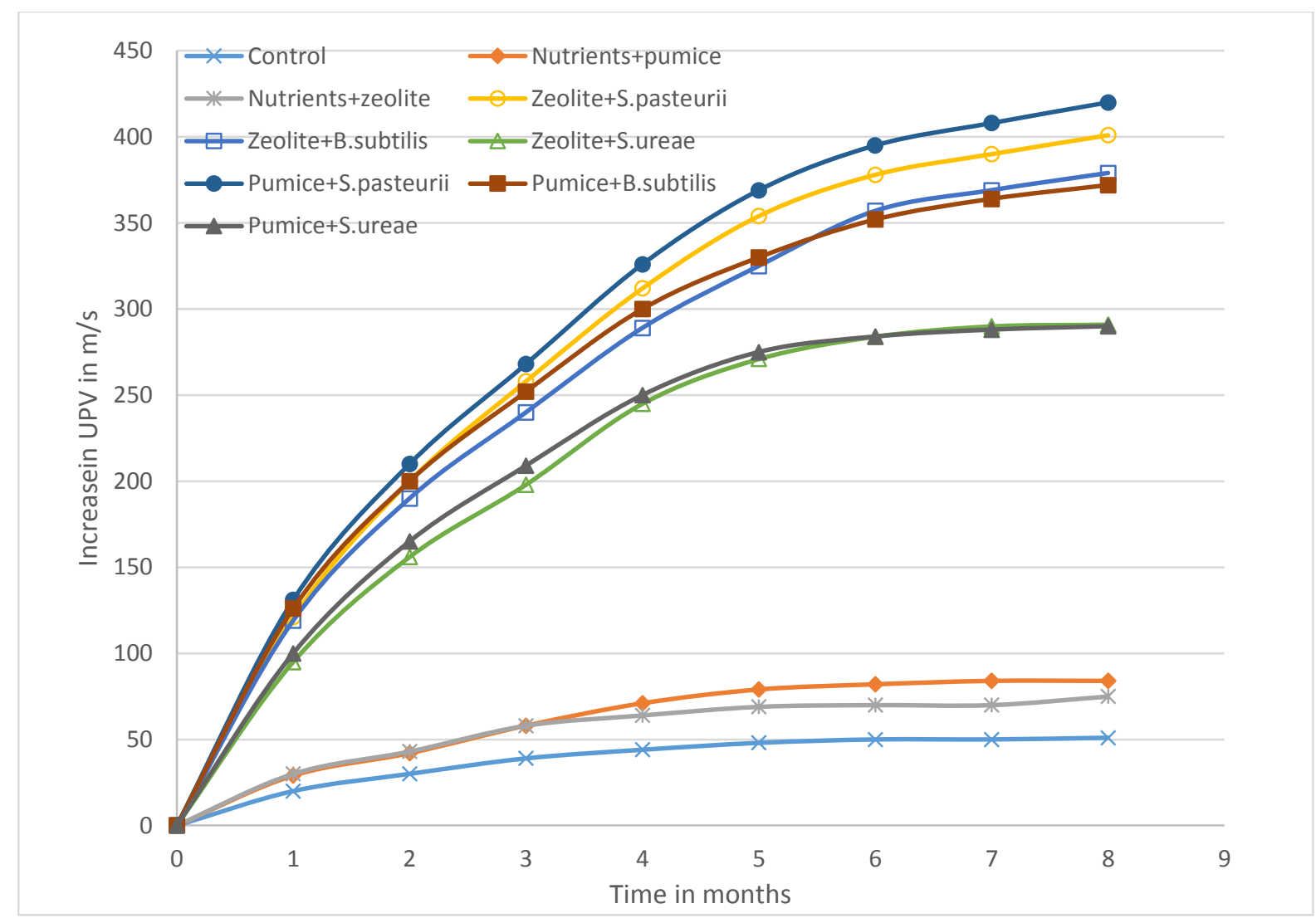

Figure 4.39: Changes in UPV values with time

\subsubsection{Photographic images to visualise self-healing}

Figure 4.40 shows the photographic images of crack healing of specimen treated with bacteria and control specimen after 4 months of healing. It can be seen that some of the wider cracks are partially filled and some of them are completely filled with bacteria. On the other hand, no crack healing was observed for control specimen. At the same time, some white precipitation can be noticed on the crack wall for the no bacteria + nutrients specimen. This might be either due to carbonation or due to the contribution from calcium lactate. The characterization of the material formed will be presented through micro-structural investigations. 

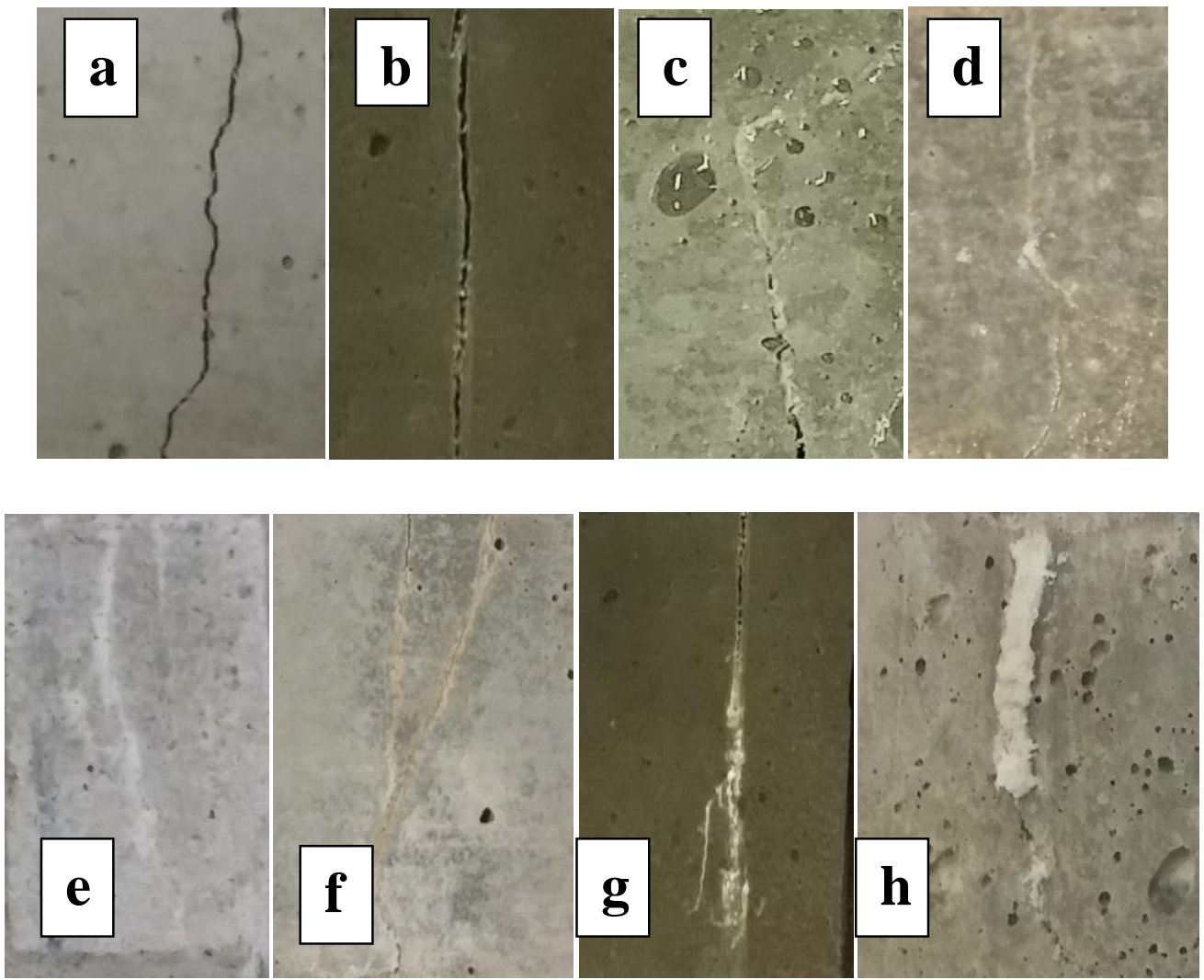

Figure 4.40: Photographic images of crack healing of specimen treated bacteria and control (a) control, (b) no bacteria + nutrients, (c) and (d) Bacillus subtilis treated (e) and (f) Sporosarcina ureae treated (g) and (h) Sporosarcina pasteurii treated

\subsubsection{SEM and EDS studies for self-healing characterization}

Microscopic visualisation and characterisation of self-healing conducted at multiple areas on the FR cracked specimens is discussed in this section.

\subsubsection{SEM and EDS analysis of crack surface}

The fibre reinforced mortar specimens incorporated with and without bacteria were observed under SEM after 8 months of healing. Figures 4.41 to 4.54 show the SEM and EDS observations of selfhealing products formed for crack filling. Specimens were analysed using EDS in order to .investigate the chemical nature of the self-healing products. 
It can be observed from the Figures 4.41 and 4.43 that the crack width of around $100 \mu \mathrm{m}$ is completely filled by calcite crystals produced by Sporosarcina pasteurii immobilised in pumice and Sporosarcina pasteurii immobilised in zeolite, respectively. Closer examination of SEM shows the presence of distinct rhombohedral shaped crystals in the crack area (Figure 4.45). Additionally, a layer of white precipitates can be found all over the surface of the specimen with bacteria. Similar crystal morphology can be observed for the precipitation of bacteria Sporosarcina pasteurii irrespective of the carrier materials. Therefore, it can be inferred that the types of carrier material do not influence the morphology of precipitated crystals.

From Figures 4.46 and 4.48, it can be seen that the crack is not completely filled with the bacteria Bacillus subtilis subsp. spizizenii immobilised in zeolite and pumice. However, abundant precipitation can be observed all over the surface. The measured crack width after cracking was $0.16 \mathrm{~mm}$ and it can be observed that almost $90 \%$ of the crack was filled by the $\mathrm{CaCO}_{3}$ precipitation in the sample with Bacillus subtilis subsp. spizizeniit zeolite. At the same time the initial crack width was around $0.18 \mathrm{~mm}$ and it can be seen that almost $75 \%$ of the cracks were healed in the specimen with Bacillus subtilis subsp. spizizenii+ pumice. It might be possible to completely heal the crack by immersing in water for a longer period of time. Similar to the case of $S$. pasteurii rhombohedral shaped crystals were observed in the crack area as well (Figure 4.49).

It can be observed from the Figure 4.51 that the crack width of around $70 \mu \mathrm{m}$ is completely filled by calcite crystals produced by Sporosarcina ureae immobilised in zeolite. Distinctly visible rhombohedral shaped crystals in the crack area were observed in this case also. However, the precipitated calcite crystals were appeared more scattered compared to that formed by the bacteria Sporosarcina pasteurii. This implies that the bonding between the crystals might be weak. Similar to the results observed by the samples with the other two bacteria, same crystal morphology can be observed for the precipitation irrespective of the carrier materials.

Energy Dispersive Spectroscopy (EDS) allows to identify the types of particular elements present and their relative proportions (Atomic \%). Initial EDS analysis usually involves the generation of an X-ray spectrum from the entire scan area of the SEM. The Y-axis shows the counts (number of $\mathrm{X}$-rays received and processed by the detector) and the $\mathrm{X}$-axis shows the energy level of those counts in $\mathrm{eV}$. The current EDS study was performed at 20keV. As shown in Figures 4.43, 4.44, 4.45, 4.50 and 4.52 EDS analysis of all the specimens with bacteria disclosed that the massively 
formed precipitates comprised of three main elements: $\mathrm{C}, \mathrm{O}$ and $\mathrm{Ca}$. Therefore, it can be confirmed that the mineral precipitates were $\mathrm{CaCO}_{3}$ based. From the SEM study, it was observed that a layer of white precipitates all over the surface of the specimen with bacteria. EDS analysis conducted away from the crack area (Spectrum 21 of Figure 4.42) confirmed this by indicating the presence of three main elements: $\mathrm{C}, \mathrm{O}$ and $\mathrm{Ca}$. By observing the dense precipitation at the crack space more closely, it can be noticed that the calcium carbonate crystals were very well developed near the surface of the crack. These crystals have clear and sharp edges which reveal a full growth of crystals. The high calcium amounts from the EDS analysis confirmed that calcite was present in the form of calcium carbonate due to the microbial induced calcite precipitation. Figure 4.42 presents the results of EDS study conducted at two different locations on the Sporosarcina pasteurii + pumice specimen. One in the crack filled area (spectrum 20) and the other, away from the crack (Spectrum 21). At the crack area, it shows high counts of $\mathrm{Ca}(14 \%), \mathrm{O}(37 \%)$ and $\mathrm{C}(44 \%)$, which indicates the formed crystals are $\mathrm{CaCO}_{3}$. The same was observed on the spectrum 21 also, which confirms the findings of the SEM that a layer of precipitation was formed on the surface. Figure 4.44 shows the EDS results on the Sporosarcina pasteurii + zeolite specimen. Similar to the previous case, presence of $\mathrm{Ca}(21 \%)$, O (48\%) and C (20\%) is confirmed. Figure 4.47 shows the EDS analysis results on Bacillus subtilis subsp. spizizenii + zeolite and similar presence of minerals was observed on both spectrums. Observation of the SEM image indicates rich and well-formed crystal growth on both spectrums. Figure 4.50 depicts the SEM image and the EDS analysis of Bacillus subtilis subsp. spizizenii + pumice specimen. SEM image shows the crystal formation in the crack area and the EDS analysis indicates the high presence of $\mathrm{Ca}, \mathrm{C}$ and O compared to other minerals. Figure 4.52 shows the SEM and EDS results for Sporosarcina ureae+zeolite zeolite and in this case, the crystals forms are found to be smaller compared to the ones formed by other bacteria, still the EDS analysis shows high percentage of $\mathrm{Ca}, \mathrm{O}$ and $\mathrm{C}$ compared to other minerals.

However, the SEM observation of control sample without bacteria and nutrients showed no sign of crystal growth (Figure 4.53). Though the samples without bacteria but with nutrients exhibited the formation of fewer amount of white crystals on the surface and along the crack wall, but not as dense as that of bacterial incorporated one. Besides, the crack closure was not much pronounced in the specimen with nutrients + pumice or nutrients + zeolite. This formation of crystals in the nutrients only specimen might be due to two kinds of reactions. First one is carbonation and the 
second one is due to the presence of calcium lactate which up on reaction with carbonate ions forms calcium carbonate.

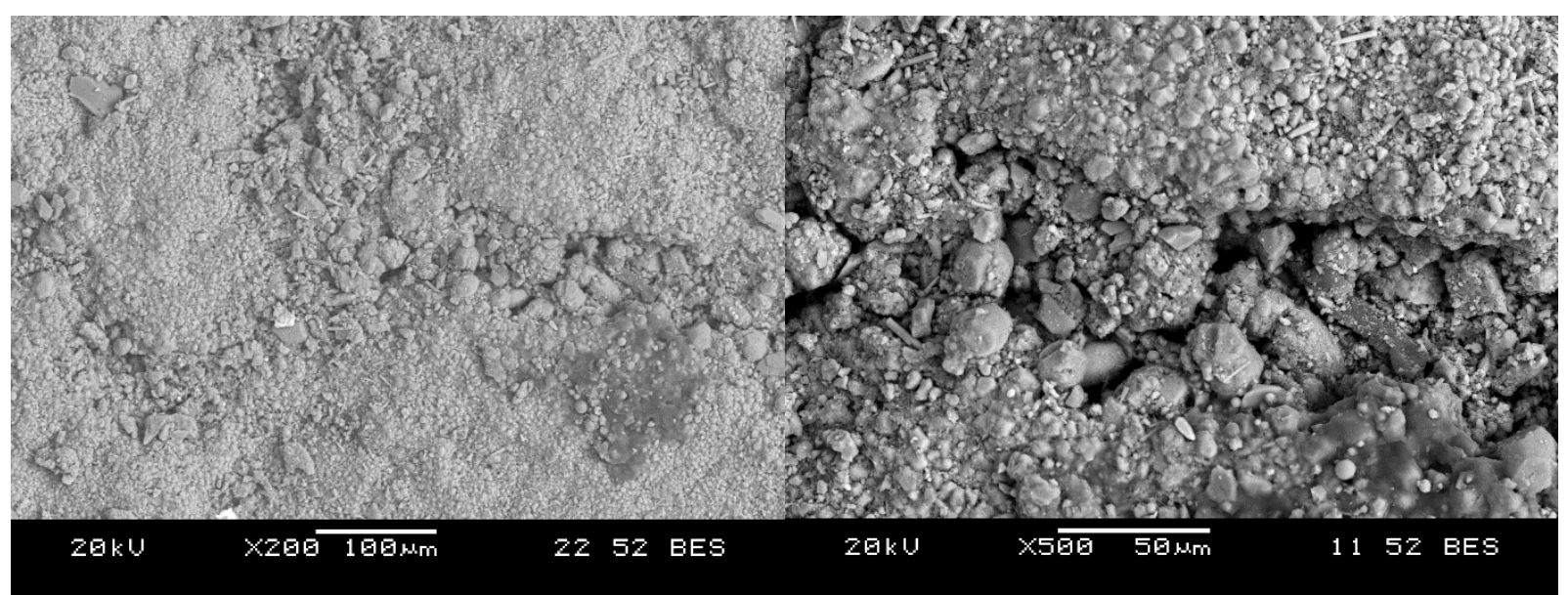

Figure 4.41: SEM observation of cracked samples with Sporosarcina pasteurii +pumice
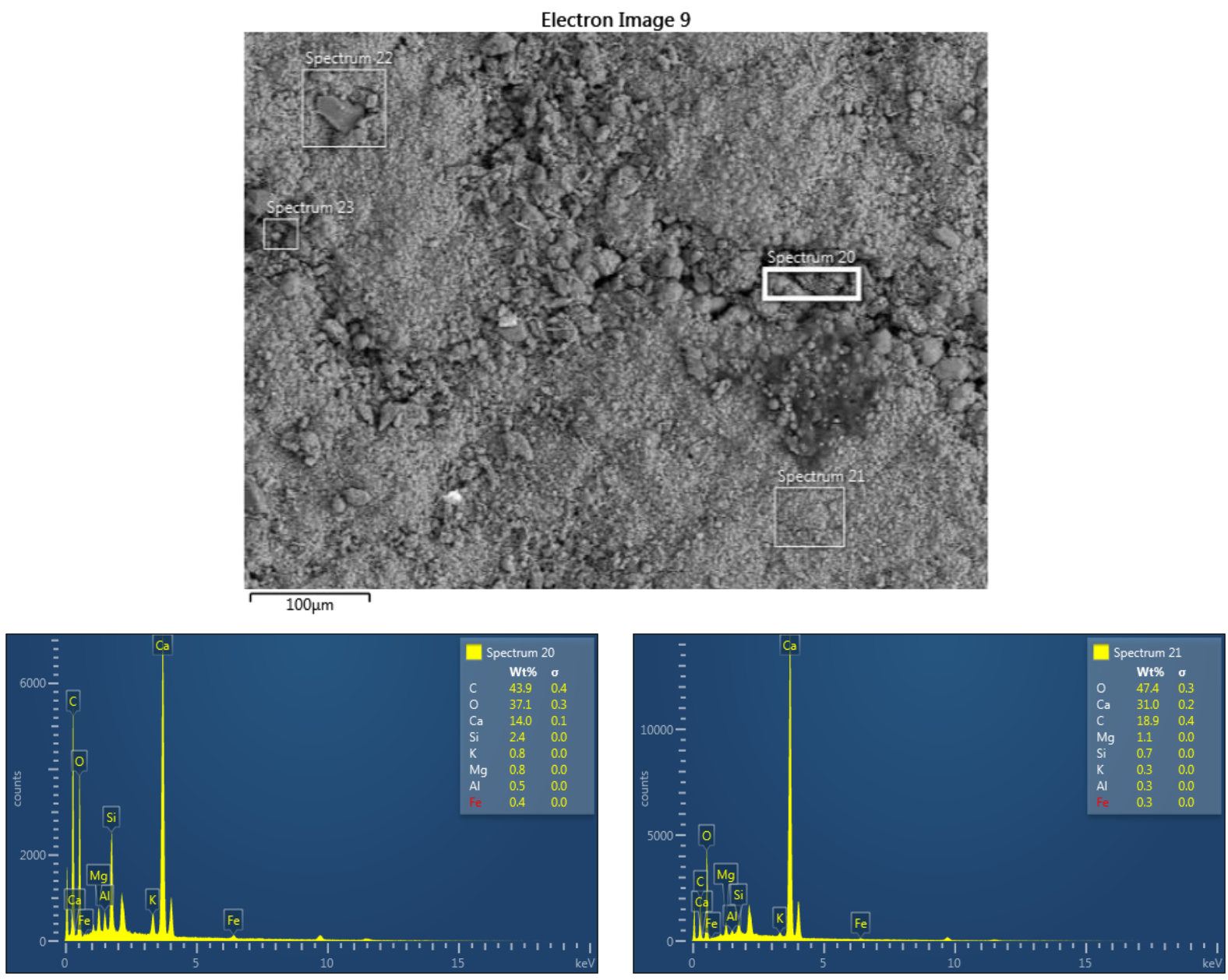

Figure 4.42: EDS analysis of Sporosarcina pasteurii + pumice 

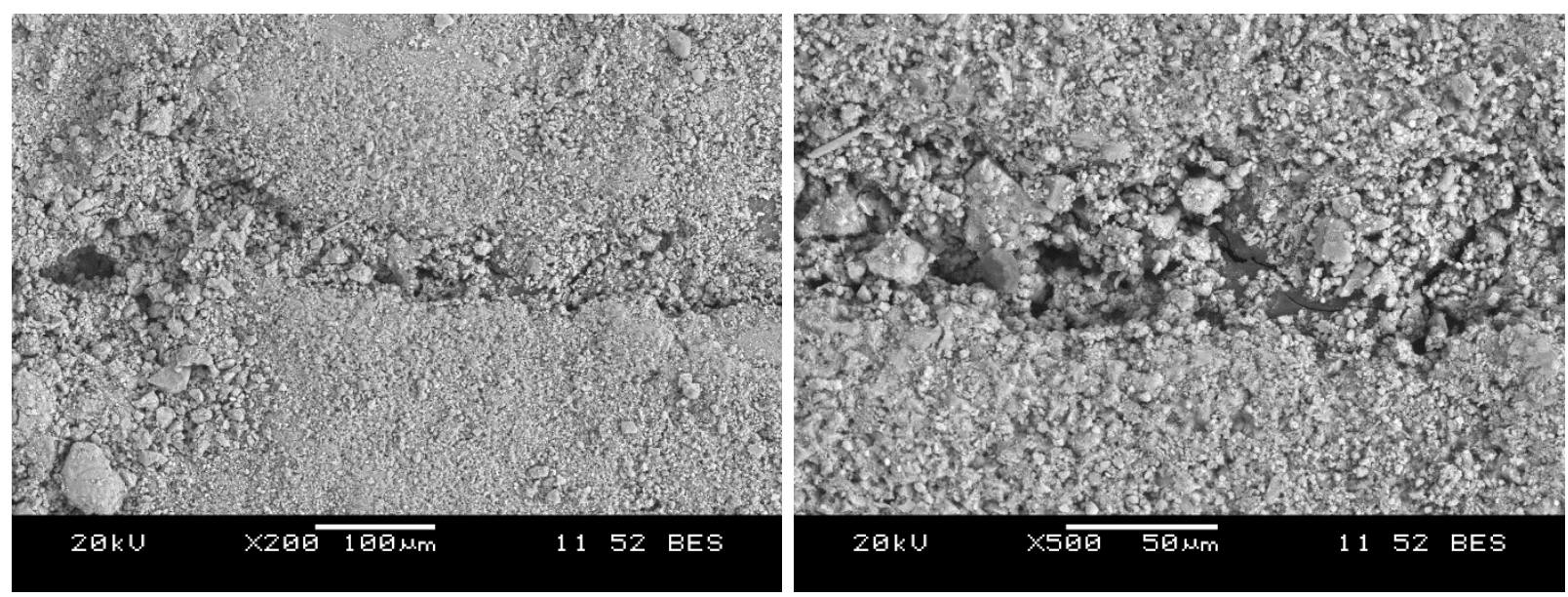

Figure 4.43: SEM observation of cracked samples with Sporosarcina pasteurii + zeolite
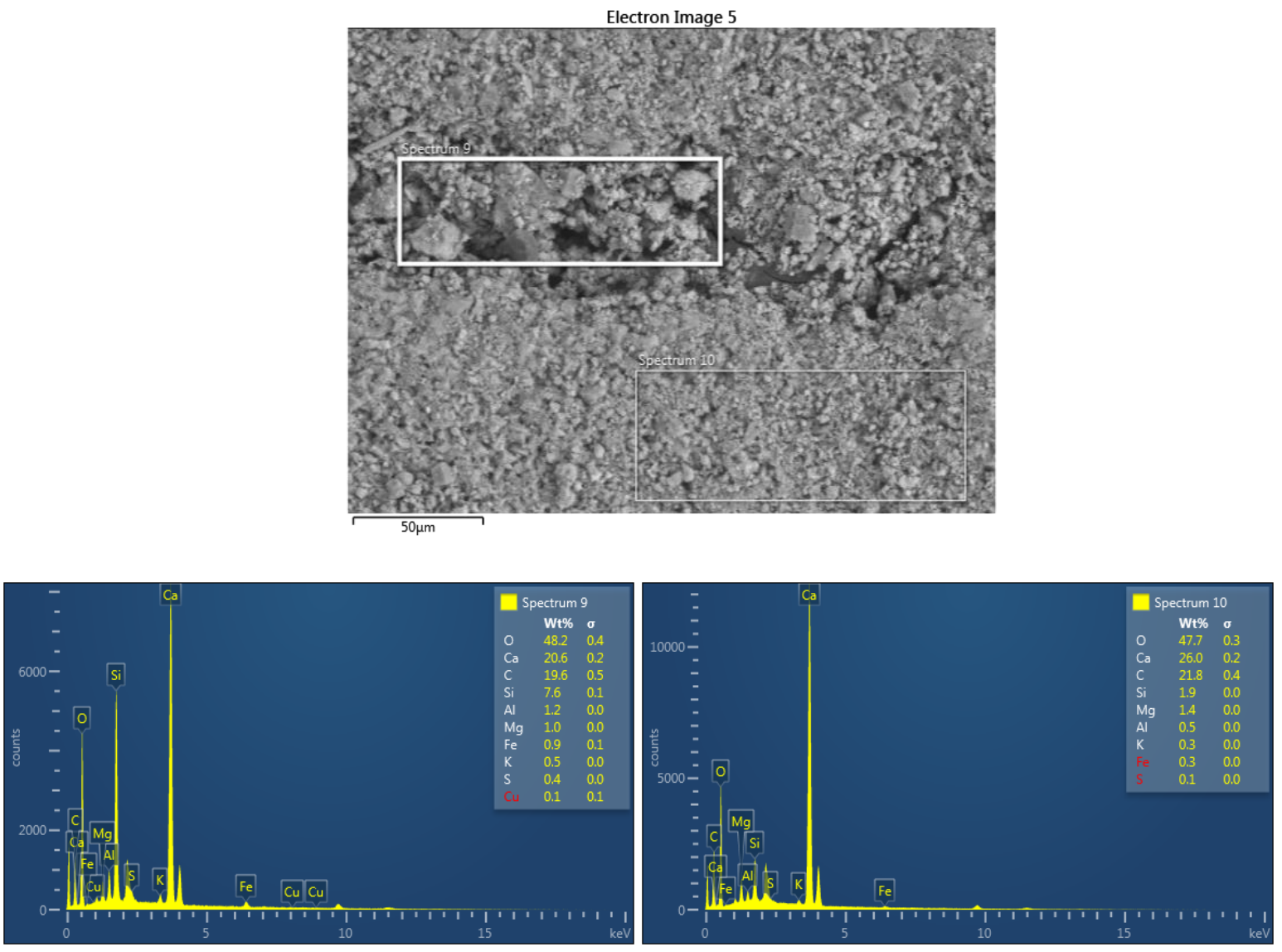

Figure 4.44: EDS analysis of Sporosarcina pasteurii + zeolite 


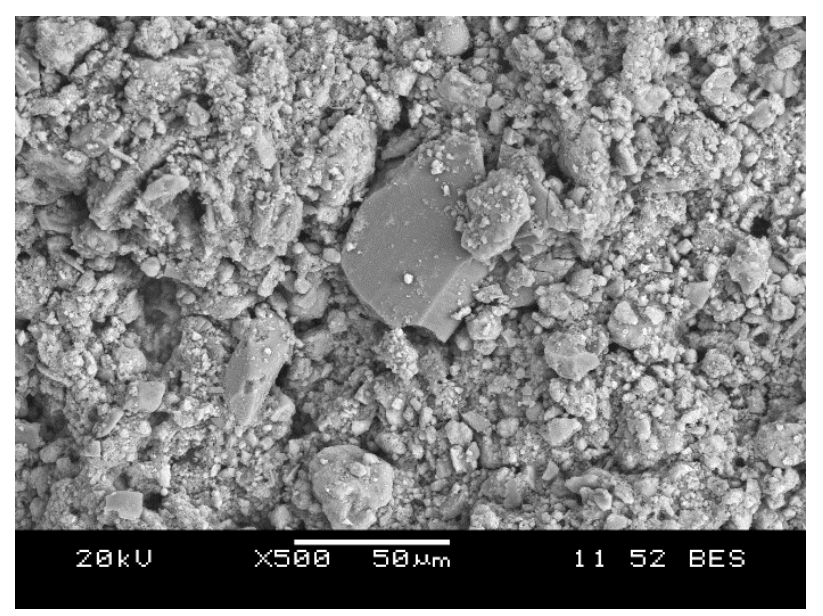

Figure 4.45: SEM observation of $\mathrm{CaCO}_{3}$ crystals formed by Sporosarcina pasteurii

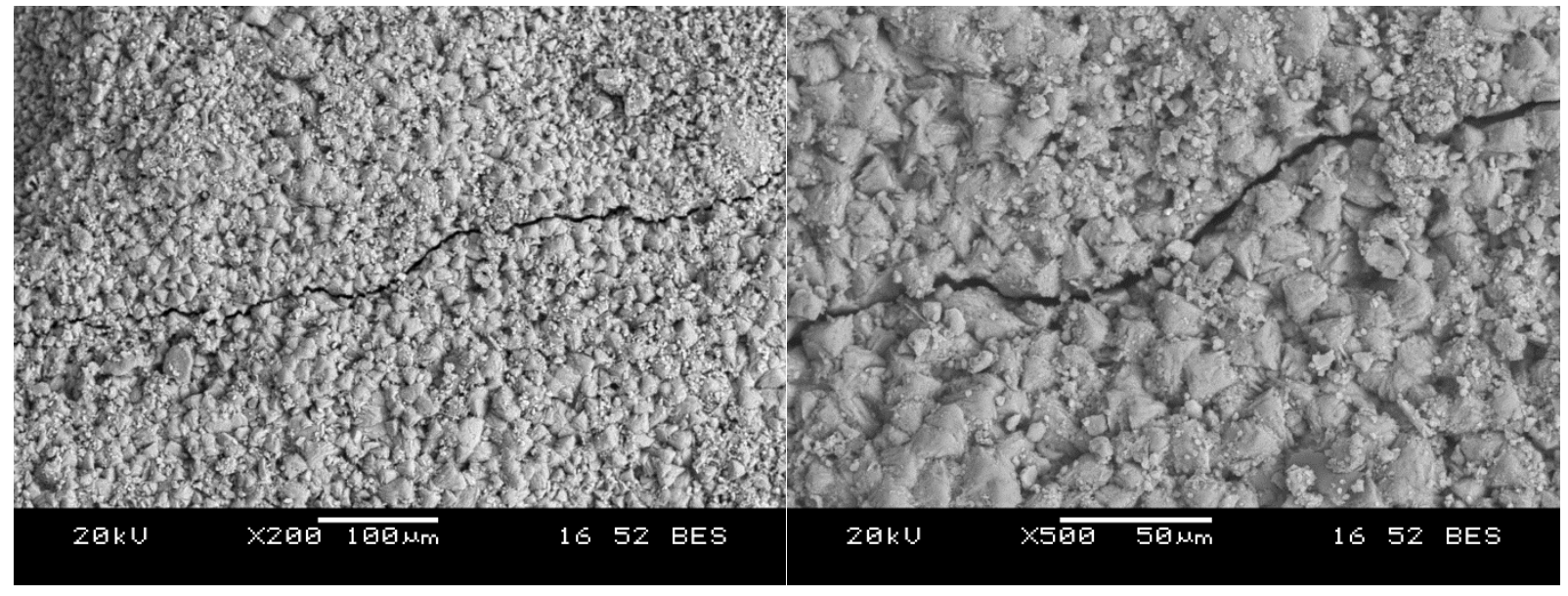

Figure 4.46: SEM observation of cracked samples with $B$. subtilis subsp. spizizenii + zeolite 

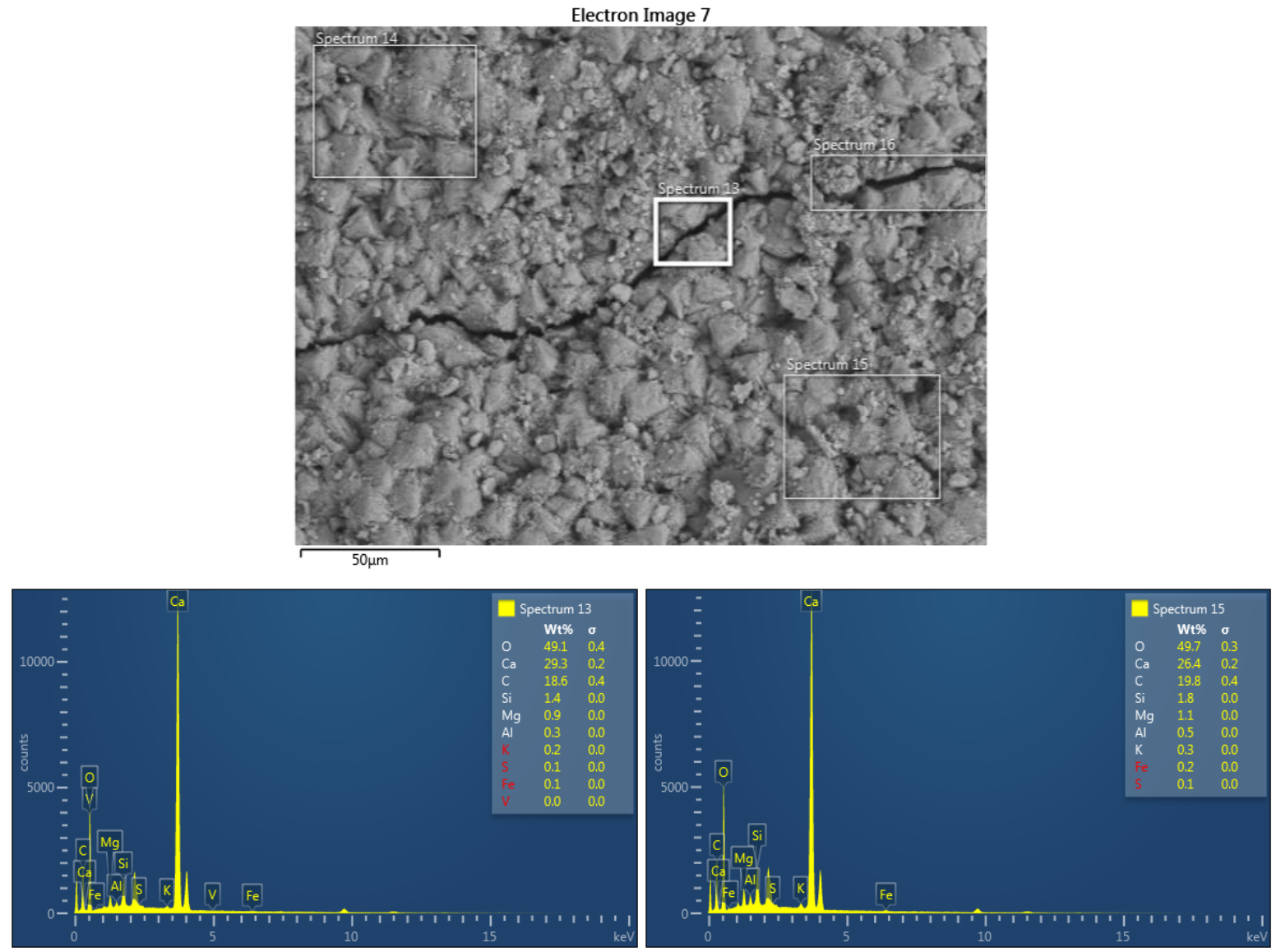

Figure 4.47: EDS analysis of $B$. subtilis subsp. spizizenii + zeolite

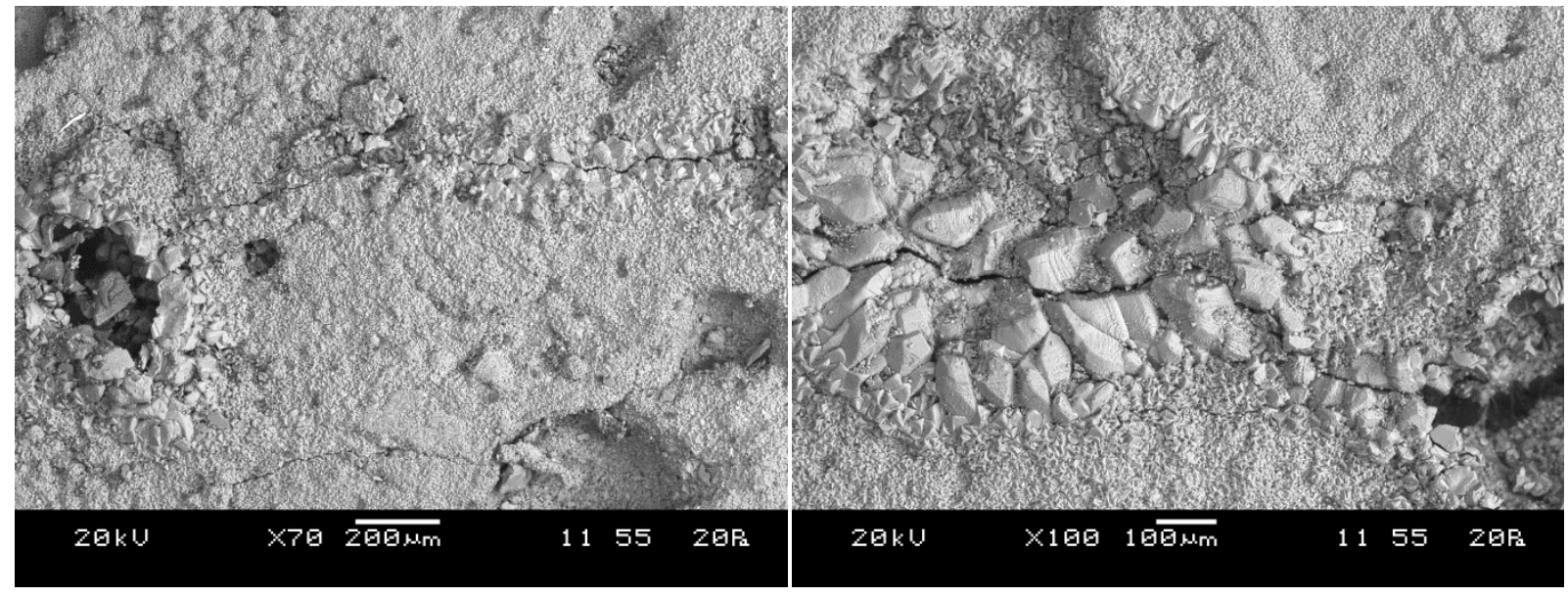

Figure 4.48: SEM observation of cracked samples with $B$. subtilis subsp. spizizenii + pumice 

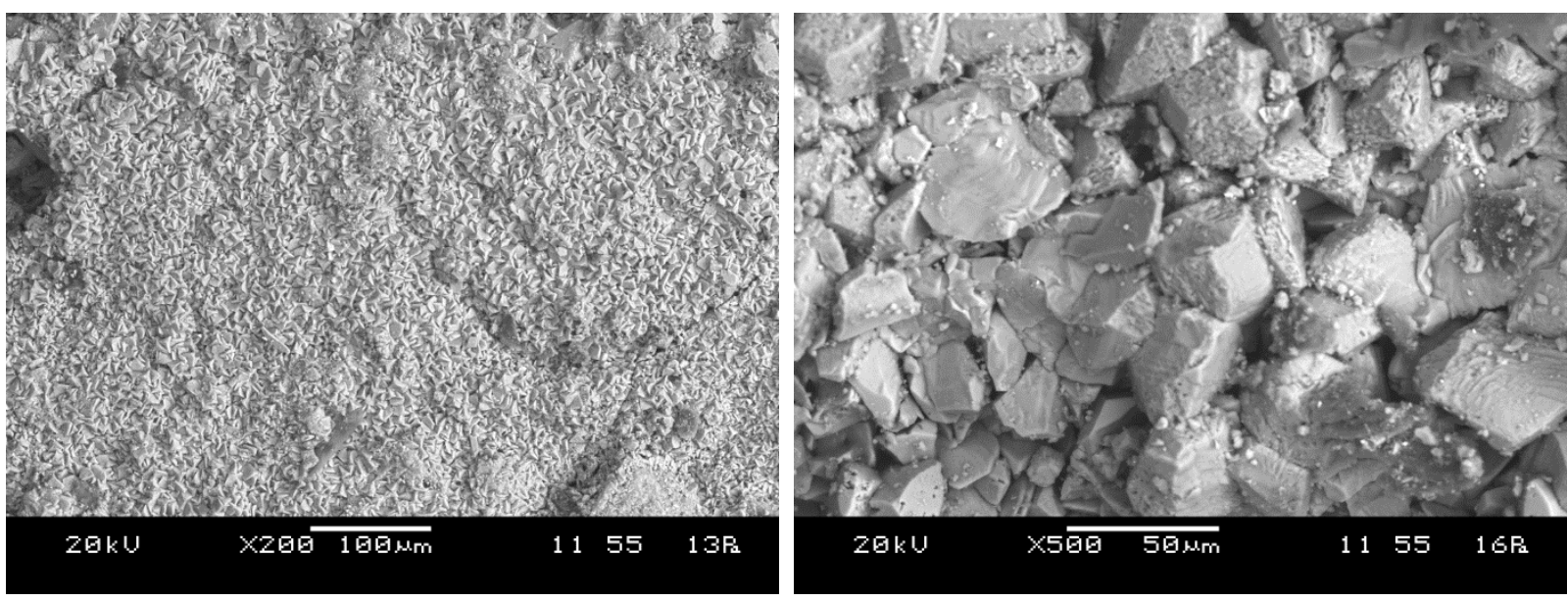

Figure 4.49: $\mathrm{SEM}$ observation of $\mathrm{CaCO}_{3}$ crystals formed by Bacillus subtilis subsp. spizizenii (thickly formed $\mathrm{CaCO}_{3}$ crystals on left, closer observation on right)
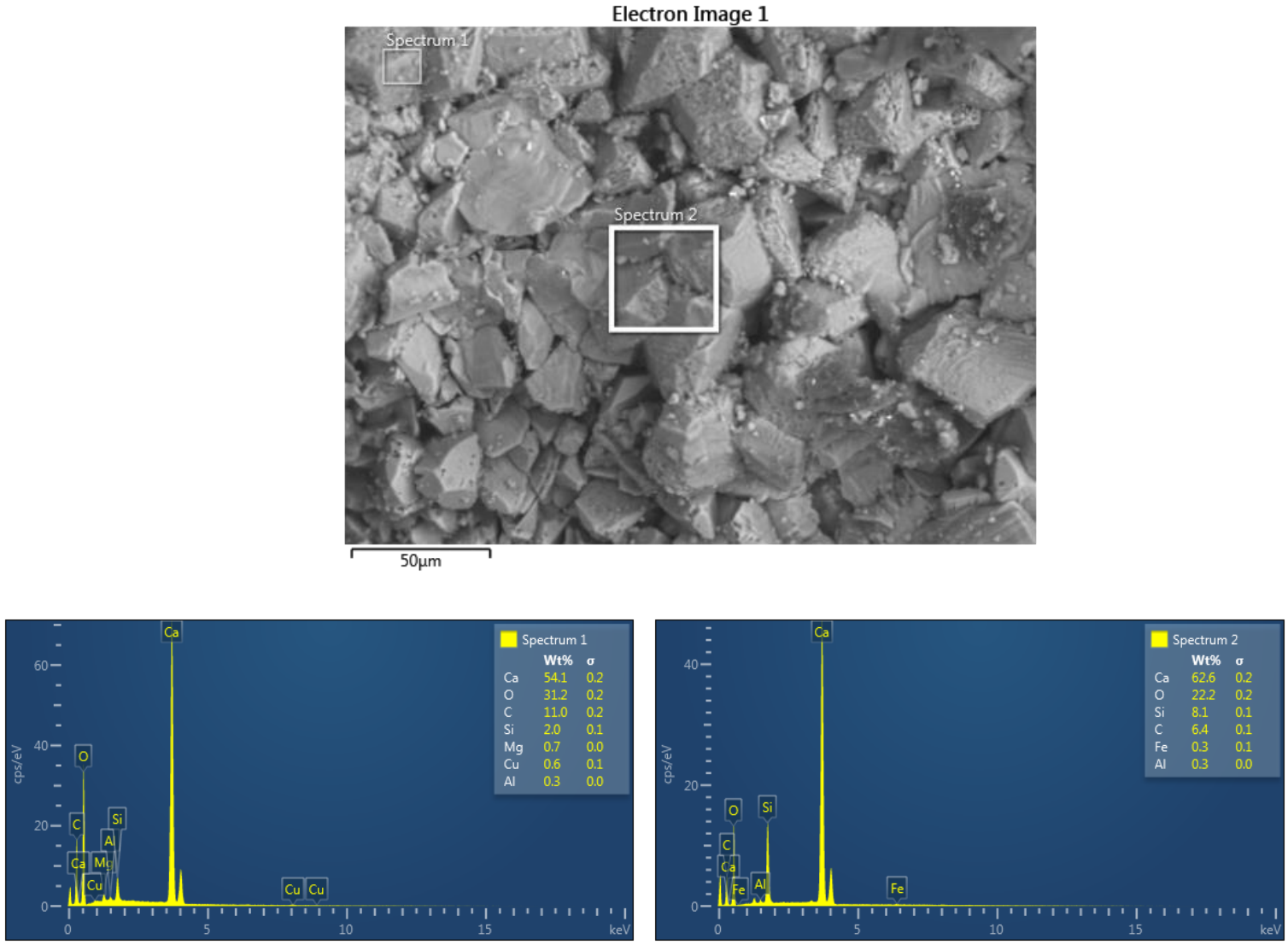

Figure 4.50: EDS analysis of B. subtilis subsp. spizizenii + pumice 

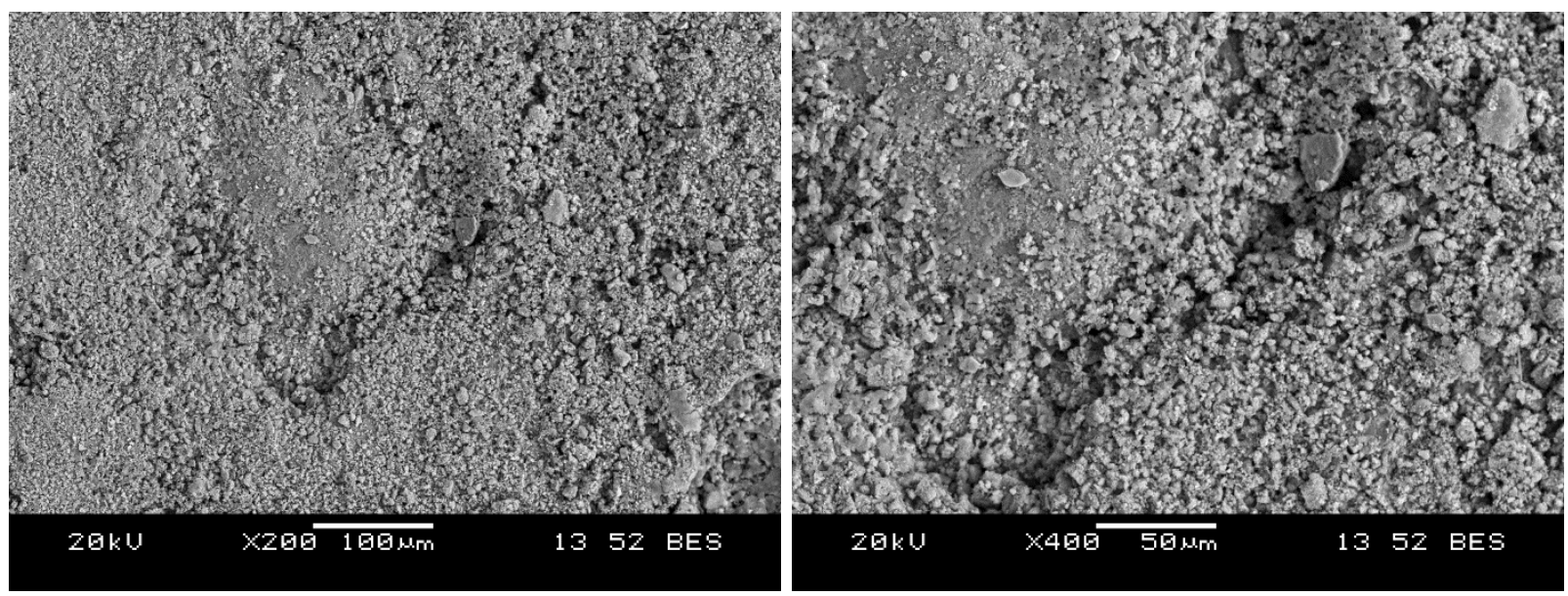

Figure 4.51: SEM observation of cracked samples with Sporosarcina ureae + zeolite
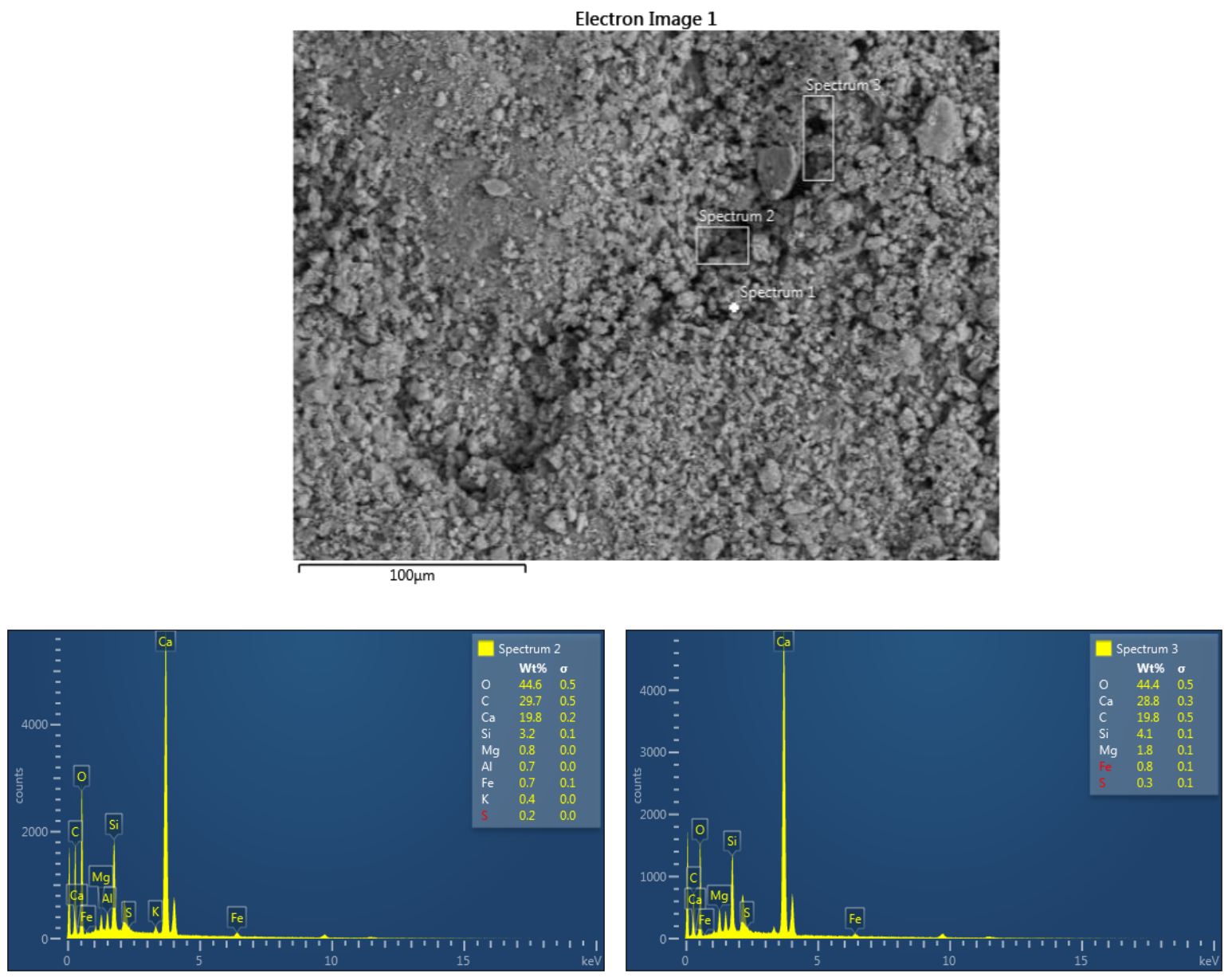

Figure 4.52: EDS analysis of Sporosarcina ureae + zeolite 


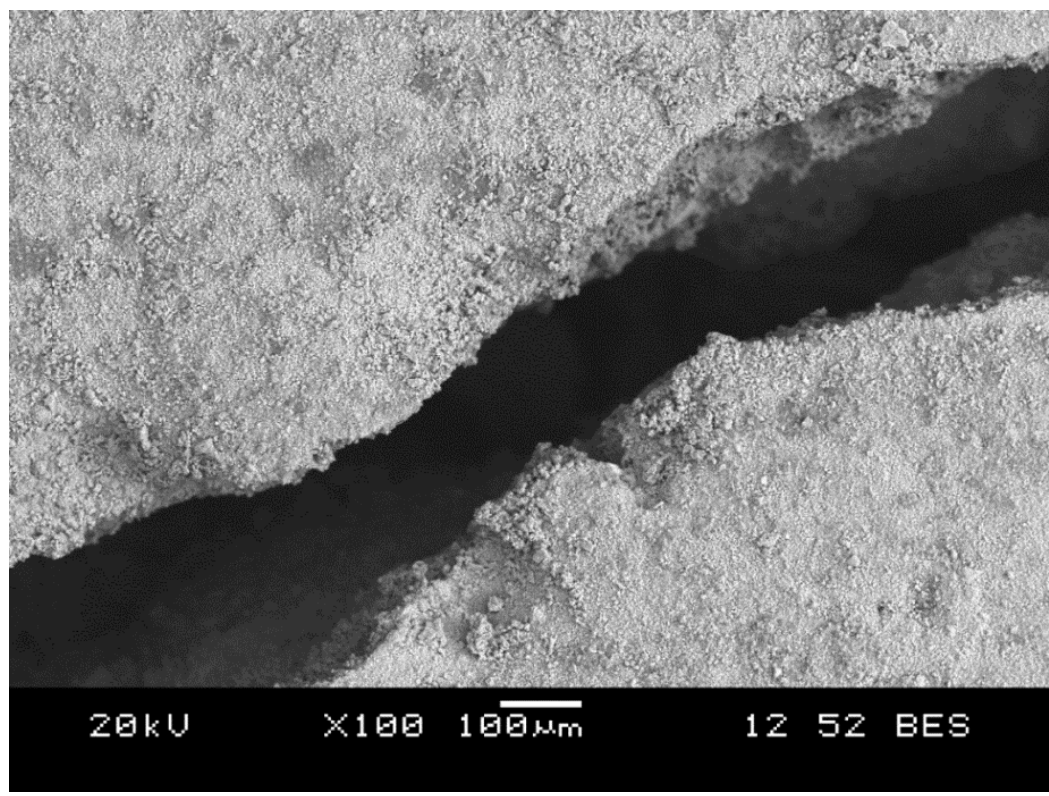

Figure 4.53: SEM observation of control sample with no healing

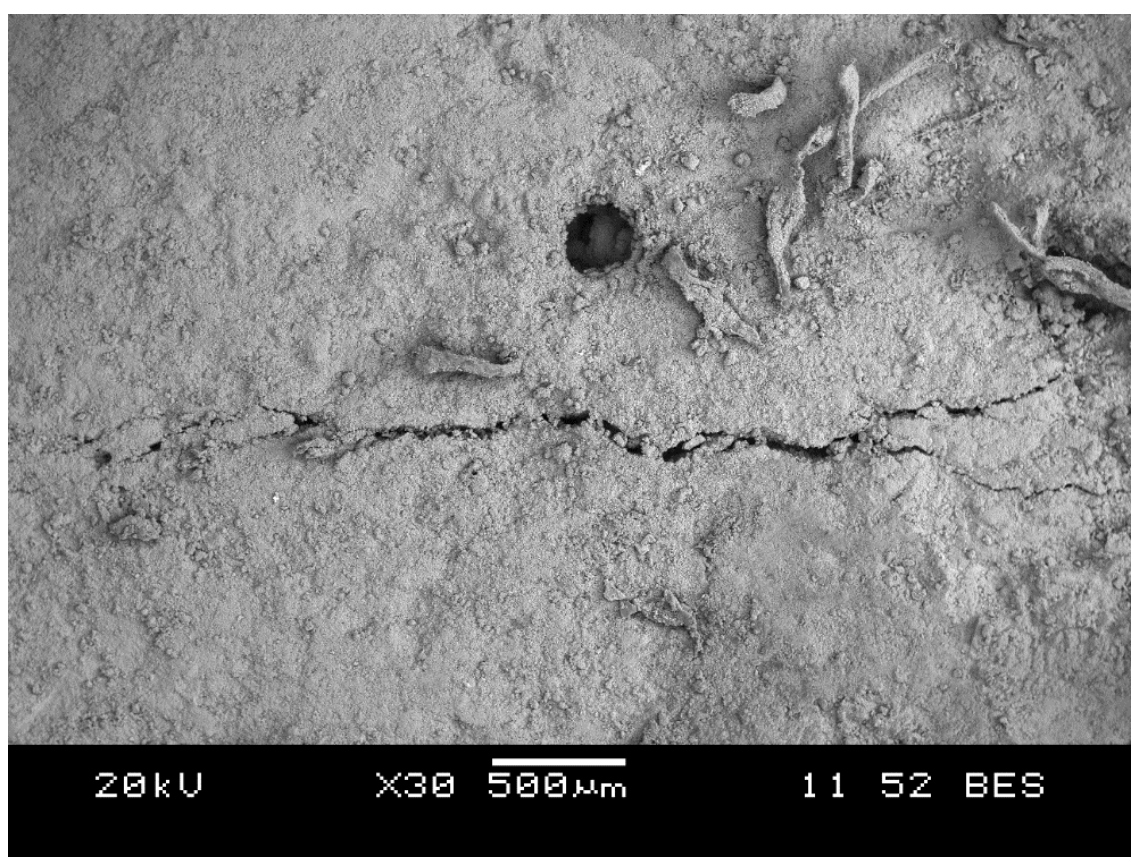

Figure 4.54: SEM observation of sample with nutrients + pumice with lesser healing

\subsubsection{SEM analysis of interior region of crack}

It was found that the calcite precipitation in the interior region of the crack was much reduced. Some amount of calcite crystals can be seen at the crack area for the specimen with Sporosarcina pasteurii (Figure 4.55) and the specimen with Bacillus subtilis subsp. Spizizenii (Figure 4.56). 
However, it was appeared to be scattered and limited. From the Figure 4.56, it was observed that calcite crystals were negligibly less for the specimen with the bacterial species Sporosarcina ureae.

Observation of the thin sections at the interior region of the bacteria based specimen revealed that the cracks which appeared to be completely closed at the surface were actually open at the interior region. The reason for this might be due to the fact that the bacteria need oxygen and water for their action which are available more at the surface compared to the interior region. Once the entrance of the crack is blocked by $\mathrm{CaCO}_{3}$ formation, the ingress of water, $\mathrm{CO}_{2}$ and oxygen is rather difficult to obtain for the bacteria to become active for the conversion of calcium lactate to $\mathrm{CaCO}_{3}$. However, with this surface covering it is possible to prevent the ingress of aggressive chemicals into the matrix and hence durability of the material can be enhanced.

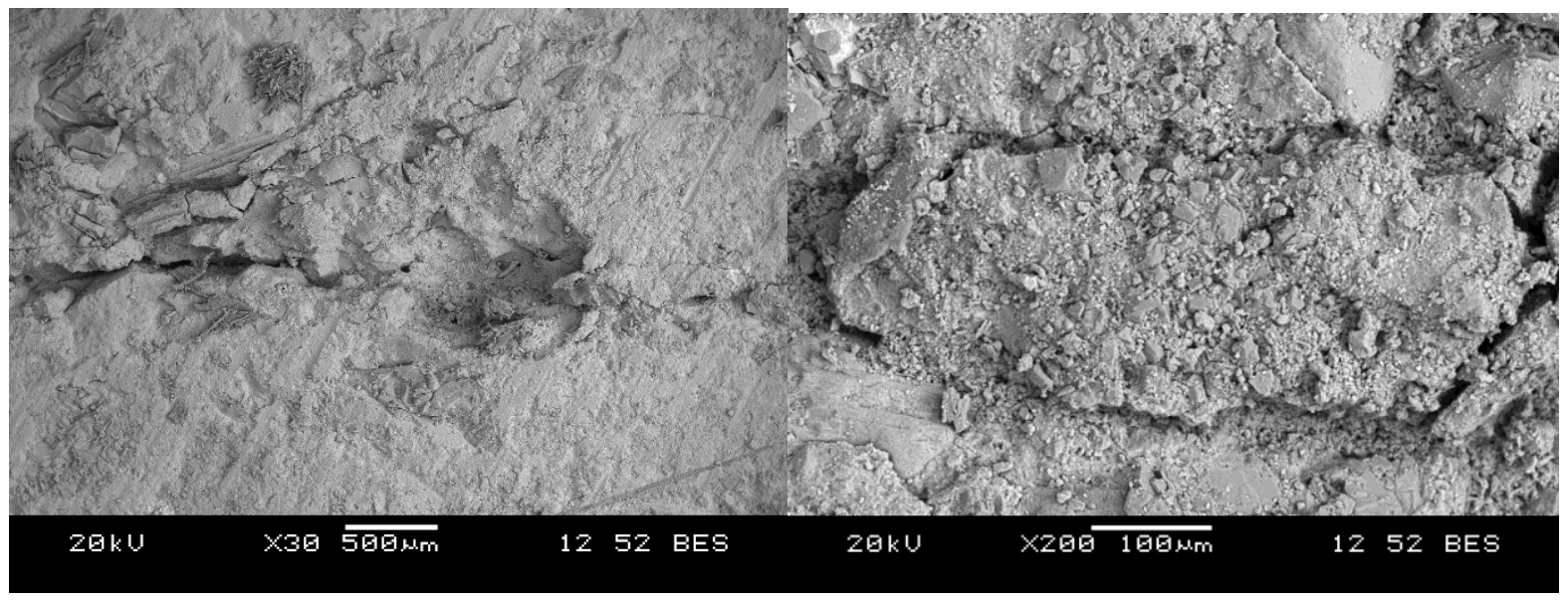

Figure 4.55: Thin section observation of crack healing at the interior region of crack (Sporosarcina pasteurii based mortar) 


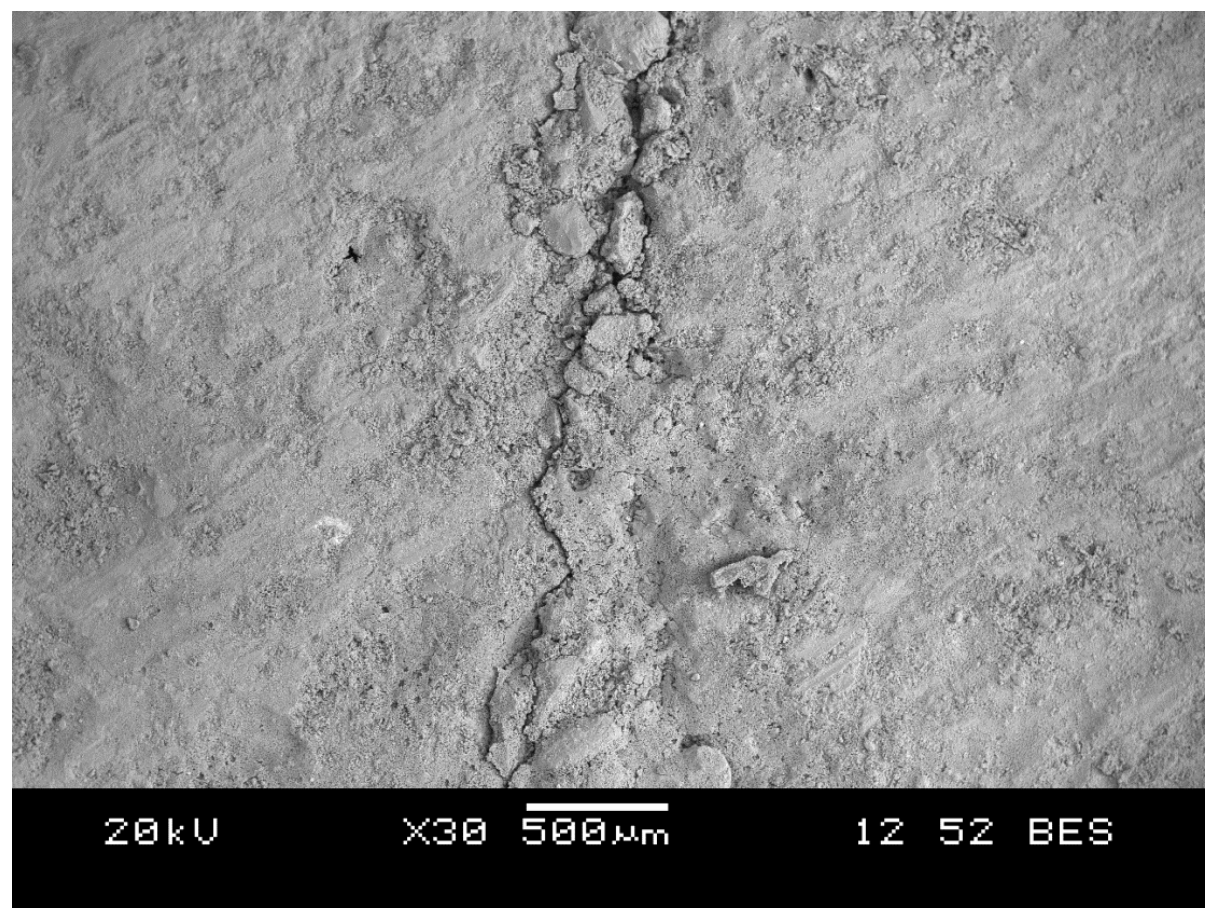

Figure 4.56: Thin section observation of crack healing at the interior region of crack (Bacillus subtilis subsp. Spizizenii based mortar)

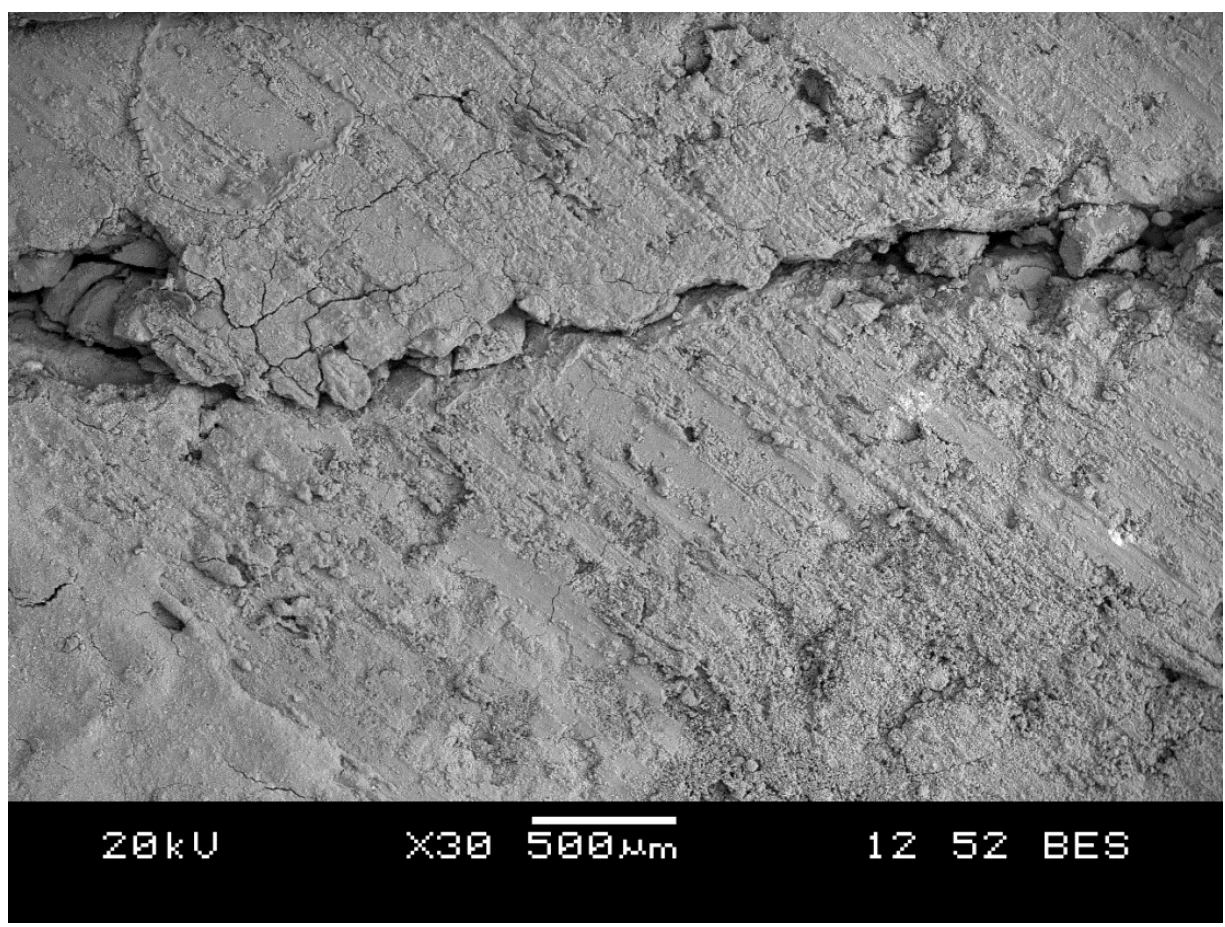

Figure 4.57: Thin section observation of crack healing at the interior region of crack (Sporosarcina ureae based mortar) 


\subsubsection{Micro structural properties of normal mortar matrix with and without bacteria}

Figures 4.58 and 4.59 show the microstructure of mortar matrix of the specimens with and without bacteria, respectively after 8 months of healing in water. A high improvement in the hydrated structure of mortar matrix can be observed in the specimen with bacteria (Figure 4.58). It can be noticed that the pores were almost filled with white crystals. On the other hand, too many pores can be observed in the specimen without bacteria. Because the specimen was immersed in water for longer periods, large number of needle like ettringite crystals was observed for the specimen without bacteria.

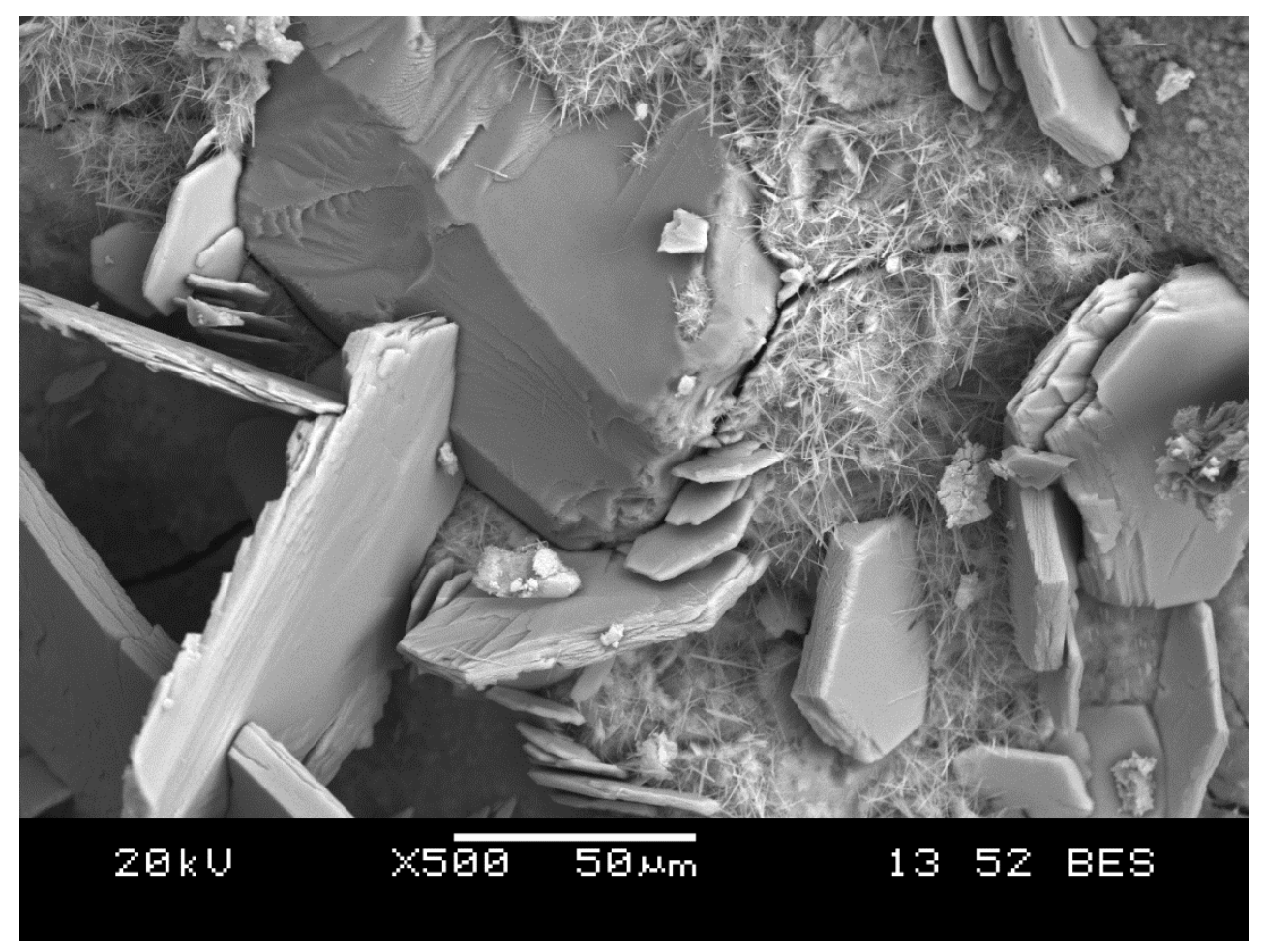

Figure 4.58: Microstructure of mortar matrix with bacteria and nutrients 


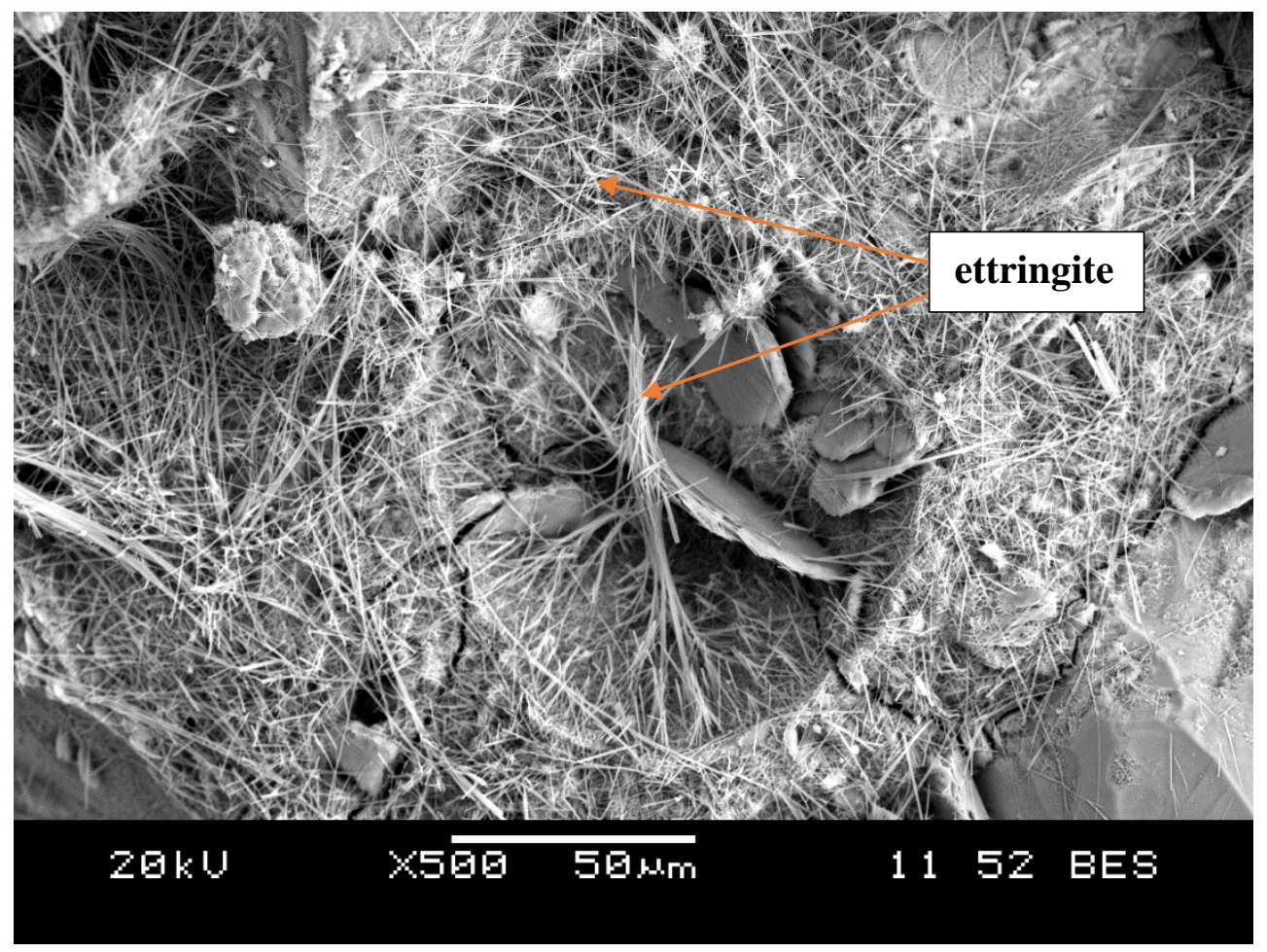

Figure 4.59: Microstructure of mortar matrix without bacteria but with nutrients

\subsubsection{XRD analysis mortar specimens}

In order to confirm that crack healing occurred due to the precipitation of calcium carbonate, XRD analysis were carried out on the mortar specimens with and without bacteria. Essentially, XRD analysis was performed to detect the nature of the crystalline materials formed in the precipitated layer. Figures 4.60 to 4.68 show the results of XRD analysis of 9 different FR mortar mixes. In the Figures 4.60 to 4.68, C stands for Calcite and S stands for Silica/Quartz). It can be observed that the crystalline materials produced on the cracked surface were calcite in bacteria based specimens. Results of XRD also confirmed the maximum number of calcite peaks in all the bacteria based mortar specimens. However, for control (Figure 4.60) and nutrients + pumice (Figure 4.61) and nutrients + zeolite specimens (Figure 4.62), most of the peaks were silica and quarts. At the same time, limited number of calcite peaks can be observed for the nutrients + pumice and nutrients + zeolite specimens.

The obtained XRD spectra were analyzed to get the specimen which gave maximum number of calcite peaks. It was observed that the maximum number of calcite peaks was obtained for mortar specimen incorporated with Sporosarcina pasteurii (figure 4.63 and 4.64). Figures 4.65 and 4.66 
respectively show the XRD analysis of $B$. subtilis with pumice and zeolite in which some extra high calcite peaks can be observed. Similar observations can be seen for the XRD analysis of $S$. ureae with pumice and zeolite (Figure 4.67 and 4.68). The X-ray analysis of the crystal powder from the specimen incorporated with bacteria shows some extra peaks compared to specimens without bacteria but with nutrients + zeolite/nutrients + pumice.

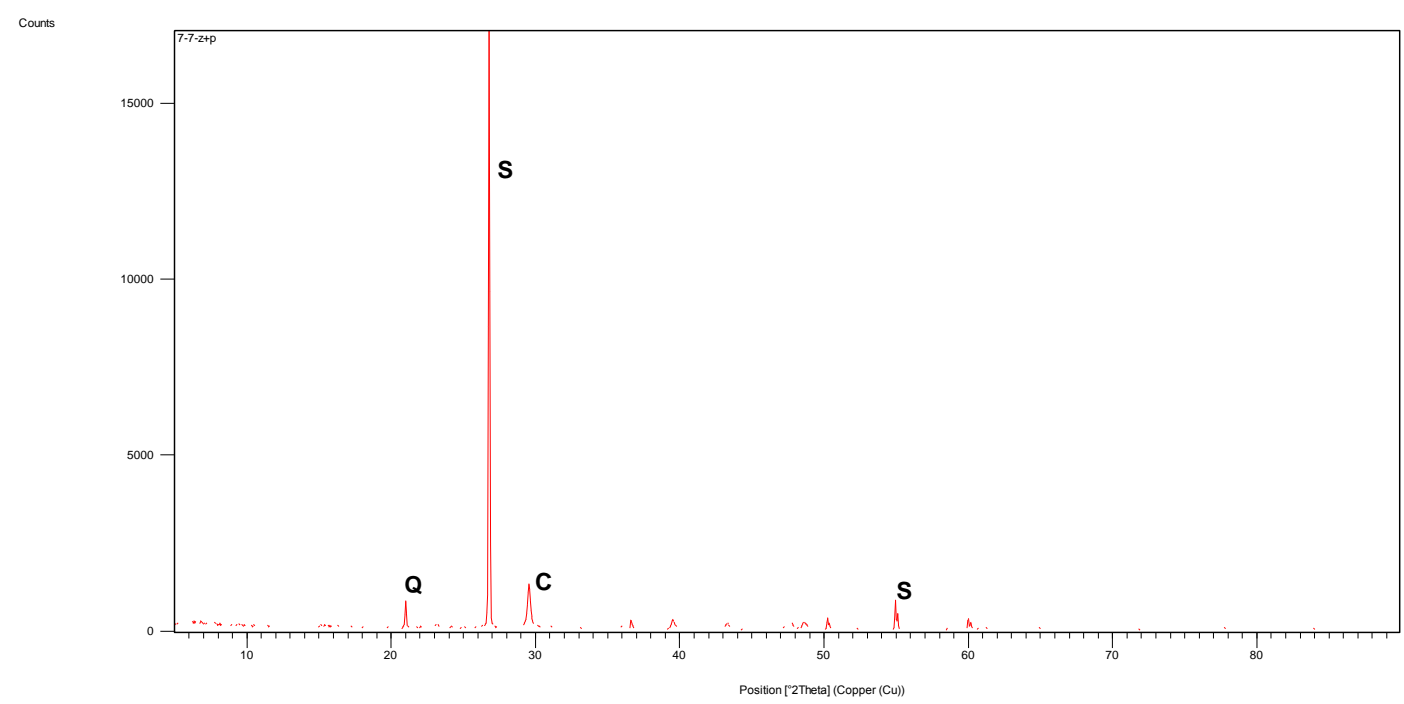

Figure 4.60: XRD analysis of Control sample (without bacteria and nutrients) (C stands for Calcite and S stands for Silica/Quartz) 


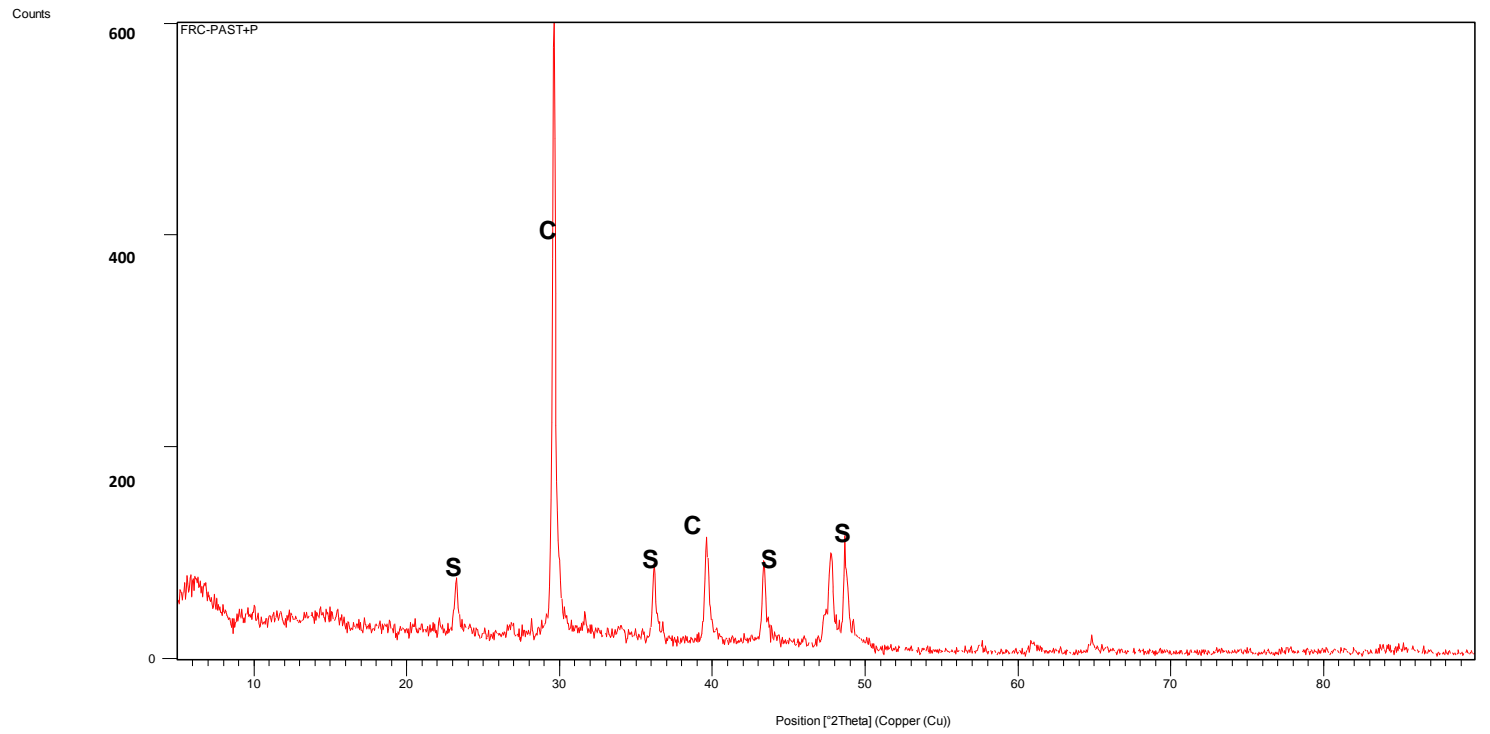

Figure 4.61: XRD analysis of mortar without bacteria + pumice

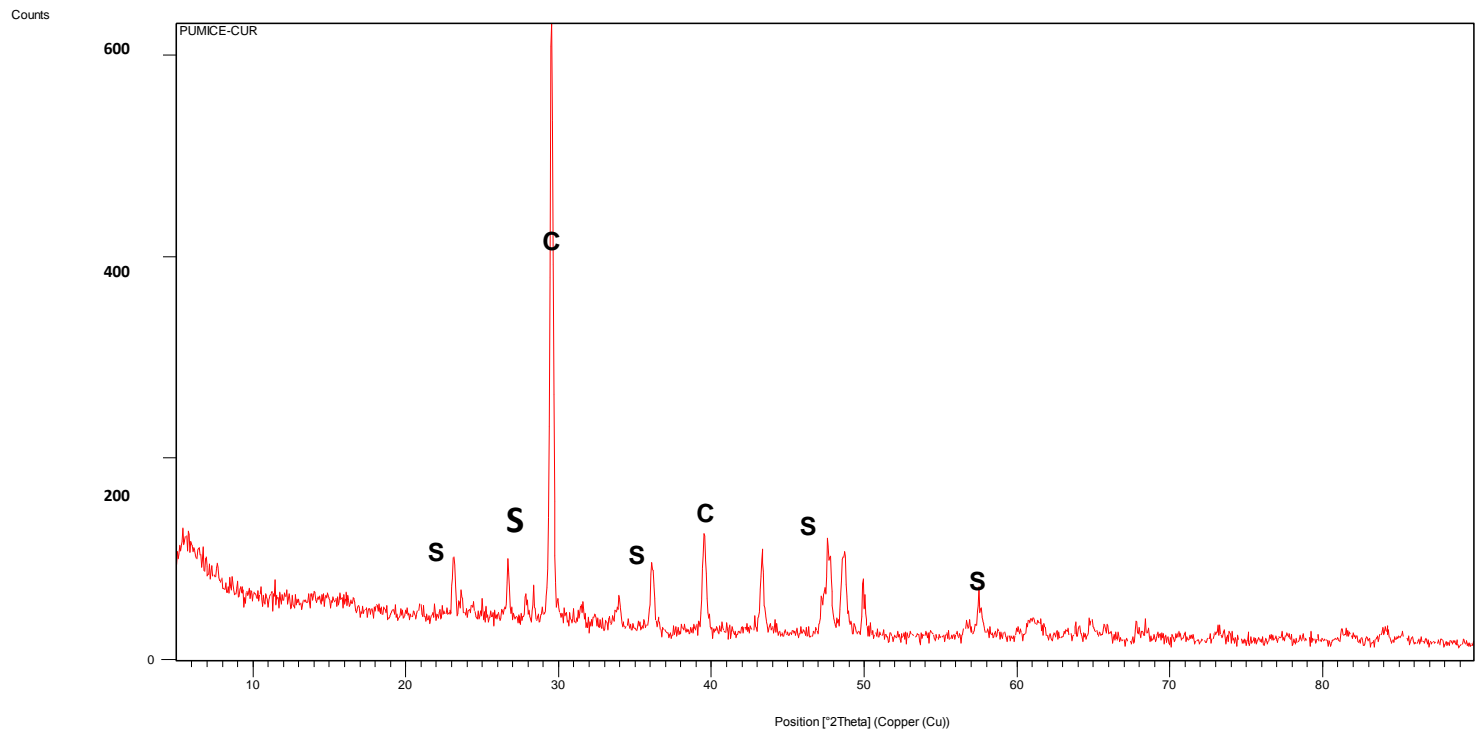

Figure 4.62: XRD analysis of mortar without bacteria + zeolite 


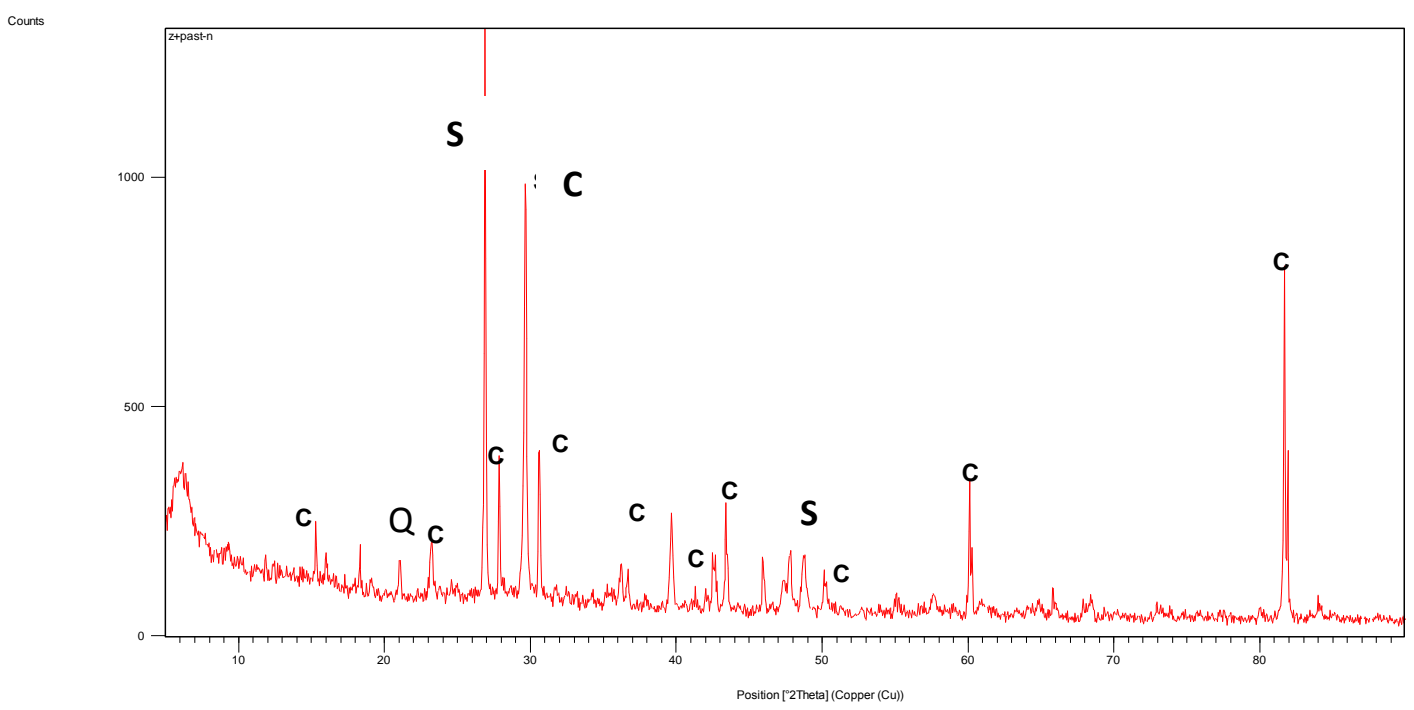

Figure 4.63: XRD analysis of mortar with Sporosarcina pasteurii + pumice

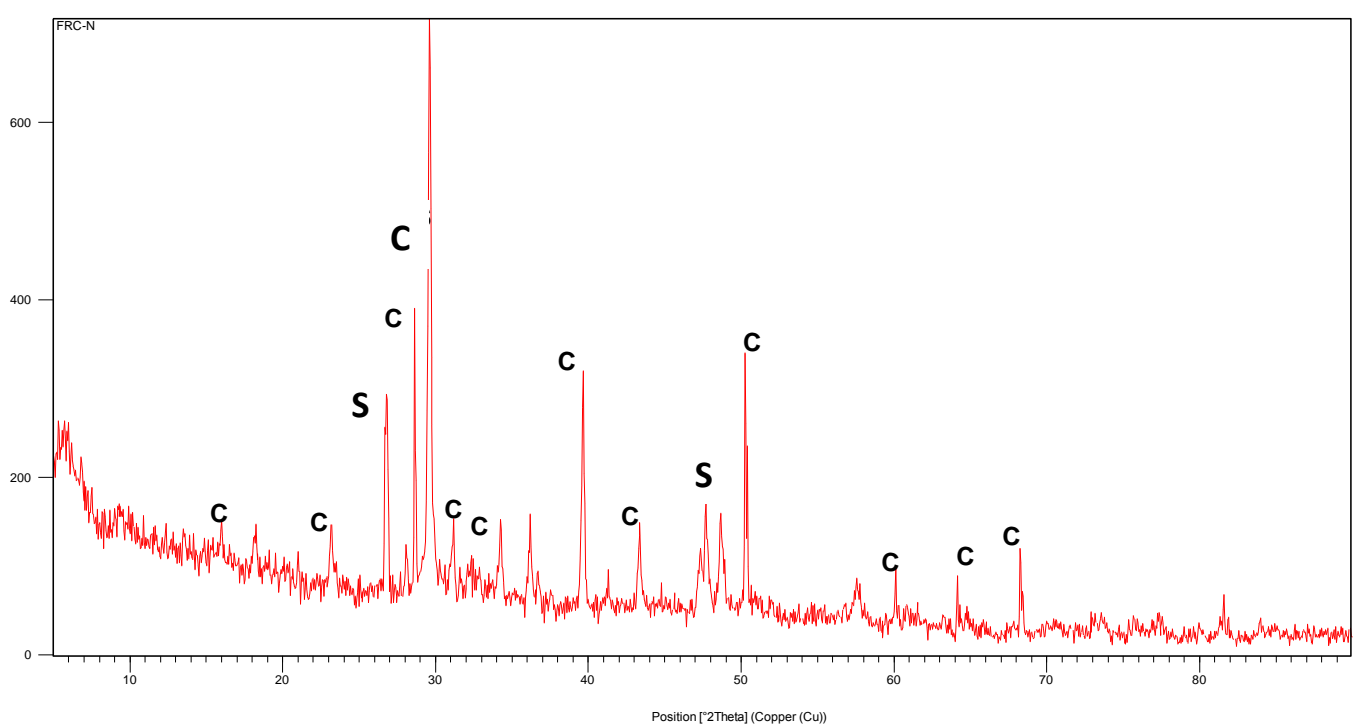

Figure 4.64: XRD analysis of mortar with Sporosarcina pasteurii + zeolite 


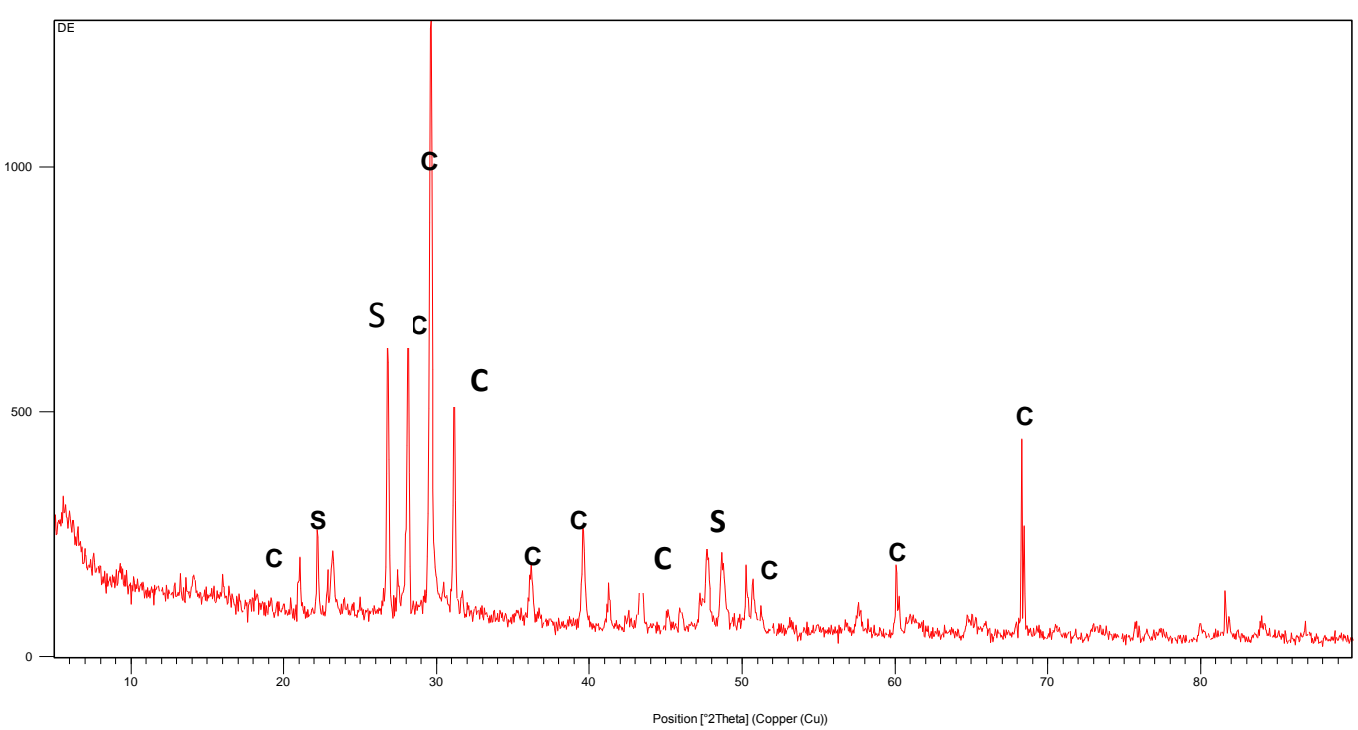

Figure 4.65: XRD analysis of mortar Bacillus subtilis + pumice

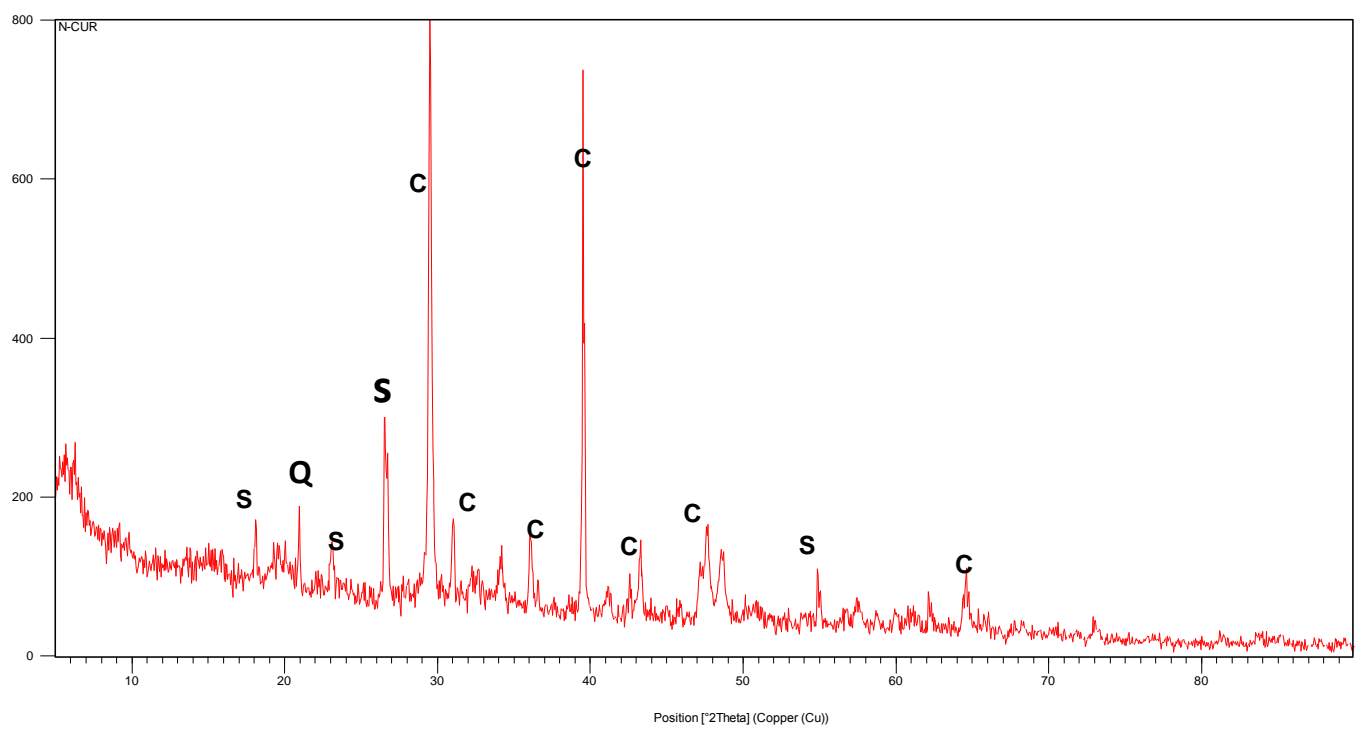

Figure 4.66: XRD analysis of mortar Bacillus subtilis + zeolite 


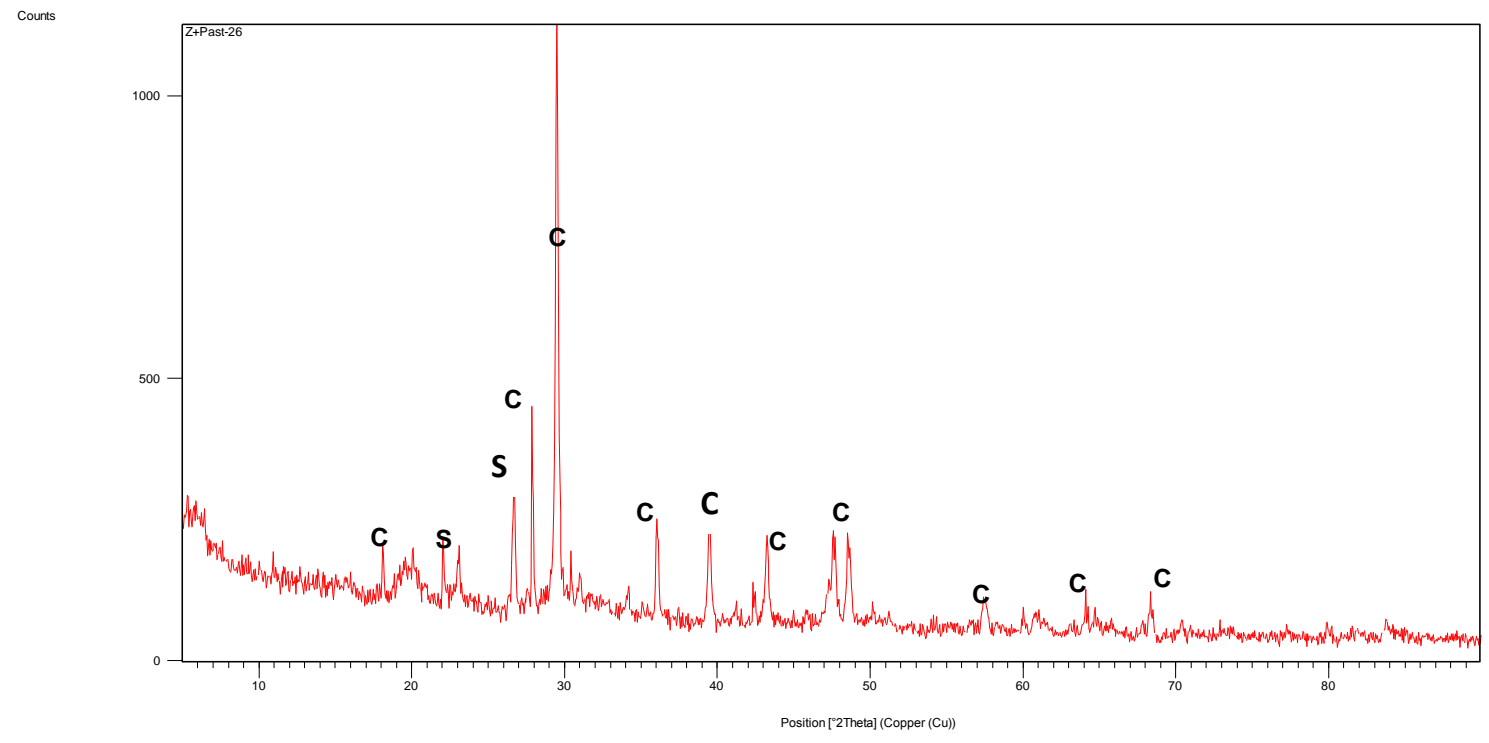

Figure 4.67: XRD analysis of mortar Sporosarcin ureae + pumice

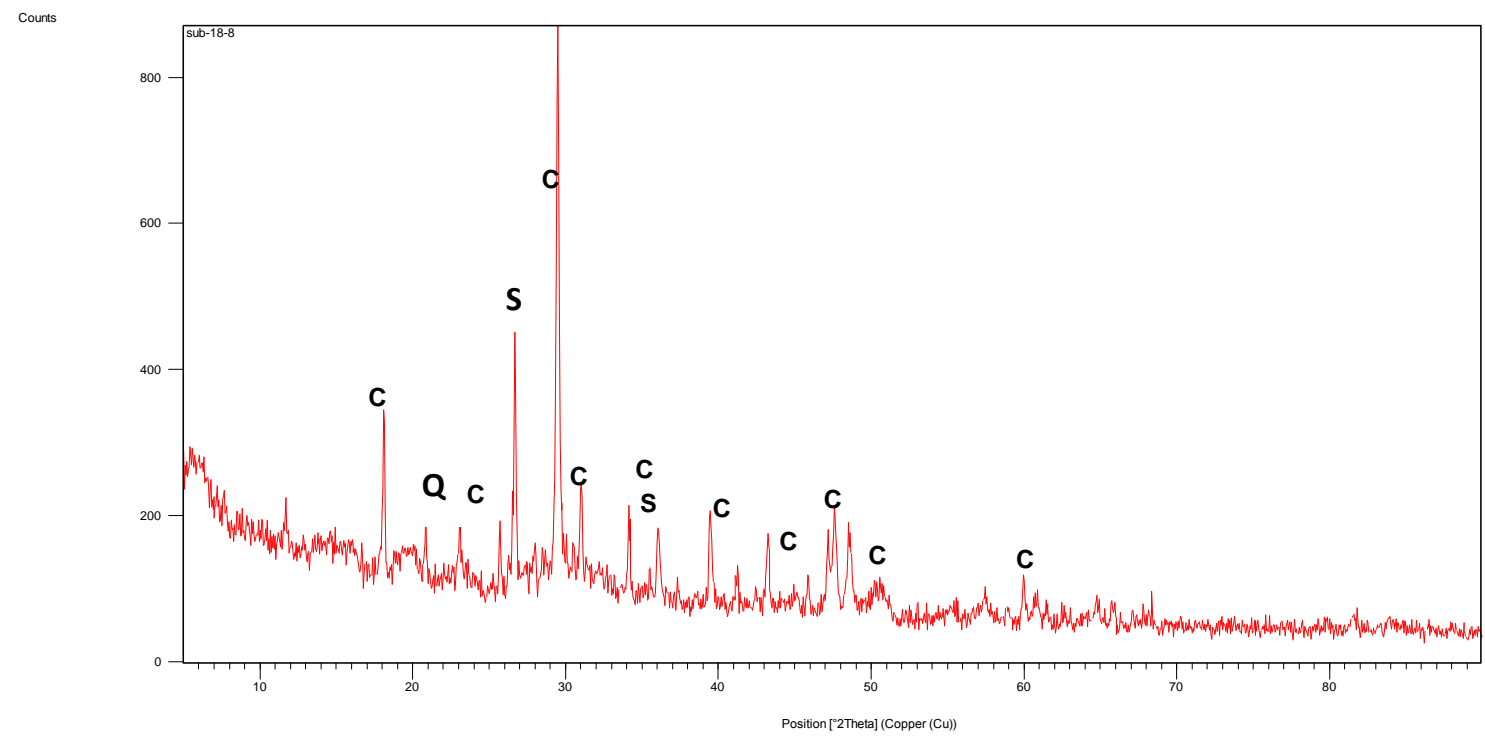

Figure 4.68: XRD analysis of mortar Sporosarcina ureae + zeolite

The most abundant mineral present was calcite crystals and was confirmed by XRD analyses for all the specimens. Also, The SEM pictures disclosed the presence of rhombohedral (calcite) and spherical crystals. Previous studies stated that the morphology of bacterially induced calcium 
carbonate precipitation is very much influenced by the source of calcium compound (De Muynck et al., 2008; Van Tittelboom et al., 2010). The production of aragonite and calcite by B.cohnii were reported in which calcium lactate was used as the calcium source (Wiktor and Jonkers, 2011). These findings led to the conclusion that the precipitation of calcium carbonate in the form of calcite might be due to the effect of calcium lactate which was used as the calcium source for the present study. Furthermore, it can be seen that there was no significant difference between the XRD analysis of the precipitated materials based on the two different carrier materials, indicating that the incorporation of bacteria in pumice or zeolite in the mortar mix does not influence the morphology of the crystals deposited.

\subsection{Research Phase 5: Self-healing efficiency of bacteria based ECC}

Controlling the crack width in FR mortar using four-point bending test turned out to be hugely challenging. This resulted in the formation of wider cracks on the specimen which proved to be hard for the bacteria to be filled completely. In order to quantify self-healing based on bending strength evolution more accurately, four point bending tests were conducted on Engineered Cementitious Composites (ECC) resulting in multiple narrow cracks. For these tests, only two bacterial species which showed promising healing potential from earlier experiments (on FR and normal mortars) and only one carrier material (zeolite) were chosen. Also an unprotected $S$. pasteurii without carrier material was used for the experimentation in order to test the self-healing efficiency of bacteria in the absence of a carrier material. The results of tests such as UPV, SEM, EDS and XRD studies are discussed in the following sections.

\subsubsection{Self-healing in terms of compressive strength recovery of ECC mix cubes}

Figure 4.69 shows the average compressive strength of 7 and 28 days old control and bacteria based ECC cubes. It can be observed that the compressive strength of specimens made of the unprotected bacteria was bit higher (71.6 MPa in 28 days) compared to that made with bacteria immobilised in to zeolite (66 MPa in 28 days). This might be due to the fact the bacterial cells can enter into the pores of the matrix and fill them contributing to the development of the additional strength. Average compressive strength at 7 and 28 days of the mixes with bacteria based agent is higher than those of the control mixes. This may be due to the presence of calcium lactate in the bacteria based agent. Similar results were reported by the other researchers (Jonkers et al., 2010; Sierra-Beltran et al., 2014). 


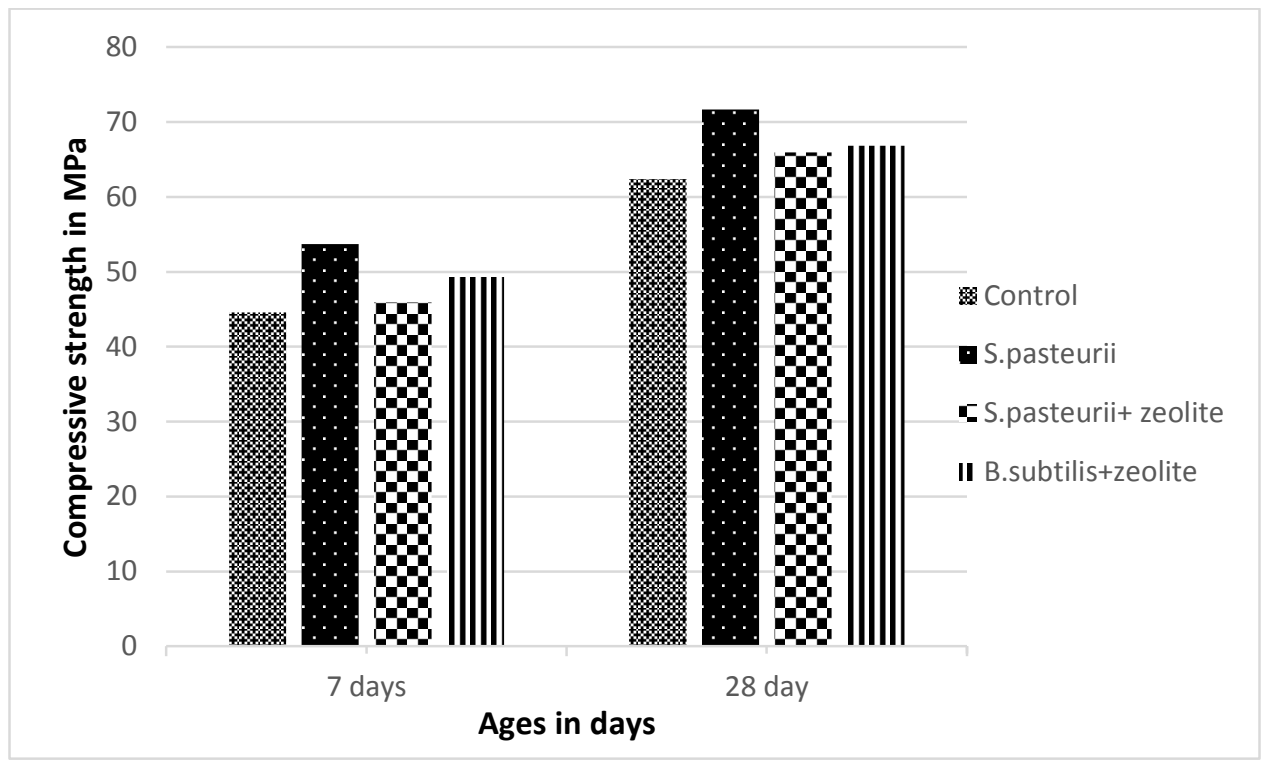

Figure 4.69: Compressive strength of bacteria based ECC mix at 7 and 28 days

\subsubsection{Self-healing in terms of flexural strength recovery of ECC mix}

The flexural behaviour of all the selected ECC mixtures is shown in Figure 4.70 to 4.73 and summarized in Tables 4.14 and 4.15. All mixtures showed good deflection capacity. Since the structural application of ECC requires high deformation and energy dissipation capacity, its deflection capacity is of major concern. The deflection capacity is defined as the deflection that corresponds to the maximal flexural stress.

Table 4.15 describes the flexural strength at preload level and recovery after healing. It was found that the average flexural strength measured at 56 days for the mix with unprotected Sporosarcina pasteurii and zeolite immobilised Bacillus subtilis found to have decreased compared to the control ECC mix (Table 4.15). However, merely a slight increase in the flexural strength was observed for the mix with zeolite immobilised Sporosarcina pasteurii. It was found that both zeolite immobilised bacteria showed an increase in flexural strength (23\% for S. pasteurii and $17 \%$ for $B$. subtilis) compared to the sound specimen after 56 days of healing. At the same time, a slight decrease in flexural strength was found for the control and specimen with unprotected bacteria. Figures 4.70 to 4.73 show the flexural stress - deflection curve of the reloading tests (flexural test conducted after healing of the cracked specimen) along with a preloading test (four point bending test conducted after 28 days to induce cracks) curve and the reference test (flexural test conducted 
on the sound specimen) to failure at 56 days for all mixes. Reloaded specimens for the zeolite immobilised S. pasteurii (Figure 4.72) and B. subtilis (Figure 4.73) mixes appeared to have a higher strength compared to the respective reference specimens. However, the reloaded strength of the mix with unprotected bacteria (Figure 4.71) and control specimens (Figure 4.70) appeared to have a slight decrease in strength than the reference specimen.

On the other hand, the deflection capacity of the ECC mix with bacteria based healing agent appeared to be increased (Table 4.16). The increase in deflection capacity might be due to the presence of particles with bacteria based healing agent. It may be illustrated that the fiber bridging capacity could be improved due to the additional bonding between self-healing products and fiber surface. It can be seen from the Table 4.16 that there is a reduction in the deflection capacity of the reloaded control ECC mixture. Conversely, the deflection capacity is almost fully recovered by the reloaded specimens of bacteria based ECC mixtures and a slight increase in the deflection capacity can be observed for the mix with zeolite immobilised Bacillus subtilis.

Figure 4.74 shows relative flexural stiffness of healed specimens as a percentage of the virgin specimens which were un-cracked and exposed to the same healing conditions. The flexural stiffness was defined as the secant of the initial rising branch of the flexural stress- deflection curve. For the data in this study, the first point is chosen at 1.5 MPa and the second point at $4 \mathrm{MPa}$. It was observed that stiffness decreased during the reloading to about half of the initial stiffness for all mixes. It might be the reason that the larger number of cracks contributed to reduced flexural stiffness. It can be seen from the Figure 4.74 that the flexural stiffness is not much recovered for bacteria based ECC mix compared to the control ECC specimen. However, the specimen treated with zeolite immobilised Sporosarcina pasteurii recovered the highest percentage of stiffness of around $61 \%$. The specimen with unprotected bacteria appeared to have lower stiffness recovery compared to control ECC mix. 


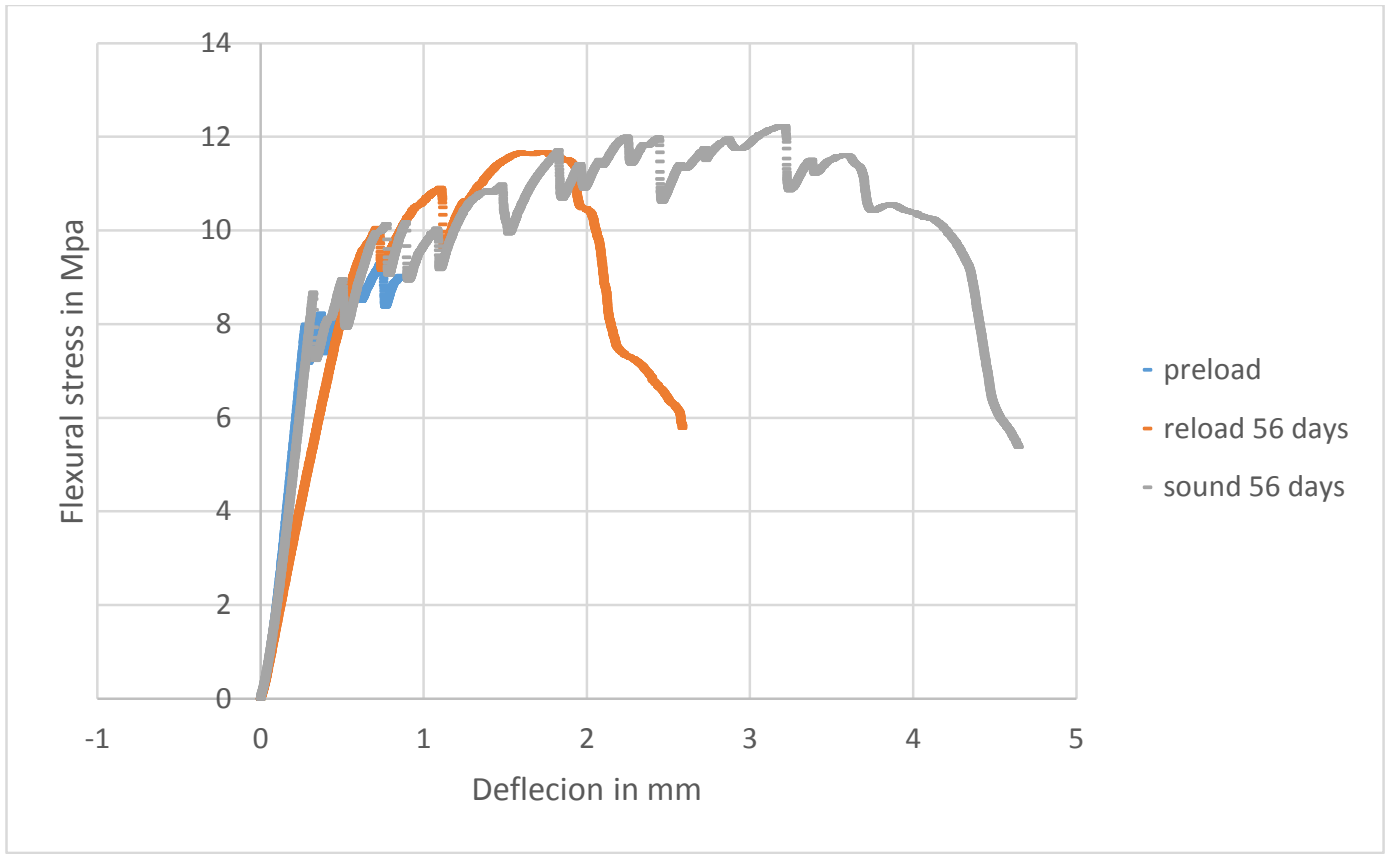

Figure 4.70: Flexural stress - deflection curve for Control ECC

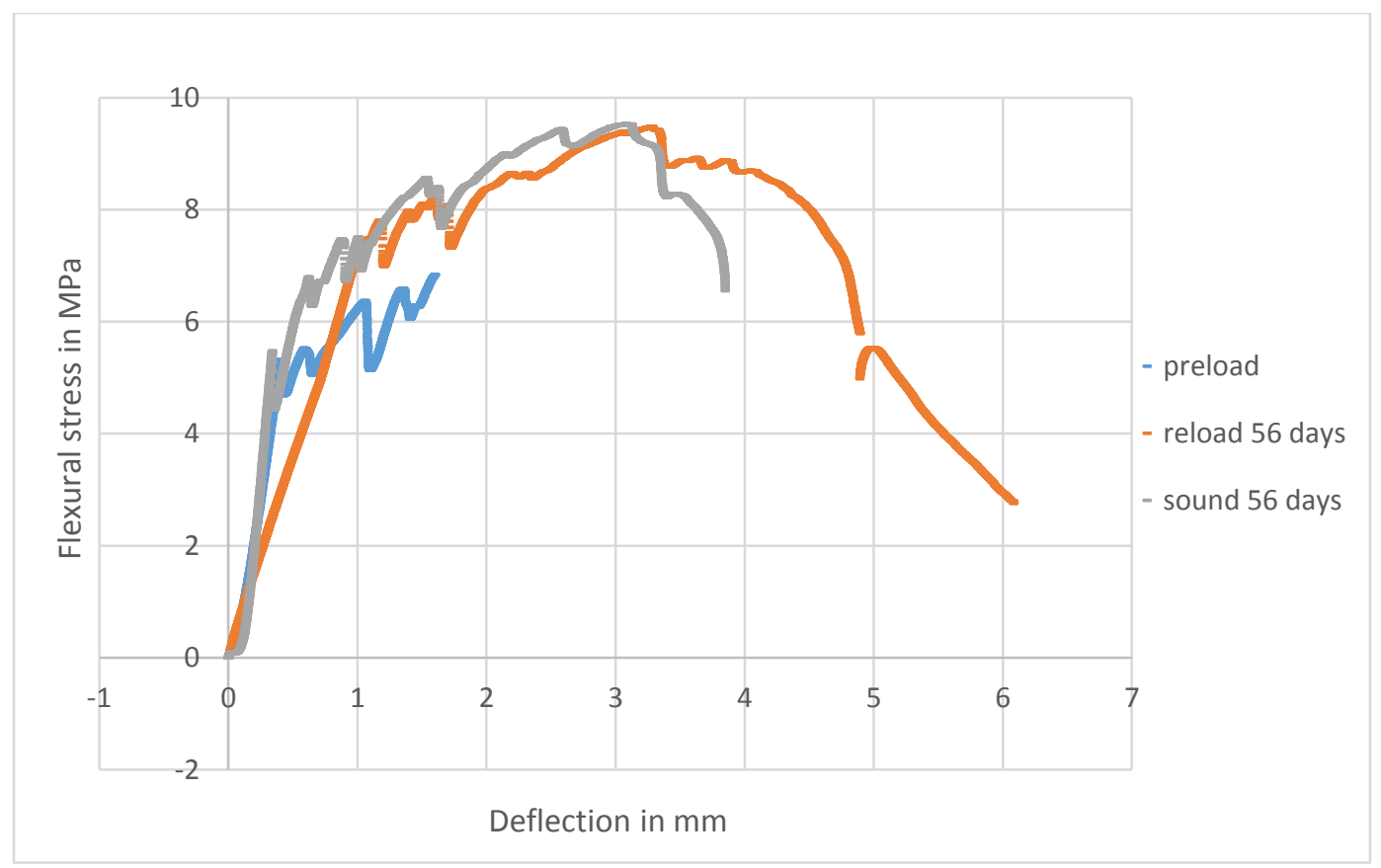

Figure 4.71: Flexural stress - deflection curve for unprotected Sporosarcina pasteurii based ECC 


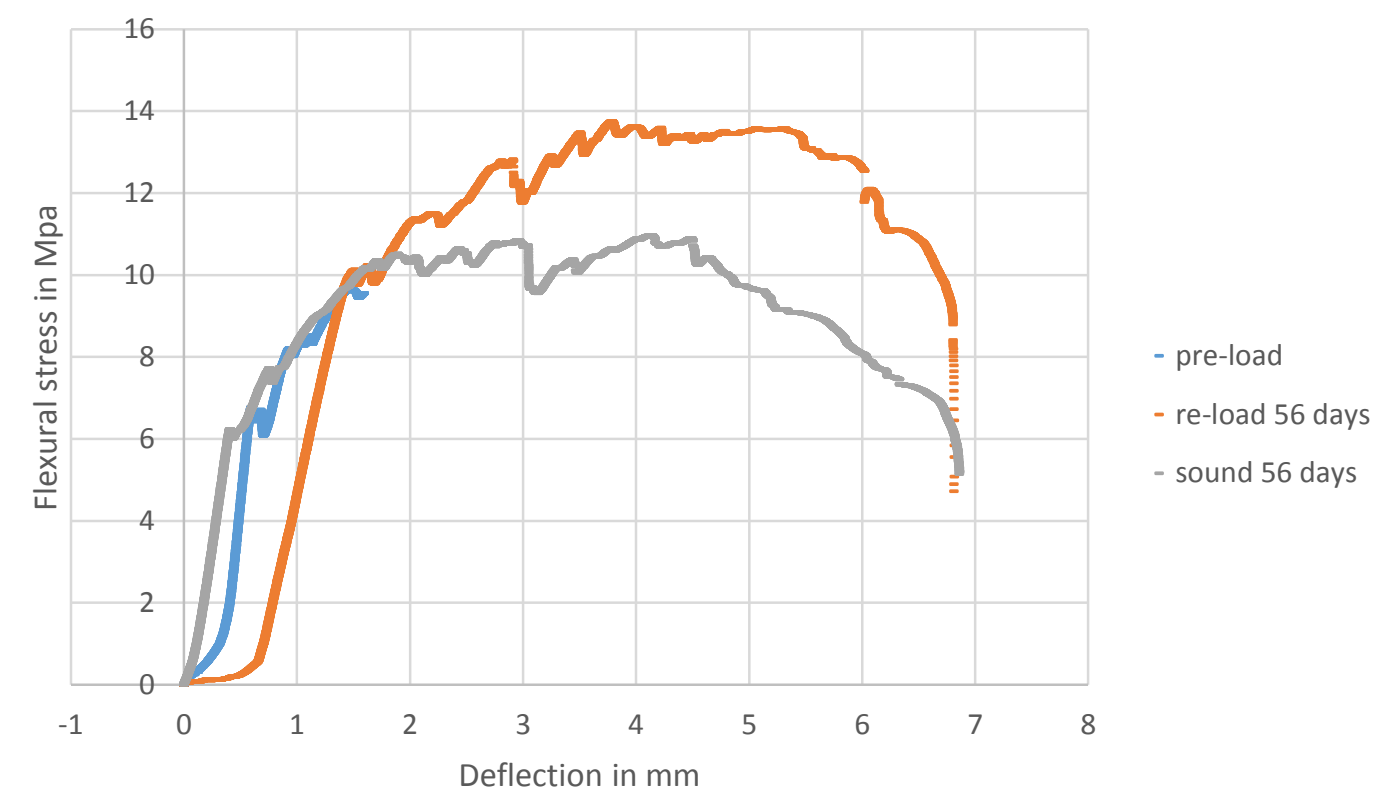

Figure 4.72: Flexural stress - deflection curve for zeolite + Sporosarcina pasteurii based ECC

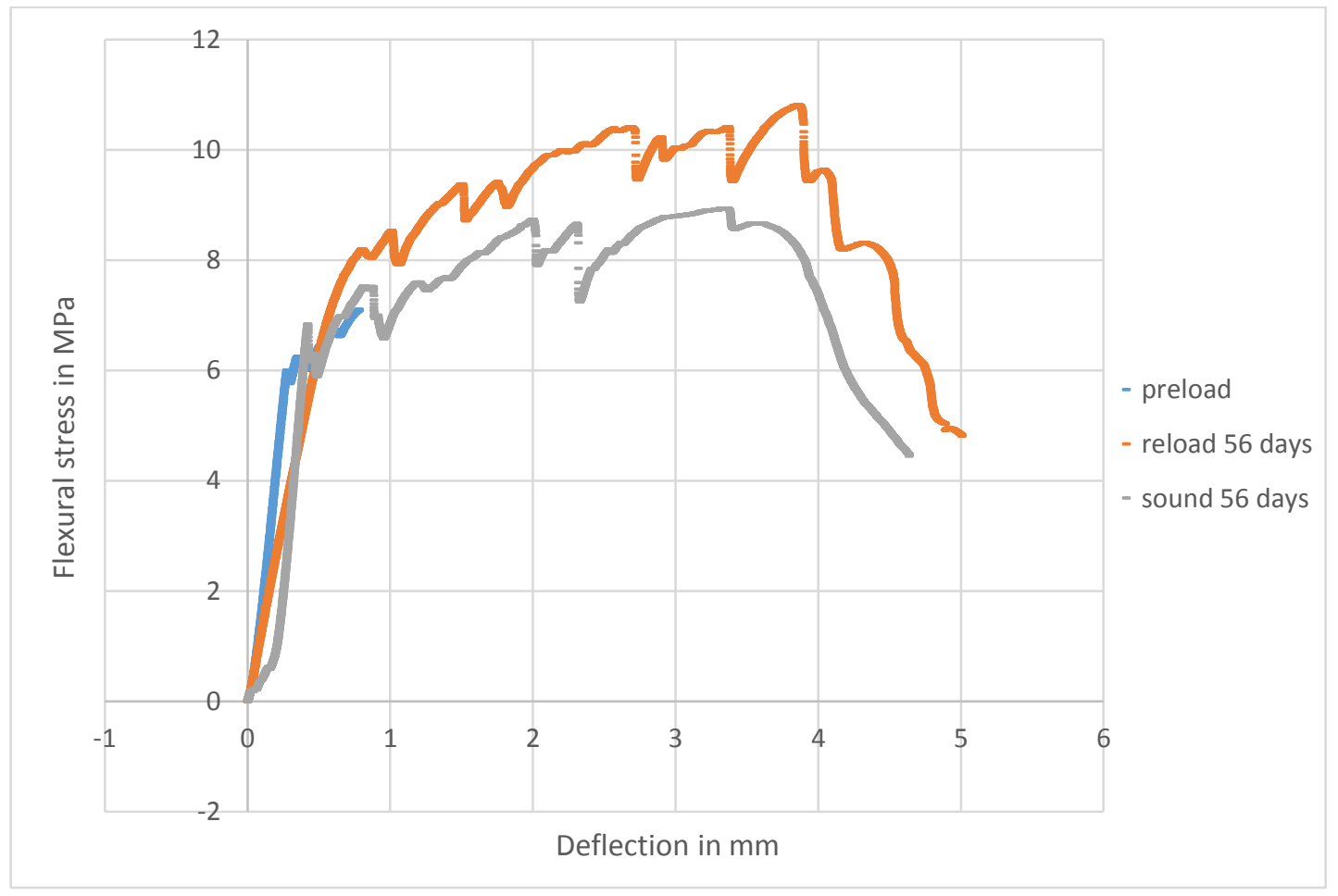

Figure 4.73: Flexural stress - deflection curve for zeolite + Bacillus subtilis based ECC 
Table 4.15: Flexural strength at preload level and recovery after healing

\begin{tabular}{|c|c|c|c|c|}
\hline Mix type & Specimen & Pre-load level (Mpa) & $\begin{array}{c}56 \text { days flexural strength } \\
\text { after } 28 \text { days of healing } \\
\text { (Mpa) }\end{array}$ & Healed/Ref \\
\hline \multirow{2}{*}{ M1 (Control) } & $\begin{array}{l}\text { Pre-loaded+reloaded } \\
\text { (healed) }\end{array}$ & 9.28 & 11.68 & 0.97 \\
\hline & Reference (sound) & - & 12 & - \\
\hline \multirow{2}{*}{$\begin{array}{c}\text { M2 } \\
\text { (unprotected } \\
\text { S.pateurii) }\end{array}$} & $\begin{array}{l}\text { Pre-loaded+reloaded } \\
\text { (sound) }\end{array}$ & 6.9 & 9.44 & 0.98 \\
\hline & Reference (sound) & - & 9.6 & - \\
\hline \multirow{2}{*}{$\begin{array}{l}\text { M3 (zeolite } \\
+S . \\
\text { pasteurii) }\end{array}$} & $\begin{array}{l}\text { Pre-loaded+reloaded } \\
\text { (sound) }\end{array}$ & 9.6 & 13.76 & 1.23 \\
\hline & Reference (sound) & - & 11.2 & - \\
\hline \multirow{2}{*}{$\begin{array}{l}\text { M4 (zeolite } \\
+ \text { B. subtilis) }\end{array}$} & $\begin{array}{l}\text { Pre-loaded+reloaded } \\
\text { (healed) }\end{array}$ & 6.8 & 10.88 & 1.17 \\
\hline & Reference (sound) & - & 9.3 & - \\
\hline
\end{tabular}

Table 4.16: Mid-span deflection at preload level and recovery after healing

\begin{tabular}{|c|c|c|c|c|}
\hline Mix type & Specimen & Pre-load level (mm) & $\begin{array}{c}\text { Mid span deflection in } 56 \\
\text { days after } 28 \text { days of } \\
\text { healing }(\mathrm{mm})\end{array}$ & Healed/Ref \\
\hline \multirow{2}{*}{ M1 (Control) } & $\begin{array}{c}\text { Pre-loaded+reloaded } \\
\text { (healed) }\end{array}$ & 1.42 & 2.59 & 0.8 \\
\hline & Reference (sound) & - & 3.21 & - \\
\hline \multirow{2}{*}{$\begin{array}{c}\text { M2 } \\
\text { (unprotected } \\
\text { S.pateurii) }\end{array}$} & $\begin{array}{l}\text { Pre-loaded+reloaded } \\
\text { (healed) }\end{array}$ & 1.6 & 3.28 & 1.02 \\
\hline & Reference (sound) & - & 3.2 & - \\
\hline \multirow{2}{*}{$\begin{array}{l}\text { M3 (zeolite } \\
\quad+S . \\
\text { pasteurii) }\end{array}$} & $\begin{array}{l}\text { Pre-loaded+reloaded } \\
\text { (healed) }\end{array}$ & 1.59 & 3.74 & 0.97 \\
\hline & Reference (sound) & - & 3.85 & - \\
\hline \multirow{2}{*}{$\begin{array}{l}\text { M4 (zeolite } \\
+ \text { B. subtilis) }\end{array}$} & $\begin{array}{l}\text { Pre-loaded+reloaded } \\
\text { (healed) }\end{array}$ & 1.44 & 3.85 & 1.14 \\
\hline & Reference (sound) & - & 3.36 & - \\
\hline
\end{tabular}




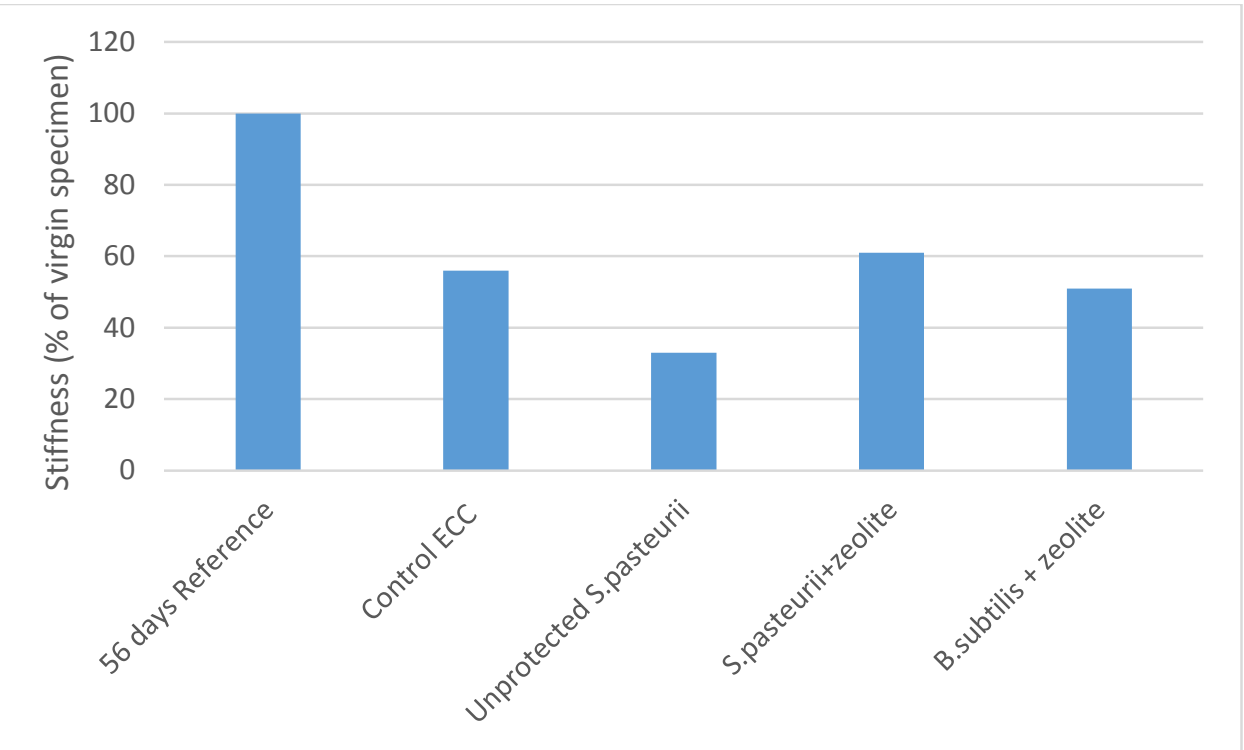

Figure 4.74: Relative flexural stiffness of healed specimens as a percentage of the reference sound specimens

By applying four point bending test, multiple cracks with different widths were generated in the beam/prism specimens. Figure 4.75 shows the typical crack pattern in the bacteria based ECC specimen subjected to around $1.5 \mathrm{~mm}$ deflection before healing and reloading to failure after 28 days of healing. Some white precipitates can be observed in the cracks and this crack closing might be mostly due to the calcium carbonate precipitation on the surface of the specimens. It was observed that in the zeolite immobilised Sporosarcina pasteurii and Bacillus subtilis specimens, some of the cracks deviated from the pre-existing healed cracks and generated new cracks while reloading after healing (Figure 4.75). However, in control specimens most of the cracks under reloading passed through the pre-existing cracks. This indicates the efficiency/capability of bacteria based ECC materials to self-heal and enhance/recover the mechanical properties. Similar results were reported by Sierra-Beltran et al, 2014. 

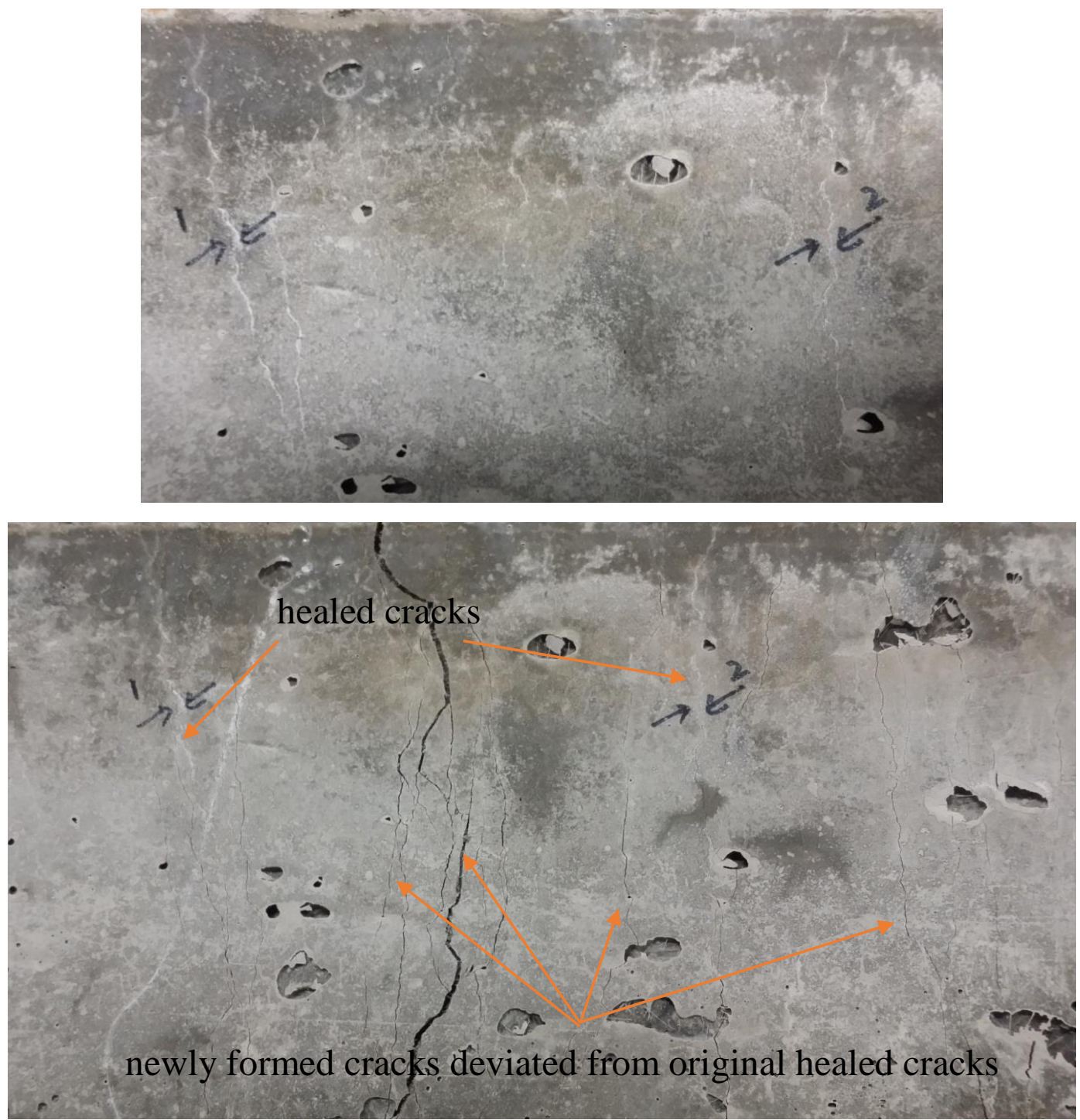

Figure 4.75: Healed specimen of zeolite immobilised Bacillus subtilis before (top) and after reloading (bottom) showing the deviation of crack formation

This study investigated self-healing capability of bacteria induced ECC to improve mechanical and durability properties. From the obtained results, it can be seen that the selected ECC mixes with bacteria based healing agent can attain robust self-healing capabilities with appropriate time period because of its intrinsic tight crack width of less than $50 \mu \mathrm{m}$. In this research, bacterial based ECC mixtures displayed considerably better recovery of both flexural strength and deflection capacity compared to control mixture (ECC mix without bacteria and nutrients) after cracking and healing. 
In the context of self-healing, ECC exhibited better overall healing efficiency compared to fibre reinforced mortar in terms of flexural strength recovery, deflection capacity recovery and crack healing efficiency. In the case of fibre reinforced mortar, crack width was difficult to control during crack formation and this resulted in much wider cracks $(0.3$ to $0.7 \mathrm{~mm})$. So, the cracks never filled completely after healing. Due to this, reloading resulted in the expansion of the pre-existing cracks. At the same time, crack control was easier in ECC and reloading resulted in formation of newer cracks and the healed cracks remained intact.

\subsubsection{Self-healing measurements of ECC using Ultrasonic Pulse Velocity (UPV)}

Results of UPV performed on beam specimens before loading, after loading to pre-defined cracking, and after healing are shown in Table 4.17. Higher the UPV values, higher is the healing efficiency. It can be seen that increase in UPV values for the bacteria based ECC specimens are higher compared to those of control. This emphasises the healing efficiency of bacteria based ECC specimen. Furthermore, the specimen with immobilised bacteria showed higher increment in UPV values compared to the one with unprotected bacteria. The increments in UPV were found to be $100 \mathrm{~m} / \mathrm{s}$ for the control, $137 \mathrm{~m} / \mathrm{s}$ for unprotected bacteria, $218 \mathrm{~m} / \mathrm{s}$ for $S$. pasteurii + zeolite and $194 \mathrm{~m} / \mathrm{s}$ for B. subtilis + zeolite after 1 month of healing.

Table 4.17: UPV performed before loading, after loading and after healing

\begin{tabular}{|c|c|c|c|c|c|c|}
\hline \multirow{2}{*}{ Specimen } & \multicolumn{2}{|c|}{ UPV performed } & \multicolumn{2}{|c|}{$\begin{array}{l}\text { UPV performed } \\
\text { after healing in }\end{array}$} & \multicolumn{2}{|c|}{$\begin{array}{c}\text { Increase in UPV } \\
\text { values due to } \\
\text { healing in }\end{array}$} \\
\hline & $\begin{array}{c}\text { before } \\
\text { cracking } \\
(\mathbf{m} / \mathbf{s})\end{array}$ & $\begin{array}{c}\text { after } \\
\text { cracking } \\
(\mathrm{m} / \mathrm{s})\end{array}$ & $\begin{array}{c}2 \\
\text { weeks } \\
(\mathrm{m} / \mathrm{s})\end{array}$ & $\begin{array}{c}4 \\
\text { weeks } \\
(\mathrm{m} / \mathrm{s})\end{array}$ & $\begin{array}{c}2 \\
\text { weeks } \\
(\mathrm{m} / \mathrm{s})\end{array}$ & $\begin{array}{c}4 \\
\text { weeks } \\
(\mathrm{m} / \mathrm{s})\end{array}$ \\
\hline Control ECC & 4200 & 4095 & 4185 & 4195 & 90 & 10 \\
\hline Unprotected $S$. pasteurii & 4230 & 4123 & 4240 & 4260 & 117 & 20 \\
\hline S. pasteurii + zeolite & 4133 & 4017 & 4186 & 4235 & 169 & 49 \\
\hline B. subtilis + zeolite & 4128 & 4031 & 4181 & 4225 & 150 & 44 \\
\hline
\end{tabular}




\subsubsection{Visualisation of crack healing of ECC materials}

Figures 4.76 to 4.79 show the photographic images of 28 days healed control and bacteria based ECC specimen. For the control ECC specimen, no visible crack healing was observed as shown in Figure 4.76. On the other hand, in the ECC specimen with unprotected Sporosarcina pasteurii, some white precipitation was found around the crack wall. However, the cracks were observed to be open as shown in Figure 4.77. At the same time, white precipitation was observed to be in the crack for the ECC specimens with Bacillus subtilis (Figure 4.79) immobilised into zeolite and Sporosarcina pasteurii (Figures 4.78).

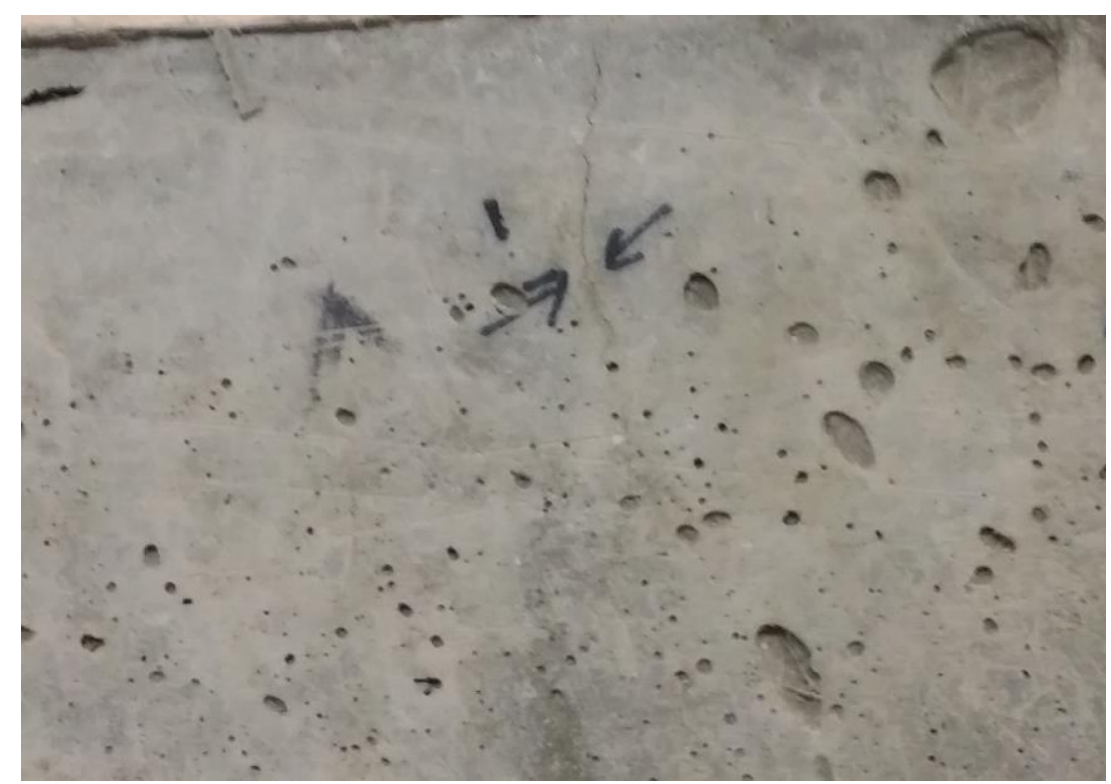

Figure 4.76: Control specimen with no visible crack healing 


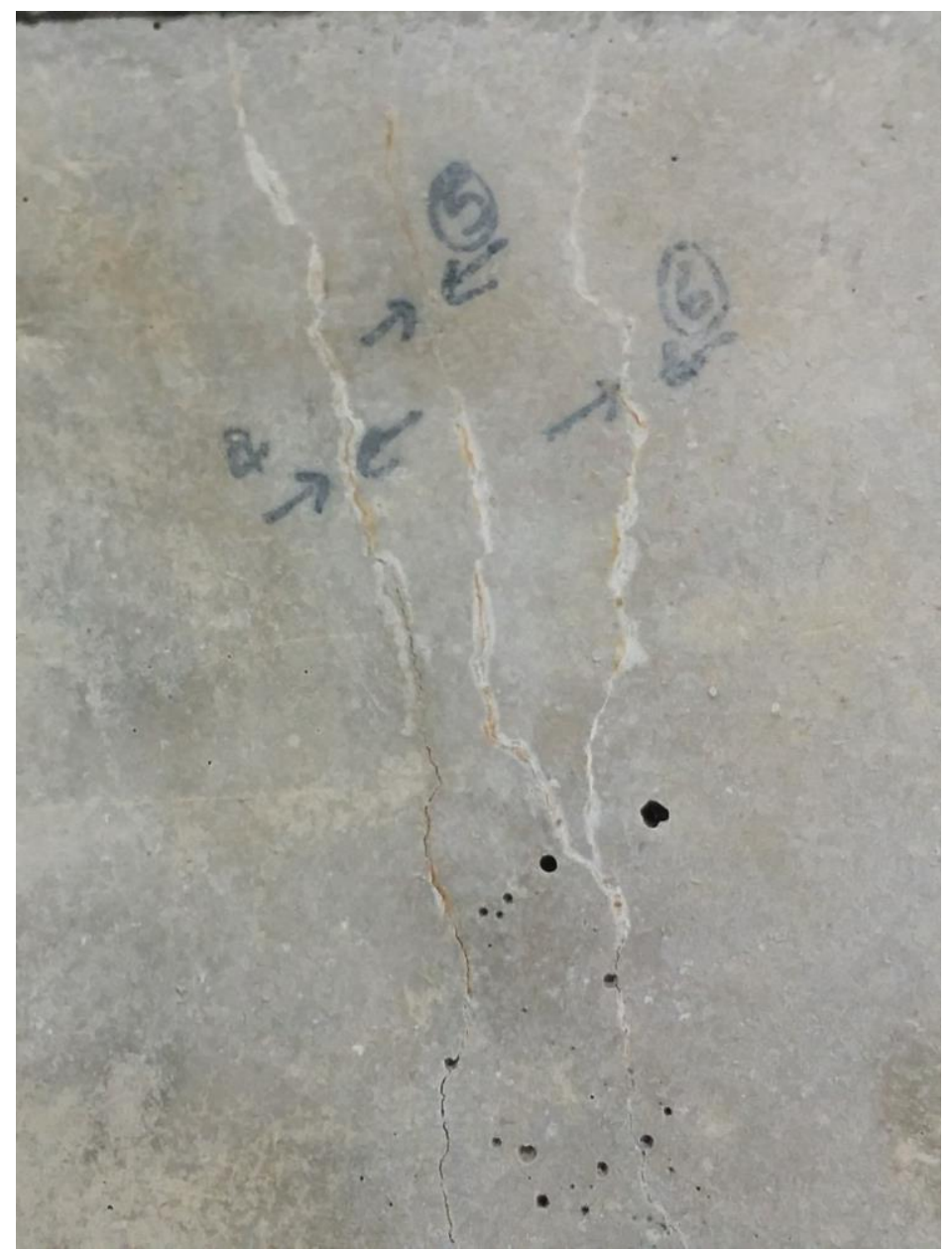

Figure 4.77: ECC specimen with unprotected Sporosarcina pasteurii 

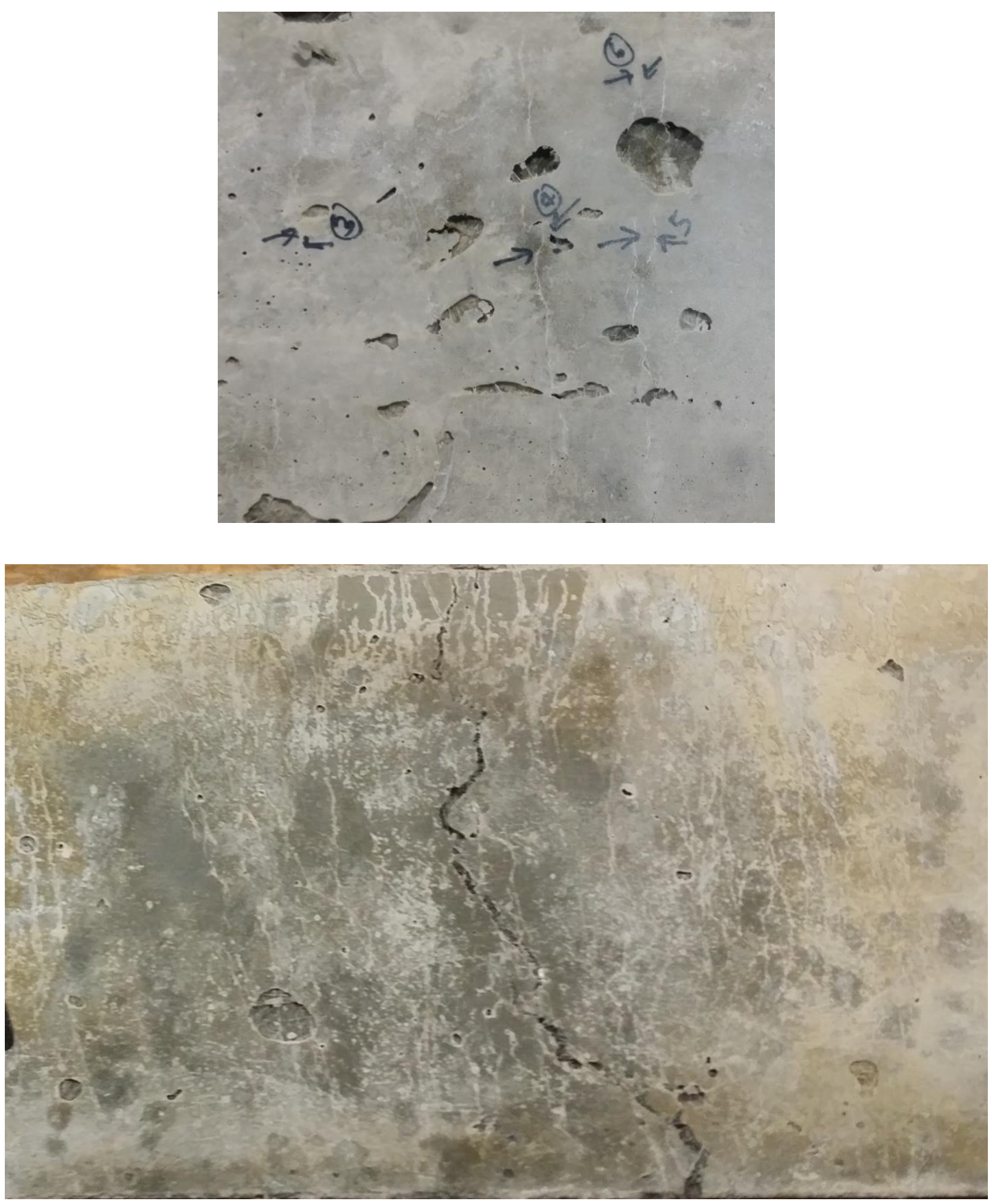

Figure 4.78: ECC specimens with Sporosarcina pasteurii immobilised into zeolite healed cracks (top) and all micro cracks appeared to be filled with white precipitates (bottom) 


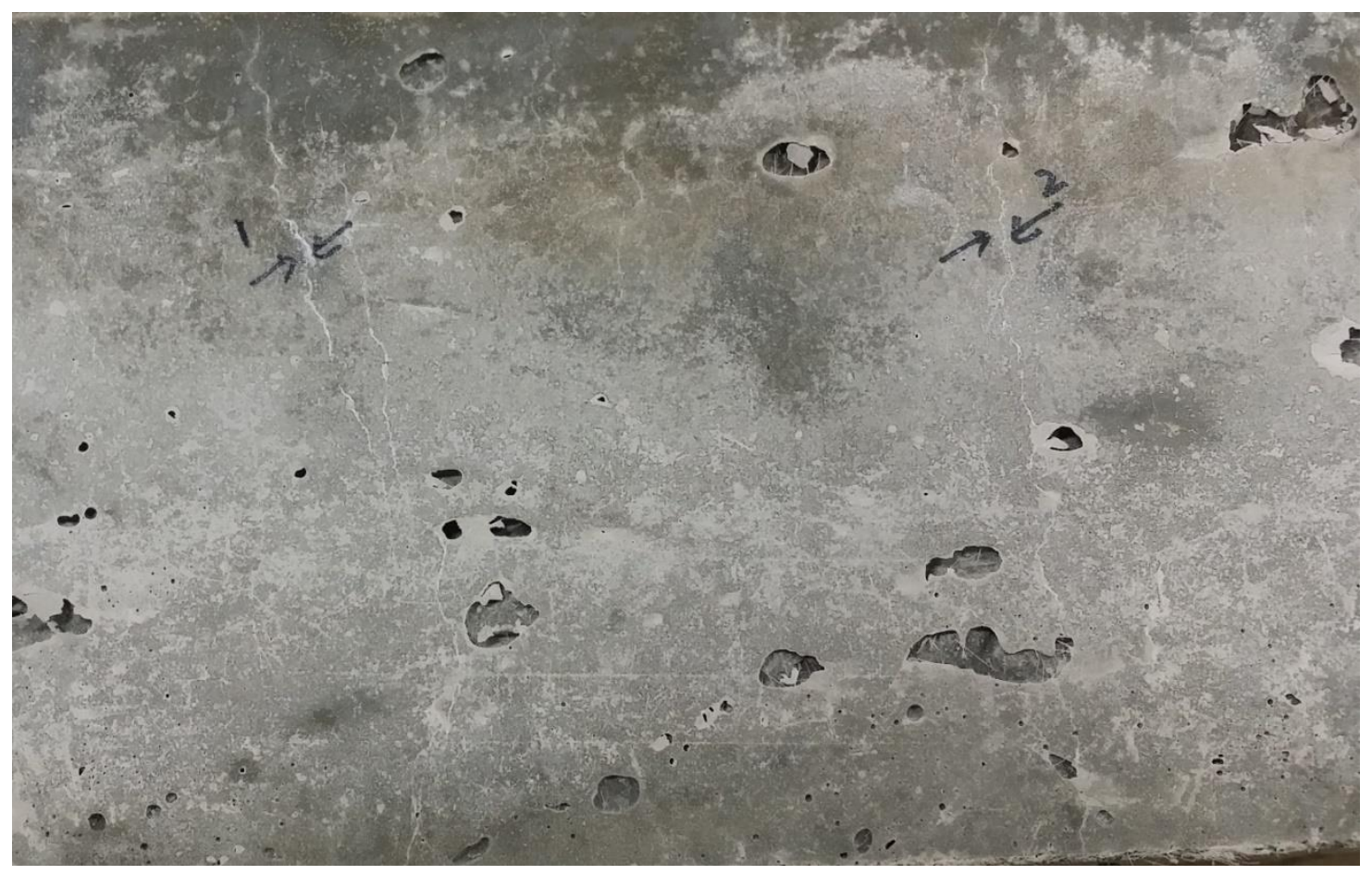

Figure 4.79: Healed specimens with Bacillus subtilis immobilised into zeolite

\subsubsection{SEM and EDS studies}

Four different specimens of ECC mix with and without bacteria were observed under SEM after 1 months of healing. Figures 4.80, 4.82, 4.84 and 4.86 present the SEM observations of precipitation of self-healing products filled at the crack area. In order to examine the chemical nature of the selfhealing products, all four specimens were analysed using EDS and the results are illustrated in Figures 4.81, 4.83, 4.85 and 4.87.

Figure 4.80 shows the SEM observation of cracked ECC specimen with S. pasteurii + zeolite. Excessive amount of crystals can be observed at the crack area, however, the crack was not completely filled. It may take more time for the crack to fill completely as it was only 1 month healed specimen. It was observed that at some locations, the crack appeared to be completely closed. Closer examination of crack area shows the presence of distinct rhombohedral shaped crystals. As mentioned previously (for the fibre reinforced mortar), a layer of white precipitates can be found all over the surface of the specimen. SEM observation of cracked ECC specimen with $B$. subtilis + zeolite is shown in Figure 4.82. It can be seen that large amount of crystals were developed from both sides of the crack walls and extending towards the centre of the crack to fill it completely. Closer observation revealed the presence of well-developed rhombohedral shaped 
crystals. $75 \%$ of the measured crack width of around $80 \mu \mathrm{m}$ was appeared to be filled with the crystals. Figure 4.84 shows the SEM observation of control ECC specimen. Compared to the bacteria treated specimen mentioned previously, visible crack healing was absent in this specimen. However, crack bridging due to fibres and small amount of crystal formation can be observed from a closer examination. Some precipitation can be observed on the crack wall in the case of ECC specimen with unprotected S. pasteurii (Figure 4.86). Picture of higher magnification (1000x) exhibited the filling of cracks with crystals in some locations while some other locations remained open. It can be observed that a crack width of $10 \mu \mathrm{m}$ was completely filled with this specimen treated with unprotected S. pasteurii. It was observed from these studies that the Bacteria immobilised in carrier material such as zeolite showed superior crack healing compared to that of specimen treated with unprotected $S$. pasteurii. These results emphasize the significance of the carrier material in bacteria based self-healing in cementitious composites.

Figures 4.81, 4.83, 4.85 and 4.87 show the EDS analysis of all the specimens. From Figures 4.81 and 4.83 it can be seen that the precipitates comprised of three main elements: $\mathrm{C}, \mathrm{O}$ and $\mathrm{Ca}$. Therefore, it can be confirmed that $\mathrm{CaCO}_{3}$ based mineral precipitates were formed at the crack area. Figure 4.84 presents the results of EDS study conducted on control ECC specimen. It can be seen that some of the crystals observed at the crack wall is silica (spectrum 41) even though they appeared to be similar to calcite crystals. However, a few $\mathrm{CaCO}_{3}$ based mineral precipitates can be observed at the crack face (spectrum 44 of Figure 4.84). Figure 4.86 presents the EDS analysis of cracked ECC specimen with unprotected S. pasteurii. Since it was observed that the precipitates at the crack filled area comprised of three main elements: $\mathrm{C}, \mathrm{O}$ and $\mathrm{Ca}$, it can be concluded that the crystals formed were $\mathrm{caCO}_{3}$ based. Therefore, it can be inferred that the unprotected bacteria has the ability to precipitate crystals while their activity was appeared to be reduced compared to that of the protected bacteria. However, the long term viability of these unprotected bacteria needs to be tested. 


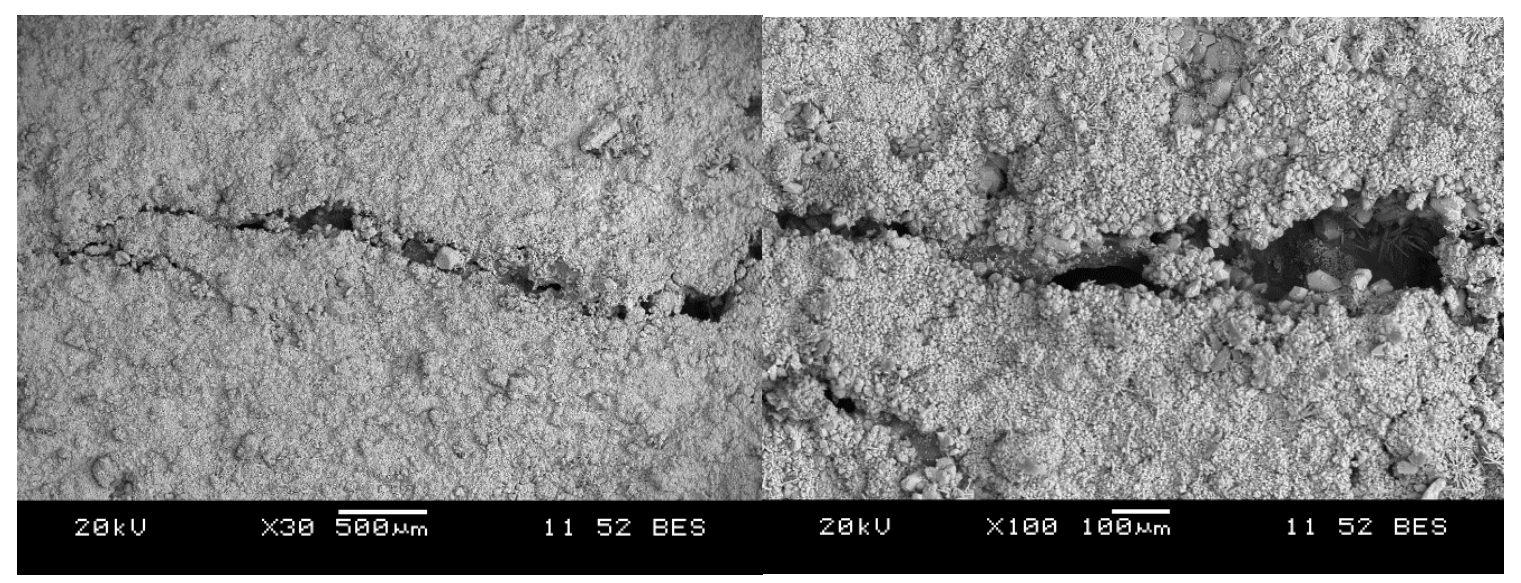

Figure 4.80: SEM observation of cracked ECC specimen with $S$. pasteurii + zeolite after 1 month of healing
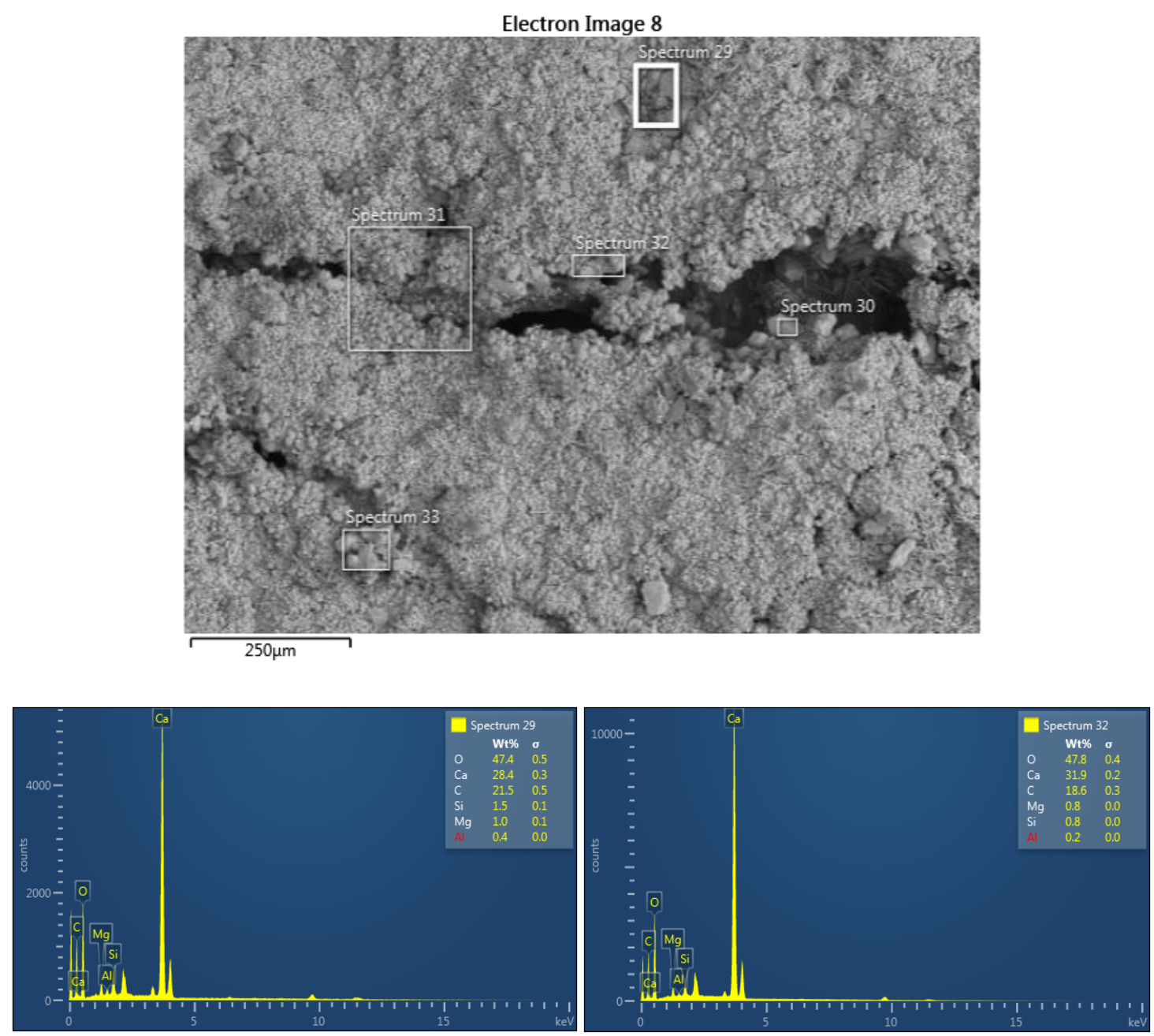

Figure 4.81: EDS analysis of cracked ECC specimen with $S$. pasteurii+zeolite after 1 month of healing 


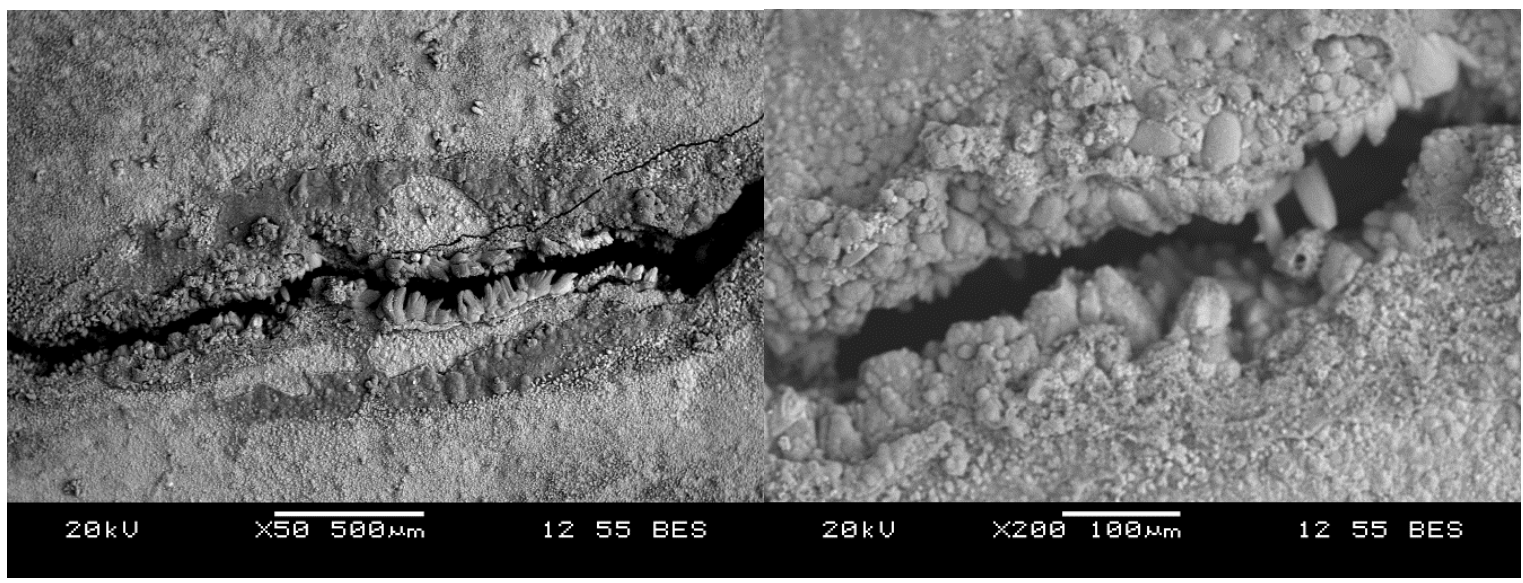

Figure 4.82: SEM observation of cracked ECC specimen with B. subtilis + zeolite after 1 month of healing
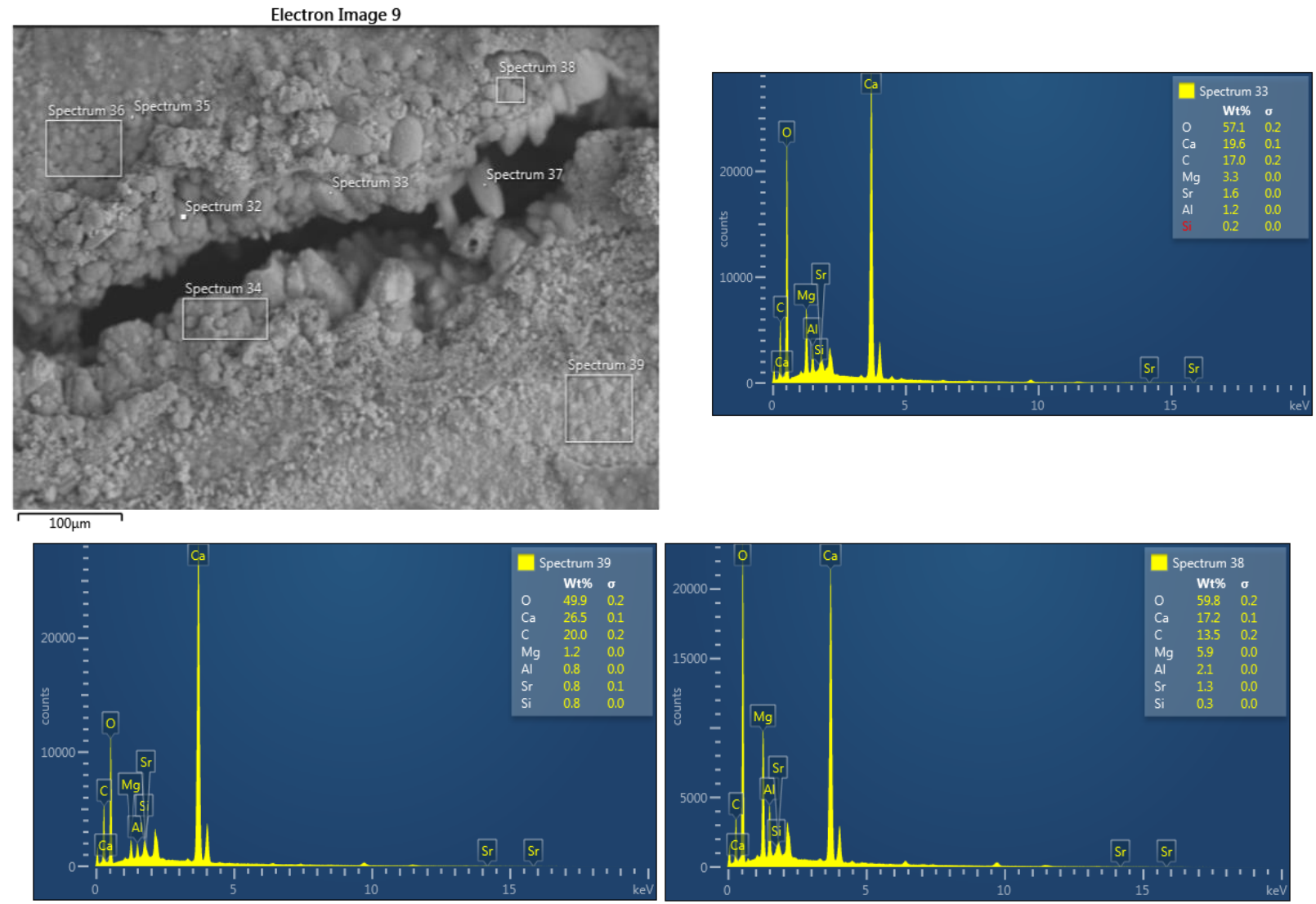

Figure 4.83: EDS analysis of cracked ECC specimen with $B$. subtilis+zeolite after 1 month of healing 


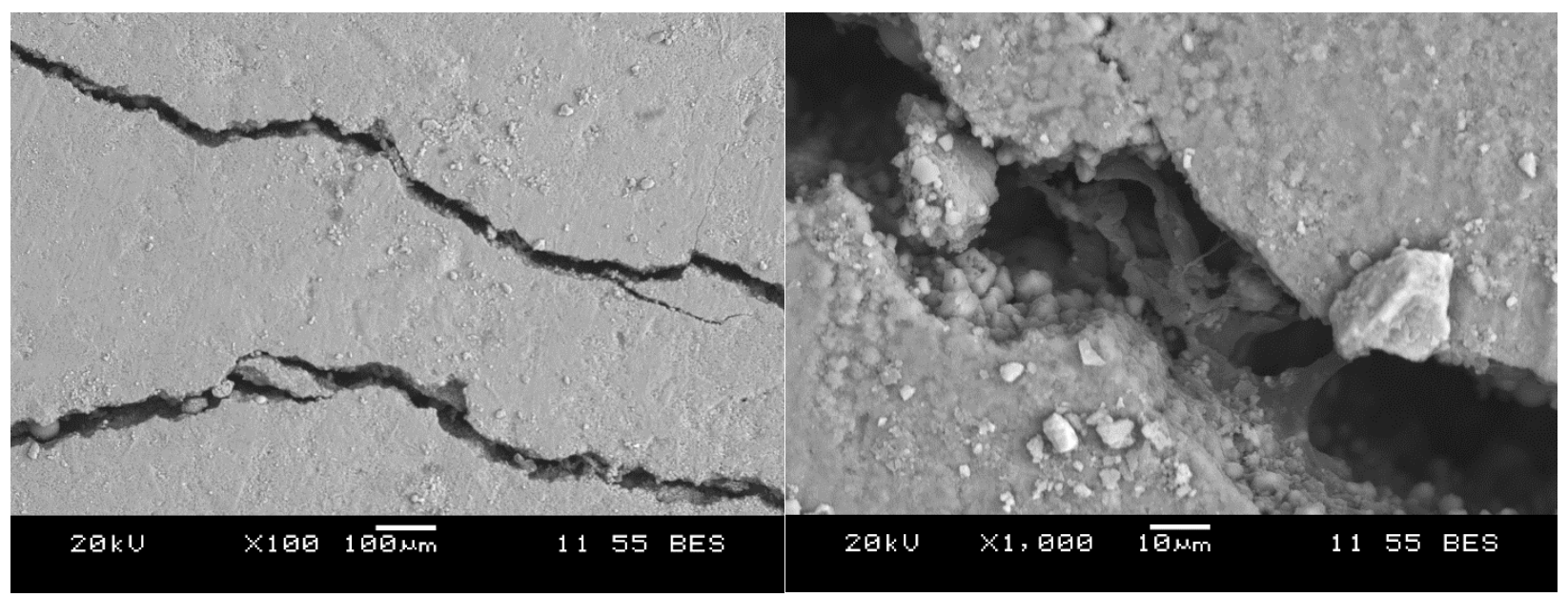

Figure 4.84: SEM observation of cracked ECC specimen (control) after 1 month of healing
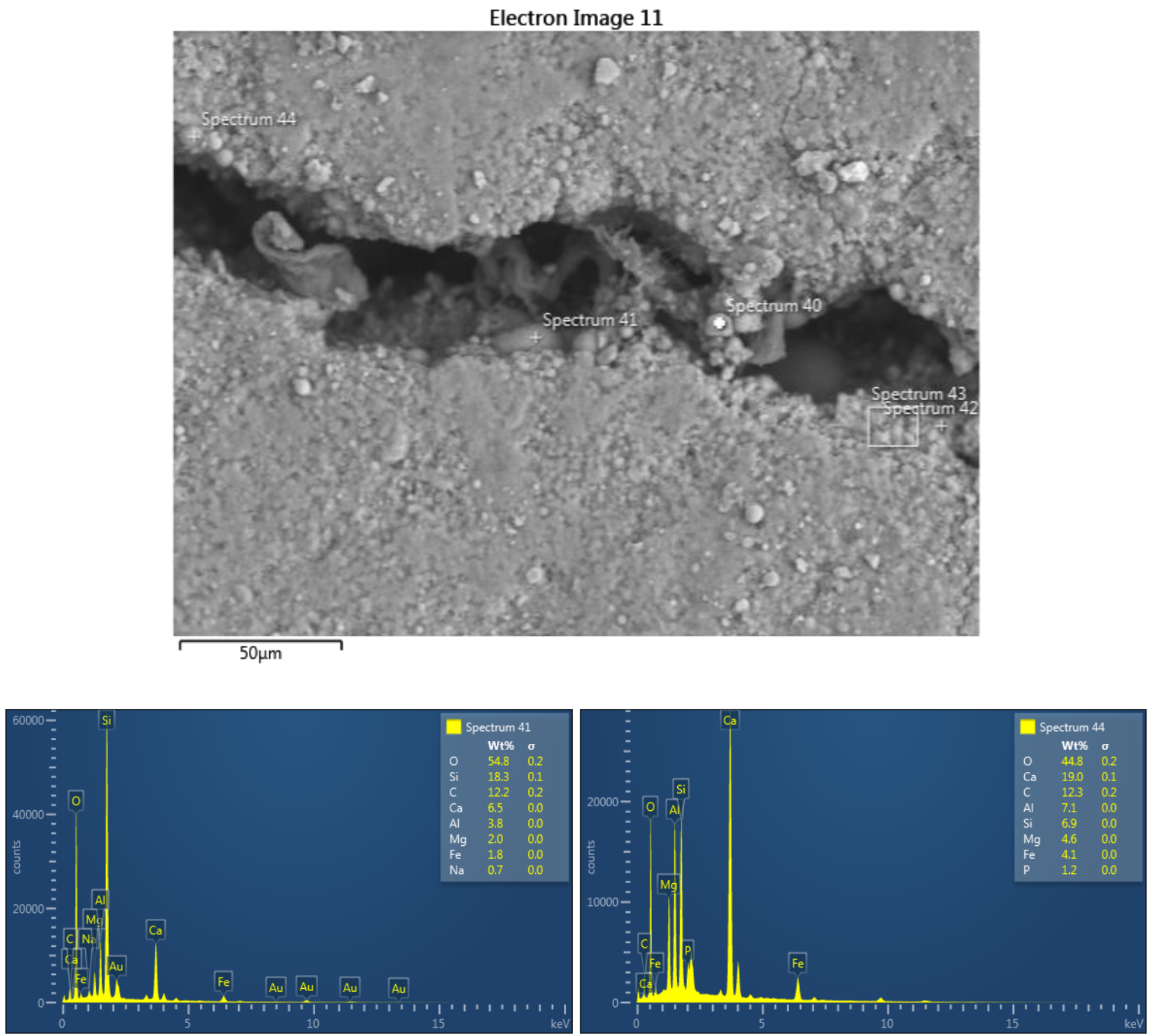

Figure 4.85: EDS analysis of cracked ECC specimen (control) after I month of healing 


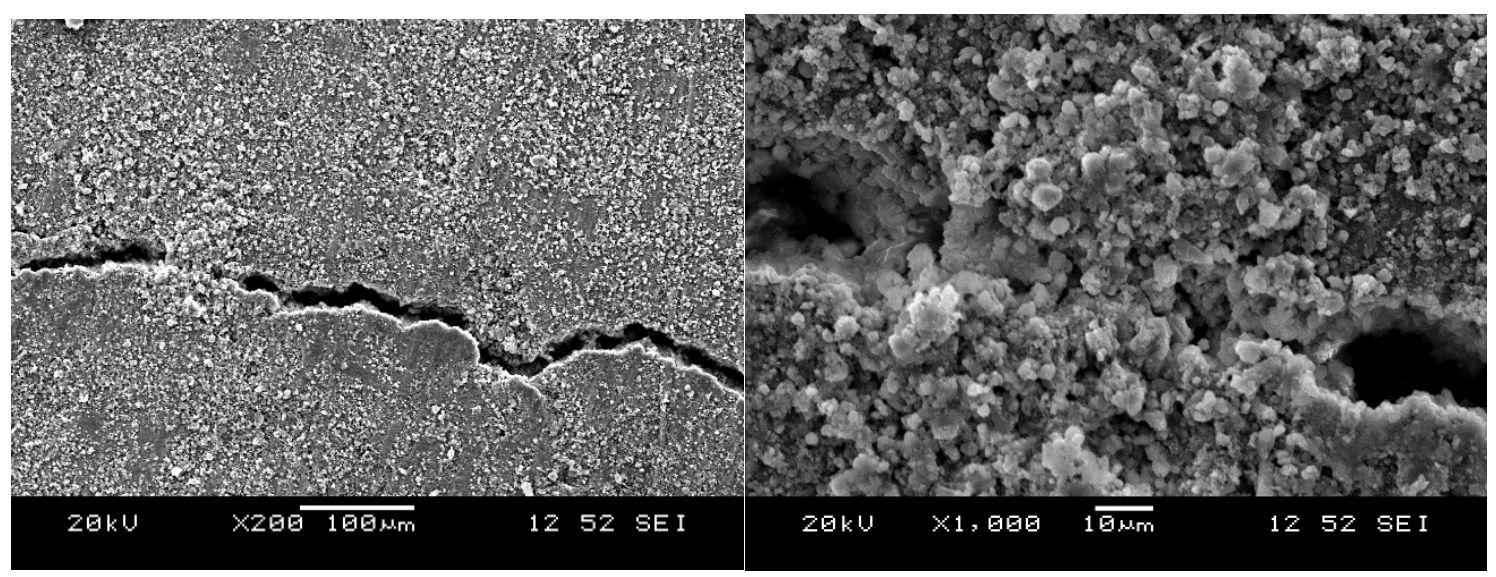

Figure 4.86: SEM observation of cracked ECC specimen with unprotected S. pasteurii after 1 month of healing
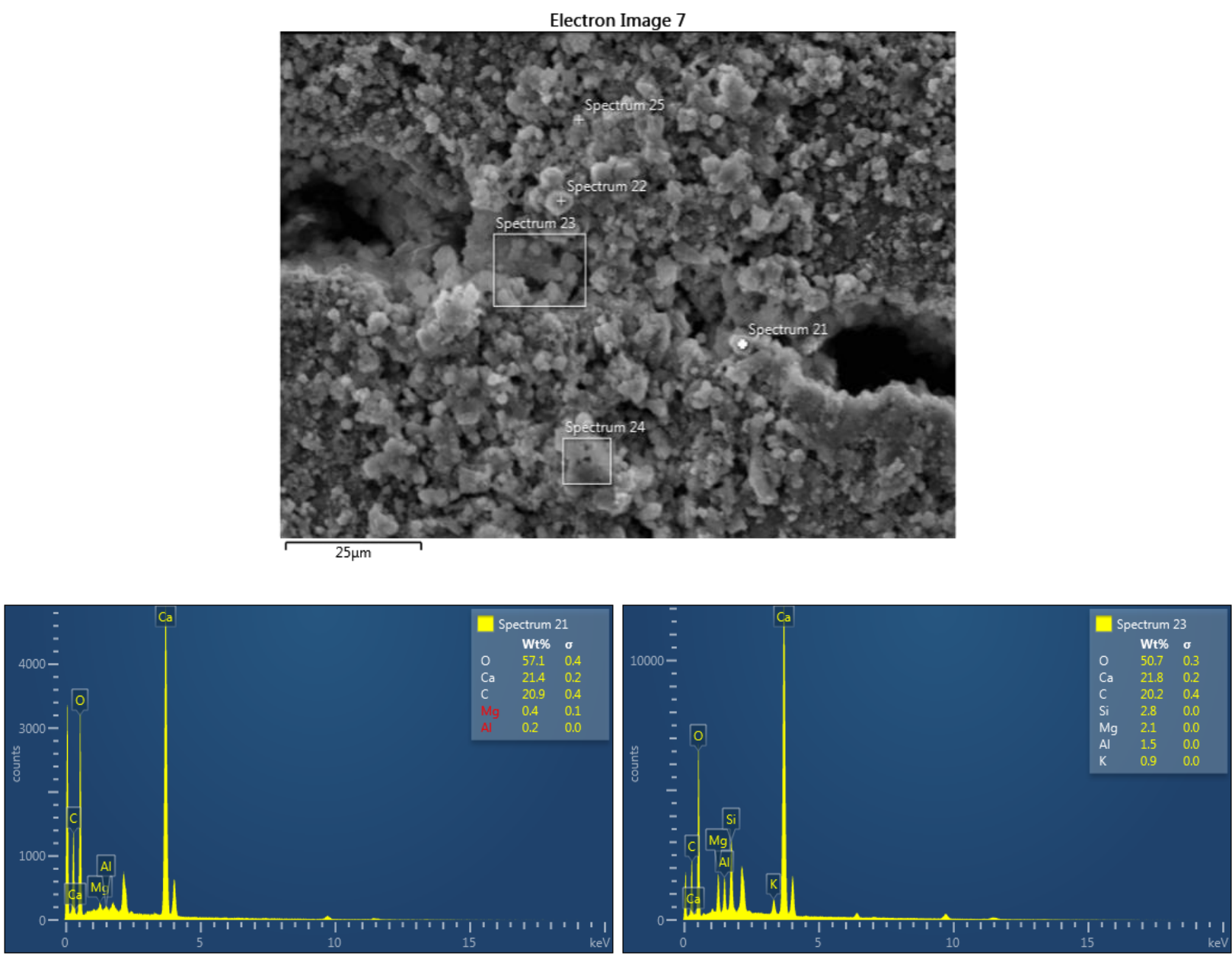

Figure 4.87: EDS analysis of cracked ECC specimen with unprotected $S$. pasteurii after 1 month of healing 


\subsubsection{XRD analysis}

XRD analyses were carried out for specimens made of four different ECC (Control ECC, ECC mix with unprotected S. pasteurii, S. pasteurii with zeolite and B. subtilis with zeolite) in order to detect the type of crystalline materials which were formed in the precipitated layer. XRD results are shown in Figures 4.88 to 4.91. It was found from this analysis that calcite was precipitated for all types of ECC mixes. However, in the case of control ECC mix with no bacteria (Figure 4.91), high amount of silicate was also detected. Excessive amount of calcite (count of around 1000) was observed in zeolite immobilised B. subtilis (Figure 4.89) and count of around 800 was observed for both zeolite + S. pasteurii (Figure 4.89) and unprotected S. pasteurii (Figure 4.88) specimens compared to the control (count of around 400) specimen(Figure 4.91).

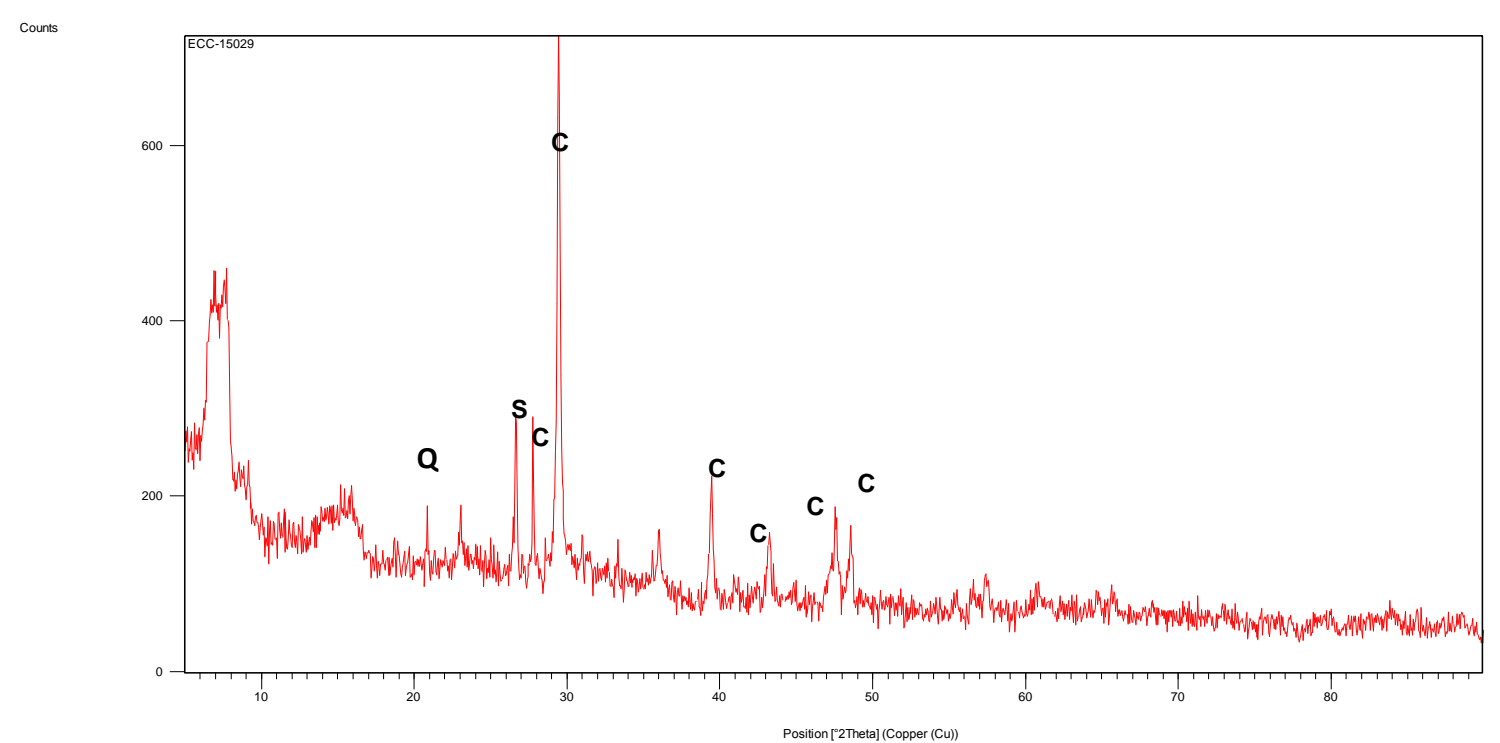

Figure 4.88: XRD analysis of ECC mix with unprotected S.pasteurii 


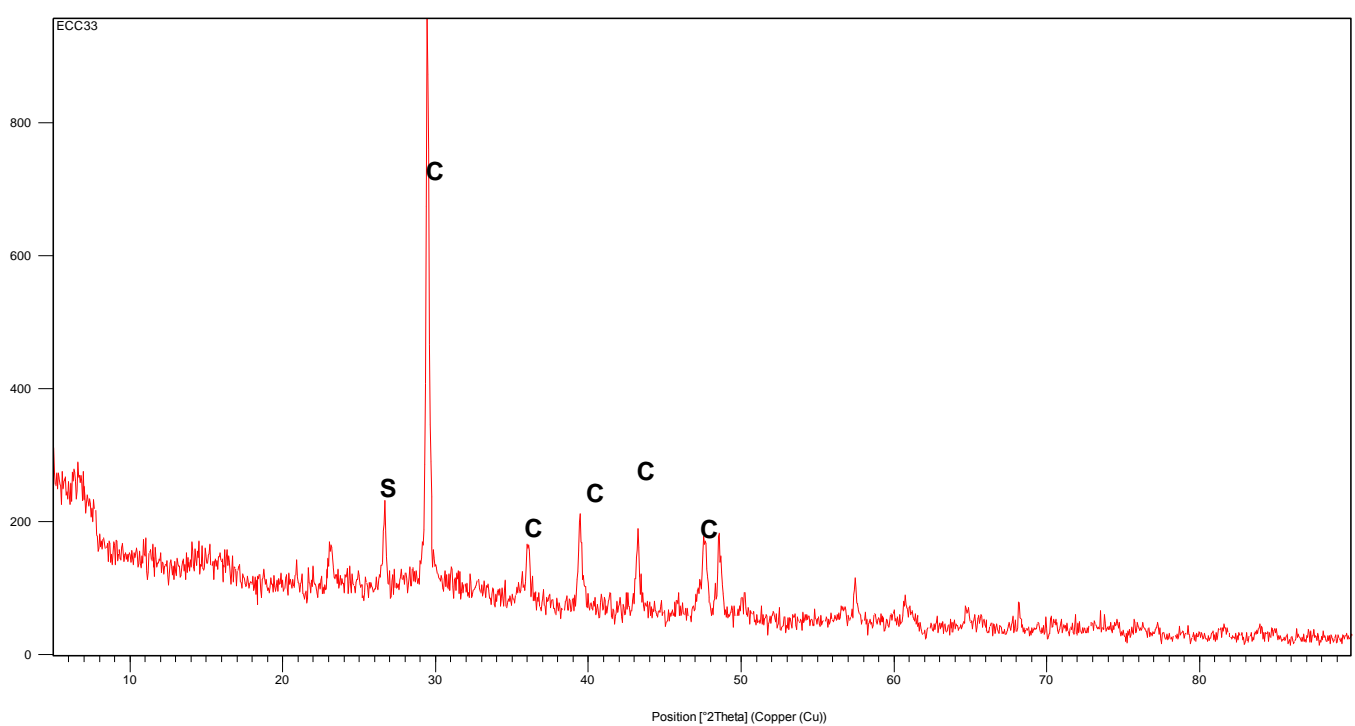

Figure 4.89: XRD analysis of ECC mix with zeolite immobilised B. subtilis

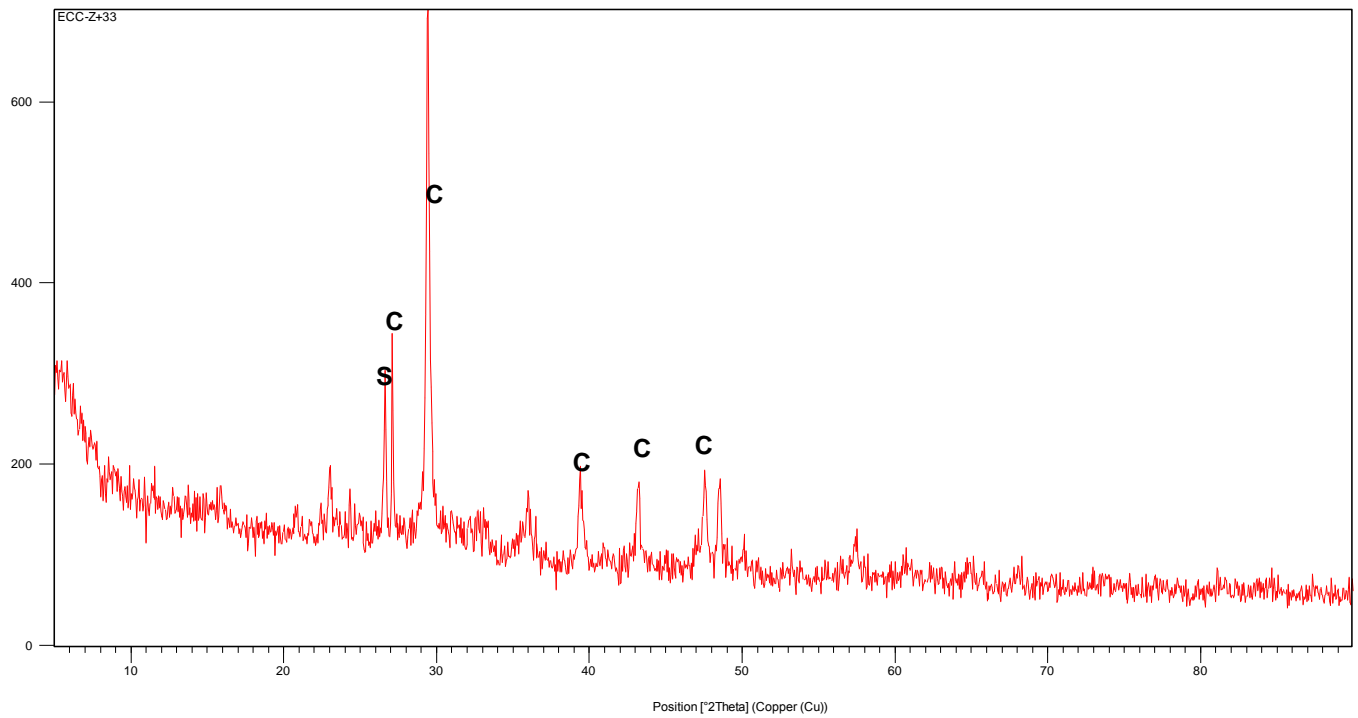

Figure 4.90: XRD analysis of ECC mix with zeolite immobilised $S$. pasteurii 


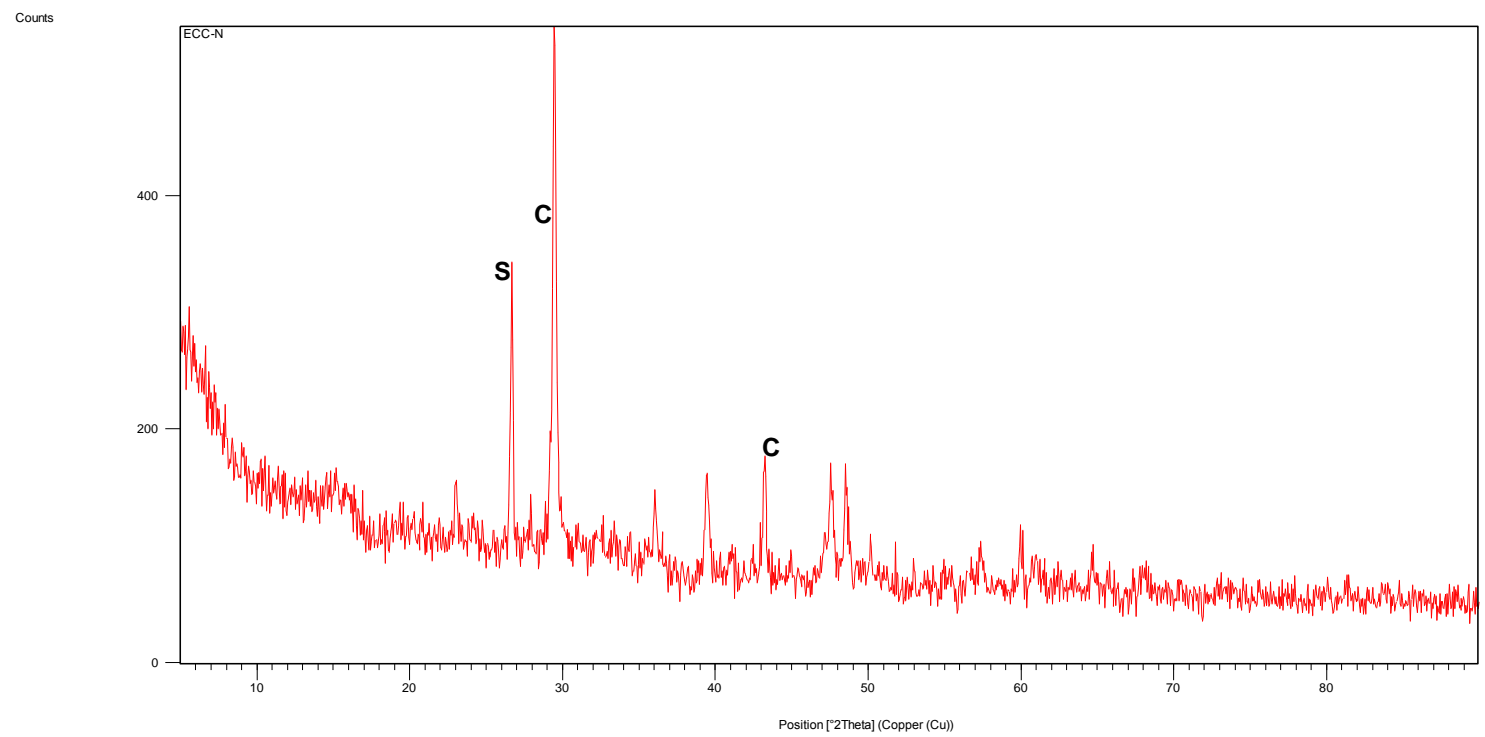

Figure 4.91: XRD analysis of control ECC mix

\subsection{Conclusions}

In this Chapter detailed analysis of self-healing was carried out for normal mortar and fibre reinforced mortar extensively as well as ECC to a certain extent. All the selected bacteria was able to exhibit significant amount of self-healing capability. Among them, S. pasteurii and B. subtilis are found to be ideal choices for bacteria based self-healing in cementitious concrete composites. Both carrier materials chosen for this study also turned out to be good protective vehicle for bacteria. The crack healing was observed using SEM/EDS examination and precipitation was tested and analysed using XRD techniques. These tests confirmed that the precipitation formed in the cracks was indeed $\mathrm{CaCO}_{3}$. SEM studies indicated that crack width up to $0.16 \mathrm{~mm}$ was completely filled with $\mathrm{CaCO}_{3}$ crystals. The quantification of self-healing performed using sorptivity, UPV, RCP, compressive strength and flexural strength - all provided convincing evidence of self-healing in different cementitious composite mixes. Evidence of self-healing was more pronounced in ECC compared to FR mortar especially in flexural tests. Therefore, it is recommended to conduct more detailed study on ECC including sorptivity and RCP tests. 


\section{CHAPTER FIVE: MATHEMATICAL MODELING OF SELF-HEALING PROPERTIES}

\subsection{Introduction}

This chapter illustrates parameter optimization in modelling self-healing characteristics in terms of compressive strength, sorptivity, Rapid Chloride Permeability (RCP) and Ultrasonic Pulse Velocity (UPV) evolution of bacteria incorporated normal and fibre reinforced mortars by statistical design and analysis of experimental results. Statistical analyses were carried out in order to model the influence of key parameters such as age of healing, types of bacteria and types of carrier materials on self-healing behavior of cracked specimens made of fibre reinforced mortar. The responses/properties considered to evaluate self-healing efficiency were RCP, primary sorptivity, UPV and compressive strength. As a first step, an attempt has been made to model the compressive strength of bacterial concrete with different concentration of bacteria and the calcium compound to identify the ideal concentration. The calcium compound used was calcium lactate, which acts as the calcium source for the bacteria to precipitate calcium carbonate. In order to determine the optimum concentration of the bacteria and calcium lactate which would aid in selfhealing, a certain set of experiment was conducted initially. This involved choosing three different concentration of bacteria (based on past studies conducted in this field) and two concentrations of calcium lactate. The concentration level which provided the maximum compressive strength was chosen for all subsequent self- healing experimentation. The statistical modelling following the laboratory experimentation enabled identifying the concentrations which were not considered in the experimental study and observing the interaction effects. This chapter mainly describes two aspects: i) modeling of compressive strength of bacteria incorporated mortar with different concentration of bacteria to identify the ideal concentration, ii) modeling of self-healing behavior of bacteria based fiber reinforced mortar. For the current study, statistical software tool MINITAB 17 has been used to perform the design of experiments and analysis. 


\subsection{Modeling of compressive strength of normal mortar with different bacterial concentration}

Design of experimental method (a statistical method) is used in this study to reduce the number of tests and increase the number of studied factors and also to study the interaction between these factors. In order to analyze the effects of all three-way, four-way and five-way interaction effects on the response it was decided to conduct all the experiments by full factorial design and analysis. Since all possible combinations of the levels of the factors are experimented, there is enough data to select a $3^{3} 2^{2}$ full factorial design and analysis for the response compressive strength. The $3^{3} 2^{2}$ full factorial design requires all possible combinations of the maximum and minimum levels of the analyzed five process parameters. It lets the analysis of all two-way, three-way, four-way and five-way factor interaction effects in addition to the main factor effects. Therefore, it needs 108 different parameter level combinations and three replicates of each experiment condition in order to take the noise factors into consideration. As a result, a total of 324 experiments were conducted for the response variable. All the 324 experiments were arranged in a completely randomized manner to avoid possible errors. Table 5.1 outlines the chosen factors and their levels. The response variable is the compressive strength.

Table 5.1: Factors and their levels

\begin{tabular}{|c|c|c|c|c|}
\hline Factor & Name & Level (-1) & Level (0) & Level (+1) \\
\hline A & Testing days & 7 days & 14 days & 28 days \\
\hline B & Concentration of bacteria & $10^{4}$ cells $/ \mathrm{ml}$ & $10^{6}$ cells $/ \mathrm{ml}$ & $10^{8}$ cells $/ \mathrm{ml}$ \\
\hline C & Concentration of calcium lactate & $1 \%$ & - & $3 \%$ \\
\hline D & Types of bacteria & S. ureae & S. pasteurii & B. subtilis \\
\hline E & Carrier material & zeolite & - & pumice \\
\hline
\end{tabular}

\subsubsection{Significant terms and their definitions}

Some of the significant terms associated with Analysis of Variance (ANOVA) and regression analysis are as follows. 
i) DF: Degrees of freedom of variables.

ii) Sum of Squares (SS) and Mean Squares (MS): In ANOVA, the total sum of squares helps to express the total variation that can be attributed to various factors. Converting the sum of squares into mean squares by dividing by the degrees of freedom allows to compare these ratios and determine whether there is a significant difference due to the factors. The larger this ratio, the more the treatments affect the outcome.

iii) F value: The test statistic used to decide whether the model as a whole has statistically significant predictive capability, that is, whether the regression SS is big enough, considering the number of variables needed to achieve it. $\mathrm{F}$ is the ratio of the Model Mean Square to the Error Mean Square.

iv) $\mathrm{P}$ value: Determine which terms to keep in the regression model. $\mathrm{P}$ value of $<0.05$ should be taken into account for the model.

v) R-squared: A statistical measure of how close the data are to the fitted regression line. $\mathrm{R}$-squared is always between 0 and $100 \%$. In general, the higher the R-squared, the better the model fits the data.

vi) Adjusted R-squared: It compares the explanatory power of regression models that contain different numbers of predictors. The adjusted R-squared is a modified version of R-squared that has been adjusted for the number of predictors in the model. The adjusted R-squared increases only if the new term improves the model more than would be expected by chance. It decreases when a predictor improves the model by less than expected by chance.

vii) Predicted R-squared: It indicates how well a regression model predicts responses for new observations. A key benefit of predicted R-squared is that it can prevent from over fitting a model.

viii) S: It represents the average distance that the observed values fall from the regression line. Smaller values are better because it indicates that the observations are closer to the fitted line

ix) Coef: It is the coefficient of the variables in the regression equation.

$\mathrm{x}) \mathrm{SE}$ : It measures the precision of the estimate of the coefficient. The smaller the standard error, the more precise the estimate. 
xi) T: Dividing the coefficient by its standard error calculates a t-value. If this value is too small, it is not possible to declare statistical significance.

\subsubsection{Compressive strength based on full factorial design}

The analysis of variance (ANOVA) table for the mean compressive strength can be seen in Table 5.2. This ANOVA table gives a summary of the main effects and interactions. Since some of the two -way, three- way and four- way factor interactions are insignificant, they were omitted from the model. It can be observed from the table that all the main effects, some of the two way interactions such as AB (Testing days*Concentration of bacteria) and AE (Testing days*Carrier material) and the three way interactions ACE (Testing days*Calcium lactate concentration*Carrier material) and the four way interaction ACDE (Testing days*Calcium lactate concentration*Types of bacteria*Carrier material) significantly affect the compressive strength. The significance AB and AE interactions can also be seen from the plot given in Figure 5.1. X-axis of each column and $\mathrm{Y}$-axis of each row represents the levels of the related factor. Each line corresponds to the different levels of the second parameter. Since the three lines in the AB and AE interaction plots are almost non-parallel, their effect on the compressive strength can be accepted as significant. The residual plots of the model for the mean compressive strength are given in Figures 5.2 and 5.3.

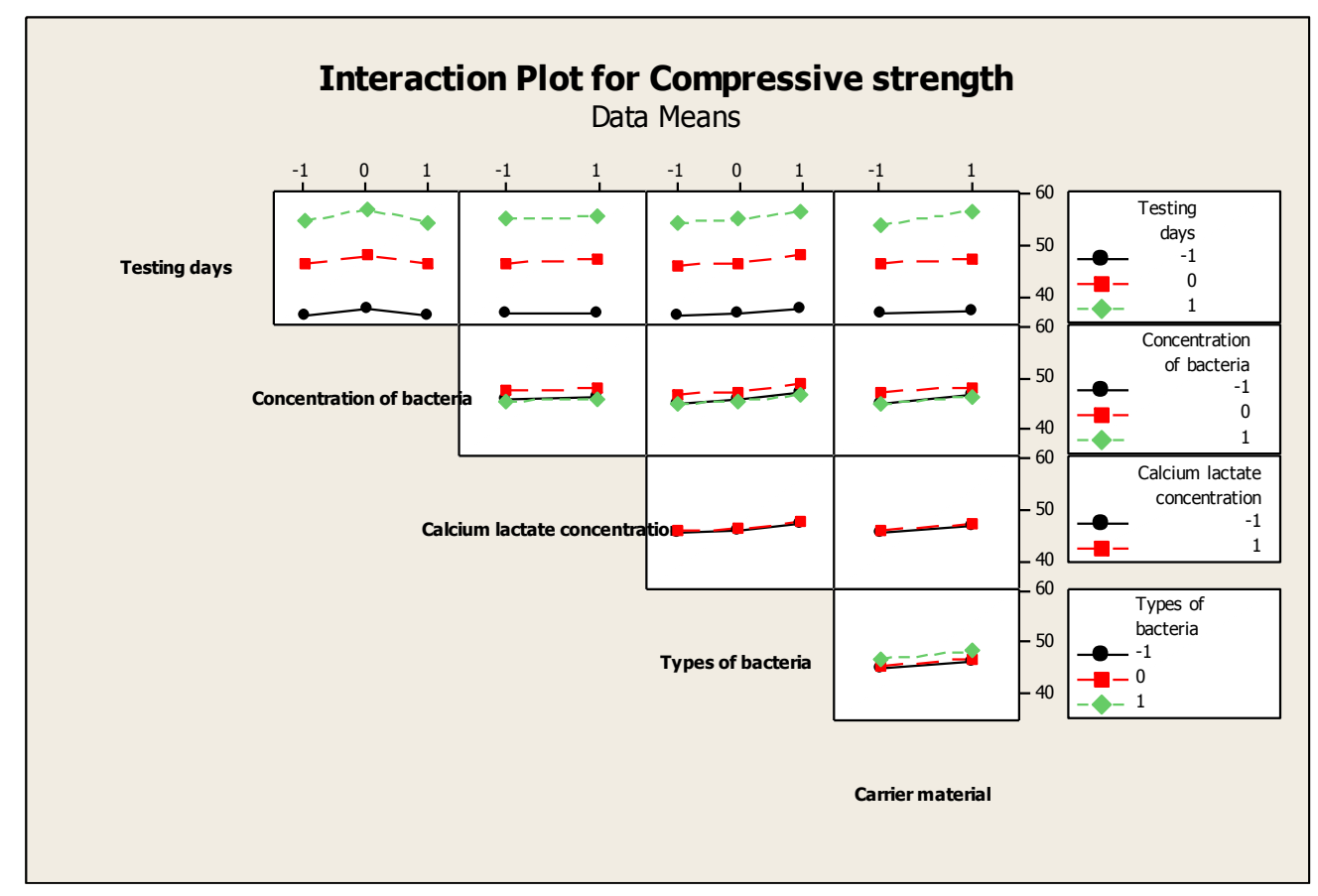

Figure 5.1: Two-way interaction plots for the compressive strength 
Table 5.2: ANOVA table for the mean compressive strength based on the full factorial design

\begin{tabular}{|c|c|c|c|c|c|}
\hline Source & DF & $\begin{array}{l}\text { Sum of } \\
\text { Squares }\end{array}$ & $\begin{array}{c}\text { Mean } \\
\text { squares }\end{array}$ & $\mathbf{F}$ & $\mathbf{P}$ \\
\hline $\mathrm{A}$ & 2 & 19467.63 & 9733.82 & 7658.47 & 0 \\
\hline B & 2 & 273.18 & 136.59 & 107.47 & 0 \\
\hline $\mathrm{C}$ & 1 & 14.61 & 14.61 & 11.49 & 0.001 \\
\hline $\mathrm{D}$ & 2 & 217.17 & 108.58 & 85.43 & 0 \\
\hline $\mathrm{E}$ & 1 & 138.72 & 138.72 & 109.14 & 0 \\
\hline $\mathrm{AB}$ & 4 & 24.26 & 6.07 & 4.77 & 0.001 \\
\hline $\mathrm{AC}$ & 2 & 4.57 & 2.29 & 1.8 & 0.168 \\
\hline $\mathrm{AD}$ & 4 & 5.95 & 1.49 & 1.17 & 0.325 \\
\hline $\mathrm{AE}$ & 2 & 80.53 & 40.27 & 31.68 & 0 \\
\hline $\mathrm{BE}$ & 2 & 4.15 & 2.07 & 1.63 & 0.198 \\
\hline $\mathrm{CD}$ & 2 & 1.86 & 0.93 & 0.73 & 0.482 \\
\hline $\mathrm{CE}$ & 1 & 2.12 & 2.12 & 1.67 & 0.198 \\
\hline $\mathrm{DE}$ & 2 & 3.15 & 1.57 & 1.24 & 0.292 \\
\hline $\mathrm{ABE}$ & 4 & 8.3 & 2.07 & 1.63 & 0.167 \\
\hline $\mathrm{ACE}$ & 2 & 9.56 & 4.78 & 3.76 & 0.025 \\
\hline $\mathrm{ADE}$ & 4 & 4.02 & 1 & 0.79 & 0.533 \\
\hline BDE & 4 & 3.82 & 0.95 & 0.75 & 0.559 \\
\hline $\mathrm{CDE}$ & 2 & 4.35 & 2.18 & 1.71 & 0.183 \\
\hline ACDE & 4 & 8.58 & 2.64 & 1.69 & 0.154 \\
\hline Error & 216 & 274.53 & 1.27 & & \\
\hline Total & 323 & 20570.71 & & & \\
\hline
\end{tabular}

\section{$\mathrm{S}=1.56956 \quad \mathrm{R}-\mathrm{Sq}=96.20 \% \quad \mathrm{R}-\mathrm{Sq}(\mathrm{adj})=96.13 \% \quad \mathrm{R}-\mathrm{Sq}(\mathrm{pred})=96.04 \%$}

The adjusted multiple coefficient of determination, $\mathrm{R}^{2}{ }_{(\mathrm{adj})}$, shows that $96.1 \%$ of the sample variation in the mean compressive strength can be explained by this model. 


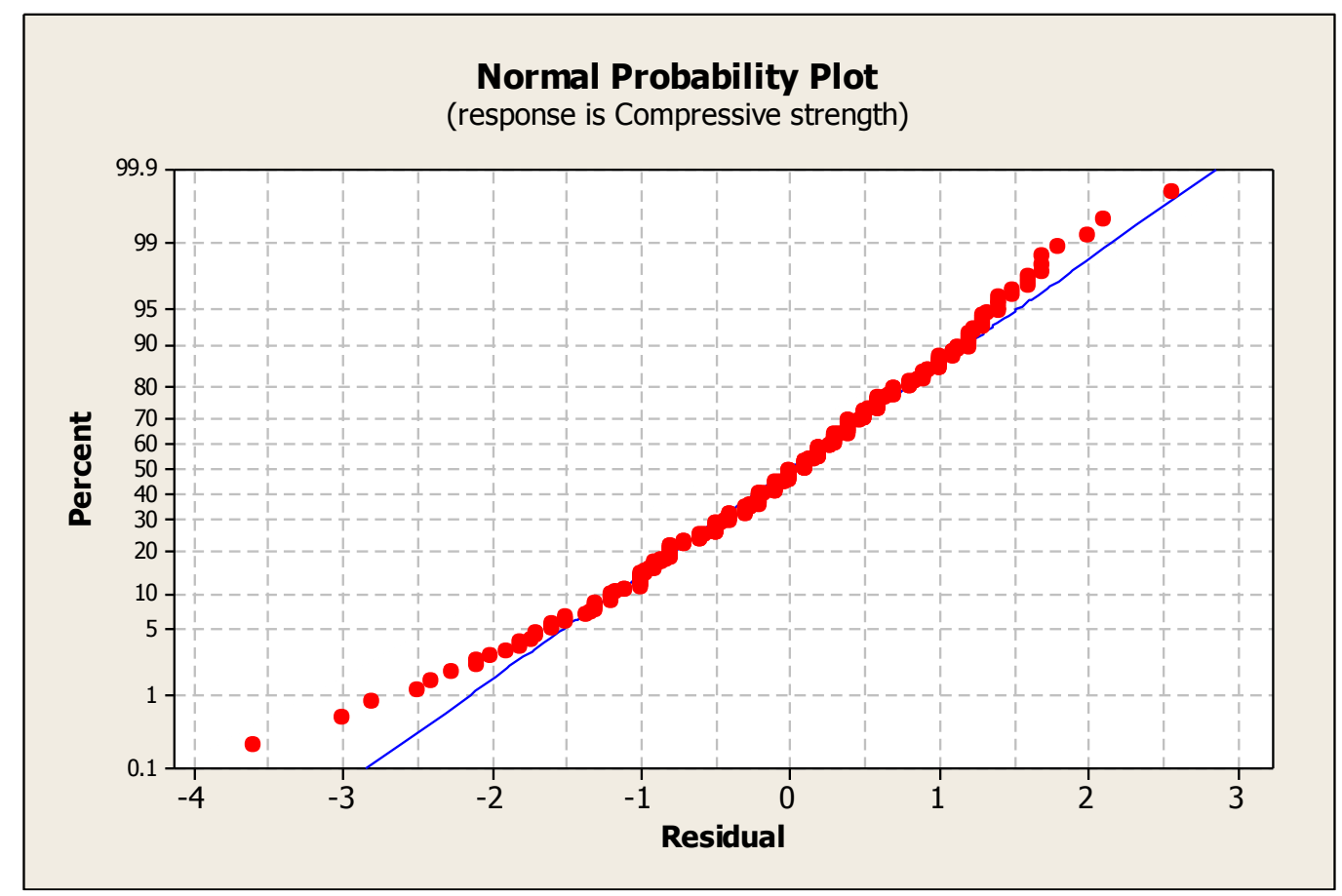

Figure 5.2: The normal probability plot for the full factorial model found by ANOVA for the compressive strength

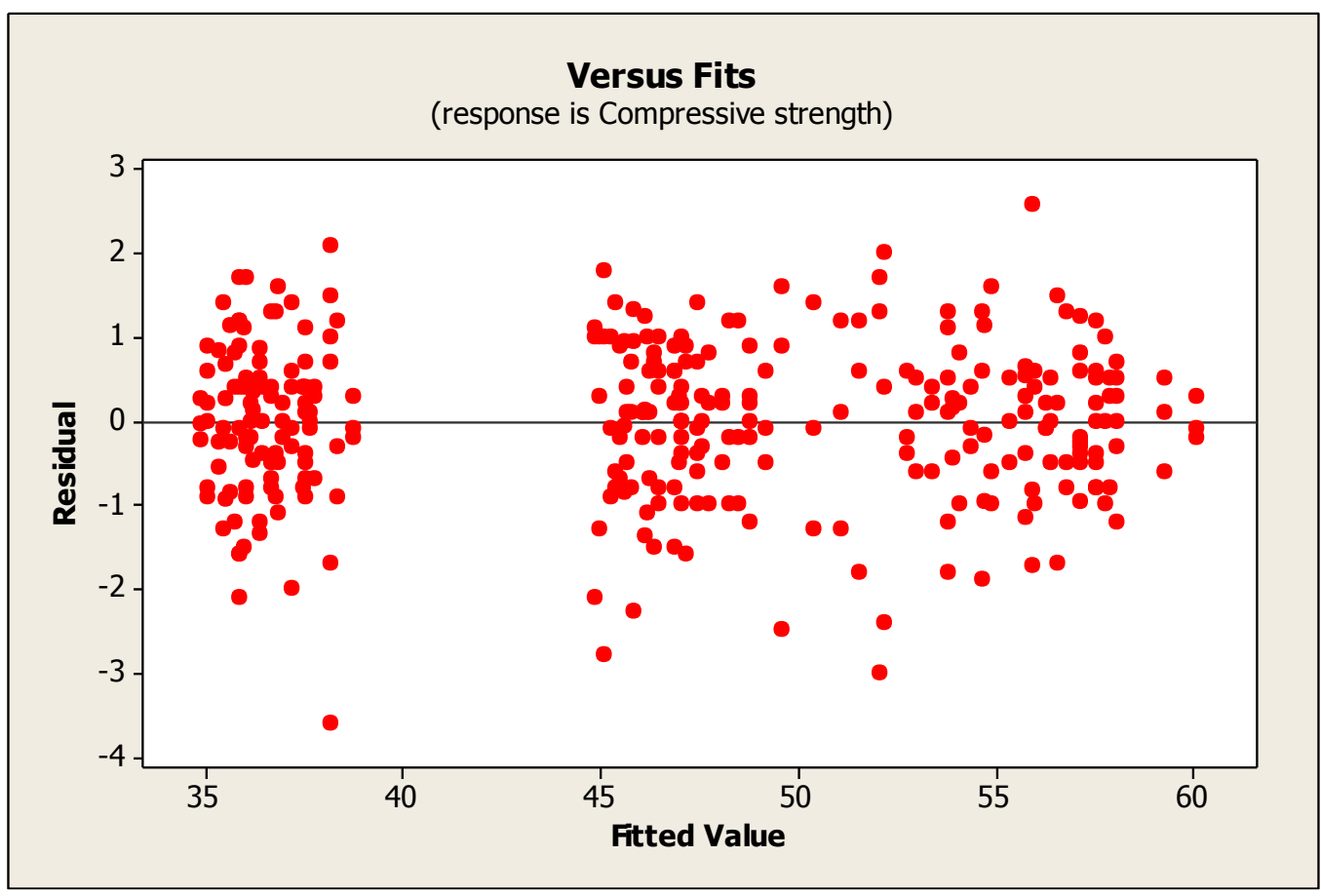

Figure 5.3: The residuals versus fitted values of the full factorial model found by ANOVA for the compressive strength 
It can be seen from Figure 5.2 that there is a linear trend on the normal probability plot which indicates that the assumption of the error term having a normal probability distribution is satisfied. It can be concluded from Figure 5.3 that the assumption of having a constant variance of the error term for all levels of the independent process parameters is not violated.

Figure 5.4 shows the main effects plot which is used for finding the optimum levels of the process parameters that increases the mean compressive strength.

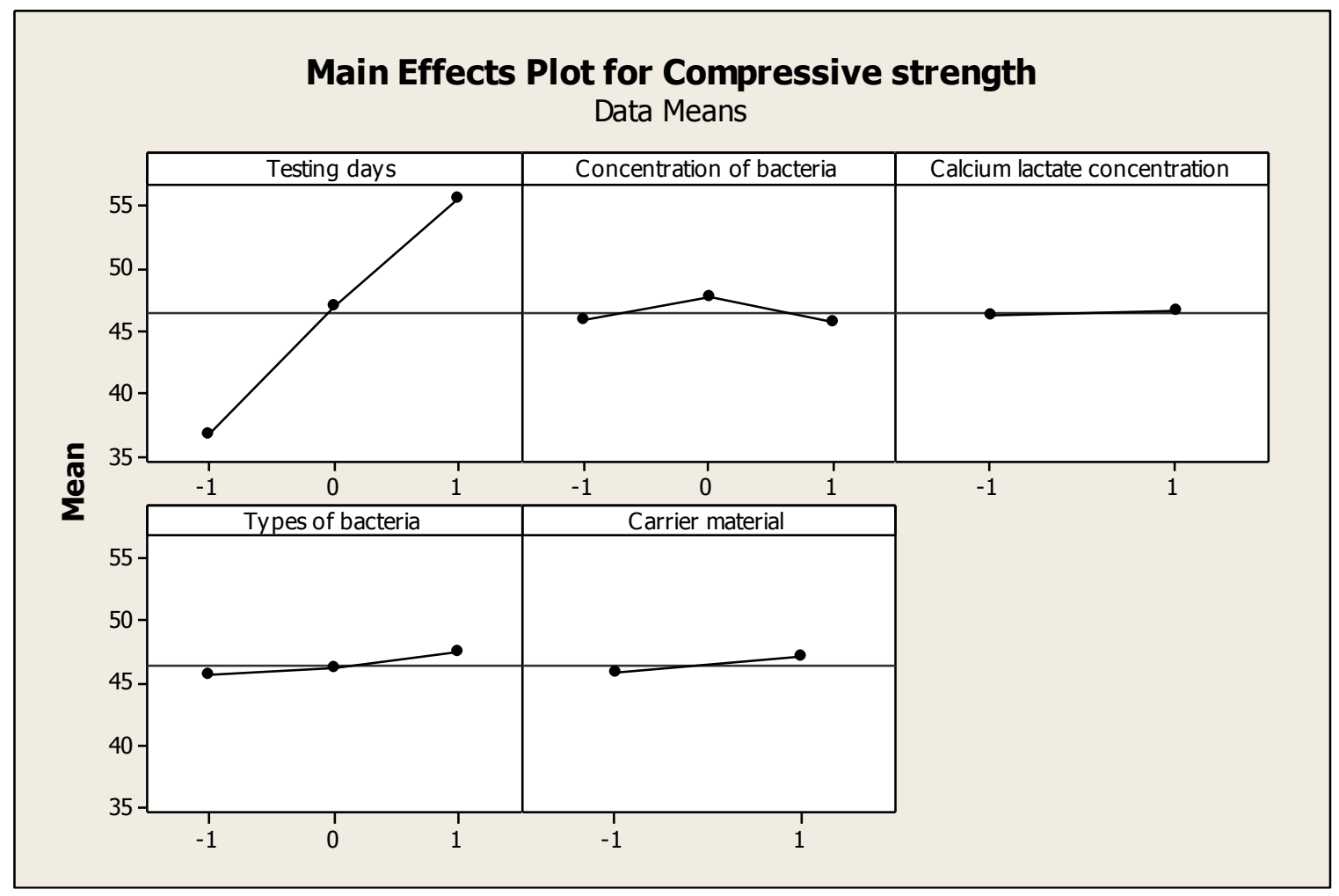

Figure 5.4: Main effects plot based on the full factorial design for the compressive strength

As it can be seen from Figure 5.4, the optimum points are $3^{\text {rd }}$ level for Age (28 days), $2^{\text {nd }}$ level for the Concentration of bacteria $\left(10^{6} \mathrm{cells} / \mathrm{ml}\right), 3^{\text {rd }}$ level for the Calcium lactate concentration $(3 \%)$, $3^{\text {rd }}$ level for the Types of bacteria (B. subtilis) and the $3^{\text {rd }}$ level for the Carrier material (pumice). Since there is only a slight difference between the $1^{\text {st }}$ level and the $3^{\text {rd }}$ level of Calcium lactate concentration, the $1^{\text {st }}$ level can also be chosen for economic considerations. Also it is necessary to consider the significant two-way factor interactions when determining the optimum condition. 
From the interaction plot (Figure 5.1) it can be seen that the optimum levels of the interaction terms are $\mathrm{A}_{1} \times \mathrm{B}_{0}$ and $\mathrm{A}_{1} \times E_{1}$ which coincide with the optimum levels of the main effects. The notation for optimum point is $\mathrm{A}_{1} \mathrm{~B}_{0} \mathrm{C}_{1} \mathrm{D}_{1} \mathrm{E}_{1}$. This corresponds to the $119^{\text {th }}$ trial run in the full factorial experiment.

Figure 5.5 shows the contour representation of the compressive strength as function of both concentration of bacteria and testing days. It represents the way compressive strength develops from low values (blue at bottom) to the optimum (dark green at top centre). It can be observed from the contour plot that the compressive strength was low (dark blue colour) when testing days was 7 days( level -1.0) and highest (dark green area) when the testing days was 28 days (level 1.0). It is quite evident that the highest compressive strength was achieved when the concentration of bacteria was $10^{6}$ cells $/ \mathrm{ml}$. (Level 0 ). This contour plot can be used to predict the compressive strengths at levels where experiment was not performed such as 10 days of healing and $10^{5}$ cells $/ \mathrm{ml}$ etc.

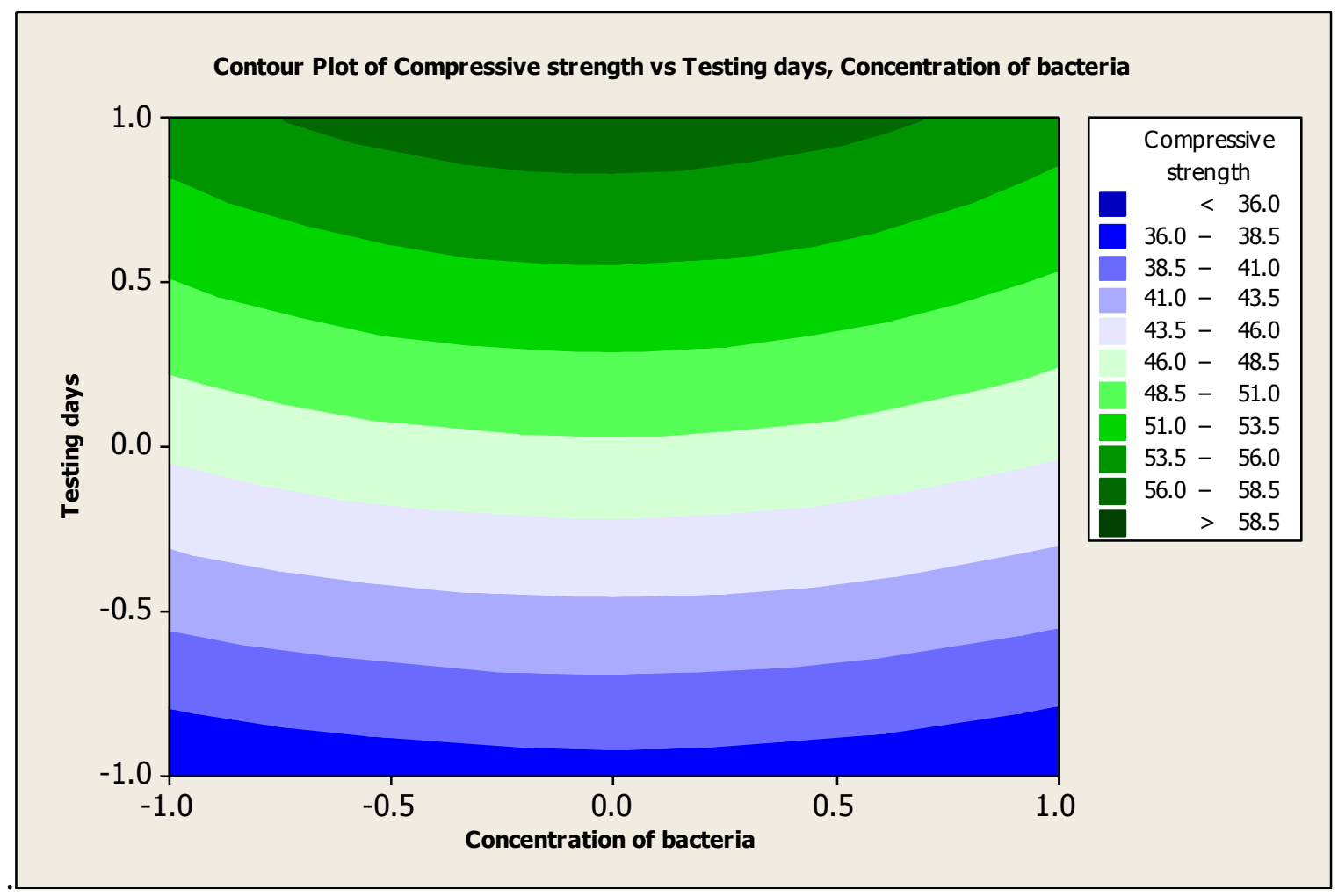

Figure 5.5: Contour plot of the compressive strength 


\subsubsection{Regression model for compressive strength}

Since the interaction between the factors are not much pronounced, the interaction between them is eliminated from the regression equation and the simplified characteristic regression model for the 28 day compressive strength is as follows in Table 5.3:

Table 5.3: Regression equation for compressive strength

\begin{tabular}{|c|c|c|}
\hline $\begin{array}{l}\text { Types of bacteria used } \\
\text { in the mortar mix }\end{array}$ & $\begin{array}{l}\text { Types of carrier } \\
\text { used in the mortar } \\
\text { mix }\end{array}$ & Regression Equation for Compressive strength \\
\hline S. ureae & zeolite & $44.8948+9.48148 \mathrm{~A}-0.72 \mathrm{~B}+0.212346 \mathrm{C} \quad$ (Eq. 5.1) \\
\hline S. ureae & pumice & $46.2034+9.48148 \mathrm{~A}-0.72 \mathrm{~B}+0.212346 \mathrm{C} \quad$ (Eq. 5.2) \\
\hline S. pasteurii & zeolite & $45.4568+9.48148 \mathrm{~A}-0.72 \mathrm{~B}+0.212346 \mathrm{C} \quad$ (Eq. 5.3) \\
\hline S. pasteurii & pumice & $46.7654+9.48148 \mathrm{~A}-0.72 \mathrm{~B}+0.212346 \mathrm{C} \quad($ Eq. 5.4) \\
\hline B. subtilis & zeolite & $46.468429+9.48148 \mathrm{~A}-0.72 \mathrm{~B}+0.212346 \mathrm{C}($ Eq. 5.5) \\
\hline B. subtilis & pumice & $48.1515+9.48148 \mathrm{~A}-0.72 \mathrm{~B}+0.212346 \mathrm{C} \quad($ Eq. 5.6) \\
\hline
\end{tabular}

\subsubsection{Validation of the proposed statistical model}

\subsubsection{Verification of the proposed model using existing data}

A total of six different simplified regression equations were developed which are given from Eq.1 through Eq.6. It was found that only slight variations were noted among the equations. These models are compared with the results of research conducted on Bacillus subtilis JC3 obtained from literature (Srinivasa et.al, 2012). Since sufficient data is unavailable to compare the models with the other bacteria used in the current study, the validation is done comparing the models with the data of Bacillus subtilis JC3. Table 5.4 shows the comparison of the predicted values of compressive strength obtained for $B$. subtilis at three different concentrations with that of Bacillus subtilis JC3. Similarly Table 5.5 shows the comparison of $S$.pasteurii with Bacillus subtilis JC3 and Table 5.6 shows S. ureae with respect to Bacillus subtilis JC3. In the Tables 5.4 to 5.6, (P) stands for predicted value and (E) stands for experimental values. Close examination of the data in the Table 5.4, 5.5 and 5.6 indicates that the percentage of variation is less than $10 \%$ for $B$. subtilis, $12 \%$ for $S$.pasteurii and $14.5 \%$ for $S$. ureae. It is worthwhile to note that the variation is 
significantly less $(0.13 \%$ to $5 \%)$ for 28 days old specimen. Figure 5.6 shows the graphical representation of predicted (B. subtilis) and experimental values (Bacillus subtilis JC3) of compressive strength. It can be observed that the predicted value curve fits well with the experimental value curve for all three concentration levels. This predicted curve can also be used to identify the compressive strength at any concentration between $10^{4}$ to $10^{8}$ cells $/ \mathrm{ml}$ for a given age. From all these results, it can be concluded that this preliminary model can be used to predict the compressive strength of any bacteria incorporated normal mortar.

Table 5.4: Comparison of Predicted strength (P) of B. subtilis and Experimental strength (E) of Bacillus subtilis JC3

\begin{tabular}{|c|c|c|c|c|c|c|c|c|c|}
\hline Age & $\begin{array}{c}\mathbf{1 0}^{\mathbf{4}}(\mathbf{P}) \\
\text { cells/ml }\end{array}$ & $\begin{array}{c}\mathbf{1 0}^{\mathbf{6}}(\mathbf{P}) \\
\text { cells/ml }\end{array}$ & $\begin{array}{c}\mathbf{1 0}^{\mathbf{8}}(\mathbf{P}) \\
\text { cells/ml }\end{array}$ & $\begin{array}{c}\mathbf{1 0}^{\mathbf{4}}(\mathbf{E}) \\
\text { cells/ml }\end{array}$ & $\begin{array}{c}\mathbf{1 0}^{\mathbf{6}}(\mathbf{E}) \\
\text { cells/ml }\end{array}$ & $\begin{array}{c}\mathbf{1 0}^{\mathbf{8}}(\mathbf{E}) \\
\text { cells/ml }\end{array}$ & $\begin{array}{c}\text { \% } \\
\text { variation } \\
\left(\mathbf{1 0}^{\mathbf{4}}\right)\end{array}$ & $\begin{array}{c}\text { \% } \\
\text { variation } \\
\left(\mathbf{1 0}^{6}\right)\end{array}$ & $\begin{array}{c}\text { \% } \\
\text { variation } \\
\left(\mathbf{1 0}^{\mathbf{8}}\right)\end{array}$ \\
\hline $\mathbf{7 ~ d a y s}$ & 39.9 & 40.42 & 39.18 & 41.68 & 43.09 & 40.11 & 4.27 & 6.20 & 2.32 \\
\hline $\mathbf{1 4}$ days & 49.4 & 50.12 & 48.68 & 45.23 & 47.69 & 45.97 & -9.22 & -5.10 & -5.90 \\
\hline 28 days & 58.16 & 58.88 & 57.44 & 58.02 & 57.21 & 54.66 & -0.24 & -2.92 & -5.09 \\
\hline
\end{tabular}

Table 5.5: Comparison of Predicted strength (P) of S. pasteurii and Experimental strength (E) of Bacillus subtilis JC3

\begin{tabular}{|c|c|c|c|c|c|c|c|c|c|}
\hline Age & $\begin{array}{l}\mathbf{1 0}^{\mathbf{4}}(\mathbf{P}) \\
\text { cells/ml }\end{array}$ & $\begin{array}{l}\mathbf{1 0}^{\mathbf{6}}(\mathbf{P}) \\
\text { cells/ml }\end{array}$ & $\begin{array}{l}\mathbf{1 0}^{\mathbf{8}}(\mathbf{P}) \\
\text { cells/ml }\end{array}$ & $\begin{array}{l}\mathbf{1 0}^{\mathbf{4}}(\mathbf{E}) \\
\text { cells/ml }\end{array}$ & $\begin{array}{l}\mathbf{1 0}^{\mathbf{6}}(\mathbf{E}) \\
\text { cells/ml }\end{array}$ & $\begin{array}{l}\mathbf{1 0}^{\mathbf{8}}(\mathbf{E}) \\
\text { cells/ml }\end{array}$ & $\begin{array}{l}\mathbf{\%} \\
\text { variation } \\
\left(\mathbf{1 0}^{\mathbf{4}}\right)\end{array}$ & $\begin{array}{l}\text { \% } \\
\text { variation } \\
\left(\mathbf{1 0}^{\mathbf{6}}\right)\end{array}$ & $\begin{array}{l}\% \\
\text { variation } \\
\left(\mathbf{1 0}^{\mathbf{8}}\right)\end{array}$ \\
\hline $\mathbf{7}$ days & 37.26 & 37.98 & 36.54 & 41.68 & 43.09 & 40.11 & 10.60 & 11.86 & 8.90 \\
\hline $\mathbf{1 4}$ days & 46.74 & 47.46 & 46.02 & 45.23 & 47.69 & 45.97 & -3.34 & 0.48 & -0.11 \\
\hline $\mathbf{2 8}$ days & 56.26 & 56.98 & 55.54 & 58.02 & 57.21 & 54.66 & 3.03 & 0.40 & -1.61 \\
\hline
\end{tabular}

Table 5.6: Comparison of Predicted strength (P) of S. ureae and Experimental strength (E) of Bacillus subtilis JC3

\begin{tabular}{|c|c|c|c|c|c|c|c|c|c|}
\hline Age & $\begin{array}{l}10^{4}(\mathrm{P}) \\
\text { cells } / \mathrm{ml}\end{array}$ & $\begin{array}{l}10^{6}(\mathrm{P}) \\
\text { cells/ml }\end{array}$ & $\begin{array}{l}10^{8}(\mathrm{P}) \\
\text { cells/ml }\end{array}$ & $\begin{array}{l}10^{4}(\mathrm{E}) \\
\text { cells } / \mathrm{ml}\end{array}$ & $\begin{array}{l}10^{6}(\mathbf{E}) \\
\text { cells/ml }\end{array}$ & $\begin{array}{l}10^{8}(\mathrm{E}) \\
\text { cells/ml }\end{array}$ & $\begin{array}{l}\% \\
\text { variation } \\
\left(10^{4}\right)\end{array}$ & $\begin{array}{l}\% \\
\text { variation } \\
\left(10^{6}\right)\end{array}$ & $\begin{array}{l}\% \\
\text { variation } \\
\left(10^{8}\right)\end{array}$ \\
\hline 7 days & 36.12 & 36.84 & 35.40 & 41.68 & 43.09 & 40.11 & 13.34 & 14.50 & 11.74 \\
\hline 14 days & 45.62 & 46.34 & 44.90 & 45.23 & 47.69 & 45.97 & -0.86 & 2.83 & 2.33 \\
\hline 28 days & 55.31 & 56.03 & 54.59 & 58.02 & 57.21 & 54.66 & 4.67 & 2.06 & 0.13 \\
\hline
\end{tabular}




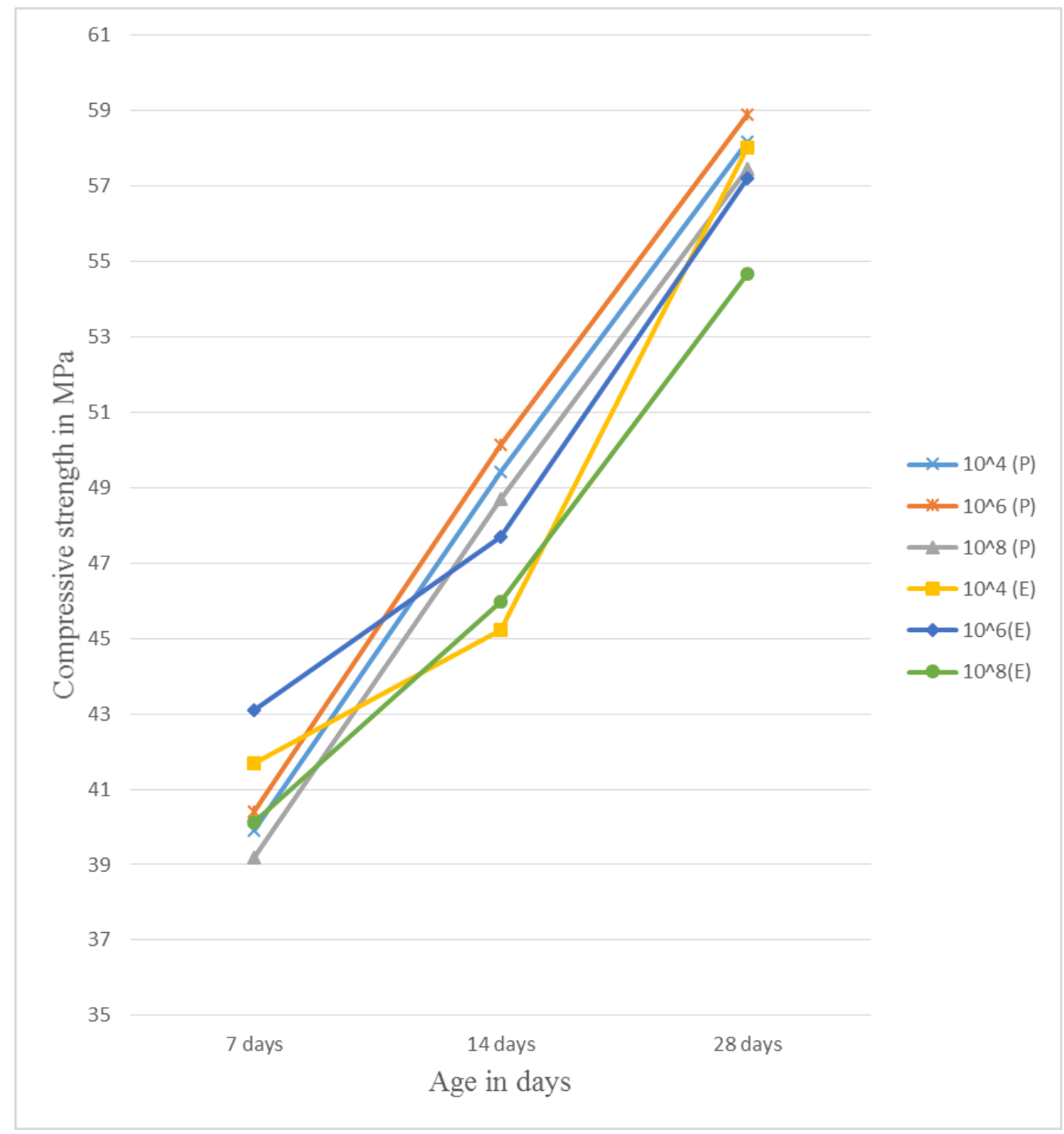

Figure 5.6: Comparison of Predicted strength of $B$. subtilis and Experimental strength of $B$. subtilis JC3

\subsubsection{Verification of the model using numerical example}

In order to verify the equations obtained for compressive strength of normal mortar, a numerical example can be put forth. All the required values for the numerical example are summarised in the Table 5.1. For example, Eq. 5.6, 48.1515 + 9.48148 A - 0.72 B + 0.212346 C will be used to predict the compressive strength of bacterial normal mortar (for B. subtilis) for the ranges of variables shown in Table 5.1. 
Now, let A (age) be 28 days, B (concentration of bacteria) be $10^{8}$ cells $/ \mathrm{ml}$ and C (calcium lactate concentration) be $1 \%$. It is important to note that the numerical value of $\mathrm{A}, \mathrm{B}$ and $\mathrm{C}$ must be chosen as $+1,+1$ and -1 respectively according to the Table 5.1.

After substituting these values, Eq. 5.6 becomes,

$48.1515+9.48148 \times(+1)-0.72 \times(+1)+0.212346 \times(-1)=56.7 \mathrm{MPa}$.

This provides the 28 days compressive strength for $B$. subtilis for $10^{8}$ cells $/ \mathrm{ml}$ for a calcium lactate concentration of 1\%. Similar results can be obtained for different bacteria using Eq 5.1 to 5.5.

These derived statistical models allow to predict compressive strength for any day between 7 and 28 days, bacterial concentration from $10^{4}$ to $10^{8} \mathrm{cells} / \mathrm{ml}$ and calcium lactate concentration from 1 to $3 \%$. As an example, it is possible to find the $21^{\text {st }}$ day compressive strength with a bacterial concentration of $10^{7}$ cells $/ \mathrm{ml}$ and calcium lactate concentration of $2 \%$ for S. pasteurii using Eq. 5.3 or 5.4 .

In this case, it is important to note that $\mathrm{A}, \mathrm{B}$ and $\mathrm{C}$ will have the values $+0.5,+0.5$ and 0 respectively. Substituting these values, Eq. 5.3 becomes,

$45.4568+9.48148 \times(0.5)-0.72 \times(0.5)+0.212346 \times(0)=49.84 \mathrm{MPa}$.

It can be concluded that regardless of the bacteria types and carrier materials, any equations from Eq. 5.1 to 5.6 may be used to generally predict the compressive strength of bacterial concrete as the variations among the obtained values are not significant.

\subsection{Modeling self-healing behavior of bacteria incorporated fiber reinforced mortar}

Three parameters among others such as: the age of healing, types of bacteria and types of carrier material that that influence all the responses/properties namely compressive strength, sorptivity, $\mathrm{RCP}$ and UPV of bacteria incorporated fiber reinforced mortar are chosen. $4^{2} 2^{1}$ full factorial experimental design results and regression model have been employed to optimise the model for each response. In other words, four levels of age of healing and types of bacteria as well as two

levels of carrier material types have been used in the conducted experiments. Three replicates of each experiment have been performed because when sample mean is used to estimate the effect of a factor in the experiment, then replication permits to obtain a more precise estimate of this effect, 
and if noise factors vary, then repeating trials may reveal their influence. ANOVA has been performed for the mean values of all the responses separately. Then, the regression analysis has been conducted and the best model has been chosen for the mean of each response variable for the design.

Moreover, this study is unique in the sense that the derived models enable the identification of underlying primary factors and their interactions that influence the modeled responses of fiber reinforced mortar.

\subsubsection{Full Factorial Experimental Design}

Since all possible combinations of the levels of the factors are experimented, there is enough data to select a $4^{2} 2^{1}$ full factorial design and analysis for the four self-healing responses: compressive strength, sorptivity, RCP and UPV. The $4^{2} 2^{1}$ full factorial design requires all possible combinations of the maximum and minimum levels of the analyzed three process parameters. It lets the analysis of all two-way, and three-way factor interaction effects in addition to the main factor effects. Therefore, it needs 32 different parameter level combinations and three replicates of each experiment condition are performed in order to take the noise factors into consideration. As a result 96 experiments are conducted for each of the response variables. This statistical modeling was a preliminary attempt to identify the self-healing behavior at different intervals of time with different kinds of bacteria and carrier materials. Main emphasis was to design the mix which would exhibit self-healing behavior. So factors selected were part of the mix design and factors which would quantify self-healing such as crack width and environmental factors like temperature and humidity were not considered in this study because this would lead to design becoming exceedingly complex. Therefore, the main factors considered were age of healing, types of bacteria used in the mortar mix and types of carrier materials used in the mortar mix. The main factors and their levels are given in Table 5.7. 
Table 5.7: Factors and their level

\begin{tabular}{|c|c|c|c|c|c|}
\hline Factor & Name & Level (1) & Level (2) & Level (3) & Level (4) \\
\hline $\mathbf{A}$ & $\begin{array}{l}\text { Age of healing } \\
\text { (days) }\end{array}$ & 7 days & 120 days & 180 days & 240 days \\
\hline B & $\begin{array}{l}\text { Types of bacteria } \\
\text { used in the mix }\end{array}$ & $\begin{array}{c}\text { No } \\
\text { bacteria }\end{array}$ & S. ureae & B. subtilis & $\begin{array}{c}\text { S. } \\
\text { pasteurii }\end{array}$ \\
\hline $\mathbf{C}$ & $\begin{array}{l}\text { Types of carrier } \\
\text { used in the mix }\end{array}$ & zeolite & pumice & - & - \\
\hline
\end{tabular}

The level assignment is done according to the increasing values of mortar compressive strength and UPV with decreasing values of sorptivity and RCPT as self-healing indicators.

For all of the responses, full factorial analysis and general linear regression analysis are performed. The MINITAB results of modeling each response/properties (indicator of self-healing) is explained in the subsequent sections, in detail.

\subsubsection{Statistical Analysis results when the response is RCP}

The ANOVA table for the mean RCP of fibre reinforced mortar is given in Table 5.8. From this table, the less significant factors can be decided by observing the $\mathrm{p}$ values which should be $<0.05$ in order to become significant at $95 \%$ confidence level. Therefore, after observing the $\mathrm{p}$ values, the analysis was done once again and a re-fit model was developed after omitting the insignificant interactions. From the Table 5.8, it can be observed that apart from the primary factors such as age of healing (A), types of bacteria (B) and types of carrier (C), only the two way interaction: age of healing * types of bacteria $(\mathrm{A} * \mathrm{~B})$ is found to be significant. Subsequently, the other two way interactions such as types of bacteria * types of carrier $(\mathrm{B} * \mathrm{C})$ and ages of healing * types of carrier $(\mathrm{A} * \mathrm{C})$ and three way interaction ages of healing * types of bacteria * types of carrier (A $* \mathrm{~B} * \mathrm{C}$ ) were omitted to re-fit the model since all those insignificantly effected the RCP values of fibre reinforced mortar. 
Table 5.8: ANOVA table for the mean RCP based on the full factorial design

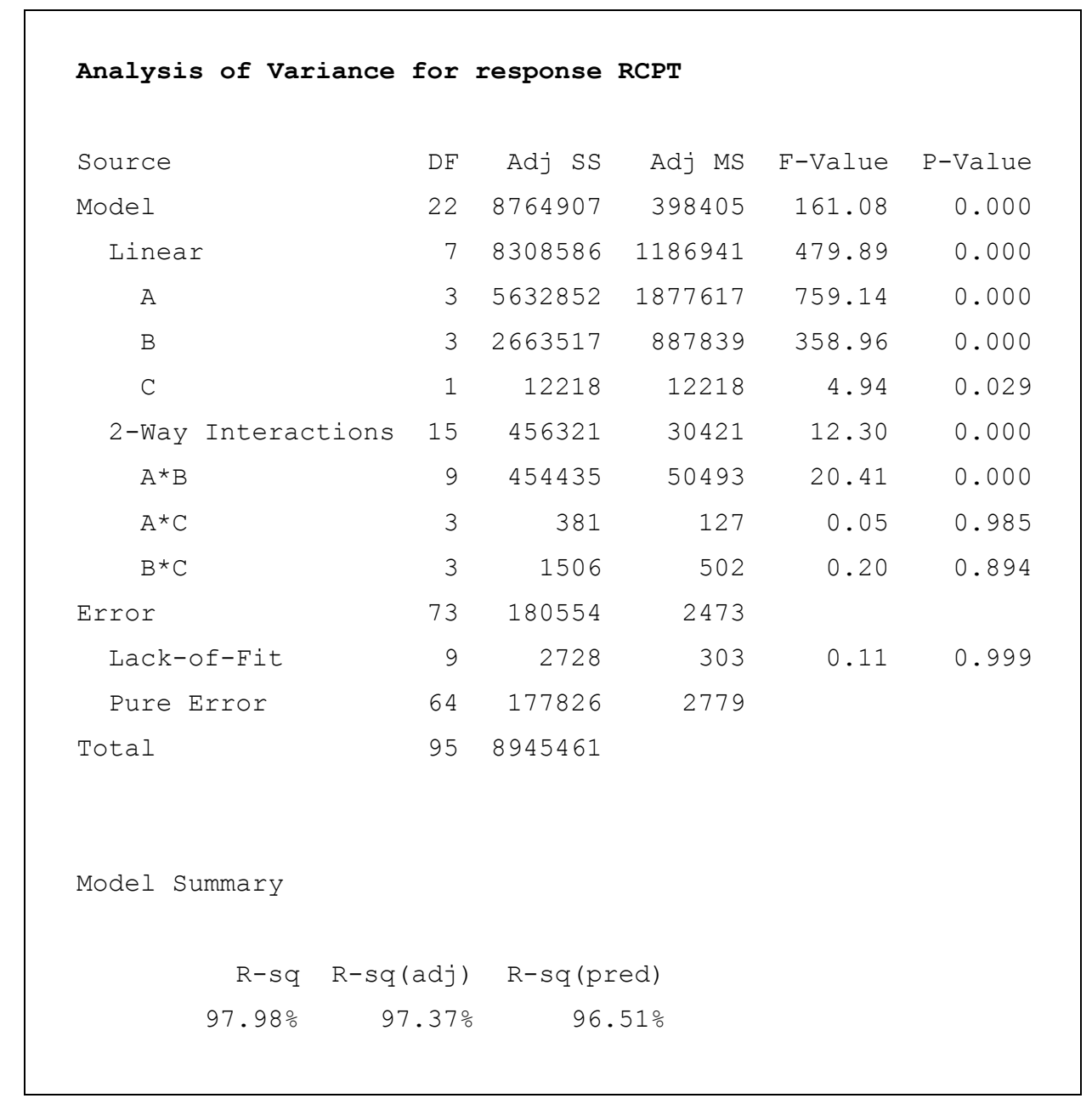

The insignificance of $\mathrm{BC}$ and $\mathrm{AC}$ interaction can also be seen from the two-way interaction plot given in Figure 5.7. $\mathrm{X}$-axis of each column and $\mathrm{y}$-axis of each row represents the levels of the related factor. Each line corresponds to the different levels of the second parameter. Since the two lines in the types of bacteria * types of carrier $(\mathrm{B} * \mathrm{C})$ and age of healing $*$ types of carrier $(\mathrm{A} *$ C) interaction plots are almost parallel/matching, their effect on the RCP can be accepted as insignificant. The relatively high $\mathrm{p}$ - values of $\mathrm{AC}$ and $\mathrm{BC}$ support this insignificance. However, the plot of age of healing * types of bacteria $(\mathrm{A} * \mathrm{~B})$ indicate a strong interaction between the parameters because of the non-parallelism/non-matching of the lines in the interaction plot thus proving the results obtained from ANOVA.

It can be noticed from the interaction between A (age of healing) and B (types of bacteria) that the deviation of the RCP values of level 1 (no bacteria) of factor B is significantly higher than levels 
2, 3 and 4 at 240 days (level 4 of factor A) compared to that at 7 days (level 1 of factor A) (Figure 5.7). This proves that the bacterial induced precipitation significantly reduced the RCP for long term thus increasing the efficiency of self-healing.

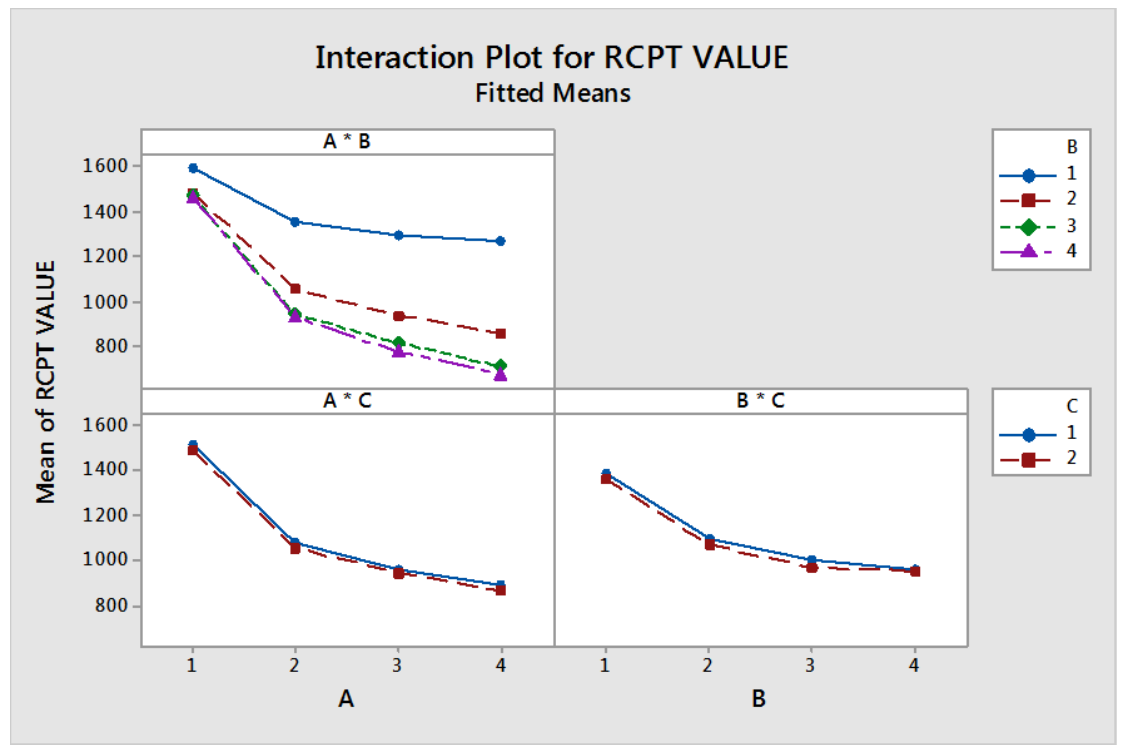

Figure 5.7: Two-way interaction plots for the mean RCP of fibre reinforced mortar

The residual plots of the model for the mean RCP values of fibre reinforced mortar are given in Figures 5.8 and 5.9.

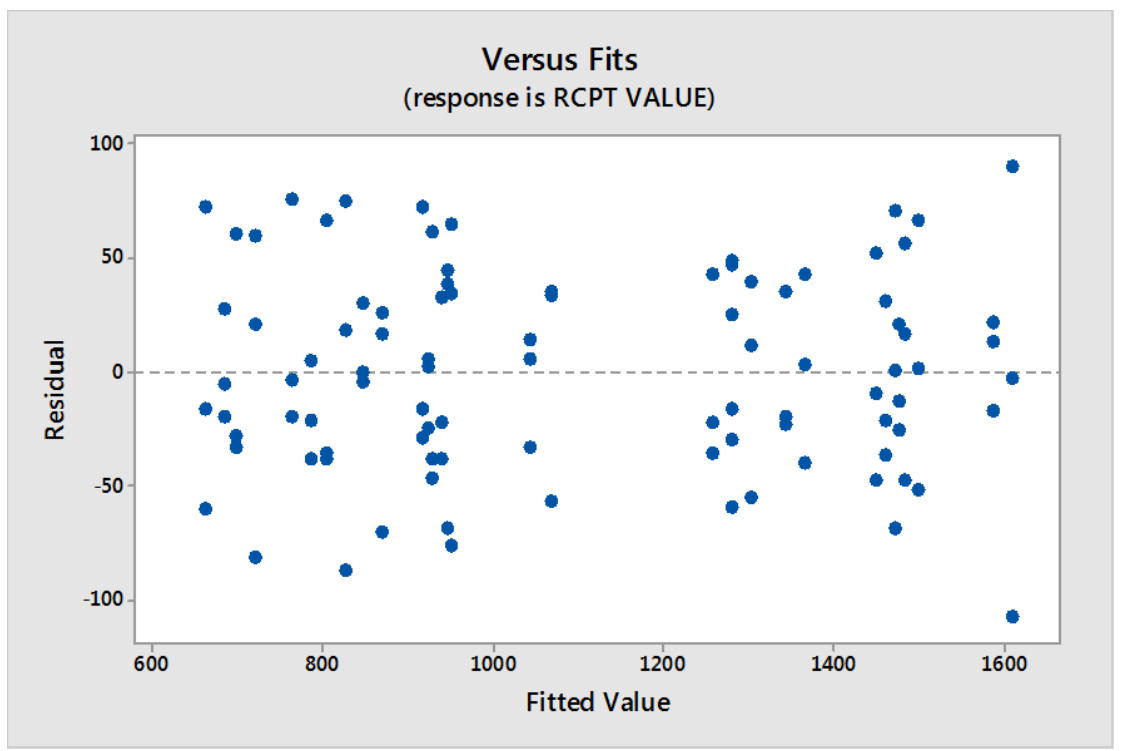

Figure 5.8: The residuals versus fitted values of the full factorial model found by ANOVA for the RCP 


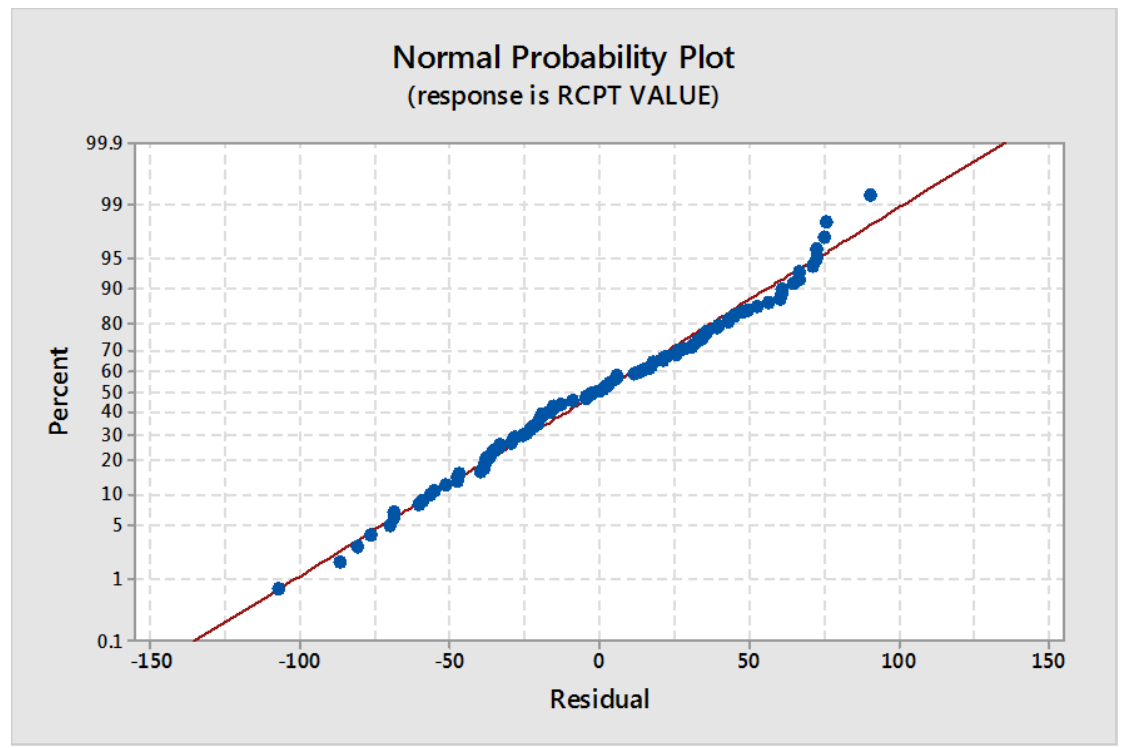

Figure 5.9: The residual normal probability plot for the full factorial model found by ANOVA for the RCP

It can be inferred from Figure 5.8 that the assumption of having a constant variance of the error term for all levels of the independent process parameters is not violated since there is no significant pattern. Also it is realised from Figure 5.9 that there is a linear trend on the normal probability plot indicating that the assumption of the error term having a normal probability distribution is satisfied.

Figure 5.10 shows the main effects plot which is a graph of the mean response values at each level of a design parameter. The two preceding figures show that the main effects, ages of healing (A) and types of bacteria (B) are found to be statistically significant for increasing the self-healing characteristics by reducing the RCP values of fibre reinforced mortar because the changes from one level to the other is high for both of the factors A and B. In addition, in the case of factor A, it can be seen that changes from level 1 (7 days) to level 2 (120 days) is very high compared to the changes from level 2 to level 3 and level 3 (180 days) to level 4 (240 days). This indicates that the main reduction in RCP values took place during the period from level 1 to level 2. In other words, as expected, most of the self-healing mechanism happened during the period of healing from 7 days to 120 days and the healing effect during the period from 120 days to 240 days was significantly reduced. The fact that the lowest RCP value was obtained for 240 days of healing confirms this. Similarly, regarding factor B, it is found that the changes from level 1 (no bacteria) 
to level 2 (S. ureae) is highly prominent. Furthermore, there is a considerable variation from level 2 to level 3 and from level 3 (B. subtilis) to level 4 (S. pasteurii). Also, it is found that the best healing efficiency was provided by the bacteria $S$. pasteurii (level 4) compared to the other two bacteria, however, the efficiency of $B$. subtilis is also remarkable since the variation from level 3 to level 4 is very less. This result signifies that the bacteria play a significant role in reducing the RCP, thereby increasing the self-healing efficiency. However, it is observed from Figure 5.4 that the types of carrier (C) do not have much influence on reducing the RCP since its variation from level 1 (zeolite) to level 2 (pumice) is negligible compared to that of A and B. Therefore, it can be concluded that selected carrier materials almost provide same kind of protection to the bacteria and the small variation might be due to the difference in their particle size distribution.

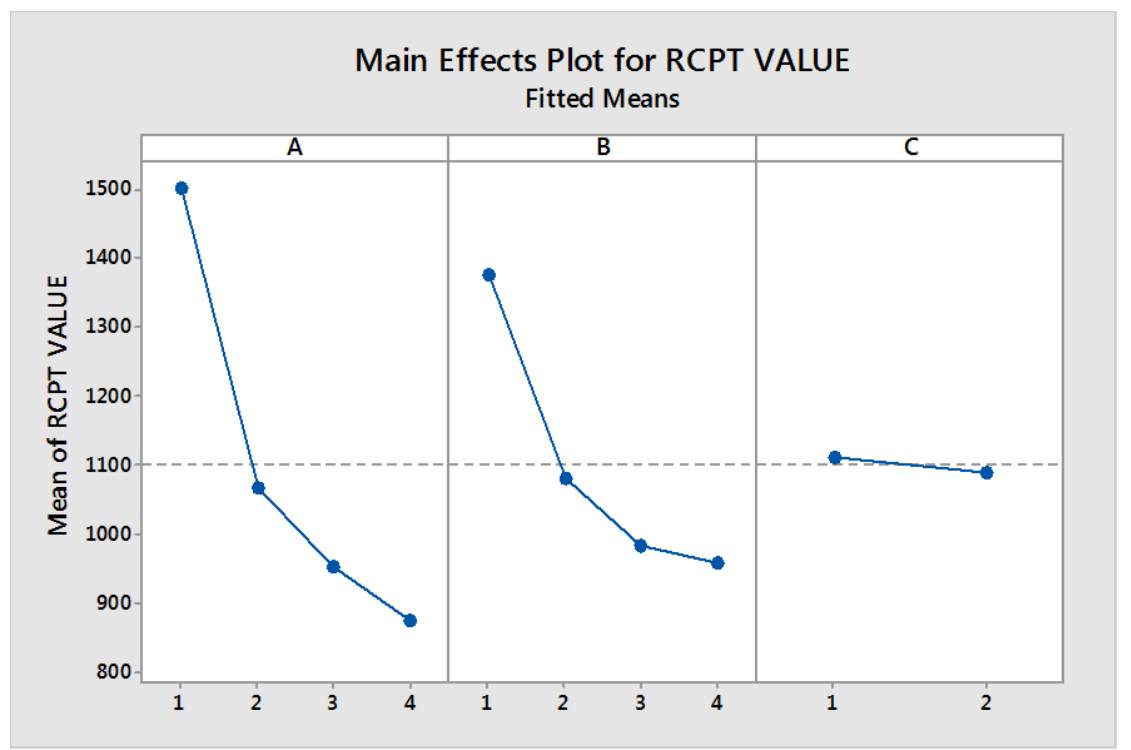

Figure 5.10: Main effect plot for the mean RCP of fibre reinforced mortar

As it is found from Figure 5.10, the optimum points are $4^{\text {th }}$ level for age of healing (240 days), $4^{\text {th }}$ level for the types of bacteria (S. pasteurii) and $2^{\text {nd }}$ level for the types of carrier (pumice). Also it is necessary to consider the significant two-way factor interactions when determining the optimum condition. From the interaction plot (Figure 5.7), it can be seen that the optimum levels for the interaction terms are $\mathrm{A}_{4} \mathrm{XB}_{4}$ which coincide with the optimum levels of the main effects. The notation for optimum points is $\mathrm{A}_{4} \mathrm{~B}_{4} \mathrm{C}_{2}$. This corresponds to the $12^{\text {th }}$ trial run in the full factorial experiment. 


\subsubsection{Regression model of the mean RCP}

Table 5.8 shows the MINITAB results of Analysis of Variance (ANOVA) for regression analysis. This re-fit model shown in the Table 5.9 is developed by omitting the insignificant interaction factors such as $\mathrm{AC}$ and $\mathrm{BC}$. Hence, characteristic regression equation (Eq. 5.7) for the current model is given below:

Table 5.9: Statistical results of analysis of variance (ANOVA) for regression analysis

\begin{tabular}{|c|c|c|c|c|c|c|}
\hline \multicolumn{7}{|c|}{ Estimated coefficients for RCPT } \\
\hline Term & Coef & $\mathrm{SE}$ & Coef & T-Value & P-Value & VIF \\
\hline Constant & 1100.97 & & 4.90 & 224.47 & 0.000 & \\
\hline \multicolumn{7}{|l|}{ A } \\
\hline 1 & 402.41 & & 8.50 & 47.37 & 0.000 & 1.50 \\
\hline 2 & -31.47 & & 8.50 & -3.70 & 0.000 & 1.50 \\
\hline 3 & -146.84 & & 8.50 & -17.29 & 0.000 & 1.50 \\
\hline \multicolumn{7}{|l|}{ B } \\
\hline 1 & 276.91 & & 8.50 & 32.60 & 0.000 & 1.50 \\
\hline 2 & -17.34 & & 8.50 & -2.04 & 0.045 & 1.50 \\
\hline 3 & -117.22 & & 8.50 & -13.80 & 0.000 & 1.50 \\
\hline \multicolumn{7}{|l|}{ C } \\
\hline 1 & 11.28 & & 4.90 & 2.30 & 0.024 & 1.00 \\
\hline \multicolumn{7}{|l|}{$A * B$} \\
\hline 11 & -183.3 & & 14.7 & -12.46 & 0.000 & 2.25 \\
\hline 12 & -0.0 & & 14.7 & -0.00 & 0.998 & 2.25 \\
\hline 13 & 84.8 & & 14.7 & 5.77 & 0.000 & 2.25 \\
\hline 21 & 8.1 & & 14.7 & 0.55 & 0.584 & 2.25 \\
\hline 22 & 3.3 & & 14.7 & 0.23 & 0.821 & 2.25 \\
\hline 23 & -12.3 & & 14.7 & -0.83 & 0.406 & 2.25 \\
\hline 31 & 61.0 & & 14.7 & 4.14 & 0.000 & 2.25 \\
\hline 32 & -1.3 & & 14.7 & -0.09 & 0.931 & 2.25 \\
\hline 33 & -22.4 & & 14.7 & -1.52 & 0.132 & 2.25 \\
\hline
\end{tabular}




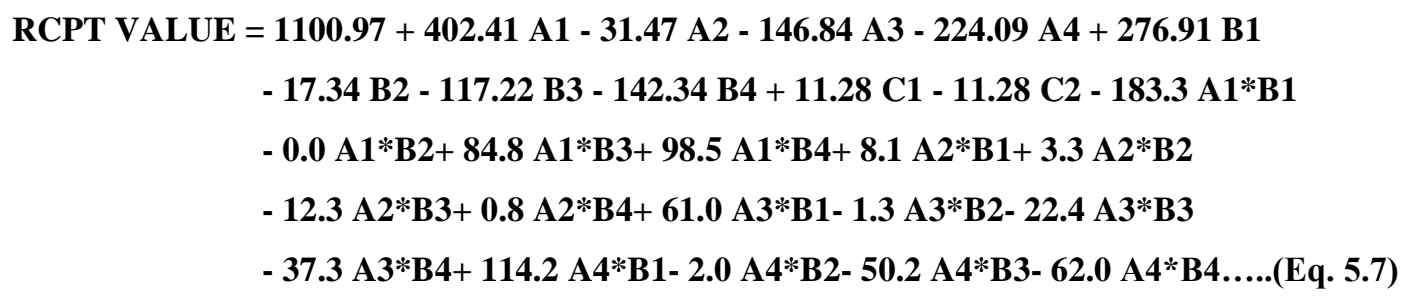

where A: age of healing, B: types of bacteria, C: types of carrier material and 1, 2, 3, 4 are levels.

\subsubsection{Statistical Analysis results when the response is mean primary sorptivity}

The ANOVA table for the mean primary sorptivity of fibre reinforced mortar is given in Table 5.10. Similar to the previous case, the less significant factors can be decided by observing the $\mathrm{p}$ values Therefore, after considering the $\mathrm{p}$ values, the analysis and a re-fit model was developed after omitting the insignificant interactions. From the Table 5.10, it can be noticed that apart from the primary factors such as ages of healing (A), types of bacteria (B) and types of carrier (C), just the two way interaction as ages of healing * types of bacteria $(\mathrm{A} * \mathrm{~B})$ is significant. At the same time, the other interactions were omitted to re-fit the model since those insignificantly effected the primary sorptivity values of fibre reinforced mortar.

Figure 5.11 depicts the 2- way interaction plots of primary sorptivity. Similar to the observations made on the RCP values, the 2-way interactions like BC and AC were found to be insignificant. The relatively high p- values of $\mathrm{AC}$ and $\mathrm{BC}$ support this insignificance. However, the plot of ages of healing * types of bacteria $(\mathrm{A} * \mathrm{~B})$ indicate a strong interaction between the parameters because of the non-parallelism of the lines in the interaction plot supporting the results obtained from ANOVA.

Similar to the RCP results, the interaction between A (age of healing) and B (types of bacteria) that the deviation for the sorptivity values of level 1 (no bacteria) of factor B is higher from level (2, 3 and 4 of factor B) at 240 days (level 4 of factor A) compared to 7 days (level 1 of factor A) (Figure 5.11). This proves that the bacteria induced precipitation of self-healing products substantially reduced the sorptivity in long term, thus increasing the efficiency of self-healing. 
Table 5.10: ANOVA table for the mean sorptivity based on the full factorial design

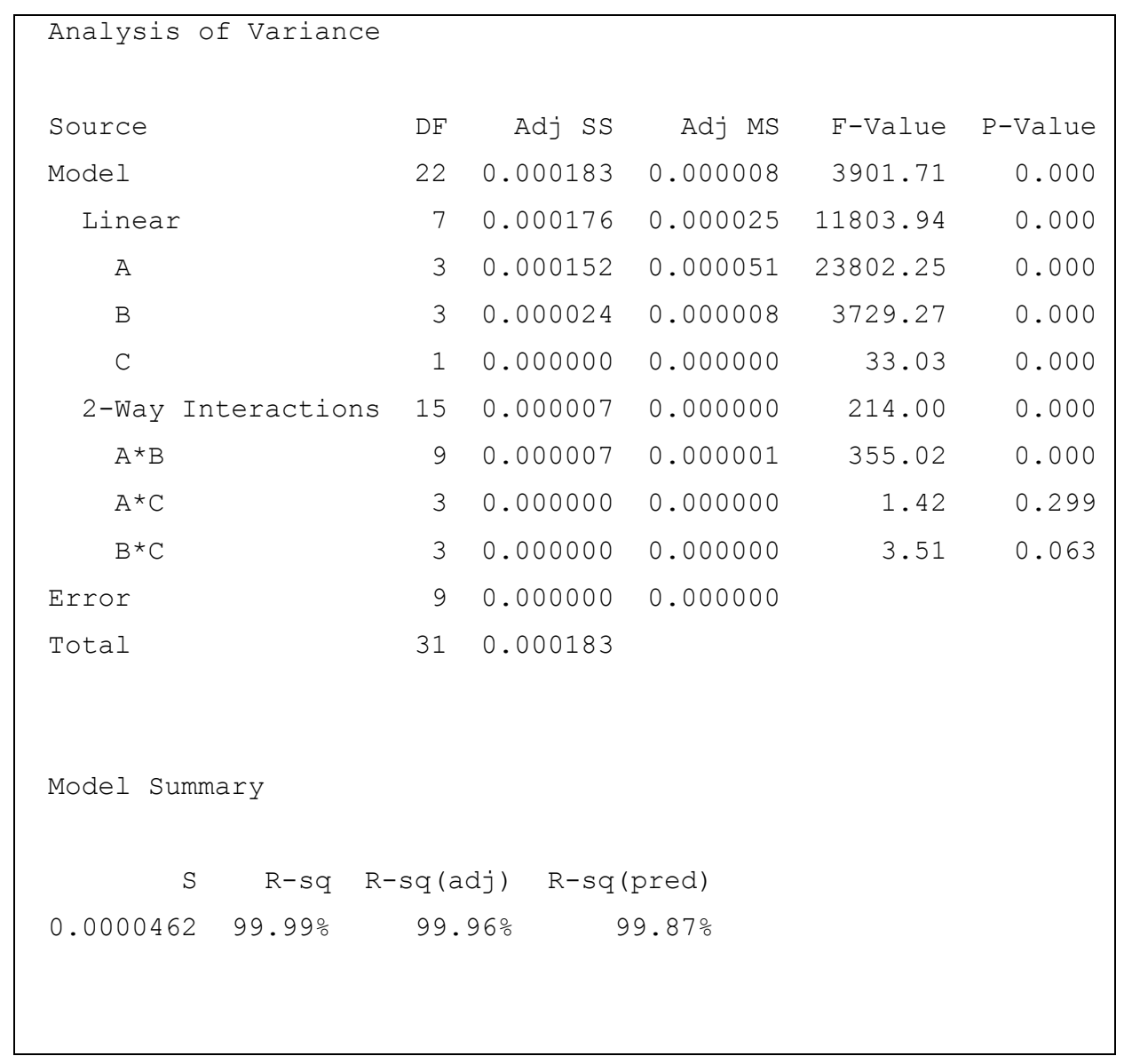

The residual plots of the model for the mean sorptivity of fibre reinforced mortar are given in Figures 5.12 and 5.13. According to Figure 5.12, the absence of a significant pattern suggests that the assumption of having a constant variance of the error term for all levels of the independent process parameters is not violated. Figure 5.13 clearly shows that normal probability distribution is satisfied. 


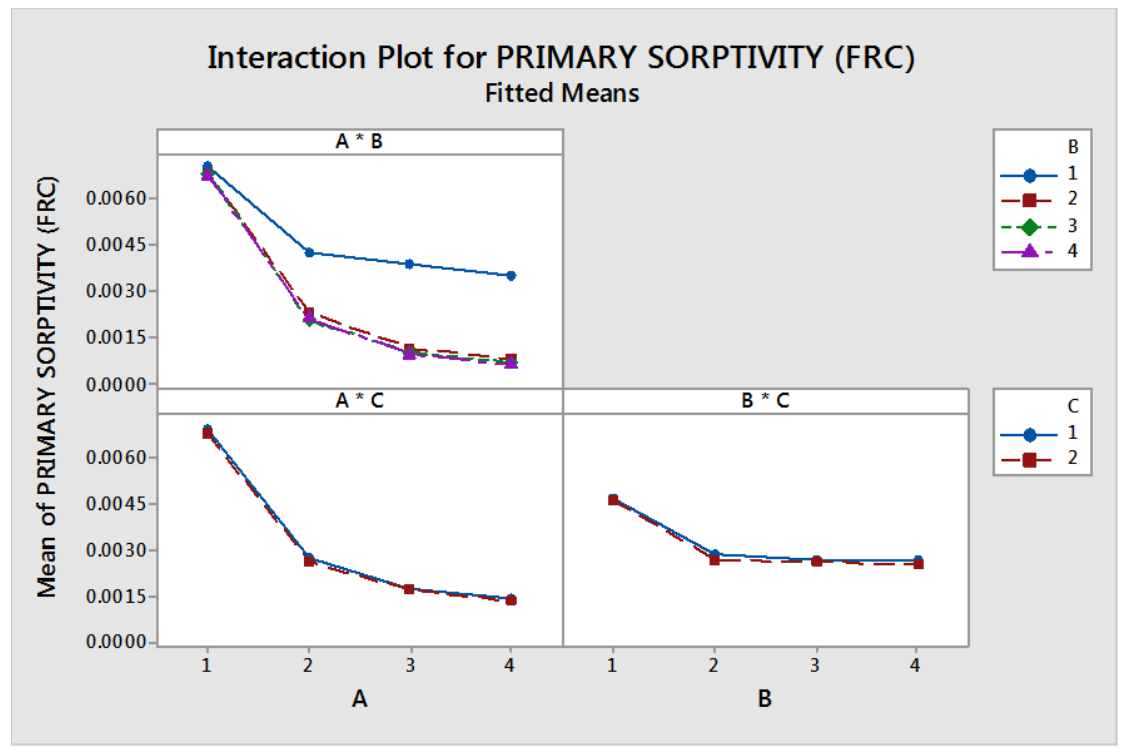

Figure 5.11: Two-way interaction plots for the mean sorptivity of fibre reinforced mortar

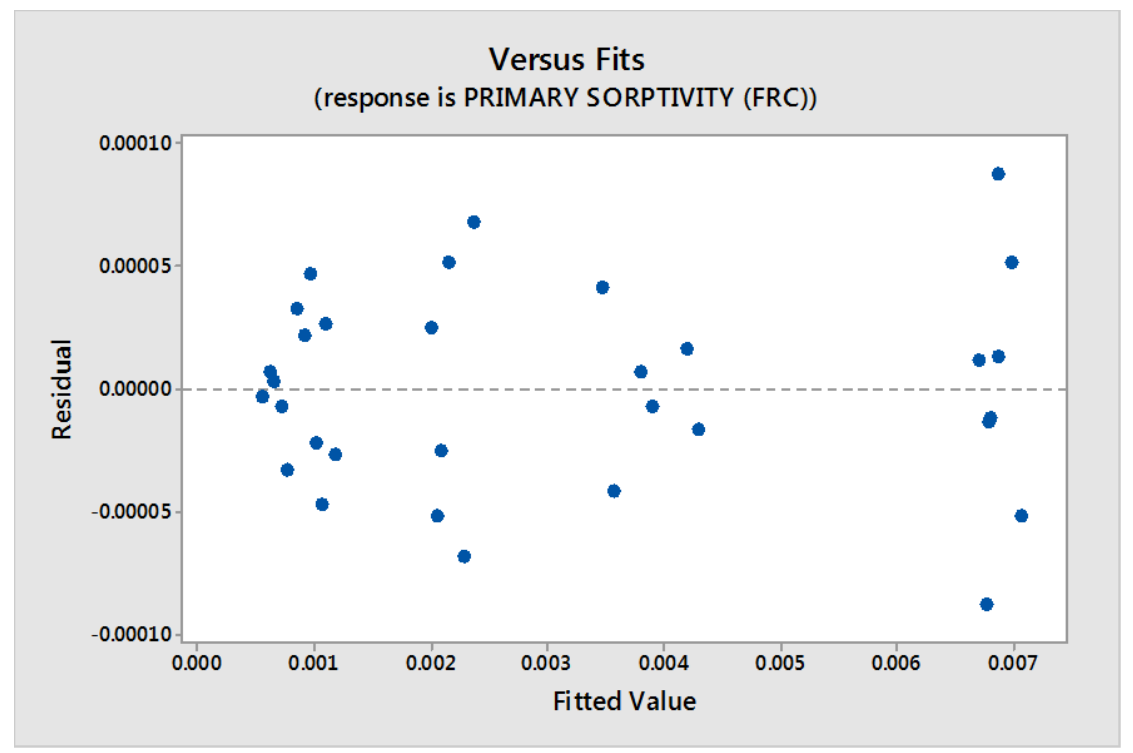

Figure 5.12: The residuals versus fitted values of the full factorial model found by ANOVA for the primary sorptivity 


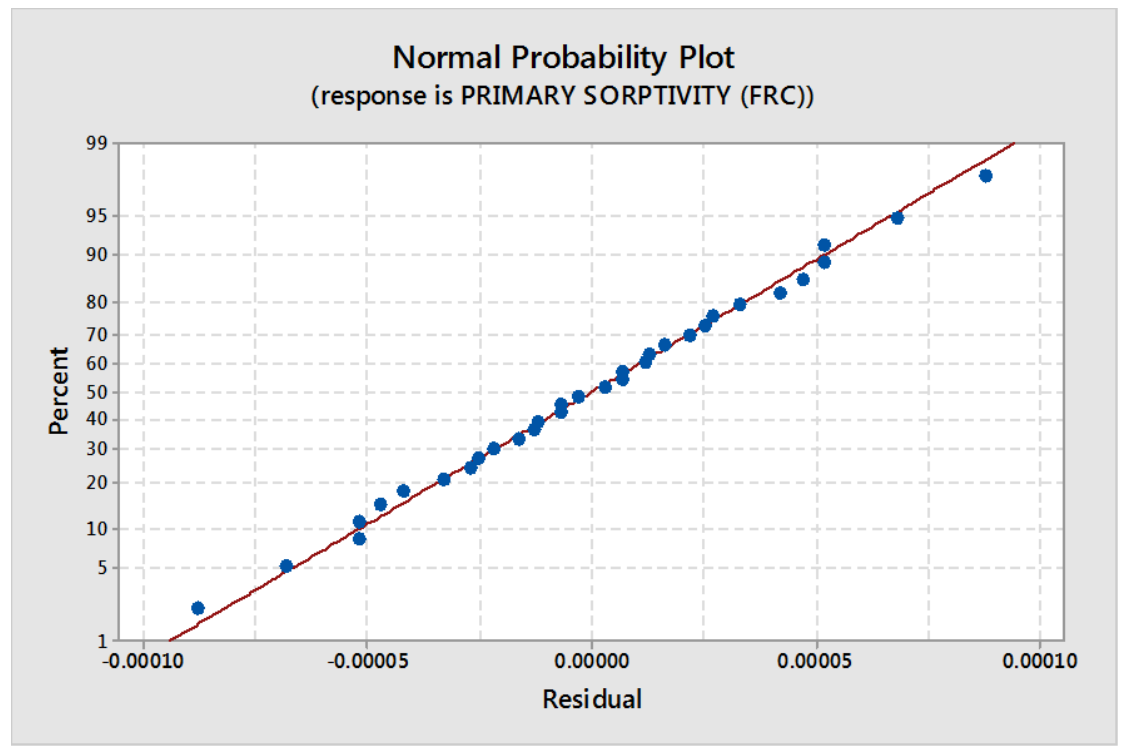

Figure 5.13: The residual normal probability plot for the full factorial model found by ANOVA for the primary sorptivity

Figure 5.14 shows the main effects plot for the mean primary sorptivity. As seen in RCPT results, the two preceding figures show that the main effects age of healing (A) and types of bacteria (B) are found to be statistically significant for increasing the self-healing characteristics by reducing the sorptivity of fibre reinforced mortar because the changes from one level to the other level is high for both of the factors A and B. Also, in the case of factor A, the changes from level 1 (7 days) to level 2 (120 days) is very high compared to the changes from level 2 to level 3 and level 3 (180 days) to level 4 (240 days). This indicates that the main reduction in sorptivity also took place during the period from level 1 to level 2. That is, similar to the RCPT results, most of the self-healing mechanism happened during the period of healing from 7 days to 120 days and the healing effect during the period from 120 days to 240 days was mostly insignificant. Evidently, the lowest sorptivity was obtained for 240 days of healing. In the case of factor B, it is found that the changes from level 1 (no bacteria) to level 2 (S. ureae) is very significant (Figure 5.14). This result proves that the bacteria play a significant role in reducing the sorptivity thereby increasing the self-healing efficiency. It can be seen that the variation of sorptivity for B. subtilis and $S$. pasteurii is almost same and variation from level 2 (S. ureae) to level 4 (B. subtilis) is very small. Hence, it can be concluded that the both of the bacterial species B. subtilis and S. pasteurii has very good healing efficiency in terms of sorptivity. Similar to RCP results, the types of carrier (C) 
do not have much influence on reducing the sorptivity because its variation from level 1(zeolite) to level 2 (pumice) is negligible. Therefore, it can be concluded that selected carrier materials do not have much influence on reducing sorptivity.

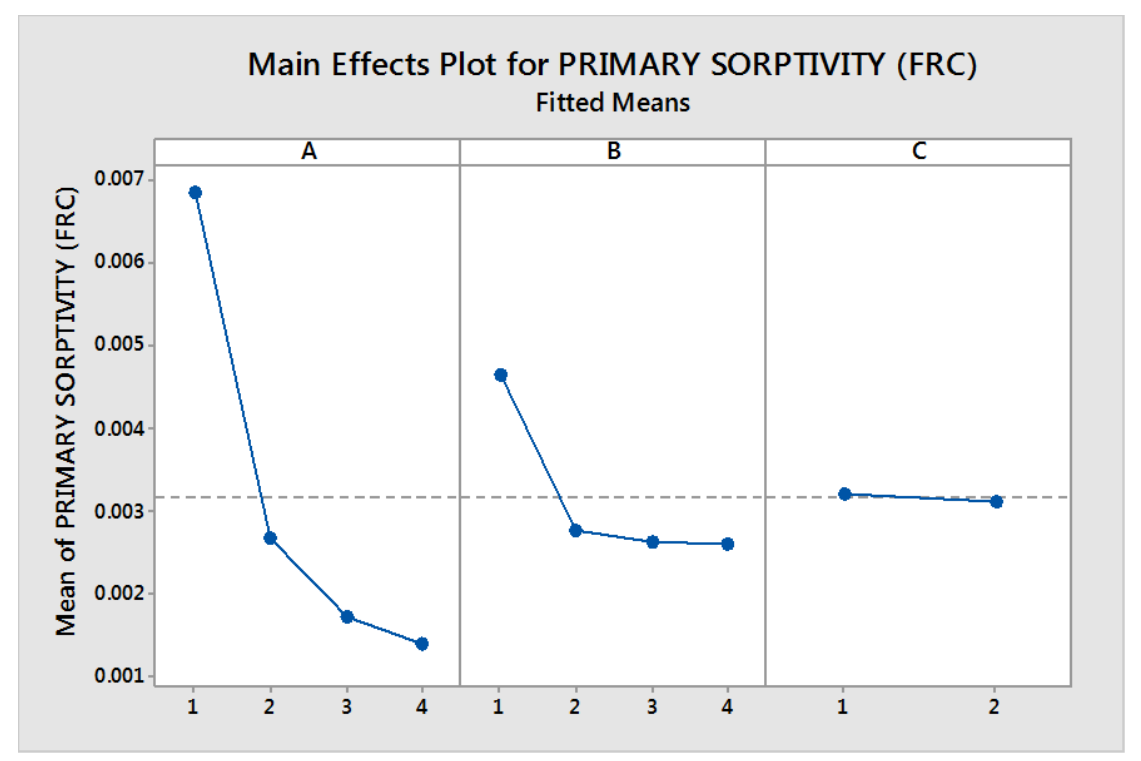

Figure 5.14: Main effect plot for the sorptivity of fibre reinforced mortar

The optimum points are $4^{\text {th }}$ level for age of healing (240 days), $4^{\text {th }}$ level for the types of bacteria (S. pasteurii) and $2^{\text {nd }}$ level for the types of carrier (pumice) (Figure 5.14). From the interaction plot (Figure 5.11) it can be seen that the optimum levels for the interaction terms are $\mathrm{A}_{4} \mathrm{xB}_{4}$ which coincide with the optimum levels of the main effects. The notation for optimum points is $\mathrm{A}_{4} \mathrm{~B}_{4} \mathrm{C}_{2}$. This corresponds to the $32^{\text {nd }}$ trial run in the full factorial experiment.

\subsubsection{Regression model of the mean sorptivity}

Characteristic regression equation (Eq. 5.8) for the sorptivity model is as follows:

PRIMARY SORPTIVITY $=0.003165+0.003691 \mathrm{~A} 1 \boldsymbol{- 0 . 0 0 0 4 9 1 ~ A 2 ~}-0.001429 \mathrm{A3}$

$$
\begin{aligned}
& -0.001771 \mathrm{~A} 4+0.001492 \mathrm{~B} 1-0.000399 \mathrm{~B} 2-0.000530 \mathrm{~B} 3 \\
& -0.000563 \mathrm{~B} 4+0.000047 \mathrm{C} 1-0.000047 \mathrm{C} 2-0.001323 \mathrm{~A} 1 * \mathrm{~B} 1 \\
& +0.000358 \mathrm{~A} 1 * \mathrm{~B} 2+0.000504 \mathrm{~A} 1 * \mathrm{~B} 3+0.000462 \mathrm{~A} 1 * \mathrm{~B} 4 \\
& +0.000073 \mathrm{~A} 2 * \mathrm{~B} 1+0.000044 \mathrm{~A} 2 * \mathrm{~B} 2-0.000104 \mathrm{~A} 2 * \mathrm{~B} 3 \\
& -0.000013 \mathrm{~A} 2 * \mathrm{~B} 4+0.000622 \mathrm{~A} 3 * \mathrm{~B} 1-0.000207 \mathrm{~A} 3 * \mathrm{~B} 2 \\
& -0.000196 \mathrm{~A} 3 * \mathrm{~B} 3-0.000218 \mathrm{~A} 3 * \mathrm{~B} 4+0.000629 \mathrm{~A} 4 * \mathrm{~B} 1 \\
& -0.000195 \mathrm{~A} 4 * \mathrm{~B} 2-0.000204 \mathrm{~A} 4 * \mathrm{~B} 3-0.000231 \mathrm{~A} 4 * \mathrm{~B} 4 \ldots . .(\mathrm{Eq} .5 .8)
\end{aligned}
$$


where A: Ages of healing, B: Types of bacteria, C: Types of carrier material and 1, 2, 3, 4 are levels.

\subsubsection{Statistical Analysis results when the response is mean UPV}

Table 5.11 shows the ANOVA table for the mean UPV. It can be seen that all two way interactions are significant because $\mathrm{p}$ value is $<0.05$.for $95 \%$ confidence level. Moreover, a strong interaction between the parameters can be noticed from the interaction plot (Figure 5.9) because of the nonparallelism of the lines in the interaction plot obtained from ANOVA.

When the interaction between A (age of healing) and B (types of bacteria) is considered, for level 1 (7days) of factor A, UPV of B. subtilis (level 3 of factor B) is slightly less than that of S. ureae (level 2 of factor B). Conversely, UPV is slightly higher for B. subtilis compared to $S$. ureae for level 3 (180 days) and level 4 (240 days) of factor A. Also, it is spotted that the variation between the UPV value of bacteria incorporated specimen and the specimen without bacteria is increased with the increase in the levels of factor A (age of healing). That is, the UPV of the bacteria incorporated 240 days water immersed specimen is very much higher than that of the specimen without bacteria. This increase might be due to the filling of crack and pores by bacterial mineral precipitation for the longer period of time.

Interaction plot (Figure 5.15) between the age of healing (A) and types of carrier (C) and also the types of bacteria (B) and types of carrier (C) shows only a slight non-parallelism. 


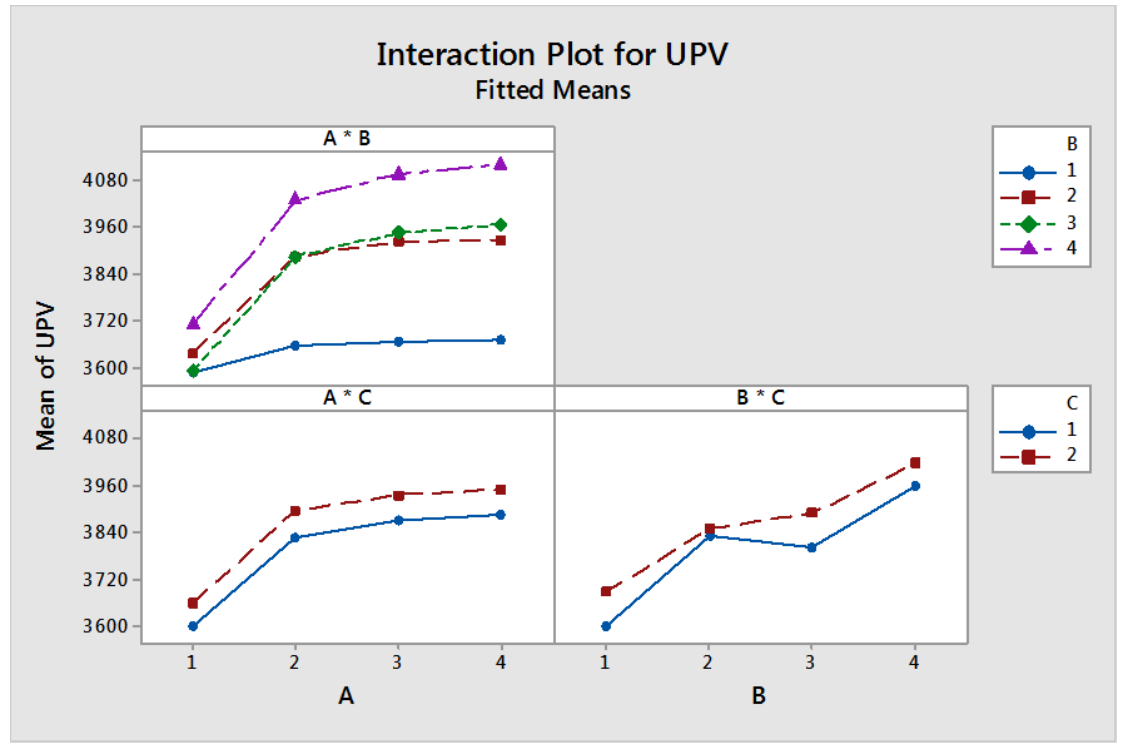

Figure 5.15: Two-way interaction plots for the UPV of fibre reinforced mortar

Table 5.11: ANOVA table for the mean UPV based on the full factorial design

\begin{tabular}{|crrrrr|}
\hline Analysis of Variance & & & & & \\
Source & & & & & \\
Model & DF & Adj SS & Adj MS & F-Value & P-Value \\
Linear & 22 & 2079240 & 94511 & 11411.59 & 0.000 \\
A & 7 & 1895615 & 270802 & 32697.63 & 0.000 \\
B & 3 & 873797 & 291266 & 35168.45 & 0.000 \\
C & 3 & 957683 & 319228 & 38544.71 & 0.000 \\
2-Way Interactions & 15 & 183625 & 12242 & 1478.10 & 0.000 \\
A*B & 9 & 169771 & 18863 & 2277.64 & 0.000 \\
A*C & 3 & 176 & 59 & 7.09 & 0.001 \\
B*C & 3 & 13678 & 4559 & 550.50 & 0.000 \\
Error & 41 & 340 & 8 & & \\
Lack-of-Fit & 9 & 340 & 38 & & \\
Pure Error & 32 & 0 & 0 & & \\
Total & 63 & 2079580 & & & \\
& & & & & \\
& & & & & \\
\end{tabular}

The residual and normal plots are shown in Figures 5.16 and 5.17 respectively, it can be seen that the normal plot is linear and the normality assumption holds. No obvious pattern is observed in 
the residuals versus the fitted values graph of the chosen model. Therefore the constant variance assumption of the error is satisfied.

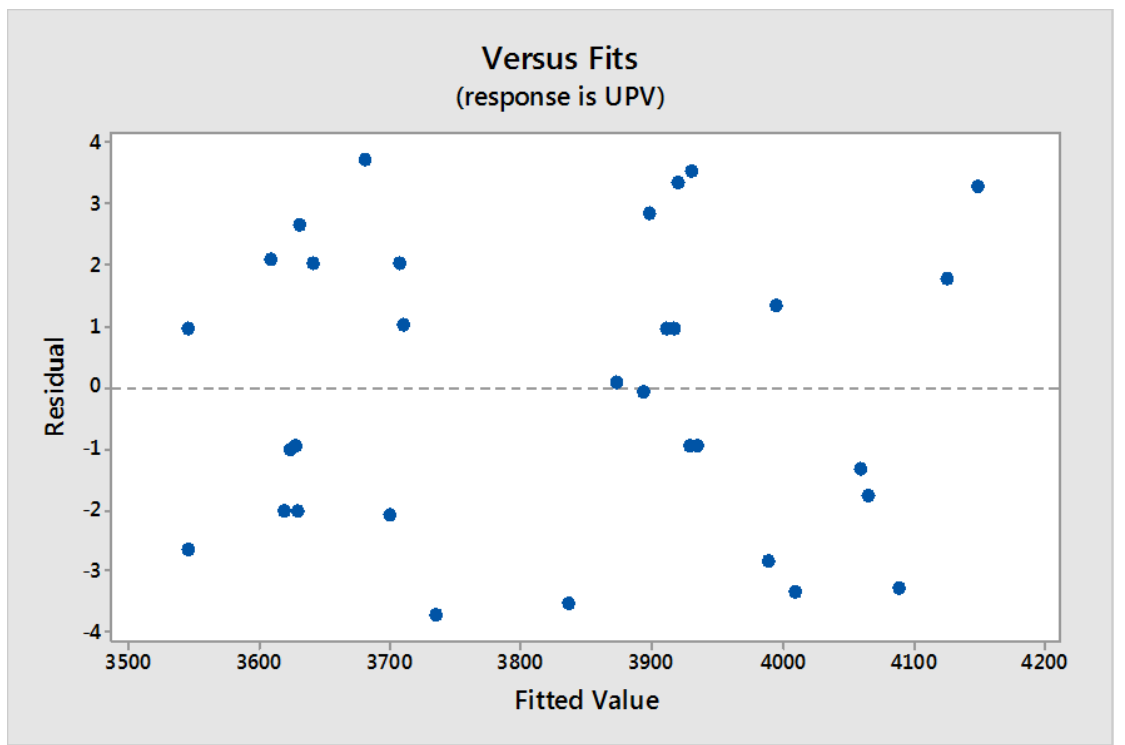

Figure 5.16: The residuals versus fitted values of the full factorial model found by ANOVA for the UPV

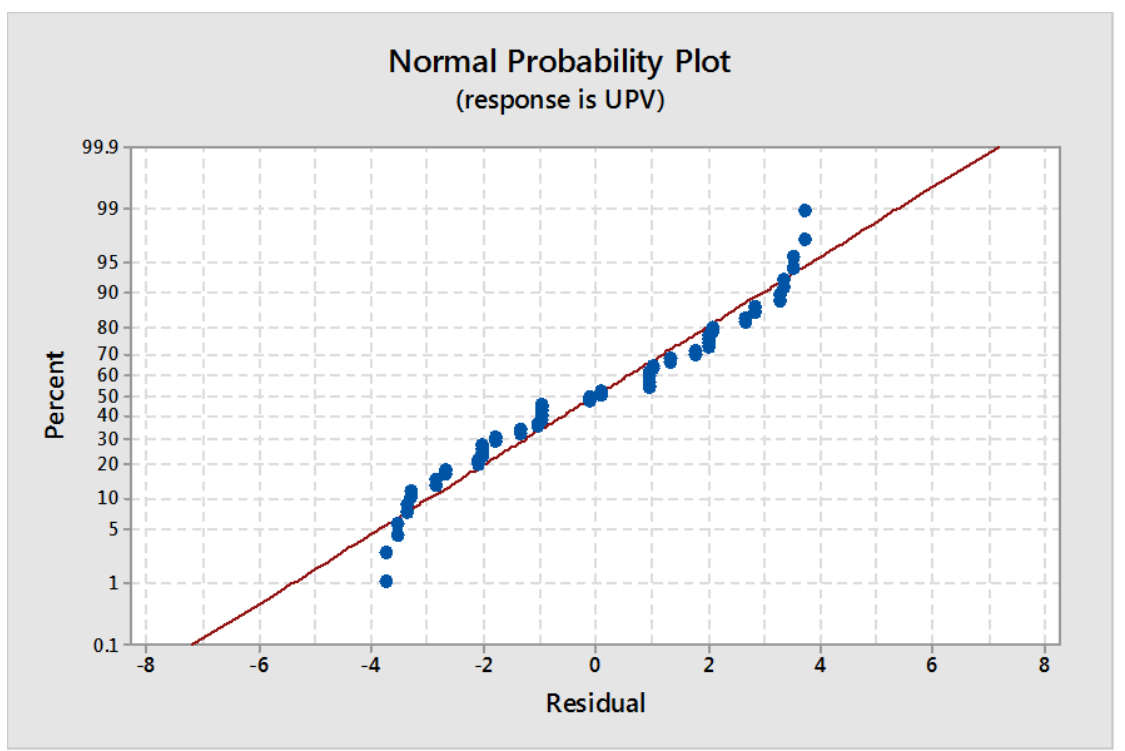

Figure 5.17: The residual normal probability plot for the full factorial model found by ANOVA for the UPV 
Figure 5.18 illustrates the main effect plot for the mean UPV. The obtained UPV value is maximum at the $4^{\text {th }}$ level (240 days) of factor A (age of healing). However, it should be noticed that the increase in UPV after 120 days of healing (level 2) is significantly reduced and the variation is almost negligible from level 3(180 days) to level 4 (240 days). This indicates that the major selfhealing occurred during the period from 7 days to 120 days. Main effect plot of factor B disclosed that the UPV of bacteria incorporated specimen (level 2, level 3 and level 4) is much higher than that of the specimen without bacteria (level 1). This result shows the role of bacteria in increasing the increasing the effectiveness of self-healing. Besides, it is found from Figure 5.18 that the UPV for S. pasteurii (level 4) is higher compared to that of the other two bacteria. At the same time, UPV value of S. ureae (level 2) and B. subtilis (level 3) appeared to be very close after considering the interaction plot (Figure 5.9) along with the main effect plot (Figure 5.18). Therefore, as far as the UPV value is concerned, it can be concluded that the bacteria $S$. pasteurii have more selfhealing efficiency. Unlike the other two responses such as RCP and sorptivity, UPV of specimen with pumice (level 2) is much higher than that of specimen with zeolite.

Therefore, the optimum points are $4^{\text {th }}$ level for age of healing (240 days), $4^{\text {th }}$ level for the types of bacteria (S. pasteurii) and $2^{\text {nd }}$ level for the types of carrier (pumice) (Figure 5.18). From the interaction plot (Figure 5.15) it can be seen that the optimum levels for the interaction terms are $\mathrm{A}_{4} \mathrm{XB}_{4}$ which coincide with the optimum levels of the main effects. The notation for optimum points is $\mathrm{A}_{4} \mathrm{~B}_{4} \mathrm{C}_{2}$. This corresponds to the $28^{\text {th }}$ trial run in the full factorial experiment. 


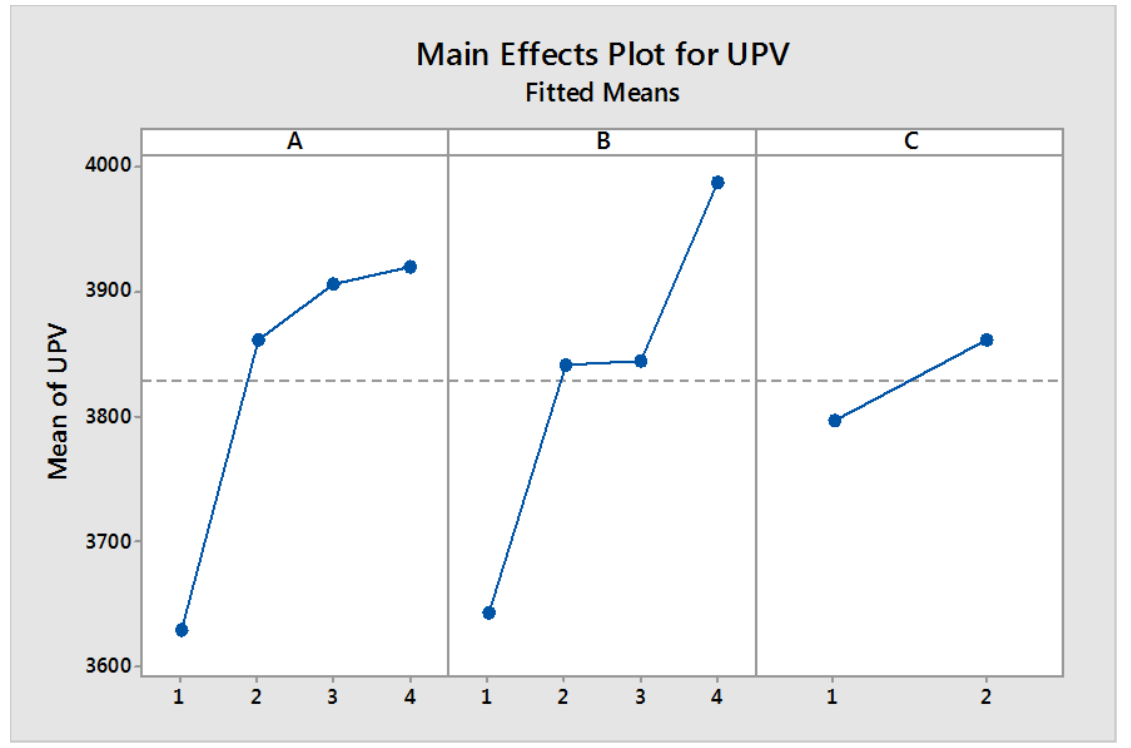

Figure 5.18: Main effect plot for the UPV of fibre reinforced mortar

\subsubsection{Regression model of the mean UPV}

Characteristic regression equation (Eq. 5.9) for the UPV model is given as follows:

$$
\begin{aligned}
\mathrm{UPV}= & 3829.47-199.094 \mathrm{~A} 1+33.031 \mathrm{~A} 2+76.156 \mathrm{A3}+89.906 \mathrm{~A} 4-185.719 \mathrm{~B} 1+12.031 \mathrm{~B} 2 \\
& +15.656 \mathrm{~B} 3+158.031 \mathrm{~B} 4-31.656 \mathrm{C} 1+31.656 \mathrm{C} 2+143.34 \mathrm{~A} 1 * \mathrm{~B} 1-6.41 \mathrm{~A} 1 * \mathrm{~B} 2 \\
& -57.03 \mathrm{~A} 1 * \mathrm{~B} 3-79.91 \mathrm{~A} 1 * \mathrm{~B} 4-21.28 \mathrm{~A} 2 * \mathrm{~B} 1+8.97 \mathrm{~A} 2 * \mathrm{~B} 2+5.34 \mathrm{~A} 2 * \mathrm{~B} 3 \\
& +6.97 \mathrm{~A} 2 * \mathrm{~B} 4-55.91 \mathrm{~A} 3 * \mathrm{~B} 1+2.34 \mathrm{A3} * \mathrm{~B} 2+22.22 \mathrm{~A} 3 * \mathrm{~B} 3+31.34 \mathrm{~A} 3 * \mathrm{~B} 4 \\
& -66.16 \mathrm{~A} 4 * \mathrm{~B} 1-4.91 \mathrm{~A} 4 * \mathrm{~B} 2+29.47 \mathrm{~A} 4 * \mathrm{~B} 3+41.59 \mathrm{~A} 4 * \mathrm{~B} 4+2.531 \mathrm{~A} 1 * \mathrm{C} 1 \\
& -2.531 \mathrm{~A} 1 * \mathrm{C} 2-2.094 \mathrm{~A} 2 * \mathrm{C} 1+2.094 \mathrm{~A} 2 * \mathrm{C} 2-0.469 \mathrm{~A} 3 * \mathrm{C} 1+0.469 \mathrm{~A} 3 * \mathrm{C} 2 \\
& +0.031 \mathrm{~A} 4 * \mathrm{C} 1-0.031 \mathrm{~A} 4 * \mathrm{C} 2-11.844 \mathrm{~B} 1 * \mathrm{C} 1+11.844 \mathrm{~B} 1 * \mathrm{C} 2+23.156 \mathrm{~B} 2 * \mathrm{C} 1 \\
& -23.156 \mathrm{~B} 2 * \mathrm{C} 2-13.219 \mathrm{~B} 3 * \mathrm{C} 1+13.219 \mathrm{~B} * \mathrm{C} 2+1.906 \mathrm{~B} 4 * \mathrm{C} 1-1.906 \mathrm{~B} 4 * \mathrm{C} 2 \ldots . .(\mathrm{Eq} .5 .9)
\end{aligned}
$$

\subsubsection{Statistical Analysis results when the response is mean compressive strength}

Unlike the other three responses such as RCP, sorptivity and UPV, for compressive strength, the factor level for the factor A (age of healing) is chosen as level 1: 7 days, level 2: 28 days, level 3: 90 days and level 4: 180 days. These ages were chosen to support the general practices of compressive strength testing. The levels for remaining two factors are same as before.

Table 5.12 shows the ANOVA table for the mean compressive strength. It can be seen that all the two-way interactions are significant in the case of mean compressive strength because the $p$ value 
is $<0.05$ (Table 5.12). Also, a strong interaction between the parameters can be noticed from the interaction plot because of the non-parallelism of the lines in the interaction plot obtained from ANOVA (Figure 5.19).

When the interaction between A (age of healing) and B (types of bacteria) is considered, for level 1 (7days) and level 2 (28 days) of factor A, compressive strength of B. subtilis (level 3 of factor B) is slightly higher than that of $S$. pasteurii (level 4 of factor B). At the same time, the compressive strgnth is slightly higher for S. pasteurii compared to B. subtilis for level 3 (90 days) and level 4 (180 days) of factor A. Also, it is found that the variation between the compressive strength of bacteria incorporated specimen and the specimen without bacteria is increased when the levels of the age of healing (A) changes from 2 (28 days) to 4 (180 days). In other words, the compressive strength of the bacteria incorporated 180 days old specimen is much higher than that of the specimen without bacteria. This increase in compressive strength might be due to the bacterial precipitation over the long term.

Interaction plot (Figure 5.19) between the age of healing (A) and types of carrier (C) shows that for 90 days (level 3) and 180 days (level 4), the specimen with zeolite showed more compressive strength compared to that of pumice, even though the difference is small. However, for 7 days (level 1) and 28 days (level 2), the specimen with pumice showed more strength. Regarding the interaction plot between the types of bacteria (B) and types of carrier (C), it is found that zeolite incorporated and pumice incorporated bacteria (for all bacteria) have almost similar strength. On the other hand, for the specimen without bacteria but with pumice showed higher strength compared to that with zeolite. 


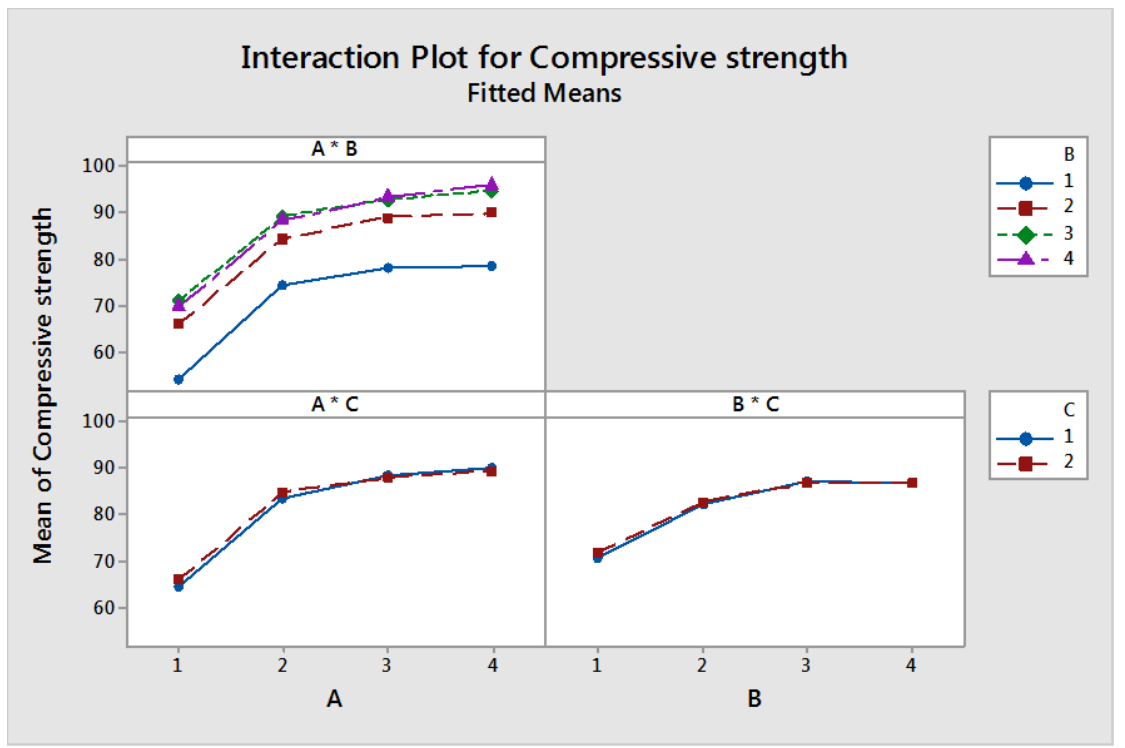

Figure 5.19: Interaction plot of compressive strength

Table 5.12: ANOVA table for the mean compressive strength

\begin{tabular}{|c|c|c|c|c|c|c|}
\hline \multicolumn{7}{|c|}{ Analysis of Variance } \\
\hline Source & & $\mathrm{DF}$ & Adj SS & Adj MS & F-Value & P-Value \\
\hline Model & & 22 & 4445.48 & 202.07 & 1431.55 & 0.000 \\
\hline Linear & & 7 & 4423.40 & 631.91 & 4476.80 & 0.000 \\
\hline A & & 3 & 3096.51 & 1032.17 & 7312.42 & 0.000 \\
\hline B & & 3 & 1325.44 & 441.81 & 3130.04 & 0.000 \\
\hline $\mathrm{C}$ & & 1 & 1.45 & 1.45 & 10.27 & 0.011 \\
\hline 2-Way & Interactions & 15 & 22.08 & 1.47 & 10.43 & 0.001 \\
\hline$A * B$ & & 9 & 9.55 & 1.06 & 7.52 & 0.003 \\
\hline$A * C$ & & 3 & 9.14 & 3.05 & 21.59 & 0.000 \\
\hline$B * C$ & & 3 & 3.39 & 1.13 & 8.00 & 0.007 \\
\hline Error & & 9 & 1.27 & 0.14 & & \\
\hline Total & & 31 & 4446.75 & & & \\
\hline \multicolumn{7}{|c|}{ Model Summary } \\
\hline S & $\mathrm{R}-\mathrm{sq}$ & \multicolumn{3}{|c|}{$\mathrm{R}-\mathrm{sq}$ (pred) } & & \\
\hline 0.375704 & $99.97 \%$ & 99.90 & & $9.64 \%$ & & \\
\hline
\end{tabular}


Residuals versus fitted values (Figure 5.20) and the normal probability plot (Figure 5.21) indicate that the error term has normal distribution with constant variance. As a result an adequate model explaining the mean response is achieved.

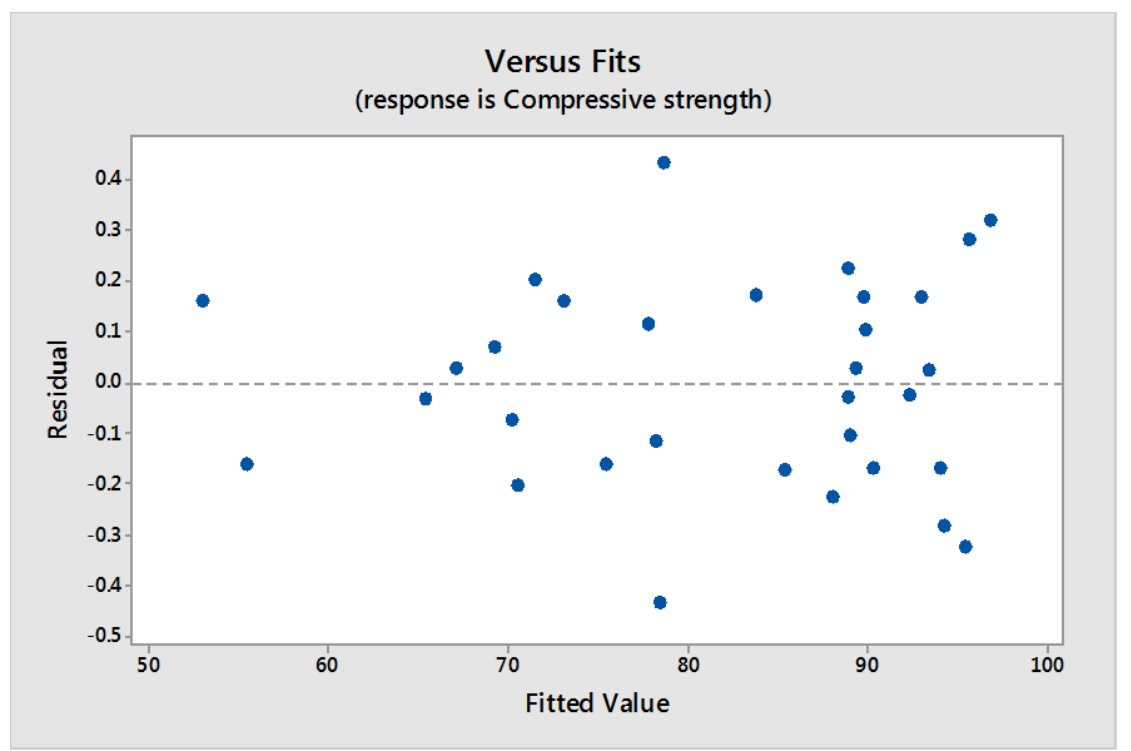

Figure 5.20: The residuals versus fitted values for the compressive strength

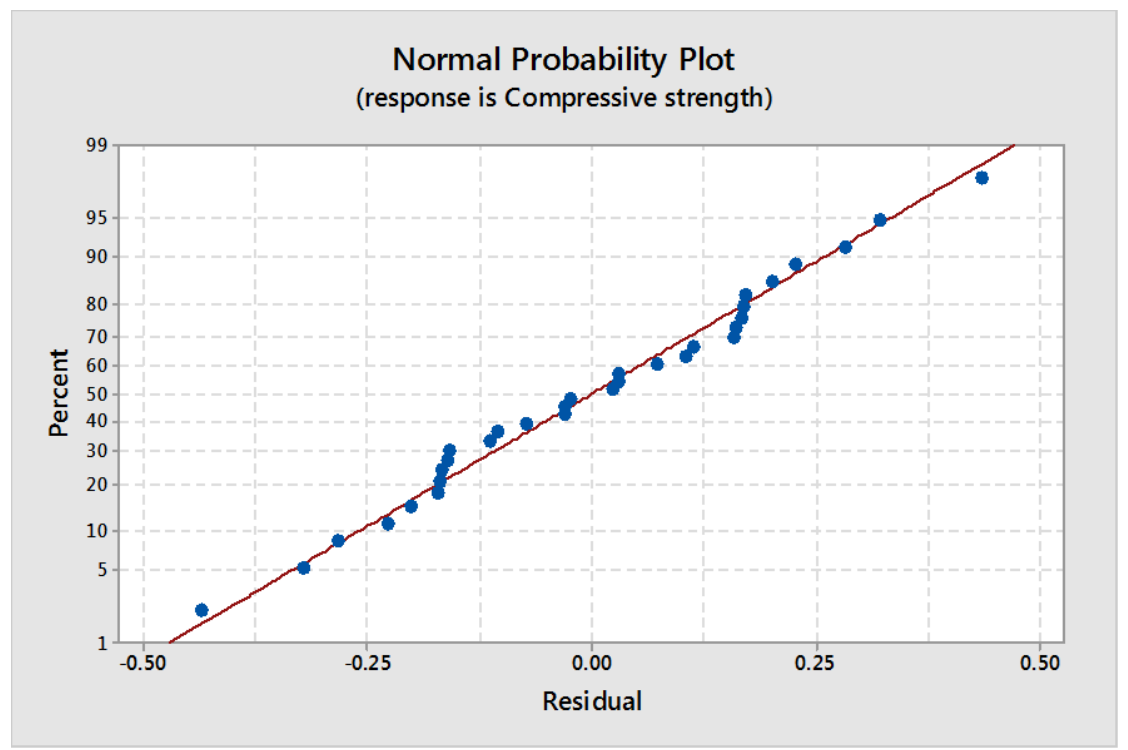

Figure 5.21: The residual normal probability plot for the compressive strength 
It can be seen from the main effect plot that (Figure 5.22), as expected, compressive strength is maximum at the $4^{\text {th }}$ level (180 days) of factor A. Increase in compressive strength after 28 days (level 2) is significantly reduced. Main effect plot of factor B revealed that the compressive strength of bacteria incorporated specimen (level 2, level 3 and level 4) is higher than that of the specimen without bacteria (level 1). This result proves that bacteria play a significant role in increasing the compressive strength thereby increasing the efficiency of self-healing. Furthermore, it can be observed from Figure 5.22 that the compressive strength for B. subtilis (level 3) is bit more than that of S. pasteurii, however, the difference is comparatively less. At the same time, in conjunction with the interaction plot (Figure 5.13), it can be noticed that the compressive strength is maximum for $B$. subtilis during 7 and 28 days and maximum for S. pasteurii during 90 and 180 days. This might be due to the action of bacteria. On the other hand, the compressive strength of the bacteria $S$. ureae is much less compared to the other two bacteria. Therefore, it can be inferred that the bacteria $B$. subtilis and $S$. pasteurii have more efficiency as a self-healing agent as far as the compressive strength is concerned. Regarding the types of carrier (factor C), it can be seen that pumice (level 2) has more compressive strength than zeolite (level), however, the deviation is very less.

Compressive strength modelling shows that, the optimum points are $4^{\text {th }}$ level for age of healing (180 days), $3^{\text {rd }}$ level for the types of bacteria (B. subtilis) and $2^{\text {nd }}$ level for the types of carrier (pumice) (Figure 5.22). The interaction plot (Figure 5.19) shows that the optimum levels for the interaction terms are $\mathrm{A}_{4} \mathrm{XB}_{3}$ in congruence with the optimum levels of the main effects. The notation for optimum points is $\mathrm{A}_{4} \mathrm{~B}_{3} \mathrm{C}_{2}$, which corresponds to the $58^{\text {th }}$ trial run in the full factorial experiment. 


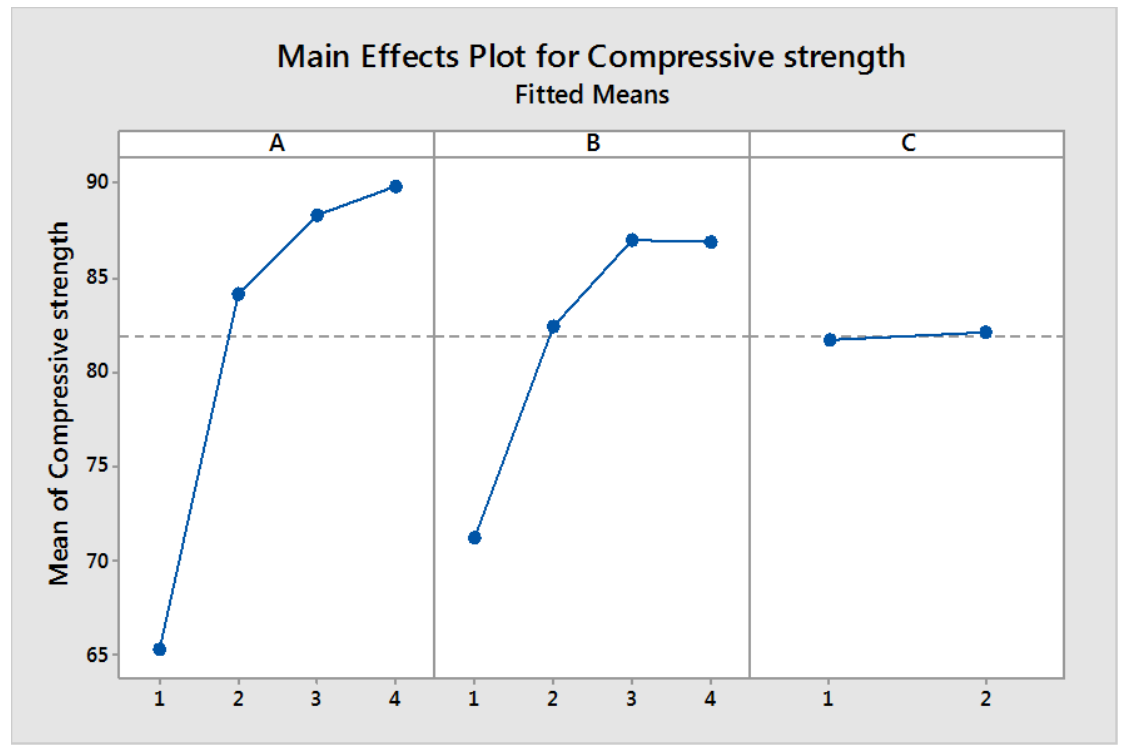

Figure 5.22: Main effect plot for compressive strength

\subsubsection{Regression model of the mean compressive strength}

Characteristic regression equation (Eq. 5.10) for the compressive strength model is as follows:

Compressive strength $=81.9372-16.647 \mathrm{~A} 1+2.244 \mathrm{~A} 2+6.435 \mathrm{~A} 3+7.968 \mathrm{~A} 4-10.677 \mathrm{~B} 1$

$$
\begin{aligned}
& +0.540 \mathrm{~B} 2+5.132 \mathrm{~B} 3+5.005 \mathrm{~B} 4-0.2128 \mathrm{C} 1+0.2128 \mathrm{C} 2 \\
& -0.393 \mathrm{~A} 1 * \mathrm{~B} 1+0.390 \mathrm{~A} 1 * \mathrm{~B} 2+0.588 \mathrm{~A} 1 * \mathrm{~B} 3-0.585 \mathrm{~A} 1 * \mathrm{~B} 4 \\
& +0.756 \mathrm{~A} 2 * \mathrm{~B} 1-0.162 \mathrm{~A} 2 * \mathrm{~B} 2+0.142 \mathrm{~A} 2 * \mathrm{~B} 3-0.737 \mathrm{~A} 2 * \mathrm{~B} 4 \\
& +0.305 \mathrm{~A} 3 * \mathrm{~B} 1+0.202 \mathrm{~A} 3 * \mathrm{~B} 2-0.634 \mathrm{A3} * \mathrm{~B} 3+0.127 \mathrm{~A} 3 * \mathrm{~B} 4 \\
& -0.668 \mathrm{~A} 4 * \mathrm{~B} 1-0.430 \mathrm{~A} 4 * \mathrm{~B} 2-0.097 \mathrm{~A} 4 * \mathrm{~B} 3+1.195 \mathrm{~A} 4 * \mathrm{~B} 4 \\
& -0.557 \mathrm{~A} 1 * \mathrm{C} 1+0.557 \mathrm{~A} 1 * \mathrm{C} 2-0.508 \mathrm{~A} 2 * \mathrm{C} 1+0.508 \mathrm{~A} 2 * \mathrm{C} 2 \\
& +0.478 \mathrm{~A} 3 * \mathrm{C} 11-0.478 \mathrm{~A} 3 * \mathrm{C} 2+0.588 \mathrm{~A} 4 * \mathrm{C} 1-0.588 \mathrm{~A} 4 * \mathrm{C} 2 \\
& -0.490 \mathrm{~B} 1 * \mathrm{C} 1+0.490 \mathrm{~B} 1 * \mathrm{C} 2-0.100 \mathrm{~B} 2 * \mathrm{C} 1+0.100 \mathrm{~B} 2 * \mathrm{C} 2 \\
& +0.292 \mathrm{~B} 3 * \mathrm{C} 1-0.292 \mathrm{~B} 3 * \mathrm{C} 2+0.298 \mathrm{~B} 4 * \mathrm{C} 1-0.298 \mathrm{~B} 4 * \mathrm{C} 2 \ldots . .(\mathrm{Eq} .5 .10)
\end{aligned}
$$

where A: Ages of healing, B: Types of bacteria, C: Types of carrier material and 1, 2, 3, 4 are levels. 


\subsubsection{Validation of the proposed model}

\subsubsection{Verification of the proposed model using existing data}

In order to validate the developed regression models, there is a general lack of availability of historical experimental research data. In the current study, regression models were developed based on the experiments conducted on three out of six replicates of fibre reinforced mortar to predict the self-healing characteristics such as RCP and primary sorptivity. The remaining three replicates of each characteristics were used to verify the accuracy of such prediction and the predicted values vs. experimental values of fibre reinforced mortar were plotted. At the same time, regression models were developed for the experimental data conducted on two replicates of fiber reinforced mortar to predict UPV. The remaining specimen were used to validate the accuracy of the model. Experimental data obtained for normal mortar with holes were also used to validate all the previously developed regression models. Compressive strength of ECC were used to validate the regression model of compressive strength. However, sufficient historical data for sorptivity RCP and UPV were not available for ECC.

The following Tables 5.13 through 5.16 show the predicted RCP values (Y') of fibre reinforced mortar compared to experimental values (Y) for both fibre reinforced and normal mortar at 7, 120, 180 and 240 days of healing. It can be observed form Tables 5.13 to 5.16 that percentage variation between the predicted and experimental values of fibre reinforced mortar ranges from $0.14-1.23 \%$ for 7 days, $0.18-2.07 \%$ for 120 days, $0.42-2.04 \%$ for 180 days and $0.45-1.94 \%$ for 240 days. This confirms that the regression modelling is ideally suited to the fibre reinforced mortar. Conversely, the percentage variation for normal mortar was found to be significantly higher (79 $\%$ ) for 7 days, $81 \%$ for 120 days, $80 \%$ for 180 days and $81 \%$ for 240 days (Table 5.13 to 5.16). This suggests that the regression model developed for fibre reinforced mortar is not suitable for normal mortar with bacteria. Therefore, a separate model may have to be developed for normal mortar. 
Table 5.13: Comparison of predicted ( $Y^{\prime}$ ) and experimental ( $\mathrm{Y}$ ) RCP of FR mortar and normal mortar for healing period of 7 days

\begin{tabular}{|c|c|c|c|c|c|c|}
\hline Specimen & \multicolumn{3}{|c|}{ FR mortar } & \multicolumn{3}{c|}{ Normal mortar } \\
\hline & $\mathbf{Y}$ & $\mathbf{Y}^{\prime}$ & Variation (\%) & $\mathbf{Y}$ & $\mathbf{Y}^{\prime}$ & Variation (\%) \\
\hline S. ureae+zeolite & 1520 & 1501.25 & 1.23 & 7213 & 1501.25 & 79.18 \\
\hline S. ureae+pumice & 1489 & 1470.83 & 1.22 & 7145 & 1470.83 & 79.41 \\
\hline B. subtilis+zeolite & 1501 & 1489.3 & 0.77 & 7201 & 1489.3 & 79.31 \\
\hline B. subtilis+pumice & 1437 & 1452.62 & -1.08 & 7138 & 1452.62 & 79.64 \\
\hline S. pasteurii+zeolite & 1481 & 1467 & 0.94 & 7189 & 1467 & 79.59 \\
\hline S. pasteurii+pumice & 1450 & 1452.08 & -0.14 & 7015 & 1452.08 & 79.30 \\
\hline
\end{tabular}

Table 5.14: Comparison of predicted (Y') and experimental (Y) RCP of FR mortar and normal mortar for healing period of 120 days

\begin{tabular}{|c|c|c|c|c|c|c|}
\hline Specimen & \multicolumn{3}{|c|}{ FR mortar } & \multicolumn{3}{c|}{ Normal mortar } \\
\hline & $\mathbf{Y}$ & $\mathbf{Y}^{\prime}$ & Variation (\%) & $\mathbf{Y}$ & $\mathbf{Y}^{\prime}$ & Variation (\%) \\
\hline S. ureae+zeolite & 1081 & 1069.55 & 1.05 & 5149 & 1069.55 & 79.22 \\
\hline S. ureae+pumice & 1060 & 1041.37 & 1.75 & 5098 & 1041.37 & 79.57 \\
\hline B. subtilis+zeolite & 942 & 957.2 & -1.61 & 4544 & 957.2 & 78.93 \\
\hline B. subtilis+pumice & 904 & 922.76 & -2.07 & 4482 & 922.76 & 79.41 \\
\hline S. pasteurii+zeolite & 936 & 934.3 & 0.18 & 4946 & 934.3 & 81.10 \\
\hline S. pasteurii+pumice & 931 & 921.62 & 1.00 & 4398 & 921.62 & 79.04 \\
\hline
\end{tabular}

Table 5.15: Comparison of predicted ( $\mathrm{Y}^{\prime}$ ) and experimental (Y) RCP of FR mortar and normal mortar for healing period of 180 days

\begin{tabular}{|c|c|c|c|c|c|c|}
\hline \multirow[t]{2}{*}{ Specimen } & \multicolumn{3}{|c|}{ FR mortar } & \multicolumn{3}{|c|}{ Normal mortar } \\
\hline & $\mathbf{Y}$ & $\mathbf{Y}^{\prime}$ & Variation $(\%)$ & $\mathbf{Y}$ & $\mathbf{Y}^{\prime}$ & Variation $(\%)$ \\
\hline S. ureae+zeolite & 951 & 945.45 & 0.58 & 4548 & 945.45 & 79.21 \\
\hline S. ureae+pumice & 939 & 925.53 & 1.43 & 4426 & 925.53 & 79.08 \\
\hline B. subtilis+zeolite & 811 & 827.6 & -2.04 & 3760 & 827.6 & 77.98 \\
\hline B. subtilis+pumice & 798 & 801.42 & -0.42 & 3693 & 801.42 & 78.29 \\
\hline S. pasteurii+zeolite & 769 & 776.7 & -1.00 & 4030 & 776.7 & 80.72 \\
\hline S. pasteurii+pumice & 759 & 772.28 & -1.74 & 3539 & 772.28 & 78.17 \\
\hline
\end{tabular}


Table 5.16: Comparison of predicted ( $\mathrm{Y}^{\prime}$ ) and experimental (Y) RCP of FR mortar and normal mortar for healing period of 240 days

\begin{tabular}{|c|c|c|c|c|c|c|}
\hline Specimen & \multicolumn{3}{|c|}{ FR mortar } & \multicolumn{3}{c|}{ Normal mortar } \\
\hline & Y & $\mathbf{Y}^{\prime}$ & Variation(\%) & Y & $\mathbf{Y}^{\prime}$ & Variation(\%) \\
\hline S. ureae+zeolite & 878 & 869.75 & 0.93 & 4198 & 869.75 & 79.28 \\
\hline S. ureae+pumice & 856 & 845.33 & 1.24 & 4081 & 845.33 & 79.28 \\
\hline B. subtilis+zeolite & 711 & 724.8 & -1.94 & 3455 & 724.8 & 79.02 \\
\hline B. subtilis+pumice & 691 & 694.12 & -0.45 & 3292 & 694.12 & 78.91 \\
\hline S. pasteurii+zeolite & 681 & 677 & 0.58 & 3574 & 677 & 81.05 \\
\hline S. pasteurii+pumice & 676 & 668.08 & 1.17 & 2990 & 668.08 & 77.65 \\
\hline
\end{tabular}

Following Tables 5.17 to 5.20 show the comparison of predicted (Y') and experimental (Y) values obtained by the sorptivity test. At 7 days, the variation found for fibre reinforced mortar is 0.14 to $1.81 \%$ (Table 5.17), and at 240 days, the maximum variation was found to be $3.0 \%$ (Table 5.20), which suggests the suitability of the model for fibre reinforced mortar. Similar to RCP studies, the variation (of sorptivity) observed for normal mortar was significantly higher compared to that of fibre reinforced mortar. The variations ranged from $31.3 \%$ (Table 5.17) to as high as $85.8 \%$ at 240 days (Table 5.20). This high variation rules out the suitability of this regression model for normal mortar.

Table 5.17: Comparison of predicted ( $\left.Y^{\prime}\right)$ and experimental (Y) primary sorptivity of FR mortar and normal mortar for healing period of 7 days

\begin{tabular}{|c|c|c|c|c|c|c|}
\hline Specimen & \multicolumn{3}{|c|}{ FR mortar } & \multicolumn{3}{c|}{ Normal mortar } \\
\hline & Y & $\mathbf{Y}^{\prime}$ & Variation (\%) & Y & $\mathbf{Y}^{\prime}$ & Variation (\%) \\
\hline S. ureae+zeolite & 0.006989 & 0.006862 & 1.81 & 0.01080 & 0.006862 & 37.00 \\
\hline S. ureae+pumice & 0.006662 & 0.006768 & -1.59 & 0.01026 & 0.006768 & 34.04 \\
\hline B. subtilis+zeolite & 0.006833 & 0.006877 & -0.64 & 0.01041 & 0.006877 & 33.94 \\
\hline B. subtilis+pumice & 0.006681 & 0.006783 & -1.52 & 0.00988 & 0.006783 & 31.37 \\
\hline S. pasteurii+zeolite & 0.006792 & 0.006802 & -0.14 & 0.01087 & 0.006802 & 37.43 \\
\hline S. pasteurii+pumice & 0.006736 & 0.006708 & 0.41 & 0.01032 & 0.006708 & 35.01 \\
\hline
\end{tabular}


Table 5.18: Comparison of predicted ( $\left.\mathrm{Y}^{\prime}\right)$ and experimental (Y) primary sorptivity of FR mortar and normal mortar for healing period of 120 days

\begin{tabular}{|c|c|c|c|c|c|c|}
\hline Specimen & \multicolumn{3}{|c|}{ FR mortar } & \multicolumn{3}{c|}{ Normal mortar } \\
\hline & Y & $\mathbf{Y}^{\prime}$ & Variation (\%) & Y & $\mathbf{Y}^{\prime}$ & Variation (\%) \\
\hline S. ureae+zeolite & 0.002349 & 0.002366 & -0.72 & 0.00551 & 0.002366 & 57.04 \\
\hline S. ureae+pumice & 0.002209 & 0.002272 & -2.85 & 0.00534 & 0.002272 & 57.47 \\
\hline B. subtilis+zeolite & 0.002111 & 0.002087 & 1.13 & 0.00490 & 0.002087 & 57.37 \\
\hline B. subtilis+pumice & 0.002006 & 0.001993 & 0.64 & 0.00457 & 0.001993 & 56.39 \\
\hline S. pasteurii+zeolite & 0.002165 & 0.002145 & 0.92 & 0.00510 & 0.002145 & 57.93 \\
\hline S. pasteurii+pumice & 0.002035 & 0.002051 & -0.78 & 0.00581 & 0.002051 & 64.71 \\
\hline
\end{tabular}

Table 5.19: Comparison of predicted ( $\left.\mathrm{Y}^{\prime}\right)$ and experimental (Y) primary sorptivity of FR mortar and normal mortar for healing period of 180 days

\begin{tabular}{|c|c|c|c|c|c|c|}
\hline Specimen & \multicolumn{3}{|c|}{ FR mortar } & \multicolumn{3}{c|}{ Normal mortar } \\
\hline & Y & $\mathbf{Y}^{\prime}$ & Variation (\%) & Y & $\mathbf{Y}^{\prime}$ & Variation (\%) \\
\hline S. ureae+zeolite & 0.001189 & 0.001177 & 1.00 & 0.00509 & 0.001177 & 76.89 \\
\hline S. ureae+pumice & 0.001106 & 0.001083 & 2.07 & 0.00499 & 0.001083 & 78.30 \\
\hline B. subtilis+zeolite & 0.001042 & 0.001057 & -1.43 & 0.00416 & 0.001057 & 74.58 \\
\hline B. subtilis+pumice & 0.000969 & 0.000963 & 0.61 & 0.00373 & 0.000963 & 74.17 \\
\hline S. pasteurii+zeolite & 0.001036 & 0.001002 & 3.28 & 0.00402 & 0.001002 & 75.09 \\
\hline S. pasteurii+pumice & 0.000936 & 0.000908 & 2.99 & 0.00364 & 0.000908 & 75.07 \\
\hline
\end{tabular}

Table 5.20: Comparison of predicted ( $\left.Y^{\prime}\right)$ and experimental (Y) primary sorptivity of FR mortar and normal mortar for healing period of 240 days

\begin{tabular}{|c|l|l|l|l|l|c|}
\hline Specimen & \multicolumn{3}{|c|}{ FR mortar } & \multicolumn{3}{c|}{ Normal mortar } \\
\hline & $\mathbf{Y}$ & $\mathbf{Y}^{\prime}$ & Variation (\%) & Y & $\mathbf{Y}^{\prime}$ & Variation (\%) \\
\hline S. ureae+zeolite & 0.000865 & 0.000847 & 2.08 & 0.00558 & 0.000847 & 84.82 \\
\hline S. ureae+pumice & 0.000731 & 0.000753 & -3.00 & 0.00441 & 0.000753 & 82.92 \\
\hline B. subtilis+zeolite & 0.000711 & 0.000707 & 0.56 & 0.00499 & 0.000707 & 85.83 \\
\hline B. subtilis+pumice & 0.000599 & 0.000613 & -2.33 & 0.00350 & 0.000613 & 82.48 \\
\hline S. pasteurii+zeolite & 0.000632 & 0.000647 & -2.37 & 0.00318 & 0.000647 & 79.67 \\
\hline S. pasteurii+pumice & 0.000549 & 0.000553 & -0.72 & 0.00295 & 0.000553 & 81.27 \\
\hline
\end{tabular}


The UPV test results which shows the comparison of predicted values to experimental results, and their variations are depicted in the following tables (Table 5.21 and 5.22). This test was carried out only on fibre reinforced specimens as generating a crack on the normal mortar specimen was difficult. From the values given in the tables, it is evident that the variation is minor between the predicted and actual experimental results. The smallest variation was $0.07 \%$ at 180 days (Table 5.22) and the highest being 3.25\% at 7 days (Table 5.21). As in the case of both RCP and sorptivity tests, the results of UPV test also indicates that the regression model holds exceptionally good capability to predict the results

Table 5.21: Comparison of predicted ( $Y^{\prime}$ ) and experimental (Y) UPV of FR mortar for healing period of 7 and 120 days

\begin{tabular}{|c|c|c|c|c|c|c|}
\hline Specimen & \multicolumn{3}{|c|}{$\mathbf{7 ~ d a y s ~}^{\prime}$} & \multicolumn{3}{c|}{ 120 days } \\
\hline & $\mathbf{Y}$ & $\mathbf{Y}^{\prime}$ & Variation (\%) & \multicolumn{1}{|c|}{$\mathbf{Y}$} & \multicolumn{1}{c|}{$\mathbf{Y}^{\prime}$} & Variation (\%) \\
\hline S. ureae+zeolite & 3589 & 3630.03 & -1.14 & 3811 & 3872.91 & -1.62 \\
\hline S. ureae+pumice & 3663 & 3641.97 & 0.57 & 3933 & 3894.1 & 0.98 \\
\hline B. subtilis+zeolite & 3612 & 3546.66 & 1.80 & 3879 & 3836.53 & 1.09 \\
\hline B. subtilis+pumice & 3721 & 3641.97 & 2.12 & 3939 & 3930.47 & 0.21 \\
\hline S. pasteurii+zeolite & 3759 & 3681.28 & 2.06 & 4010 & 3995.66 & 0.35 \\
\hline S. pasteurii+pumice & 3618 & 3735.72 & -3.25 & 4101 & 4059.35 & 1.01 \\
\hline
\end{tabular}

Table 5.22: Comparison of predicted ( $Y^{\prime}$ ) and experimental (Y) UPV of FR mortar for healing period of 180 and 240 days

\begin{tabular}{|c|c|c|c|c|c|c|}
\hline Specimen & \multicolumn{3}{|c|}{ 180 days } & \multicolumn{3}{c|}{ 240 days } \\
\hline & $\mathbf{Y}$ & $\mathbf{Y}^{\prime}$ & Variation (\%) & $\mathbf{Y}$ & $\mathbf{Y}^{\prime}$ & Variation (\%) \\
\hline S. ureae+zeolite & 3801 & 3911.03 & -2.89 & 3932 & 3918.03 & 0.35 \\
\hline S. ureae+pumice & 3965 & 3928.97 & 0.90 & 3939 & 3934.97 & 0.10 \\
\hline B. subtilis+zeolite & 3901 & 3898.16 & 0.07 & 3879 & 3919.66 & -1.04 \\
\hline B. subtilis+pumice & 3886 & 3988.85 & -2.64 & 3946 & 4009.35 & -1.60 \\
\hline S. pasteurii+zeolite & 3979 & 4064.78 & -2.15 & 4019 & 4089.28 & -1.74 \\
\hline S. pasteurii+pumice & 4103 & 4125.22 & -0.54 & 4151 & 4148.72 & 0.05 \\
\hline
\end{tabular}


Tables 5.23, 5.24 and 5.25 are the comparison of the compressive strength at various ages. For normal mortar, the minimum variation between predicted (Y') and experimental (Y) values was found to be $39 \%$ for 28 days, $32.6 \%$ for 90 days, and $35.5 \%$ for 180 days. These high variations suggests that the developed regression model is not suitable for predicting the compressive strength for normal mortar. A new model is required to predict the characteristics of normal mortar with more experimental data. The variation between predicted and experimental values for ECC is found to be smaller - in the range of 9-16\% (Tables 5.23, 5.24 and 5.25). So this regression model may be used to predict the compressive strength of ECC to a certain extent. More experimental data is still required to improve the model.

Table 5.23: Comparison of predicted ( $\left.\mathrm{Y}^{\prime}\right)$ and experimental (Y) compressive strength of normal mortar and ECC for 28 days

\begin{tabular}{|c|c|c|c|c|c|c|}
\hline Specimen & \multicolumn{3}{|c|}{ Normal mortar } & \multicolumn{3}{c|}{ ECC } \\
\hline & $\mathbf{Y}$ & $\mathbf{Y}^{\prime}$ & Variation (\%) & $\mathbf{Y}$ & $\mathbf{Y}^{\prime}$ & Variation (\%) \\
\hline S. ureae+zeolite & 57.3 & 81.74 & -42.65 & - & 81.74 & - \\
\hline S. ureae+pumice & 60 & 83.38 & -38.96 & - & 83.38 & - \\
\hline B. subtilis+zeolite & 60.3 & 85.03 & -41.01 & 75.8 & 85.03 & -12.17 \\
\hline B. subtilis+pumice & 63.1 & 87.88 & -39.27 & - & 87.88 & - \\
\hline S. pasteurii+zeolite & 59.7 & 84.03 & -40.75 & 73.6 & 84.03 & -14.17 \\
\hline S. pasteurii+pumice & 62.6 & 86.87 & -38.76 & - & 86.87 & - \\
\hline
\end{tabular}

Table 5.24: Comparison of predicted ( $\left.\mathrm{Y}^{\prime}\right)$ and experimental (Y) compressive strength of normal mortar and ECC for 90 days

\begin{tabular}{|c|c|c|c|c|c|c|}
\hline Specimen & \multicolumn{3}{|c|}{ Normal mortar } & \multicolumn{3}{c|}{ ECC } \\
\hline & $\mathbf{Y}$ & $\mathbf{Y}^{\prime}$ & Variation (\%) & $\mathbf{Y}$ & $\mathbf{Y}^{\prime}$ & Variation (\%) \\
\hline S. ureae+zeolite & 62.2 & 87.28 & -40.32 & - & 87.28 & - \\
\hline S. ureae+pumice & 64.3 & 86.95 & -35.22 & - & 86.95 & - \\
\hline B. subtilis+zeolite & 65.1 & 89.43 & -37.37 & 81.6 & 89.43 & -9.59 \\
\hline B. subtilis+pumice & 68.1 & 90.31 & -32.61 & - & 90.31 & - \\
\hline S. pasteurii+zeolite & 66.4 & 92.07 & -38.65 & 79.3 & 92.07 & -16.10 \\
\hline S. pasteurii+pumice & 67.9 & 90.94 & -33.93 & - & 90.94 & - \\
\hline
\end{tabular}


Table 5.25: Comparison of predicted (Y') and experimental (Y) compressive strength of normal mortar and ECC for 180 days

\begin{tabular}{|c|c|c|c|c|c|c|}
\hline Specimen & \multicolumn{3}{|c|}{ Normal mortar } & \multicolumn{3}{c|}{ ECC } \\
\hline & \multicolumn{1}{|c|}{ Y } & \multicolumn{1}{|c|}{$\mathbf{Y}^{\prime}$} & Variation (\%) & Y & $\mathbf{Y}^{\prime}$ & Variation (\%) \\
\hline S. ureae+zeolite & 62.2 & 89.29 & -43.55 & - & 89.29 & - \\
\hline S. ureae+pumice & 64.3 & 87.74 & -36.45 & - & 87.74 & - \\
\hline B. subtilis+zeolite & 65.1 & 93.61 & -43.79 & 85.8 & 93.61 & -9.10 \\
\hline B. subtilis+pumice & 68.1 & 92.27 & -35.49 & - & 92.27 & - \\
\hline S. pasteurii+zeolite & 66.4 & 94.78 & -42.74 & 84.38 & 94.78 & -12.32 \\
\hline S. pasteurii+pumice & 67.9 & 93.43 & -37.59 & - & 93.43 & - \\
\hline
\end{tabular}

\subsubsection{Illustration of models showing numerical examples}

The use statistical models for fiber reinforced mortar for predicting self-healing in terms of RCP, primary sorptivity, UPV and compressive strength are illustrated with following numerical examples.

Example1: Prediction of RCP - let us assume A (healing days) be 120 days, B (bacterial types) be $S$. ureae and C (carrier types) be zeolite. Required values for the A, B and C are taken as 2, 2 and 1, respectively according to Table 5.7. Substituting these values in Eq. 5.7, the resulting equation for RCP becomes:

$\mathrm{RCP}=1100.97-31.47 \mathrm{~A} 2-17.34 \mathrm{~B} 2+11.28 \mathrm{C} 1+3.3 \times \mathrm{A} 2 \times \mathrm{B} 2$

Now substituting the values of A, B and C in Eq. 5.11, RCP value can be obtained as:

$\mathrm{RCP}=1100.97-31.47 \times 2-17.34 \times 2+11.28 \times 1+3.3 \times 2 \times 2=1027.83$ Coulombs

Example 2: Prediction of primary sorptivity - let us assume A (healing days) be 120 days, B (bacterial types) be S. ureae and C (carrier types) be zeolite. Required values for the A, B and C are taken as 3, 2 and 1, respectively according to Table 5.7. Substituting these values in Eq. 5.8, the resulting equation for primiary sorptivity becomes:

Primary sorptivity $=0.003165-0.000491 \mathrm{A3}-0.000399 \mathrm{~B} 2+0.000047 \mathrm{C} 2-0.000044 \mathrm{A3} * \mathrm{~B} 2$

Now substituting the values of A, B and C in Eq. 5.12, primary sorptivity can be obtained as: 
Primary sorptivity $=0.003165-0.000491 \times 2-0.000399 \times 2+0.000047 \times 1-0.000044 \times 2 \times 2$ $=0.001256 \mathrm{~mm} / \mathrm{sec}^{-1 / 2}$

Example 3: Prediction of UPV - let us assume A (healing days) be 120 days, B (bacterial types) be S. ureae and C (carrier types) be zeolite. Required values for the A, B and C are taken as 2, 3 and 2, respectively according to Table 5.7. Substituting these values in Eq. 5.9, the resulting equation for UPV becomes:

$\mathrm{UPV}=3829.47+33.031 \mathrm{~A} 2+15.656 \mathrm{B3}+31.656 \mathrm{C} 2+5.34 \mathrm{~A} 2 * \mathrm{~B} 3+2.094 \mathrm{~A} 2 * \mathrm{C} 2+13.219 \mathrm{B3} * \mathrm{C} 2$

Substituting the values of A, B and C in Eq. 5.13, the UPV value can be obtained as:

$\mathrm{UPV}=3829.47+33.031 \times 2+15.656 \times 3+31.656 \times 1+5.34 \times 2 \times 3+2.094 \times 2 \times 1+13.219 \times 3 \times 2=4050 \mathrm{~m} / \mathrm{s}$

Example 4: Prediction of compressive strength of fiber reinforced mortar- let us assume A (healing days) be 60 days, B (bacterial types) be $S$. pasteurii and C (carrier types) be pumice. Required values for $\mathrm{B}$ and $\mathrm{C}$ can be taken from Table 5.7. However, in the case of compressive strength, the value for $\mathrm{A}$ is slightly different from previous cases. Therefore, it is taken as 2 since in this case, 1 is for 28 days, 2 is for 60 days, 3 is for 90 days and 4 is for 180 days. Substituting the respective values in Eq. 5.10, the resulting equation for compressive strength becomes:

Compressive strength $=81.9372+2.244 \mathrm{~A} 2+5.132 \mathrm{B3}+0.2128 \mathrm{C} 2+0.142 \mathrm{~A} 2 * \mathrm{~B} 3+0.508 \mathrm{~A} 2 * \mathrm{C} 2$

$$
\text { - } 0.292 \mathrm{B3} * \mathrm{C} 2
$$

Substituting the values of A, B, and C as 2, 3, 2 respectively, in Eq. 5.14, compressive strength can be obtained as:

Compressive strength $=81.9372+2.244 \times 2+5.132 \times 3+0.2128 \times 2+0.142 \times 2 \times 3+0.508 \times 2 \times 2$

$$
-0.292 \times 3 \times 2=102.25 \mathrm{MPa}
$$

\subsection{Conclusion}

Statistical modelling and design of experiment methodology were successfully used in the past in various Civil Engineering applications. An attempt has been made in this chapter to incorporate the principles of Design of Experiment to statistically model the self-healing characteristics in bacteria incorporated fibre reinforced mortar. This methodology was initially used to identify the optimum concentration of bacteria which would provide the maximum compressive strength. The results obtained turned out to be close to the experimental results. Statistical models are developed 
to predict the self-healing characteristics in terms of compressive strength, RCP, sorptivity and UPV. The performance of the models are validated through experimental results. Models are found to predict reasonably the properties of fiber reinforced mortar but failed to predict the properties of normal mortar. This was reasonable as they were derived based on test results of fibre reinforced mortar. Developed statistical models can be used a useful tool for quantifying the selfhealing capability of bacteria incorporated fiber reinforced mortar in terms of illustrated properties. 


\section{CHAPTER SIX: CONCLUSIONS AND RECOMMENDATIONS}

\subsection{Introduction}

This research was intended to study the self-healing efficiency of bacteria incorporated cementitious concrete composites. A two-component self-healing system was used in this research, Bacteria was considered as one of the components while the nutrients required for keeping bacteria active are considered as the second component. Both components were incorporated during casting of three types of cementitious composite (normal mortar, fiber reinforced mortar and engineered cementitious composite 'ECC') specimens. Three different bacterial species (Sporosarcina ureae, Sporosarcina pasteurii and Bacillus subtilis subsp. spizizenii) were selected. One of the objectives was to investigate the self-healing ability/efficiency of these selected bacterial species and to find the best amongst them. Two different materials (zeolite and pumice) were selected as the carrier vehicle for the bacteria and their efficiency as a protective vehicle in high $\mathrm{pH}$ concrete environment was determined. The effect of different mineral substrates on cementitious concrete composite properties such as compressive strength was tested to observe the adverse effect of healing agent addition on the matrix. Various permutations and combinations in terms of the bacterial concentration and amount of minerals substrates to be added into the matrix were carried out in this study. This was performed in order to determine the optimum quantity of healing agent addition that gave the best result in inducing self-healing.

In this study, self-healing efficiency of the bacterial concrete composites was mainly investigated by measuring properties at different ages such as: compressive strength, flexural/bending strength, sorptivity and rapid chloride permeability (RCP) and ultrasonic pulse velocity (UPV) for sound, cracked and healed specimens. To investigate the morphology and chemical constituents of selfhealing products and to observe the self-healing process, selected specimens were examined by scanning electron microscope (SEM) and energy dispersive spectroscopy (EDS). In order to detect the nature of the crystalline materials formed in the precipitated layer, X-ray diffraction (XRD) analysis was also performed. The self-healing efficiency of bacteria incorporated ECC, normal mortar and fiber reinforced mortar specimens was also compared. 
An attempt had been made to employ statistical models for parameter optimization of self-healing characteristics in terms of compressive strength, sorptivity, RCP and UPV of bacteria incorporated fibre reinforced mortar by design and analysis of experiments. In addition, statistical modeling of compressive strength of bacterial concrete (with different concentration of bacteria and calcium compound) was carried out in order to determine the optimum quantity of healing agent that yield best self-healing.

\subsection{Conclusion}

The following conclusions are drawn from the study:

- One of the objectives of the study was to investigate the efficiency of zeolite and pumice as a carrier or protective vehicle for bacteria in high $\mathrm{pH}$ concrete environment. The present study proved that in order for the bacteria to become metabolically active in high $\mathrm{pH}$ environment, some kind of protection to be provided for them. Zeolite and pumice showed profound protective effect for all the three selected bacterial species in high $\mathrm{pH}$ cement environment. This can be attributed to the strong capacity of these materials to adsorb bacterial cells on the surfaces and to provide a kind of micro environment around the bacteria, in which the local $\mathrm{pH}$ was less than that in the cementitious environment.

- Regarding the urease activity of the urease positive bacterial species such as Sporosarcina pasteurii and Sporosarcina ureae - both species displayed very high urease activity. However, Sporosarcina pasteurii showed the highest activity. The difference in this activity could be attributed to different capabilities of these species to provide varied types of urease enzymes and the difference in the level of urease enzyme among these strains.

- A slight decrease in the compressive strength of the normal mortar specimen was noticed with the addition of nutrients in the absence of bacteria. This might be due to the effect of yeast extract for which a negative effect on concrete composites have been reported. The calcium lactate addition as $2 \%$ of cement weight was accepted as the optimum concentration which gave the maximum strength without affecting the mortar characteristics. It was also found that more than $4 \%$ of calcium lactate addition greatly affected (weaken) the strength properties in which it was difficult for the mortar to build the bond between components of the matrix. 
- Compressive strength was found to increase with bacterial addition and this increase is mainly due to deposition of microbial induced calcium carbonate precipitation on the microorganism cell surfaces and within the pores of the mortar. It was noticed that in normal mortar, the compressive strength was increased with the increase in bacterial cell concentration up to $10^{6}$ cells $/ \mathrm{ml}$, and then there was reduction in the strength at $10^{8}$ cells $/ \mathrm{ml}$. Maximum increase in compressive strengths was achieved at $10^{6}$ cells $/ \mathrm{ml}$.

- As far as the efficiency of selected bacterial strains was concerned, both normal mortar and FR mortar specimens incorporated with Bacillus subtilis showed highest compressive strength followed by Sporosarcina pasteurii until the curing period of 90 days and afterwards specimens with Sporosarcina pasteurii appeared to attain the maximum strength. At the same time, the compressive strength of specimens made with Sporosarcina ureae was found to be less than those made with other two bacterial strains. Hence, it can be concluded that both bacterial species: Sporosarcina pasteurii and Bacillus subtilis, have excellent self-healing potential in terms of compressive strength gain. For the long term, Sporosarcina pasteurii appeared to be the best healing agent among the selected bacterial strains.

- The presence of bacteria resulted in a significant decrease in the rate of water uptake compared to control specimens. Normal mortar with holes and cracked fibre reinforced mortar containing bacteria along with nutrients showed good resistance against chloride penetration. The deposition of a layer of calcium carbonate on the surface and inside the pores of the mortar specimens resulted in a decrease of water absorption. When the pores were impeded by materials such as calcium carbonate, the passage for water, air and other pollutants was sealed. Consequently, it reduced the permeation of water and chloride in the mortar specimens.

- From the sorptivity and RCP results of both normal and FR mortar, it was found that the pronounced self-healing occurred in the specimens during the initial 120 days of healing period. Less self-healing activity occurred in the later stage as evident from the no significant change in sorptivity and RCP values.

- For the cracked and healed specimens, the decrease in chloride ion permeability of around $13 \%$ in 4 months, $18 \%$ in 6 months and $20 \%$ in 8 months were observed for control specimen whereas, nutrients with zeolite and nutrients with pumice have $16 \%$ in 4 months, 
$22 \%$ in 6 months, $25 \%$ in 8 months and $17 \%$ in 4 months, $22 \%$ in 6 months, $25 \%$ in 8 months, respectively. However, the specimens with bacteria, showed an average decrease in permeability of around 32\% in 4 months, $46 \%$ in 6 months and 57\% in 8 months. Out of the 6 mixes with bacteria, pumice with Sporosarcina pasteurii showed the maximum reduction in chloride ion permeability of around $65 \%$ in 8 months.

- Regarding the healing efficiency of carrier materials (zeolite and pumice immobilised bacteria), pumice gave better sorptivity and RCP value than zeolite for both normal and FR mortar. The reason for this might be the difference in their particle size distribution- in this study, the particle size distribution of pumice was finer than that of zeolite.

- Results of the four-point bending tests performed on fibre reinforced mortar prisms before and after healing indicated that the material was able to recover some of its strength after experiencing some kind of damage. The control specimens (without bacteria) had about $20-23 \%$ of its initial strength recovered in 8 months after the damage occurred. At the same time, the specimens containing the bacteria recovered $41-48 \%$ of its strength in 4 months and about $49-59 \%$ of its strength in 8 months after the damage. This is an evidence of the superior self- healing of the specimens treated with bacteria compared to control ones.

- UPV results also suggested better healing characteristics for bacteria incorporated specimens compared to the control ones. It is worth noting that highest healing ratio in terms of UPV was obtained for the specimen with Sporosarcina pasteurii immobilised into zeolite.

- Quantification of crack filling in each month (performed using UPV measurements) indicated that most of the crack filling was occurred during the first and second month of healing period. After two months of healing the rate of crack filling was significantly reduced. One possible reason could be the lack of availability of the calcium compound for the bacteria as this compound may became the part of the mortar matrix and therefore, inaccessible for the bacteria to precipitate.

- SEM and EDS studies on FR mortar specimens proved visually that the incorporated bacteria can produce copious amounts of minerals which can potentially seal freshly formed cracks. It was observed that the cracks with a width of $0.13-0.16 \mathrm{~mm}$ in mortar specimens were completely filled by the zeolite/pumice immobilized bacteria. At the same time, only a thin lining of mineral precipitates on the crack wall were observed for 
specimen with nutrients in the absence of bacteria and almost negligible precipitation or a thin lining on the crack wall were observed in the control specimen (normal mortar/fibre reinforced mortar).

- Although the cracks appeared to be completely healed from the surface, they remained partially or completely open for both control and bacteria based mortar for the remaining inner portions of the crack. However, at the same time it can be seen from the results that the bacteria based self-healing agent can be successfully applied to enhance the self-healing capacity since it results in the formation of a dense layer of precipitates along the crack wall and all over the surface of the specimen as well.

- XRD analysis indicated that the dominant crystal morphology for all the three selected bacterially treated mortar specimens was calcite. It has been reported previously as the source of calcium compound significantly influence the formation of calcium carbonate. Therefore, this same kind of mineral precipitation in the form of calcite by the three different bacterial species might be due to the influence of the calcium lactate which was chosen as the calcium source for the present study. The percentage of calcium carbonate in the precipitate was significantly higher in the case of bacteria incorporated specimens than those without bacteria.

- It was also sighted that the type of bacterial strains had a profound impact on crystal morphology. The fact that all the samples delivered similar XRD results, though clear morphological differences were apparent, indicating that the differences were a result of variations in crystal growth rates along different planes of the crystal structure. Another possible explanation for the differences in crystal morphology obtained with different bacterial cultures could be due to the level of the actual urease activity.

- All the selected bacterial species showed good self-healing efficiency in terms of sorptivity and rapid chloride permeability in which both Sporosarcina pasteurii and Bacillus subtilis appeared to have excellent healing efficiency compared to that of Sporosarcina ureae. Chemical and morphological analyses disclosed that $\mathrm{CaCO}_{3}$ formation and depth of cementation were more intense for Sporosarcina pasteurii compared to Sporosarcina ureae.

- Calcite formation was more pronounced for the specimen incorporated with Sporosarcina pasteurii compared to Sporosarcina ureae. The difference in $\mathrm{CaCO}_{3}$ precipitation between 
the two bacterial species Sporosarcina pasteurii and Sporosarcina ureae might be due to the different capabilities of these species to provide various types of urease enzymes.

- One of the main objectives of the study was to elucidate whether bacteria based self-healing by means of bacteria-mediated calcium carbonate precipitation will bring about enhanced mechanical properties and bonding strength of the ECC mixes. The results showed that material can accomplish the requirements in terms of compressive and bonding strength.

- Self-healed ECC shows substantial recovery of mechanical properties such as flexural strength, flexural stiffness and deformation capacity. Various degrees of recovery were found for mechanical properties such as flexural strength, flexural deformation and stiffness due to self-healing and better self-healing induced mechanical recovery was observed for specimen treated with zeolite immobilised bacteria compared to the specimen with unprotected bacteria and control specimen.

- Results of the flexural tests conducted on fibre reinforced mortar and ECC revealed that bacteria offered better efficiency of healing with smaller cracks because it might be hard for the bacteria to heal wider cracks. Healed cracks stayed as the weakest link and was damaged again without forming new cracks in the case of fibre reinforced mortar specimens where the crack widths were relatively high. Conversely, in the case of bacteria based ECC specimens, some of the cracks were deviated from the pre-existing healed cracks and new cracks were generated while reloading. This observation confirmed the argument that the bacteria based self-healing is more powerful and capable of yielding excellent efficiency for healing cracks with smaller crack widths.

- Based on the self-healing studies conducted on properties like compressive strength, rapid chloride permeability, water absorption and flexural strength, the microbial mineral precipitation appeared to be a promising practice. The bacterial precipitation may lead to lower amount of capillary pores and clogging of the pores, which substantially reduces the penetration of aggressive chemicals in concrete and hence increases the durability. Use of bacteria in concrete composites may be highly desirable because the calcite precipitation induced by the metabolic activities is natural and pollution free.

- It is believed that bacterial based self-healing technique can be employed to concrete structures that are not easily accessible for maintenance and repair such as underground structures, bridges, and dams. As the cracks can be immediately sealed, the maintenance 
costs can be reduced and the service life of the structures may be extended even though the initial costs are assumed to be higher.

- Statistical modelling using Design of Experiment (DOE) methodology is an ideal choice for modelling self-healing characteristics. This methodology enabled identifying the optimal combination of ingredients thus evolving superior healing efficiency. DOE eliminated a great deal of redundancy and provided characteristic equations for properties of cementitious composites to quantify self-healing. Characteristic equations were developed with the help of statistical tools such as regression analysis and analysis of variance (ANOVA). Statistical models were developed to predict the self-healing characteristics in terms of compressive strength, RCP, sorptivity and UPV for fiber reinforced mortar. The performance of the models were validated through experimental results. Developed statistical models can be used a useful tool for quantifying the selfhealing capability of bacteria incorporated fiber reinforced mortar in terms of illustrated properties.

\subsection{Recommendations for future studies}

It is recommended to:

- Conduct investigations on understanding the impact of relative humidity and temperature on the self-healing efficiency of bacteria. A range of relative humidity and temperature should be taken into account to realize their impact on the self-healing capability of bacteria in concrete composites.

- Study the influence of different calcium sources such as calcium nitrate, calcium acetate, calcium glutamate, calcium chloride etc. on bacteria based self-healing concrete composites as the type of source of calcium compound may greatly influence the rate of formation, morphology, crystalline nature and chemical composition of the mineral precipitation.

- Conduct more detailed self-healing behavior study on bacteria incorporated ECC specimens giving emphasis on durability properties such as gas permeability, water permeability, RCPT, sorptivity etc.

- Carry out investigations using various mix designs of bacteria incorporated ECC materials to identify the best mix which provide the best healing efficiency. Various permutations 
and combinations needs to be done in order to obtain the optimum mix design. Design of experiments and analysis statistical tools can be used to optimize the mix design and selfhealing efficiency.

- Carry out a detailed investigation on several other characteristics such as long term durability amounting to many years and cost efficiency of this innovative type of bacteria incorporated concrete before considering its commercial application. 


\section{APPENDICES}

Appendix A: Preparation of calibration curve and ammonium concentration determination Ammonium concentration was determined calorimetrically by the method of Nessler's method as described in the section 3.3.1 and 3.3.4. Prepared standard calibration curve is shown in Figure A.1.

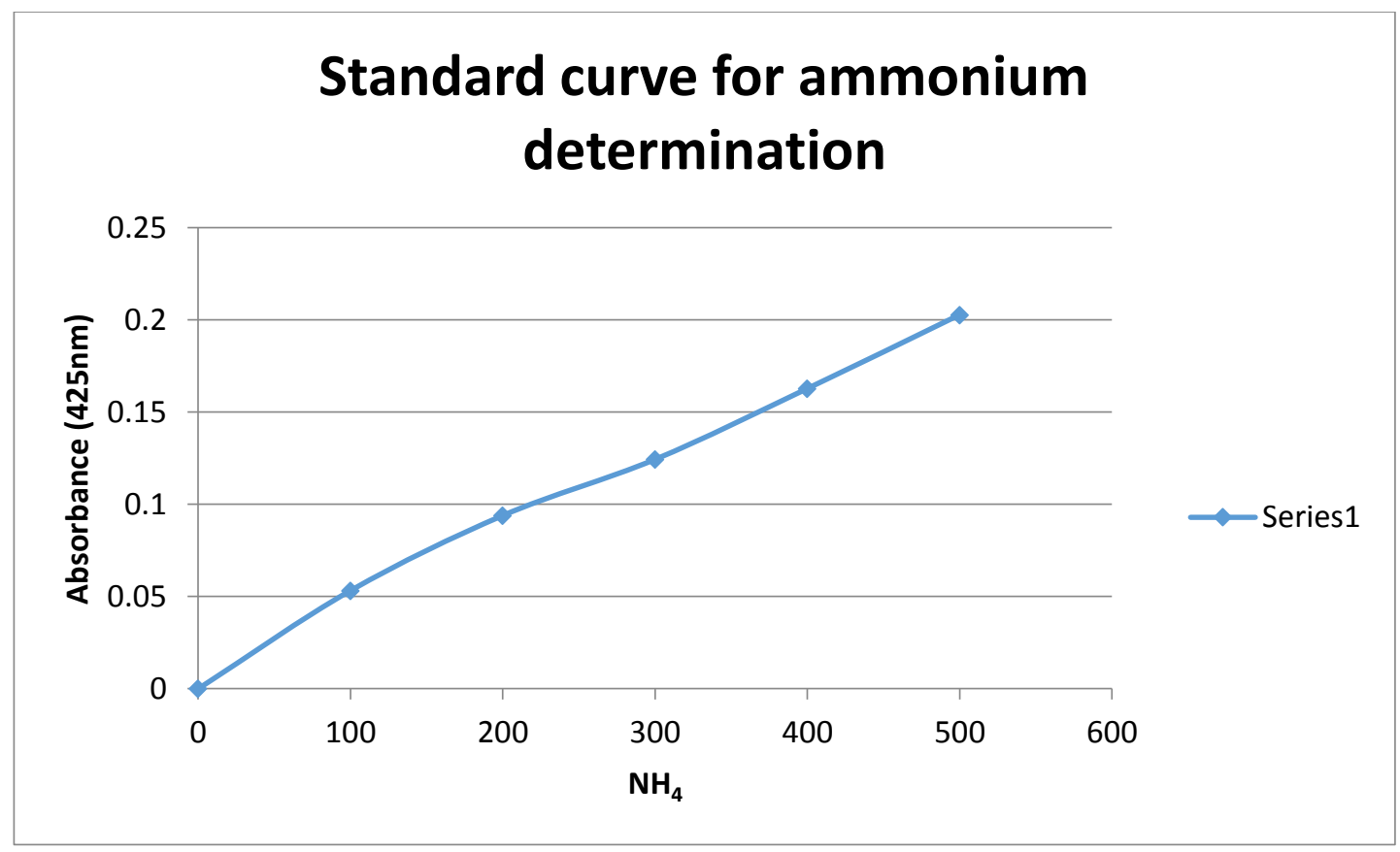

Figure A.1: Standard curve for ammonium determination versus absorbance for Nessler's method 


\section{Appendix B}

Appendix B.1: $3^{3} 2^{2}$ Full factorial design matrix for the response compressive strength

\begin{tabular}{|c|c|c|c|c|c|c|c|}
\hline \multicolumn{8}{|c|}{$3^{3} 2^{2}$ Full factorial design for compressive strength } \\
\hline Sl.No & StdOrder & $\begin{array}{c}\text { Testing } \\
\text { days }\end{array}$ & $\begin{array}{c}\text { Concentration } \\
\text { of bacteria }\end{array}$ & $\begin{array}{c}\text { Calcium } \\
\text { lactate } \\
\text { concentration }\end{array}$ & $\begin{array}{c}\text { Types } \\
\text { of } \\
\text { bacteria }\end{array}$ & $\begin{array}{c}\text { Carrier } \\
\text { material }\end{array}$ & $\begin{array}{l}\text { Compressive } \\
\text { strength }\end{array}$ \\
\hline 1 & 100 & 1 & 1 & -1 & 0 & 1 & 56.4 \\
\hline 2 & 196 & 1 & 0 & -1 & 0 & 1 & 56.9 \\
\hline 3 & 283 & 0 & 1 & 1 & -1 & -1 & 44.8 \\
\hline 4 & 16 & -1 & 0 & -1 & 0 & 1 & 37.1 \\
\hline 5 & 197 & 1 & 0 & -1 & 1 & -1 & 57.8 \\
\hline 6 & 210 & 1 & 1 & -1 & 1 & 1 & 56.8 \\
\hline 7 & 35 & -1 & 1 & 1 & 1 & -1 & 36.4 \\
\hline 8 & 308 & 1 & 0 & 1 & -1 & 1 & 56.2 \\
\hline 9 & 234 & -1 & 0 & -1 & 1 & 1 & 38.7 \\
\hline 10 & 305 & 1 & 0 & -1 & 1 & -1 & 58.4 \\
\hline 11 & 78 & 1 & -1 & -1 & 1 & 1 & 58.8 \\
\hline 12 & 71 & 0 & 1 & 1 & 1 & -1 & 45.7 \\
\hline 13 & 43 & 0 & -1 & 1 & -1 & -1 & 45.2 \\
\hline 14 & 292 & 1 & -1 & -1 & 0 & 1 & 56.2 \\
\hline 15 & 130 & -1 & 0 & 1 & 0 & 1 & 37.1 \\
\hline 16 & 287 & 0 & 1 & 1 & 1 & -1 & 46.3 \\
\hline 17 & 211 & 1 & 1 & 1 & -1 & -1 & 53.6 \\
\hline 18 & 117 & -1 & -1 & 1 & 0 & -1 & 35.3 \\
\hline 19 & 87 & 1 & 0 & -1 & 0 & -1 & 58.5 \\
\hline 20 & 51 & 0 & 0 & -1 & 0 & -1 & 47.1 \\
\hline 21 & 141 & -1 & 1 & 1 & 0 & -1 & 35.8 \\
\hline 22 & 128 & -1 & 0 & 1 & -1 & 1 & 36.9 \\
\hline 23 & 84 & 1 & -1 & 1 & 1 & 1 & 58.4 \\
\hline 24 & 64 & 0 & 1 & -1 & 0 & 1 & 45.9 \\
\hline 25 & 98 & 1 & 1 & -1 & -1 & 1 & 55.9 \\
\hline 26 & 222 & -1 & -1 & -1 & 1 & 1 & 37.6 \\
\hline 27 & 59 & 0 & 0 & 1 & 1 & -1 & 47.1 \\
\hline 28 & 225 & -1 & -1 & 1 & 0 & -1 & 35.2 \\
\hline 29 & 291 & 1 & -1 & -1 & 0 & -1 & 49.8 \\
\hline 30 & 11 & -1 & -1 & 1 & 1 & -1 & 35.2 \\
\hline 31 & 166 & 0 & 0 & 1 & 0 & 1 & 48.1 \\
\hline 32 & 169 & 0 & 1 & -1 & -1 & -1 & 45.3 \\
\hline 33 & 134 & -1 & 1 & -1 & -1 & 1 & 36.6 \\
\hline
\end{tabular}




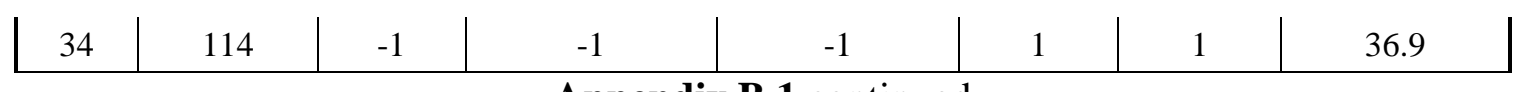

Appendix B.1 continued

\begin{tabular}{|c|c|c|c|c|c|c|c|}
\hline \multicolumn{8}{|c|}{$3^{3} 2^{2}$ Full factorial design for compressive strength } \\
\hline Sl.No & StdOrder & $\begin{array}{c}\text { Testing } \\
\text { days }\end{array}$ & $\begin{array}{c}\text { Concentration } \\
\text { of bacteria }\end{array}$ & $\begin{array}{c}\text { Calcium } \\
\text { lactate } \\
\text { concentration }\end{array}$ & $\begin{array}{c}\text { Types } \\
\text { of } \\
\text { bacteria }\end{array}$ & $\begin{array}{l}\text { Carrier } \\
\text { material }\end{array}$ & $\begin{array}{l}\text { Compressive } \\
\text { strength }\end{array}$ \\
\hline 35 & 157 & 0 & 0 & -1 & -1 & -1 & 47.9 \\
\hline 36 & 132 & -1 & 0 & 1 & 1 & 1 & 39.7 \\
\hline 37 & 34 & -1 & 1 & 1 & 0 & 1 & 36 \\
\hline 38 & 188 & 1 & -1 & 1 & -1 & 1 & 53.8 \\
\hline 39 & 281 & 0 & 1 & -1 & 1 & -1 & 44.9 \\
\hline 40 & 29 & -1 & 1 & -1 & 1 & -1 & 36.1 \\
\hline 41 & 36 & -1 & 1 & 1 & 1 & 1 & 37.8 \\
\hline 42 & 235 & -1 & 0 & 1 & -1 & -1 & 38.5 \\
\hline 43 & 323 & 1 & 1 & 1 & 1 & -1 & 54.1 \\
\hline 44 & 120 & -1 & -1 & 1 & 1 & 1 & 37.9 \\
\hline 45 & 282 & 0 & 1 & -1 & 1 & 1 & 47.3 \\
\hline 46 & 170 & 0 & 1 & -1 & -1 & 1 & 42.8 \\
\hline 47 & 10 & -1 & -1 & 1 & 0 & 1 & 34.5 \\
\hline 48 & 184 & 1 & -1 & -1 & 0 & 1 & 56.5 \\
\hline 49 & 121 & -1 & 0 & -1 & -1 & -1 & 33.8 \\
\hline 50 & 33 & -1 & 1 & 1 & 0 & -1 & 36.4 \\
\hline 51 & 185 & 1 & -1 & -1 & 1 & -1 & 56.5 \\
\hline 52 & 73 & 1 & -1 & -1 & -1 & -1 & 49.8 \\
\hline 53 & 240 & -1 & 0 & 1 & 1 & 1 & 34.6 \\
\hline 54 & 247 & -1 & 1 & 1 & -1 & -1 & 34.7 \\
\hline 55 & 136 & -1 & 1 & -1 & 0 & 1 & 36.3 \\
\hline 56 & 143 & -1 & 1 & 1 & 1 & -1 & 38.1 \\
\hline 57 & 25 & -1 & 1 & -1 & -1 & -1 & 34.8 \\
\hline 58 & 314 & 1 & 1 & -1 & -1 & 1 & 55.4 \\
\hline 59 & 9 & -1 & -1 & 1 & 0 & -1 & 37.8 \\
\hline 60 & 82 & 1 & -1 & 1 & 0 & 1 & 56.9 \\
\hline 61 & 4 & -1 & -1 & -1 & 0 & 1 & 37.3 \\
\hline 62 & 217 & -1 & -1 & -1 & -1 & -1 & 34.3 \\
\hline 63 & 220 & -1 & -1 & -1 & 0 & 1 & 35.1 \\
\hline 64 & 309 & 1 & 0 & 1 & 0 & -1 & 56 \\
\hline 65 & 190 & 1 & -1 & 1 & 0 & 1 & 56.4 \\
\hline 66 & 238 & -1 & 0 & 1 & 0 & 1 & 38.2 \\
\hline 67 & 12 & -1 & -1 & 1 & 1 & 1 & 37.9 \\
\hline
\end{tabular}


Appendix B.1 continued

\begin{tabular}{|c|c|c|c|c|c|c|c|}
\hline \multicolumn{8}{|c|}{$3^{3} 2^{2}$ Full factorial design for compressive strength } \\
\hline Sl.No & StdOrder & $\begin{array}{c}\text { Testing } \\
\text { days }\end{array}$ & $\begin{array}{c}\text { Concentration } \\
\text { of bacteria }\end{array}$ & $\begin{array}{c}\text { Calcium } \\
\text { lactate } \\
\text { concentration }\end{array}$ & $\begin{array}{c}\text { Types } \\
\text { of } \\
\text { bacteria }\end{array}$ & $\begin{array}{l}\text { Carrier } \\
\text { material }\end{array}$ & $\begin{array}{c}\text { Compressive } \\
\text { strength }\end{array}$ \\
\hline 68 & 319 & 1 & 1 & 1 & -1 & -1 & 52.8 \\
\hline 69 & 30 & -1 & 1 & -1 & 1 & 1 & 37.6 \\
\hline 70 & 165 & 0 & 0 & 1 & 0 & -1 & 48.9 \\
\hline 71 & 92 & 1 & 0 & 1 & -1 & 1 & 58.4 \\
\hline 72 & 88 & 1 & 0 & -1 & 0 & 1 & 58.8 \\
\hline 73 & 103 & 1 & 1 & 1 & -1 & -1 & 53.8 \\
\hline 74 & 142 & -1 & 1 & 1 & 0 & 1 & 36.2 \\
\hline 75 & 186 & 1 & -1 & -1 & 1 & 1 & 56.8 \\
\hline 76 & 161 & 0 & 0 & -1 & 1 & -1 & 49.7 \\
\hline 77 & 46 & 0 & -1 & 1 & 0 & 1 & 47.5 \\
\hline 78 & 204 & 1 & 0 & 1 & 1 & 1 & 60 \\
\hline 79 & 7 & -1 & -1 & 1 & -1 & -1 & 34.3 \\
\hline 80 & 182 & 1 & -1 & -1 & -1 & 1 & 55.4 \\
\hline 81 & 123 & -1 & 0 & -1 & 0 & -1 & 36 \\
\hline 82 & 294 & 1 & -1 & -1 & 1 & 1 & 57.8 \\
\hline 83 & 45 & 0 & -1 & 1 & 0 & -1 & 45.1 \\
\hline 84 & 174 & 0 & 1 & -1 & 1 & 1 & 47.6 \\
\hline 85 & 113 & -1 & -1 & -1 & 1 & -1 & 38 \\
\hline 86 & 39 & 0 & -1 & -1 & 0 & -1 & 45 \\
\hline 87 & 203 & 1 & 0 & 1 & 1 & -1 & 58.8 \\
\hline 88 & 303 & 1 & 0 & -1 & 0 & -1 & 55.1 \\
\hline 89 & 193 & 1 & 0 & -1 & -1 & -1 & 54.2 \\
\hline 90 & 44 & 0 & -1 & 1 & -1 & 1 & 44.8 \\
\hline 91 & 245 & -1 & 1 & -1 & 1 & -1 & 36.5 \\
\hline 92 & 321 & 1 & 1 & 1 & 0 & -1 & 52.4 \\
\hline 93 & 264 & 0 & -1 & 1 & 1 & 1 & 48.6 \\
\hline 94 & 202 & 1 & 0 & 1 & 0 & 1 & 57.1 \\
\hline 95 & 149 & 0 & -1 & -1 & 1 & -1 & 45.5 \\
\hline 96 & 122 & -1 & 0 & -1 & -1 & 1 & 38.6 \\
\hline 97 & 48 & 0 & -1 & 1 & 1 & 1 & 48.8 \\
\hline 98 & 145 & 0 & -1 & -1 & -1 & -1 & 46.3 \\
\hline 99 & 239 & -1 & 0 & 1 & 1 & -1 & 36.5 \\
\hline 100 & 85 & 1 & 0 & -1 & -1 & -1 & 54.1 \\
\hline 101 & 37 & 0 & -1 & -1 & -1 & -1 & 45.2 \\
\hline
\end{tabular}


Appendix B.1 continued

\begin{tabular}{|c|c|c|c|c|c|c|c|}
\hline \multicolumn{8}{|c|}{$3^{3} 2^{2}$ Full factorial design for compressive strength } \\
\hline Sl.No & StdOrder & $\begin{array}{c}\text { Testing } \\
\text { days }\end{array}$ & $\begin{array}{c}\text { Concentration } \\
\text { of bacteria }\end{array}$ & $\begin{array}{c}\text { Calcium } \\
\text { lactate } \\
\text { concentration }\end{array}$ & $\begin{array}{c}\text { Types } \\
\text { of } \\
\text { bacteria }\end{array}$ & $\begin{array}{c}\text { Carrier } \\
\text { material }\end{array}$ & $\begin{array}{c}\text { Compressive } \\
\text { strength }\end{array}$ \\
\hline 102 & 243 & -1 & 1 & -1 & 0 & -1 & 35.1 \\
\hline 103 & 257 & 0 & -1 & -1 & 1 & -1 & 47.1 \\
\hline 104 & 28 & -1 & 1 & -1 & 0 & 1 & 37.1 \\
\hline 105 & 317 & 1 & 1 & -1 & 1 & -1 & 52 \\
\hline 106 & 226 & -1 & -1 & 1 & 0 & 1 & 36.4 \\
\hline 107 & 253 & 0 & -1 & -1 & -1 & -1 & 44.4 \\
\hline 108 & 156 & 0 & -1 & 1 & 1 & 1 & 49 \\
\hline 109 & 172 & 0 & 1 & -1 & 0 & 1 & 46.2 \\
\hline 110 & 230 & -1 & 0 & -1 & -1 & 1 & 37.8 \\
\hline 111 & 266 & 0 & 0 & -1 & -1 & 1 & 47.5 \\
\hline 112 & 62 & 0 & 1 & -1 & -1 & 1 & 45.9 \\
\hline 113 & 124 & -1 & 0 & -1 & 0 & 1 & 38 \\
\hline 114 & 111 & -1 & -1 & -1 & 0 & -1 & 34.6 \\
\hline 115 & 194 & 1 & 0 & -1 & -1 & 1 & 56.8 \\
\hline 116 & 265 & 0 & 0 & -1 & -1 & -1 & 48.1 \\
\hline 117 & 15 & -1 & 0 & -1 & 0 & -1 & 37 \\
\hline 118 & 229 & -1 & 0 & -1 & -1 & -1 & 37.1 \\
\hline 119 & 312 & 1 & 0 & 1 & 1 & 1 & 60.4 \\
\hline 120 & 256 & 0 & -1 & -1 & 0 & 1 & 44.6 \\
\hline 121 & 3 & -1 & -1 & -1 & 0 & -1 & 36.6 \\
\hline 122 & 60 & 0 & 0 & 1 & 1 & 1 & 51.8 \\
\hline 123 & 41 & 0 & -1 & -1 & 1 & -1 & 46.9 \\
\hline 124 & 14 & -1 & 0 & -1 & -1 & 1 & 35.2 \\
\hline 125 & 201 & 1 & 0 & 1 & 0 & -1 & 56.3 \\
\hline 126 & 21 & -1 & 0 & 1 & 0 & -1 & 37 \\
\hline 127 & 27 & -1 & 1 & -1 & 0 & -1 & 34.2 \\
\hline 128 & 221 & -1 & -1 & -1 & 1 & -1 & 36.2 \\
\hline 129 & 83 & 1 & -1 & 1 & 1 & -1 & 54.3 \\
\hline 130 & 271 & 0 & 0 & 1 & -1 & -1 & 47.1 \\
\hline 131 & 38 & 0 & -1 & -1 & -1 & 1 & 42.3 \\
\hline 132 & 163 & 0 & 0 & 1 & -1 & -1 & 46.1 \\
\hline 133 & 258 & 0 & -1 & -1 & 1 & 1 & 48.4 \\
\hline 134 & 53 & 0 & 0 & -1 & 1 & -1 & 47.6 \\
\hline 135 & 6 & -1 & -1 & -1 & 1 & 1 & 37.1 \\
\hline
\end{tabular}


Appendix B.1 continued

\begin{tabular}{|c|c|c|c|c|c|c|c|}
\hline \multicolumn{8}{|c|}{$3^{3} 2^{2}$ Full factorial design for compressive strength } \\
\hline Sl.No & StdOrder & $\begin{array}{c}\text { Testing } \\
\text { days }\end{array}$ & $\begin{array}{c}\text { Concentration } \\
\text { of bacteria }\end{array}$ & $\begin{array}{c}\text { Calcium } \\
\text { lactate } \\
\text { concentration }\end{array}$ & $\begin{array}{c}\text { Types } \\
\text { of } \\
\text { bacteria }\end{array}$ & $\begin{array}{c}\text { Carrier } \\
\text { material }\end{array}$ & $\begin{array}{c}\text { Compressive } \\
\text { strength }\end{array}$ \\
\hline 136 & 94 & 1 & 0 & 1 & 0 & 1 & 57.6 \\
\hline 137 & 137 & -1 & 1 & -1 & 1 & -1 & 36.9 \\
\hline 138 & 286 & 0 & 1 & 1 & 0 & 1 & 46.5 \\
\hline 139 & 8 & -1 & -1 & 1 & -1 & 1 & 34.8 \\
\hline 140 & 106 & 1 & 1 & 1 & 0 & 1 & 56.4 \\
\hline 141 & 280 & 0 & 1 & -1 & 0 & 1 & 46.2 \\
\hline 142 & 26 & -1 & 1 & -1 & -1 & 1 & 35.8 \\
\hline 143 & 107 & 1 & 1 & 1 & 1 & -1 & 54.8 \\
\hline 144 & 164 & 0 & 0 & 1 & -1 & 1 & 48.6 \\
\hline 145 & 189 & 1 & -1 & 1 & 0 & -1 & 52.6 \\
\hline 146 & 2 & -1 & -1 & -1 & -1 & 1 & 36.2 \\
\hline 147 & 65 & 0 & 1 & -1 & 1 & -1 & 47.1 \\
\hline 148 & 262 & 0 & -1 & 1 & 0 & 1 & 45.4 \\
\hline 149 & 277 & 0 & 1 & -1 & -1 & -1 & 44.8 \\
\hline 150 & 105 & 1 & 1 & 1 & 0 & -1 & 53.5 \\
\hline 151 & 310 & 1 & 0 & 1 & 0 & 1 & 58.1 \\
\hline 152 & 69 & 0 & 1 & 1 & 0 & -1 & 46.4 \\
\hline 153 & 207 & 1 & 1 & -1 & 0 & -1 & 49.1 \\
\hline 154 & 20 & -1 & 0 & 1 & -1 & 1 & 37.2 \\
\hline 155 & 296 & 1 & -1 & 1 & -1 & 1 & 54.6 \\
\hline 156 & 249 & -1 & 1 & 1 & 0 & -1 & 36.6 \\
\hline 157 & 90 & 1 & 0 & -1 & 1 & 1 & 59.4 \\
\hline 158 & 108 & 1 & 1 & 1 & 1 & 1 & 56.7 \\
\hline 159 & 300 & 1 & -1 & 1 & 1 & 1 & 58.2 \\
\hline 160 & 151 & 0 & -1 & 1 & -1 & -1 & 45.8 \\
\hline 161 & 261 & 0 & -1 & 1 & 0 & -1 & 46.3 \\
\hline 162 & 218 & -1 & -1 & -1 & -1 & 1 & 34.6 \\
\hline 163 & 154 & 0 & -1 & 1 & 0 & 1 & 47.8 \\
\hline 164 & 295 & 1 & -1 & 1 & -1 & -1 & 52.4 \\
\hline 165 & 284 & 0 & 1 & 1 & -1 & 1 & 43.6 \\
\hline 166 & 251 & -1 & 1 & 1 & 1 & -1 & 35.9 \\
\hline 167 & 316 & 1 & 1 & -1 & 0 & 1 & 56.3 \\
\hline 168 & 5 & -1 & -1 & -1 & 1 & -1 & 35.9 \\
\hline 169 & 18 & -1 & 0 & -1 & 1 & 1 & 38.6 \\
\hline
\end{tabular}


Appendix B.1 continued

\begin{tabular}{|c|c|c|c|c|c|c|c|}
\hline \multicolumn{8}{|c|}{$3^{3} 2^{2}$ Full factorial design for compressive strength } \\
\hline Sl.No & StdOrder & $\begin{array}{c}\text { Testing } \\
\text { days }\end{array}$ & $\begin{array}{c}\text { Concentration } \\
\text { of bacteria }\end{array}$ & $\begin{array}{c}\text { Calcium } \\
\text { lactate } \\
\text { concentration }\end{array}$ & $\begin{array}{c}\text { Types } \\
\text { of } \\
\text { bacteria }\end{array}$ & $\begin{array}{c}\text { Carrier } \\
\text { material }\end{array}$ & $\begin{array}{c}\text { Compressive } \\
\text { strength }\end{array}$ \\
\hline 170 & 125 & -1 & 0 & -1 & 1 & -1 & 38.1 \\
\hline 171 & 301 & 1 & 0 & -1 & -1 & -1 & 53.5 \\
\hline 172 & 236 & -1 & 0 & 1 & -1 & 1 & 38.7 \\
\hline 173 & 173 & 0 & 1 & -1 & 1 & -1 & 47.2 \\
\hline 174 & 49 & 0 & 0 & -1 & -1 & -1 & 45.6 \\
\hline 175 & 200 & 1 & 0 & 1 & -1 & 1 & 56.9 \\
\hline 176 & 40 & 0 & -1 & -1 & 0 & 1 & 44.8 \\
\hline 177 & 212 & 1 & 1 & 1 & -1 & 1 & 52.8 \\
\hline 178 & 139 & -1 & 1 & 1 & -1 & -1 & 34.9 \\
\hline 179 & 116 & -1 & -1 & 1 & -1 & 1 & 36.8 \\
\hline 180 & 129 & -1 & 0 & 1 & 0 & -1 & 36.8 \\
\hline 181 & 263 & 0 & -1 & 1 & 1 & -1 & 47.5 \\
\hline 182 & 307 & 1 & 0 & 1 & -1 & -1 & 54.9 \\
\hline 183 & 180 & 0 & 1 & 1 & 1 & 1 & 48.3 \\
\hline 184 & 246 & -1 & 1 & -1 & 1 & 1 & 37.7 \\
\hline 185 & 297 & 1 & -1 & 1 & 0 & -1 & 54.9 \\
\hline 186 & 213 & 1 & 1 & 1 & 0 & -1 & 53.1 \\
\hline 187 & 267 & 0 & 0 & -1 & 0 & -1 & 47.3 \\
\hline 188 & 324 & 1 & 1 & 1 & 1 & 1 & 58 \\
\hline 189 & 241 & -1 & 1 & -1 & -1 & -1 & 35.1 \\
\hline 190 & 140 & -1 & 1 & 1 & -1 & 1 & 34.2 \\
\hline 191 & 93 & 1 & 0 & 1 & 0 & -1 & 58.1 \\
\hline 192 & 153 & 0 & -1 & 1 & 0 & -1 & 47.2 \\
\hline 193 & 289 & 1 & -1 & -1 & -1 & -1 & 51.2 \\
\hline 194 & 275 & 0 & 0 & 1 & 1 & -1 & 51.2 \\
\hline 195 & 55 & 0 & 0 & 1 & -1 & -1 & 48.1 \\
\hline 196 & 77 & 1 & -1 & -1 & 1 & -1 & 54.3 \\
\hline 197 & 61 & 0 & 1 & -1 & -1 & -1 & 46.4 \\
\hline 198 & 24 & -1 & 0 & 1 & 1 & 1 & 40.3 \\
\hline 199 & 260 & 0 & -1 & 1 & -1 & 1 & 46.3 \\
\hline 200 & 259 & 0 & -1 & 1 & -1 & -1 & 46.1 \\
\hline 201 & 67 & 0 & 1 & 1 & -1 & -1 & 45.6 \\
\hline 202 & 233 & -1 & 0 & -1 & 1 & -1 & 37.5 \\
\hline 203 & 58 & 0 & 0 & 1 & 0 & 1 & 49.5 \\
\hline
\end{tabular}


Appendix B.1 continued

\begin{tabular}{|c|c|c|c|c|c|c|c|}
\hline \multicolumn{8}{|c|}{$3^{3} 2^{2}$ Full factorial design for compressive strength } \\
\hline SI.No & StdOrder & $\begin{array}{c}\text { Testing } \\
\text { days }\end{array}$ & $\begin{array}{c}\text { Concentration } \\
\text { of bacteria }\end{array}$ & $\begin{array}{c}\text { Calcium } \\
\text { lactate } \\
\text { concentration }\end{array}$ & $\begin{array}{c}\text { Types } \\
\text { of } \\
\text { bacteria }\end{array}$ & $\begin{array}{l}\text { Carrier } \\
\text { material }\end{array}$ & $\begin{array}{c}\text { Compressive } \\
\text { strength }\end{array}$ \\
\hline 204 & 187 & 1 & -1 & 1 & -1 & -1 & 52.6 \\
\hline 205 & 199 & 1 & 0 & 1 & -1 & -1 & 56.8 \\
\hline 206 & 255 & 0 & -1 & -1 & 0 & -1 & 45.9 \\
\hline 207 & 118 & -1 & -1 & 1 & 0 & 1 & 37.1 \\
\hline 208 & 160 & 0 & 0 & -1 & 0 & 1 & 46.9 \\
\hline 209 & 152 & 0 & -1 & 1 & -1 & 1 & 47.4 \\
\hline 210 & 205 & 1 & 1 & -1 & -1 & -1 & 49.8 \\
\hline 211 & 250 & -1 & 1 & 1 & 0 & 1 & 36.4 \\
\hline 212 & 56 & 0 & 0 & 1 & -1 & 1 & 46.8 \\
\hline 213 & 178 & 0 & 1 & 1 & 0 & 1 & 47.2 \\
\hline 214 & 168 & 0 & 0 & 1 & 1 & 1 & 49.1 \\
\hline 215 & 22 & -1 & 0 & 1 & 0 & 1 & 38.1 \\
\hline 216 & 274 & 0 & 0 & 1 & 0 & 1 & 47.3 \\
\hline 217 & 209 & 1 & 1 & -1 & 1 & -1 & 55.1 \\
\hline 218 & 95 & 1 & 0 & 1 & 1 & -1 & 57.2 \\
\hline 219 & 138 & -1 & 1 & -1 & 1 & 1 & 37.8 \\
\hline 220 & 102 & 1 & 1 & -1 & 1 & 1 & 57 \\
\hline 221 & 99 & 1 & 1 & -1 & 0 & -1 & 53.4 \\
\hline 222 & 144 & -1 & 1 & 1 & 1 & 1 & 38.3 \\
\hline 223 & 74 & 1 & -1 & -1 & -1 & 1 & 55.9 \\
\hline 224 & 288 & 0 & 1 & 1 & 1 & 1 & 47.5 \\
\hline 225 & 191 & 1 & -1 & 1 & 1 & -1 & 53.1 \\
\hline 226 & 171 & 0 & 1 & -1 & 0 & -1 & 45.3 \\
\hline 227 & 231 & -1 & 0 & -1 & 0 & -1 & 37.1 \\
\hline 228 & 176 & 0 & 1 & 1 & -1 & 1 & 47.2 \\
\hline 229 & 126 & -1 & 0 & -1 & 1 & 1 & 39.1 \\
\hline 230 & 215 & 1 & 1 & 1 & 1 & -1 & 54.3 \\
\hline 231 & 119 & -1 & -1 & 1 & 1 & -1 & 36.9 \\
\hline 232 & 279 & 0 & 1 & -1 & 0 & -1 & 46 \\
\hline 233 & 278 & 0 & 1 & -1 & -1 & 1 & 46 \\
\hline 234 & 293 & 1 & -1 & -1 & 1 & -1 & 53.9 \\
\hline 235 & 13 & -1 & 0 & -1 & -1 & -1 & 36.8 \\
\hline 236 & 42 & 0 & -1 & -1 & 1 & 1 & 47.6 \\
\hline 237 & 135 & -1 & 1 & -1 & 0 & -1 & 36 \\
\hline
\end{tabular}


Appendix B.1 continued

\begin{tabular}{|c|c|c|c|c|c|c|c|}
\hline \multicolumn{8}{|c|}{$3^{3} 2^{2}$ Full factorial design for compressive strength } \\
\hline Sl.No & StdOrder & $\begin{array}{c}\text { Testing } \\
\text { days }\end{array}$ & $\begin{array}{c}\text { Concentration } \\
\text { of bacteria }\end{array}$ & $\begin{array}{c}\text { Calcium } \\
\text { lactate } \\
\text { concentration }\end{array}$ & $\begin{array}{c}\text { Types } \\
\text { of } \\
\text { bacteria }\end{array}$ & $\begin{array}{l}\text { Carrier } \\
\text { material }\end{array}$ & $\begin{array}{l}\text { Compressive } \\
\text { strength }\end{array}$ \\
\hline 238 & 110 & -1 & -1 & -1 & -1 & 1 & 35.8 \\
\hline 239 & 272 & 0 & 0 & 1 & -1 & 1 & 48 \\
\hline 240 & 127 & -1 & 0 & 1 & -1 & -1 & 35.8 \\
\hline 241 & 254 & 0 & -1 & -1 & -1 & 1 & 46.9 \\
\hline 242 & 89 & 1 & 0 & -1 & 1 & -1 & 58.1 \\
\hline 243 & 273 & 0 & 0 & 1 & 0 & -1 & 46.5 \\
\hline 244 & 104 & 1 & 1 & 1 & -1 & 1 & 55.3 \\
\hline 245 & 244 & -1 & 1 & -1 & 0 & 1 & 34.3 \\
\hline 246 & 228 & -1 & -1 & 1 & 1 & 1 & 36.7 \\
\hline 247 & 214 & 1 & 1 & 1 & 0 & 1 & 55 \\
\hline 248 & 315 & 1 & 1 & -1 & 0 & -1 & 53.8 \\
\hline 249 & 155 & 0 & -1 & 1 & 1 & -1 & 47.1 \\
\hline 250 & 32 & -1 & 1 & 1 & -1 & 1 & 36.9 \\
\hline 251 & 81 & 1 & -1 & 1 & 0 & -1 & 53.9 \\
\hline 252 & 276 & 0 & 0 & 1 & 1 & 1 & 50.3 \\
\hline 253 & 112 & -1 & -1 & -1 & 0 & 1 & 36.9 \\
\hline 254 & 86 & 1 & 0 & -1 & -1 & 1 & 57.8 \\
\hline 255 & 66 & 0 & 1 & -1 & 1 & 1 & 47.9 \\
\hline 256 & 147 & 0 & -1 & -1 & 0 & -1 & 46.5 \\
\hline 257 & 192 & 1 & -1 & 1 & 1 & 1 & 57.1 \\
\hline 258 & 318 & 1 & 1 & -1 & 1 & 1 & 57.8 \\
\hline 259 & 285 & 0 & 1 & 1 & 0 & -1 & 45.6 \\
\hline 260 & 304 & 1 & 0 & -1 & 0 & 1 & 58.6 \\
\hline 261 & 159 & 0 & 0 & -1 & 0 & -1 & 46.9 \\
\hline 262 & 52 & 0 & 0 & -1 & 0 & 1 & 48.2 \\
\hline 263 & 76 & 1 & -1 & -1 & 0 & 1 & 56.2 \\
\hline 264 & 131 & -1 & 0 & 1 & 1 & -1 & 39.2 \\
\hline 265 & 320 & 1 & 1 & 1 & -1 & 1 & 56 \\
\hline 266 & 31 & -1 & 1 & 1 & -1 & -1 & 35.2 \\
\hline 267 & 208 & 1 & 1 & -1 & 0 & 1 & 54.6 \\
\hline 268 & 162 & 0 & 0 & -1 & 1 & 1 & 48.7 \\
\hline 269 & 298 & 1 & -1 & 1 & 0 & 1 & 55.9 \\
\hline 270 & 54 & 0 & 0 & -1 & 1 & 1 & 49.1 \\
\hline 271 & 179 & 0 & 1 & 1 & 1 & -1 & 47.5 \\
\hline
\end{tabular}


Appendix B.1 continued

\begin{tabular}{|c|c|c|c|c|c|c|c|}
\hline \multicolumn{8}{|c|}{$3^{3} 2^{2}$ Full factorial design for compressive strength } \\
\hline Sl.No & StdOrder & $\begin{array}{c}\text { Testing } \\
\text { days }\end{array}$ & $\begin{array}{c}\text { Concentration } \\
\text { of bacteria }\end{array}$ & $\begin{array}{c}\text { Calcium } \\
\text { lactate } \\
\text { concentration }\end{array}$ & $\begin{array}{c}\text { Types } \\
\text { of } \\
\text { bacteria }\end{array}$ & $\begin{array}{c}\text { Carrier } \\
\text { material }\end{array}$ & $\begin{array}{l}\text { Compressive } \\
\text { strength }\end{array}$ \\
\hline 272 & 177 & 0 & 1 & 1 & 0 & -1 & 46.9 \\
\hline 273 & 223 & -1 & -1 & 1 & -1 & -1 & 35.8 \\
\hline 274 & 148 & 0 & -1 & -1 & 0 & 1 & 46.8 \\
\hline 275 & 23 & -1 & 0 & 1 & 1 & -1 & 38.9 \\
\hline 276 & 232 & -1 & 0 & -1 & 0 & 1 & 37.7 \\
\hline 277 & 306 & 1 & 0 & -1 & 1 & 1 & 59.8 \\
\hline 278 & 270 & 0 & 0 & -1 & 1 & 1 & 49.8 \\
\hline 279 & 322 & 1 & 1 & 1 & 0 & 1 & 56.6 \\
\hline 280 & 175 & 0 & 1 & 1 & -1 & -1 & 46.6 \\
\hline 281 & 290 & 1 & -1 & -1 & -1 & 1 & 56.1 \\
\hline 282 & 133 & -1 & 1 & -1 & -1 & -1 & 36.2 \\
\hline 283 & 313 & 1 & 1 & -1 & -1 & -1 & 52.2 \\
\hline 284 & 158 & 0 & 0 & -1 & -1 & 1 & 47.1 \\
\hline 285 & 72 & 0 & 1 & 1 & 1 & 1 & 49.7 \\
\hline 286 & 19 & -1 & 0 & 1 & -1 & -1 & 36.4 \\
\hline 287 & 47 & 0 & -1 & 1 & 1 & -1 & 46.7 \\
\hline 288 & 96 & 1 & 0 & 1 & 1 & 1 & 59.9 \\
\hline 289 & 101 & 1 & 1 & -1 & 1 & -1 & 54.3 \\
\hline 290 & 97 & 1 & 1 & -1 & -1 & -1 & 52.8 \\
\hline 291 & 91 & 1 & 0 & 1 & -1 & -1 & 58.1 \\
\hline 292 & 268 & 0 & 0 & -1 & 0 & 1 & 47.4 \\
\hline 293 & 57 & 0 & 0 & 1 & 0 & -1 & 47.1 \\
\hline 294 & 219 & -1 & -1 & -1 & 0 & -1 & 36.2 \\
\hline 295 & 206 & 1 & 1 & -1 & -1 & 1 & 54.9 \\
\hline 296 & 227 & -1 & -1 & 1 & 1 & -1 & 37.1 \\
\hline 297 & 269 & 0 & 0 & -1 & 1 & -1 & 49.1 \\
\hline 298 & 248 & -1 & 1 & 1 & -1 & 1 & 35.4 \\
\hline 299 & 242 & -1 & 1 & -1 & -1 & 1 & 35.9 \\
\hline 300 & 50 & 0 & 0 & -1 & -1 & 1 & 46.1 \\
\hline 301 & 181 & 1 & -1 & -1 & -1 & -1 & 52.3 \\
\hline 302 & 195 & 1 & 0 & -1 & 0 & -1 & 54.2 \\
\hline 303 & 198 & 1 & 0 & -1 & 1 & 1 & 58.7 \\
\hline 304 & 70 & 0 & 1 & 1 & 0 & 1 & 47.3 \\
\hline 305 & 109 & -1 & -1 & -1 & -1 & -1 & 35.3 \\
\hline
\end{tabular}


Appendix B.1 continued

\begin{tabular}{|c|c|c|c|c|c|c|c|}
\hline \multicolumn{2}{|c|}{$\mathbf{3}^{32}$ Full factorial design for compressive strength } \\
\hline Sl.No & StdOrder & $\begin{array}{c}\text { Testing } \\
\text { days }\end{array}$ & $\begin{array}{c}\text { Concentration } \\
\text { of bacteria }\end{array}$ & $\begin{array}{c}\text { Calcium } \\
\text { lactate } \\
\text { concentration }\end{array}$ & $\begin{array}{c}\text { Types } \\
\text { of } \\
\text { bacteria }\end{array}$ & $\begin{array}{c}\text { Carrier } \\
\text { material }\end{array}$ & $\begin{array}{c}\text { Compressive } \\
\text { strength }\end{array}$ \\
\hline 306 & 1 & -1 & -1 & -1 & -1 & -1 & 35.7 \\
\hline 307 & 80 & 1 & -1 & 1 & -1 & 1 & 55.9 \\
\hline 308 & 63 & 0 & 1 & -1 & 0 & -1 & 43.7 \\
\hline 309 & 183 & 1 & -1 & -1 & 0 & -1 & 52.6 \\
\hline 310 & 115 & -1 & -1 & 1 & -1 & -1 & 37.6 \\
\hline 311 & 167 & 0 & 0 & 1 & 1 & -1 & 50.5 \\
\hline 312 & 150 & 0 & -1 & -1 & 1 & 1 & 48.3 \\
\hline 313 & 311 & 1 & 0 & 1 & 1 & -1 & 56.8 \\
\hline 314 & 216 & 1 & 1 & 1 & 1 & 1 & 56.9 \\
\hline 315 & 146 & 0 & -1 & -1 & -1 & 1 & 46.1 \\
\hline 316 & 302 & 1 & 0 & -1 & -1 & 1 & 58.2 \\
\hline 317 & 299 & 1 & -1 & 1 & 1 & -1 & 54.9 \\
\hline 318 & 237 & -1 & 0 & 1 & 0 & -1 & 37.2 \\
\hline 319 & 252 & -1 & 1 & 1 & 1 & 1 & 36.7 \\
\hline 320 & 17 & -1 & 0 & -1 & 1 & -1 & 39.6 \\
\hline 321 & 224 & -1 & -1 & 1 & -1 & 1 & 35.4 \\
\hline 322 & 68 & 0 & 1 & 1 & -1 & 1 & 46.8 \\
\hline 323 & 75 & 1 & -1 & -1 & 0 & -1 & 54.2 \\
\hline 324 & 79 & 1 & -1 & 1 & -1 & -1 & 53.4 \\
\hline
\end{tabular}


Appendix B.2: $4^{2} 2^{1}$ Full factorial design matrix for the response RCPT

\begin{tabular}{|c|c|c|c|c|c|}
\hline \multicolumn{6}{|c|}{$4^{2} 2^{1}$ Full factorial design for RCPT } \\
\hline Sl.No & StdOrder & $\begin{array}{l}\text { Testing } \\
\text { age }\end{array}$ & $\begin{array}{l}\text { Types of } \\
\text { bacteria }\end{array}$ & $\begin{array}{l}\text { Types of } \\
\text { carrier }\end{array}$ & $\begin{array}{l}\text { RCPT } \\
\text { value }\end{array}$ \\
\hline 1 & 87 & 3 & 4 & 1 & 765 \\
\hline 2 & 4 & 1 & 2 & 2 & 1462 \\
\hline 3 & 94 & 4 & 3 & 2 & 759 \\
\hline 4 & 3 & 1 & 2 & 1 & 1499 \\
\hline 5 & 74 & 2 & 1 & 2 & 1320 \\
\hline 6 & 28 & 4 & 2 & 2 & 846 \\
\hline 7 & 47 & 2 & 4 & 1 & 917 \\
\hline 8 & 57 & 4 & 1 & 1 & 1327 \\
\hline 9 & 51 & 3 & 2 & 1 & 986 \\
\hline 10 & 56 & 3 & 4 & 2 & 839 \\
\hline 11 & 81 & 3 & 1 & 1 & 1343 \\
\hline 12 & 96 & 4 & 4 & 2 & 734 \\
\hline 13 & 82 & 3 & 1 & 2 & 1265 \\
\hline 14 & 27 & 4 & 2 & 1 & 895 \\
\hline 15 & 36 & 1 & 2 & 2 & 1449 \\
\hline 16 & 31 & 4 & 4 & 1 & 679 \\
\hline 17 & 15 & 2 & 4 & 1 & 972 \\
\hline 18 & 9 & 2 & 1 & 1 & 1409 \\
\hline 19 & 46 & 2 & 3 & 2 & 990 \\
\hline 20 & 16 & 2 & 4 & 2 & 989 \\
\hline 21 & 7 & 1 & 4 & 1 & 1472 \\
\hline 22 & 45 & 2 & 3 & 1 & 986 \\
\hline 23 & 64 & 4 & 4 & 2 & 645 \\
\hline 24 & 23 & 3 & 4 & 1 & 791 \\
\hline 25 & 68 & 1 & 2 & 2 & 1496 \\
\hline 26 & 80 & 2 & 4 & 2 & 901 \\
\hline 27 & 66 & 1 & 1 & 2 & 1569 \\
\hline 28 & 49 & 3 & 1 & 1 & 1315 \\
\hline 29 & 91 & 4 & 2 & 1 & 886 \\
\hline 30 & 61 & 4 & 3 & 1 & 742 \\
\hline 31 & 63 & 4 & 4 & 1 & 712 \\
\hline 32 & 48 & 2 & 4 & 2 & 888 \\
\hline 33 & 83 & 3 & 2 & 1 & 992 \\
\hline 34 & 41 & 2 & 1 & 1 & 1369 \\
\hline
\end{tabular}


Appendix B.2 continued

\begin{tabular}{|c|c|c|c|c|c|}
\hline \multicolumn{6}{|c|}{$4^{2} 2^{1}$ Full factorial design for RCPT } \\
\hline Sl.No & StdOrder & $\begin{array}{c}\text { Testing } \\
\text { age }\end{array}$ & $\begin{array}{l}\text { Types of } \\
\text { bacteria }\end{array}$ & $\begin{array}{l}\text { Types of } \\
\text { carrier }\end{array}$ & $\begin{array}{c}\text { RCPT } \\
\text { value }\end{array}$ \\
\hline 35 & 75 & 2 & 2 & 1 & 1101 \\
\hline 36 & 62 & 4 & 3 & 2 & 665 \\
\hline 37 & 73 & 2 & 1 & 1 & 1326 \\
\hline 38 & 32 & 4 & 4 & 2 & 601 \\
\hline 39 & 29 & 4 & 3 & 1 & 640 \\
\hline 40 & 85 & 3 & 3 & 1 & 844 \\
\hline 41 & 89 & 4 & 1 & 1 & 1305 \\
\hline 42 & 88 & 3 & 4 & 2 & 760 \\
\hline 43 & 5 & 1 & 3 & 1 & 1499 \\
\hline 44 & 70 & 1 & 3 & 2 & 1491 \\
\hline 45 & 17 & 3 & 1 & 1 & 1248 \\
\hline 46 & 92 & 4 & 2 & 2 & 842 \\
\hline 47 & 13 & 2 & 3 & 1 & 875 \\
\hline 48 & 79 & 2 & 4 & 1 & 901 \\
\hline 49 & 84 & 3 & 2 & 2 & 930 \\
\hline 50 & 37 & 1 & 3 & 1 & 1435 \\
\hline 51 & 35 & 1 & 2 & 1 & 1564 \\
\hline 52 & 76 & 2 & 2 & 2 & 1050 \\
\hline 53 & 33 & 1 & 1 & 1 & 1699 \\
\hline 54 & 24 & 3 & 4 & 2 & 744 \\
\hline 55 & 14 & 2 & 3 & 2 & 891 \\
\hline 56 & 22 & 3 & 3 & 2 & 870 \\
\hline 57 & 40 & 1 & 4 & 2 & 1501 \\
\hline 58 & 69 & 1 & 3 & 1 & 1539 \\
\hline 59 & 21 & 3 & 3 & 1 & 739 \\
\hline 60 & 55 & 3 & 4 & 1 & 748 \\
\hline 61 & 71 & 1 & 4 & 1 & 1542 \\
\hline 62 & 10 & 2 & 1 & 2 & 1379 \\
\hline 63 & 59 & 4 & 2 & 1 & 799 \\
\hline 64 & 19 & 3 & 2 & 1 & 878 \\
\hline 65 & 67 & 1 & 2 & 1 & 1446 \\
\hline 66 & 8 & 1 & 4 & 2 & 1401 \\
\hline 67 & 11 & 2 & 2 & 1 & 1102 \\
\hline
\end{tabular}


Appendix B.2 continued

\begin{tabular}{|c|c|c|c|c|c|}
\hline \multicolumn{6}{|c|}{$4^{2} 2^{1}$ Full factorial design for RCPT } \\
\hline Sl.No & StdOrder & $\begin{array}{c}\text { Testing } \\
\text { age }\end{array}$ & $\begin{array}{l}\text { Types of } \\
\text { bacteria }\end{array}$ & $\begin{array}{l}\text { Types of } \\
\text { carrier }\end{array}$ & $\begin{array}{c}\text { RCPT } \\
\text { value }\end{array}$ \\
\hline 68 & 1 & 1 & 1 & 1 & 1606 \\
\hline 69 & 93 & 4 & 3 & 1 & 781 \\
\hline 70 & 86 & 3 & 3 & 2 & 768 \\
\hline 71 & 60 & 4 & 2 & 2 & 877 \\
\hline 72 & 38 & 1 & 3 & 2 & 1439 \\
\hline 73 & 42 & 2 & 1 & 2 & 1324 \\
\hline 74 & 43 & 2 & 2 & 1 & 1010 \\
\hline 75 & 50 & 3 & 1 & 2 & 1330 \\
\hline 76 & 65 & 1 & 1 & 1 & 1501 \\
\hline 77 & 78 & 2 & 3 & 2 & 882 \\
\hline 78 & 30 & 4 & 3 & 2 & 670 \\
\hline 79 & 58 & 4 & 1 & 2 & 1235 \\
\hline 80 & 52 & 3 & 2 & 2 & 900 \\
\hline 81 & 44 & 2 & 2 & 2 & 1011 \\
\hline 82 & 54 & 3 & 3 & 2 & 765 \\
\hline 83 & 72 & 1 & 4 & 2 & 1439 \\
\hline 84 & 53 & 3 & 3 & 1 & 901 \\
\hline 85 & 77 & 2 & 3 & 1 & 1016 \\
\hline 86 & 39 & 1 & 4 & 1 & 1402 \\
\hline 87 & 25 & 4 & 1 & 1 & 1220 \\
\hline 88 & 12 & 2 & 2 & 2 & 1059 \\
\hline 89 & 2 & 1 & 1 & 2 & 1599 \\
\hline 90 & 95 & 4 & 4 & 1 & 664 \\
\hline 91 & 6 & 1 & 3 & 2 & 1423 \\
\hline 92 & 90 & 4 & 1 & 2 & 1300 \\
\hline 93 & 18 & 3 & 1 & 2 & 1251 \\
\hline 94 & 20 & 3 & 2 & 2 & 927 \\
\hline 95 & 34 & 1 & 1 & 2 & 1608 \\
\hline 96 & 26 & 4 & 1 & 2 & 1221 \\
\hline
\end{tabular}


Appendix B.3: $4^{2} 2^{1}$ Full factorial design matrix for the response primary sorptivity

\begin{tabular}{|c|c|c|c|c|c|}
\hline \multicolumn{6}{|c|}{$4^{2} 2^{1}$ Full factorial design for primary sorptivity } \\
\hline Sl.No & StdOrder & $\begin{array}{c}\text { Testing } \\
\text { age }\end{array}$ & $\begin{array}{l}\text { Types of } \\
\text { bacteria }\end{array}$ & $\begin{array}{l}\text { Types of } \\
\text { carrier }\end{array}$ & $\begin{array}{c}\text { Primary } \\
\text { sorptivity }\end{array}$ \\
\hline 1 & 75 & 3 & 1 & 2 & 0.0038100 \\
\hline 2 & 62 & 4 & 1 & 2 & 0.0035100 \\
\hline 3 & 73 & 1 & 2 & 1 & 0.0069500 \\
\hline 4 & 32 & 2 & 4 & 2 & 0.0020000 \\
\hline 5 & 29 & 4 & 3 & 2 & 0.0006200 \\
\hline 6 & 85 & 3 & 3 & 1 & 0.0010100 \\
\hline 7 & 89 & 1 & 4 & 1 & 0.0067900 \\
\hline 8 & 88 & 2 & 4 & 1 & 0.0021979 \\
\hline 9 & 5 & 2 & 3 & 2 & 0.0020183 \\
\hline 10 & 70 & 4 & 2 & 1 & 0.0008800 \\
\hline 11 & 17 & 1 & 3 & 2 & 0.0067700 \\
\hline 12 & 92 & 1 & 1 & 1 & 0.0070200 \\
\hline 13 & 13 & 2 & 2 & 1 & 0.0024349 \\
\hline 14 & 79 & 3 & 4 & 2 & 0.0009300 \\
\hline 15 & 84 & 4 & 3 & 1 & 0.0007000 \\
\hline 16 & 37 & 2 & 2 & 2 & 0.0022051 \\
\hline 17 & 35 & 3 & 4 & 1 & 0.0009800 \\
\hline 18 & 76 & 4 & 1 & 1 & 0.0035200 \\
\hline 19 & 33 & 3 & 3 & 2 & 0.0010100 \\
\hline 20 & 24 & 3 & 2 & 2 & 0.0011100 \\
\hline 21 & 14 & 1 & 3 & 1 & 0.0068900 \\
\hline 22 & 22 & 4 & 4 & 2 & 0.0005500 \\
\hline 23 & 40 & 4 & 2 & 2 & 0.0007200 \\
\hline 24 & 69 & 4 & 4 & 1 & 0.0006500 \\
\hline 25 & 21 & 1 & 1 & 2 & 0.0070300 \\
\hline 26 & 55 & 3 & 1 & 1 & 0.0038900 \\
\hline 27 & 71 & 1 & 4 & 2 & 0.0067200 \\
\hline 28 & 10 & 2 & 3 & 1 & 0.0020614 \\
\hline 29 & 59 & 2 & 1 & 2 & 0.0042091 \\
\hline 30 & 19 & 2 & 1 & 1 & 0.0042700 \\
\hline 31 & 67 & 3 & 2 & 1 & 0.0011500 \\
\hline 32 & 8 & 1 & 2 & 2 & 0.0066800 \\
\hline 33 & 11 & 3 & 1 & 2 & 0.0038100 \\
\hline 34 & 87 & 4 & 1 & 2 & 0.0035100 \\
\hline
\end{tabular}


Appendix B.3 continued

\begin{tabular}{|c|c|c|c|c|c|}
\hline \multicolumn{6}{|c|}{$4^{2} 2^{1}$ Full factorial design for primary sorptivity } \\
\hline Sl.No & StdOrder & $\begin{array}{l}\text { Testing } \\
\text { age }\end{array}$ & $\begin{array}{l}\text { Types of } \\
\text { bacteria }\end{array}$ & $\begin{array}{l}\text { Types of } \\
\text { carrier }\end{array}$ & $\begin{array}{c}\text { Primary } \\
\text { sorptivity }\end{array}$ \\
\hline 35 & 4 & 1 & 2 & 1 & 0.0069500 \\
\hline 36 & 94 & 2 & 4 & 2 & 0.0020000 \\
\hline 37 & 3 & 4 & 3 & 2 & 0.0006200 \\
\hline 38 & 74 & 3 & 3 & 1 & 0.0010100 \\
\hline 39 & 28 & 1 & 4 & 1 & 0.0067900 \\
\hline 40 & 47 & 2 & 4 & 1 & 0.0021979 \\
\hline 41 & 57 & 2 & 3 & 2 & 0.0020183 \\
\hline 42 & 51 & 4 & 2 & 1 & 0.0008800 \\
\hline 43 & 56 & 1 & 3 & 2 & 0.0067700 \\
\hline 44 & 81 & 1 & 1 & 1 & 0.0070200 \\
\hline 45 & 96 & 2 & 2 & 1 & 0.0024349 \\
\hline 46 & 82 & 3 & 4 & 2 & 0.0009300 \\
\hline 47 & 27 & 4 & 3 & 1 & 0.0007000 \\
\hline 48 & 36 & 2 & 2 & 2 & 0.0022051 \\
\hline 49 & 31 & 3 & 4 & 1 & 0.0009800 \\
\hline 50 & 15 & 4 & 1 & 1 & 0.0035200 \\
\hline 51 & 9 & 3 & 3 & 2 & 0.0010100 \\
\hline 52 & 46 & 3 & 2 & 2 & 0.0011100 \\
\hline 53 & 16 & 1 & 3 & 1 & 0.0068900 \\
\hline 54 & 7 & 4 & 4 & 2 & 0.0005500 \\
\hline 55 & 45 & 4 & 2 & 2 & 0.0007200 \\
\hline 56 & 64 & 4 & 4 & 1 & 0.0006500 \\
\hline 57 & 23 & 1 & 1 & 2 & 0.0070300 \\
\hline 58 & 68 & 3 & 1 & 1 & 0.0038900 \\
\hline 59 & 80 & 1 & 4 & 2 & 0.0067200 \\
\hline 60 & 66 & 2 & 3 & 1 & 0.0020614 \\
\hline 61 & 49 & 2 & 1 & 2 & 0.0042091 \\
\hline 62 & 91 & 2 & 1 & 1 & 0.0042700 \\
\hline 63 & 61 & 3 & 2 & 1 & 0.0011500 \\
\hline 64 & 63 & 1 & 2 & 2 & 0.0066800 \\
\hline 65 & 48 & 3 & 1 & 2 & 0.0038100 \\
\hline 66 & 83 & 4 & 1 & 2 & 0.0035100 \\
\hline 67 & 41 & 1 & 2 & 1 & 0.0069500 \\
\hline
\end{tabular}


Appendix B.3 continued

\begin{tabular}{|c|c|c|c|c|c|}
\hline \multicolumn{6}{|c|}{$4^{2} 2^{1}$ Full factorial design for primary sorptivity } \\
\hline Sl.No & StdOrder & $\begin{array}{c}\text { Testing } \\
\text { age }\end{array}$ & $\begin{array}{l}\text { Types of } \\
\text { bacteria }\end{array}$ & $\begin{array}{l}\text { Types of } \\
\text { carrier }\end{array}$ & $\begin{array}{l}\text { Primary } \\
\text { sorptivity }\end{array}$ \\
\hline 68 & 87 & 3 & 1 & 2 & 0.0038100 \\
\hline 69 & 4 & 4 & 1 & 2 & 0.0035100 \\
\hline 70 & 94 & 1 & 2 & 1 & 0.0069500 \\
\hline 71 & 65 & 2 & 4 & 2 & 0.0020000 \\
\hline 72 & 78 & 4 & 3 & 2 & 0.0006200 \\
\hline 73 & 30 & 3 & 3 & 1 & 0.0010100 \\
\hline 74 & 58 & 1 & 4 & 1 & 0.0067900 \\
\hline 75 & 52 & 2 & 4 & 1 & 0.0021979 \\
\hline 76 & 44 & 2 & 3 & 2 & 0.0020183 \\
\hline 77 & 54 & 4 & 2 & 1 & 0.0008800 \\
\hline 78 & 72 & 1 & 3 & 2 & 0.0067700 \\
\hline 79 & 53 & 1 & 1 & 1 & 0.0070200 \\
\hline 80 & 77 & 2 & 2 & 1 & 0.0024349 \\
\hline 81 & 39 & 3 & 4 & 2 & 0.0009300 \\
\hline 82 & 25 & 4 & 3 & 1 & 0.0007000 \\
\hline 83 & 12 & 2 & 2 & 2 & 0.0022051 \\
\hline 84 & 2 & 3 & 4 & 1 & 0.0009800 \\
\hline 85 & 95 & 4 & 1 & 1 & 0.0035200 \\
\hline 86 & 6 & 3 & 3 & 2 & 0.0010100 \\
\hline 87 & 90 & 3 & 2 & 2 & 0.0011100 \\
\hline 88 & 18 & 1 & 3 & 1 & 0.0068900 \\
\hline 89 & 20 & 4 & 4 & 2 & 0.0005500 \\
\hline 90 & 34 & 4 & 2 & 2 & 0.0007200 \\
\hline 91 & 26 & 4 & 4 & 1 & 0.0006500 \\
\hline 92 & 1 & 1 & 1 & 2 & 0.0070300 \\
\hline 93 & 93 & 3 & 1 & 1 & 0.0038900 \\
\hline 94 & 86 & 1 & 4 & 2 & 0.0067200 \\
\hline 95 & 60 & 2 & 3 & 1 & 0.0020614 \\
\hline 96 & 38 & 2 & 1 & 2 & 0.0042091 \\
\hline
\end{tabular}


Appendix B.4: $4^{2} 2^{1}$ Full factorial design matrix for the response UPV

\begin{tabular}{|c|c|c|c|c|c|}
\hline \multicolumn{6}{|c|}{$4^{2} 2^{1}$ Full factorial design for UPV } \\
\hline Sl.No & StdOrder & $\begin{array}{c}\text { Testing } \\
\text { age }\end{array}$ & $\begin{array}{l}\text { Types of } \\
\text { bacteria }\end{array}$ & $\begin{array}{l}\text { Types of } \\
\text { carrier }\end{array}$ & UPV \\
\hline 1 & 87 & 4 & 3 & 1 & 3923 \\
\hline 2 & 4 & 1 & 1 & 1 & 3548 \\
\hline 3 & 94 & 1 & 2 & 2 & 3644 \\
\hline 4 & 65 & 3 & 4 & 2 & 4127 \\
\hline 5 & 78 & 2 & 2 & 2 & 3894 \\
\hline 6 & 30 & 2 & 4 & 1 & 3997 \\
\hline 7 & 58 & 4 & 4 & 1 & 4086 \\
\hline 8 & 52 & 1 & 2 & 1 & 3628 \\
\hline 9 & 44 & 4 & 3 & 2 & 4006 \\
\hline 10 & 54 & 1 & 1 & 2 & 3628 \\
\hline 11 & 72 & 3 & 4 & 1 & 4063 \\
\hline 12 & 53 & 1 & 4 & 2 & 3732 \\
\hline 13 & 77 & 2 & 2 & 2 & 3894 \\
\hline 14 & 39 & 3 & 1 & 2 & 3710 \\
\hline 15 & 25 & 1 & 2 & 2 & 3644 \\
\hline 16 & 12 & 1 & 2 & 1 & 3628 \\
\hline 17 & 2 & 3 & 2 & 1 & 3912 \\
\hline 18 & 95 & 2 & 2 & 1 & 3873 \\
\hline 19 & 6 & 2 & 4 & 2 & 4058 \\
\hline 20 & 90 & 4 & 2 & 1 & 3919 \\
\hline 21 & 18 & 2 & 1 & 1 & 3612 \\
\hline 22 & 20 & 1 & 3 & 1 & 3544 \\
\hline 23 & 34 & 3 & 4 & 2 & 4127 \\
\hline 24 & 26 & 2 & 4 & 2 & 4058 \\
\hline 25 & 1 & 4 & 4 & 2 & 4152 \\
\hline 26 & 93 & 1 & 1 & 2 & 3628 \\
\hline 27 & 86 & 4 & 4 & 1 & 4086 \\
\hline 28 & 60 & 1 & 1 & 1 & 3548 \\
\hline 29 & 38 & 2 & 3 & 2 & 3934 \\
\hline 30 & 87 & 2 & 2 & 1 & 3873 \\
\hline 31 & 4 & 3 & 1 & 1 & 3618 \\
\hline 32 & 94 & 3 & 2 & 1 & 3912 \\
\hline 33 & 65 & 1 & 4 & 1 & 3685 \\
\hline 34 & 78 & 1 & 3 & 1 & 3544 \\
\hline
\end{tabular}


Appendix B.4 continued

\begin{tabular}{|c|c|c|c|c|c|}
\hline \multicolumn{6}{|c|}{$4^{2} 2^{1}$ Full factorial design for UPV } \\
\hline Sl.No & StdOrder & $\begin{array}{c}\text { Testing } \\
\text { age }\end{array}$ & $\begin{array}{l}\text { Types of } \\
\text { bacteria }\end{array}$ & $\begin{array}{l}\text { Types of } \\
\text { carrier }\end{array}$ & UPV \\
\hline 35 & 96 & 1 & 3 & 2 & 3634 \\
\hline 36 & 82 & 4 & 1 & 1 & 3623 \\
\hline 37 & 27 & 3 & 3 & 2 & 3986 \\
\hline 38 & 36 & 2 & 1 & 1 & 3612 \\
\hline 39 & 31 & 2 & 1 & 2 & 3699 \\
\hline 40 & 15 & 3 & 3 & 2 & 3986 \\
\hline 41 & 9 & 4 & 1 & 1 & 3623 \\
\hline 42 & 46 & 4 & 2 & 1 & 3919 \\
\hline 43 & 16 & 3 & 3 & 1 & 3901 \\
\hline 44 & 7 & 4 & 2 & 2 & 3934 \\
\hline 45 & 45 & 2 & 3 & 1 & 3833 \\
\hline 46 & 64 & 3 & 2 & 2 & 3928 \\
\hline 47 & 23 & 3 & 1 & 2 & 3710 \\
\hline 48 & 68 & 1 & 4 & 2 & 3732 \\
\hline 49 & 80 & 2 & 3 & 2 & 3934 \\
\hline 50 & 66 & 3 & 3 & 1 & 3901 \\
\hline 51 & 49 & 2 & 4 & 1 & 3997 \\
\hline 52 & 91 & 3 & 2 & 2 & 3928 \\
\hline 53 & 61 & 4 & 1 & 2 & 3712 \\
\hline 54 & 63 & 2 & 1 & 2 & 3699 \\
\hline 55 & 48 & 4 & 3 & 2 & 4006 \\
\hline 56 & 83 & 4 & 2 & 2 & 3934 \\
\hline 57 & 41 & 4 & 3 & 1 & 3923 \\
\hline 58 & 81 & 3 & 1 & 1 & 3618 \\
\hline 59 & 96 & 1 & 4 & 1 & 3685 \\
\hline 60 & 82 & 2 & 3 & 1 & 3833 \\
\hline 61 & 27 & 1 & 3 & 2 & 3634 \\
\hline 62 & 36 & 3 & 4 & 1 & 4063 \\
\hline 63 & 31 & 4 & 1 & 2 & 3712 \\
\hline 64 & 15 & 4 & 3 & 1 & 3923 \\
\hline 65 & 75 & 1 & 1 & 1 & 3548 \\
\hline 66 & 62 & 1 & 2 & 2 & 3644 \\
\hline 67 & 73 & 3 & 4 & 2 & 4127 \\
\hline
\end{tabular}


Appendix B.4 continued

\begin{tabular}{|c|c|c|c|c|c|}
\hline \multicolumn{7}{|c|}{$\mathbf{4}^{\mathbf{2}}{ }^{\mathbf{1}}$ Full factorial design for UPV } \\
\hline S1.No & StdOrder & $\begin{array}{c}\text { Testing } \\
\text { age }\end{array}$ & $\begin{array}{c}\text { Types of } \\
\text { bacteria }\end{array}$ & $\begin{array}{c}\text { Types of } \\
\text { carrier }\end{array}$ & UPV \\
\hline 68 & 32 & 2 & 2 & 2 & 3894 \\
\hline 69 & 29 & 2 & 4 & 1 & 3997 \\
\hline 70 & 85 & 4 & 4 & 1 & 4086 \\
\hline 71 & 89 & 1 & 2 & 1 & 3628 \\
\hline 72 & 88 & 4 & 3 & 2 & 4006 \\
\hline 73 & 5 & 1 & 1 & 2 & 3628 \\
\hline 74 & 70 & 3 & 4 & 1 & 4063 \\
\hline 75 & 17 & 1 & 4 & 2 & 3732 \\
\hline 76 & 92 & 2 & 2 & 2 & 3894 \\
\hline 77 & 13 & 3 & 1 & 2 & 3710 \\
\hline 78 & 79 & 1 & 2 & 2 & 3644 \\
\hline 79 & 84 & 1 & 2 & 1 & 3628 \\
\hline 80 & 37 & 3 & 2 & 1 & 3912 \\
\hline 81 & 35 & 2 & 2 & 1 & 3873 \\
\hline 82 & 76 & 2 & 4 & 2 & 4058 \\
\hline 83 & 33 & 4 & 2 & 1 & 3919 \\
\hline 84 & 24 & 2 & 1 & 1 & 3612 \\
\hline 85 & 14 & 1 & 3 & 1 & 3544 \\
\hline 86 & 22 & 3 & 4 & 2 & 4127 \\
\hline 87 & 40 & 2 & 4 & 2 & 4058 \\
\hline 88 & 69 & 4 & 4 & 2 & 4152 \\
\hline 89 & 21 & 1 & 1 & 2 & 3628 \\
\hline 90 & 55 & 4 & 4 & 1 & 4086 \\
\hline 91 & 71 & 1 & 1 & 1 & 3548 \\
\hline 92 & 10 & 2 & 3 & 2 & 3934 \\
\hline 93 & 59 & 2 & 2 & 1 & 3873 \\
\hline 94 & 19 & 3 & 1 & 1 & 3618 \\
\hline 95 & 67 & 3 & 2 & 1 & 3912 \\
\hline
\end{tabular}




\section{REFERENCES}

AASHTO T277, (1993), "Electrical Indication of Concrete's Ability to Resist Chloride", American Association of State Highway and Transport Officials.

Achal, V., Mukherjee, A., Basu, P.C. and Reddy, M.S., (2009), "Lactose mother liquor as an alternative nutrient source for microbial concrete production by Sporosarcina pasteurii. Journal of Industrial Microbiology Biotechnology, Vol. 36, No. 3, pp. 433-438.

Achal, V., Mukherjee, A., Reddy, M.S., (2010a), "Biocalcification by Sporosarcina pasteurii using corn steep liquor as nutrient source", Industrial Biotechnology, Vol. 6, No. 3, pp. 170174.

Achal, V., (2010b), "Microbial remediation of defects in building materials and structures", $\mathrm{PhD}$ thesis, Thapar University, Patiala, India, pp. 1-263.

Achal, V., Mukherjee, A. and Reddy, M.S., (2011a), "Microbial concrete: A way to enhance the durability of concrete buildings", Journal of Materials in Civil engineering, Vol. 23, No.6, pp. 730-734.

Achal, V., Mukherjee, A., and Reddy, M.S., (2011b), "Effect of calcifying bacteria on permeation properties of concrete structures", Journal of Industrial Microbiology Biotechnology, Vol. 38, No. 9, pp. 1229-1234.

Ahmad, S., and Alghamdi, (2014), "A statistical approach optimizing concrete mix design", The Scientific World Journal, Vol. 14, No. 10, pp. 1-7

Arabi, N.S., Mustapha, K.N.B., Al Mattarneh, H., and Al kadi, Q.N.S., (2009), "Statistical models for hardened properties of self-compacting concrete", American Journal of Engineering and Applied Sciences, Vol.2, No. 4, pp.764-770.

ASTM C39, (2001), "Standard test method for compressive strength of cylindrical concrete specimens", American Society for Testing and Materials.

ASTM C87, (2002), "Standard test method for flexural strength of concrete (using simple beam with third-point loading)", American Society for Testing and Materials. 
ASTM C117, (2004), "Standard test method for materials finer than 75- $\mu \mathrm{m}$ (No.200) sieve in mineral aggregates by washing, American Society for Testing and Materials.

ASTM C136, (2005), "Standard test method for sieve analysis of fine and coarse aggregates", American Society for Testing and Materials.

ASTM C496, (2001), "Standard test method for splitting tensile strength of cylindrical concrete specimens", American Society for Testing and Materials.

ASTM C618, (2012), "Standard specifications for coal fly ash and raw or calcined natural pozzolan for use in concrete", American Society for Testing and Materials.

ASTM C642, (1997), "Standard test method for density, water absorption and voids in hardened concrete", American Society for Testing and Materials.

ASTM C1202, (1997), "Standard test method for electrical indication of concrete's ability to resist chloride ion penetration", American Society for Testing and Materials.

ASTM C1585, (2004), "Standard Test Method for Measurement of Rate of Absorption of Water by Hydraulic Cement Concretes", American Society for Testing and Materials.

Bang, S.S., Galinat, J.K., and Ramakrishnan, V., (2001), "Calcite precipitation induced by polyurethane immobilised Sporosarcina pasteurii”, Enzyme and Microbial Technology, Vol.28, No. 4, pp. 404-409.

Bachmeier, K.L., Williams, A.E., Warmington, J.R., and Bang, S.S., (2002), "Urease activity in microbiologically-induced calcite precipitation", Journal of Biotechnology, Vol.93, No. 2, pp. 171-181.

Castanier S., Le Métayer-Levrel, G., and Perthuisot, J.P., (1999), "Carbonates precipitation and limestone genesis-the microbiologist point of view", Sedimentary Geology, Vol. 126, No. 1, pp. 9-23.

Cihan, M.T., Guner, A., and Yuzer, N., (2013), "Response surfaces for compressive strength of concrete", Construction and Building Materials, Vol.40, No. 3, pp.763-774. 
Claudia, S., Ricardo, S., Loreto, M.V., and Mauricio, L., (2014), “Quantification of chemical and biological calcium carbonate precipitation: Performance of self-healing in reinforced mortar containing chemical admixtures", Cement and Concrete Composites, Vol. 50, No. 7 , pp. 10-15.

De Muynck, W., Cox, K., Belie, N.D., and Verstraete, W., (2008a), "Bacterial carbonate precipitation as an alternative surface treatment for concrete", Construction Building Materials, Vol. 22, No. 5, pp. 875-885.

De Muynck, W., Debrouwer, D., De Belie, N., and Verstraete, W., (2008b), "Bacterial carbonate precipitation improves the durability of cementitious materials", Cement and Concrete Research, Vol. 38, No. 7, pp. 1005-1014.

De Muynck W., De Belie N., and Verstraete W., (2010), "Microbial carbonate precipitation in construction materials: A review", Ecological Engineering, Vol. 36, No. 2, pp. 118-136.

Edvardsen, C., (1999), "Water permeability and autogenous healing of cracks in concrete", ACI Materials Journal, Vol. 96, No. 7, pp.448-454.

Emmons, P.H. and Sordyl, D.J., (2006), "The state of the concrete repair industry, and a vision for its future," Concrete Repair Bulletin, pp. 7-14.

Ersan, Y.C., Wang, J.Y., Boon, N., and De Belie, N., (2014), "Ureolysis and denitrification based microbial strategies for self-healing concrete", Concrete Solutions - Grantham et al. (Eds), Taylor \& Francis Group, London, pp. 59-64.

Farmani, F., Bonakdarpour, B. and Ramezanianpour, A., (2014), "pH reduction through amendment of cement mortar with silica fume enhances its biological treatment using bacterial carbonate precipitation", Materials and Structures, Vol. 10, No. 10, pp. 3205-3215.

Figurovskaya, V.N., Ivanov, V.M., Barbalat Yu, A., and Ershova, N.I., (2005), "Chromaticity characteristics of $\mathrm{NH} 2 \mathrm{Hg} 2 \mathrm{I} 3$ and I2: molecular Iodine as a test form alternative to Nessler's reagent", Journal of Analytical Chemistry, vol. 60, No. 7, pp. 707710. 
Freyermuth, C.L., (2001), "Life-cycle cost analysis for large segmental bridges," Concrete International, Vol. 23, No. 2, pp. 89-95.

Ghosh, P., Mandal, S., Chattopadhyay, B.D., and Pal, S., (2005), "Use of microorganism to improve the strength of cement mortar", Cement and Concrete Research, Vol. 35, No. 10, pp.1980-1983.

Guadalupe Sierra-Beltran, M., Jonkers, H.M., and Schlangen, E., (2014), “Characterization of sustainable bio-based mortar for concrete repair", Construction and Building Materials, Vol. 67, No. 9, pp. 344-352.

Hammes, F. and Verstraete, W., (2002), "Key roles of $\mathrm{pH}$ and calcium metabolism in microbial carbonate precipitation", Reviews in Environmental Science and Biotechnology, Vol. 1, No. 1, pp. 3-7.

Hossain, K.M.A., (2004), "Properties of volcanic pumice based cement and lightweight concrete", Cement and Concrete Research, Vol. 34, No. 2, pp. 283-291.

Hua Xia., (2010), "Self-healing of Engineered Cementitious Composites (ECC) in Concrete Repair System”, MASc thesis, Delft University of Technology, Netherlands, pp. 1-56.

ITT Total Quality Management Group, (1992), “Taguchi Methods”, Vol. 2, pp. 3-97.

Jagadeesha, Kumar, B.G., Prabhakara, R., and Pushpa, H., (2013), "Bio mineralisation of calcium carbonate by different bacterial strains and their application in concrete crack remediation", International Journal of Advances in Engineering and Technology, Vol. 6, No.1, pp. 201-213.

Jonkers, H.M., (2007), “Self-healing concrete: a biological approach, Self-healing Materials: An Alternative Approach to 20 Centuries of Materials Science”, Springer, pp. 195-204.

Jonkers, H.M., and Schlangen, E., (2008), "Development of a bacteria-based self-healing concrete", Tailor Made Concrete Structures- New Solution for Society, pp. 425-30.

Jonkers, H.M. and Schlangen, E., (2009), "A two component bacteria based self-healing concrete", Concrete Repair, Rehabilitation and Retrofitting II, No. 3, pp. 215-220. 
Jonkers, H.M., Thijssen, A., Muyzer, G., Copuroglu, O., and Schlangen, E., (2010), "Application of bacteria as self-healing agent for the development of sustainable concrete", Ecological engineering, Vol. 36, No. 2, pp. 230-235.

Kamada, T., and Li, V, C., (2000), "The effects of surface preparation on the fracture behavior of ECC/concrete repair system", Cement and Concrete Composites, Vol. 22, No. 6, pp. 422-431.

Kim, H.K., Park, S.J., Han, J.I., and Lee, H.K., (2013), "Microbially mediated calcium carbonate precipitation on normal and lightweight concrete", Construction Building Materials, Vol. 38, No. 1, pp. 1073-82.

Kitis, M., Kaplan, S.S., Karakaya, E., Yigit, N.O., and Civelekoglu, G., (2007), “Adsorption of natural organic matter from waters by iron coated pumice”, Chemosphere, Vol. 66, No.1, pp. 130-138.

Koch, G., Brongers, M., Thompson, N., Virmani, Y., and Payer, J., (2002), “Corrosion cost and preventive strategies in the United States," Technical report, Turner-Fairbank Highway Research Center.

Le Metayer, L., Castanier, C.G., Orial, G., Loubiere, J.F and Perthuisot, J.P., (1999), "Applications of bacterial carbonatogenesis to the protection and regeneration of limestones in buildings and historic patrimony", Sediment Geology, Vol. 126, No 1-4, pp. 25-34.

Li, V.C., (1998), "Engineered cementitious composites - tailored composites through micromechanical modeling, in fiber reinforced concrete: present and the future", Canadian Society for Civil Engineering, Montreal, pp. 64-97.

Li, V.C., and Yang, E.H., (2007), "Self-healing materials: an alternative approach to 20 centuries of material science, In: Zwaag S van der, editor, Springer, pp. 161-93.

Ma, Hui., Qian, S., and Zhang, Z., (2014), "Effect of self-healing on water permeability and mechanical property of Medium-Early-Strength Engineered Cementitious Composites", Construction and Building Materials, Vol. 68, No. 10, pp. 92-101. 
Lofty, A., Hossain, K.M.A., Lachemi, M., (2015), "Light eight self-consolidating concrete with expanded shale aggregates: modelling and optimization", International Journal of concrete Structures and Materials, Vol. 9, No. 2, pp. 185-206.

Mendenhall W., Sincich T., (1996), "A Second Course in Statistics: Regression Analysis", Prentice Hall Inc., pp.96-435.

Montgomery D.C., (1997), "Design and Analysis of Experiments", John Wiley \& Sons Inc., Fourth Edition, pp.2-234.

Nabil, B., Aissa, A., and Aguida, B.I., (2011), "Use of a new approach (Design of experiments method) to study different procedures to avoid plastic shrinkage cracking of concrete in hot climates", Journal of Advanced concrete technology, Vol.9, No.2, pp. 149157.

Navneet, C., Rafat, S., and Anita, R., (2012), "Influence of bacteria on the compressive strength, water absorption and rapid chloride permeability of fly ash concrete", Construction and Building Materials, Vol. 28, No. 1, pp. 351-356.

Navneet C., and Rafat S., (2013), "Permeation properties of concrete made with fly ash and silica fume: Influence of ureolytic bacteria", Construction and Building Materials, Vol. 49, No. 12, pp. 161-174.

Nemati, M., Greene, E.A. and Voordouw, G., (2005), "Permeability profile modification using bacterially formed calcium carbonate: comparison with enzymic option", Process Biochemistry, Vol. 40, No. 2, pp. 925-33.

Neville A.M., (2002), “Autogenous healing - A concrete miracle?” Concrete International, Vol. 24, No.11, pp. 76-82.

Palin, D., Wiktor, V., and Jonkers, H.M., (2014), “Towards cost efficient bacteria based selfhealing marine concrete", Concrete Solutions - Grantham et al. (Eds), Taylor \& Francis Group, London, pp. 105-108. 
Patel, R., Hossain, K.M.A., Shehata, M., Bouzoubaa, N., and Lachemi, M., (2004), "Development of Statistical Models for Mixture Design of High-Volume Fly Ash Self-Consolidating Concrete”, ACI Materials Journal, Vol. 101, No. 4, pp. 294-302.

Rafat, S., and Navneet Kaur C., (2011), "Effect of ureolytic bacteria on concrete properties", Construction and Building Materials, Vol. 25, No. 10, pp. 3791-3801.

Raijiwala D.B., (2008), "Bacterial Concrete: A Self-Healing Concrete" The ICFAI University Journal of Structural Engineering, No. 2, pp. 56-63.

Ramachandran, S.K., Ramakrishnan, V. and Bang, S.S., (2001), "Remediation of concrete using microorganisms”, ACI Materials Journal, Vol. 98, No. 1, pp. 3-9.

Ramakrishnan, V., Bang, S.S., Deo, K.S., (1998), "A novel technique for repairing cracks in high performance concrete using bacteria", In: Proc international conference on high performance high strength concrete, Perth, Australia, pp. 597-618.

Rodriguez-Navarro, C., Rodriguez-Gallego, M., Chekroun, K.B. and Gonzalez-Munoz, M.T., (2003), "Conservation of ornamental stone by Myxococcus xanthus-induced carbonate biomineralization”, Applied Environmental Microbiology, Vol. 69, No. 4, pp. 2182-2193.

Sahmaran, M., Keskin, S.B., Ozerkan, G., and Yaman, I.O., (2008), "Self-healing of mechanically-loaded self-consolidating concretes with high volumes of fly ash", Cement and Concrete Composites, Vol. 30, No. 10, pp. 872-879.

Sahmaran, M., and Li, V.C., (2009), "Durability properties of micro-cracked ECC containing high volumes fly ash", Cement and Concrete Research, Vol. 39, N0.11, pp. 10331043

Sahmaran, M., Lachemi, M., Hossain, K.M.A., Ranade, R., and Li, V.C., (2009), "Influence of aggregate type on ductility and mechanical properties of engineered cementitious composites", ACI Materials Journal, Vol. 106, No. 3, pp. 1-9. 
Sarmast, M., Farpoorcal, M.H., Sarcheshmehpoor, M., and Eghbal, M.K., (2014), "Micromorphological and biocalcification effects of sporosarcina pasteurii and Sporosarcina ureae in sandy soil columns”, Journal of Agricultural Science and Technology, No. 14, pp. 681-693.

Sierra-Beltran, M.G., Jonkers, H.M., and Schlangen, E., (2014), "Charecterization of sustainable bio-based mortar for concrete repair", Construction and Building Materials, Vol. 67, No. 9, pp. 344-352.

Srinivasa Reddy, V., Jyothi Kumar, K.S., Seshagiri Rao, M.V., and Sasikala., (2011), "Strength enhancement of cement mortar using microorganisms - an experimental study", International Journal of Earth Sciences and Engineering, Vol. 4, No. 6, pp. 933-936.

Srinivasa Reddy, V., Jyothi Kumar, K.S., Seshagiri Rao, M.V., and Sasikala., (2013), Characteristics studies on permeability of self-healing built-in bacteria concrete" International Journal of Recent Technology and Engineering, Vol. 6, No.1, pp. 119-125.

Srinivasan, C.B., Narasimhan, N.L., and Ilango, S.V., (2003), "Development of rapid-set high-strength cement using statistical experimental design”, Cement and Concrete Research, Vol.23, No.11, pp.1-6.

Stocks-Fischer S., Galinat J.K., and Bang S.S., (1999), "Microbiological precipitation of $\mathrm{CaCO}_{3}$ ", Soil Biology and Biochemistry, Vol. 31, No. 11, pp. 1563-1571.

Sunil Pratap Reddy, S., Seshagiri Rao, M.V., Aparna, P., and Sasikala, (2010), "Characteristics performance of ordinary grade bacterial (Bacillus subtilis) concrete", International Journal of Earth Sciences and Engineering, Vol. 3, No. 1, pp. 116-124.

van der Zwaag, S., van Dijk, N.H., Jonkers, H.M., Mookhoek, S.D., and Sloof, W.G., (2009), Self-healing behaviour in man-made engineering materials: bioinspired but taking into account their intrinsic character", Philosophical Transactions A: Mathematical Physical and Engineering Science, Vol. 367, No. 3, pp. 1689-704. 
Van Tittelboom, K., De Belie, N., De Muynck, W., and Verstraete, W., (2010), "Use of bacteria to repair cracks in concrete", Cement Concrete Research, Vol.40, No. 1, pp.157166.

Vandamme, E. J., De Baets, S., Vanbaelen, A., Joris, K., De Wulf, P., (1998), "Improved production of bacterial cellulose and its application potential", Polymer Degradation and Stability, Vol. 59, No. 1, pp. 93-99.

Wang, J.Y., De Belie, N., and Verstraete, W., (2012a), "Diatomaceous earth as a protective vehicle for bacteria applied for self-healing concrete”, Journal of Industrial Microbiology and Biotechnology, Vol. 39, No. 4, pp. 567-577.

Wang, J.Y., Van Tittelboom, K., De Belie N, and Verstratete, W., (2012b), "Use of silica gel or polyurethane immobilized bacteria for self-healing concrete”, Construction Building Materials, Vol.26, No 1, pp. 532-40.

Wang, J.Y., Jan Dewanckele, J., Cnudde, V., Sandra Van Vlierberghe, S.V., Verstraete, W., and De Belie, N., (2014a), "X-ray computed tomography proof of bacterial-based selfhealing in concrete", Cement and Concrete Composites, Vol. 53, No. 10, pp. 289-304.

Wang, J.Y., Snoeck, D., Vlierberghe, S.V., Verstraete, W., and De Belie, N., (2014b), "Application of hydrogel encapsulated carbonate precipitating bacteria for approaching a realistic self-healing in concrete", Construction and Building Materials, Vol. 68, pp. 110119.

Whiffin, V.S., (2004), "Microbial $\mathrm{CaCO}_{3}$ Precipitation for the production of bio cement," PhD thesis, Murdoch University, Western Australia, pp. 1-154.

Wiktor, V., and Jonkers, H.M., (2011), "Quantification of crack healing in novel bacteriabased self-healing concrete", Cement Concrete Composite, Vol. 33, No. 7, pp. 763-770

$\mathrm{Xu}$, Jing., and Yao, Wu., (2014), "Multiscale mechanical quantification of self-healing concrete incorporating non-ureolytic bacteria-based healing agent", Cement and Concrete Research, Vol. 64, No. 10, pp. 1-10. 
$\mathrm{Xu}$, Jing., Yao, Wu., and Jiang, Zhengwu., (2014), "Non-Ureolytic Bacterial Carbonate Precipitation as a Surface Treatment Strategy on Cementitious Materials", Journal of Materials in Civil Engineering, Vol. 26, No. 5, pp. 983-991.

Ying, Yang., Lepech, M.D., and Li, V.C., (2005) "Self-healing of engineered cementitious composites under cyclic wetting and drying", Proc. International workshop on Durability of reinforced concrete under combined mechanical and climatic loads, Qingdao, China, pp. 231-242.

Zemskov, S.V., Jonkers, H.M., and Vermolen, F.J., (2011), "Two analytical models for the probability characteristics of a crack hitting encapsulated particles: application to selfhealing materials", Computational Materials Science, Vol. 50, No. 12, pp. 3323-3333.

Zemskov, S.V., Jonkers, H.M., and Vermolen, F.J., (2012), “A mathematical model for bacterial self-healing of cracks in concrete", Journal of Intelligent Material Systems and Structures, Vol. 25, No. 1, pp. 4-12.

Zhong, W., and Yao, W., (2003), "Influence of damage degree on self-healing of concrete", Construction Building Materials, Vol. 22, No. 2, pp.1137-42.

Zhou, J., (2011), "Performance of engineered cementitious composites for concrete repairs", $\mathrm{PhD}$ thesis, Delft University of Technology, Netherlands. 